UNIVERSIDAD DE BURGOS

FACULTAD DE CIENCIAS

Departamento de Química

Área de Química Orgánica

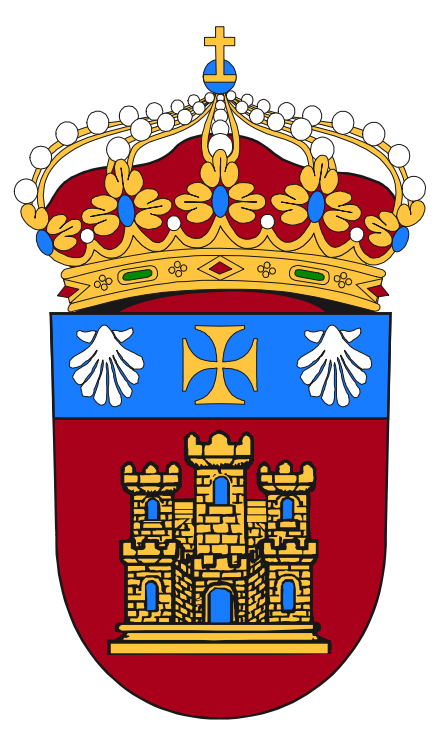

\title{
Fluorescent probes and nanostructured materials for the detection of environmental toxins and catalysts development
}

A thesis submitted for the degree of Doctor of Philosophy

\author{
by \\ José García Calvo
}

Burgos, 2018 



\section{UNIVERSIDAD DE BURGOS}

FACULTAD DE CIENCIAS - DEPARTAMENTO DE QUÍMICA

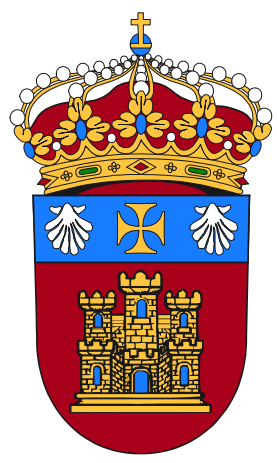

Dr. TOMÁS TORROBA PÉREZ, Professor of Organic Chemistry at the Organic Chemistry Department of the University of Burgos,

HEREBY CERTIFIES:

That the research work included in the dissertation: "Fluorescent probes and nanostructured materials for the detection of environmental toxins and catalysts development" performed by Mr. José García Calvo, Bsc. (Hons) Msc., in order to apply for the degree of Doctor of Philosophy $(\mathrm{PhD})$ in Chemistry by the University of Burgos, constitutes an original the research contained in the $\mathrm{PhD}$ Thesis has been developed under my supervision and I give my approval for its submission to be defended as a $\mathrm{PhD}$ Thesis.

Burgos, $15^{\text {th }}$ June 2018

SGD: Tomás Torroba Pérez

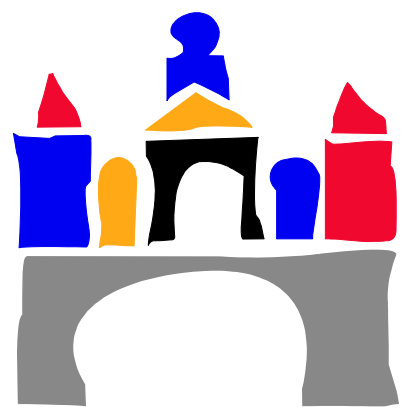





\section{Agradecimientos}

Han sido muchos años desde que comencé el doctorado y mucho trabajo el que he realizado en este tiempo hasta completar la tesis. Por todo ello, a continuación, quiero agradecer a todas las personas que han ayudado o participado en el desarrollo de la misma. Comenzando con mi director de tesis, Tomás Torroba, siempre disponible para discutir cualquier duda y cuya dirección, ayuda y paciencia me han permitido llegar hasta aquí.

Para mi es muy importante agradecer también a todos los miembros y exmiembros del grupo con los que he trabajado directamente; a Patricia Calvo, con la cual compartí muchos años de tesis y cuya ayuda ha sido fundamental; a Borja Díaz de Greñu. por enseñarme a trabajar en el laboratorio cuando estaba empezando y a Alberto Díez por su ayuda en los análisis de masas y muchas otras dudas en los últimos años. Por supuesto, también quiero expresar mi agradecimiento a los muchos otros compañeros que he tenido durante estos años, a Daisy C. Velásquez. con la que he compartido mucho tiempo como doctorandos; a los estudiantes que realizaron sus trabajos de Fin de grado y/o Máster en el grupo, Clara Antón, Marcos Ibáñez, Virginia Renuncio, Cristina Viyuela y Andrea Revilla; así como a los técnicos, cuya ayuda es inestimable, Sergio Domingo, Nerea Jalón, Miryam Asensio y los últimos en unirse Juan Ingelmo e Irene Abajo.

También al resto de compañeros que me han ayudado y/o con los que he colaborado y trabajado en el laboratorio en distintas partes de la tesis, Pablo Peña, Saúl Vallejos, Andrea Sancho o Natalia Busto entre otros muchos. Especialmente, también quiero mencionar y agradecer a James D. Wilton-Ely y los miembros de su grupo que me permitieron realizar la estancia en el Imperial College, que siempre se mostraron amables y con ganas de ayudar.

Por último y más importante, todo esto no sería posible sin mi familia, mis padres, mi hermana y, sobre todo, mi hermano Víctor García, que trabajó en laboratorio conmigo y me ha ayudado siempre que lo necesito; y a mi pareja, Esther López, sin la cual no hubiera sido posible esta tesis, no sólo por leérsela y corregirla, sino por su apoyo diario, su ayuda y ánimo.

Gracias a todos. 



\section{ABBREVIATIONS}

\section{SOLVENTS:}

\section{Ac: Acetone}

1,2-DCE: 1,2-Dichloroethane.

$\mathrm{CH}$ : Cyclohexane.

DCM: Dichloromethane.

DMF: N,N-Dimethylformamide.

DME: Dimethoxyethane.

DMSO: Dimethyl sulfoxide.

EtOAc: Ethyl acetate.

EtOH: Ethanol.

$\mathrm{Et}_{2} \mathrm{O}$ : Diethylether.

Hex: Hexane.

MCH: Methylcyclohexane.

$\mathrm{MeCN}$ : Acetonitrile.

MeOD: Deuterated methanol.

MeOH: Methanol.

NMP: N-Methylpyrrolidone.

$\mathrm{PhCl}$ : Chlorobenzene.

THF: Tetrahydrofuran.

Tol: Toluene.

\section{REAGENTS:}

DABCO: 1,4-Diazabicyclo[2.2.2] octane.

DADP: Diacetone diperoxyde.

DCTB: Trans-2-[3-(4-tert-Butylphenyl)-2-methyl-2-propenylidene]malononitrile.

DIPEA: N,N-Diisopropylethylamine.

DIT: Dithranol.

DMAP: 4-Dimethylaminopyridine.

DMPA: 2,2-Dimethoxy-2-phenylacetophenone.

EDCI: N-(3-Dimethylaminopropyl)-N'-ethylcarbodiimide.

$\mathrm{Et}_{3} \mathrm{~N}$ : Triethylamine.

HEPES: 2-[4-(2-Hydroxyethyl)piperazin-1-yl]ethanesulfonic acid.

HOBt: Hydroxybenzotriazole.

HMTD: Hexamethylene triperoxidediamine.

NBS: N-Bromosuccinimide.

MCPBA: 3-Chloroperoxybenzoic acid.

NIS: N-Iodosuccinimide.

Oxone: Potassium peroxymonosulfate.

PBS: Phosphate buffer solution.

PDA: Perylenedianhydride.

PDI: Perylenediimide.

PMI: Perylenemonoimide.

pTsOH: p-Toluenesulfonic acid.

PyBOP: Benzotriazol-1-yl-oxytripyrrolidinophosphonium hexafluorophosphate. 
TATP: Triacetone triperoxide.

TAMRA: Tetramethylrhodamine.

TBTA: Tris[(1-benzyl-1H-1,2,3-triazol-4-yl)methyl]amine.

TFA: Trifluoroacetic acid.

\section{TECHNIQUES.}

${ }^{1}$ H NMR: Proton Nuclear Magnetic Resonance.

${ }^{13} \mathrm{C}$ NMR: Carbon Nuclear Magnetic Resonance.

${ }^{19}$ F NMR: Fluorine Nuclear Magnetic Resonance

AFM: Atomic Force Microscopy.

EDX: Energy Dispersive X-Ray spectroscopy.

ESI: Electrospray Ionization.

ETAAS: Electrothermal Atomic Absorption Spectrometry.

FAAS: Fame atomic absorption spectrometry.

FT-IR: Fourier-Transform Infrared Spectroscopy.

HRMS: High Resolution Mass Spectrometry.

MALDI: Matrix-Assisted Laser Desorption/Ionization.

TOF: Time-of-Flight. $\quad$ LSIMS: Liquid Secondary Ion Mass Spectrometry.

HMBC: Heteronuclear Multiple Bond Correlation.

HMQC: Heteronuclear Multiple-Quantum Correlation.

ICP-MS: Inductively Coupled Plasma Mass Spectrometry.

ICP-OES: Inductively Coupled Plasma Optical Emission Spectrometry.

HPLC: High Performance Liquid Chromatography.

COSY: Correlation Spectroscopy. (NMR analysis)

NOESY: Nuclear Overhauser Spectroscopy. (NMR analysis)

DEPT: Distortionless Enhancement by Polarization Transfer. $\left({ }^{13} \mathrm{C}-\mathrm{NMR}\right.$ analysis $)$

TEM Transmission Electron Microscopy.

TGA: Thermogravimetric Analysis.

UPLC: Ultra Performance Liquid Chromatography.

TLC: Thin Layer Chromathography.

XPS: X-Ray Photoelectron Microscopy.

\section{OTHER ABBREVIATIONS:}

CORM: CO Releasing Molecules.

$\mathrm{C}_{\mathbf{q}}$ : Quaternary Carbon.

DFT: Density Functional Theory.

EDG: Electron Donating Group.

EWG: Electron Withdrawing Group.

FBS: Fetal Bovine Serum (for cell culture).

FRET: Förster Resonance Energy Transfer.

NPs: Nanoparticles.

PET: Photoelectronic Transference.

PCT: Photoinduced Charge Transfer.

Ps: Particles.

$\tau=$ FLD: Fluorescence Lifetime Decay.

$\Phi_{\mathrm{F}}=$ FQY: Fluorescence Quantum Yield. 


\section{INDEX}

\section{INTRODUCTION. CONTEXT AND OBJECTIVES OF THE THESIS ..............................................................1-6}

\section{CHAPTER 0.}

1. SENSORS, DEFINITION AND TYPES

1.1. Types of chemical sensors

1.2. Sensors, characteristics and advantages. The role of Absorbance + Photoluminescence sensors.

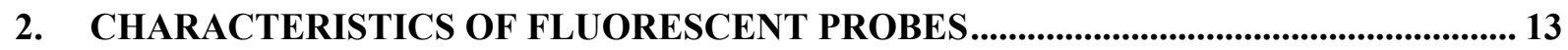

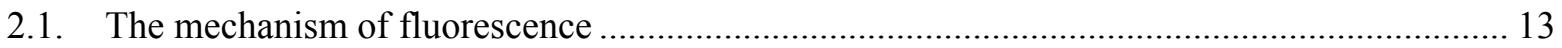

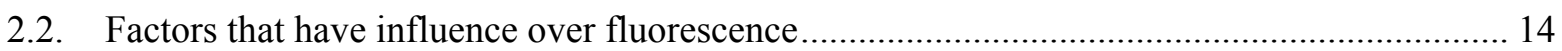

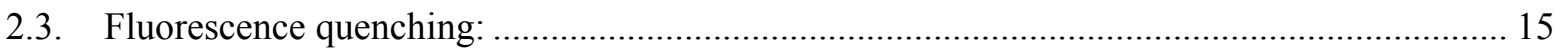

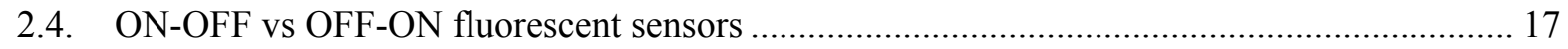

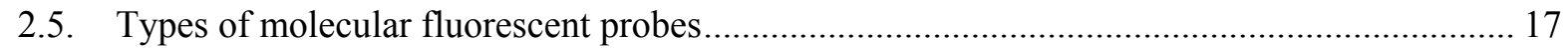

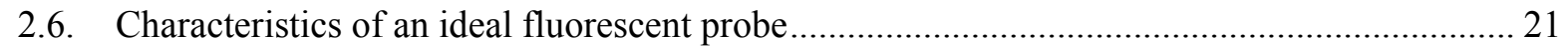

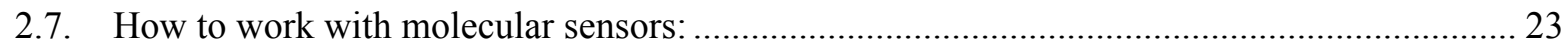

3. PURPOSE AND PROCEDURE WHEN PERFORMING FLUORESCENT STUDIES ..... 24

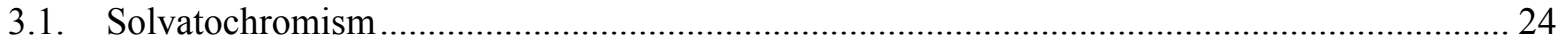

3.2. Tests with different species; cations, anions, oxidative, reductive species, amines............... 26

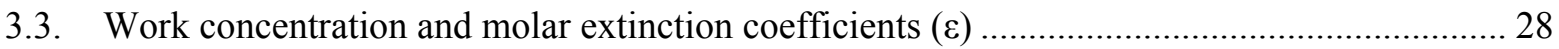

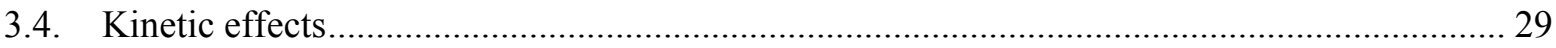

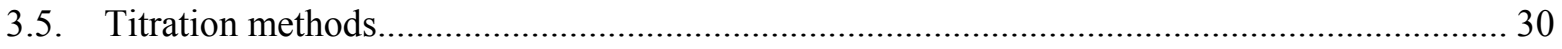

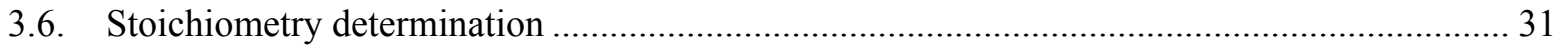

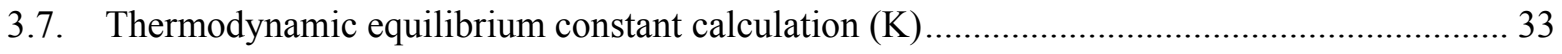

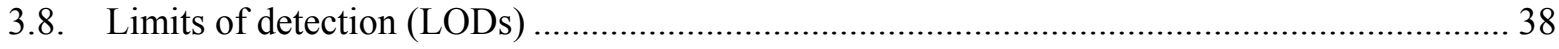

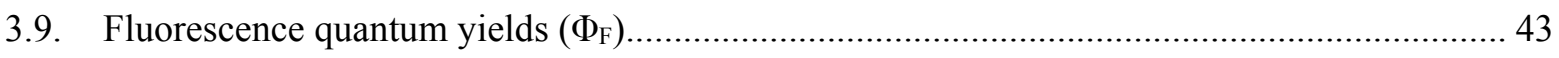

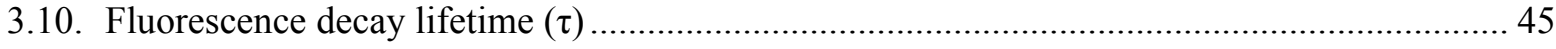

4. GENERAL SCHEME OF FLUORESCENT PROBES; PHOTOPHYSICS AND SUPRAMOLECULAR CHEMISTRY ........................................................................................... 47

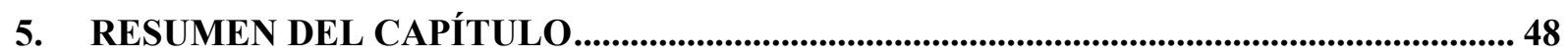




\section{CHAPTER 1.}

FLUORESCENT PROBES FOR DETECTION OF Hg(II)

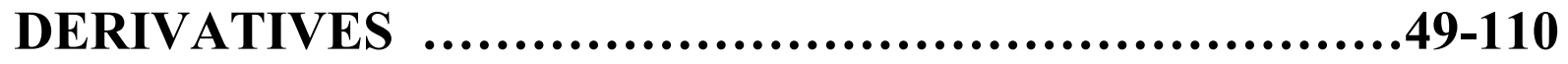

1. INTRODUCTION. THE IMPORTANCE OF MERCURY ..............................................51

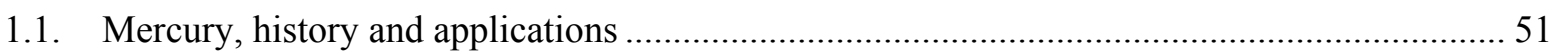

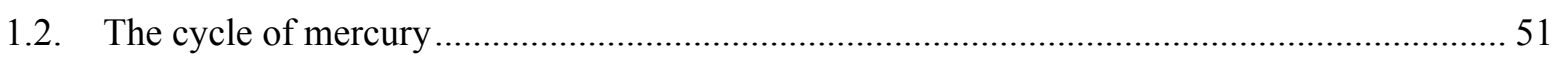

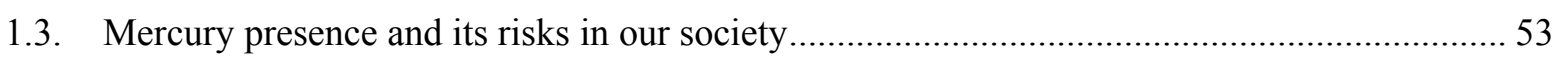

1.4. Standardized techniques for detecting Mercury presence in a sample ..................................... 54

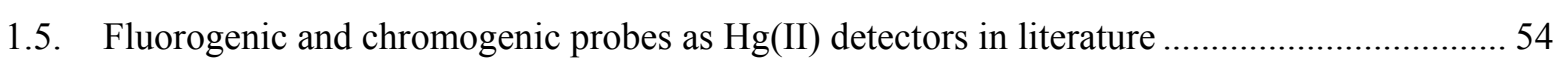

2. ANTECEDENTS FROM THE GROUP IN MERCURY PROBES DEVELOPMENT....... 58

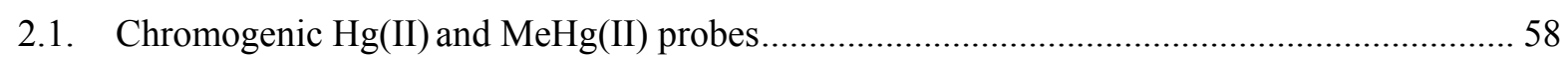

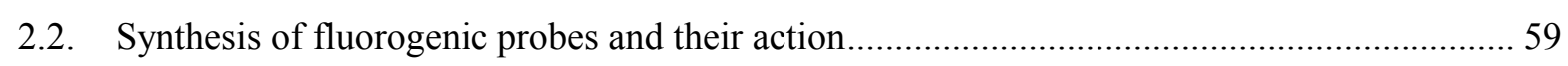

3. OBJECTIVES. Hg(II) AND MeHg(II) FLUOROGENIC PROBES USING 100\% WATER

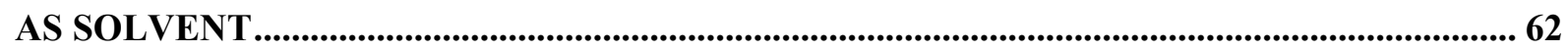

3.1. Designing molecular probes for detection of $\mathrm{Hg}(\mathrm{II})$ and $\mathrm{MeHg}(\mathrm{II})$ in water solution.............. 62

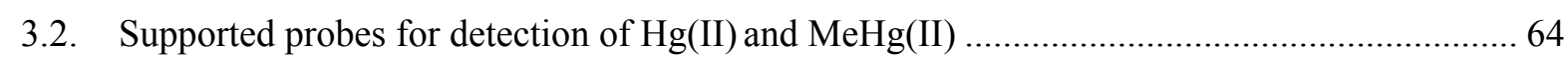

4. SYNTHETIC PROCEDURE OF MOLECULAR PROBES ................................................65

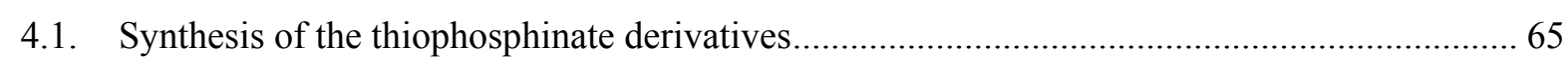

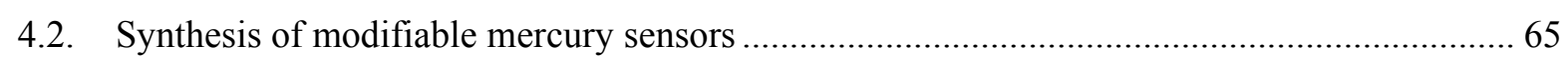

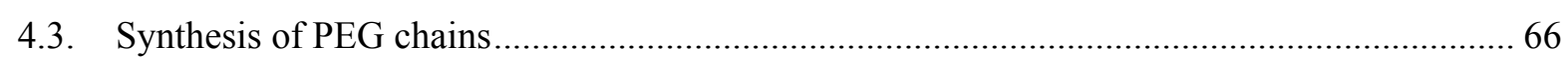

4.4. Click chemistry for developing water soluble molecules based on PEG chains ....................... 67

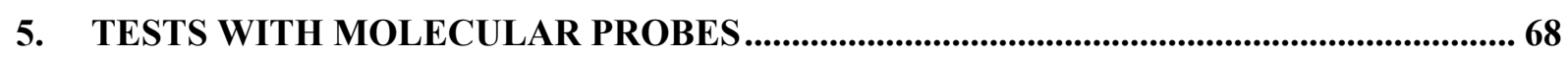

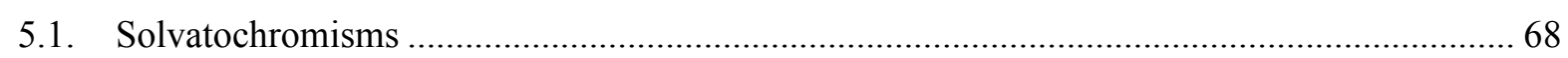

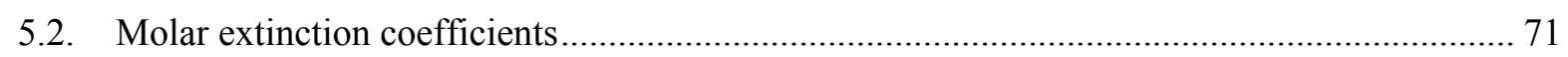

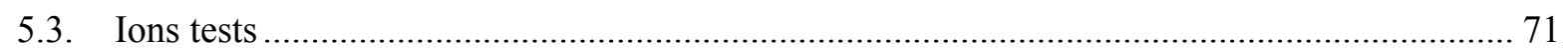

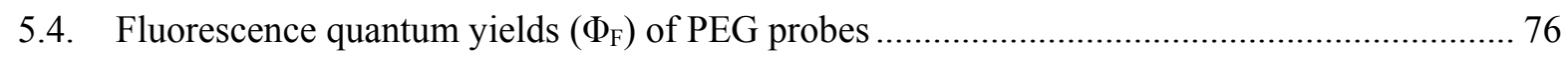

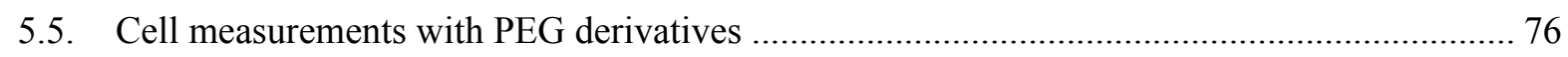

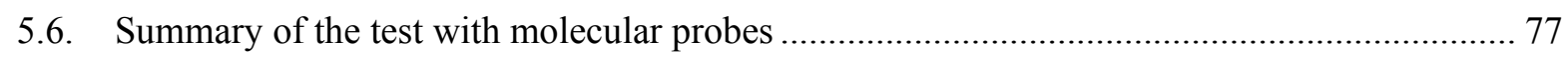

6. SYNTHESIS OF MODIFIED MATERIALS FOR THE DETECTION OF Hg(II) AND

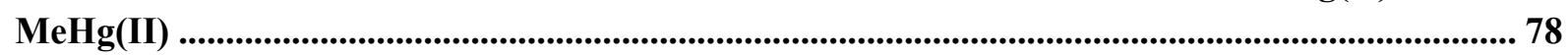

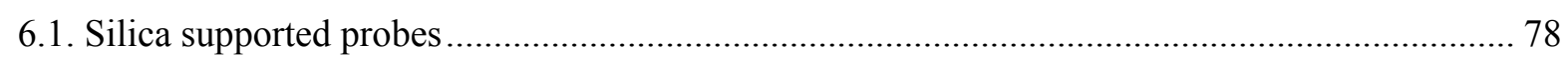

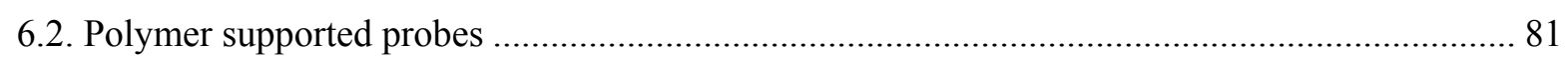

7. QUALITATIVE TESTS OF MATERIAL SUPPORTED PROBES...................................... 86

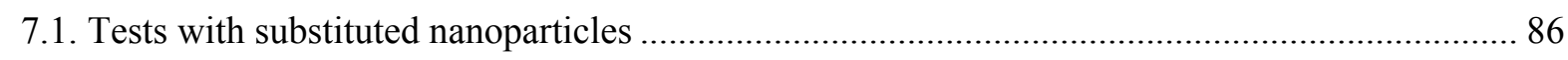


7.2. Tests with soluble polymer JG32.

7.3. Tests with polymeric film JG25.

8. QUANTITATIVE TESTS OF MERCURY(II) SENSITIVE POLYMERS ......................... 91

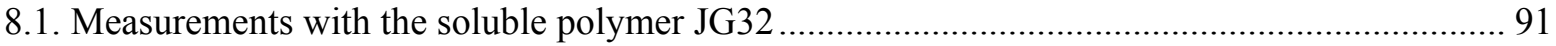

8.2. Measurements with the solid crosslinked polymer JG25 .......................................................... 95

9. ANALYSIS OF FISH SAMPLES .............................................................................................. 100

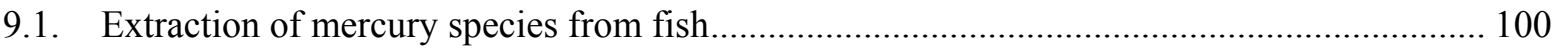

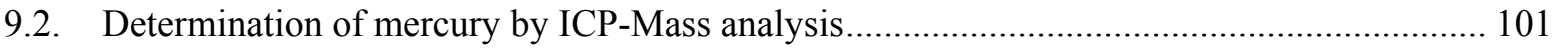

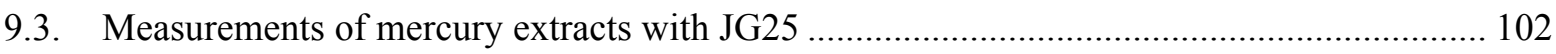

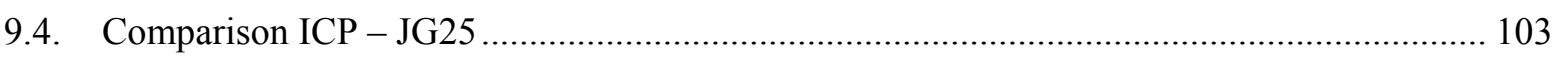

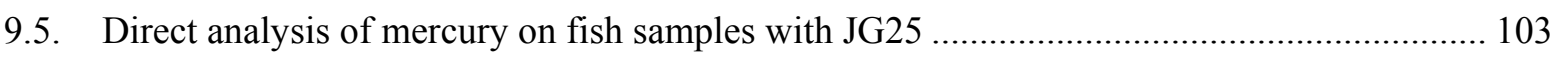

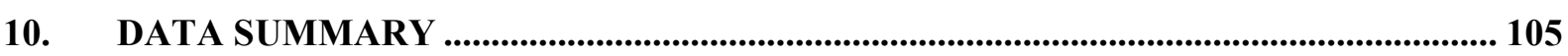

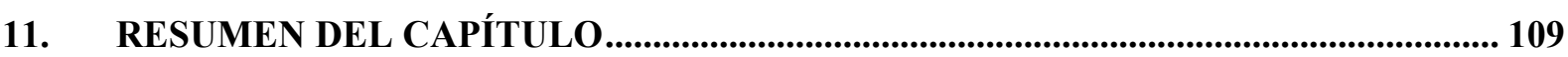

\section{CHAPTER 2.}

\section{PERYLENEMONOIMIDES. INTRODUCTION AND}

GENERAL PROPERTIES ....................................111-154

1. INTRODUCTION. PERYLENE DERIVATIVES, STRUCTURE AND PROPERTIES.. 113

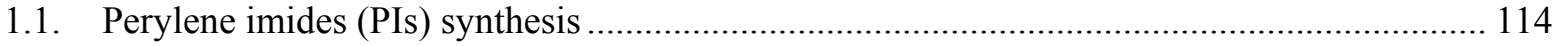

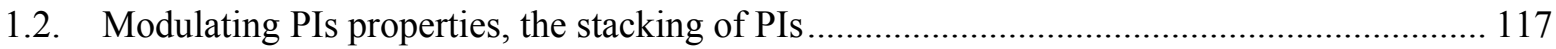

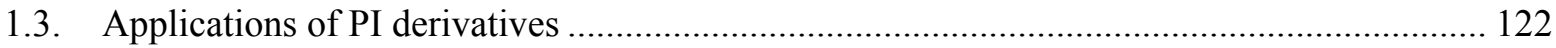

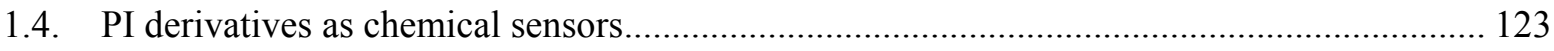

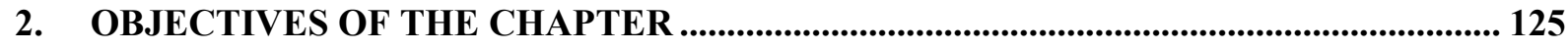

3. OPTIMIZED SYNTHESIS PROCEDURE FOR PERYLENEMONOIMIDES (PMIS) ... 126

3.1. Imidization of the PDA to Perylenemonoimide (PMI) ..................................................... 126

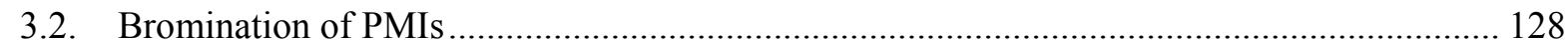

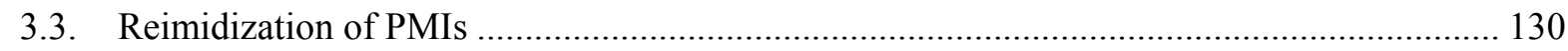

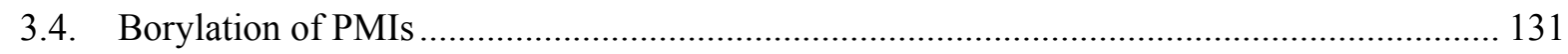

3.5. Introduction of groups in peri position by Suzuki reaction ............................................... 131

4. RELATION STRUCTURE-LUMINESCENCE OF THE SYNTHETIZED PMI

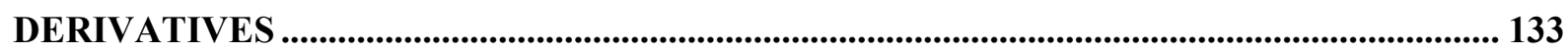

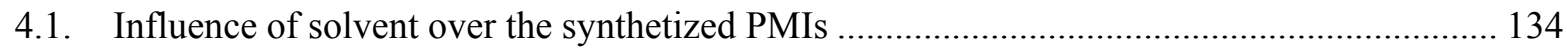


4.2. PMI-Bodipy combinations for creation of FRET systems ................................................. 145

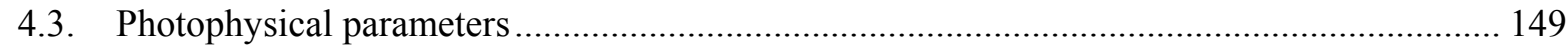

5. SPECIFIC APPLICATIONS OF THE SYNTHETIZED PMI DERIVATIVES ................. 150

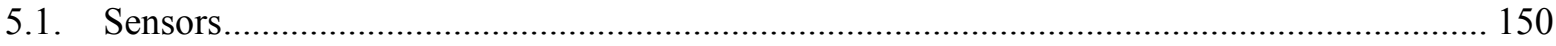

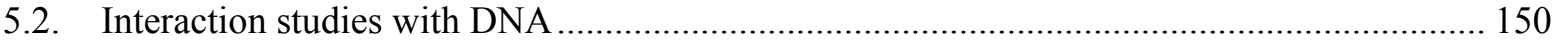

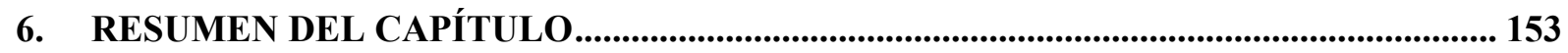

\section{CHAPTER 3A.}

\section{PMI DERIVATIVES FOR DETECTION OF}

EXPLOSIVES ..............................................155-184

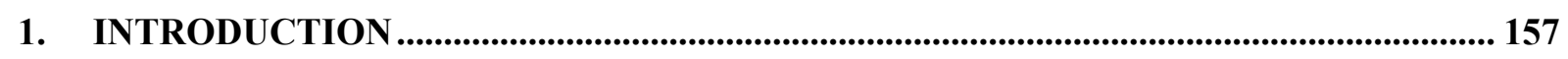

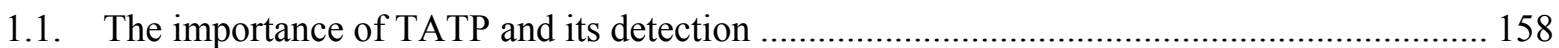

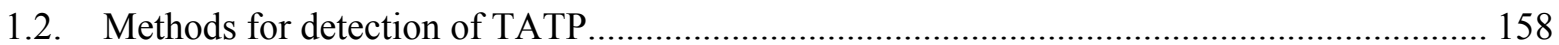

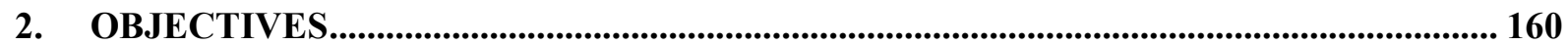

3. SYNTHESIS OF PMI DERIVATIVES SENSITIVE TO TATP ...................................... 162

3.1. Synthesis of the PMI molecular derivatives and election of the probe .................................. 162

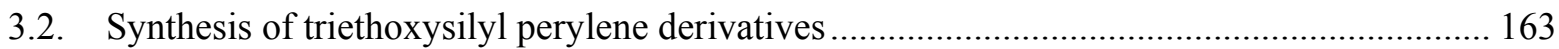

3.3. Synthesis of silica materials with supported perylene derivatives ......................................... 164

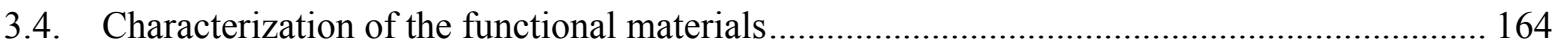

4. QUALITATIVE AND QUANTITATIVE MEASUREMENTS OF JG125 WITH

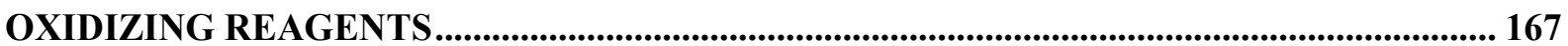

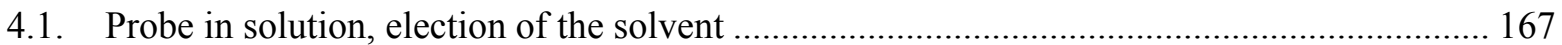

4.2. Qualitative response of solutions to different oxidizers ...................................................... 168

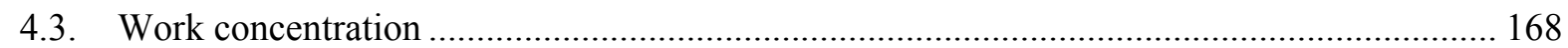

4.4. Quantitative measurements of JG125 vs TATP / MCPBA in solution ................................. 170

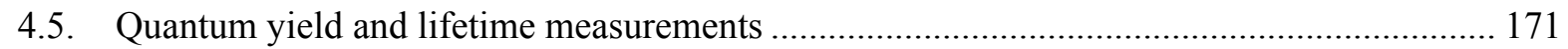

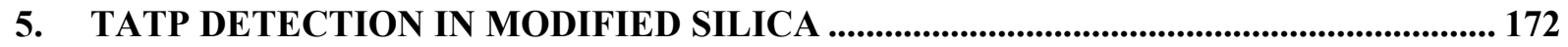

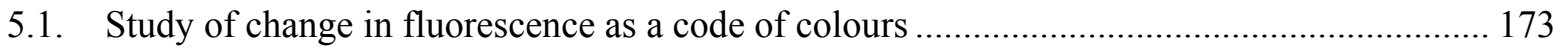

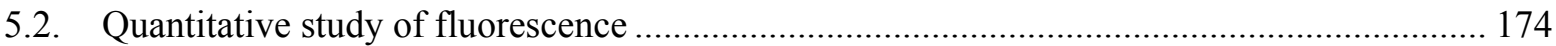

5.3. Change in Fluorescence Quantum Yield with TATP …...................................................... 176

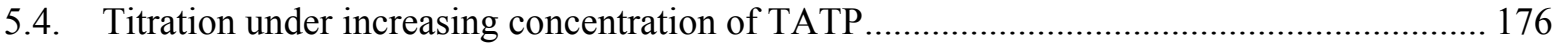


5.5. Summary of fluorescence measurements

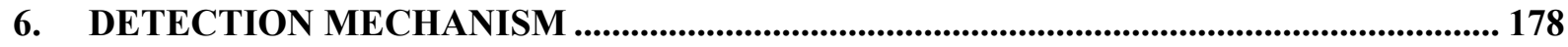

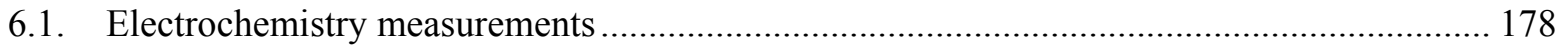

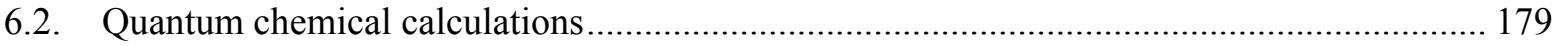

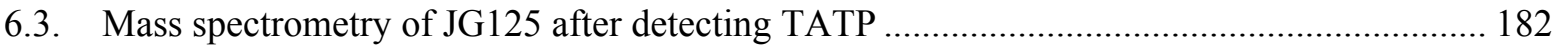

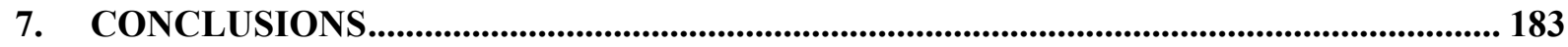

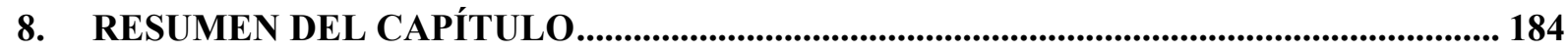

\section{CHAPTER 3B.}

PMI-Ru(II) COMPLEXES FOR CO DETECTION.........185-208

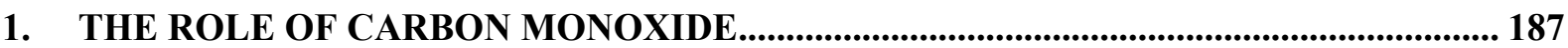

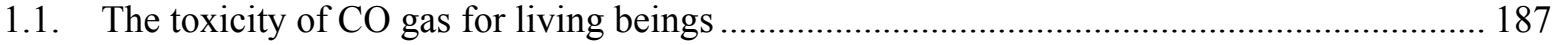

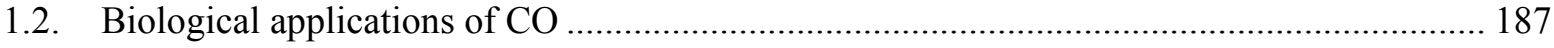

1.3. Detection of $\mathrm{CO}$

2. Ru(II) COMPLEXES FOR CO DETECTION, ANTECEDENTS AND POSSIBLE

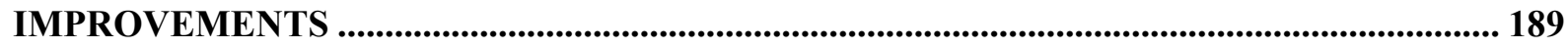

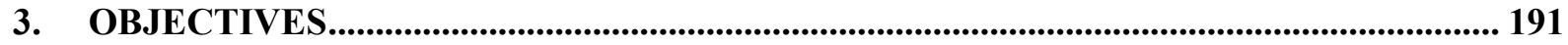

4. DESIGN OF THE PMI-Ru(II) COMPLEXES ........................................................................ 192

5. SYNTHESIS OF PMI-Ru(II) COMPLEXES.............................................................................. 194

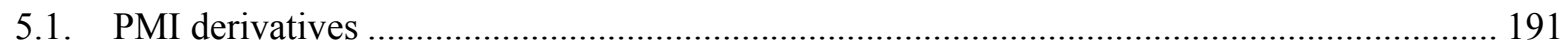

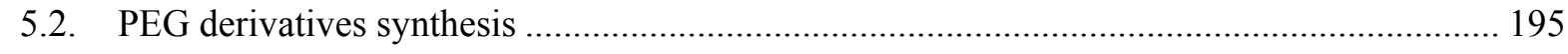

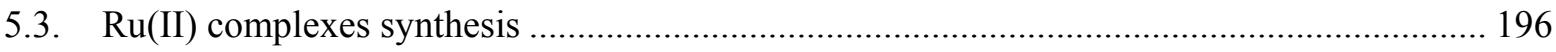

6. ABSORBANCE AND FLUORESCENCE INTERPRETATION ....................................... 197

7. TESTS WITH PMI-Ru(II) PROBES ..................................................................................... 200

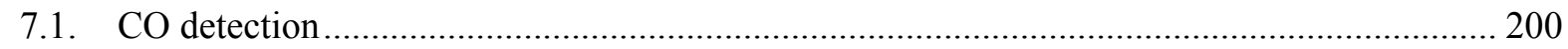

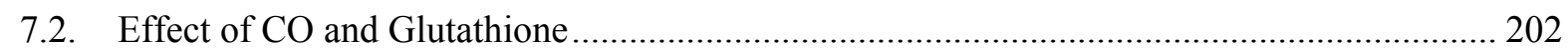

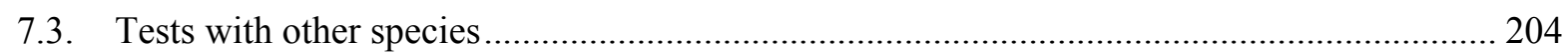

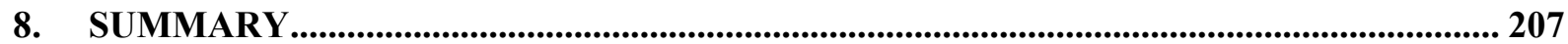

9. RESUMEN DEL CAPÍTULO 


\section{CHAPTER 3C.}

\section{INTRODUCTION, PMIS MODIFIED RECEPTORS FOR FLUORESCENT}

1. DETECTION OF POTASSIUM CATIONS.

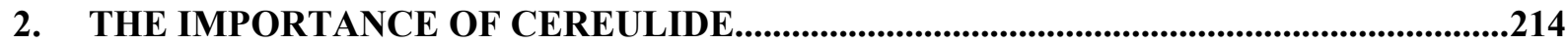

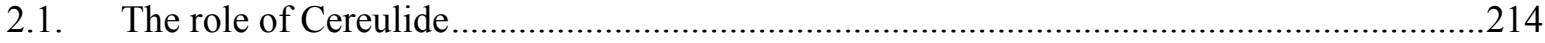

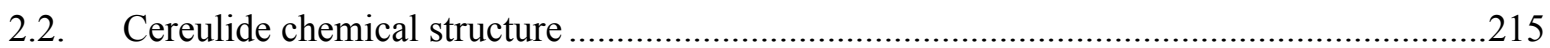

2.3. Toxicity comparison Valinomycin-Cereulide and structural variations...............................215

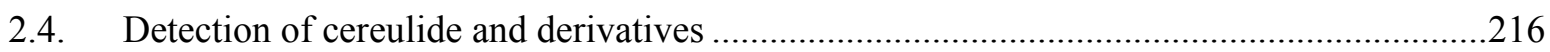

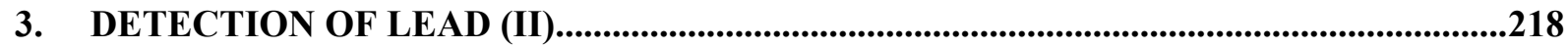

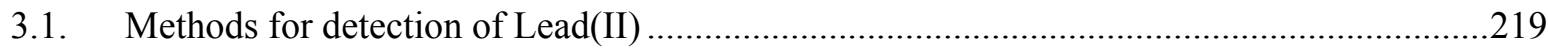

3.2. Colorimetric and fluorometric Lead(II) sensors in literature ..........................................221

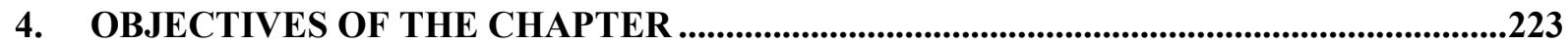

5. SYNTHESIS OF FLUORESCENT MOLECULAR SENSORS WITH CRYPTAND AND

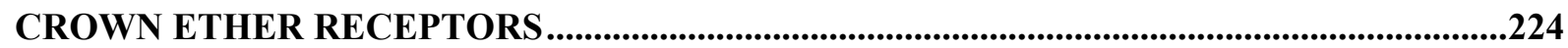

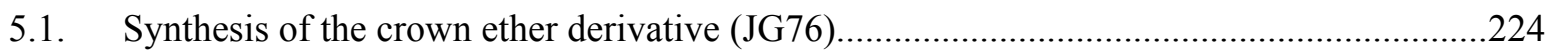

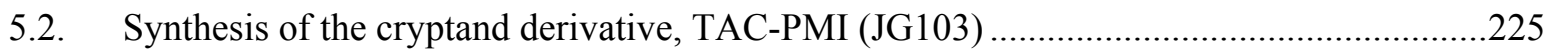

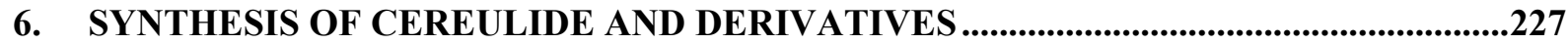

7. SOLVATOCHROMIC STUDIES OF CROWN ETHER (JG76) AND TAC (JG103)

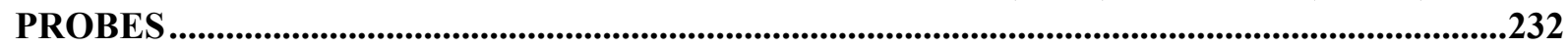

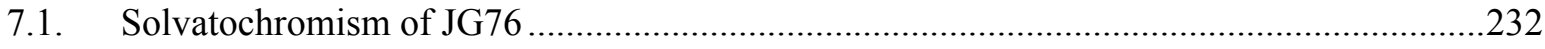

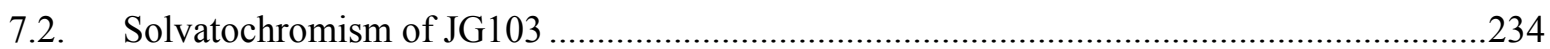

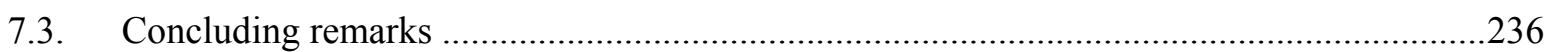

8. EFFECT OF IONS, pH AND WATER PERCENTAGE ON CROWN ETHER PROBE

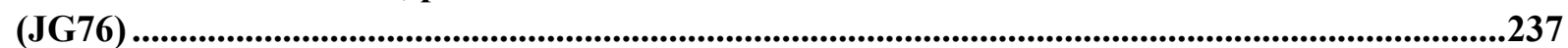

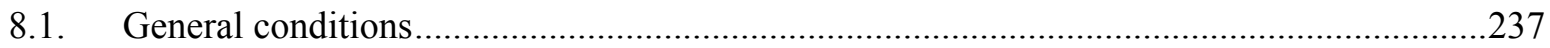

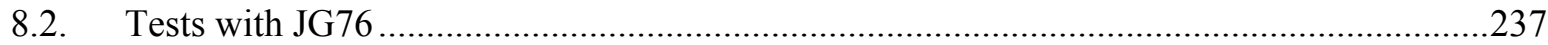

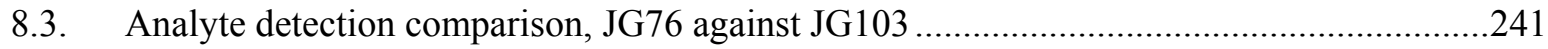

9. DETECTION TESTS OF $\mathrm{K}^{+}, \mathrm{Pb}^{2+}$ AND CEREULIDE IN SOLUTION ..............................242

9.1. JG76 Job's Plot, stoichiometric determination of the complex ...........................................242

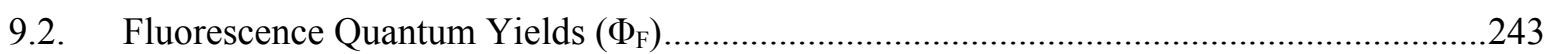

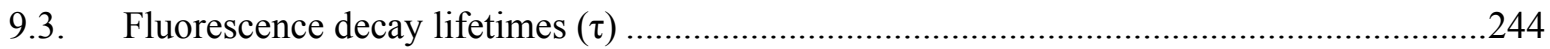

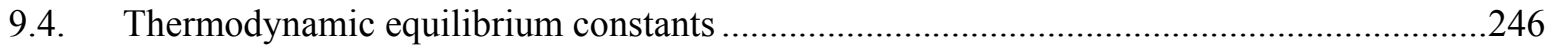

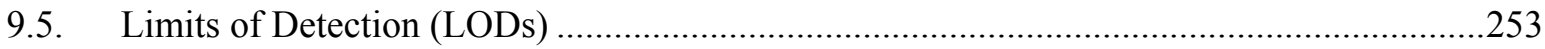


10. MEASUREMENTS OF EXTRACTED CEREULIDE SAMPLES BY

FLUORESCENCE

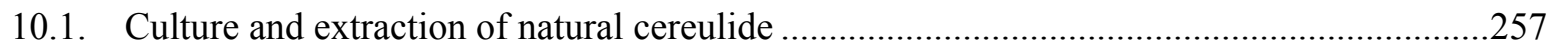

10.2. Fluorescence measurements of natural cereulide extracts...................................................257

11. BIOLOGICAL DETECTION OF POTASSIUM, COMPARISON BETWEEN

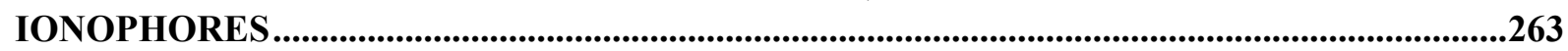

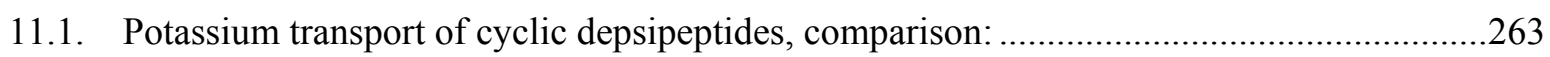

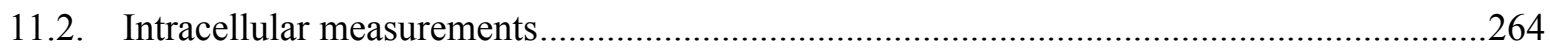

12. SYNTHESIS OF A MATERIAL FOR DETECTION OF LEAD (II) ..................................268

12.1. Synthesis of a PMI-crown ether derivative containing a free amino group ........................268

12.2. Composition and synthesis of the modified polymer .........................................................268

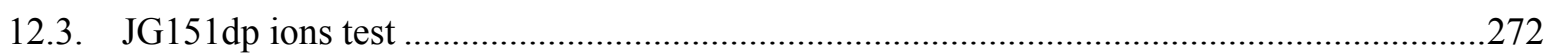

12.4. Fluorescence quantum yields $\left(\Phi_{\mathrm{F}}\right)$ and decay lifetimes $(\tau)$ of JG151dp ............................274

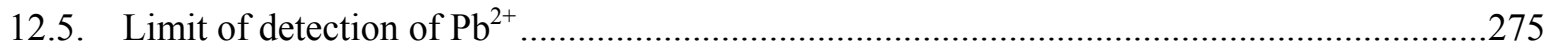

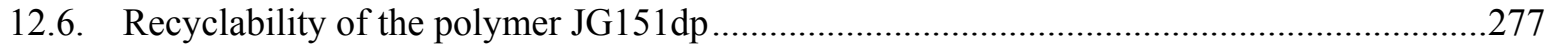

13. DETECTION OF Pb(II) IN COLOURED SOLUTIONS .....................................................278

14. SUMMARY OF THE CHAPTER ….....................................................................................280

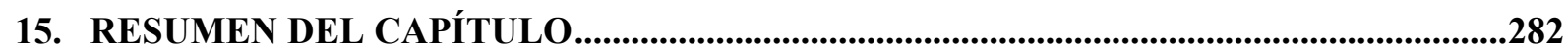

\section{CHAPTER 4.}

SUPPORTED GOLD AND PALLADIUM NANOPARTICLES

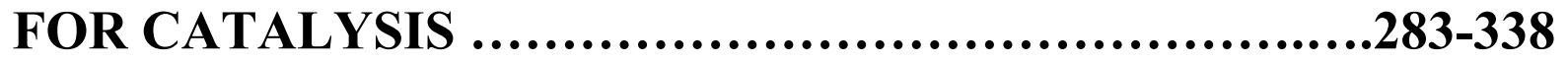

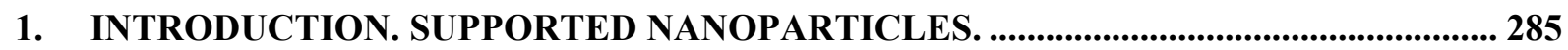

2. GOLD NANOPARTICLES.................................................................................................... 287

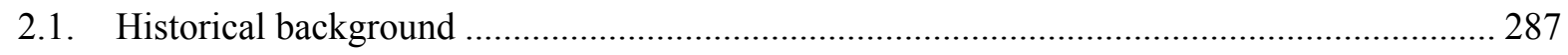

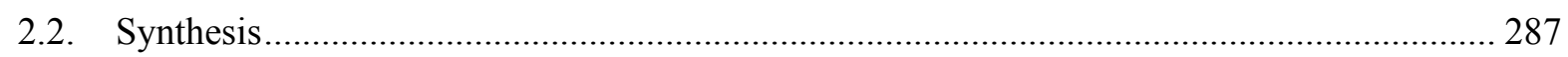

2.3. Physical and chemical properties-applications of gold nanoparticles ................................... 288

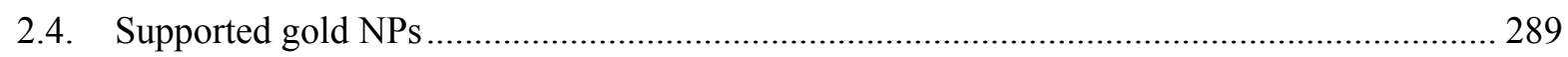

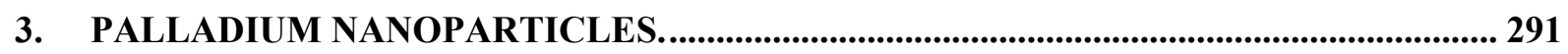

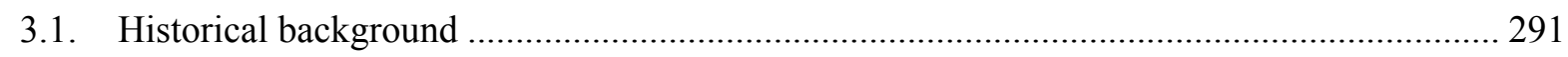

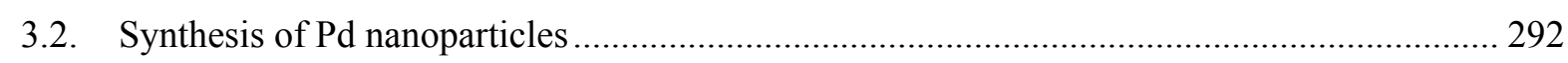

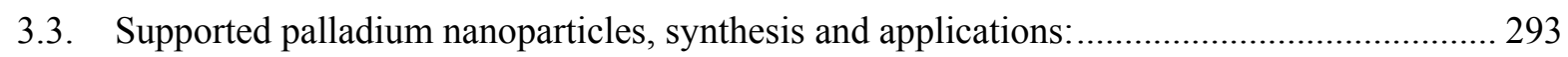

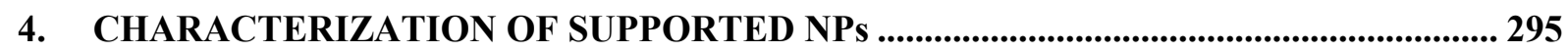

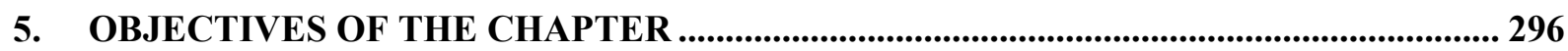




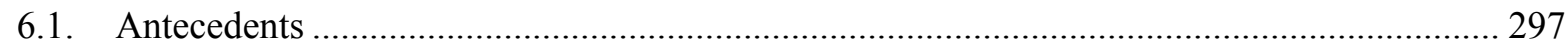

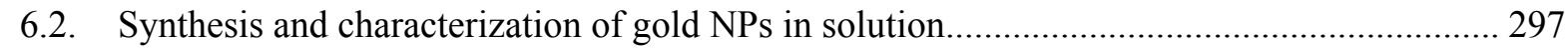

6.3. Synthesis and characterization of supported gold particles .................................................. 299

7. APPLICATION IN CATALYSIS OF GOLD-NPs .................................................................... 307

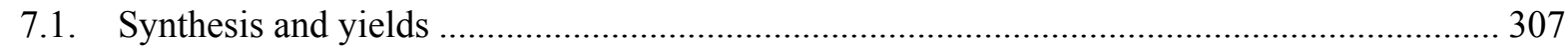

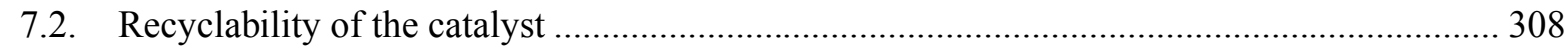

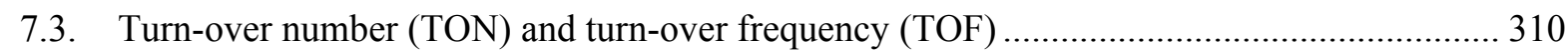

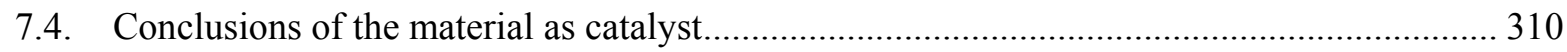

8. EXPERIMENTAL SYNTHESIS OF PALLADIUM-NPs...................................................... 311

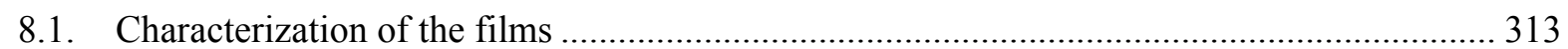

8.2. Deep study of PB20_80A1 and PB80_20 modifications with palladium ............................. 315

9. PALLADIUM SUPPORTED POLYMERS AS CATALYSTS.......................................... 322

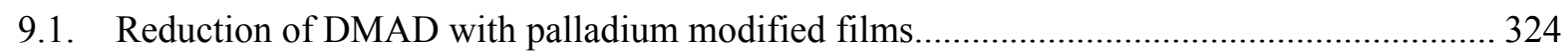

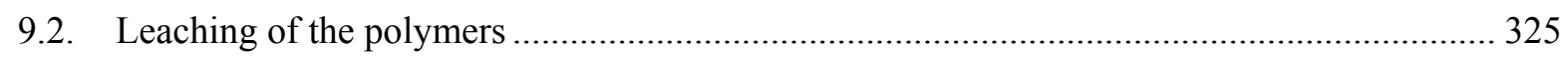

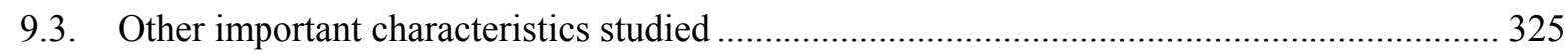

10. CATALYTIC REDUCTION OF COMPOUNDS WITH BIOLOGICAL INTEREST . 326

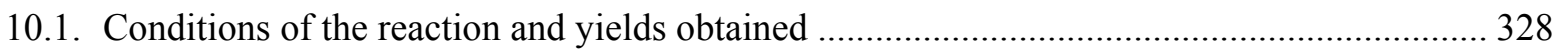

10.2. Results for Pd films reduction and comparison with $\mathrm{Pd} / \mathrm{C}$ catalyst....................................... 328

11. PALLADIUM-SUPPORTED REDUCTION. MECHANISM AND CALCULATIONS334

12. SUPPORTED GOLD AND PALLADIUM PARTICLES. SUMMARY ........................... 336

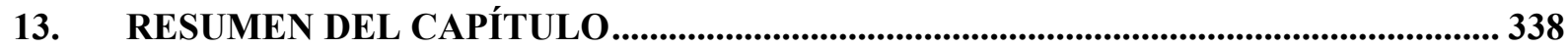

FINAL CONCLUSIONS..................................339-342

ANNEX....................................................343-354

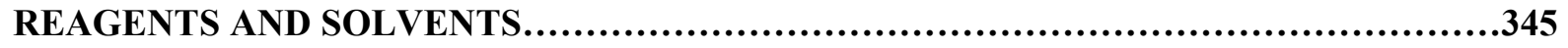

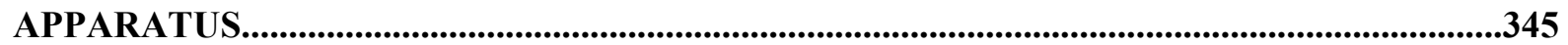

MATERIAL AND SAMPLES FOR SENSORING STUDIES........................................................346

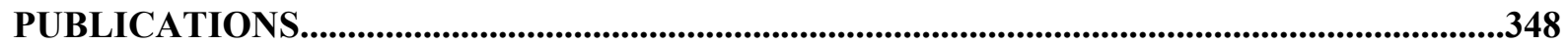




\section{INTRODUCTION. CONTEXT AND OBJECTIVES OF THE THESIS}





\section{CONTEXT}

Throughout the years, the research topics of the group have changed so as to achieve the objectives of different projects and to improve previous results. In this way, the work has been characterized for being open to explore new areas of chemistry instead of being focused in one topic. Therefore, this thesis explores the applications of new dyes in some research areas such as chemical sensors, catalysis and new materials.

Initially, the main scope of the research group was the development of fluorescent sensors for detection of environmental contaminants and dangerous/toxic substances. In this regard, the research was devoted to the elaboration of fluorescent chemical probes for a quick, easy and cheap detection. This was the topic of the first chapter of the thesis (Chapter 0) which includes a background search to understand how fluorescence works, what are their advantages and which would be the proper way to use fluorescent probes for sensing. Thus, the laboratory work of this thesis started by the development and improvement of fluorescent probes for detection of $\mathrm{Hg}(\mathrm{II})$ derivatives. Previous members of the group had synthetized and tested blue/yellow fluorescent probes soluble in organic-aqueous media. However, the research needed for completion, which was achieved by synthetizing a derivative soluble in water. Furthermore, the possibility of creating a material with water affinity and enhanced sensitivity to $\mathrm{Hg}(\mathrm{II})$ was explored. The development of the study is thoroughly explained in Chapter 1.

Alongside the development of the probes for $\mathrm{Hg}$ (II) detection, the research group became involved in a European Research Project. The project was entitled "Sensory devices network for food supply chain security", SNIFFER, whose objectives were focused on the development of tools for detection of CBR agents (chemical, biological and radiological). As a consequence, and taking into account previous results, two objectives were raised for the rest of the research: the improvement of the characteristics of the fluorogenic probes (emission, solubility and selectivity) and their adaptation for detection of some chemical and biological threats.

First synthetized probes (Hg(II) selective probes) were based on the fluorescence of indanone derivatives. As a consequence, the working range was very limited (generally blue fluorescence) and the emission intensity was quite poor (inferior to $10 \%$ in fluorescence quantum yield). To solve these issues, the first step was the search for a fluorogenic backbone with more suitable characteristics. The selected dye was the perylenemonoimide (PMI) family of dyes. These structures are usually fluorescent in the region of green-red and show fluorescence quantum yields close to $100 \%$, in most cases; therefore, they are perfect candidates for fluorescent probes. Chapter $\mathbf{2}$ describes the properties, advantages and some of the applications of this kind of derivatives.

Using the new fluorescent backbones in the synthesis of sensors was the next task to address. It was divided into three parallel research topics. First, a PMI was adapted for the detection of explosives, specifically TATP (Chapter 3A). Second, PMI derivatives were used as a metallic ligand so as to develop sensitive probes to carbon monoxide (in collaboration with the Imperial College London, Chapter 3B). Finally, they were modified to be sensitive to potassium and lead cations, and indirectly, for detecting foodborne toxins, such as cereulide (Chapter 3C), being an objective directly associated to the SNIFFER project. This part represents the core of the thesis covering all the studied applications for the synthetized PMIs and presenting the results in Chapters 2 and 3.

Additionally, during the search for specific materials for new $\mathrm{Hg}(\mathrm{II})$ sensors, some outstanding properties of several of the modified materials were discovered. They possessed the ability to perform 
one-step synthesis and modification of the surface with metallic nano- and microparticles. Hence, the work proceeded throughout the optimization of such materials for their modification, due to the lack of previous results in literature about such properties in a material. Furthermore, the study was complemented with a research about the applications of these materials, mainly for heterogeneous organic catalysis (Chapter 4).

Finally, and complementary to all the results explained in the different chapters of the thesis, two extra parts are included. On one side, the Annex contains the data about reagents, materials and technical characteristics of the used equipment, as well as a summary of publications and symposiums in which this work was presented. On the other side, and in order to clarify some parts, the Experimental Appendix (digital format) includes all the reaction schemes, a complete characterization of the different compounds and materials, some extra experiments (from Chapters 1, 3B and 3C) and the DFT calculation parameters.

\section{OBJECTIVES}

Different aims were proposed for the different chapters of the thesis:

\section{Chapter 0: Introduction to fluorescent probes.}

- Explanation about the different kinds of chemical sensors.

- Introduction to fluorescent sensors.

- Elaboration of a protocol to work with fluorescent probes.

Chapters 1, 2 and 3 of the thesis are oriented to the development of new fluorescent probes for several applications.

\section{Chapter 1: Fluorescent probes for the detection of $\mathrm{Hg}(\mathrm{II})$ derivatives.}

- Introduction of $\mathrm{Hg}(\mathrm{II})$ and its derivatives, the importance of $\mathrm{MeHg}(\mathrm{II})$.

- Description of new fluorescent probes for detection of Mercury(II) derivatives.

- Synthesis of water soluble probes for $\mathrm{Hg}(\mathrm{II})$ detection.

- Detection of $\mathrm{Hg}(\mathrm{II})$ and $\mathrm{MeHg}(\mathrm{II})$ in cellular environments.

- Creation of materials that are capable to detect $\mathrm{Hg}(\mathrm{II})$ presence in water.

- Detection and quantification of $\mathrm{Hg}(\mathrm{II})$ from fish samples.

\section{Chapter 2: Perylenemonoimides. Introduction and general properties.}

- Introduction to the properties of perylene imide derivatives

- Optimization of the synthesis of PMIs.

- Comparative study of PMIs substituted in different ways.

- Introduction to some general applications for PMIs: cellular imaging, solar cells, sensors and biological markers. 


\section{Chapter 3A. PMI derivatives for detection of explosives.}

- Introduction to the interests of detecting TATP.

- Synthesis of a fluorescent probe with a selective response for TATP detection.

- Optimization of a material to perform the detection of TATP in the gas phase.

- Evaluation of the properties of the material for TATP detection.

\section{Chapter 3B. PMI-Ru(II) complexes for detection of CO.}

- Introduction to the advantages of $\mathrm{Ru}(\mathrm{II})$ complexes for $\mathrm{CO}$ sensing.

- Synthesis of complexes Ru(II)-PMI.

- Evaluation of the properties / sensitivity of the complexes.

- Application of the complexes in CO detection.

\section{Chapter 3C. PMI derivatives for $\mathrm{K}^{+}$and $\mathrm{Pb}^{2+}$ sensing.}

- Introduction to the importance of $\mathrm{K}^{+}, \mathrm{Pb}^{2+}$, cereulide and derivatives; their role and the interest of their detection.

- Synthesis of fluorescent PMI probes for $\mathrm{K}^{+}$and $\mathrm{Pb}^{2+}$.

- Synthesis of cereulide, a natural ionophore of $\mathrm{K}^{+}$.

- Evaluation of the sensors properties.

- Detection of cereulide from B. cereus cultures.

- Preparation of cereulide derivatives and comparison of their properties.

- Design and evaluation of a material for sensing of cations, intended for $\mathrm{Pb}^{2+}$.

\section{Chapter 4. Supported gold and palladium nanoparticles for catalysis.}

- Introduction to the applications of metal-nanoparticles modified materials.

- Modification of a material with metal nanoparticles in one-step.

- Optimization of conditions for getting homogeneous surfaces covered by gold and palladium nanoparticles and characterization of the materials.

- Using the materials for heterogeneous catalytic processes. 


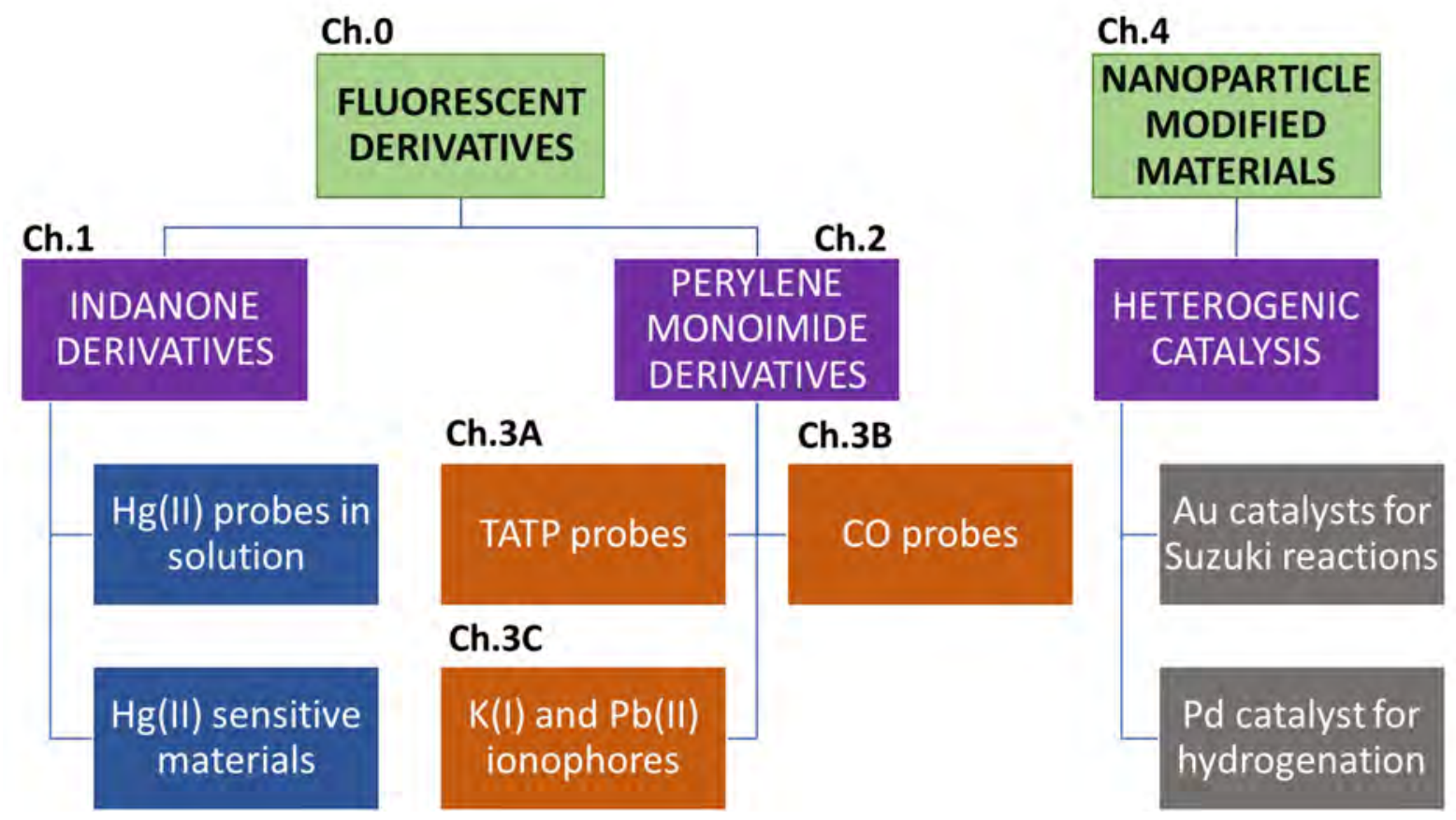

General scheme of the Chapters of the thesis and their content 


\title{
CHAPTER 0
}

\section{INTRODUCTION TO FLUORESCENT PROBES}

\begin{abstract}
Due to their many applications, one of the aims of this thesis was the development of highly efficient fluorogenic probes, which may be useful for detection of different species or as biological markers, amongst other possibilities. With this aim, this chapter is intended to define what the fluorescent sensors are, what properties they have, how to design them properly and what data can be obtained from them, being an introduction for Chapters 1, 2 and 3.
\end{abstract}





\section{SENSORS, DEFINITION AND TYPES}

A sensor is defined as an interface capable of receiving and translating information across physical, chemical and biological samples. Chemical sensors are characterized for being based on a chemical interaction that leads to one or several analytical responses; therefore, a molecular sensor ${ }^{1}$ is a system that is capable of detecting the presence of a specie in a molecular level by a signal that can be measured.

Throughout history there have been many methods to perform sensing procedures and some of them, such as liquid or gas chromatography, have become standards. Although such methods are very sensitive, their cost is usually prohibitive to low scale industries, for instance in some food and pharmaceutical quality control. As a consequence, since 1970s there has been an increasing interest in developing methods with lower costs, faster response times, possibility of miniaturization and greater accessibility. All these characteristics may be achieved by designing new and more efficient molecular sensors, which has become a very important issue not only in the field of chemistry but in industry too, fundamentally because of the high level of different substances they handle.

The main purpose for molecular sensors is detecting the presence of some toxic or dangerous species in waste but it can be extended to other purposes. Some examples of what molecular sensors could perform are the detection of changes in the DNA structure or the distribution of metabolites within cells. In conclusion, research in this field is of high interest for biomedical, environmental, safety or food related purposes.

\subsection{Types of chemical sensors ${ }^{2}$}

There are many ways of developing and classifying chemical sensors. Nevertheless, the process followed for detection is common for all of them and is divided into three steps: recognition of the analyte, transduction of the signal and measurement/analysis (Figure 1).

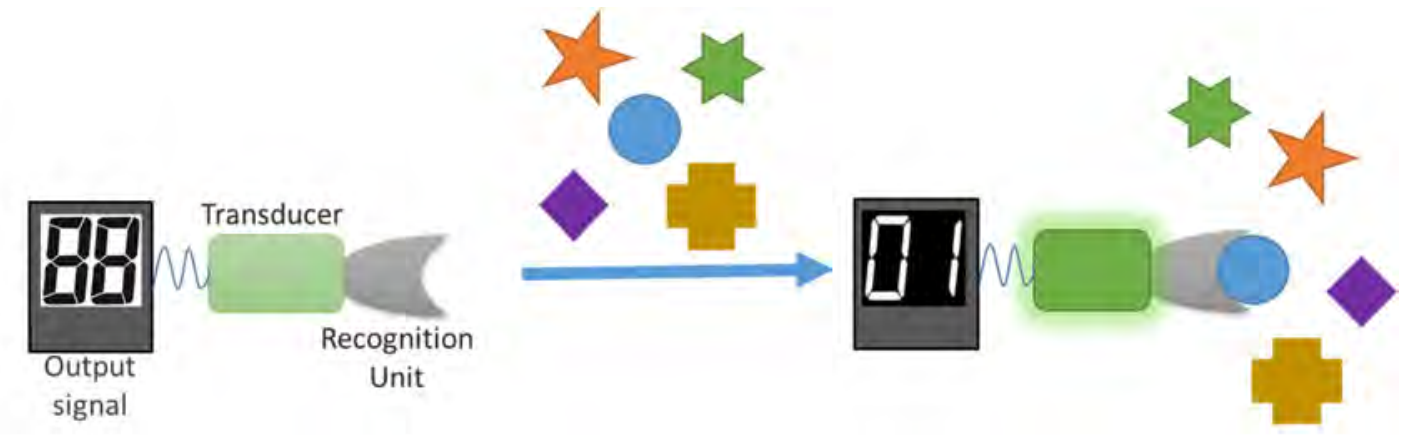

Figure 1. Recognition process of a sensor.

\footnotetext{
${ }^{1}$ J. X. J. Zhang, K. Hoshino, Molecular Sensors and Nanodevices: Principles, Designs and Applications in Biomedical Engineering, Elsevier, Chapter 1, pp. 1-42, 2014.

${ }^{2}$ P. Gründler, Chemical Sensors: An Introduction for Scientists and Engineers, Springer-Verlag Berlin Heidelberg, Chapter 1, pp. 1-13, 2007.
} 
As a consequence, the differences between sensors may be found on the signal/signals that are measured and/or how are they transduced and analysed. Depending on it, a standardised way to classify sensors may be done as follows.

Optical sensors: the detection process is based on the interaction light-matter. The presence of one analyte produces a change in the optical signal received. This group can be subdivided into several groups, being possible to measure several properties at the same time (Figure 2).

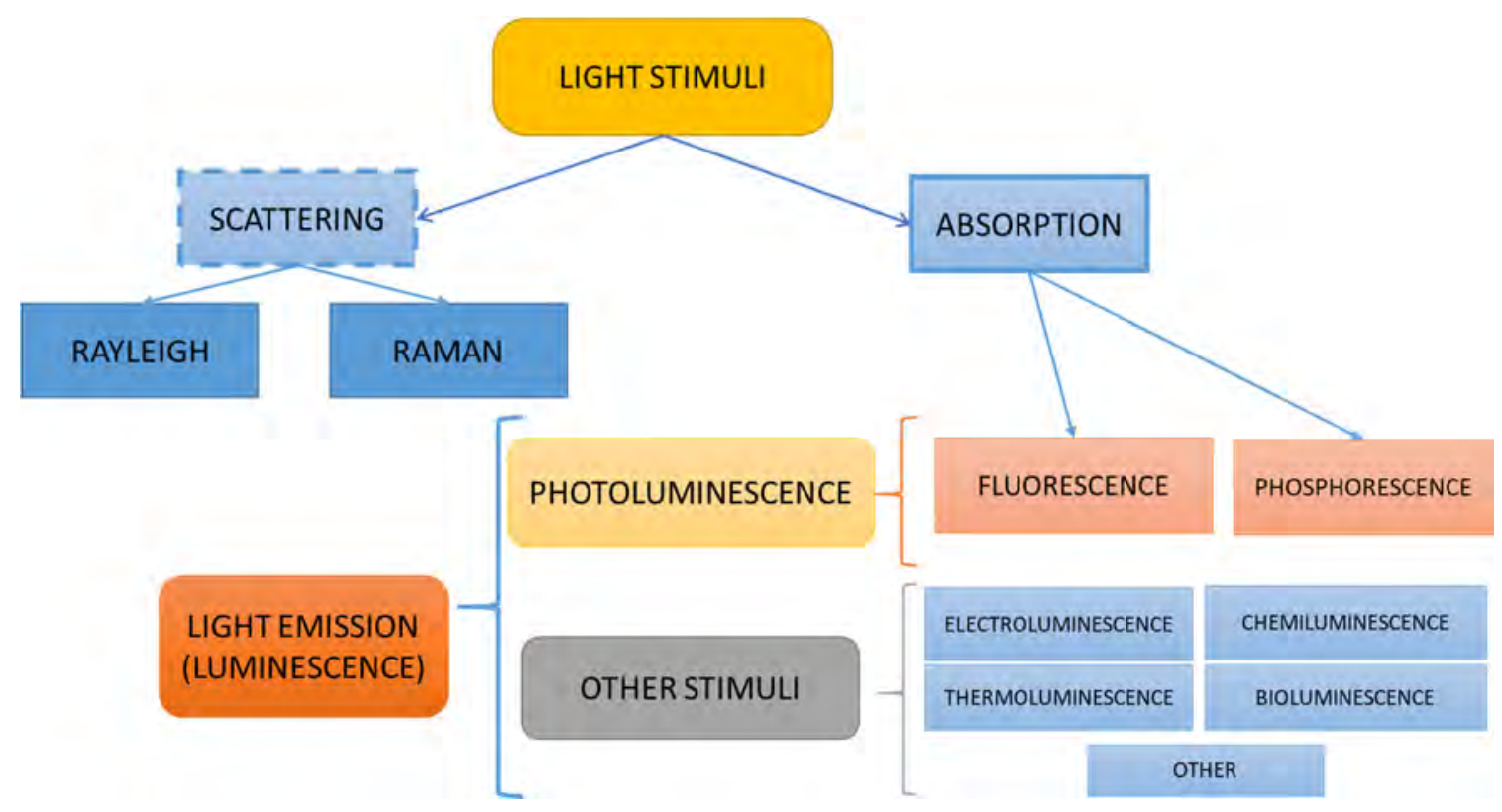

Figure 2. Scheme of different properties that may be measured with optical sensors.

Electrochemical sensors: ${ }^{3}$ they respond to changes in electric current, when the analyte interacts with an electrode. Within this group there are multiple options depending on what is measured. From the many possibilities, they may be classified in voltammetric (that measure the current between the electrode and the media), with constant potential or potentiometric (that measure the changes of potentials between the electrode and a reference).

Electrical sensors: they work by measuring any change in intensity, voltage and/or electric resistance, when in the presence of the analyte. There are several types depending on how they work; metal oxide semiconductor sensors (a redox processes of analyte gas components, for gas detection), organic semiconductor sensors (modifying the charge carrier density), electrolytic conductivity sensors (conductivity of the solution related with interactions with the analyte) and electric permittivity sensors.

Mass sensitive sensors: they are capable of emitting different signals by a change in the electrical current, in response to a variation in mass. They work because of the piezoelectric effect: the use of a piezo crystal material whose oscillation varies with the mass of the analyte over the plate. ${ }^{4}$

Thermometric sensors: this kind of sensors usually consist of a semiconductor surface with a temperature dependent conductivity. It makes them extremely sensitive to any change in temperature. For instance, they may be useful to follow how temperature changes during a chemical reaction.

\footnotetext{
${ }^{3}$ E. Bakker, Y. Qin, Analytical Chem. 2006, 78, 3965-3984.

${ }^{4}$ P. Gründler, Chemical Sensors: An Introduction for Scientists and Engineers, Springer-Verlag Berlin Heidelberg, Chapter 4, pp. 119-122, 2007.
} 
Magnetic sensors: they are based on changes in the magnetic field; mostly used for detection of paramagnetic substances such as oxygen.

Other sensors: there are many other properties, such as the emission of radiation $\gamma$ or $\beta^{-}$radiation or the release of gas, that may be also measured, and used as chemical sensors.

Apart from this, Biosensors are usually classified as a different group of sensors. Nevertheless, the mechanism is similar to that of any other chemical sensor, but with biological elements: organisms, tissues, cells, organelles, membranes, enzymes, antibodies, etc.

All this kind of measurements may give information about changes at a molecular level, although the quality and quantity of this information depends on many factors. In consequence, the type of sensor and the method of analysis are associated to specific accuracy, reusability or selectivity, being some of the parameters that determine how good is a sensor.

\subsection{Sensors, characteristics and advantages. The role of Absorbance + Photoluminescence sensors}

The parameters that determine how a sensor works, and the suitability of them for different purposes, have been summarized and given by many organizations, such as the International Union of Pure and Applied Chemistry, known as IUPAC. ${ }^{5}$ Their work has been to compile each type of sensor and their different advantages and drawbacks.

Taking this into consideration, this work is focused on the development and study of probes to be used as optical sensors, which are ideal for molecular recognition and widely studied in literature. In addition, several properties may be measured simultaneously with optical sensors, giving complementary information and having many advantages over other techniques. For instance, Raman is useful for characterization, while absorption, fluorescence or phosphorescence have more applications in quantification processes. For these reasons, the optical methods are one of the most used and most promising type of sensors.

Specifically, within the optical sensors, the ones based on measuring ultraviolet, visible or infrared emissions from electronically excited species, are called luminescent sensors. From all the possibilities, this thesis is focused on the study of sensors that work by photoluminescence processes (Figure 2), meaning a photo-excitation by light stimuli to study its emission of fluorescence and/or phosphorescence. Therefore, the study of absorption, fluorescence and phosphorescence (luminescence) of a system having light stimuli (photoluminescence) is the core of the sensors developed throughout the thesis.

In the first place, it is important to know the analytical parameters that define a sensor, and what advantages the absorption - photoluminescence sensors have:

- High Specificity: the sensor should have a number of false positives-negatives as low as possible.

\footnotetext{
${ }_{5}^{5}$ IUPAC. Compendium of Chemical Terminology, 2.3.3. (the "Gold Book"). Compiled by A. D. McNaught and A. Wilkinson. Blackwell Scientific Publications, Oxford, 1997. XML on-line corrected version: http://goldbook.iupac.org, 2014, created by M. Nic, J. Jirat, B. Kosata; updates compiled by A. Jenkins.
} 
- High Sensitivity: compared with other optical and non-optical sensors, fluorescent sensors have lower detection limits, hence, the capability to detect smaller changes. Moreover, in comparison, fluorescent sensors are usually between 2 or 3 orders of magnitude more sensitive than colorimetric (absorbance) measurements.

- Reutilization: photoluminescence detection processes are, usually, non-destructive techniques, not requiring from high energy sources or destructive pre-treatment.

- Low cost per operation.

- Fast operation mechanisms.

- Cheap maintenance capabilities.

- Portability: it is possible to develop small devices for measuring absorbance-fluorescence; usually, thanks to the use of optical fibre.

Although nowadays there are plenty of fluorescent sensors, it would be also applicable to phosphorescence methods. This is not usual because only a few materials/molecules present this property, and it is generally quenched by oxygen presence. Therefore, it is difficult to develop a sensor working this way and it would be also more cost effective, having more restrictive conditions for using them. 


\section{CHARACTERISTICS OF FLUORESCENT PROBES}

Due to the characteristics of fluorescent optical sensors, they are a potential opportunity for the development of new sensory devices. Their many advantages over other sensors make them a state of the art tool in many fields of application. In this regard, it is always needed a background knowledge about fluorescence, which clarifies why it is an ideal technique for elaborating sensors and what are its potential issues.

\subsection{The mechanism of fluorescence}

The process of fluorescence is defined in a simplistic way as the emission of light after irradiation at a defined wavelength, provided that the emitted light has higher wavelength and it is emitted within a very short delay (without intersystem crossing).

Fluorescence is usually explained by understanding the Jablonski diagram (Figure 3). ${ }^{6}$ When light is absorbed, the electron in the highest energy level increases its energy, going from the ground state to an excited state with the same multiplicity. Then, there are many ways to return to the ground state: by vibrational relaxation and internal conversion (without emitting), fluorescence (return to the fundamental state without change, emitting energy without intersystem crossing) and phosphorescence (when there is interconversion between systems before the emission). In consequence, the relaxation processes are related with the emission lifetime decay, which is a characteristic parameter that gives information about the time between the excitation and the emission, since having interconversion increase the emission lifetime decay.

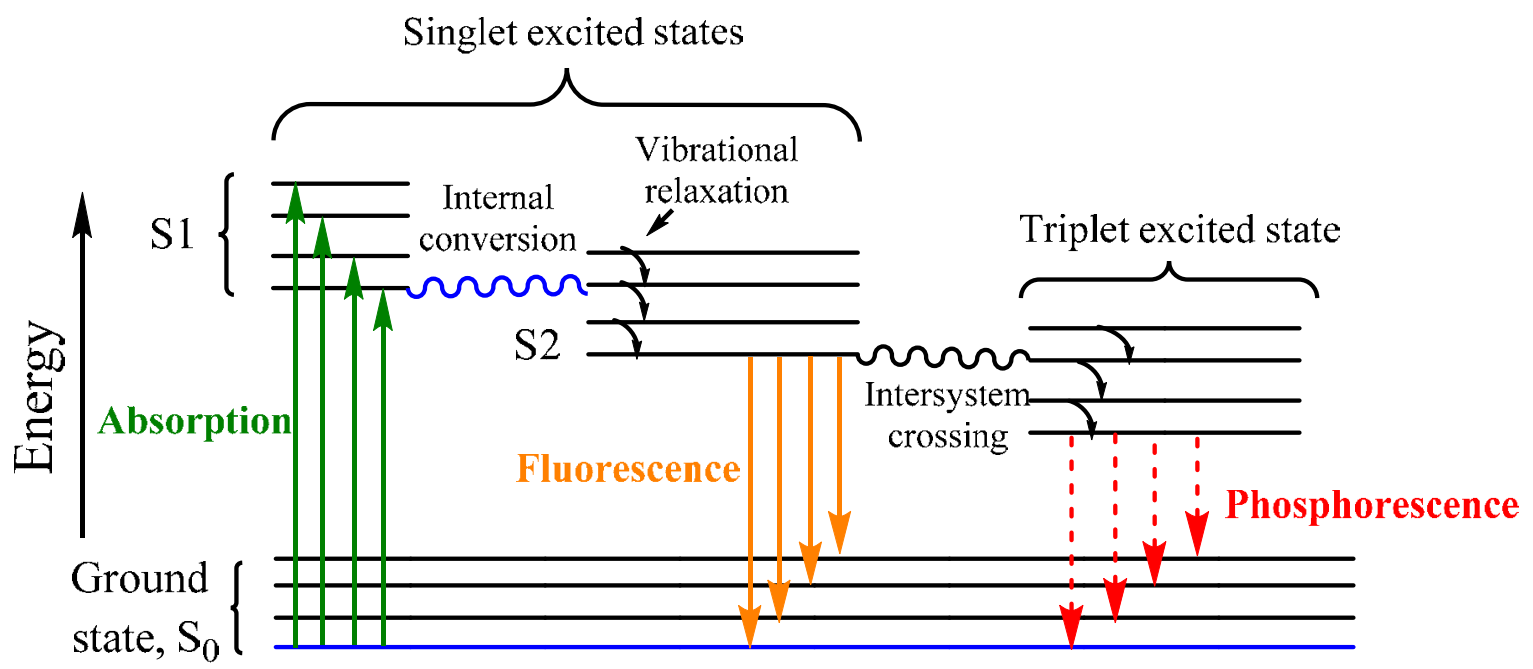

Figure 3. Jablonski diagram of radiative relaxation of excited electrons.

\footnotetext{
${ }^{6}$ P. Elumalai, P. Atkins, J. de Paula, Atkins, Physical Chemistry, Oxford University Press, Oxford, 2002.
} 


\subsection{Factors that have influence over fluorescence}

Due to the nature of fluorescence and how it works, there are many parameters that affect it directly. The fluorescent emission is only one of the many ways in which an electron in an excited state can relax to the ground state, having several competitive processes that could occur (Figure 4).

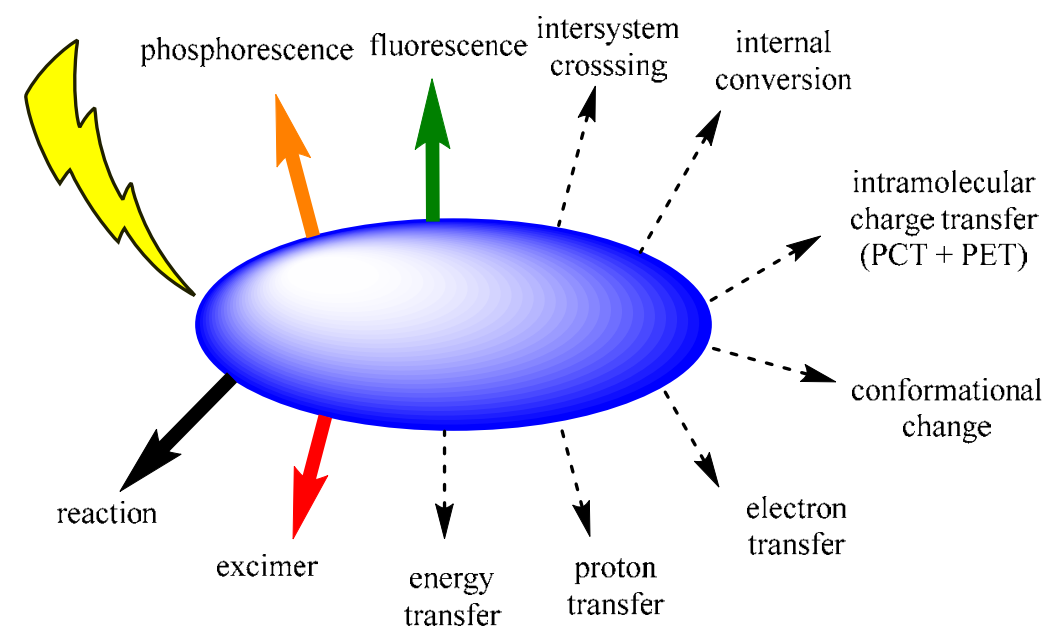

Figure 4. Schematic representation of the ways in which the absorption of a photon affects a molecule.

The characteristics of the fluorophore and the media (physical and chemical conditions) in which the fluorescence is measured determine which other processes are competitive and to what extent. The influence of the media is defined by a series of parameters that are usually listed in several ways. For instance, in solution, (Figure 5) parameters such as the concentration of the fluorophore, polarity of the solvent, $\mathrm{pH}$ or viscosity are of upmost importance. Furthermore, others parameters such as the presence of ions, the possibility of giving hydrogen bonds, the aggregation of the species, the pressure or the temperature, also have direct influence over the signal, being closely related between each other.

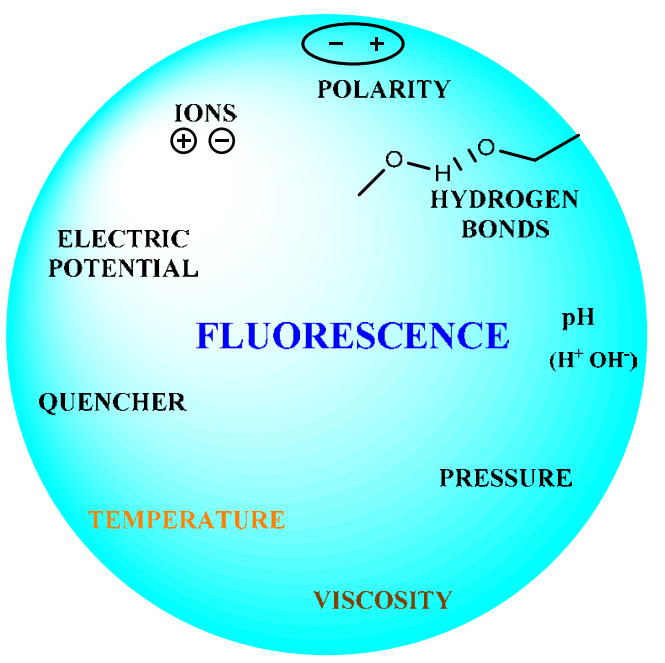

Figure 5. Parameters that affect fluorescence.

As a consequence, the conditions when fulfilling an experiment that involves studying fluorescence, must be controlled (Figure 5). Therefore, the parameters that may have influence in measurements must be always known and taken into account. 
In a regular experiment in solution, the parameters that are usually controlled are temperature pressure (usually constant), solvent (polarity, $\mathrm{pH}$ or viscosity) and the species in the solution (quenchers and other interactions between species); which are deeply correlated between them.

- Temperature and pressure: they have direct influence over all the other parameters. The interaction between molecules, mobility and internal vibration, is directly related with them. In addition, it is important to remark that physical parameters affect directly the properties of the solvent, changing the interaction between species in solution too. Because of that, they are usually predetermined and always given as general data when measuring fluorescence.

- Solvent: Fluorescence is either in solid state, in which the interaction with itself is high but steady, or in solution. When using different solvents and studying how the fluorescent response changes, it is easy to figure out the importance of it. This kind of studies are called solvatochromism, which usually show dramatic changes in the fluorescent response. In summary, depending on solvents, variations in polarity may tune their intermolecular (aggregation) and fluorophore-solvent (solvation sphere) interactions. In addition, the response to protic or aprotic solvents is usually very different; being always greatly influenced by $\mathrm{pH}$. Different behaviours are more widely explained in Chapter 2, considering that, when studying the influence of solvents, perylene derivatives are some of the most noteworthy examples.

- Species in solution: as it was stated before, every species in contact with a fluorophore has influence over the final fluorescence. In spite of this fact, if the species in solution have no specific interaction with the fluorophore and they are presented diluted (usually, $<10^{-5} \mathrm{M}$ ), their influence over fluorescence is usually negligible. It makes the method very sensitive, although it is easy to get interferents if there are any concentrated species and the media is not strictly controlled.

The interaction between the fluorophore and other agents may be classified within three types:

o Static interactions: the interaction between the fluorophore and the analyte occurs by direct electrostatic forces; which usually alters the electronical properties and, as a consequence, fluorescence.

o Reaction: the interaction between species may lead to a new one, which potentially alters the fluorescence.

o Dynamic interactions (dynamic quenching): when not working at "infinite dilution" conditions, the interaction by collision or proximity of molecules is always important. It is strongly dependent on concentration, viscosity of the media and temperaturepressure.

\subsection{Fluorescence quenching}

In order to perform fluorescence measurements, it is important to know the factors that affect the fluorescence, not only how to increase it but what tampers it. In this regard, the inhibition of fluorescence is also known as fluorescence quenching.

Every species is a potential quencher, which is the major difficult to overcome when using fluorescence as a detection system. Ions, heavy metals and highly conjugated molecules are strong fluorescence quenchers, due to the high mobility of their electrons; however, specific interactions may decrease fluorescence too. Therefore, to distinguish between the causes of a quenching process they are divided into two: dynamic and static quenching (Figure 6). 

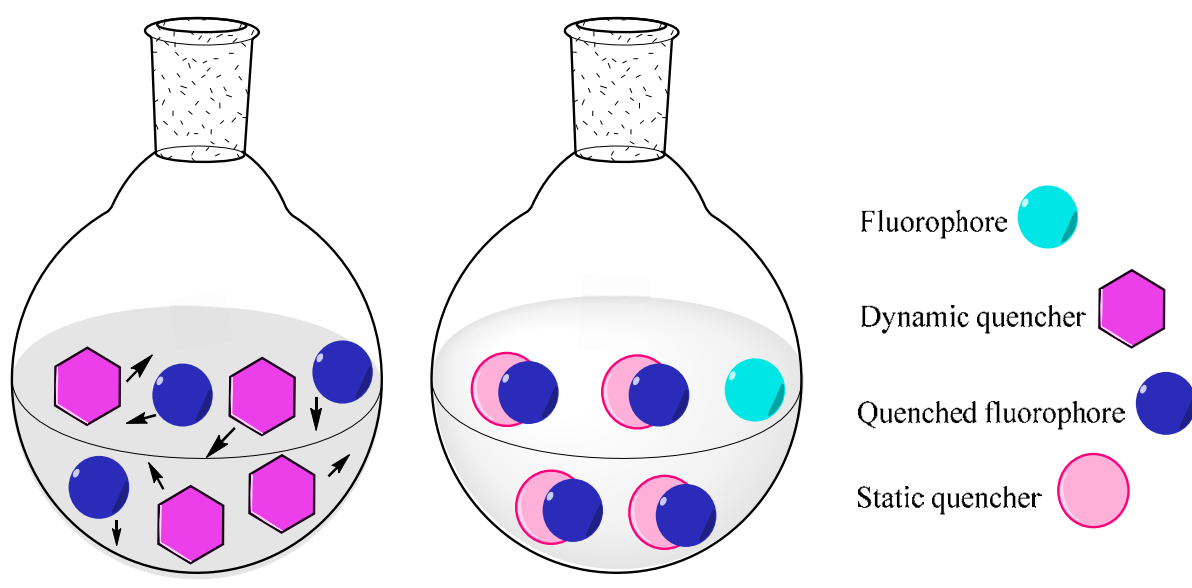

Figure 6. Scheme of the two different kinds of fluorescent quenching and how they work.

Dynamic quenching is something intrinsic to every fluorescent material or solution. It is defined as the decrease in fluorescence because of collisions in a molecular level. Thus, if there are many species, the final fluorescence decreases, and if the species have accessible energy levels (high molecular weight, transition metals, ions, very conjugated...) the fluorescence decreases even faster with concentration. Moreover, because of how it works, it is highly affected by temperature and viscosity of the media.

The variation on the emission intensity is related to the concentration of the quencher by the SternVolmer equation: ${ }^{7}$

$$
\begin{aligned}
\frac{\Phi_{0}}{\Phi}=\frac{I_{0}}{I} & =1+k_{q} \tau_{0}[Q] & & \text { Equation [A] } \\
k_{q} & =8 R T / 3 \eta & & \text { Equation [B] }
\end{aligned}
$$

Where $\Phi$ is the quantum yield, I the emission intensity, $\tau$ the fluorescence decay lifetime, [Q] the concentration of the quencher and $\mathrm{k}_{\mathrm{q}}$ the quenching constant. While $\mathrm{k}_{\mathrm{q}}$ is directly proportional to temperature and inversely to the viscosity of the reaction $(\eta)$.

In consequence, this previous equation gives a linear dependence between the variation in emission and the concentration of the quencher, provided that the solvent and temperature are constant.

Static quenching consists of the specific interaction between the fluorophore and the analyte. When it occurs, it is far more noticeable, compared to dynamic quenching, and less dependent on temperature. An equation that relates the molecular interaction in the equilibrium with the variation of the emission and concentration may be defined to calculate the thermodynamic equilibrium constant (Section 3.6).

In practice, there are several experiments that may be performed so as to distinguish static from dynamic quenching. For example, when it is dynamic, the fluorescence decreases linearly with the quencher concentration (except if the variation in quencher concentration is too high) but, when it is static, it has downward curvature when having enough amount of the quencher. Another way to distinguish between them is the fact that dynamic quenching is highly affected by temperature. What is more, some techniques, such as the study of the lifetime of the fluorophore in the presence of the analyte, are useful to distinguish between dynamic and static quenching (Section 3.10).

\footnotetext{
${ }^{7}$ J.R. Lakowicz, G. Weber, Biochem. 1973, 12, 4161-4170.
} 


\subsection{ON-OFF vs OFF-ON fluorescent sensors}

Detecting the presence of an analyte by fluorescence may be done by two processes:

- By a decrease in fluorescence (ON-OFF). This method is based on the specific quench of fluorescence by interaction with the analyte, static quenching. The main drawback is that quenching is not a selective process. In fact, the presence of other substances (such as cations and/or anions) or the probe in high concentration leads to OFF processes (dynamic quenching). As a consequence, all of them must be taken into account in the results, eventually leading to possible false positive results if they are not considered.

- By an increase in fluorescence (OFF-ON). It is associated to an interaction with specific analytes, provided that temperature and solvent are set. It may occur through different mechanisms; an increase from a non-fluorescent species, a decrease in fluorescence by increasing in another wavelength, or just an increase in different wavelengths to the initial one. This is the most trustworthy method because of the easy distinction from quenching effects. In addition, the selectivity may give different responses depending on the analyte/analytes.

Some authors may consider a third variation when measuring fluorescence. A change in the wavelength of emission. However, this might be considered as an OFF-ON probe, being an increase of fluorescence at a different wavelength.

Besides that, it is important to remark that there are ways in which the fluorescent properties of the probe may be tuned differently. For example, there are some OFF-ON processes that work through a mechanism with lower selectivity than some ON-OFF processes. To illustrate the idea, it might be the case of an isolated non-fluorescent complex in which a ligand is displaced by the analyte under study, increasing the final fluorescence, and this process could be performed with very low selectivity.

\subsection{Types of molecular fluorescent probes}

There are many ways to classify molecular fluorescent probes, depending on how they work. Most authors classify fluorescent probes by the interaction Fluorophore-Receptor, being the most straightforward distinction. There are two main possible interactions, if the probe works by chemical reaction or by complexation. In addition, at the same time, they may be classified depending on the type of complexation or reaction, as it is explained in this section.

First, and in order to simplify the classification, this section is described from the point of view of OFF-ON probe. The equivalent process may be done the other way around, for the ON-OFF samples, with the differences previously explained. 


\subsubsection{The recognition process is based on a complexation ${ }^{8}$}

a) Photoelectron transfer (PET): ${ }^{9}$

Fluorescent PET molecular sensors consist of a fluorophore linked to a recognition unit via a non-conjugated sigma bond (spacer). When the receptor interacts with its analyte, the electron transfer is hindered and an enhancement of fluorescence is observed (Figure 7A). From the point of view of the orbitals (Figure 7B) of the molecule, the electrons from the HOMO level of the receptor are between the HOMO level and the LUMO level of the fluorophore, inhibiting the fluorescence from the excited state by non-radiative relaxation processes; until the analyte is recognized.

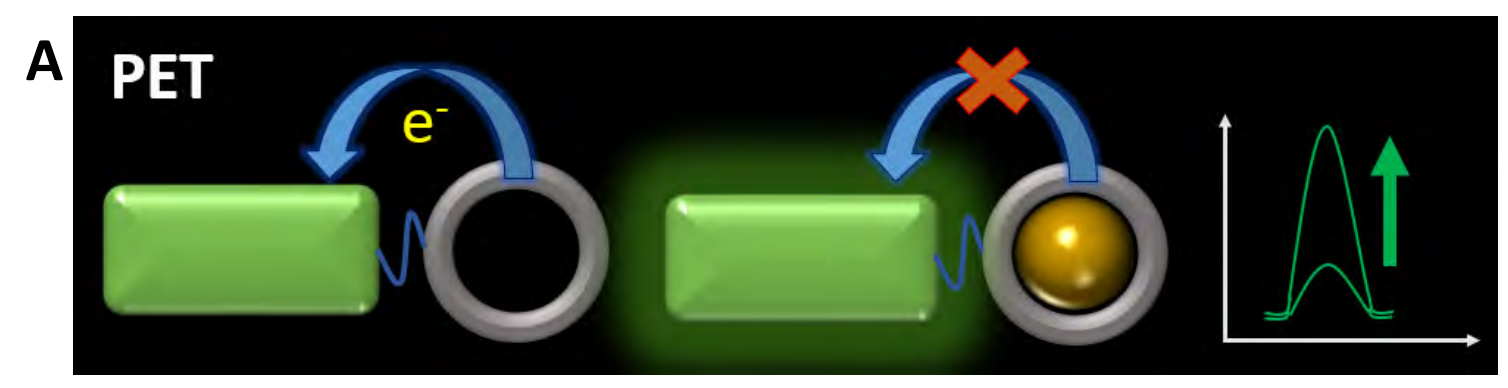

B

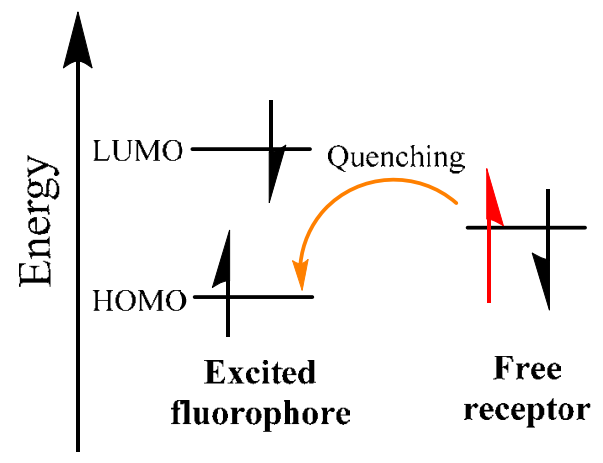

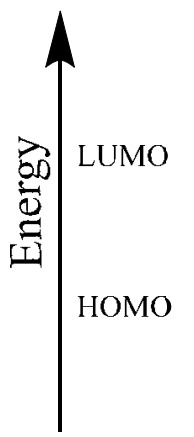

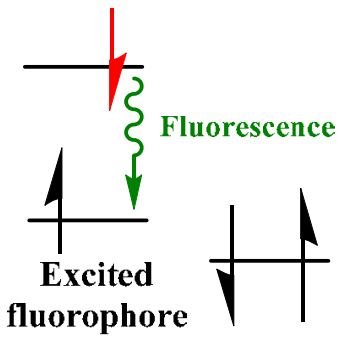

Bound receptor

Figure 7. PET process mechanism and fluorescent response (A) and orbitals diagram (B).

b) Photoinduced charge transfer (PCT):

PCT molecular sensors consist of a sensor in which the recognition unit is part of the fluorophore. Usually, the fluorophore contains an electron - donating group (for example an amino group) conjugated to an electron - withdrawing group. As a consequence, after excitation with light, it undergoes intramolecular charge transfer from the donor to the acceptor. (Figure 8)

The change in dipole moment (change in polarity/conjugation), when recognizing a species by the receptor unit, results in a Stokes shift that depends on the fluorophore, giving the opportunity to design probes on this basis.

\footnotetext{
${ }^{8}$ B. Valeur, Molecular Fluorescence: Principles and Applications, Wiley-VCH, Weinheim, Chapter 14, 2002.

${ }^{9}$ A. Prasanna de Silva, H. Q. N. Gunaratne, T. Gunnlaugsson, A. J. M. Huxley, C. P. McCoy, J. T. Rademacher, T. E. Rice; Chem. Rev. 1997, 97, 1515-1566.
} 


\section{PCT (Blue shifted)}

Charge transfer

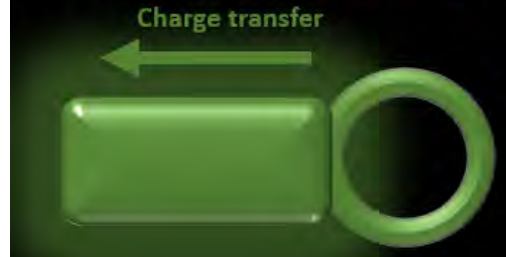

PCT (Red shifted)

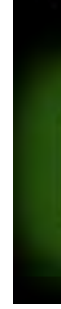

Charge transfer

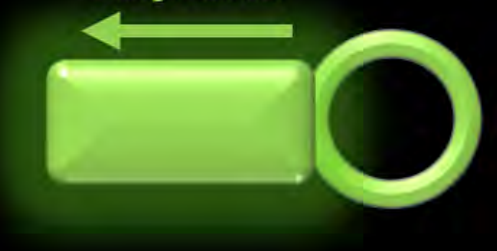

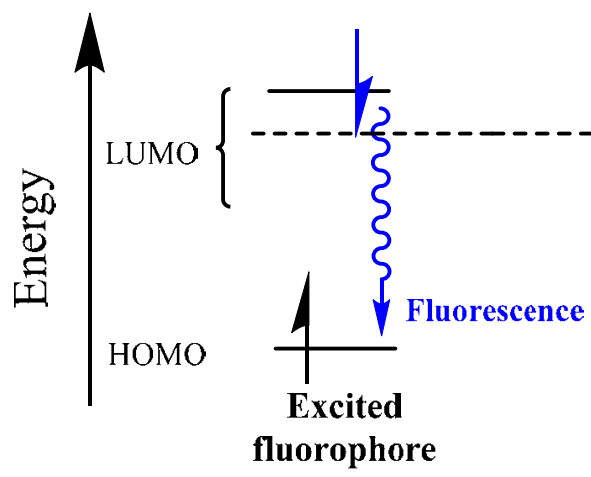

Blue-shifted
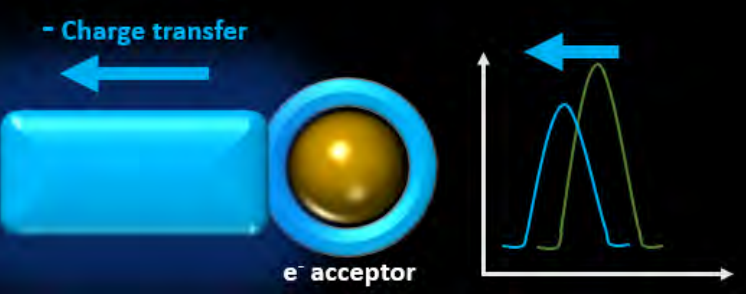

+ Charge transfer
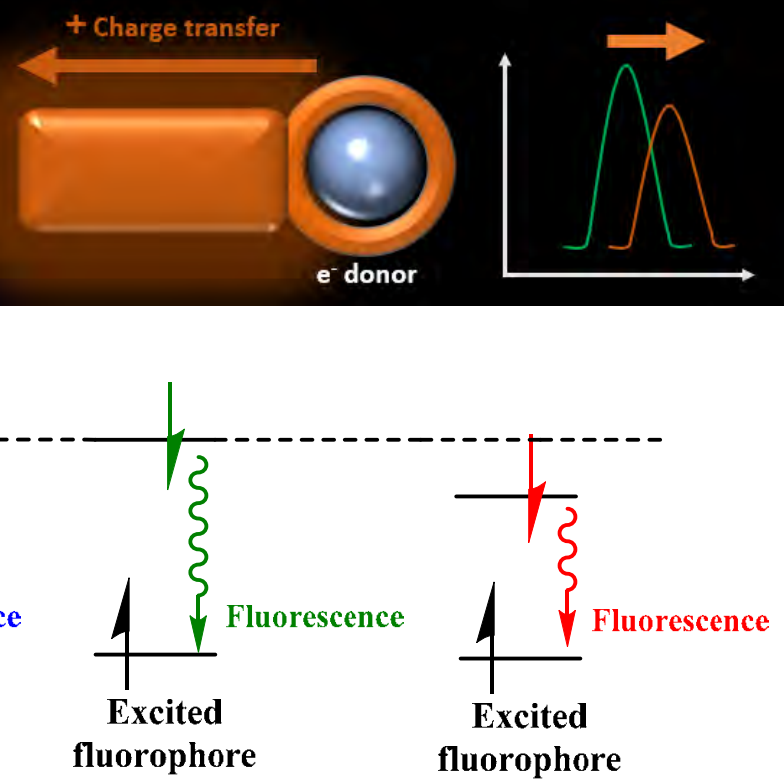

Red-shifted

Figure 8. PCT process mechanism and fluorescent response (up) and energy levels of frontier orbitals diagram (down).

\section{c) Excimer formation:}

This case involves two or more side chains bounded to the recognition unit, usually two fluorophores, that interact by $\pi-\pi$ stacking or other polarity involved forces. The fluorophores are joined by the recognition unit and the presence of the analyte modifies the interaction between them, altering the fluorescence. When this interaction between fluorophores gives a different wavelength of emission to the monomers it is called excimer, which allows ratiometric measurements. (Figure 9)

When developing sensors with this kind of mechanism the fluorophores must be carefully chosen. Groups with high likeness to interact with themselves are usually the best to choose, groups such as perylene derivatives. ${ }^{10}$

\footnotetext{
${ }^{10}$ K. V. Balakin, V. A. Korshun, I. I. Mikhalev, G. V. Maleev, A. D. Malakhov, I. A. Prokhorenko, A. Y. Berlin, Biosensors Bioelectron. 1998, 13, 771-778.
} 


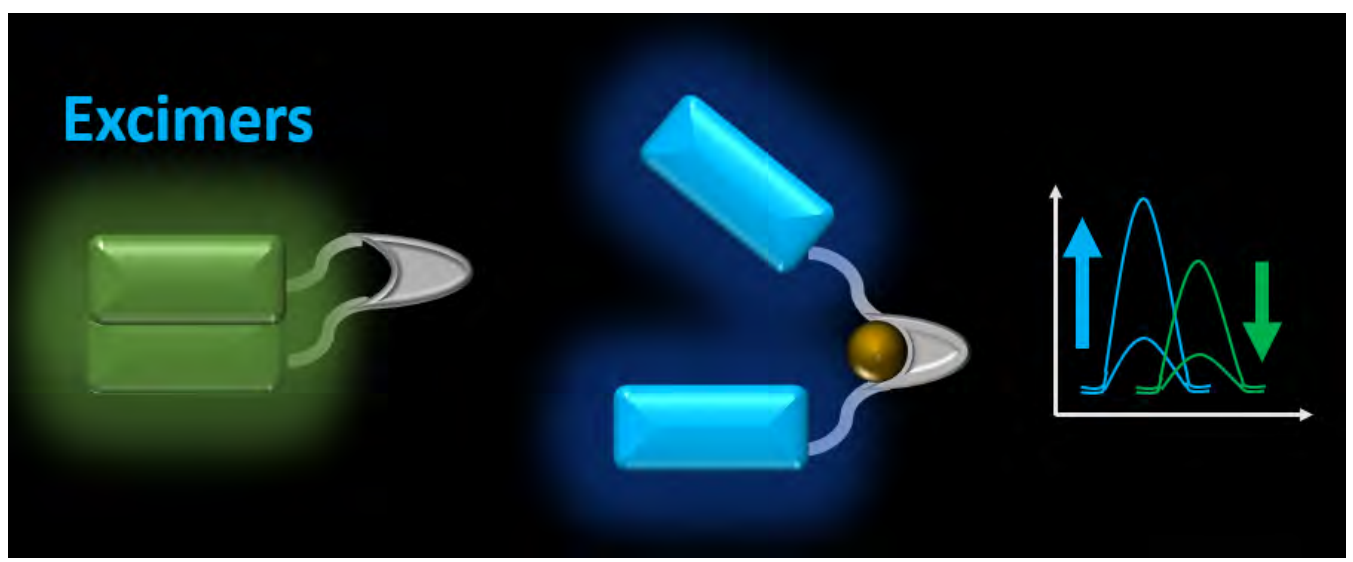

Figure 9. Excimers separation process mechanism when recognising an analyte and its fluorescent response.

\section{d) Förster Resonance Energy Transfer (FRET)}

In a similar way to excimers, there are two joined species involved, two different fluorophores in this case. One of the fluorophores acts as an acceptor, absorbing the light and transferring the energy to the other, which emits. The presence of a recognition unit between both fluorophores may alter the conformation and, as a consequence, the response. (Figure 10)

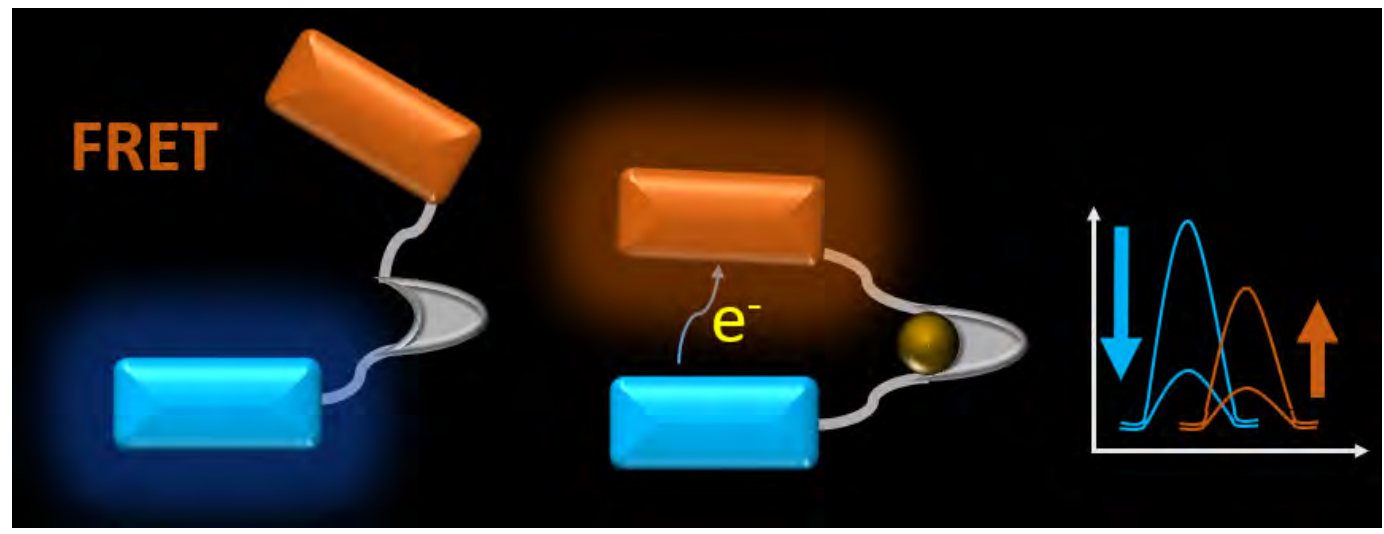

Figure 10. FRET process mechanism and fluorescent response.

e) Combination of several processes:

It is not usually taken into account when explaining how to design a molecular probe, but most of them work by several of the previously explained mechanism at once. Many probes are a mixture between PET a PCT processes, in which the fluorescence is inhibited by electron transfer (PET) but when the analyte is recognized the conjugation of the system also changes (PCT) what varies the emission wavelength. This is the case of the probe JG76, explained in Chapter 3C. 


\subsubsection{The recognition process is based on a reaction:}

It is also possible to detect the presence of analytes by having species that modify their fluorescence after a chemical reaction with the analyte. This kind of method is not usual for quantifying and detecting analytes because of its characteristics. A reaction destroys the samples, and the media must be strictly controlled to assure reproducibility. It usually has greater time dependence and it is very uncommon not having by-products.

In spite of the drawbacks, they present some advantages, such as greater changes in the signal, usually much more than in complexation processes. This characteristic leads to the possibility of lower limits of detection under ideal conditions. Undoubtedly, a particular case is based on the existence of catalytic reactions; it may lead to limits of detection far lower than any complexation reaction could reach. However, the quantification would not be so reliable, being capable of detecting traces of an analyte, but not being possible to determine exact quantities with certainty.

\subsection{Characteristics of an ideal fluorescent probe ${ }^{11}$}

In order to develop fluorescent probes that fulfil their purpose appropriately, they must reach certain standards, and possess suitable photophysical and analytical properties to the role they have:

\subsubsection{Photophysical properties:}

- High thermal and photochemical stability, to avoid easy and/or fast degradation.

- High molar absorptivity $(\varepsilon)$ : this value shows the absorption of the solution, if it is high, it means that it is easier to promote the electrons from the ground state, which could allow higher sensitivity and higher fluorescence.

- High final fluorescence quantum yield $\left(\Phi_{\mathrm{F}}\right)$, when the analyte is detected. It decreases the influence of dynamic quenching effects, and the measurements are more accurate and with less background noise.

- Excitation wavelength superior to $400 \mathrm{~nm}$, far for UV - blue excitation wavelengths. That is because high energy irradiation is most likely to degrade some samples, especially when they are biological.

- Emission wavelength, for experimental measurements it is better when the emission is above $450 \mathrm{~nm}$. The emission detectors are more sensitive in the region until $750 \mathrm{~nm}$ and, biological samples, have less interferents between $500-900 \mathrm{~nm}$ (many proteins emit in the UV-blue region).

- Large stokes shift between excitation - emission spectra. It would avoid reabsorption processes and simplify data treatment (overlapped signals). It becomes of upmost importance in cases such as FRET systems, to avoid HOMO-FRET between fluorophores.

- High fluorescent increase: so as to measure the fluorescence properly, the fluorescence must increase typically more than $100 \%$ in intensity; at some wavelength, once the analyte is detected.

In literature, it is very common to find publications in which the most remarked fact is how much increases the fluorescence, frequently underestimating the rest of the parameters. In reality, having 2

\footnotetext{
${ }^{11}$ M. D. Heagy, Chemosensors 2011, 13, 253-273.
} 
or 40-fold increase in fluorescence does not make it better or worse without taking into account the proper additional data. Figure 11 illustrates a representative example.
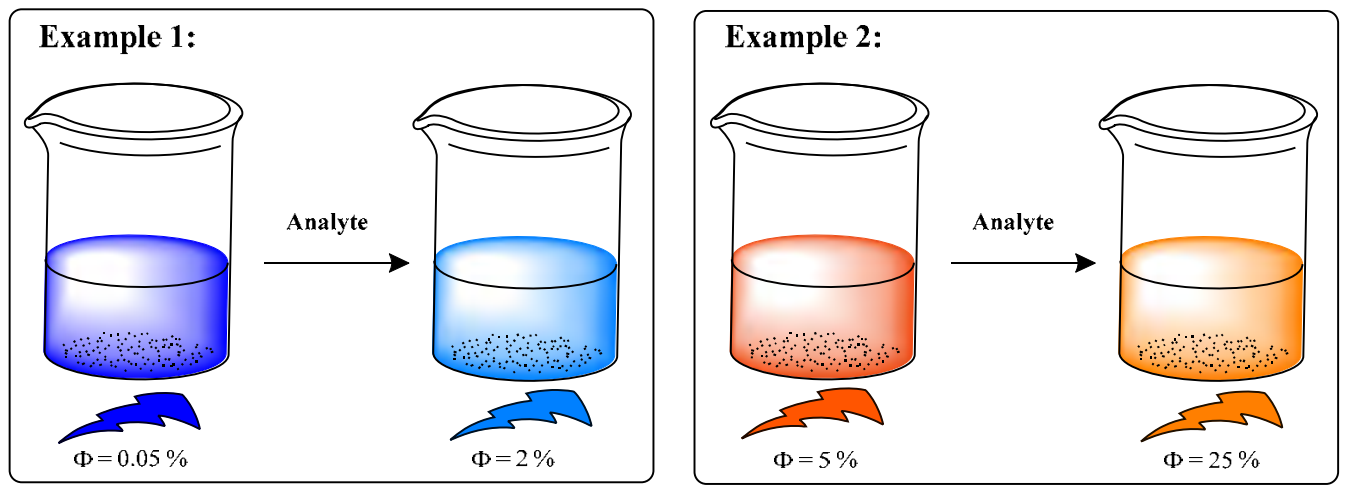

Figure 11. Two examples of fluorescence increase. Example 1: 40-fold increase (from $0.05 \%$ ).

Example 2: 5-fold increase (from $5 \%$ ).

In the example 1, a blue fluorescent probe has an initial Fluorescence Quantum Yield $\left(\Phi_{\mathrm{F}}\right)$ very low $(0.05 \%)$ which increases to $2 \%$, meaning 40 times increase, although with a very low fluorescent emission. In consequence the experimental error in measurements is likely to be very high and the possibility of having interferents would be high too.

On the other side, the example 2 shows an orange fluorescent probe with a 5-fold increase in fluorescence $\left(\Phi_{\mathrm{F}}\right.$ from 5 to $\left.25 \%\right)$. Apparently, the increase is much lower, mainly because the initial fluorescence was already high. However, if the fluorescence quantum yield is taken into account, the fluorescence is much higher and the possible experimental errors in the measurements far lower, being likely to be a much better probe than the one from Example 1. Additionally, as it was previously explained, a yellow-orange fluorescent probe is more appealing than a blue one, in regard to possible applications in biological samples and sensitivity of the detection systems.

\subsubsection{Analytical properties:}

- Direct measurements: The measurements are more reliable if they occur after the interaction molecule-analyte without needing a third specie. Having more than two species in equilibrium hinders the interpretation and decreases the reliability of the results.

- High selectivity: The probe has to be as selective as possible. Although $100 \%$ selectivity is not possible for any probe, especially when based on a complexation process, the best conditions must be studied and always taken into account.

- High sensitivity, reaching a low limit of detection. It is usually related with the selectivity to a certain extent, being less trustworthy when working close to the limit. For this reason, it is important that the detection limit was low, in order to work outside the limit conditions.

- ON fluorescence: The fluorescence is preferred to be $\mathrm{ON}$, as it is more selective and trustworthy than OFF, because the fluorescence can be always inhibited by many species, especially when working with concentrated solutions, as it was previously explained (Section 2.4).

- Water solubility (for most probes): water soluble probes are necessary for bio-applications. For instance, measuring cations in water (from rivers or living organisms) or to introduce them into cells. Despite the difficulties of doing it, because of the high hydration spheres of many ions and molecules in water, they are very interesting. 
- Recyclability: the probes that work by a complexation process are reusable in many occasions, by displacing the equilibrium. However, it is not usually possible, or much more difficult, when working with probes that react with the analyte.

Taking into account all these characteristics, the probes have to fulfil as many of them as possible. The importance of each one would be different depending on the final application.

\subsection{How to work with molecular sensors}

In the development of this thesis the processes followed to develop fluorogenic sensors may be summarized into a series of steps that were followed for every probe developed. In addition, although the study is about designing fluorescent molecular probes, many steps are the same for other type of sensors, especially colorimetric probes.

1) Synthesis of a fluorescent backbone.

2) Synthesis of the recognition part.

3) Joining the fluorescent moiety with the recognition part.

4) Testing and choosing an appropriate material as support (if required).

5) Characterization of the compounds, using techniques such as NMR, IR, melting point, Mass spectrometry analysis, elemental analysis, IR, SEM or EDX.

6) Studies of fluorescence: This part may be different depending on what is the purpose for the synthetized molecule or material. In general, for sensors that are useful in solution, the process starts with a solvatochromism; after that, some qualitative tests with different species, followed by the calculation of the work concentration, and next the existence of possible kinetic effects. Finally, the qualitative tests are performed, for instance calculation of the stoichiometry, and some other parameters, such as the limits of detection for different analytes, equilibrium constants, fluorescence quantum yields or fluorescence lifetimes decays are measured. 


\section{PURPOSE AND PROCEDURE WHEN PERFORMING FLUORESCENT STUDIES}

When working with fluorescent sensors is recommendable to follow a series of rules and to use probes adapted to the purpose they have. Hence, during the development of this thesis, a protocol has been developed for fluorescent measurements. ${ }^{12}$ It was elaborated and followed in order to ensure that all measurements were performed to obtain all required information through a useful, trustworthy and efficient method.

\subsection{Solvatochromism}

Whenever the probe is going to be used in solution, the first step is to check the solubility and behaviour in different solvents, which is fundamental to optimize the probe for the applications that are pursued.

As a consequence, there is a double purpose:

- Finding out the best solvent: the method is useful for studying the solubility in different solvents, which gives an idea about the possibilities of the probe. That is of upmost importance when the probes are made to work in specific solvents, (such as water). Furthermore, it is interesting for studying possible mixtures of solvents, whenever the desired is not possible. For example, mixtures Ethanol:Water.

- Studying the behaviour in different solvents: once the probes are dissolved, measuring the spectra of absorption and fluorescence and taking pictures under visible and UV-light, the change in colour and fluorescence, with polarity or using protic or aprotic solvents, is shown.

Along the development of the Thesis, a general procedure was followed:

For each compound, a mother solution was prepared, the solvent had to be quite volatile and one in which the probe was highly soluble and stable; chloroform was a usual candidate when working with organic molecules. From this solution, an aliquot was evaporated in different vials, one for each solvent that was measured. Once evaporated, the probe was redissolved in each one of the chosen solvents, under sonication if the probe was not soluble by stirring, and there was no risk of degradation. The final concentration was selected between $5-100 \mu \mathrm{M}$, depending on the characteristics of the probe. Finally, the absorbance and the fluorescence spectra were measured.

As an example, the study of the highly solvatochromic compound JG125 (deeply studied in Chapters 2 and 3A) is showed in Figures 12, 13 and 14. It was performed in different solvents (ordered by the Snyder polarity index):

\footnotetext{
${ }^{12}$ Several pictures are taken from the results shown in the next chapters, as an example to illustrate what kind of information is obtained. This is only a previous example for a better understanding and hence, they are deeply explained in their corresponding chapters.
} 

1. Water
2. $\mathrm{MeOH}$
3. DMSO
4. DMF
5. $\mathrm{MeCN}$
6. Acetone
7. AcOEt
8. THF
9. $\mathrm{CHCl}_{3}$
10. $\mathrm{CH}_{2} \mathrm{Cl}_{2}$
11. Toluene
12. $\mathrm{Et}_{2} \mathrm{O}$
13. Hexane
14. Cyclohexane

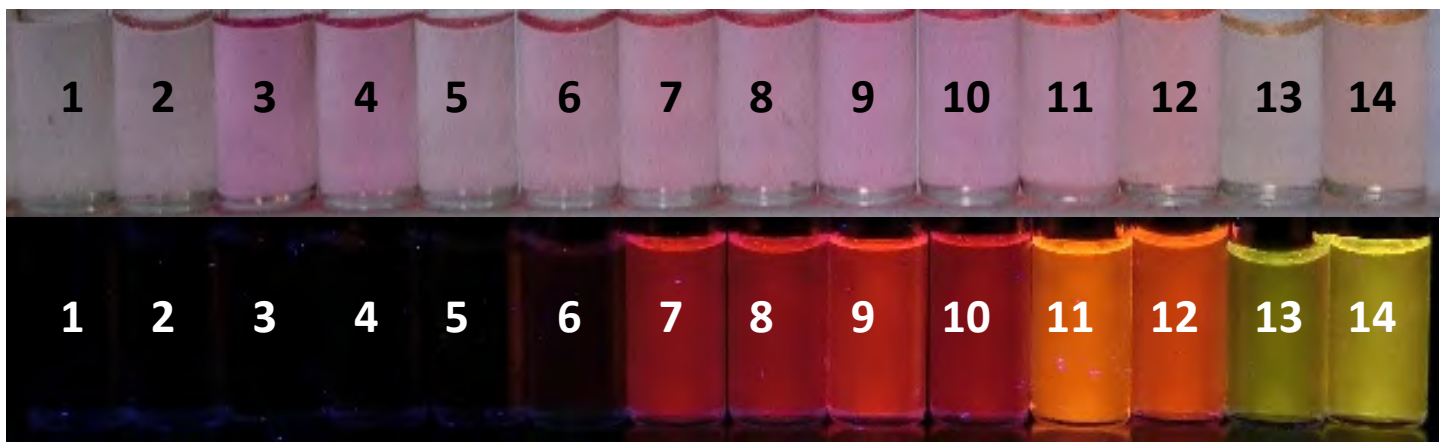

Figure 12. Solvatochromism of JG125 (10 $\mu \mathrm{M}$ solutions), pictures under visible (up) and UV light (down) in different solvents.
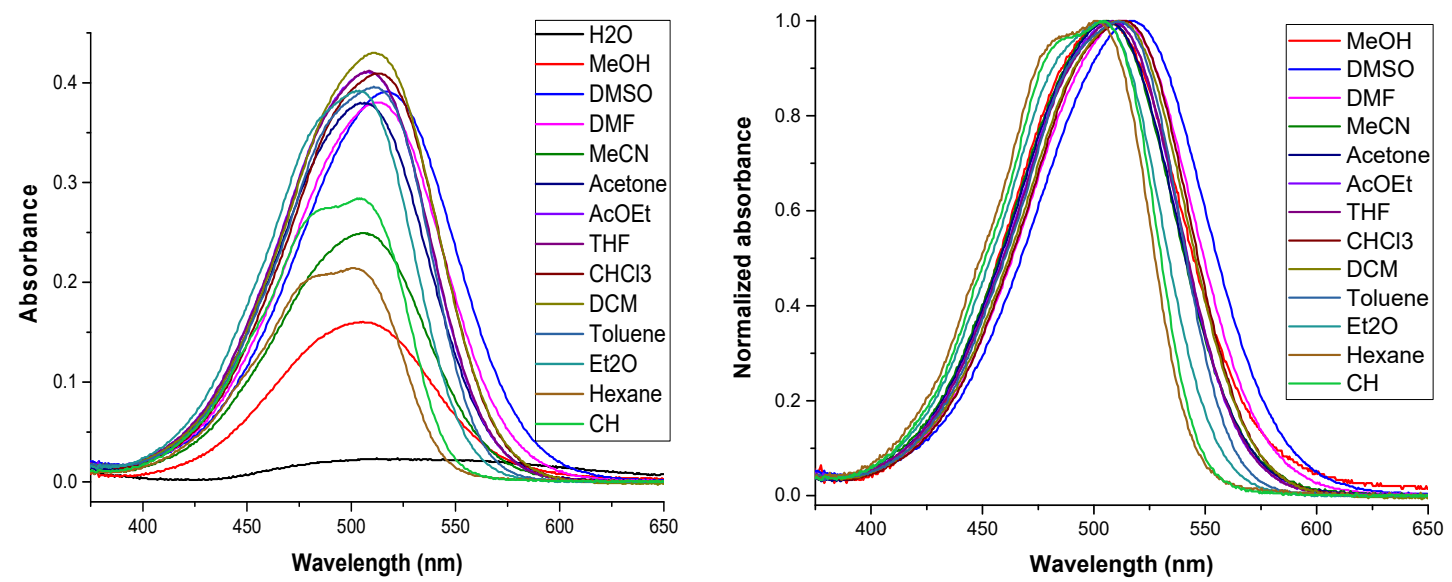

Figure 13. Solvatochromism of JG125 (10 $\mu \mathrm{M}$ solutions), absorbance in different solvents.

The fluorescence was measured by excitation at $512 \mathrm{~nm}$ :
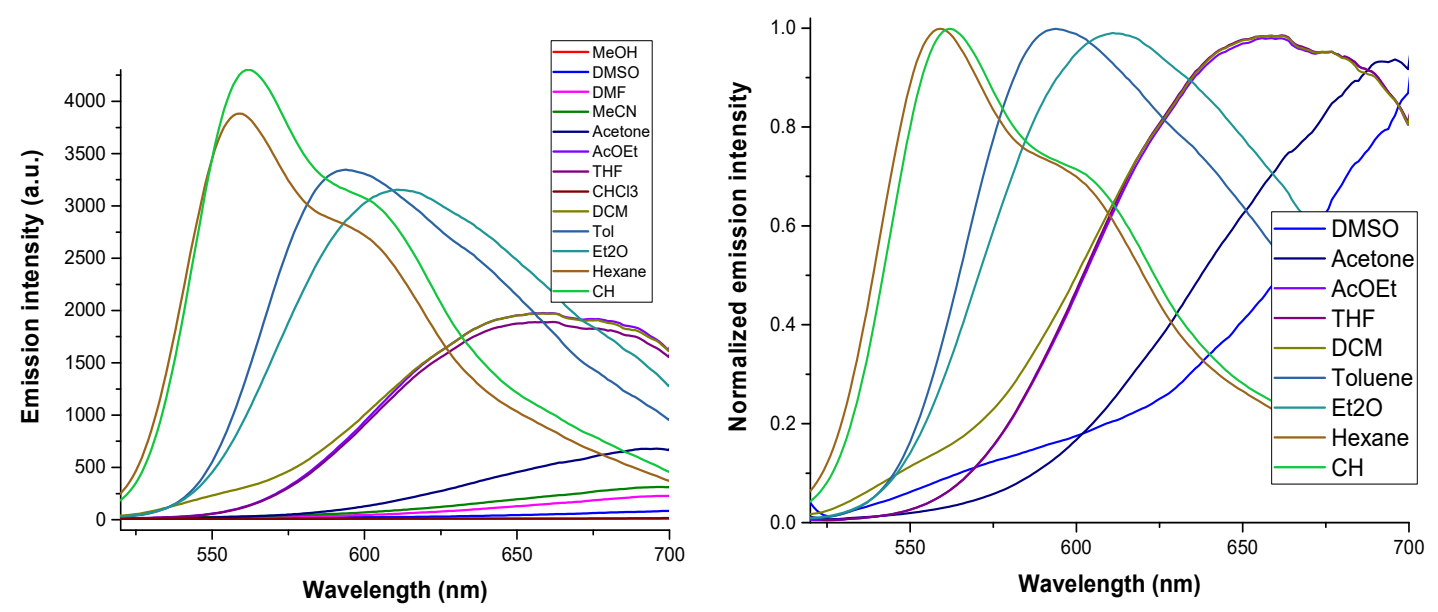

Figure 14. Solvatochromism of JG125 $\left(10 \mu \mathrm{M}\right.$ solutions), fluorescent response when $\lambda_{\mathrm{exc}}=512 \mathrm{~nm}$, in different solvents. 
Taking the previous example as reference, the conditions to select the most suitable solvent are summarized as follows:

- The solubility of the probe must be high. "High" is understood as enough to prepare solutions at least close to $0.1 \mathrm{mM}$ solutions. Having lower solubility implies an added difficulty to perform titrations.

- The solvent must be miscible with water, if the purpose is to analyse water samples or in/from living beings. The importance may vary depending on what is being measured and how.

- Low fluorescence. The ideal probes are OFF-ON, so the initial fluorescence must be low. Considering an initial quantum yield high, for example, $90 \%$, would not let a margin for any noticeable fluorescent improvement. This condition does not apply when the recognition takes place by the appearance of a new band at another wavelength.

Once the solvatochromism is finished, the best solvent would be the one that has better outcomes fulfilling the photophysical, analytical and specific conditions that are searched for. However, it is not easily chosen until the next steps are performed. Usually, from solvatochromism studies, the most likely solvents are chosen and, after several of the subsequent tests, the best one is selected.

For the given example, JG125, it was not soluble in water, it was highly solvatochromic and it was used for detection of explosives. Hence, it was not important to dissolve the probe in water; moreover, the solubility and fluorescence in solvents such as chloroform was enough for using it as sensor in solution.

\subsection{Tests with different species; cations, anions, oxidative, reductive species, amines...}

For a start, qualitative tests with several species are usually performed to check the ability of the probe as a selective detector. There are several characteristics that define a probe and may be obtained from these tests. For instance, an ideal probe will respond with:

1) High selectivity. There should be a noteworthy difference in the response under the presence of the analyte, significantly different from possible interferents.

2) High emission when the analyte is recognized. The final fluorescence should not be negligible, fluorescence quantum yields $\left(\Phi_{\mathrm{F}}\right)$ superior to $3 \%$ at least. Having lower fluorescence quantum yield would make the measurements unreliable and, probably, it would require to open the fluorometer slits, which would increase the errors and possible interferents exponentially.

3) High increase in emission. Around a $100 \%$ increase in fluorescence is required for a good quantification, although there is always interferents that affect the measurements. In this regard, it is quite common that, for example, acid pH may increase fluorescence. In any case, the interferents must be significantly lower than the response to the analyte.

Taking into account the ideal characteristics of a probe, the procedure followed for laboratory testing is described as: 
The probe of interest was dissolved in the solvent previously chosen. The concentration usually varied between $0.01-0.1 \mathrm{mM}$, depending on the solubility-fluorescence of the compound. The species to test were added from concentrated solutions, usually 10 microliters from mother solutions in water, and the change in colour and fluorescence was recorded by taking pictures under visible and UV light (366 nm), with different concentrations of the species and at different time periods.

In addition to the requirements of the procedure, the possibility of having solvent mixtures is also important; moreover, buffered solutions may be used for distinguishing $\mathrm{pH}$ effect from other recognition processes in case of necessity.

As an example, Figure 15 shows the compound JG76, a sensor used for potassium and lead cations detection (deeply studied in Chapter 3C). JG76 was first dissolved in EtOH, from which a final solution was JG76, $20 \mu \mathrm{M}$, in $70 \% \mathrm{EtOH}-30 \% \mathrm{H}_{2} \mathrm{O}(\mathrm{v} / \mathrm{v})$ buffer solution, $20 \mathrm{mM}$ of HEPES.

Sequence: Reference - Water $-\mathrm{Zn}\left(\mathrm{ClO}_{4}\right)_{2}-\mathrm{K}\left(\mathrm{CF}_{3} \mathrm{SO}_{3}\right)-\mathrm{Fe}\left(\mathrm{ClO}_{4}\right)_{3}-\mathrm{Sn}\left(\mathrm{ClO}_{4}\right)_{2}-\mathrm{Ba}\left(\mathrm{ClO}_{4}\right)_{2}$ $\mathrm{Pb}\left(\mathrm{ClO}_{4}\right)_{2}-\mathrm{Be}\left(\mathrm{NO}_{3}\right)_{2}-\mathrm{Cu}\left(\mathrm{ClO}_{4}\right)_{2}-\mathrm{Al}\left(\mathrm{ClO}_{4}\right)_{3}$

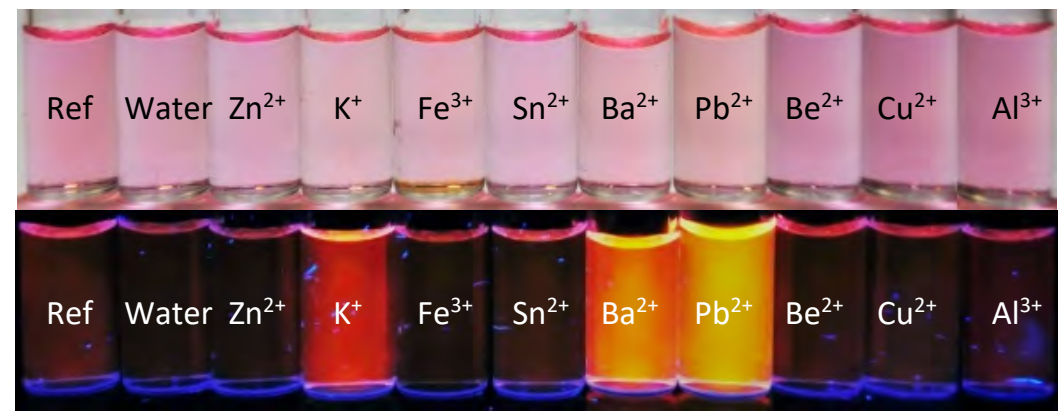

Figure 15. JG76 in ethanol $(20 \mu \mathrm{M})$, in a mixture with a buffer solution of different $\mathrm{pH}, 20 \mathrm{mM}$ of HEPES, pH 7. Adding different cations $0.1 \mathrm{mM}$. Under visible and $366 \mathrm{~nm}$ UV light.

The conditions for qualitative studies should be optimized, depending on the specifics of each probe, to the best conditions for quantitative acquisition of the absorbance-fluorescence measurements. Nevertheless, it is not less important to notice that making such amount of measurements with different analytes quantitatively and in optimal conditions will be a tedious and very long task from which only a small part of the data would have some interest. It should be kept in mind that these results are preliminary to find possible analytes of interest and their interferents.

From the previous example some cations were of particular interest, therefore their fluorescent spectra were registered and compared, as it is shown in Figure 16.
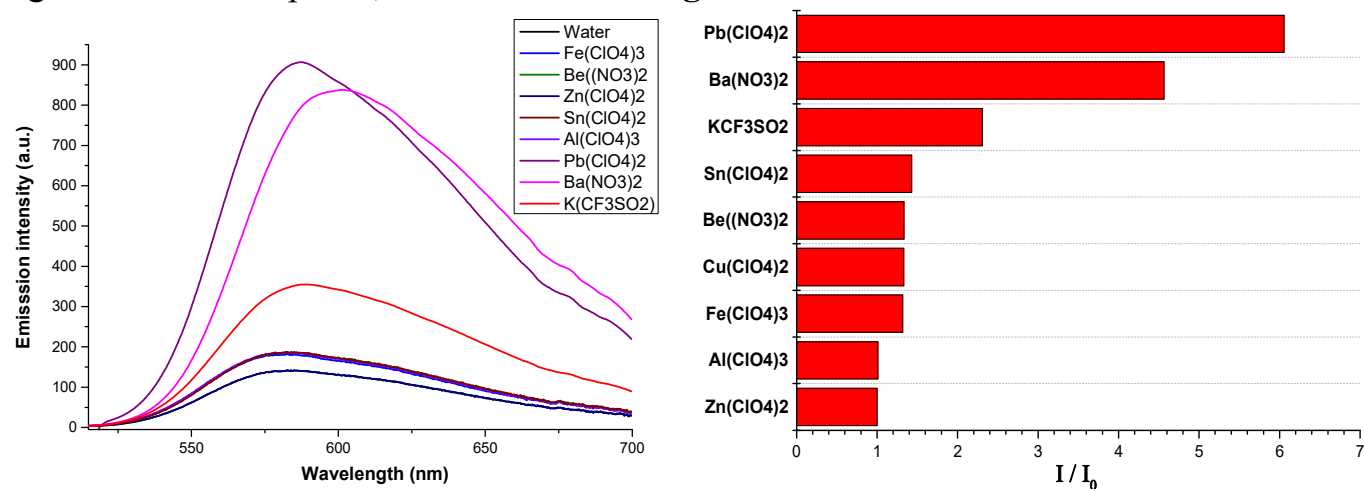

Figure 16. JG76 in ethanol $(20 \mu \mathrm{M})$, in a mixture with a buffer solution of different $\mathrm{pH}, 20 \mathrm{mM}$ of HEPES, pH 7. Adding different cations $0.1 \mathrm{mM}$. Fluorescence spectra (left) and increase in total emission (right). $\lambda_{\mathrm{exc}}=500 \mathrm{~nm} . \lambda_{\mathrm{em}}=580 \mathrm{~nm}$ 
In this case, it was concluded that there was selectivity for potassium, lead and barium cations, increasing fluorescence between 3 to 7 -fold. $\left(\Phi_{\mathrm{F}}(\mathbf{J G 7 6}-\mathrm{EtOH})=0.12\right)$

In fact, it is usual to show a comparison between different possible interferents and the analytes, an example is shown in Figure 17 for JG76.

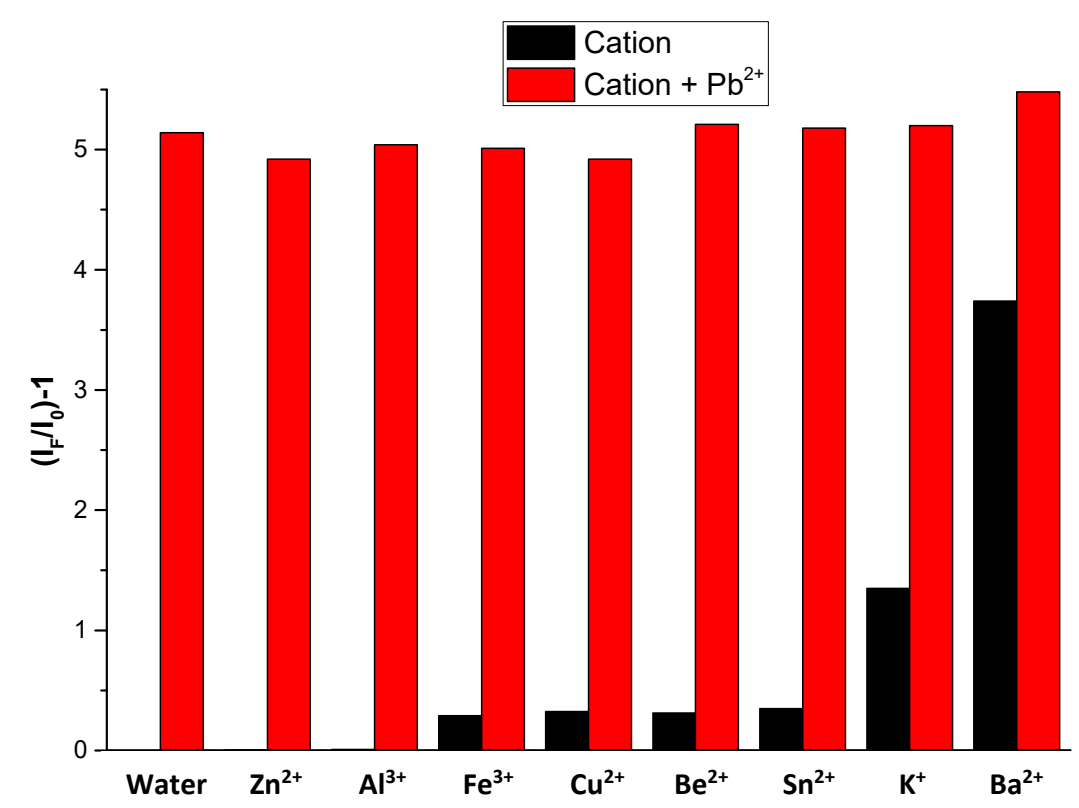

Figure 17. JG76 in EtOH:Buffer $(20 \mu \mathrm{M})$, the buffer solution is, $10 \mathrm{mM}$ of HEPES, pH 7. Adding different cations $0.1 \mathrm{mM}+\mathrm{Pb}^{2+} 0.05 \mathrm{mM}$. Increase in total emission. $\lambda_{\mathrm{exc}}=500 \mathrm{~nm} . \lambda_{\mathrm{em}}=580 \mathrm{~nm}$.

This kind of tests are usually explained to give an idea of how much the fluorescence increases. However, it is common to find that some authors show the increase in fluorescence directly related with how good the probe is, which could be not only misleading but false, if parameters such as the fluorescence quantum yield are not taken into account.

\subsection{Work concentration and molar extinction coefficients $(\varepsilon)$}

Before doing any calculation it is not only desirable but necessary to check the behaviour of the probe at different work concentrations. There are several reasons to perform this test. On one hand, because working at "high concentrations" may be misleading in the determination of some characteristic values of the probe, in fact, it is possible to have competitive processes such as dimerization or dynamic quenching. On the other hand, working at too low concentrations may be subject to signal-noise issues, more influenced by possible impurities or the absence of signal if the sample is slightly diluted. Of course, what is called "high concentration" or "low concentration" is dependent on many factors, which mainly are the system (spectrophotometer of absorbance and fluorescence), the probe, the analyte and the media. So that, it is the reason why, in order to avoid possible extra parameters and/or possible undesired side processes, the conditions are usually high dilution and constant ionic force.

In working conditions, little variations in concentration should change the absorption fluorescence slightly and linearly and for absorbance, the ordinate in the origin should be 0 , which is called fulfilling the Lambert-Beer law. 
The Lambert-Beer equation is defined as:

$$
\mathrm{A}=\log \frac{I-I_{0}}{I_{0}}=\varepsilon \times c \times l \quad \text { Equation }[\mathrm{C}]
$$

Where $\mathrm{A}$ is the absorbance at a given wavelength, $\mathrm{I}_{0}$ is the intensity of the light at the chosen wavelength, I the intensity that reaches the detector, $\varepsilon$ the molar extinction coefficient and 1 the width of the cuvette.

Typically, when working with absorbance-fluorescent probes, the response to different concentrations is evaluated around $0.1-10 \mu \mathrm{M}$. Afterwards, it was represented and adjusted to a linear regression around the chosen value. The concentrations between the change in the signal is linear is considered the working range. This working range would allow variations in concentration of the probe without having changes in the signal that would not be associated to analyte-probe interaction. In addition, after choosing the desired solvent or solvents, the molar extinction coefficients $(\varepsilon)$ may be calculated throughout the formula adjusted to a linear regression, what is useful to know how much radiation is absorbed by the probes in solution. For instance, higher coefficients are useful for applications such as solar cell electron sensitizers.

Additionally, with fluorescent probes, this process is double checked by absorbance and fluorescence (pseudo Lambert-Beer linear behaviour), because of the higher sensitivity of the second. In practice, it usually means having absorptions around 0.1 , and fluorescence with high signal-noise ratio, without opening the slits too much (it depends on the specific properties of the fluorometer).

\subsection{Kinetic effects}

When working with molecular sensors, the factor time is important so as to detect an analyte. Usually, the complexation equilibrium in solution is reached very fast, as much as within the range of seconds. This simplifies the study of the compound by titration and increases the applications as a probe.

In a 1:1 equilibrium it was represented such as:

$$
\mathrm{P}+\mathrm{A} \underset{\mathrm{k}_{2}}{\stackrel{\mathrm{k}_{1}}{\rightleftharpoons}} \mathrm{PA}
$$

Where $\mathrm{k}_{1}$ and $\mathrm{k}_{2}$ are the kinetic association and dissociation constants respectively

Being the thermodynamic equilibrium constant

$$
K_{e q}=\frac{k_{1}}{k_{2}} \quad \text { Equation [D] }
$$

As a consequence, in order to perform a titration in which calculations are performed in the equilibrium, it is necessary to know how fast the equilibrium is reached before giving data about thermodynamic constants, stoichiometry or other equilibrium dependent factors. In any case, if it is necessary, there is plenty of literature ${ }^{13}$ and methods about how to perform the calculations of kinetic constants or other kinetic parameters. In this regard, it is common to make calculations for first order

\footnotetext{
${ }_{13}$ J. I. Steinfeld, J. S. Francisco, W. L Hase, Chemical Kinetics and Dynamics, Prentice Hall, Upper Saddle River, New Jersey, $2^{\text {nd }}$ edition, 1999.
} 
interaction (1:1) analyte-probe. To do so, techniques such as the T-Jump can measure equilibria reached in microseconds.

In contrast, this is not the case when working with materials. For most materials, factors such as the swelling, the diffusion of the analyte within the material, or the thickness, have direct influence over the results. However, it is not possible, or too complicated, to calculate thermodynamic or kinetic constants. In any case, the determination of other parameters such as the limit of detection or the fluorescence quantum yield are dependent on the kinetics too, hence the conditions should be always specified.

\subsection{Titration methods}

Once the work concentration is chosen, it is possible to perform a titration by any of two processes (Figure 18), and it may be extrapolated to most spectrometric or spectrophotometric measurements (absorption, fluorescence, NMR titration...):

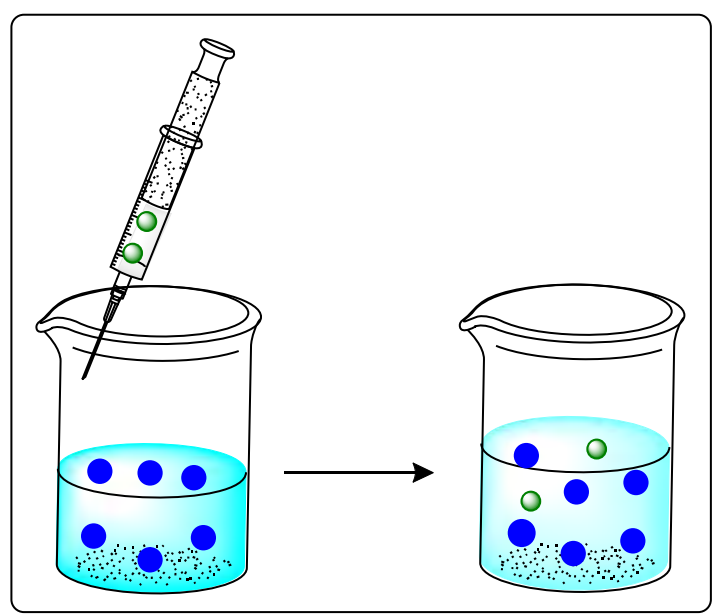

Method 1: No probe in the additions

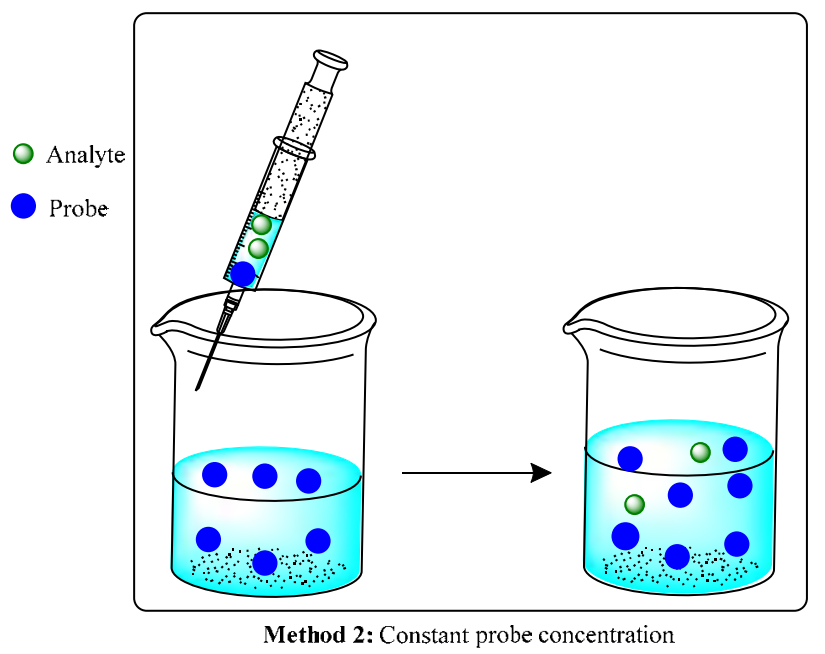

Method 2: Constant probe concentration

Figure 18. Scheme of two methods for adding analyte to solution for titrations.

A) Method 1: adding concentrated analyte to a diluted solution of the probe. It has to be performed always within the range in which the Lambert-Beer law is fulfilled. When performing titrations this way, the analyte is added dissolved in the same solvent (it is possible not to, but the conditions must be carefully explained and it would add more factors of uncertainty to the titration).

Many authors dislike this method because of the dilution effect. Consequently, it is very important to check that Lambert-Beer law (absorbance) and pseudo-Lambert-Beer (fluorescence) conditions are fulfilled in the working range of the titration. Some analysis like NMR titrations are not so much dependent on variations in concentrations of probeanalyte; however, it may have side effects such as an increase in the relation signal-noise or the broadness of the signals.

B) By adding the concentrated analyte previously dissolved in a solution of the probe: it is the most standardized method. It eliminates the problem of changing the concentration of the probe, reducing possible errors derived from it and simplifying the interpretation of the data. 
Nevertheless, there are also several issues that must be taken into account when using this method. In doing so, the analyte is preconcentrated in probe solution so:

a. It is not possible to follow the process if it is a reaction, because it would start at the moment that probe and analyte are mixed.

b. Although the concentration of probe does not change, the possibility of having dimers or other processes should be studied (Lambert-Beer behaviour). For instance, it could happen that the analyte triggers a disaggregation process that causes ratiometric response and, as a consequence, the treatment of the data might be different.

c. The amount of probe consumed is high (important when the amount is very limited), it could make it more desirable to perform the tests using as low quantity of product as possible.

There are other factors to take into account, besides the consequences of performing the titration one way or another, such as the kinetic response probe-analyte or the changes in solubility of the probe when the analyte is added. Furthermore, when the response is highly dependent on time (within the range of several minutes or more) calculating the equilibrium constants or limits of detection becomes a much more difficult task, as it is explained in sections 3.7 and 3.8.

\subsection{Stoichiometry determination}

Until the last few years, in literature, the standardized method for calculating stoichiometry complexations in a titration analyte-probe was the continuous variation method, also known as Job's Plot analysis. ${ }^{14}$ This process consists of a representation of the molar fraction of the analyte against the signal ratio per molar fraction.

$$
x_{A} \text { vs } x_{A} \times|\Delta S|
$$

Where $\mathrm{x}_{\mathrm{A}}$ is the molar fraction of the analyte and $|\Delta \mathrm{S}|$ is the absolute value of the variation of the signal.

Theoretically, when it is graphically represented, a parabolic figure indicates the stoichiometry depending on the position of the maximum of the quadratic function (see example in Figure 19). If 0.5 , the complex would be 1:1 (probe:analyte), 0.66 implies 1:2, 0.33 would be $2: 1$ and so on. Additionally, it should indicate even more complex processes (such as 2:3 or 1:4), but they are less common and it would be even more difficult to demonstrate them as correct, as it is explained in the next paragraphs.

Provided that the working conditions have no interferents and that the ionic strength of the media is controlled, this process should be enough to determine the stoichiometry by fluorescence; or by other techniques based on measuring the changes caused by the interactions of probe-analyte, such as NMR. Specifically, in the case of fluorescence/absorbance, the concentration of probe has to be placed between the range when the Lambert-Beer law is fulfilled and linear, of course, the fluorescence has to be measured with fixed $\lambda_{\text {exc }}$ and $\lambda_{\text {em }}$ (or the $\Delta$ of the emission area).

\footnotetext{
${ }^{14}$ J. S. Renny, L. L. Tomasevich, E. H. Tallmadge, D. B. Collum, Angew. Chem.Int. Ed. 2013, 52, 11998-12013.
} 


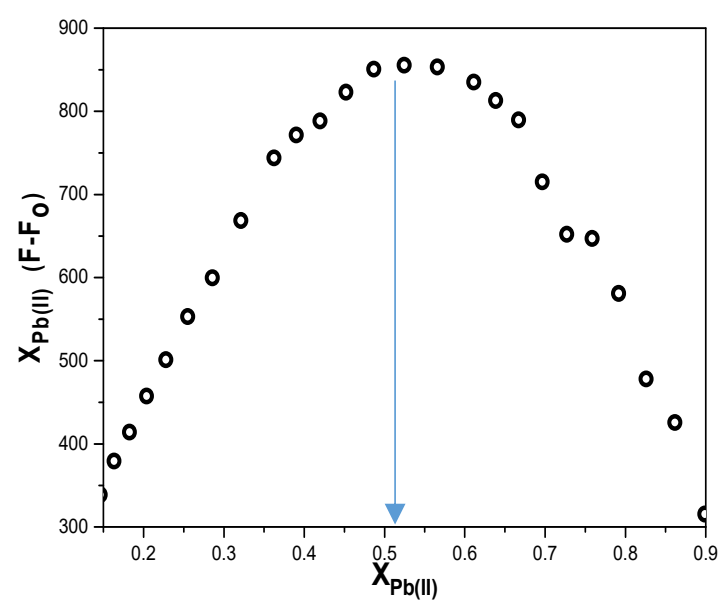

Figure 19. Job's plot of a 1:1 process, probe JG76 $(5 \mu \mathrm{M})$ for $\mathrm{Pb}^{2+}$ detection in $\mathrm{EtOH}($ Chapter 3C).

Nonetheless, in the last years the validity of the Job's plot method for calculating stoichiometry has been often questioned. In this regard, several papers have been published showing experimental results and simulations that demonstrate the limitations of this method, and how it is usually applied incorrectly. ${ }^{15}$ In the paper by Hibbert and Thordarson ${ }^{15}$ it is thoroughly explained why it is not possible to apply this method in every case. In particular, by referring to the work of Jurczak and coworkers, ${ }^{16}$ they simulated the results of Job's Plot 1:2 and 2:1 with different relations between (molar fraction):(equilibrium constants). Surprisingly, the Job's Plot analysis gave information about the relation between constants, but the condition in which it is related to stoichiometry is limited, and impossible to predict without knowing the equilibrium constant previously to the titration. Additionally, some authors point out that the results may still have some reliability when the relation between the equilibrium constant and the concentration is:

$$
\text { Keq }>>1 /[\text { Host }]
$$

In practice, where either both $\mathrm{K}_{1}$ and $\mathrm{K}_{2}$ (hypothetical 1:2 equilibrium) are large or one of them is relatively large compared to the other, Job-plots appear to be valid. But this would imply knowing the stoichiometry before calculating it, which makes the method quite unfit for such calculations. Considering the results, the authors conclude that the best way to proceed is to calculate the stoichiometry by fitting the titrations to different models $1: 1,1: 2,2: 1 \ldots$ The one with the "best-fitting regression model" is likely to be the real stoichiometry.

In order to clarify concepts, the results are considered as the "best-fitting regression model" when the error is low, there is no tendency in the residues (the scatter of the residual plot) and statistic tests, that validate the relation data-fitting (such as the F-test for the sum-of squares regression), are performed with positive results.

In conclusion, the best method to determine the stoichiometry proceed by adjusting the equilibrium constant to several possible models. The most likely correct results are related to the best fitting, being the Job's Plot a reassuring calculation but never determining.

\footnotetext{
${ }^{15}$ D. B. Hibbert, P. Thordarson, Chem. Commun. 2016, 52, 12792-12805.

${ }^{16}$ F. Ulatowski, K. Dabrowa, T. Balakier and J. Jurczak, J. Org. Chem. 2016, 81, 1746-1756.
} 


\subsection{Thermodynamic equilibrium constant calculation (K)}

The equilibrium constants between analyte-probe in solution may be measured under different conditions. Equilibrium constants not only depend on the solvent, but also on the ionic strength and temperature. In order to ensure reliability in their determination, the ionic strength and temperature must be constant during the titration and the concentrations must be as low as possible (avoiding dimers or other interactions).

As it was previously explained, the applied methods for titrations work at high dilution conditions by increasing the concentration of the analyte. In addition, the results may be compared by using different variations such as changing the concentration of the probe, the analyte additions or even doing the titration of the analyte with probe. In any case, if the probe is not likely to have side processes, such as aggregation, the results are usually comparable and with high repeatability and robustness. Moreover, the calculation of equilibrium constants when having slow association and/or dissociation constants must be done by waiting the necessary amount of time until the species reach equilibrium, taking into account that the necessary amount of time is usually dependent on the concentration of the species (first order or more).

There are several methods for performing experimental calculations, what is more, there is plenty of software available ${ }^{17}$ to make this kind of calculation fast and simple. The software is continuously updated and with plenty of explanations about how to adapt it to the most common applications, such as fluorescence, absorbance, circular dichroism or NMR titrations. These methods work by iterative fitting, however, there are some alternatives. Nowadays, the use of outdated calculation methods that rely in approximations is still very spread. The most common is based on Benesi-Hildebrand equation. ${ }^{18}$ Nevertheless, their use makes no sense nowadays, because of the easy access to more accurate methods without needing for relying on approximations; surprisingly, BenesiHildebrand and similar equations are frequently found in recent publications in the topic of sensors. ${ }^{19}$

The next part will consist on the theory behind the constant calculation, briefly explained from the point of view of a fluorescent probe, starting with 1:1 complexes.

\subsubsection{Equilibrium constant of the complex ML (1:1)}

The complexation reaction can be stated by the following equilibrium:

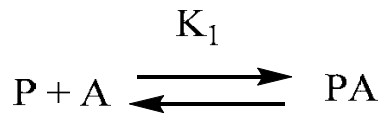

Where $\mathrm{P}$ represents the probe and A the analyte

By taking into account the mass balance and the fluorescence:

$$
C_{P}=[P]+[P A]
$$

17 An example of webpage adapted to calculate equilibrium constants by iterative methods: http://supramolecular.org, accessed $23^{\text {th }}$ June 2018. It might be also calculated by software, such as the used for calculations: Origin v2016. The equations were adapted for the software with the help of professor Saturnino Ibeas Cortés from University of Burgos.

${ }^{18}$ B. H. Hildebrand. J. Am. Chem. Soc. 1949, 71, 2703-07.

${ }^{19}$ Two examples, A) A. Ghosh, S. Das, S. Kundu, P. K. Maiti, P. Sahoo, Sensors and Actuators B: Chem. 2018, 266, 80-85. B) S. Fernández-Alonso, T. Corrales, J. L. Pablos, F. Catalina, Sensors and Actuators B: Chem. 2018, 270, 256-262. 


$$
\begin{gathered}
C_{A}=[A]+[P A] \\
I_{F}=f_{P}[P]+f_{P A}[P A]
\end{gathered}
$$

Where $C_{A}, C_{P}, I_{F}, f_{P}$ and $f_{P A}$ are the total concentrations of the probe $\left(C_{P}\right)$ and analyte $\left(C_{A}\right)$, the intensity of fluorescence and the proportional fluorescence factors of the probe and the complex PA, respectively. The rest of the parameters are the concentrations of the species in the equilibrium. By calculating the concentration of the probe in the equilibrium on the equation (1) and substituing on the equation (3), the following equation is obtained:

$$
I_{F}=f_{P} C_{P}+\left(f_{P A}-f_{P}\right)[P A]
$$

The definition of the equilibrium constant states that:

$$
K_{1}=\frac{[P A]}{[P][A]}=\frac{[P A]}{\left(C_{P}-[P A]\right)\left(C_{P}-[P A]\right)}
$$

By calculating $[\mathrm{PA}]$ on the previous equation (5), it may be replaced on equation (4), obtaining equation (6):

$$
I_{F}=f_{P} C_{P}+\frac{f_{P A}-f_{P}}{2}\left[C_{P}+C_{A}+\frac{1}{K_{1}}-\sqrt{\left(C_{p}+C_{A}+\frac{1}{K_{1}}\right)^{2}-4 C_{P} C_{A}}\right]
$$

This equation is used by iterative fitting in function of $\mathrm{C}_{\mathrm{A}}-\mathrm{I}_{\mathrm{F}}$ (experimental data), solving a nonlinear least square regression, starting by giving initial values of $K_{1}, f_{P}$ and $f_{P A}$. The results obtained are similar to the example from Figure 20 (JG76, Chapter 3C).

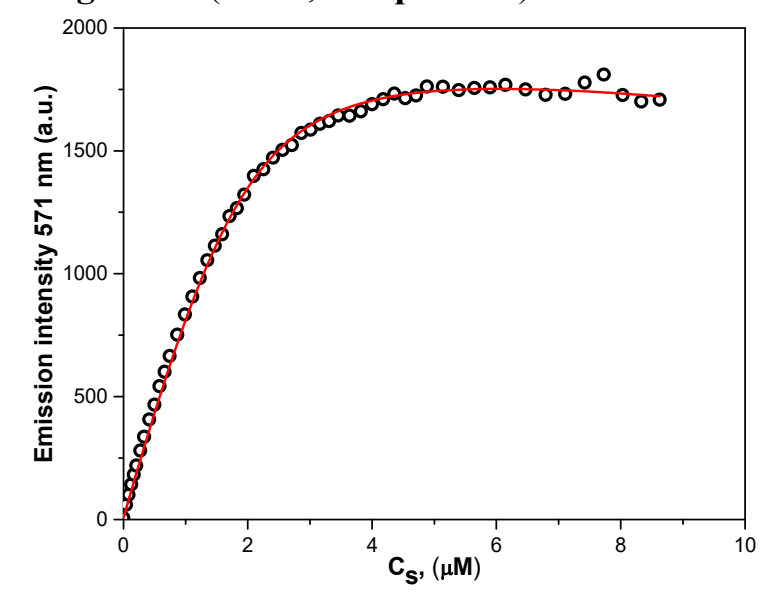

Figure 20. Fitted fluorescent emission of a titration with $\mathrm{K}\left(\mathrm{CF}_{3} \mathrm{SO}_{3}\right)$ of a $2 \mu \mathrm{M}$ solution of JG76 in EtOH.

In practice, the fitting calculation of the complexation constants is repeated several times and the value of $\mathrm{K}$ is estimated from it. 


\subsubsection{Complexes $\mathrm{M}_{2} \underline{\underline{L}(2: 1)}$}

Similarly, the equations for the equilibria 1:2 (analyte:probe) and 2:1 might be calculated, an extensive explanation might be found in literature. ${ }^{20}$ This leads to obtain the expressions:

$$
\begin{array}{cc}
\mathrm{M}+\mathrm{L} \rightleftharpoons \mathrm{ML} & K_{1}=\frac{[M L]}{[M][L]} \\
\mathrm{ML}+\mathrm{M} \rightleftharpoons \mathrm{M}_{2} \mathrm{~L} & K_{2}=\frac{\left[M_{2} L\right]}{[M][M L]} \\
A=\frac{A_{0}+C_{0} b K_{1}[M]+A_{\text {lim }} \beta[M]^{2}}{1+K_{1}[M]+\beta[M]^{2}} & \text { (Equation [E]) }
\end{array}
$$

Where $\beta$ represents the cumulative or overall constant. This $\beta$ is the constant for the formation of a complex from reagents and can be expressed as the product of each constant, which considers the formation of the complex step by step. For instance, the cumulative constant for the formation of $\mathrm{M}_{2} \mathrm{~L}$ is given by $\beta=\mathrm{K}_{1} \mathrm{~K}_{2}$, b is the molar extinction coefficient of the intermediate complex ML.

\subsubsection{Complexes $\mathrm{ML}_{2}(1: 2)$}

$$
\begin{gathered}
\mathrm{M}+\mathrm{L} \rightleftharpoons \mathrm{ML} \quad K_{1}=\frac{[M L]}{[M][L]} \\
\mathrm{ML}+\mathrm{L} \rightleftharpoons \mathrm{ML}_{2} \quad K_{2}=\frac{\left[M L_{2}\right]}{[L][M L]} \\
A=\frac{N}{4 K_{1} K_{2}[M]}\left(\frac{A_{0}}{C_{0}}+b K_{1}\left[M+\frac{A_{\text {lim }} N}{2 C_{0}}\right]\right) \text { (Equation [F]) } \\
\text { where } N=-1-K_{1}[M]+\sqrt{\left(1+K_{1}[M]\right)^{2}+8 C_{0} K_{1} K_{2}[M]}
\end{gathered}
$$

\subsubsection{Indirect calculation for thermodynamic equilibrium constant:}

During the development of the thesis it was reached a point in which it was interesting to calculate the equilibrium constant of a complex which was not fluorescent. To do so, the same equation may be applied without major changes in, for example, NMR titrations or circular dichroism. However, the main drawback of these methods is the high work concentration, which usually makes the results not valid when diluted, and that they are not always applicable.

In this regard it has been developed a new way to calculate thermodynamic equilibrium constants, due to an indirect calculation with a fluorogenic probe throughout a displacement in the complexation equilibrium.

\footnotetext{
${ }^{20}$ K. A. Connors, Binding Constants: The Measurement of Molecular Complex Stability, J. Wiley \& Sons, New York, 1987. b) Doctoral Thesis performed by Dr. Daniel Moreno. Supervised by Prof. Tomás Torroba. Nuevas sondas cromo-fluorogénicas a partir de derivados de organopaladio y compuestos indénicos, Universidad de Burgos (Spain), February 2011.
} 
In the work performed for this Thesis, this method has been successfully applied along Chapter 3C. The calculation was performed for compounds that acted as ligands of potassium cation, a synthetic fluorescent crown ether $(\mathbf{J G 7 6}=\mathrm{P})$ and some non-fluorescent natural depsipeptides, such as valinomycin and cereulide (X). Then, the thermodynamic equilibrium constant $\mathbf{J G}^{-} \mathbf{7 6}-\mathrm{K}^{+}(\mathrm{P}-\mathrm{A})$ was studied and calculated. With the data it was proposed the possibility of having an equilibrium to calculate the constant $\mathrm{X}-\mathrm{K}^{+}(\mathrm{X}-\mathrm{A})$.

Now it was necessary to include a new equilibrium:

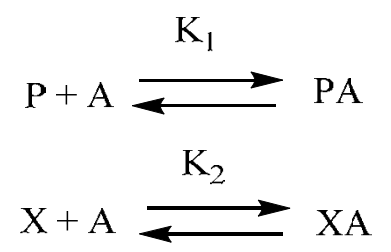

Mass and fluorescence balance:

$$
\begin{gathered}
C_{P}=[P]+[P A] \\
C_{A}=[A]+[X A]+[P A] \\
C_{X}=[X]+[X A] \\
I_{F}=f_{P}[P]+f_{P A}[P A]
\end{gathered}
$$

Where $C_{P}, C_{A}, C_{X}, I_{F}, f_{P} y f_{P A}$ are, concentrations of probe, analyte and not fluorescent ligand, the fluorescence intensity (only depends on the probe and the complex probe-potassium) and the factors of proportion between probe and PA complex. The rest are the concentration of the species on the equilibrium. By taking into account $K_{1}$ and $K_{2}$ and the equations (7) and (9):

$$
\begin{aligned}
& K_{1}=\frac{[P A]}{[P][A]}=\frac{[P A]}{\left(C_{P}-[P A]\right)\left(C_{A}-[P A]-[X A]\right)} \\
& K_{2}=\frac{[X A]}{[X][A]}=\frac{[X A]}{\left(C_{X}-[X A]\right)\left(C_{A}-[X A]-[P A]\right)}
\end{aligned}
$$

a) First option, solving the equation:

By solving (11), the concentration [VK], in (12):

$$
\begin{gathered}
{[P A]^{3}\left(K_{1} K_{2}-K_{1}^{2}\right)+[P A]^{2}\left(\left(1+K_{1}\left(C_{A}+2 C_{P}\right)\right) K_{1}-\left(1+K_{1}\left(C_{P}+C_{A}-C_{X}\right)\right) K_{2}\right)-} \\
{[P A] K_{1} C_{P}\left(1+K_{1}\left(2 C_{A}+C_{P}\right)+K_{2}\left(C_{X}-C_{A}\right)\right)+\left(K_{1} C_{P}\right)^{2} C_{A}=0}
\end{gathered}
$$

This cubic function could be solved by the next procedure:

Being the equation (14):

$$
a x^{3}+b x^{2}+c x+d=0
$$

Dividing between " $\mathrm{a}$ " and replacing $\mathrm{x}=\mathrm{z}-\mathrm{b} / 3 \mathrm{a}, \mathrm{z}^{3}+\mathrm{pz}+\mathrm{q}=0$ (Tschirnhaus transformation); where $\mathrm{p}$ and $q$ are calculated as: 


$$
\begin{gathered}
p=\frac{3 a c-b^{2}}{3 a^{2}} \\
q=\frac{2 b^{2}-9 a b c+27 a^{2} d}{27 a^{3}}
\end{gathered}
$$

To know the number of real roots, the discriminant $\Delta$ is calculated as:

$$
\Delta=-4 p^{3}-27 q^{2}
$$

With these data, further calculations for the constant were done, but at the end:

$>$ The equation turned out to be too much complicated, with many parameters to adjust.

$>$ The results of adjusting data to the equation were more dependent on the initial values than on the variation of them.

It was decided that, in order to make a more accurate calculation of the constant, it was necessary to make some approximations and simplify the equation.

\section{b) Simplification of the equation:}

The experiment started with a solution of not fluorescent compound (X) and the analyte (A), subsequently at the start of the titration there was complex (XA) in the equilibrium. When the probe (P) was added it formed a complex with the free analyte (A), creating the new complex (PA) and replacing the previous complex (XA). In conclusion, the concentration of XA decreased, whereas the concentration of $\mathrm{X}$ increased. Afterwards, a possible approximation could be done:

$$
\mathrm{C}_{\mathrm{X}}-[\mathrm{XA}] \approx \mathrm{C}_{\mathrm{X}}
$$

And this simplification was more realistic when the initial proportion $\mathrm{X} / \mathrm{A}$ was as high as possible.

The new equation obtained, from (11) and (12) was:

$$
[P A]=\frac{\left(C_{P}+C_{A}+\frac{1+K_{2} C_{X}}{K_{1}}\right)-\sqrt{\left(C_{P}+C_{A}+\frac{1+K_{2} C_{X}}{K_{1}}\right)^{2}-4 C_{P} C_{A}}}{2}
$$

As it is explained in Chapter 3, this method was developed specifically for a potassium probe that increases its fluorescence in presence of potassium cations, although it can be applied to other equilibria.

The validity of the method was evaluated:

First, the test was repeated several times with different initial proportions of $\mathbf{X}: \mathbf{A}$, a proportion 1:1, 1:0.25 and 1:0.1. It was checked that the results were slightly different, but it had the best fitting when the proportions were 1:0.1, in which the approximation was more valid, because of the simplification.

$>$ Second, the method was also compared with values calculated by other methods from literature. In our case, there were plenty of studies with circular dichroism. The results were comparable and even more reliable, due to some issues that were found in the literature calculations. 
The equilibrium constant with the fluorescent molecule $\left(K_{1}\right)$ and with the nonfluorescent one $\left(\mathbf{K}_{2}\right)$ should of the same order. Methods for calculating equilibrium constants have usually errors and deviations of at least $10 \%$. If the previously calculated $\mathrm{K}_{1}$ was much bigger than $\mathrm{K}_{2}$ the error would be higher than the value of the constant. In addition, if it was the other way around $\left(\mathrm{K}_{2}>>\mathrm{K}_{1}\right)$ the approximation would not be valid.

As an example, the graphs in Figure 21 were obtained by changing between cereulide/valinomycin and $\mathrm{EtOH}$ (see Chapter $\mathbf{3 C}$ for further information) getting very similar results to the already studied circular dichroism: ${ }^{21}$
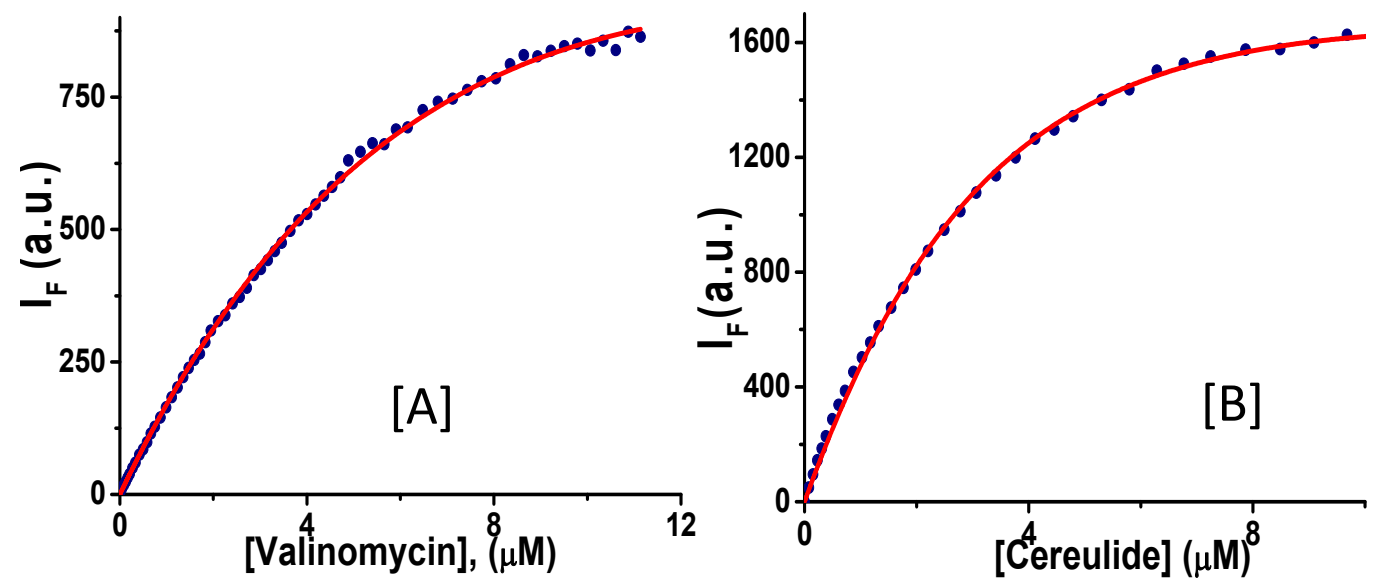

Figure 21. Fitted fluorescent titration emissions with $\mathbf{J G 7 6}$ of a $2 \mu \mathrm{M}$ solution of $\mathrm{K}\left(\mathrm{CF}_{3} \mathrm{SO}_{3}\right)$ and 20 $\mu \mathrm{M}$ of valinomycin and cereulide solution in $\mathrm{EtOH}$ (A and B).

\subsection{Limits of detection (LODs)}

The IUPAC defines the detection limit as the smallest quantity of an analyte that can be detected with reasonable certainty for a given analytical procedure, being distinguishable from the blank.

The method used during this thesis is not widely spread among scientist working in the field of sensors. Therefore, the reasons for using it are explained by giving an example of one limit of detection calculated by this method, and compared with the results of some of the most common methods found in literature. The explanation about how the expressions used to calculate the LODs work are deeply elaborated and discussed in literature, being beyond the aim of the thesis.

Figure 22 represents an example for the titration of probe JG25 with $\mathrm{Hg}^{2+}$ (from Chapter 1); the measurements at low concentrations of $\mathrm{Hg}^{2+}$ were fitted to a linear regression in order to calculate the LODs.

\footnotetext{
${ }^{21}$ M. C. Rose and R. W. Henkens, Biochim. Biophys. Acta 1974, 372, 426-435.
} 

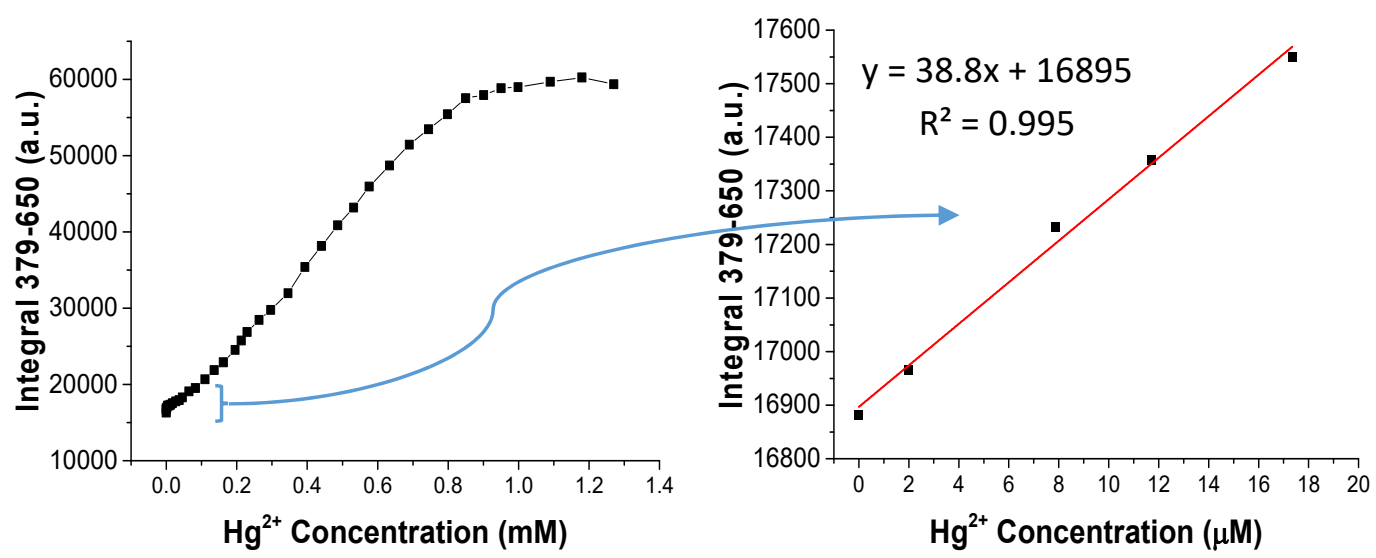

Figure 22. Titration of $\mathbf{J G 2 5}$ with adding $\mathrm{Hg}^{2+}$. Global titration (left) and linear regression for calculation of the LOD (right).

In addition, the blank (sample only containing probe) was measured three times obtaining an intensity value of 16876 a.u. and a standard deviation of 15.7 a.u.

\section{A) Method 1: Calculation of the LOD based on measurements of the blank.}

The method is based in what IUPAC defines as the equation so as to measure LODs:

$$
L O D=x_{b}+k s_{b}
$$

Where $\mathrm{x}_{\mathrm{b}}$ is the media of the measurements of the blank (only containing probe), $\mathrm{k}$ is a constant dependent on the reliability given to the method (usually 3 ) and $\mathrm{s}_{\mathrm{b}}$ is the standard deviation of a region with low concentration of the analyte.

$$
L O D=16876+3 \cdot 15.7=16923
$$

This equation gives the LOD in signal units. This signal is transformed into concentration by adjusting it to the fitted linear regression with the analyte.

$$
\begin{gathered}
\mathrm{y}=38.8 \mathrm{x}+16895 \\
\mathrm{x}=\mathbf{L O D}=\mathbf{0 . 7} \boldsymbol{\mu M}
\end{gathered}
$$

\section{B) Method 2: By using a linear regression at low concentrations of the analyte.}

In most papers from literature, ${ }^{22}$ the limit of detection (LOD) was estimated by the following equation:

$$
\mathrm{LOD}=3.3 \times \mathrm{SD} / \mathrm{s}
$$

Where SD is the standard deviation of a blank figure sample and $\mathrm{s}$ is the slope of the calibration curve in a region of low analyte content. Depending on the specific paper, they also use SD as the standard deviation of the linear regression.

A) Using $\mathrm{SD}$ as the standard deviation of the blank:

\footnotetext{
${ }^{22}$ S. Ahuja, M. Dong, Handbook of Pharmaceutical Analysis by HPLC, Elsevier, New York, 2005.
} 


$$
\text { LOD }=3.3 \times \frac{15.7}{38.8}=1.33 \mu \mathrm{M}
$$

B) Using $\mathrm{SD}$ as the standard deviation of the linear regression:

$$
\text { LOD }=3.3 \times \frac{16.75}{38.8}=\mathbf{1 . 4 2} \boldsymbol{\mu M}
$$

Furthermore, some authors also distinguish between limit of detection and limit of quantification, substituting the factor 3.3 by 10 , to give more reliability to the calculation.

\section{C) Method 3: By linear regression + False positive and negative.}

Based on the results of several authors ${ }^{23}$ there are many factors that must be taken into account when calculating the limit of detection:

- $\quad$ Adjusting to a mean square linear regression.

- Removing the "outliers". Understanding them as points that are significantly different from the rest, within a $95 \%$ of probability. If it is not possible to fulfil this part, the measurements should be repeated.

- $\quad$ Adjusting to a linear regression and checking that the slope is significantly different from 0 (95\%). P-Value $>0.05$.

- $\quad$ Calculation of the LOD when the probability of false positive $(\alpha)$ and false negative $(\beta)$ is equal or inferior to $5 \%$; or the value that the author is looking for. To do so, some software such as, " $R$ " could provide the fitting and give the results.

This method allowed to obtain a $\mathbf{L O D}=\mathbf{6 . 6} \boldsymbol{\mu M}$.

From the comparison of the methods a discrepancy may be seen between the most commonly used in literature and the method used in this thesis.

1) The obtained values of the LODs from most of the literature methods are, at least, between 5 to 10 times lower than the ones obtained by Method 3. The main issue is the dependence on the blank. Between the many issues with literature methods, in the first place, the deviation of the blank may not be the same when adding the analyte. In addition, it should be possible to get far lower values when measuring it repeatedly. Sometimes it should be theoretically possible to get values 100 or even 1000 lower than the values calculated by other methods.

2) Many of the methods from literature have not measured the quantity they put as a limit of detection. If they are not capable of measuring it in laboratory conditions, then it shouldn't be given as the LOD. As a general rule, the LOD is higher or around the value of the secondthird point given when presenting a reliable linear regression used for the calculation, and always higher than the first value that is considered different from 0 .

3) Papers usually do not give details of many of the parameters they calculate. For example, about how many times they have measured the blank, or they do not specify the way in which they have calculated the LOD.

\footnotetext{
${ }^{23}$ M. C. Ortiz, L. A. Sarabia, M. S. Sánchez, Anal. Chim. Acta. 2010, 674, 123-142.
} 
In addition, there are more methods apart from the ones exposed, and some of them may be trustworthy if correctly explained. In order to compare results between probes there are many factors that should be taken into account, not only the numbers, but also the method used to get the values and the data that is provided.

Deeper information about this topic has been studied by many analytical chemists. In fact, it has been recently published a book in which many conclusions fit to the findings explained in this thesis. ${ }^{24}$ The publication is focused in the analytical procedures to perform limits of detection correctly. Of course, it is a much deeper analysis that delves into mathematical explanations to face this issue. However, although it might be very complex for many chemists with no experience in the area, some of the conclusions about the mistakes when determining LODs are easily understandable and may be summarized as: $:^{25}$

Using the statistical parameters wrongly. In this part are included practices such as:

o Defining the LODs as signal noise ratio $=3$.

o Ignoring possible errors in estimating the value of the blank.

o Using the IUPAC as guidance, being barely updated since 1970s.

0 In the formula from the IUPAC, defining " $k$ " as 3 without particular justification.

o Accepting previous results from literature without checking.

$>$ Calculating LODs out of the Content Domain. Inferred estimations of the LOD would not be valid; only numerical values recorded and reported might be taken as true. Meaning that the LODs must be values within the region measured, always superior to the lowest measurement that is different from the blank (with no analyte).

In general, the methods used in literature to perform LODs are very controversial, especially for chromo-fluorogenic probes. Introducing more reliable methods is the first step for the development of standardized procedures for the future, but it must start by rejecting outdated old techniques, that give unreliable information, and giving more data about the procedures followed for the calculations.

\section{* A particular case of LOD calculation, when the thermodynamic equilibrium is not reached:}

Another topic that could be addressed when calculating LODs is what to do when the titration and calculations are performed for species that had not reached the equilibrium (usually because it would take minutes or hours). In most papers it is an issue that is ignored, although it is a problem easily solved if the data are properly explained for the conditions in which the limit is valid. This kind of titrations (equilibrium reached after too long periods of time) may be addressed in several ways.

First, by waiting the necessary amount of time; the different points of the titrations are prepared at the same time and after waiting the necessary amount of time, it may give reliable results. However, this could be not possible when the quantity of probe is limited or the kinetic is unknown to a certain extent (which could be a consequence of working with supported probes, Chapter 1). Furthermore, LODs that are very low but need several hours/days to give signal could not be useful. In spite of the difficulties, there are some alternatives. For example, performing the titrations similarly to when there is no time dependence but with constant time lapse between additions.

\footnotetext{
${ }^{24}$ E. Voigtman, Limits of detection in chemical analysis, John Wiley \& Sons, Inc: Hoboken, New Jersey, $1^{\text {st }}$ Edition, Chapter 1, 2017.

${ }^{25}$ E. Voigtman, Limits of detection in chemical analysis, John Wiley \& Sons, Inc; Inc: Hoboken, New Jersey, $1^{\text {st }}$ Edition, Chapter 24, 2017.
} 
a) Method 1: No probe in the additions. (Figure 23, The first method explained in section 3.5.). The limit of detection observed will be calculated giving higher values than if the equilibrium is reached. Moreover, the time in which the calculated limit is reached must be taken into account.

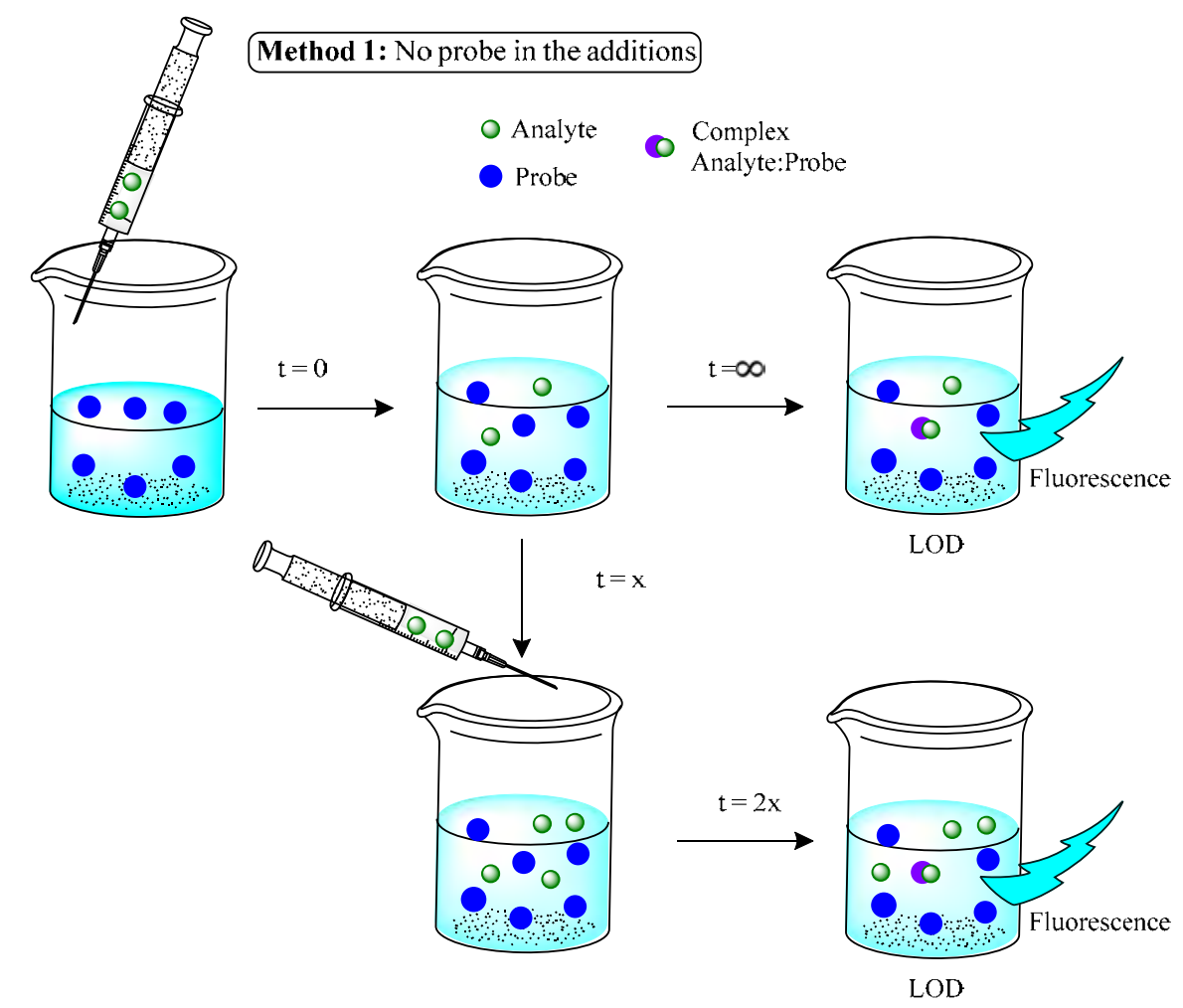

Figure 23. Titration method for calculation of LOD by fluorescence. Experimental method waiting until equilibrium (up) or by additions at constant time (down).

b) Method 2: When the titration is performed by adding analyte dissolved in probe solution (Figure 24). The opposite process occurs, having the probe complexed from the very beginning. In contrast with the other method, the LOD would be inferior to the one in the equilibrium; what is more, it could be the consequence of increasing the quantity of complexed product, which would lead to unrealistic results because of the reliability of the system, but not the probe. The equilibrium and the additions would compete between increase-decrease fluorescence (Figure 24) which makes this method unsuitable to calculate LODs when the equilibrium is not reached.

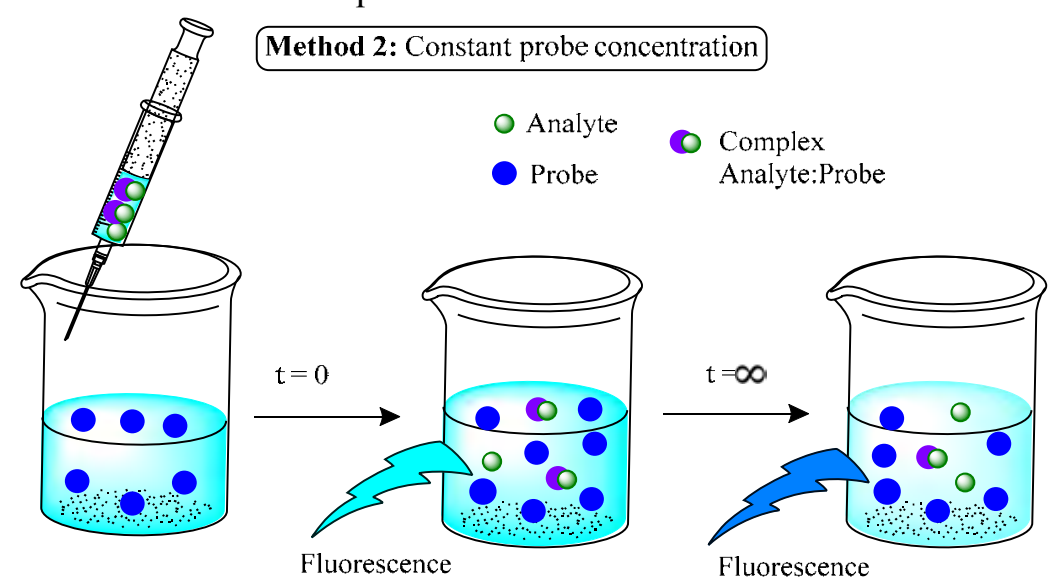

Figure 24. Titration addition under constant probe concentration and influence of the time. 
In conclusion, the LOD calculation may be performed by many processes, but the conditions should be carefully explained as it was stated. If the LOD is calculated far from the equilibrium, the time periods must be specified, giving a pseudo-LOD, valid under restricted conditions.

\subsection{Fluorescence quantum yields $\left(\Phi_{\mathrm{F}}\right)$}

Fluorescence quantum yield is defined as the fraction of excited molecules that return to the ground state $\mathrm{S}_{0}$ with emission of fluorescence photons. In other words, the ratio between emitted photons by fluorescence and absorbed photons.

The expression may be explained this way, when working in a system with no phosphorescence processes:

$$
\Phi_{F}=\frac{\text { Emitted photons }}{\text { Absorbed photons }}=\frac{k_{r}^{S}}{k_{r}^{S}+k_{n r}^{S}}=k_{r}^{S} \tau_{S} \quad \text { Equation [G] }
$$

Where $k_{r}^{S}$ is the radiative rate constant, $k_{n r}^{S}$ the non-radiative rate constant and $\tau_{\mathrm{S}}$ the fluorescence decay lifetime.

As a result, this value gives an idea of the efficiency absorbance-fluorescence, that characterizes a system. The purpose of evaluating the fluorescence quantum yields is obtaining an objective comparison of how much fluorescence do we have. In addition, in the field of fluorescent probes, it is interesting because of the possibility to compare fluorescence with and without the analyte, by giving absolute values.

There are several parameters that have influence over the fluorescence quantum yield. To begin with, an increase in temperature results in a decrease in the fluorescence quantum yield because of the nonradiative processes related to thermal agitation (collisions with solvent molecules, intramolecular vibrations and rotations, etc.) that are more efficient at higher temperatures. To avoid quenching process, the quantum yield associated to molecule should be done in absence of interferents, at controlled temperature and low concentrations (avoiding possible homo-interactions such as dimers).

\section{Calculation methods: ${ }^{26}$}

Nowadays there are two methods to calculate quantum yields, the relative calculation of quantum yields (by comparison with a standard) ${ }^{27}$ and the absolute determination.

Relative Fluorescence Quantum yield was the most classical and accessible way to determine Fluorescence quantum yields, and it is expressed by its general equation:

$$
\Phi_{F}=\Phi_{F, R} \frac{n^{2}}{n_{R}^{2}} \frac{1-10^{-A} R}{1-10^{-A}} \frac{F}{F_{R}} \text { Equation }[\mathbf{H}]
$$

Where

$\Phi_{\mathrm{F}}$ is the quantum yield.

${ }^{26}$ C. Würth, M. Grabolle, J. Pauli, M. Spieles, U. Resch-Genger, Nat. Prot. 2013, 8, 1535-1550.

${ }^{27}$ A. M. Brouwer, Pure Appl. Chem. 2011, 83, 2213-2228. 
$>\mathrm{n}$ represents the refractive index of the solvent.

$>\mathrm{A}$ is the absorbance.

$>\mathrm{F}$ is the integral of the whole fluorescent emission.

$>\mathrm{R}$ means that the parameter is associated to a reference sample.

Specific parameters and rules:

$>$ The chosen reference has to be as similar as possible to the probe in absorbance and emission.

$>$ The parameter $\left(1-10^{-\mathrm{A}}\right)$ is called optical density. To have valid results, the absorbance must be around 0.1 (high dilution to have the precision of the system maximized) and should be as similar as possible when comparing between standard-probe values.

$>$ The excitation wavelength has to be the same for both samples. The maximum value for the probe must be as similar as possible (in shape and height) and as close to the reference as possible.

$>$ The integral has to be done for the whole emission registered of both the reference and the sample.

Figure 25 shows an example for the synthetized probe JG76 (Chapter 3C); the integral was done between $480-700 \mathrm{~nm}$, and the Rayleigh signal was deconvoluted.
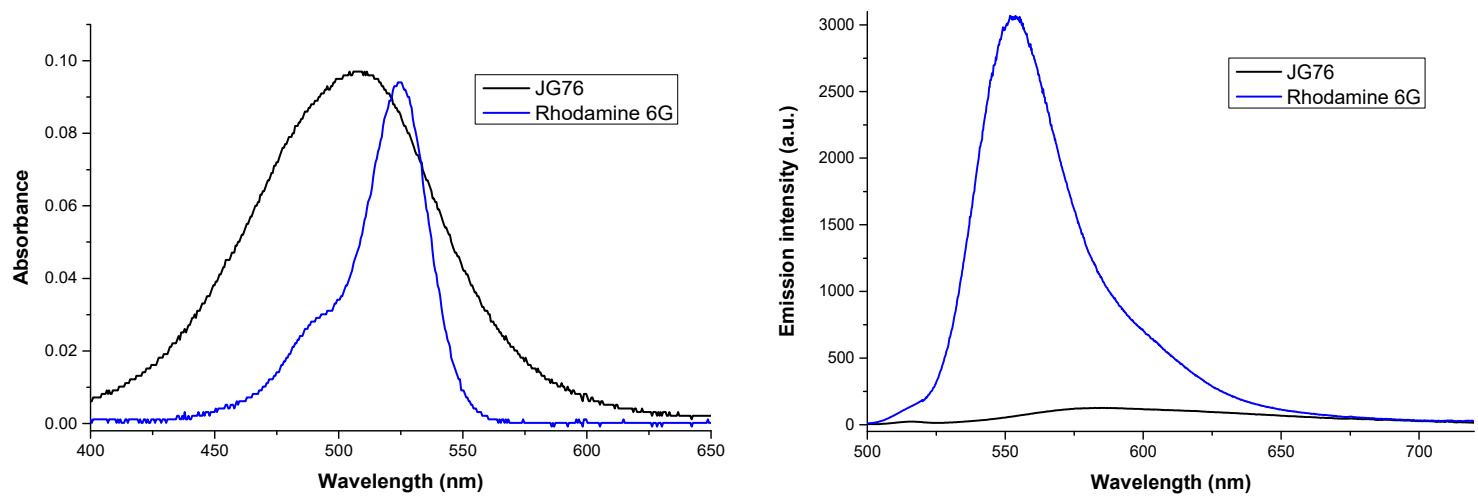

Figure 25. Absorbance of JG76-Rhodamine 6G (left), fluorescence of JG76-Rhodamine 6G with $\lambda_{\text {exc }}=515 \mathrm{~nm}$ (right); dissolved in EtOH.

The process was repeated three times to obtain the average value and a confidence interval:

$\Phi_{\mathrm{F} J G 76}($ EtOH $)=0.11 \pm 0.01$

Absolute fluorescence quantum yield may be determined by using an integration sphere.

Specific parameters and rules:

The sample is introduced in the chamber in the selected mode, liquid or solid.

$>$ The response, including the Rayleigh emission, of the media is measured (solvent or air).

$>$ The response, including the Rayleigh emission, of the sample is measured.

The process consists of comparing the results of solvent and sample to give the fluorescence quantum yield. Several replications and different acquisition times increase the reliability of the measurements. The limitations of the method always give an experimental error of the procedure 1-2 \% as minimum; similar to the relative method. 
In practice, this method should give equivalent results to relative measurements (see example in Figure 26).

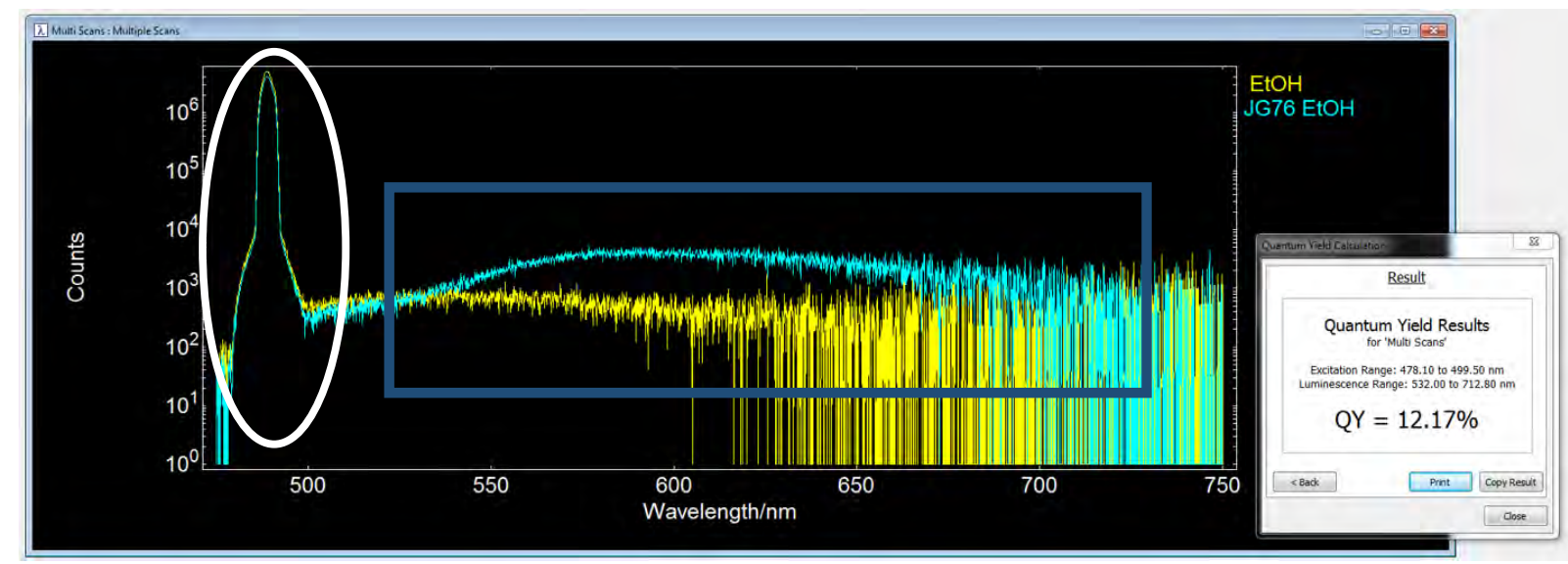

Figure 26. Example of a measurement performed with an integration sphere of the software from FLS980 Edinburgh instruments to measure a quantum yield. Compound JG76 in EtOH solution.

Keeping in mind that the error associated to the method is at least $1 \%$, the samples were repeated three times obtaining variations in the results around this number. It is remarkable the coincident value of both methods, 11-12\%, within the experimental error. Moreover, in regard of other kind of samples, the integration sphere is especially important for solid materials. It allows comparisons between the emission of solid samples, whose fluorescence values are much more difficult to evaluate with precision and repeatability than for dissolved analytes.

In addition, the same equations may be used for the calculation of phosphorescence quantum yields, although they are usually very low and it is not uncommon that they reach values close to the limit of the technique (1-2\%).

\subsection{Fluorescence decay lifetime $(\tau)$}

Fluorescence decay lifetimes are defined as the time between a system absorbs a photon and it emits it by fluorescence, returning the electronic structure to its ground state. Their values are calculated by using a time-correlated single photon counting instrument ${ }^{28}$ with several pulsed LED. The fluorescence decay lifetimes of the probes were analysed using the software provided by the Fluorometer.

Decays were recorded at the wavelength that best fits the probe (excitation-emission). Without going into details, a thorough discussion which can be found in literature, ${ }^{29}$ the software uses an iterative re-convolution of the source time profile of the instrument, IRF, or an iterative tail fitting of the sample by avoiding the overlapping between IRF and the lifetime of the sample, which is

\footnotetext{
${ }^{28}$ FLS980 Series, Edinburgh instruments. Software FAST 3.4.2 (Edinburgh instruments). Specifics are detailed in the Annex.

29 a) J. R. Lakowicz, Principles of Fluorescence Spectroscopy, Springer USA, 3rd Edition, 2006. b) B. Valeur, Molecular Fluorescence: Principles and Applications, Wiley-VCH, Weinheim, Chapter 10, 2002 c) B. Herman. Fluorescence Microscopy, $2^{\text {nd }}$ Edition, Springer-Verlag Berlin, 1998. D) W. R. G. Baeyens, D. de Keukeleire, K. Korkidis. Luminescence techniques in chemical and biochemical analysis, M. Dekker, New York, 1991. E) D. M. Jameson, T. L. Hazlett, (T.G. Dewey), Time-Resolved Fluorescence in Biology and Biochemistry, in Biophysical and Biochemical Aspects of Fluorescence Spectroscopy, Springer, Denver, Colorado 1991.
} 
necessary when the lifetime is low. The theoretical fluorescence decay is modelled as a sum of exponentials:

$$
F(t)=\sum_{i} A_{i} e^{-\frac{t}{\tau_{i}}} \quad \text { Equation }[\mathbf{I}]
$$

Where $\mathrm{Ai}$ is the weighted amplitude (fractional value between 0 and 1 ) and $\tau \mathrm{i}$ is the lifetime of the $i$-th fluorescent component. IRF is recorded from a scattering sample that does not fluoresce (in this case LUDOX dispersion). Both $\mathrm{F}(\mathrm{t})$ and IRF are recorded at a sampling rate adapted to the repetition rate of the pulsed source in order to avoid non-linear effects in the acquisition. The convolution of $\mathrm{F}(\mathrm{t})$ is fitted to the experimental decay curve, $\mathrm{I}(\mathrm{t})$, by iterative change of the amplitude and lifetime parameters, using the least-squares method to optimize the fitting parameters. The quality of the fit is determined by ensuring that the $\chi^{2}$ statistical parameter is between 1 and 1.3 there is no deviation or tendencies in the residues. (See example in Figure 27).
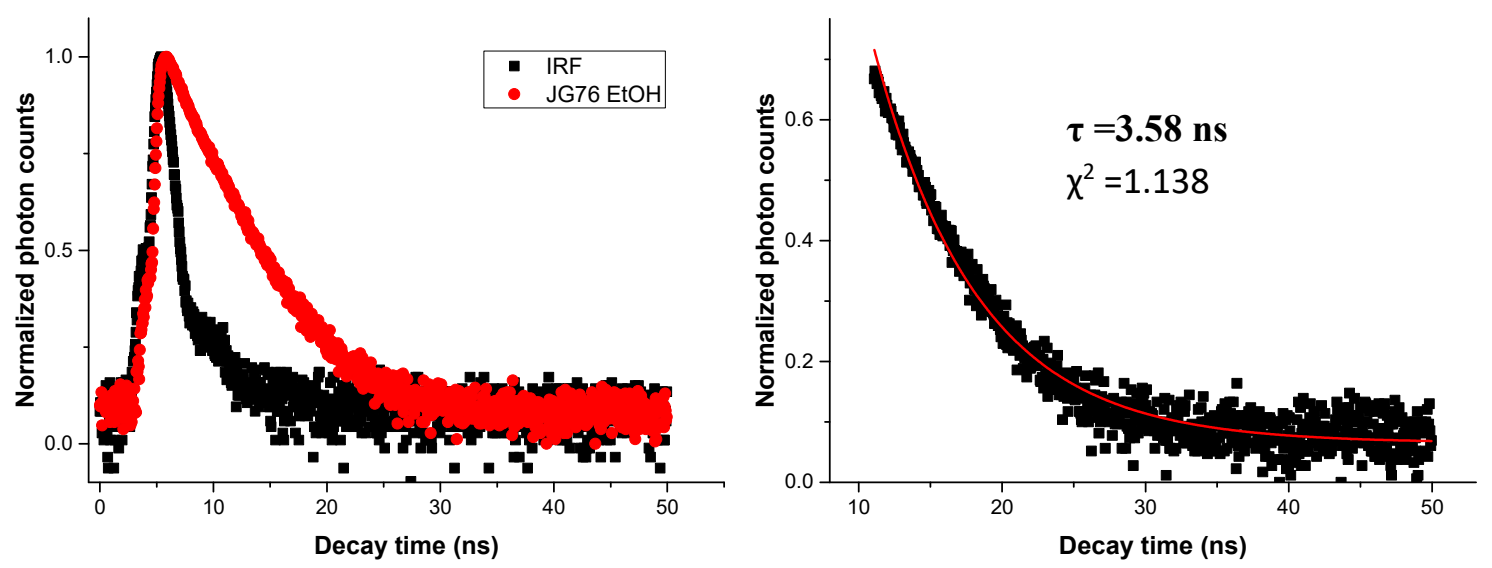

Figure 27. Lifetime decay representation of JG76 in EtOH; comparison with the IRF (left) and fitted to an exponential decay (right).

The applicability of this kind of analysis is high. First of all, it is a parameter useful in order to compare properties of a system, from which it is possible to obtain radiative and non-radiative constants and everything associated with this phenomenon. Second, the order (in seconds) gives information about the emission, if it is fluorescence the decay is less than microseconds, in phosphorescence it reaches much higher values.

As an example, one of the applications is to distinguish between dynamic and static quenching, ${ }^{30}$ which may be of upmost interest when developing a turn-off probe. Other application comes from the relation between radiative and non-radiative decays which may be interesting for photodynamic therapy studies. ${ }^{31}$

${ }^{30}$ J. H. Gutow, J. Chem. Educ. 2005, 82, 302-305.

${ }^{31}$ A. Kamkaew, S. H. Lim, H. B. Lee, L.V. Kiew, L.Y. Chung, K. Burgess. Chem. Soc. Rev. 2013, 42, 77-88. 


\section{GENERAL SCHEME OF FLUORESCENT PROBES; PHOTOPHYSICS AND SUPRAMOLECULAR CHEMISTRY}

In a schematic way, probes can be also classified depending on their photophysical properties and supramolecular interactions.

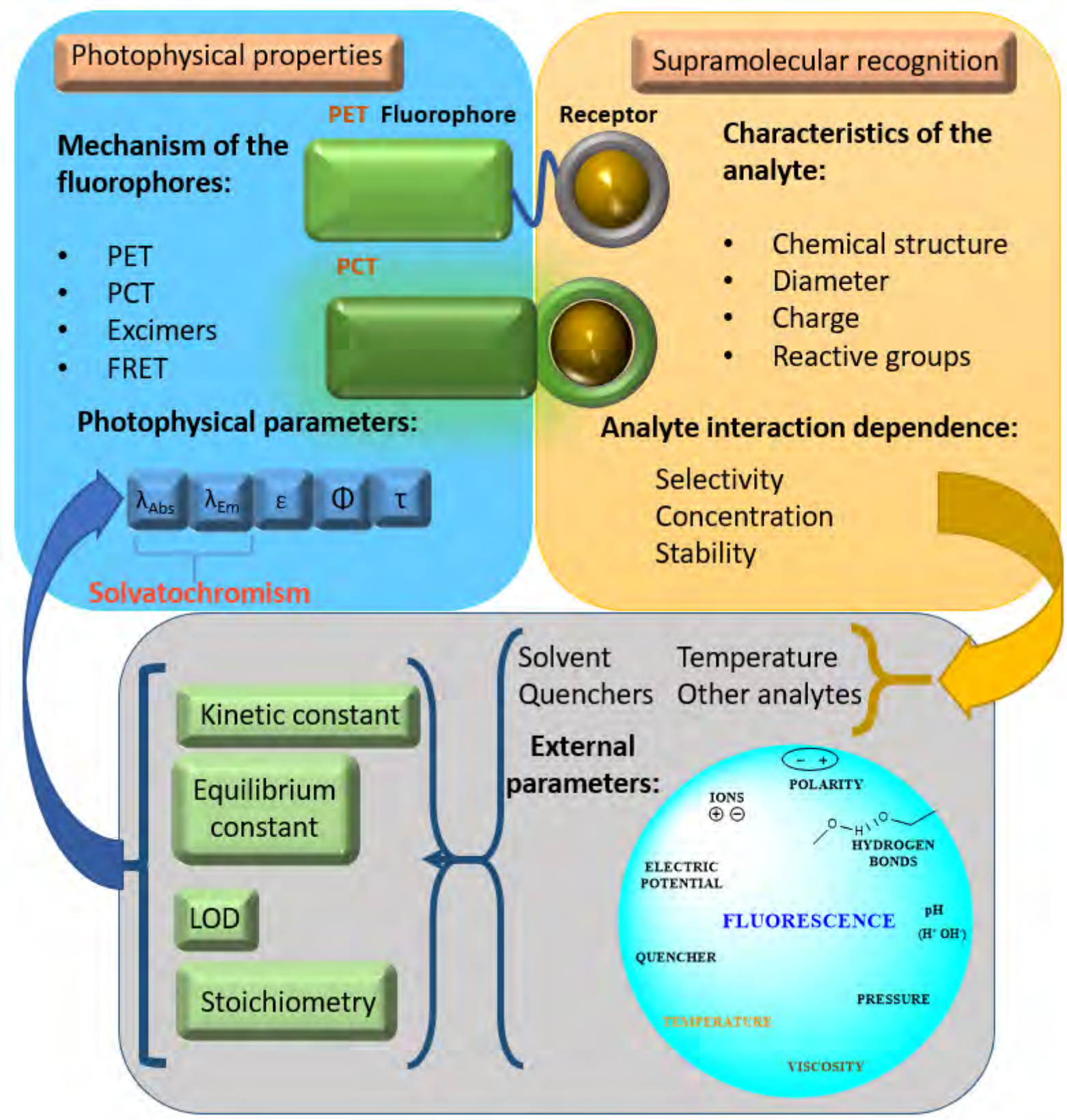

Figure 28. Scheme illustrating photophysical properties and supramolecular recognition. 


\section{RESUMEN DEL CAPÍTULO}

El propósito de este capítulo es introducir el uso de sensores fluorescentes como método para la detección de analitos de interés. Para alcanzar este objetivo, se muestra que este tipo de sensores presentan ventajas en factores importantes como el tiempo o el coste, respecto a otros métodos clásicos analíticos. También se hace hincapié en el mecanismo de fluorescencia, las propiedades, los tipos, las distintas formas de crear sensores moleculares fluorescentes y sus limitaciones.

Complementando todo lo anterior, se describen los procesos para el monitoreo de las distintas propiedades y parámetros que definen a las sondas fluorescentes. Al mismo tiempo, se explica el modo en que se han evaluado estas propiedades y qué clase de experimentos en el laboratorio y en el tratamiento de datos son necesarios para ello. Algunos de estos experimentos son el estudio del solvatocromismo para evaluar el comportamiento en distintos disolventes, métodos para el cálculo de la estequiometría de un complejo y cálculo de distintas constantes y parámetros propios (constantes de equilibrio, límites de detección, rendimientos cuánticos de fluorescencia o tiempos de vida).

En resumen, este capítulo sirve de introducción para los Capítulos 1, 2 y 3 en los cuales se diseñaron sondas moleculares y materiales fluorescentes modificados para la detección de especies de interés, así como su utilización como marcadores biológicos, para terapia fotodinámica o en otras posibles aplicaciones. 


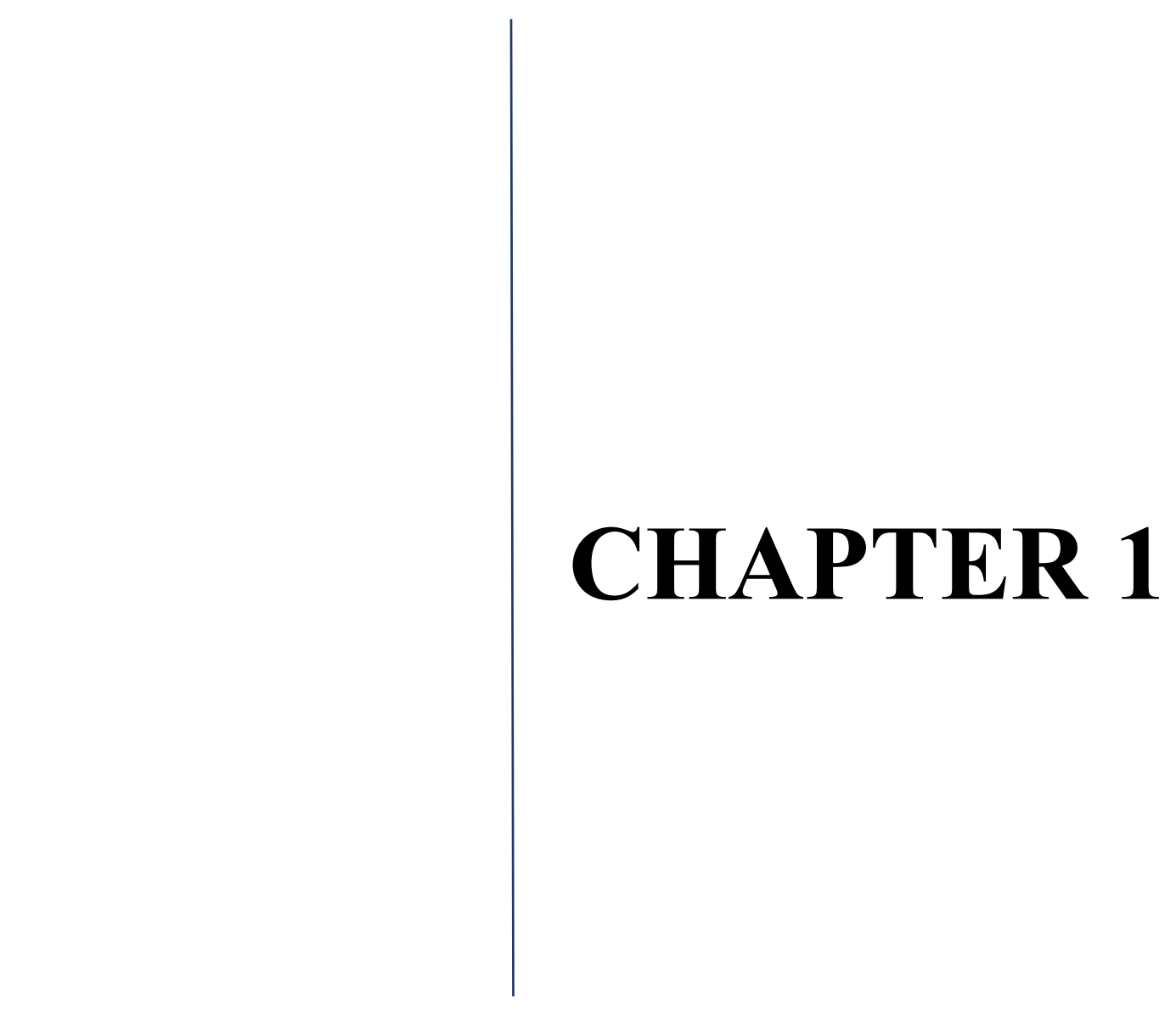

\section{FLUORESCENT PROBES FOR THE DETECTION OF Hg(II) DERIVATIVES}

\section{OBJECTIVES}

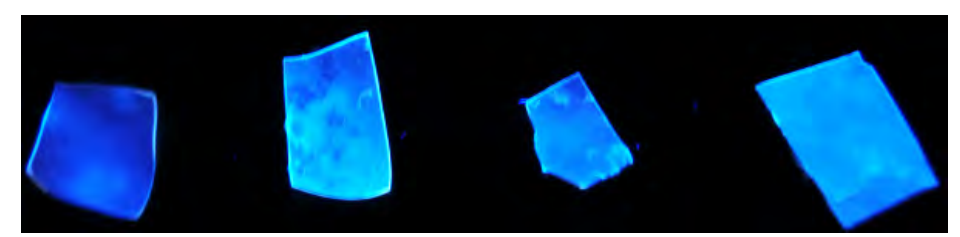

This chapter aims to explain the work performed in the search of new fluorogenic sensors for the detection of mercury cationic species, with high environmental impact because of their toxicity. Specifically, $\mathrm{Hg}$ (II) and $\mathrm{MeHg}$ (II) fluorescent sensors were synthetized; based on indanone derivatives, working in water solution (soluble or supported sensor), in cellular media ${ }^{1}$ and in fish extracts. ${ }^{2}$

\footnotetext{
${ }^{1}$ B. Díaz de Greñu, J. García-Calvo, J. Cuevas, G. García-Herbosa, B. García, N. Busto, S. Ibeas, T. Torroba, B. Torroba, A. Herrera, S. Pons, Chem. Sci., 2015, 6, 3757-3764.

2 J. García-Calvo, S. Vallejos, F. C. García, J. Rojo, J. M. García, T. Torroba, Chem. Commun., 2016, 52, 1191511918.
} 



\section{INTRODUCTION. THE IMPORTANCE OF MERCURY}

\subsection{Mercury, history and applications}

Mercury, as many other heavy elements of the periodic table, is often associated to danger and toxicity. Although it is usually true, mercury also has many applications and has proven to be very useful when correctly handled. Mercury derivatives, in the history of humankind, have been used in paintings, jars or even with medicinal purposes, generally leading to little results in terms of health. Old applications still remain in use, such as the chlor-alkali industry, the use in batteries or the mercury lamps although they are now banned or disappearing. ${ }^{3}$ Because of the many applications of the metal derivatives and the toxicity for living organisms of mercury compounds, it is of great importance to know where the different mercury species come from and detecting and quantifying their presence, which is critical for toxicological and environmental issues.

The origins of mercury species in the environment are mainly two, volcanic and human action. ${ }^{4}$ Whereas volcanic processes occur without human interference and have not had significant increase, the concentration of mercury species in the sea has tripled compared to pre-anthropogenic interference, ${ }^{5}$ which gives an idea of the impact of human action.

Mercury salts and sulfurs are the main source when mining this element for different purposes, however, the disposals have not always been correctly treated, specially until the first news about their dangerousness came to light in the $20^{\text {th }}$ century. Furthermore, over the last years, the realization of the consequences of dealing with high levels of mercury, and the development of new ways to detect and stop irresponsible disposals, have been very important for avoiding massive introduction of this kind of waste in the environment. Although it is also true that sometimes the information is confusing and it is not clear how dangerous it could be, generating unnecessary social alarm. ${ }^{6}$

\subsection{The cycle of mercury}

There are two very important organometallic derivatives of mercury, methylmercury ( $\mathrm{MeHg}(\mathrm{II}))$ and dimethylmercury $\left(\mathrm{Me}_{2} \mathrm{Hg}\right)$. Dimethylmercury is a liquid and very volatile species that possesses the highest toxicity for animals of all mercury derivatives, a single drop of this organometallic compound can go through most of the clothes and laboratory protection and it is immediatelly absorbed through the skin, causing neuronal damage and being mortal within a few days after skin absorption. ${ }^{7}$ For this reason, and being the less common of all the species, it is not studied for detection purposes.

\footnotetext{
${ }^{3}$ a) M. S. Bank, Mercury in the Environment: Pattern and Process. 1st ed., University of California Press, 2012. b) G. Liu, Y. Cai, N. O’Driscoll, X. Feng, G. Jiang. Environmental Chemistry and Toxicology of Mercury, G. Liu, Y. Cai, N. O'Driscoll, (Editors), John Wiley \& Sons, Hoboken, New Jersey, Chapter 1, pp. 1-12, 2012.

${ }^{4}$ P. A. Ariya, M. Amyot, A. Dastoor, D. Deeds, A. Feinberg, G. Kos, A. Poulain, A. Ryjkov, K. Semeniuk, M. Subir, K. Toyota, Chem. Rev. 2015, 115, 3760.

${ }^{5}$ C. H. Lamborg, C. R. Hammerschmidt, K. L. Bowman, G. J. Swarr, K. M. Munson, D. C. Ohnemus, P. J. Lam, L.-E. Heimbürger, M. J. A. Rijkenberg, M. A. Saito, Nature 2014, 512, 65.

${ }^{6}$ J. Burger, M. Gochfeld, Rev. Environ. Health. 2013, 28, 129-143.

${ }^{7}$ D. W. Nierenberg, R. E. Nordgren, M. B. Chang, R. W. Siegler, M. B. Blayney, F. Hochberg, T. Y. Toribara,

E. Cernichiari, T. Clarkson, N. Eng. J. Med. 1998, 338, 1672-1676.
} 
From the other different mercury species, apart from metallic mercury, which also emits toxic vapours, the cationic $\mathrm{Hg}(\mathrm{II})$ and $\mathrm{MeHg}(\mathrm{II})$ are of upmost importance. The cation $\mathrm{Hg}(\mathrm{II})$ has been widely studied and controlled. Nevertheless, $\mathrm{Hg}$ (II) is an inorganic salt, being barely absorbed by living beings. As a consequence, its toxicity is far lower than the organometallic cations, from what the most representative is $\mathrm{MeHg}(\mathrm{II})$.

The existence of $\mathrm{MeHg}$ (II) comes from the mercury cycle, which explains how this lypophilic cation is introduced in the trophic chain. Mercury cycle consists of an abiotic sulfuration-desulfuration equilibrium in bacteria (Figure 1) that transforms $\mathrm{Hg}$ (II) in water to $\mathrm{MeHg}(\mathrm{II})$; after that, some plants absorb this compound. The lipophilicity of $\mathrm{MeHg}$ (II) makes it easy to be absorbed by living beings. In addition, since this complex of $\mathrm{MeHg}$ (II) and cysteine resembles the structure of large and neutral amino acid methionine, it can enter the cell and exit as a complex with reduced glutathione, thus forming water soluble complexes in tissues. ${ }^{8}$ The special characteristics of this cation makes it bioaccumulative, which means that going up in the trophic chain, the concentration is bigger each time, reaching dangerous concentrations in some big aquatic animals, such as swordfish or shark.

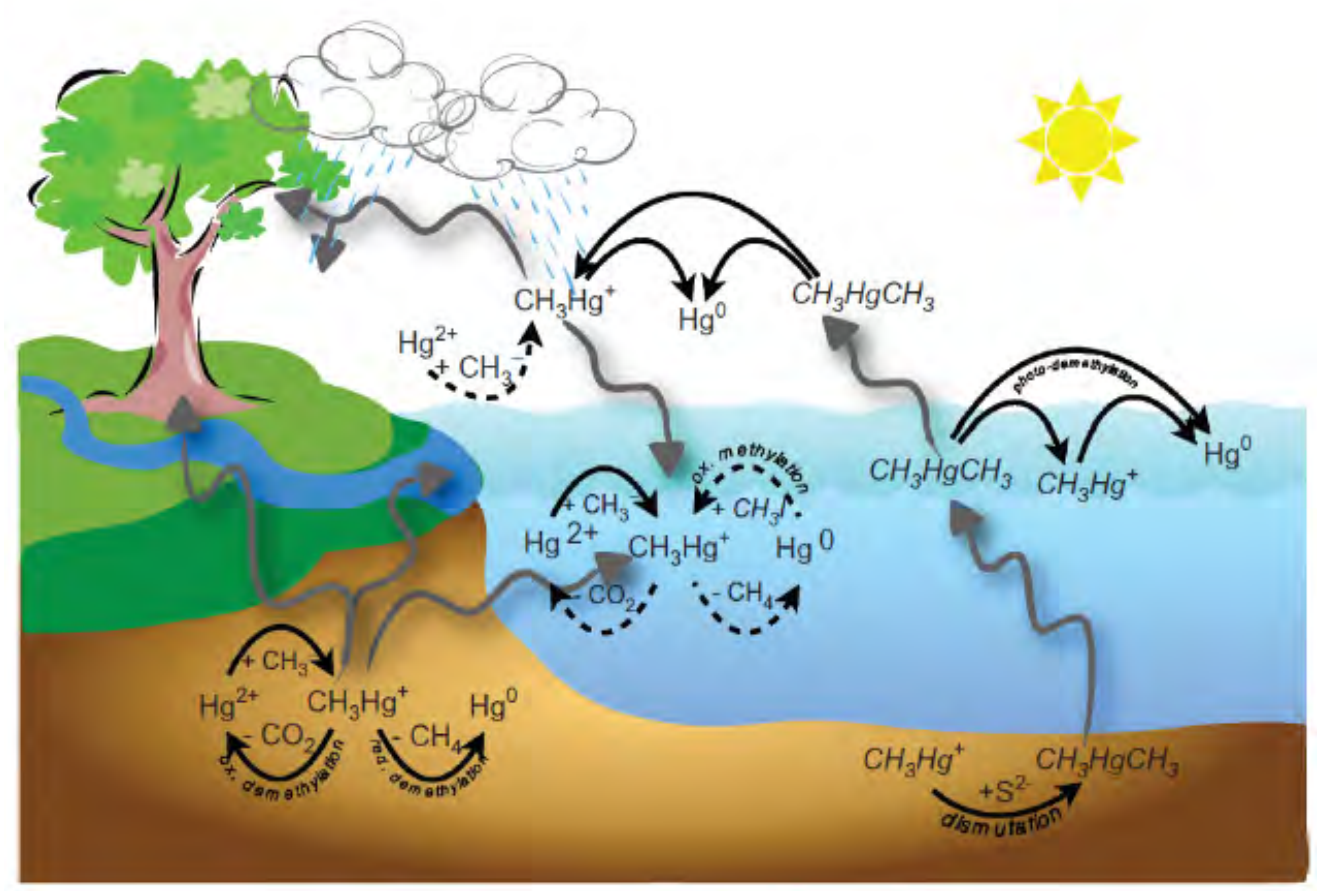

Figure 1. Cycle of $\mathrm{Hg}(\mathrm{II})-\mathrm{MeHg}(\mathrm{II}){ }^{9}$

\subsection{Mercury presence and its risks in our society}

There are records of several disasters related with massive contamination with mercury cationic species, the two examples with more impact were, the leaking of $\mathrm{MeHg}(\mathrm{II})$ in Minamata's river in the 1950s and the contamination of seed grain in Iraq in 1971, caused by its use as pesticide. As a result of these disasters, the consequences of introducing $\mathrm{Hg}(\mathrm{II})$ and $\mathrm{MeHg}(\mathrm{II})$ to the environment are widely

\footnotetext{
${ }^{8}$ Reviews: (a) M. Farina, J. B. T. Rocha, M. Aschner, Life Sci. 2011, 89, 555-563; b) M. Yamashita, Y. Yamashita, T. Suzuki, Y. Kani, N. Mizusawa, S. Imamura, K. Takemoto, T. Hara, M. A. Hossain, T. Yabu, Touhata, Mar. Biotechnol. 2013, 15, 559-570; c) G. J. Lu, Y. Tian, N. Vora, F. M. Marassi, S. J. Opella; J. Am. Chem. Soc. 2013, 135, 9299-9302.

${ }^{9}$ H. Hintelmann. Metal. Ions Life Sci. 2010, 7, 365-401.
} 
documented and studied. ${ }^{10}$ In spite of these facts, the most important and constant source of mercury cationic species in food, in our society, comes from fish. ${ }^{11}$ The explanation for it comes from the already explained cycle of mercury, so that some fish are capable to accumulate concentrations close, or even superior to $5 \mathrm{ppm}$ of this element.

The concentration of the different mercury species in living beings consists of the highly toxic derivative, $\mathrm{MeHg}(\mathrm{II}),(70-90 \%)$ and only a $10-30 \%$ of $\mathrm{Hg}(\mathrm{II})$. Food with mercury concentrations in the range of ppm is toxic if regularly introduced on human diet, even if it is eaten once a week. ${ }^{12}$ As a consequence, many American and European international institutions do not recommend to eat fish with more than $1 \mathrm{ppm}$ of this metal. The results of a long period of ingestion go from long-lasting neurological damage to physical and psychological deficiencies $;{ }^{13}$ being especially toxic for children and the foetus ${ }^{14}$ in pregnant women.

For all these reasons, nowadays detection of mercury cationic derivatives has drawn a lot of attention for health, environmental and industrial issues. In this regard, many examples of detection may be found in literature, especially for $\mathrm{Hg}(\mathrm{II})$, in spite of the greater importance of the other species. That is the reason because the efforts in this area of research aim to develop new selective, highly accurate and fast methods to detect and discriminate different mercury species.

\subsection{Standardized techniques for detecting mercury presence in a sample}

There are many ways to quantify $\mathrm{Hg}(\mathrm{II})$ derivatives present in solutions, or extracted from a sample to a solution, the most common are analytical techniques based on the use of classical analytical devices:

- Inductive coupled plasma mass spectrometry (ICP-MS): it is the most common and reliable. This method is applied after the degradation of the sample in concentrated acid

\footnotetext{
${ }^{10}$ See for example: a) T. A. Douglas, L. L. Loseto, R. W. Macdonald, P. Outridge, A. Dommergue, A. Poulain, M. Amyot, T. Barkay, T. Berg, J. Chetelat, P. Constant, M. Evans, C. Ferrari, N. Gantner, M. S. Johnson, J. Kirk, N. Kroer, C. Larose, D. Lean, T. G. Nielsen, L. Poissant, S. Rognerud, H. Skov, S. Sørensen, F. Wang, S. Wilson, C. M. Zdanowicz, Environ. Chem. 2012, 9, 321-355; b) C. R. Hammerschmidt, M. B. Finiguerra, R. L. Weller, W. F. Fitzgerald, Environ. Sci. Technol. 2013, 47, 3671-3677; c) A. L. Soerensen, R. P. Mason, P. H. Balcom, E. M. Sunderland, Environ. Sci. Technol. 2013, 47, 7757-7765; d) D. Liu, S. Wang, M. Swierczewska, X. Huang, A. A. Bhirde, J. Sun, Z. Wang, M. Yang, X. Jiang, X. Chen, ACS Nano 2012, 6, 10999-11008; e) J. Chen, S. Zhou, J. Wen, J. Anal. Chem. 2014, 86, 3108-3114.

11 a) R. Wang, X. B. Feng, W. X. Wang, Environ. Sci. Technol. 2013, 47, 7949-7957; b) W. F. Fitzgerald, C. H. Lamborg, C. R. Hammerschmidt, Chem. Rev. 2007, 107, 641-662, c) H. Hintelmann, Organomercurials. Their Formation and Pathways in the Environment, in A. Sigel, H. Sigel, R. K. O. Sigel: Organometallics in Environment and Toxicology: Metal Ions in Life Sciences, Chapter 11, vol. 7, pp. 365-401; 2010; d) I. Lehnherr, V. L. St. Louis, H. Hintelmann, J. L. Kirk, Nature Geosci. 2011, 4, 298-302; e) J. M. Parks, A. Johs, M. Podar, R. Bridou, R. A. Hurt, S. D. Smith, S. J. Tomanicek, Y. Qian, S. D. Brown, C. C. Brandt, A. V. Palumbo, J. C. Smith, J. D.Wall, D. A. Elias, L. Liang, Science 2013, 339, 1332-1335.

$12 \mathrm{https} / / /$ www.fda.gov/food/foodborneillnesscontaminants/metals/ucm2006760.htm; accessed on June 2018.

13 a) J. Z. Byczkowski, Methyl Mercury Toxicity: Pharmacokinetics and Toxicodynamic Aspects, in R. R. Watson, V. R. Preedy, Reviews in Food and Nutrition Toxicity, CRC Press, Taylor \& Francis Group, Boca Raton, Florida, Chapter 2, Vol. 3, 2005; b) M. Aschner, N. Onishchenko, S. Ceccatelli, Toxicology of Alkylmercury Compounds, in: A. Sigel, H. Sigel, R. K. O. Sigel, Organometallics in Environment and Toxicology: Metal Ions in Life Sciences, Chapter 12, vol. 7, pp. 403-434, 2010.

${ }^{14}$ Reviews: a) P. Aggarwal, S. Gaur, P. Gauba, Environ. Dev. Sustain. 2014, 16, 71-78; b) S. Díez, Rev. Environ. Contam. Toxicol. 2009, 198, 111-132; c) J. E. Sonke, L.-E. Heimbürger, A. Dommergue, C. R. Geoscience 2013, 345, 213-224.
} 
solution (usually nitric acid). A plasma source ionizes the sample and allows the quantification of elemental ions, the results show a relation molecular weight - signal intensity.

- Flame atomic absorption spectroscopy (FAAS): it is performed by the sample ionization, measuring the absorption of the sample when it is transformed to gaseous state by a flame. It is capable of measuring elemental composition.

Both analytical techniques are the most reliable way to detect the presence of an element in a sample and quantify it. Nevertheless, they also have important drawbacks such as the needing for a specialist to perform the measurements, the high price, long time, necessity of a pre-treatment of the samples (generally with strong acids) or the impossibility to distinguish between the different derivatives, which might be of extreme importance in the case of mercury. In contrast, the use of fluorescent and colorimetric methods for the quantification have increased its popularity because of their simplicity, lower prices, faster results and the potential for discriminating between derivatives.

\subsection{Fluorogenic and chromogenic probes for $\mathrm{Hg}$ (II) detection in literature ${ }^{15}$}

Mercury cations are considered as big, soft and metallic because of the atomic weight of mercury and the position in the periodic table. This makes them a good target for detection, since there are many possibilities to design probes with high affinity. There is a huge number of publications about detecting mercury cations by using colorimetric and/or fluorometric probes. So then, the next part serves as an example of some common characteristics from the most cited probes in literature.

- Probes containing sulfur atoms. ${ }^{16}$ Most probes for mercury cations contain several sulfur atoms because of the high affinity between mercury-sulfur.

- Probes based on macrocycles. There are many examples in literature (see Figure 2) and they work by many different mechanisms. There are examples of ON-OFF ${ }^{17}$ or OFF-ON systems, ${ }^{18}$ working usually by PET or PCT mechanisms. In addition, using macrocycles with $\mathrm{O} / \mathrm{S}$ atoms increases the solubility in water and they are easy to combine with fluorescent molecules.

\footnotetext{
${ }^{15}$ Chromogenic or fluorogenic probes for $\mathrm{Hg}(\mathrm{II})$ and other cations: Reviews: a) X. Li, X. Gao, W. Shi, H. Ma, Chem. Rev. 2014, 114, 590-659; b) D. Sareen, P. Kaur, K. Singh, Coord. Chem. Rev. 2014, 265, 125-154; c) M. Formica, V. Fusi, L. Giorgi, M. Micheloni, Coord. Chem. Rev. 2012, 256, 170-192;

Specific fluorogenic probes for $\mathrm{Hg}(\mathrm{II})$ : Reviews: d) M. J. Culzoni, A. Muñoz de la Peña, A. Machuca, H. C. Goicoechea, R. Babiano, Anal. Methods. 2013, 5, 30-49. e) M. Tian, L. Liu, Y. Li, R. Hu, T. Liu, H. Liu, S. Wang, Y. Li, Chem. Commun. 2014, 50, 2055-2057;

Colorimetric nanoprobes for $\mathrm{Hg}(\mathrm{II})$ : f) I. Ratera, A. Tárraga, P. Molina, J. Veciana, in T. Torres, G. Bottari, Editors: Organic Nanomaterials: Synthesis, Characterization, and Device Applications, John Wiley \& Sons, Hoboken, New Jersey, Chapter 24, pp. 529-548, 2013.

Recent examples: g) Q. Wei, R. Nagi, K. Sadeghi, S. Feng, E. Yan, S. J. Ki, R. Caire, D. Tseng, A. Ozcan, ACS Nano 2014, 8, 1121-1129; h) Q. Yue, T. Shen, J. Wang, L. Wang, S. Xu, H. Li, J. Liu, Chem. Commun. 2013, 49, $1750-1752$ (and references therein);

Lifetime fluorescence: i) D. Huang, C. Niu, M. Ruan, X. Wang, G. Zeng, C. Deng, Environ. Sci. Technol. 2013, 47, 4392-4398.

${ }^{16}$ D. Riccardi, H. B. Guo, J. M. Parks, B. Gu, A. O. Summers, S. M. Miller, L. Liang, J. C. Smith, J. Phys. Chem. Lett. 2013, 4, 2317-2322.

${ }_{17}$ A. B. Descalzo, R. Martínez-Máñez, R. Radeglia, K. Rurack, J. Soto, J. Am. Chem. Soc. 2003, 125, 34183419.

${ }^{18}$ S. Yoon, A. E. Albers, A. P. Wong, C. J. Chang, J. Am. Chem. Soc. 2005, 127, 16030-16031.
} 

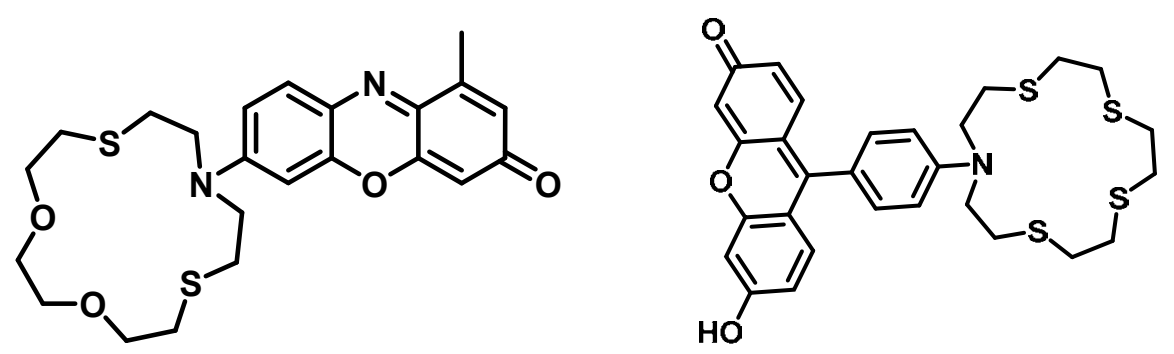

Figure 2. Examples of molecular probes with macrocycles containing sulfur atoms for the detection of mercury cations described in publications from Martínez-Máñez ${ }^{17}$ and Chang, ${ }^{18}$ respectively.

- Probes based on recognition by a specific PET - excimer system. ${ }^{19}$ Some groups have designed molecules with special affinity for mercury cations because of supramolecular recognition and specific changes in the structure, affecting fluorescence. For instance, Guo and coworkers developed a probe (Figure 3) as an V shape coordinating molecule capable of changing its fluorescence with high selectivity in water buffered solution.

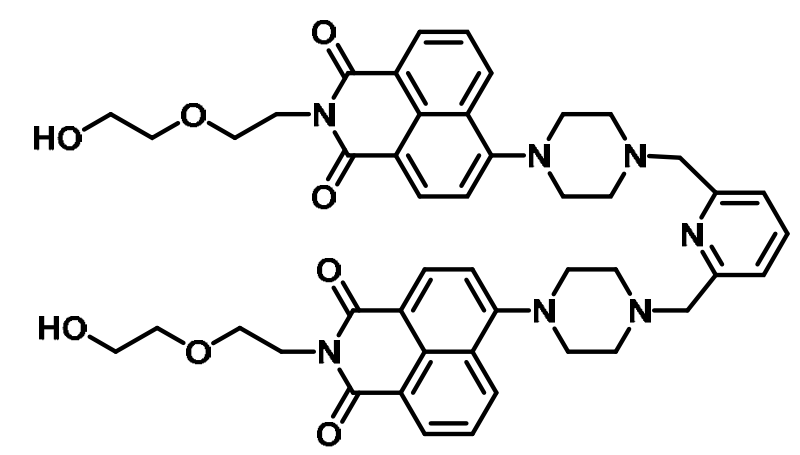

Figure 3. Structure of the molecule synthesized by Guo and co-workers for the identification of $\mathrm{Hg}$ (II) in solution.

- Probes that work by a chemical reaction, ${ }^{20}$ in which, for instance, the mercury cations may work by producing a redox reaction and a complexation. The molecules of Figure 4, which contain an azadiene group, are capable of complexing selectively $\mathrm{Hg}(\mathrm{II})$ over other cations. The fluorescence of the product increases by adding $\mathrm{Hg}(\mathrm{II})$.

\footnotetext{
${ }^{19}$ X. Guo, X. Qian, L. Jia, J. Am. Chem. Soc. 2004, 126, 2272-2273.

${ }^{20}$ Fluorimetric chemodosimeters: Example: A. Caballero, R. Martínez, V. Lloveras, I. Ratera, J. Vidal-Gancedo, K. Wurst, A. Tárraga, P. Molina, J. Veciana, J. Am. Chem. Soc. 2005, 127, 15666-15667. Reviews: a) Yang, Y.; Zhao, Q.; Feng, W.; Li, F. Chem. Rev. 2013, 113, 192-270; b) X. Chen, T. Pradhan, F. Wang, J. S. Kim, Q. Yoon, J. Chem. Rev. 2012, 112, 1910-1956; c) D. T. Quang, J. S. Kim, Chem. Rev. 2010, 110, 6280-6301; d) K. Kaur, R. Saini, A. Kumar, V. Luxami, N. Kaur, P. Singha, S. Kumar, Coord. Chem. Rev. 2012, 256, 1992-2028; e) J. Du, M. Hu, J. Fan, X. Peng, Chem. Soc. Rev. 2012, 41, 4511-4535; desulfurization in cells: K. Bera, A. K. Das, M. Nag, S. Basak, Anal. Chem. 2014, 86, 2740-2746.
} 

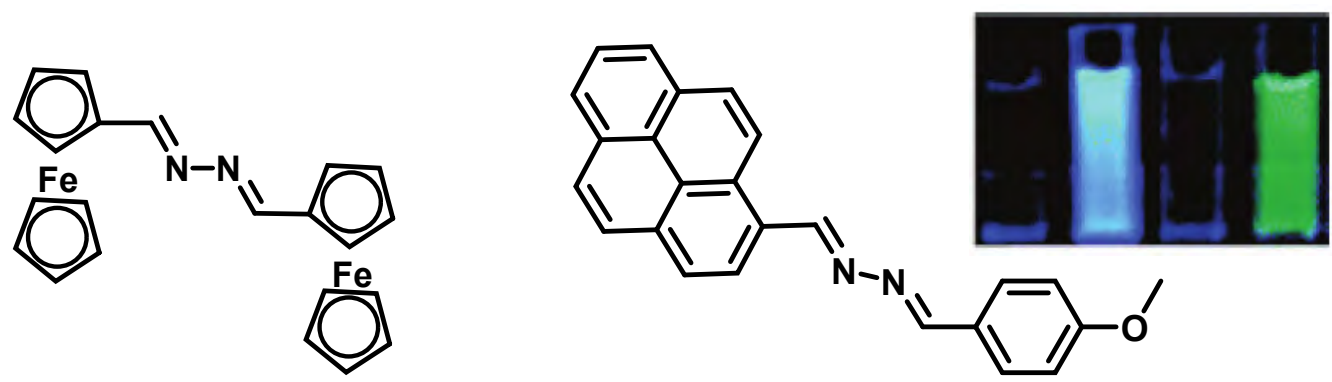

Figure 4. Fluorescent molecules described in the paper from Caballero et al, emission in the presence of $\mathrm{Hg}(\mathrm{II})$ in $\mathrm{MeCN}: \mathrm{H}_{2} \mathrm{O}$ 7:3 solutions (blue) or just $\mathrm{MeCN}$ (green).

- Probes using nanoparticles or nanosystems. ${ }^{21}$ They are characterized for having molecular recognition subunits, such as aptamers joined to highly fluorescent molecules / nanomaterials. In particular, there are aptamers with specific interactions with mercury cations, being capable to develop nanosystems with high sensitivity.

In addition to fluorescent detection of the substance in a quantitative way, some of the probes have also application in cellular imaging; which might be useful to visualize the probes in cells, as it is shown in Figure 5.<smiles>CC(=O)/C(C)=N/N1C(=S)c2ccccc2C12c1ccc(N(C)C)cc1Oc1cc(N(C)C)ccc12</smiles>

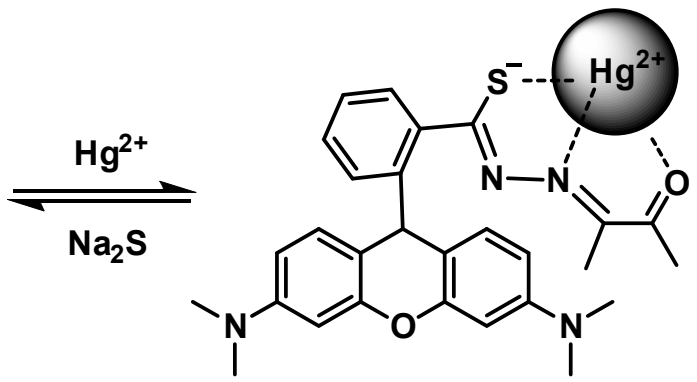
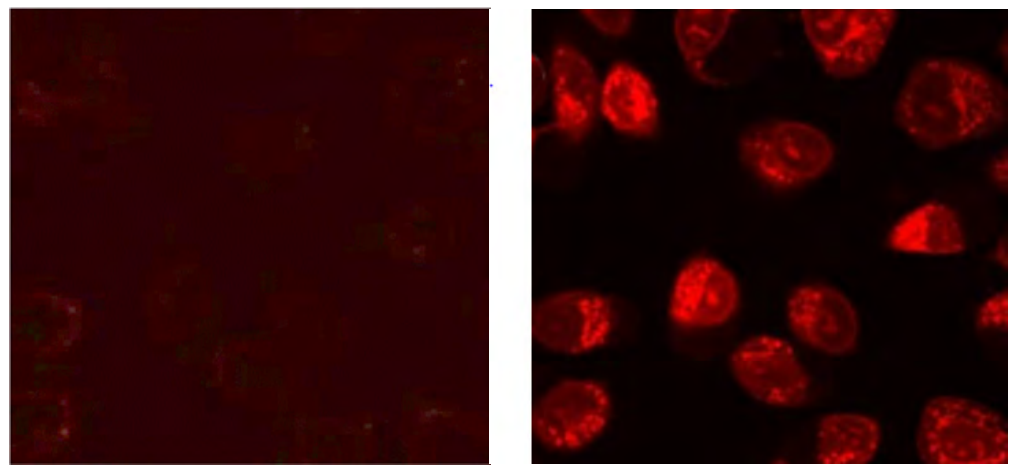

Figure 5. Rhodamine sensor ThioRh-1 in A549 cells $10 \mu \mathrm{M}$ probe, $30 \mu \mathrm{M} \mathrm{Hg}(\mathrm{II})$, synthesized and tested by the group of Jiang and coworkers. ${ }^{22}$

In spite of the great amount of papers in the topic, there was a series of common problems for most of the probes from literature:

\footnotetext{
${ }^{21}$ Graphene aptamers/current change: a) J. H. An, S. J. Park, O. S. Kwon, J. Bae, J. Jang, ACS Nano 2013, 7, 10563-10571; b) J. Li, W. Tu, H. Li, M. Han, Y. Lan, Z. Dai, J. Bao, Anal. Chem. 2014, 86, 1306-1312;

Fluorogenic arrays: c) S. S. Tan, S. J. Kim, E. T. Kool, J. Am. Chem. Soc. 2011, 133, 2664-2671;

up-conversion nanophosphors: d) X. Li, Y. Wu, Y. Liu, X. Zou, L. Yao, F. Lia, W. Feng, Nanoscale 2014, 6, 1020-1028; e) Q. Liu, J. Peng, L. Sun, F. Li, ACS Nano 2011, 5, 8040-8048.

${ }^{22}$ H. H. Wang, L. Xue, C. L. Yu, Y. Y. Qian, H. Jiang, Dye. Pigment. 2011, 91, 350-355.
} 
$>$ There were no probes $100 \%$ soluble in water media, some of them were soluble in waterorganic solvent mixtures with high percentages of water but there were no examples of $100 \%$ water soluble probes.

$>$ The LODs calculated in most of the papers are not reliable, usually the values are calculated by outdated methods that give unreliable results.

$>$ There are only a few examples of using probes for detecting both $\mathrm{Hg}$ (II) and $\mathrm{MeHg}(\mathrm{II}),{ }^{23}$ and none of them were able to speciate between both until the publication of our research was done ${ }^{24}$ which is in strong contrast to the enormous interest that $\mathrm{MeHg}(\mathrm{II})$-induced neurotoxicity promotes ${ }^{25}$ and its imaging in living systems. ${ }^{26}$

There were no examples of materials that change its colour/fluorescence in presence of $\mathrm{MeHg}(\mathrm{II})$ cations. $^{2}$

In this regard, the synthesized systems had the objective to overcome these issues.

\footnotetext{
${ }^{23}$ Fluorogenic probes for MeHg(II): a) M. Santra, D. Ryu, A. Chatterjee, S. K. Ko, I. Shin, K. H. Ahn, Chem. Commun. 2009, 2115-2117; b) I. Costas-Mora, V. Romero, I. Lavilla, C. Bendicho, Anal. Chem. 2014, 86, 4536-4543 (and referenced methods therein); d) E. Climent, M. D. Marcos, R. Martínez-Máñez, F. Sancenón, J. Soto, K. Rurack, P. Amorós, Angew. Chem. Int. Ed. 2009, 48, 8519-8522; C. Coll, A. Bernardos, R. MartínezMáñez, F. Sancenón, Acc. Chem. Res. 2013, 46, 339-349.

${ }^{24}$ Speciation $\mathrm{Hg}[\mathrm{II}] / \mathrm{MeHg}[\mathrm{II}]$ : Y. Li, Y. Yin, G. Liu, Y. Cai, in: Environmental Chemistry and Toxicology of Mercury, G. Liu, Y. Cai, N. O'Driscoll, (Editors), John Wiley \& Sons, Hoboken, New Jersey, Chapter 2, pp. 15$58,2012$.

${ }^{25}$ S. Ceccatelli, M. Aschner, Editors: Methylmercury and Neurotoxicity, Current Topics in Neurotoxicity, Vol. 2, Springer, New York, 2012.

${ }^{26}$ Methylmercury imaging: reaction-based examples in reviews: a) M. J. Pushie, I. J. Pickering, M. Korbas, M. J. Hackett, G. N. George, Chem. Rev. 2014, 114, 8499-8541; b) Z. Guo, S. Park, J. Yoon, I. Shin, Chem. Soc. Rev. 2014, 43, 16-29; an example of upconversion bioimaging of $\mathrm{MeHg}(\mathrm{II})$ : c) Y. Liu, M. Chen, T. Cao, Y. Sun, C. Li, Q. Liu, T. Yang, L. Yao, W. Feng, F. Li. J. Am. Chem. Soc. 2013, 135, 9869-9876.
} 


\section{ANTECEDENTS FROM THE GROUP IN MERCURY PROBES DEVELOPMENT}

\subsection{Chromogenic $\mathrm{Hg}$ (II) and $\mathrm{MeHg}(\mathrm{II})$ probes}

Years ago, the group started working with some variations of mercury probes based on colorimetric analysis and detection ${ }^{27}$ of $\mathrm{Hg}$ (II) and $\mathrm{MeHg}(\mathrm{II})$. This first probe was a palladium complex capable to methylate $\mathrm{Hg}$ (II) salts in a water:ethanol solution leading to a change in the colour of the solution. In addition, the process was reversible by adding a dithiol compound. (Figure 6)

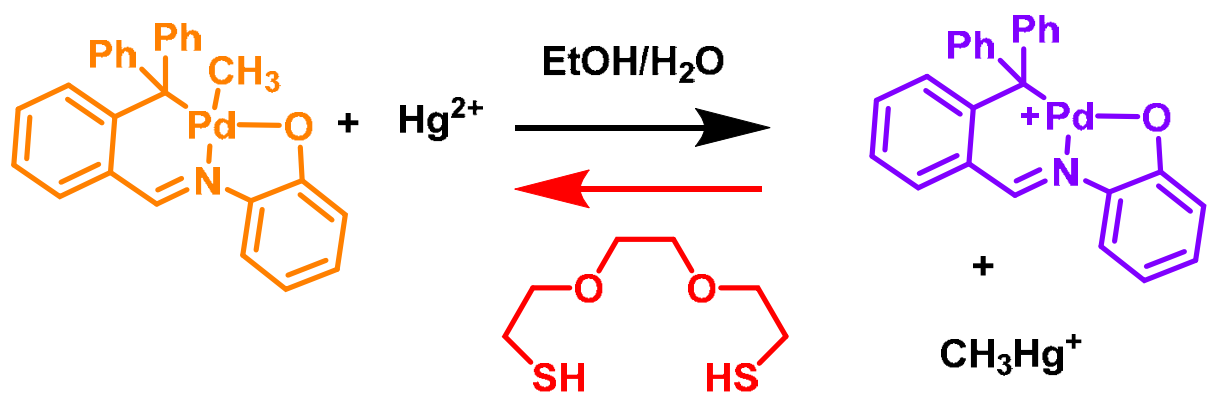

Figure 6. Chromogenic prober for detection of $\mathrm{Hg}(\mathrm{II})$ and $\mathrm{MeHg}(\mathrm{II})$

After that, the group also worked in other chromogenic probes that changed its colour in presence of $\mathrm{Hg}(\mathrm{II})$ solutions in buffer, MeCN:HEPES 1:1, through complexation of the cation to sulfur atoms in polycyclic dithiolethiones, a class of polysulfur-nitrogen heterocycles. Then, years later, other probes based on similar systems, pyrrolidine-fused 1,3-dithiolane oligomers, were tested and optimized for colorimetric response to the presence of $\mathrm{Hg}$ (II) cations.

Due to the positive results and the interest of detecting very low quantities of mercury cationic species, the next idea was to use more versatile molecules, that were able to increase their signal ratio in the presence of the analytes with higher selectivity and sensitivity. Simultaneously, the group had been also working with fluorescent indanone derivatives ${ }^{28}$ as chemical sensors, which had applications for analysis of warfare agents, ${ }^{29}$ encouraging us to follow this path in order to develop new cationic mercury probes.

\footnotetext{
${ }^{27}$ a) P. Fuertes, M. García-Valverde, J. V. Cuevas, B. Díaz de Greñu, T. Rodríguez, J. Rojo, T. Torroba, J. Org. Chem. 2014, 79, 2213-2225; b) P. Fuertes, D Moreno, J. V. Cuevas, M. García-Valverde, T. Torroba, Chem. Asian J. 2010, 5, 1692-1699; c) O. del Campo, A. Carbayo, J. V. Cuevas, A. Muñoz, G. García-Herbosa, D. Moreno, E. Ballesteros, S. Basurto, T. Gómez, T. Torroba, Chem. Commun. 2008, 0, 4576-4578.

28 a) T. Gómez, D. Moreno, B. Díaz de Greñu, A. C. Fernández, T. Rodríguez, J. Rojo, J. V. Cuevas, T. Torroba, Chem. Asian J. 2013, 8, 1271-1278; b) D. Moreno, B. Díaz de Greñu, B. García, S. Ibeas, T. Torroba, Chem. Commun. 2012, 48, 2994-2996; c) D. Moreno, J. V. Cuevas, G. García-Herbosa, T. Torroba, Chem. Commun. 2011, 47, 3183-3185; d) E. Ballesteros, D. Moreno, T. Gómez, T. Rodríguez, J. Rojo, M. García-Valverde, T. Torroba, Org. Lett. 2009, 11, 1269-1272.

${ }_{29}$ B. Díaz de Greñu, D. Moreno, T Torroba, A. Berg, J. Gunnars, T. Nilsson, R. Nyman, M. Persson, J. Pettersson, I. Eklind, P. Wästerby, J. Am. Chem. Soc. 2014, 136, 4125-4128.
} 


\subsection{Synthesis of fluorogenic probes and their action}

The group started working with several fluorescent derivatives of the pyrimidine and pyridine indanone for synthesizing PET fluorescent sensors. Figure 7 shows the indanone backbones (fluorophore) that were attached to a thiophosphinate (receptor) through a piperazine group (spacer) anticipating high sensitivity, typical for fluorescent probes, to the presence of mercury and methylmercury cations.

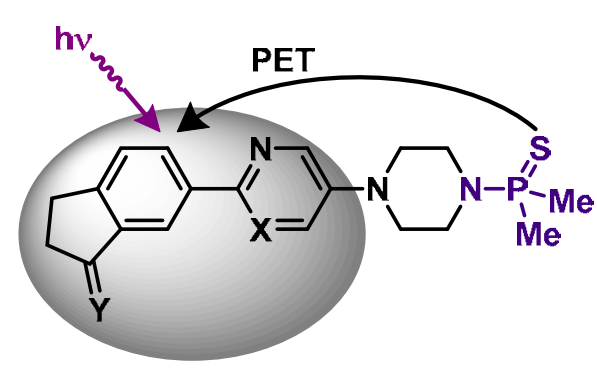

$\mathrm{X}=\mathrm{CH}$ or $\mathrm{N} ; \mathrm{Y}=\mathrm{O}$ or $\mathrm{C}(\mathrm{CN})_{2}$

Fluorophore - Spacer-Receptor

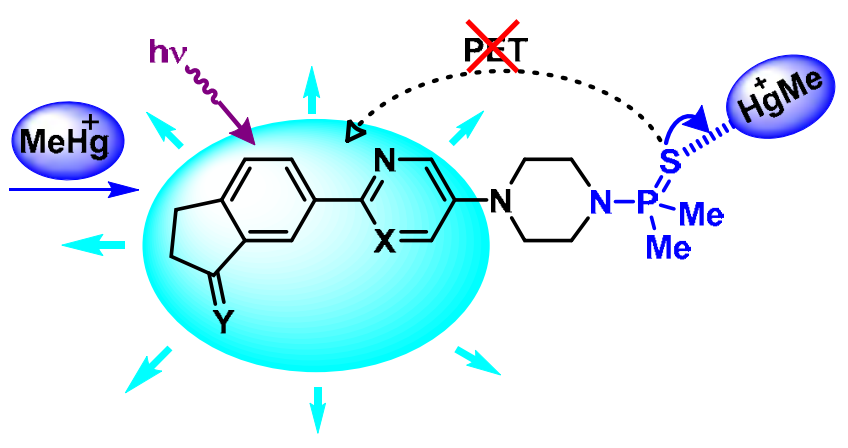

Fluorophore - Spacer-Receptor

Figure 7. Mechanism of PET in the indanone based mercury cation probes.

The synthesis of this kind of sensors was already optimized in previous publications and consisted of two steps for the ketone derivative (Figure 8) and, when needed, a third step to modify it to the dicyanomethylene derivative (Figure 9). Finally, $\mathrm{Hg}(\mathrm{II})$ receptor was added in the free amino group.

(Figure 10):

1) Synthesis of the pyridine/pyrimidine indanone joined to a piperazine group:

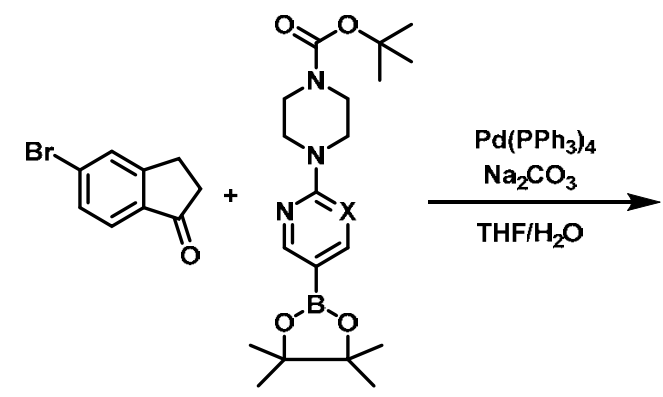<smiles>CC(C)(C)OC(=O)N1CCN(c2ncc(-c3ccc4c(c3)CCC(=O)C4)cn2)CC1</smiles><smiles>O=C1CCc2cc(-c3ccc(N4CCNCC4)cn3)ccc21</smiles>

Figure 8. Synthesis of the pyridine/pyrimidine indanone joined to a piperazine group.

The commercial reagents, 5-bromoindanone and the pyridine/pyrimidine piperazine bocprotected boronate react $(1: 1)$ through a Suzuki coupling under nitrogen atmosphere to give the boc protected products. The conditions for these reactions were optimized in THF/Water (10:1) mixture and catalyzed by $\mathrm{Pd}\left(\mathrm{PPh}_{3}\right)_{4}$ in presence of a weak base, $\mathrm{Na}_{2} \mathrm{CO}_{3}$. The reactions were escalated up to 1 $\mathrm{g}$ and gave quantitative yields ( $>95 \%$ ) as a white solid, only by removing the starting material in diethylether.

In a second step, the amine group of the product was easily deprotected in strong acidic solution with excess of trifluoroacetic acid in DCM. The trifluoroacetic acid was added dropwise to the solution while under stirring $(\mathrm{DCM} \approx 20 \mathrm{ml}$, probe $\approx 1 \mathrm{~g}$, TFA $\approx 10 \mathrm{ml}$ ), then stirring for 15 minutes was enough to obtain the product. Afterwards, it was neutralized to $\mathrm{pH} 8-10$, extracted with dichloromethane and evaporated quantitatively obtaining the amine as a white solid. 


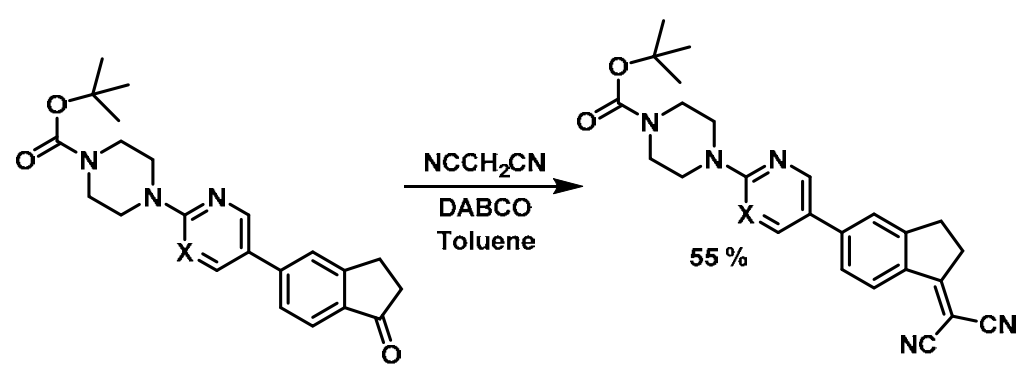

Figure 9. Knoevenagel reaction over the indanone products.

For the preparation of the derivative containing a dicyanomethylene group, the protected probe was dissolved in toluene and excess of malononitrile (1:3) and DABCO (1:2.5) were added to the solution. This mixture was heated under reflux for 24 hours and the product was extracted from a mixture DCM-water $(3 \times \mathrm{DCM})$ and purified by column chromatography in DCM mixture with $\mathrm{MeOH}$ $1.5 \%$. The process gave a yellow solid in $55 \%$ yield.

2) Synthesis of the thiophosphinate probe:<smiles></smiles>

1a-d

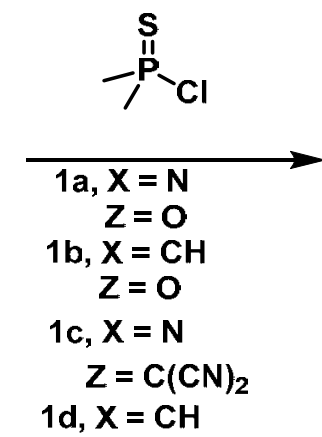

$\mathrm{Z}=\mathrm{C}(\mathrm{CN})_{2}$<smiles>[Z]C1=Cc2ccc(-c3c[X]c(N4CCN(P(C)(=S)S)CC4)nc3)cc2CC1</smiles>

Fluorogenic probes

Figure 10. Scheme of the $\mathrm{S}_{\mathrm{N}} 2$ reaction to synthesize the probes containing dimethylthiophosphinate.

The amine group from the piperazine reacted with a commercial thiophosphinate chloride giving a $\mathrm{S}_{\mathrm{N}} 2$ substitution of the chloride in DCM at room temperature in 24 hours.

In this way, previous members of the group had synthetized and tested the probes in Figure 11.<smiles>CP(C)(=S)P(C)(=S)N1CCN(c2ncc(-c3ccc4c(c3)CCC4=O)cn2)CC1</smiles>

BD116

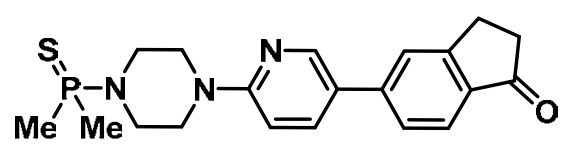

BD118<smiles>CP(C)(=S)N1CCN(c2ncc(-c3ccc4c(c3)CCC4=C(C#N)C#N)cn2)CC1</smiles>

BD119

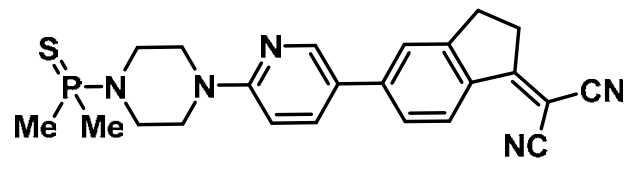

BD117

Figure 11. Molecular structures of BD116, BD117, BD118 and BD119. 
These probes were fully characterized and tested as $\mathrm{Hg}(\mathrm{II})$ sensors, the process and measurements are fully explained within the published paper, ${ }^{1}$ belonging to the thesis of Borja Díaz de Greñu. $^{30}$

Summarizing these previous results is useful as context and introduction of the results presented in the present thesis. Therefore, in subsequent Sections 3 and 4, the fluorogenic probes were tested in mixtures of different solvents (DMSO, DMF, acetone, $\mathrm{MeCN}, \mathrm{MeOH})$ and water $(100 \%, 80: 20,60: 40$ and 20:80). After that, several cations, as perchlorate or triflate salts, or anions, as tetrabutylammonium salts, dissolved in water were added to the solutions (see Annex for more information). The changes were followed for every analyte; pictures of probes BD116 and BD119 are shown in Figure 12.

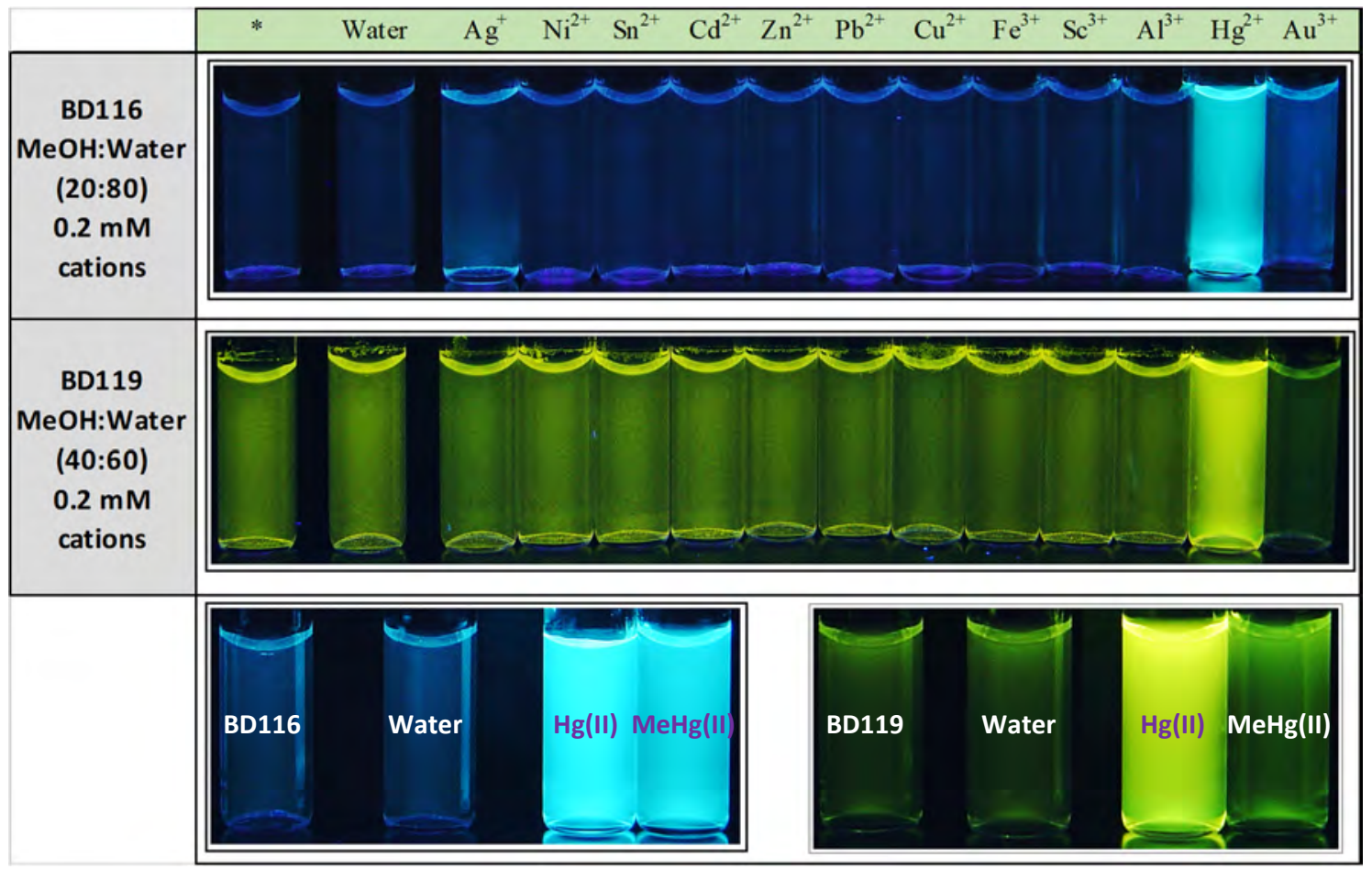

Figure 12. Observable colours under $366 \mathrm{~nm}$ UV light. The samples contain BD116 (upper) or BD119 (down) $0.1 \mathrm{mM}$ solutions in $\mathrm{MeOH} / \mathrm{H}_{2} \mathrm{O} 20: 80$ mixed with $0.2 \mathrm{mM}$ solutions of common cations, from $5 \mathrm{mM}$ solutions in water.

These experiments demonstrated that the probes with a pyrimidine group BD116 and BD119 were OFF-ON highly fluorogenic probes for the selective detection of $\mathrm{Hg}$ (II) cations in mixtures of methanol/water 20:80 V/V or acetonitrile/water $20: 80 \mathrm{~V} / \mathrm{V}$, and that characteristic was the basis for the rest of the studies.

\footnotetext{
${ }^{30} \mathrm{PhD}$ Thesis of B. Díaz de Greñu Puertas, Detection and discrimination of organic contaminants of high environmental impact by means of fluorogenic probes. Director: Tomás Torroba Pérez. University of Burgos, 2014.
} 


\section{OBJECTIVES. Hg(II) AND MeHg(II) FLUOROGENIC PROBES USING 100\% WATER AS SOLVENT}

The aim of this chapter is to improve and complement the previous results obtained using indanone derivatives as fluorescent backbones, developing new molecular sensors for the detection of $\mathrm{Hg}$ (II) and derivatives. In this regard, Section 3 is a summary of the objectives and the steps followed to achieve these probes, which are extensively explained in subsequent sections.

\subsection{Designing molecular probes for detection of $\mathrm{Hg}$ (II) and $\mathrm{MeHg}(\mathrm{II})$ in water solution}

The results of previous tests were satisfactory and deeply studied qualitatively and quantitively. Thereafter, in order to improve the number of probes, some derivatives were synthetized.

The work is divided into some parts:

- Synthesis of the molecules (Section 4, soluble probes, and Section 6, materials).

- Characterization (Detailed in Experimental Appendix 1).

- Tests with probes (Section 5, soluble probes, and Section 7, materials):

o Solvatochromism.

o Tests with cations and anions.

o Qualitative and quantitative tests with $\mathrm{Hg}$ (II) and $\mathrm{MeHg}(\mathrm{II})$.

o Response of the probes to the presence of $\mathrm{Hg}$ (II) and $\mathrm{MeHg}$ (II) within cells.

A) Direct variations from previous probes.

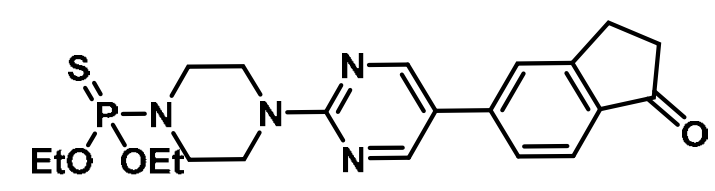

JG7<smiles>CCOP(=S)(OCC)N1CCN(c2ncc(-c3ccc4c(c3)CCC4=C(C#N)C#N)cn2)CC1</smiles>

JG30

Figure 13. Structure of the molecules JG7 and JG30.

Although BD116, BD119, JG7 and JG30 presented excellent properties as $\mathrm{Hg}(\mathrm{II})$ and $\mathrm{MeHg}(\mathrm{II})$ detectors and discriminators; the main disadvantage was their limitation to be used with no more than $60 \%$ - $80 \%$ water/solvent mixtures in the best case. Therefore, different approaches were proposed in order to synthesize derivatives with better water solubility. 
B) Synthesis and development of $100 \%$ water soluble probes.

B.1) Synthesis of modifiable fluorescent precursors:
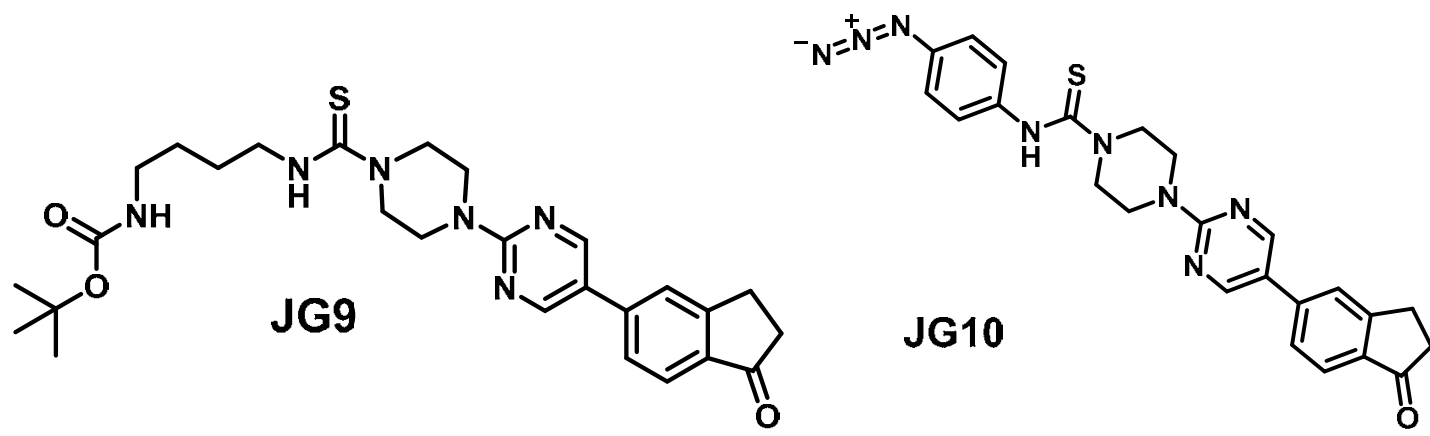

Figure 14. Structure of the molecules JG9 and JG10.

JG9 had poor selectivity and a turn OFF fluorescence in the presence of $\mathrm{Hg}(\mathrm{II})$, which led to focus the research in the modification of JG10.

B.2) Increased solubility by adding a commercial PEG to the molecule:
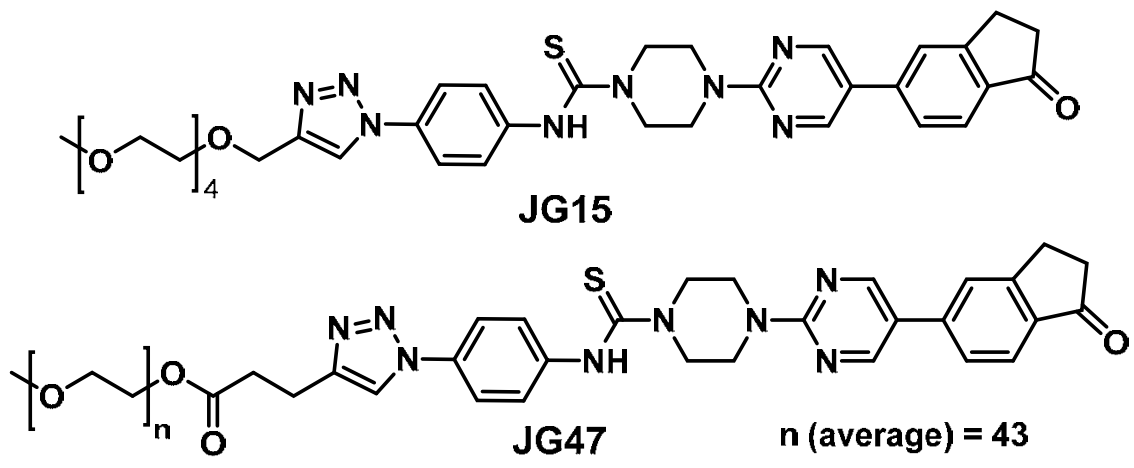

Figure 15. Structure of the molecules JG15 and JG47.

JG15 was not soluble in $100 \%$ water and JG47 was a mixture between PEGs with a molecular weight around $5000 \mathrm{~g} / \mathrm{mol}$.

B.3) Development and synthesis of a tri-PEG chain derivative.

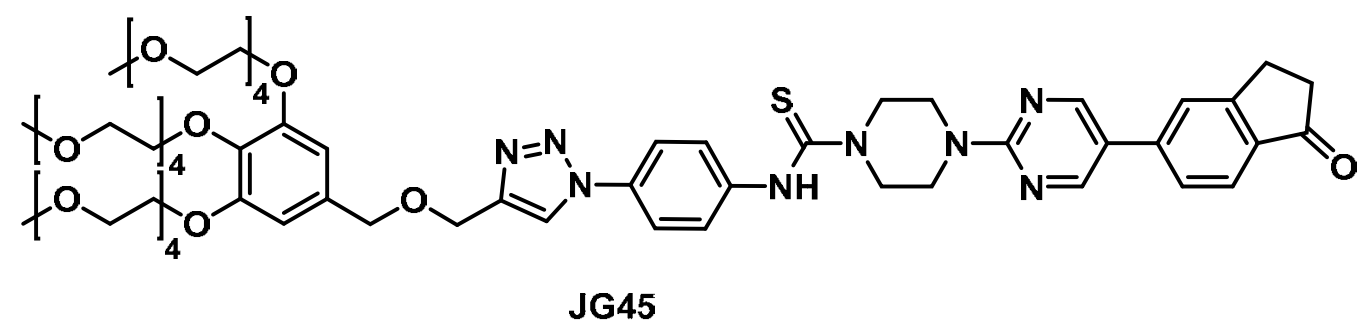

Figure 16. Structure of the molecules JG45.

In this way, JG45 was $100 \%$ soluble in water media. 


\subsection{Supported probes for detection of $\mathrm{Hg}$ (III) and $\mathrm{MeHg}$ (II)}

Next step after designing water soluble molecular probes was to develop materials with similar properties, which would improve the applications significantly. In this regard, the work was oriented in using two kinds of materials; modified silica nanoparticles and polymeric films. In the same way used for molecular probes, they were fully tested following the next steps:

- Synthesis and characterization.

- Evaluation of the qualitative properties in water, response to cations and anions.

- Quantitative analysis with $\mathrm{Hg}(\mathrm{II})$ and $\mathrm{MeHg}(\mathrm{II})$, kinetic effect, titrations, LODs calculation and Fluorescence Quantum Yield.

- Measurements with fish samples.

\section{Synthesized probes:}

First, silica NPs were modified with JG10 (Figure 17).

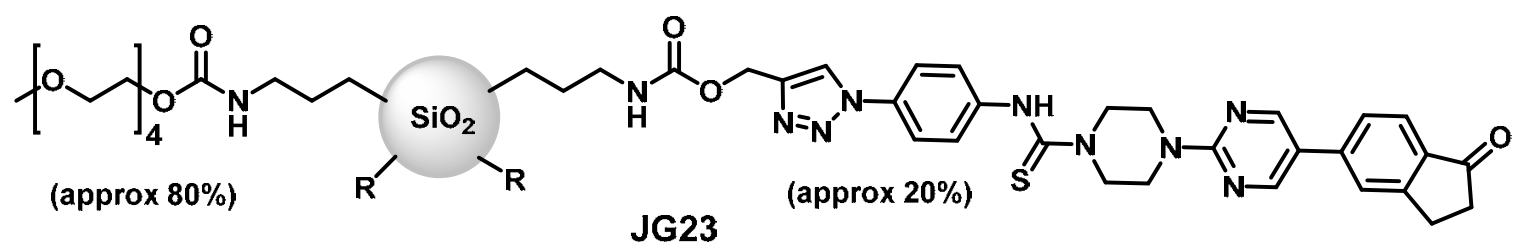

Figure 17. Scheme of silicon nanoparticles JG23, which were modified with PEG chains and JG10.

The results were not satisfactory for mercury detection in preliminary tests. Hence, the next step was to improve the sensitivity and water affinity by using other materials, hydrophilic polymers. Two kinds of polymers were synthetized that reached optimal results (Figure 18).
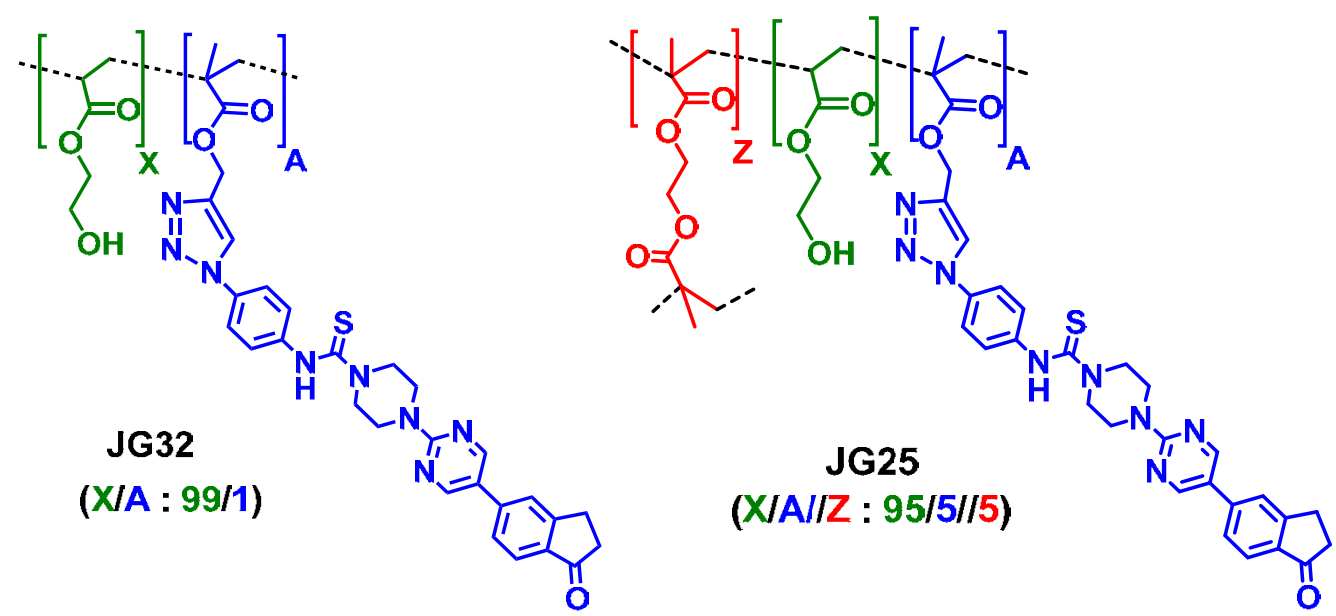

Figure 18. Structure of the polymers JG32 and JG25.

JG32 is a polymer slime soluble in water, whereas JG25 is a film with water affinity. 


\section{SYNTHETIC PROCEDURE FOR MOLECULAR PROBES}

\subsection{Synthesis of the thiophosphinate derivatives}

The first step was the development of derivatives from the probes BD116 and BD119, following the same synthetic route than their precursors, a $\mathrm{S}_{\mathrm{N}} 2$ reaction of the thiophosphinate chloride with the amine group (Figure 19).
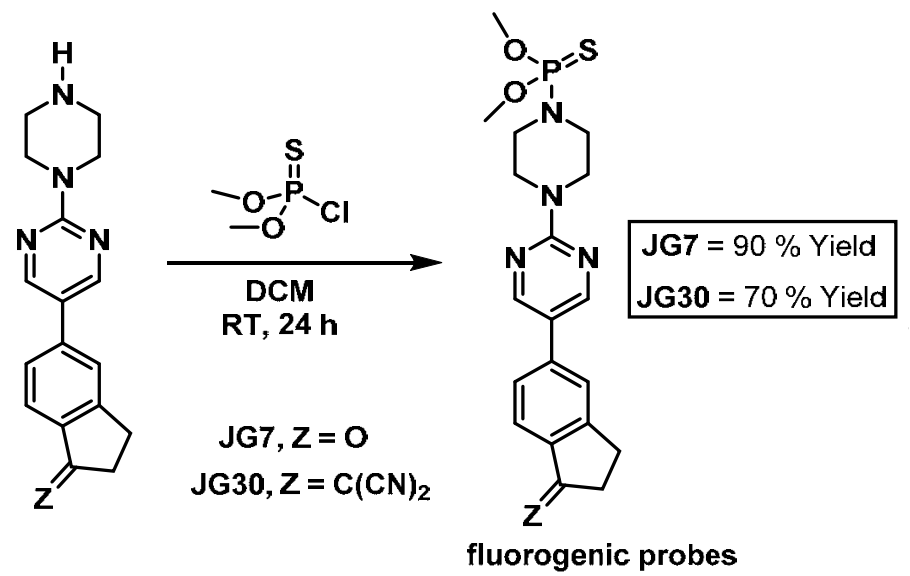

Figure 19. Synthesis scheme of JG7 and JG30.

The ketone derivative JG7 was, as BD116, a white creamy solid that gave colourless solutions whereas the dicyanomethylene derivative JG30, as BD119, was a yellow powder which gave very solvatochromic pale yellow solutions in most organic solvents.

\subsection{Synthesis of modifiable mercury sensors:}

In the same way used for thiophosphinate derivatives, the reaction was performed at room temperature by dissolving the reagents in dichloromethane or chloroform (Figure 20).<smiles>O=C1CCc2cc(-c3cnc(N4CCNCC4)nc3)ccc21</smiles>

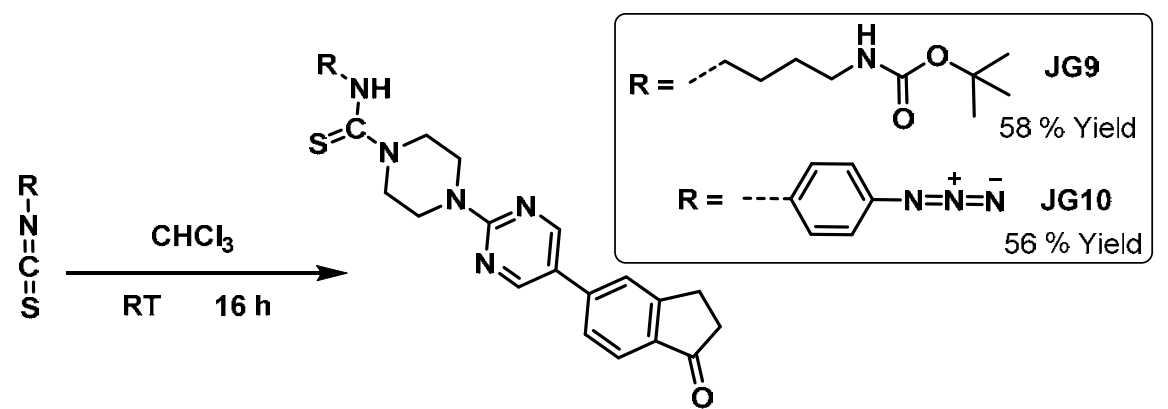

Figure 20. Synthesis of JG9 and JG10.

The reaction takes place through a nucleophilic addition of the amine to the isothiocyanate. The purification was performed by column chromatography in DCM:MeOH $(2 \%)$ obtaining the products as white powders in $56-58 \%$ yields. 


\subsection{Synthesis of PEG chains}

Two synthetic PEGs and one commercial (Figure 21) were used in order to obtain water soluble compounds and, at the same time, to increase the permeability in cells as it had been reported previously by other authors: ${ }^{31}$

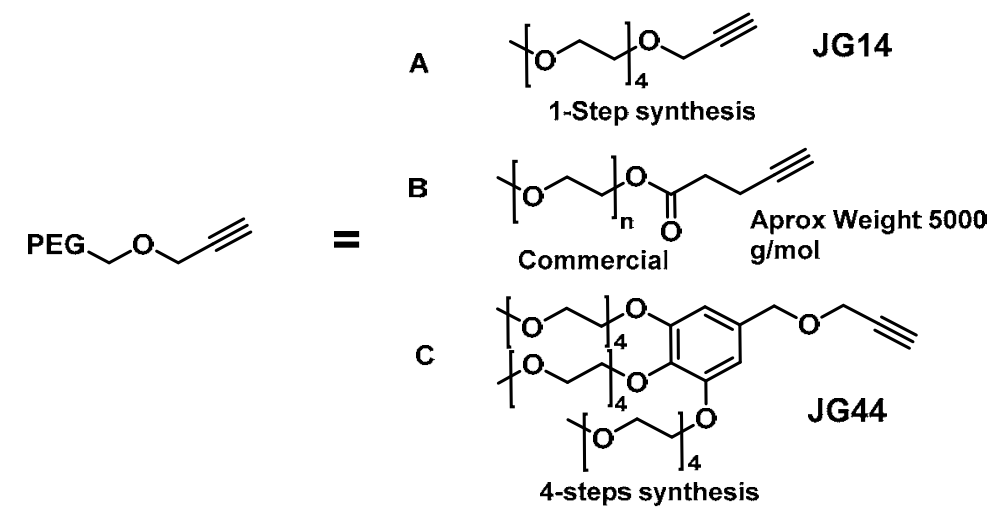

Figure 21. Triple bond substituted PEGs used for water-soluble derivatives.

Tri-PEG JG44 was synthesized starting from tetraethylene glycol monomethyl ether and methyl gallate ester, following the scheme explained in Figure 22.
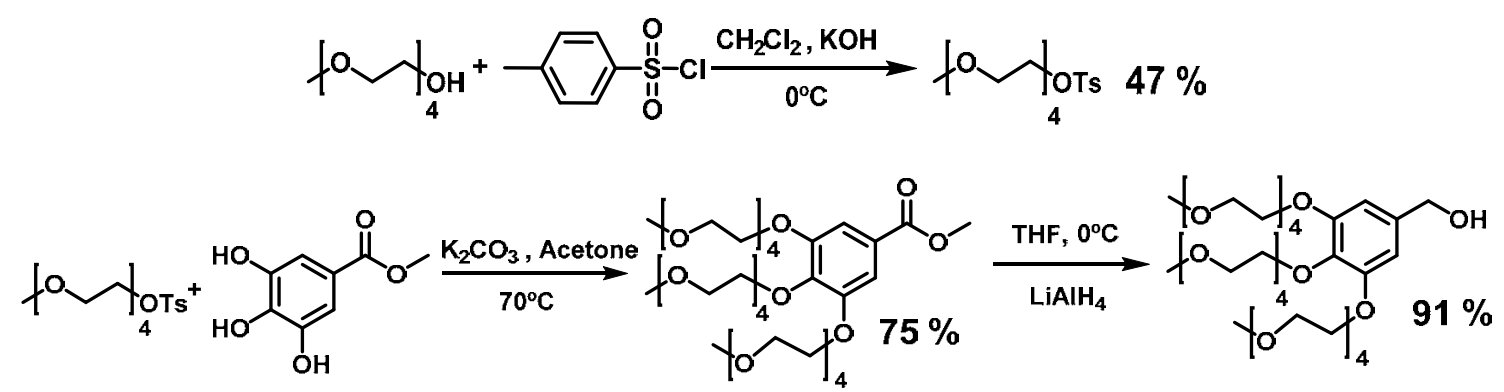

Figure 22. Synthesis of the 3,4,5-tri-PEG benzyl alcohol for the synthesis of tri-PEG JG44.

The reaction started with a nucleophilic addition of the alcohol to $p$-toluenesulfonyl chloride, under basic conditions in DCM solution. The product was purified by column chromatography DCM:MeOH (4\%) as eluent. Afterwards, a nucleophilic substitution of the toluenesulfonic acid group by the alcohol group and potassium carbonate as a base, under heating, led to the tri-PEG with an ester group derivative. The ester was also purified by column chromatography, DCM:MeOH (4\%) as eluent. Finally, the ester group was reduced to alcohol by lithium aluminium hydride in THF solution, obtaining the tri-PEG with a hydroxyl group in almost quantitative yield as a pale yellow oil, by filtration of the product and evaporation of solvent.

Finally, the triple bond-containing PEGs were synthesized from propargyl bromide, Figure 23.

\footnotetext{
31 a) A. X. Zhang, R. P. Murelli, C. Barinka, J. Michel, A. Cocleaza, W. L. Jorgensen, J. Lubkowski, D. A. Spiegel, J. Am. Chem. Soc. 2010, 132, 12711-12716. b) D. K. Tosh, K. Phan, F. Deflorian, Q. Wei, L. S. Yoo, Z.-G. Gao, K. A. Jacobson, Bioconjugate Chem. 2012, 23, 232-247. C) C. Deraedt, N. Pinaud, D. Astruc, J. Am. Chem. Soc. 2014, 136, 12092-12098.
} 


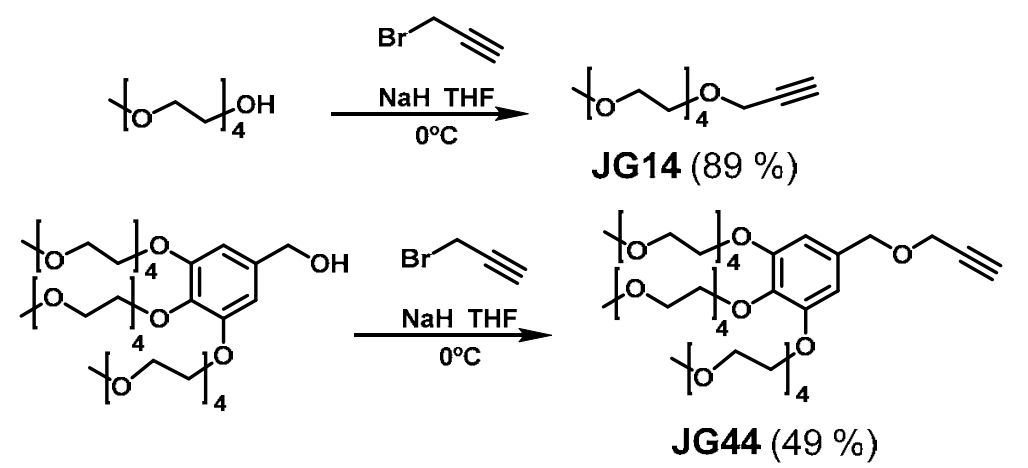

Figure 23. Synthesis of JG14 and JG44, PEG chains with a triple bond.

Propargyl bromide (excess) was dissolved in THF under nitrogen. Next, sodium hydride was added at $0{ }^{\circ} \mathrm{C}$ and after five minutes the PEG was also added. Then, it was stirred for 45 minutes and heated under reflux overnight. The product was purified by neutralization with $\mathrm{HCl}$, extraction with DCM-Water $(3 \times \mathrm{DCM})$ and column chromatography DCM:MeOH $(6 \%)$ of the residue, obtaining the product as a yellow-orange liquid.

\subsection{Click chemistry for developing water soluble compounds based on PEG chains}

The click reactions (Figure 24) were performed between the azide group JG10 and the triple bond from the PEG chain.
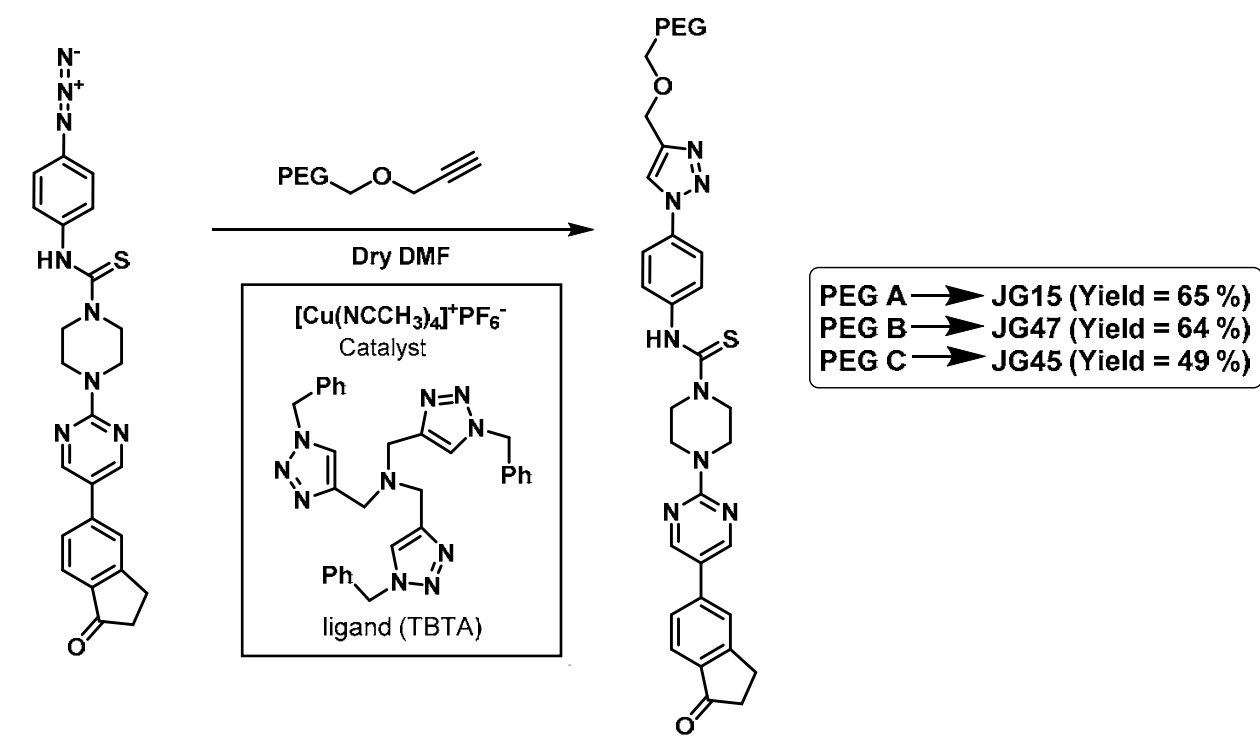

Figure 24. Synthesis scheme of JG15, JG47 and JG45, PEG substituted indanone molecular derivatives.

The reaction was performed in DMF, under nitrogen atmosphere and in presence of a $\mathrm{Cu}(\mathrm{I})$ catalyst $(5 \%)$, stirred at $30^{\circ} \mathrm{C}$ overnight. The reaction yields were improved by adding TBTA ligand to the reaction for avoiding copper retention in the final product ${ }^{32}$ in the same molar concentration than the catalyst. The purification was performed by column chromatography DCM: $\mathrm{MeOH}$ and the products obtained in $49-65 \%$ were waxy solids.

${ }^{32}$ P. S. Donnelly, S. D. Zanatta, S. C. Zammit, J. M. White, S. J. Williams. Chem. Commun., 2008, 21, 24592461. 


\section{TESTS WITH MOLECULAR PROBES}

PEG molecular probes were developed to increase the final solubility in water media and the cell permeability. With this aim, the studies started by registering the response to the presence of $\mathrm{Hg}$ (II) and $\mathrm{MeHg}$ (II) (selectivity and sensitivity) and checking the intracellular response to the presence of these cations. Different experiments were performed.

\subsection{Solvatochromisms}

In order to characterize the properties, the first step was to perform a solvatochromism study of the mercury sensing products JG7, JG30, JG15, JG47 and JG45. The conditions were as follows:

Solvents in order of polarity:
1. Water
2. $\mathrm{MeOH}$
3. DMSO
4. DMF
5. $\mathrm{MeCN}$
6. Acetone
7. AcOEt
8. THF
9. $\mathrm{CHCl}_{3}$
10. $\mathrm{CH}_{2} \mathrm{Cl}_{2}$
11. Toluene
12. $\mathrm{Et}_{2} \mathrm{O}$
13. Hexane
14. Cyclohexane

The solvatochromic studies (Figures 25 and 26) were performed for the probes, they showed a clear influence from polarity and hydrogen bonding interactions, contributing to the Stokes shifts.

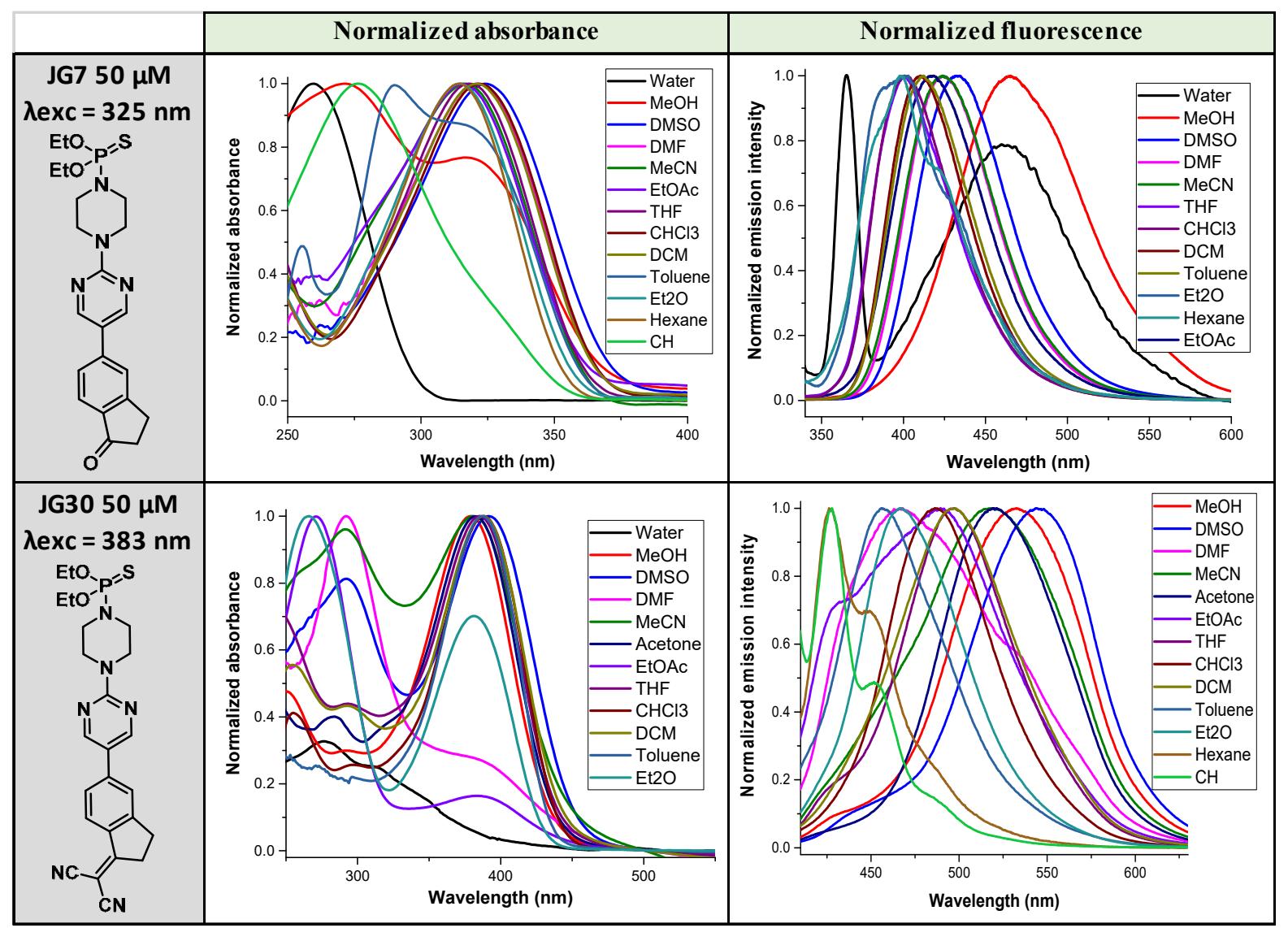

Figure 25. Normalized absorbance and emission spectra of JG7and JG30, $50 \mu \mathrm{M}$ in different solvents. 


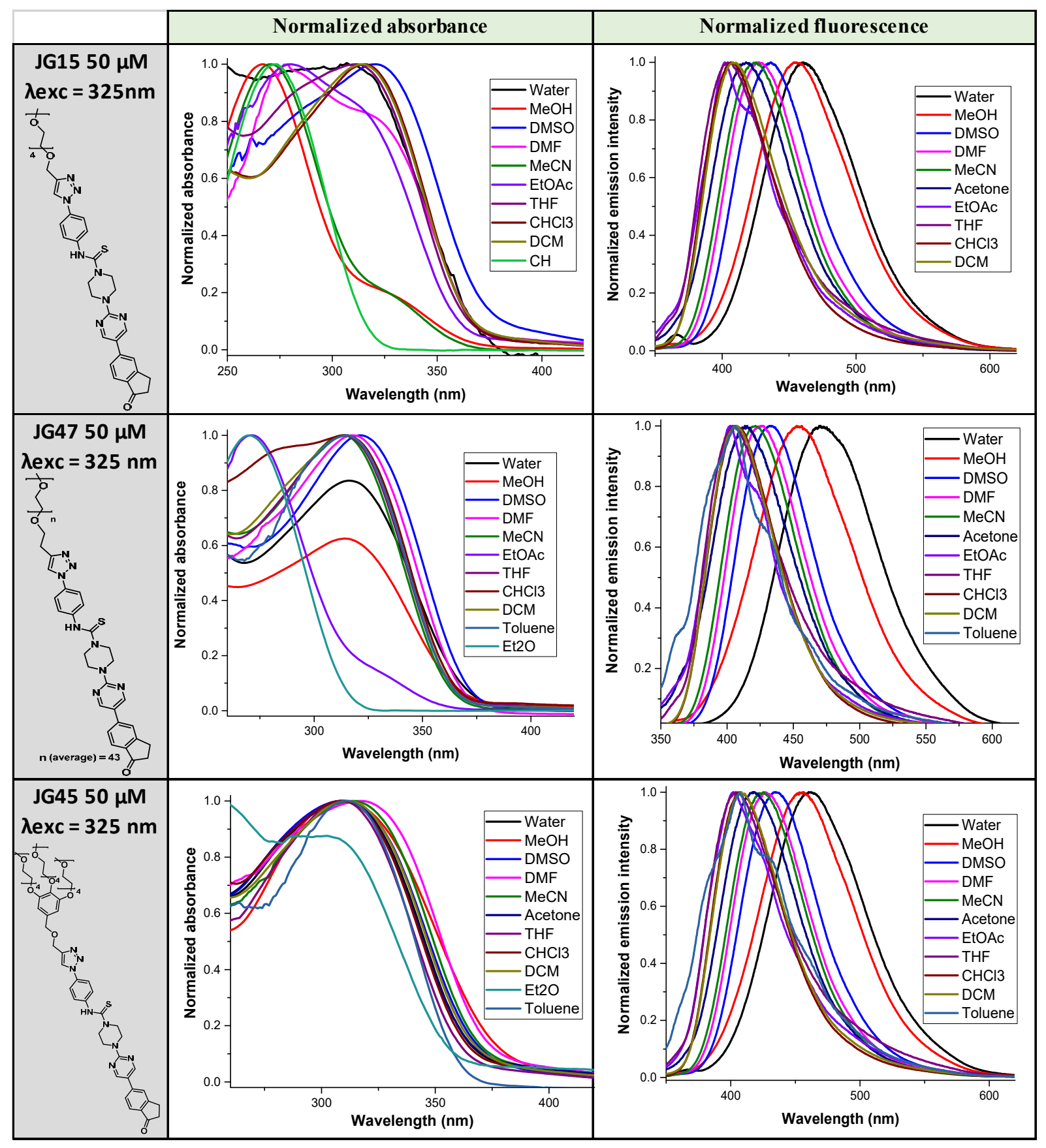

Figure 26. Normalized absorbance and emission spectra of JG15, JG47 and JG45, $50 \mu \mathrm{M}$ in different solvents.

Pictures of the solvatochromism were also taken under visible and UV light (366 nm); Figure 27. In this case, they were colourless under visible light, therefore, it was only showed the response under UV light. 


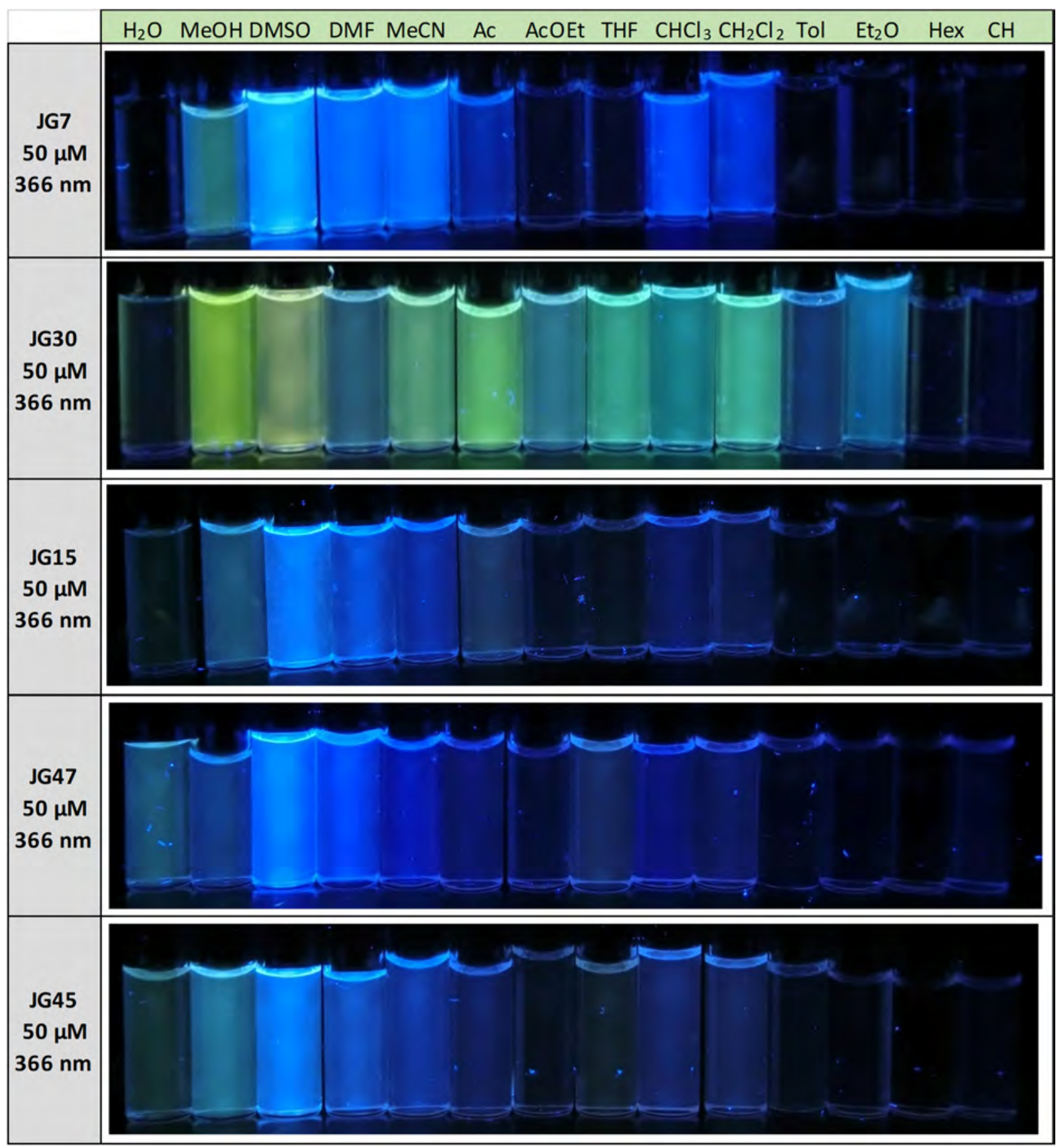

Figure 27. Pictures of probes of JG7, JG30, JG15, JG47 and JG45, $50 \mu \mathrm{M}$ in different solvents under UV light at $366 \mathrm{~nm}$.

From these tests a series of conclusions were obtained:

- Less polar solvents such as hexane or cyclohexane were not capable of solubilising the products.

- All the products had a hypsochromic effect with the decrease of polarity, insignificant for absorbance and as much as $80 \mathrm{~nm}$ for fluorescence, in the range MeOH-Toluene.

- JG30 was also characterized for having a stokes shift that changes up to $150 \mathrm{~nm}$ depending on the solvent.

- In diethyl ether and toluene, compounds showed fluorescence within the long UV for some of the derivatives, for that reason their emission was not observed by the naked eye. 
- Only JG45 and JG47 were soluble in water, JG15 was not soluble in spite of having a PEG chain, it was not enough to solubilise the molecule, which was the main reason for synthesizing JG47 and JG45.

- The probes with a PEG chain had a hypsochromic shift compared with JG7.

- The fluorescence was maximized in DMSO for all the probes, where there was no increase independently of the analyte added.

- The best solvents, high solubility and not very high fluorescence, were methanol and acetonitrile.

\subsection{Molar extinction coefficients}

To get a more accurate value, each of the molar extinction coefficients for the different probes were calculated by doing a regression between 10 to $50 \mu \mathrm{M}$ concentrations in $\mathrm{MeOH}$.

$$
\begin{array}{ll}
\text { JG7 MeOH } \lambda(\varepsilon)=318 \mathrm{~nm}\left(33000 \mathrm{M}^{-1} \mathrm{~cm}^{-1}\right) & \log (\varepsilon)=4.52 \\
\text { JG30 MeOH } \lambda(\varepsilon)=383 \mathrm{~nm}\left(38000 \mathrm{M}^{-1} \mathrm{~cm}^{-1}\right) & \log (\varepsilon)=4.58 \\
\text { JG15 MeOH } \lambda(\varepsilon)=320 \mathrm{~nm}\left(27000 \mathrm{M}^{-1} \mathrm{~cm}^{-1}\right) & \log (\varepsilon)=4.43 \\
\text { JG47 MeOH } \lambda(\varepsilon)=315 \mathrm{~nm}\left(25000 \mathrm{M}^{-1} \mathrm{~cm}^{-1}\right) & \log (\varepsilon)=4.40 \\
\text { JG45 MeOH } \lambda(\varepsilon)=310 \mathrm{~nm}\left(37000 \mathrm{M}^{-1} \mathrm{~cm}^{-1}\right) & \log (\varepsilon)=4.57
\end{array}
$$

\subsection{Ions tests}

As it was explained in Chapter 0, the probes were tested following a general procedure, taking pictures of solutions of the probes in the presence of different cations and anions under visible and ultraviolet light (366 nm):

- The concentration of the probes was $0.1 \mathrm{mM}$.

- The solvents were methanol and water or mixtures between them; while having as high percentage of water as possible, without experimenting precipitation.

- It was also tested the mixture acetonitrile:water, but with worse results in terms of solubilitydetection capabilities (See Experimental Appendix 5)

- Non-coordinant counterions were used; perchlorate, triflate and tetrabutylammonium, except for the salt $\mathrm{AuCl}_{3}$ (See Annex).

The probes did not have any visible colour nor change under ambient light, as in solvatochromisms, with the exception of $\mathrm{Au}^{3+}$. After adding ever cation, the pictures are showed only under UV light at $366 \mathrm{~nm}$, Figures 28, 29 and 30. The case of $\mathrm{Au}^{3+}$ is special and it was treated separately in section 5.3.2. 
5.3.1. Response of the probes to cations in water or mixtures MeOH:water:

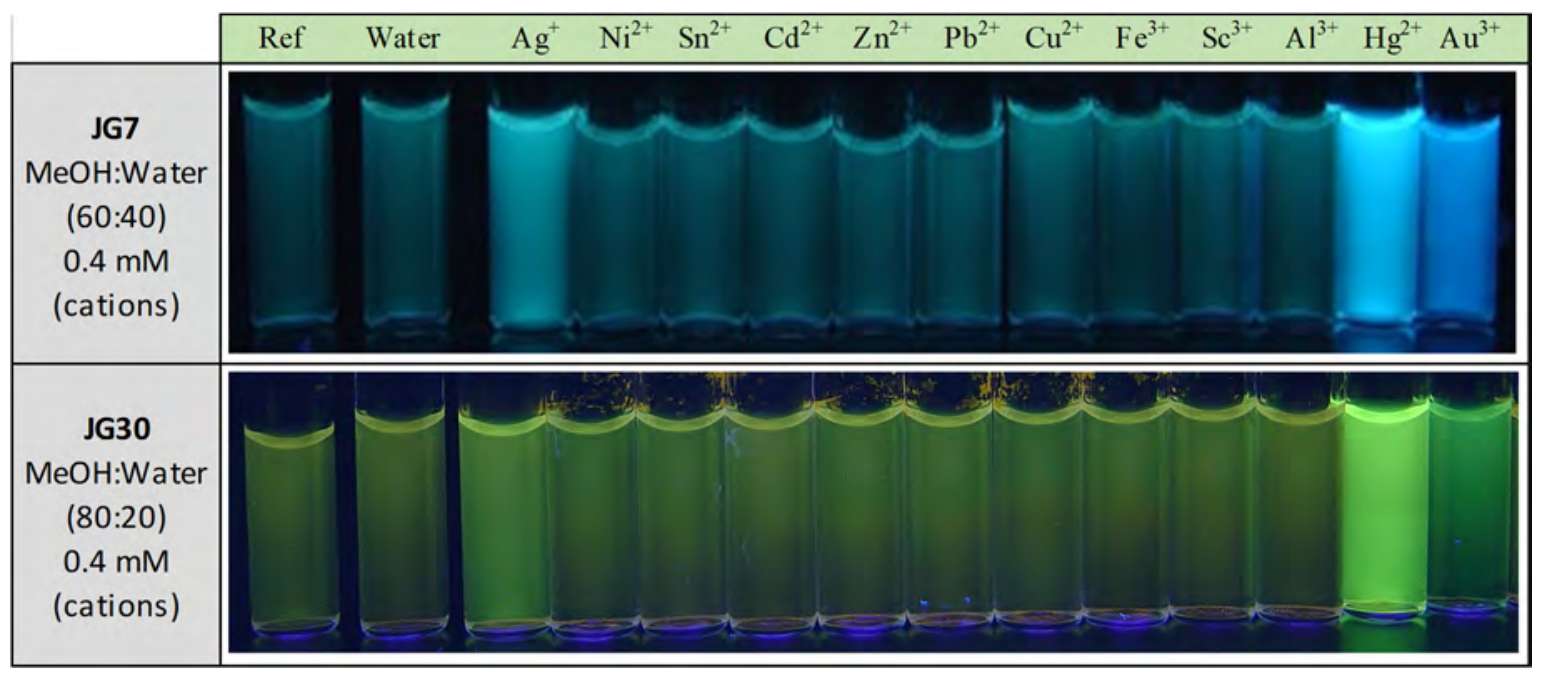

Figure 28. Response to the presence of different cations by probes JG7 and JG30 in solutions $0.1 \mathrm{mM}$, in mixtures water/methanol under a $366 \mathrm{~nm}$ light.

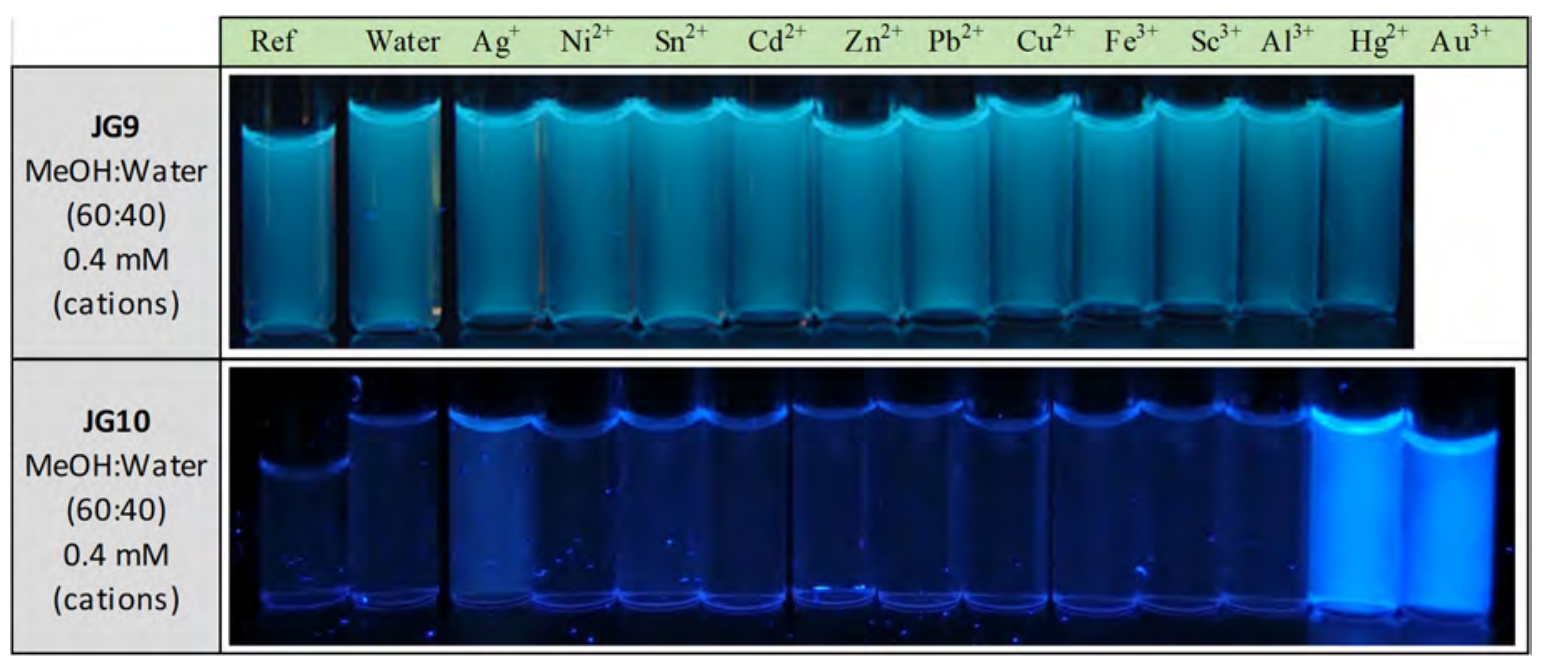

Figure 29. Response to the presence of different cations by probes JG9 and JG10 in solutions $0.1 \mathrm{mM}$, in mixtures water/methanol under $366 \mathrm{~nm}$ light.

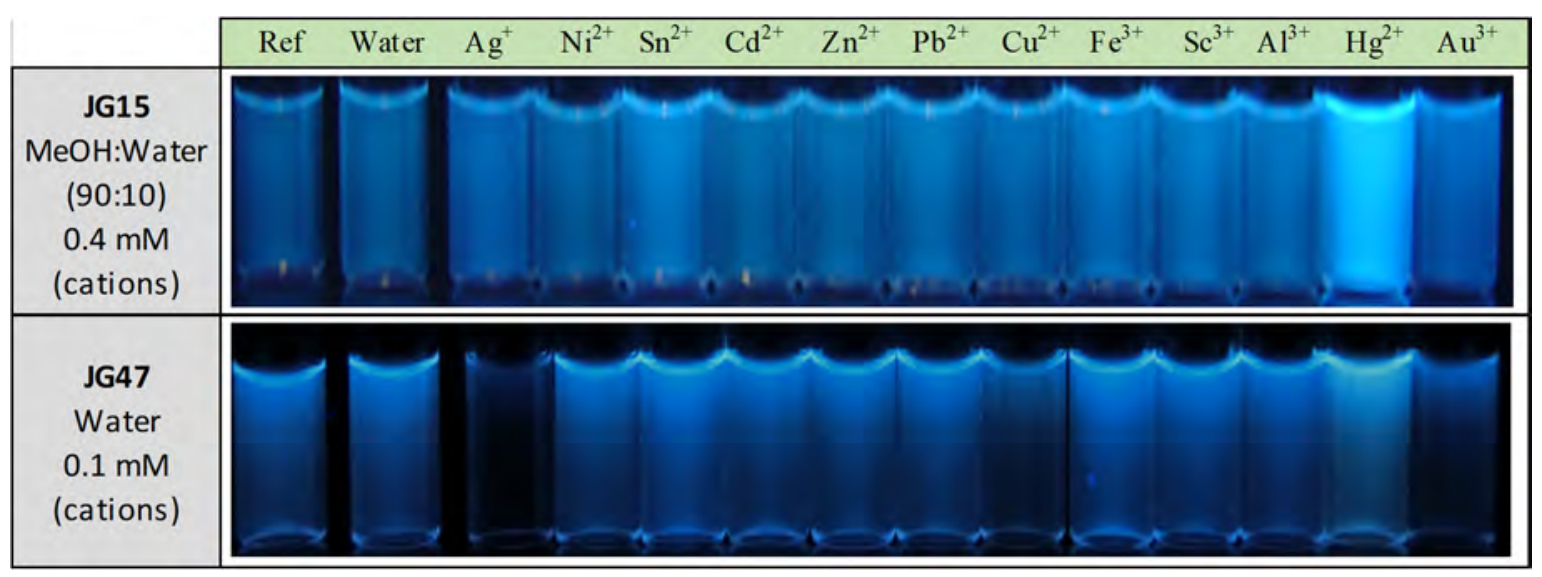

Figure 30. Response to the presence of different cations by probes JG15, JG47 and JG45 in solutions $0.1 \mathrm{mM}$, in mixtures water/methanol under $366 \mathrm{~nm}$ light. 
From these images many conclusions were obtained.

- All of the probes, with the exception of JG9, had selective response to $\mathrm{Hg}(\mathrm{II})$.

- JG7 and JG30 had similar response to the previously synthesized compounds bearing a dimethylthiophosphinate (BD116 and BD119). However, the solubility in water was lower, precipitating when there was more than $40 \%$ water in the mixture of solvents. As a consequence, they were not so deeply studied as the previous probes.

- The fluorescence also increased in presence of silver and gold cations, except for the PEG derivatives in which decreased (JG45 and JG47) or remained constant (JG15). The reason is explained in Section 5.3.2.

- PEG derivatives presented a side effect, greater $\mathrm{pH}$ dependence, being very sensitive to the presence of Lewis acid cations such as Fe(III) or $\mathrm{Sn}(\mathrm{II})$.

- PEG derivatives presented kinetic effects in presence of $\mathrm{Hg}(\mathrm{II})$, in which the fluorescence increased upon the addition of $\mathrm{Hg}(\mathrm{II})$.

- Adding high excess of $\mathrm{Hg}(\mathrm{II})$ to the PEG solution caused the precipitation of the compound (providing an apparent decrease in fluorescence).

\subsection{2. $\mathrm{Au}^{3+}$ and $\mathrm{Ag}^{+}$cations effect in water solution containing PEG probes:}

Concurrently to the studies of $\mathrm{Hg}(\mathrm{II})$ effect, it was observed an interaction effect of some probes with gold and silver cations. In the case of the thiophosphinate probes and the one with an azide group, only an increase in fluorescence was observed. This fact was easily explained by different interaction processes, what is normally due to $\mathrm{pH}$ effect and the thiophilicity of gold and silver cations. In contrast, additional changes occurred for the solutions that possessed a PEG chain, having a decrease in fluorescence instead of an increase and, what is more remarkable, a change of colour (Figures 31 and 32).

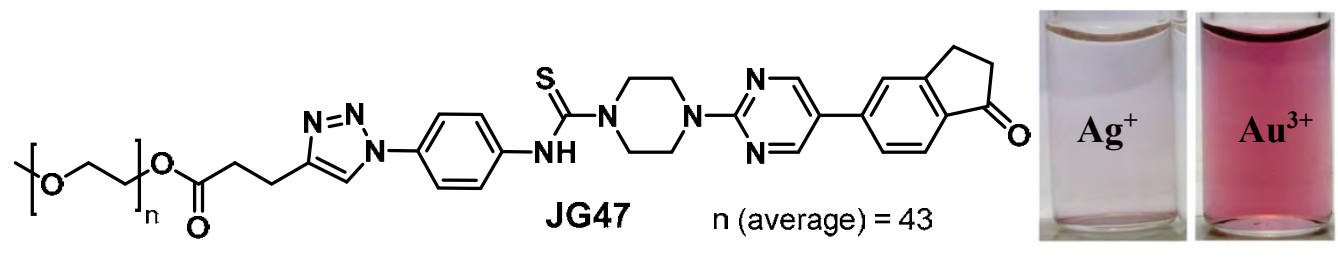

Figure 31. Silver (left) and gold (right) cations, $0.2 \mathrm{mM}$, in JG47 solution, $0.5 \mathrm{mM}$. After 48 hours.
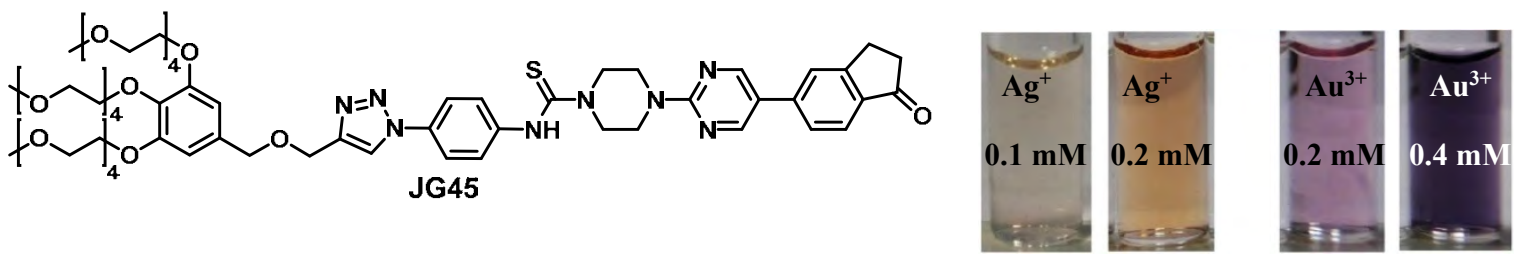

Figure 32. Silver ( 0.1 and $0.2 \mathrm{mM}$, left) and gold ( 0.2 and $0.4 \mathrm{mM}$, right) cations in JG45 solution, $0.1 \mathrm{mM}$. After 24 hours.

Moreover, these probes were useful not only for the formation of gold nanoparticles (deeply explained in Chapter 4), but in order to stabilise both gold and silver nanoparticles. It can be justified due to the presence of PEG chains and a reductive agent, presumably the carbothioamide moiety. 


\subsubsection{Response of the PEG containing probes to $\mathrm{Hg}(\mathrm{II})$ and $\mathrm{MeHg}(\mathrm{II})$ in different solvents:}

The objective was to find a probe soluble in water and capable of detecting the presence of $\mathrm{Hg}$ (II) or $\mathrm{MeHg}$ (II) as perchlorate and chloride in water, respectively. However, it seemed that the solvent was decisive in the results. Therefore, a study was performed in which, over the samples used for the solvatochromism, $\mathrm{Hg}$ (II) water solution was added to the solution in excess, $0.4 \mathrm{mM}$; Figures 33, 34 and 35.

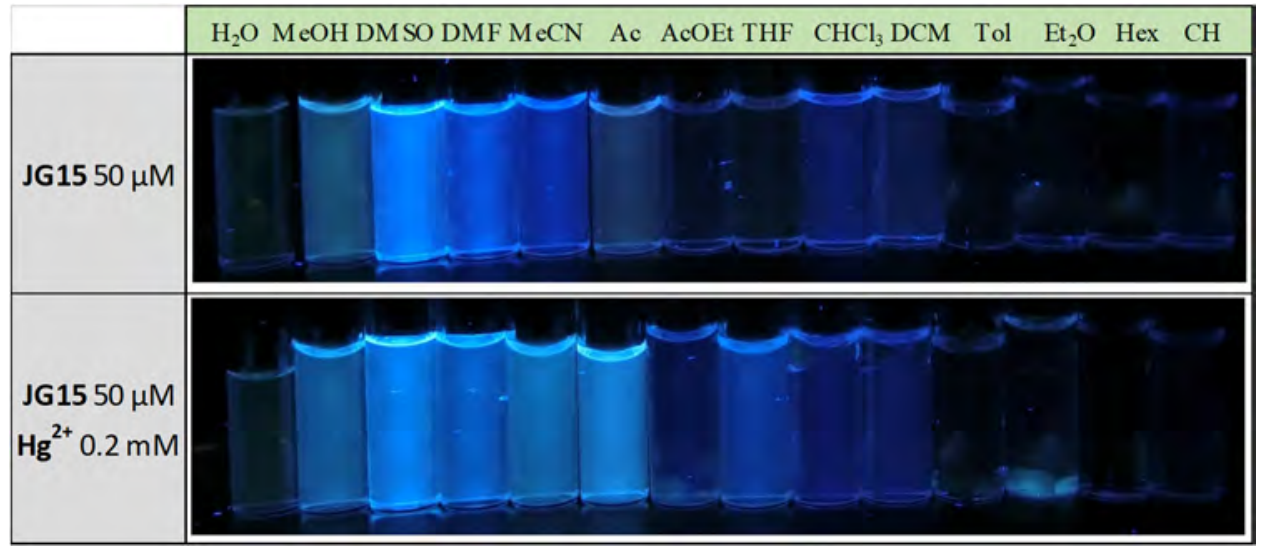

Figure 33. JG15 $50 \mu \mathrm{M}$ solvatochromism and effect of adding concentrated $\mathrm{Hg}\left(\mathrm{ClO}_{4}\right)_{2}$ solutions in water under a $366 \mathrm{~nm}$ light.

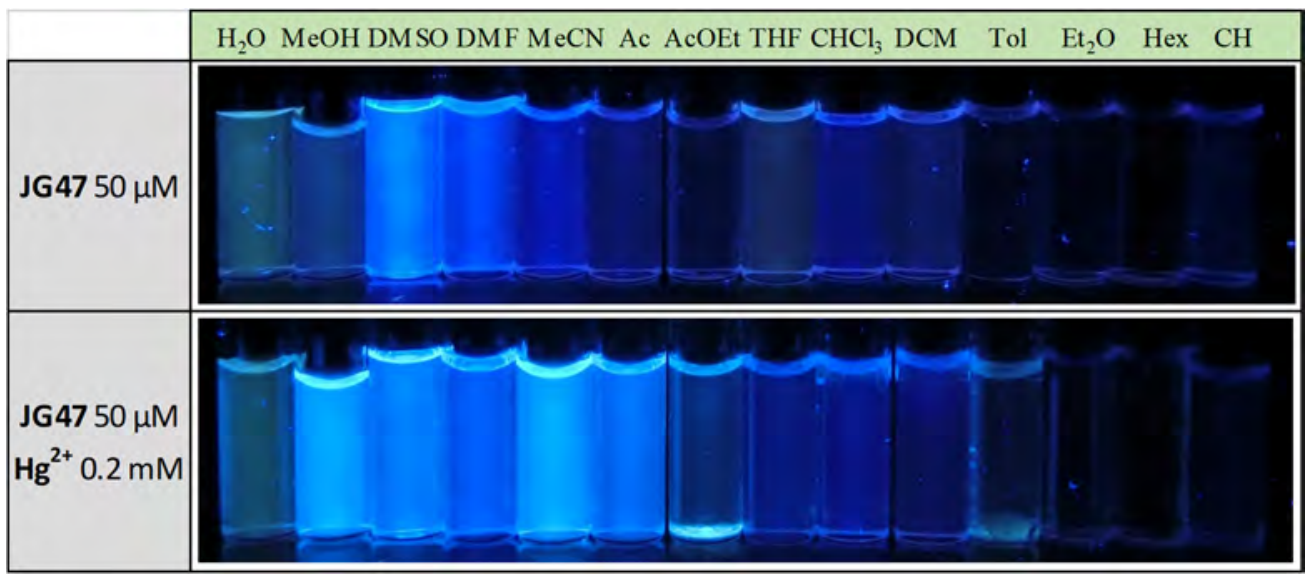

Figure 34. JG47 $50 \mu \mathrm{M}$ solvatochromism and effect of adding concentrated $\mathrm{Hg}\left(\mathrm{ClO}_{4}\right)_{2}$ solutions in water under a $366 \mathrm{~nm}$ light.

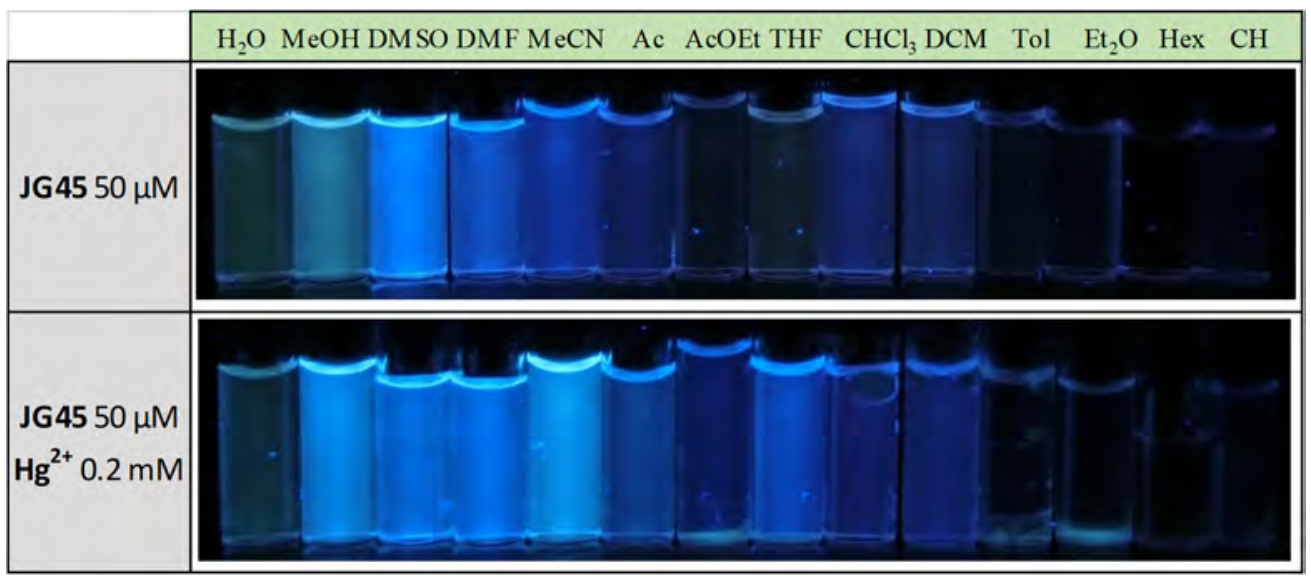

Figure 35. JG45 $50 \mu \mathrm{M}$ solvatochromism and effect of adding concentrated $\mathrm{Hg}\left(\mathrm{ClO}_{4}\right)_{2}$ solutions in water under a $366 \mathrm{~nm}$ light. 
It was concluded that, by adding $\mathrm{Hg}(\mathrm{II})$ cations:

- All of them underwent an increase of fluorescence when dissolved in $\mathrm{MeCN}$ and acetone.

- JG47 had a very high increase in fluorescence when dissolved in MeOH, JG15 and JG45 also increased the fluorescence, but less.

- The fluorescence in DMSO did not increase, it was already very high at the beginning.

- The fluorescence in water of JG45 and JG47 increased, but it was a less remarkable change.

JG47 and JG45 were of particular interest for studying $\mathrm{Hg}(\mathrm{II})$ an $\mathrm{MeHg}(\mathrm{II})$ and because of that, a comparative of the response with the cations was also performed. (Figures 36 and 37)

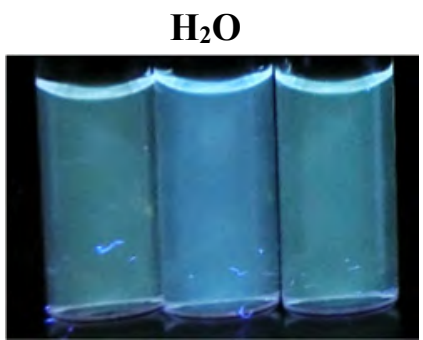

Ref - Hg(II) - MeHg(II)

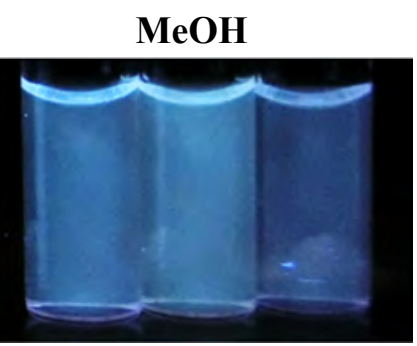

Ref- $\mathrm{Hg}(\mathrm{II})-\mathrm{MeHg}(\mathrm{II})$

Figure 36. JG47 in water (left) and in $\mathrm{MeOH}$ (right) $100 \mu \mathrm{M}, 0.3 \mathrm{mM}$ of $\mathrm{Hg}(\mathrm{II})$ and $\mathrm{MeHg}(\mathrm{II}) .366$ nm light.

For JG47, the fluorescence decreased or did not change in presence of $\mathrm{MeHg}(\mathrm{II})$ but increased in presence of $\mathrm{Hg}(\mathrm{II})$.

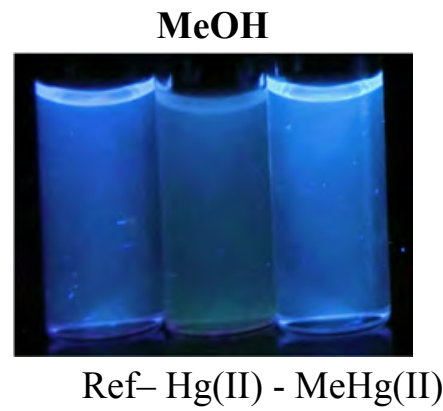

Figure 37. JG45 in $\mathrm{MeOH} 50 \mu \mathrm{M}$ with $0.8 \mathrm{mM}$ of $\mathrm{Hg}(\mathrm{II})$ and $\mathrm{MeHg}(\mathrm{II}) .366 \mathrm{~nm}$ light.

For JG45, the fluorescence decreased in the presence of very high excess of $\mathrm{Hg}$ (II), giving a yellow precipitate that interfered in the fluorescence. However, for the cation $\mathrm{MeHg}(\mathrm{II})$ there was an increase, and it did not decrease when in higher excess. Other solvents were also tested with JG45 but the fluorescence did not increase significantly in the presence of $\mathrm{MeHg}$ (II). This different behaviour was associated to a dual behaviour in the PEGylated probes,${ }^{33}$ a complexation when in presence of $\mathrm{MeHg}(\mathrm{II})$ or in low concentrations of $\mathrm{Hg}(\mathrm{II})$, but the compound reacted in high concentration of $\mathrm{Hg}(\mathrm{II})$, which was important to explain the results when different tests were performed in cell cultures.

\footnotetext{
${ }^{33}$ The tests performed to demonstrate that was a dual process (complexation + reaction) are detailed in the
} Experimental Appendix 5. 


\subsection{Fluorescence quantum yields $\left(\Phi_{\mathrm{F}}\right)$ of PEG probes}

The fluorescence quantum yield of the PEG probes was calculated using an integration sphere. The process followed was detailed in Chapter 0. The emission was compared with and without adding $\mathrm{Hg}(\mathrm{II})$ as it is shown in Table 2.

\begin{tabular}{|l|c|c|c|c|}
\multicolumn{1}{c|}{} & $\mathbf{M e O H}$ & & $\mathbf{H}_{2} \mathbf{O}$ & \multicolumn{1}{c}{} \\
\hline \multicolumn{1}{c|}{$\boldsymbol{\Phi}_{\mathbf{F}}$} & + & $\mathbf{H g}\left(\mathbf{C l O}_{4}\right)_{\mathbf{2}}$ & + & $\mathbf{H g}\left(\mathbf{C l O}_{4}\right)_{\mathbf{2}}$ \\
\hline JG15 & 0.18 & 0.44 & --- & --- \\
\hline JG47 & 0.08 & 0.64 & 0.1 & 0.16 \\
\hline JG45 & 0.18 & 0.15 & 0.09 & 0.11 \\
\hline Error & 0.02 & 0.02 & 0.02 & 0.02 \\
\hline
\end{tabular}

Table 2. Quantum yield of PEG derivatives.

JG15 increased its $\Phi_{\mathrm{F}}$, because the quantity added was only one equivalent and without waiting. The rest of the tests were performed by adding $\mathrm{Hg}(\mathrm{II})(10 \mu \mathrm{M})$ to a $5 \mu \mathrm{M}$ solution of the probes. The change in fluorescence for JG45 was not significant, or even a decrease; which was in agreement with the qualitative results; no significant change was observed in $\mathrm{MeOH}$ or water.

\subsection{Cell measurements with PEG derivatives}

Previously, the probe BD116 was tested in HEK cells, getting good results in the detection of $\mathrm{Hg}(\mathrm{II})$ by an increase in fluorescence. Instead, JG47 and JG45 showed a similar sensitivity for mercury(II) cation in water as solvent, albeit JG45 was sensitive to methylmercury(II) cation in organic solvents such as methanol; therefore, these two probes were tested for the speciation of mercury(II) species in image microscopy.

\section{Cell conditions:}

HEK293 cells were incubated with JG47 or JG45 solutions ${ }^{34}\left(100 \mu \mathrm{M}\right.$ in PBS with $\mathrm{Ca}^{2+}$ and $\mathrm{Mg}^{2+}$ ) for $1 \mathrm{~h}$ at $37^{\circ} \mathrm{C}$. Then, the plates were washed three times with PBS and incubated with $\mathrm{Hg}$ (II) $(100 \mu \mathrm{M}$ to $500 \mu \mathrm{M} \mathrm{HgClO} 4)$ or $\mathrm{MeHg}(\mathrm{II})(100 \mu \mathrm{M}$ to $400 \mu \mathrm{M} \mathrm{MeHgCl})$ in $\mathrm{PBS}+\mathrm{Ca}^{2+}+\mathrm{Mg}^{2+}$ for $1 \mathrm{~h}$ and the fluorescent emission was measured by exciting at $388 \mathrm{~nm}$ wavelength.

\section{Results:}

Cells remained viable after incubation in the presence of the probes, which were permeable to the cellular membrane. In the case of cells without probe, they were measured as blank and the relative intensity of intracellular $\mathrm{Hg}(\mathrm{II})$ and $\mathrm{MeHg}$ (II) fluorescence was compared.

In an initial test, probes JG47 or JG45 showed low levels of background intracellular fluorescence in the absence of $\mathrm{Hg}(\mathrm{II}) / \mathrm{MeHg}(\mathrm{II})$. From the different tests, there was very little change in the

\footnotetext{
${ }^{34}$ These tests were performed by the group of Sebastians Pons, in the University of Barcelona; the conditions, measurements and analysis were performed by the group with the probes synthesized in Burgos.
} 
intracellular fluorescence when JG47 was tested in the presence of $\mathrm{Hg}$ (II) or $\mathrm{MeHg}$ (II) and when JG45 was in presence of $\mathrm{Hg}$ (II). In contrast, the intracellular fluorescence suffered a dramatical increase by addition of $\mathrm{MeHg}(\mathrm{II})$ up to $400 \mu \mathrm{M}$ to the HEK293 cells incubated with JG45. The emission was observed almost exclusively in the nucleus of cells (Figure 38) with very dim fluorescence in the cytoplasm of cells.

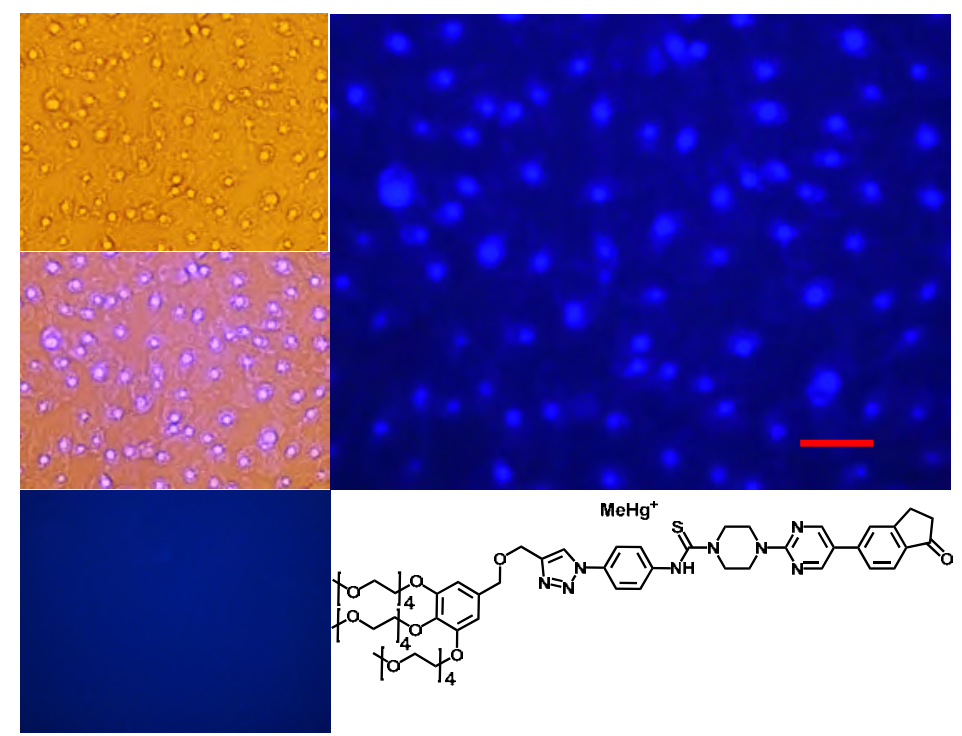

Figure 38. Fluorescence imaging of HEK293 cells incubated in the presence of JG45 and MeHg(II) $400 \mu \mathrm{M}$. Upper left: Cells under visible light. Middle left: Overlay maximum UV-Vis light. Down left: cells with JG45 only. Right: cells with JG45 and $400 \mu \mathrm{M} \mathrm{MeHg(II)} \mathrm{Scale} \mathrm{bar:} 40 \mu \mathrm{m}$

The selective enhancement of fluorescence of probe $\mathbf{J G 4 5}$ in the presence of $\mathrm{MeHg}$ (II) inside the nuclei of HEK293 cells was justified due to lipophilicity of both $\mathrm{MeHg}$ (II) and JG45, which tended to concentrate in the nucleus of cells, acting as an optimum lipophilic environment for interaction of probe and cation. This fact was a reflection of the previously observed behaviour of JG45 in organic solvents and constitutes a new paradigm for the design of selective fluorescent probes for imaging methylmercury(II) cation on the basis of a sensitivity linked to lipophilicity.

\subsection{Summary of the test with molecular probes}

New molecular fluorogenic probes were prepared. These probes interacted in different ways with two closely related cations of high environmental concern, $\mathrm{Hg}$ (II) and $\mathrm{MeHg}(\mathrm{II})$; having enough water affinity and being suitable to work in a cellular environment. First, the thiophosphinate derivatives were used for the chemical speciation of both cations in aqueous-organic solvents as well as $\mathrm{Hg}$ (II) in salts. Next, the PEG substituted derivatives were proven to be useful in water solutions and for the selective detection of $\mathrm{MeHg}(\mathrm{II})$ in HEK293 cells.

In consequence, the best selective speciation of $\mathrm{Hg}$ (II) and $\mathrm{MeHg}$ (II) cations was achieved by in vitro approaches based on the fluorogenic probes supported in cultured cells, due to the particular sensitivity of the HEK293 cells to $\mathrm{MeHg}$ (II) permeation. These achievements provided the biochemical bases to the understanding of $\mathrm{MeHg}$ (II) selective detection, contributing to the discovery of endogenous and exogenous molecular probes that provide efficient means for speciation between mercury(II) species. 


\section{SYNTHESIS OF MODIFIED MATERIALS FOR THE DETECTION OF Hg(II) AND MeHg(II)}

With the purpose of preparing new portable fluorescent sensors for practical applications, the most suitable fluorogenic probe (JG10) was bonded to the surface of different materials. Apart from the molecular probes, the modified materials offered new possibilities and more industrial applications. With that aim in mind, two synthetic modified materials were planned, a modified silica and a polymeric material.

\subsection{Silica supported probes}

With the objective of synthesizing materials for the detection of $\mathrm{Hg}(\mathrm{II})$ the first step was to develop silica nanoparticles modified with PEG chains, (giving water affinity) and a colorant with proven sensitivity to $\mathrm{Hg}$ (II) (JG10). Silica nanoparticles (10-20 nm, Sigma Aldrich) are capable of accepting a substitution within the external hydroxyl groups of $0.5 \mathrm{mmol}$ each gram of nanoparticles (experimental result).

A) Introduction of triple bonds in the nanoparticles (JG19)

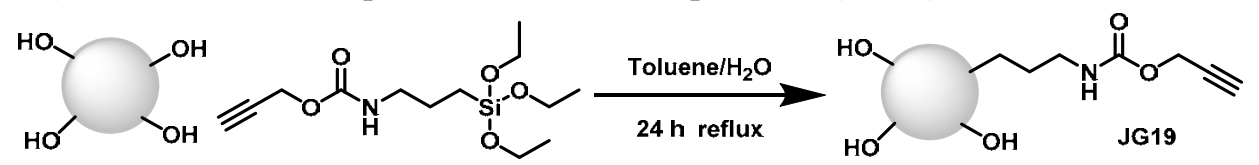

Figure 39. Scheme for the synthesis of modified silica nanoparticles JG19.

In a flask, ethynyl (3-(triethoxysilyl)propyl)carbamate (20\% substitution) was dissolved in toluene $(100 \mathrm{~mL}$ each $0.5 \mathrm{~g}$ silica). Thereafter, silica nanoparticles were dispersed in solution. Water $(0.4 \mathrm{ml} / \mathrm{g}$ of silica) was added to solution and put under reflux for 24 hours. The product was centrifuged and $4500 \mathrm{rpm}$ for 15 minutes and the supernatant retired. Then, it was cleaned with $2 \times$ Toluene, $2 \times \mathrm{DCM}$ and $2 \times \mathrm{Et}_{2} \mathrm{O}$.

B) Introduction of the fluorogenic molecule (JG22)

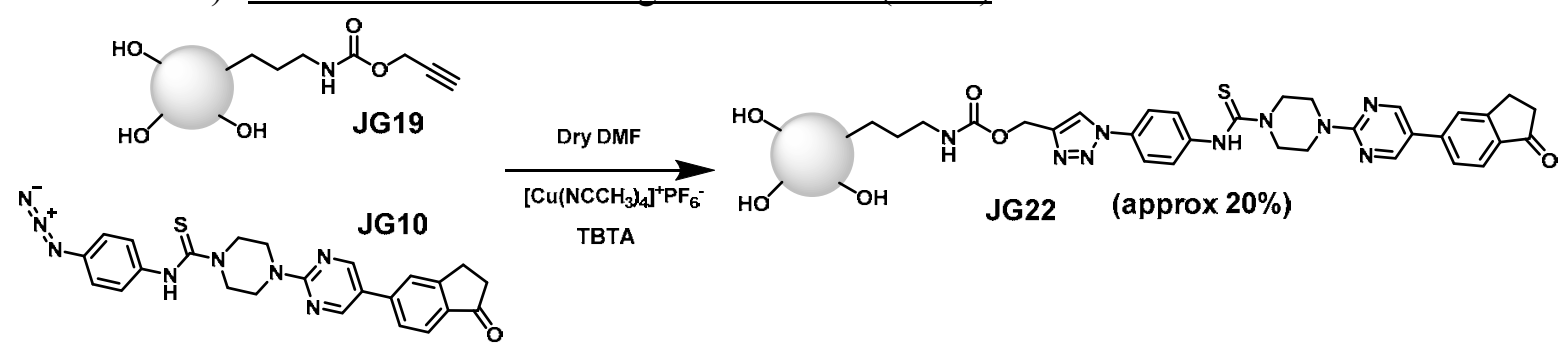

Figure 40. Scheme for the synthesis of modified silica nanoparticle JG22.

In a Schlenk, JG10 was put under nitrogen atmosphere. The probe containing an azide group JG10 (1:1 triple bond) was dissolved in dry DMF (160 mL $1 \mathrm{~g}$ of silica). Thereafter, JG19 silica nanoparticles were dispersed in the solution and the copper catalyser $\left[\mathrm{Cu}\left(\mathrm{NCCH}_{3}\right)_{4}\right]^{+}\left[\mathrm{PF}_{6}^{-}\right](5 \%$ molar/Triple bonds) was added. The product was centrifuged at $4500 \mathrm{rpm}$ for 15 minutes and the supernatant retired. Then, it was cleaned with $50 \mathrm{~mL}$ of $2 \times \mathrm{DMF}, 2 \times \mathrm{DCM}$ and $2 \times \mathrm{Et}_{2} \mathrm{O}$. The resulting nanoparticles presented blue fluorescence under UV light. 
C) Synthesis of 2-methoxyethyl (3-(triethoxysilyl)propyl)carbamate (JG20):<smiles>CCO[Si](CCCN=C=O)(OCC)OCC</smiles><smiles>CCCCCCCCC(C)(C)C(=O)NCCC[Si](OCC)(OCC)OCC</smiles>

Figure 41. Scheme for the synthesis of the triethoxysilane tetraPEG JG20.

In a flask, $1 \mathrm{~g}$ of 3-(triethoxysilyl)propyl)isocyanate $(4.04 \mathrm{mmol})$ and $0.92 \mathrm{~g}$ of tetraethylene glycol monomethylether were dissolved in $5 \mathrm{ml}$ of DCM and stirred for 24 hours at room temperature. The product obtained was a colourless liquid that was used without further purification.

D) Introduction of the fluorogenic molecule (JG23):

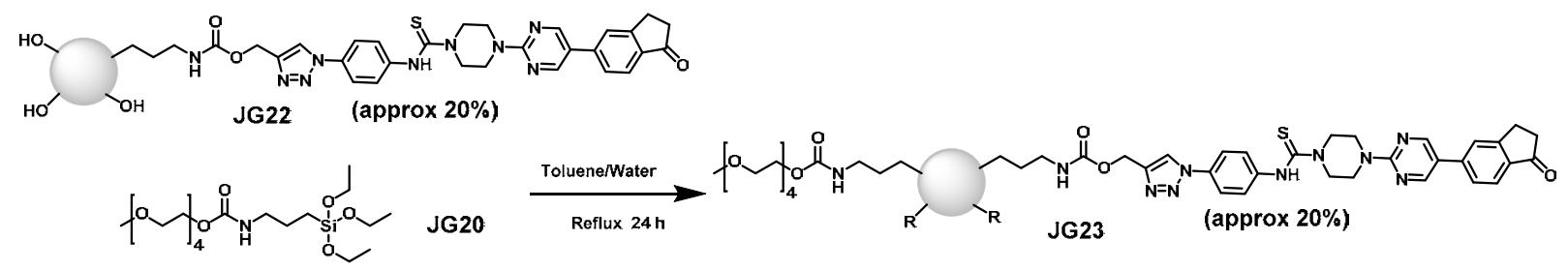

Figure 42. Scheme for the synthesis of modified silica nanoparticle JG23.

In a flask, 2-methoxyethyl (3-(triethoxysilyl)propyl)carbamate, JG21, (0.5 mmol/1g of silica) was dissolved in toluene (100 ml/1 g of silica). Thereafter, modified silica nanoparticles JG22 were dispersed in the solution. Water $(0.4 \mathrm{ml} / \mathrm{lg}$ of silica) was added and the solution was put to reflux for 24 hours. For purification, the product was centrifuged at $4500 \mathrm{rpm}$ for 15 minutes and the supernatant retired. Then, it was cleaned with $2 \times$ toluene, $2 \times \mathrm{DCM}$ and $2 \times \mathrm{Et}_{2} \mathrm{O}$. Obtaining blue fluorescent silica nanoparticles, under UV light.

\section{E) Characterization (IR): Figures 43, 44 and 45:}

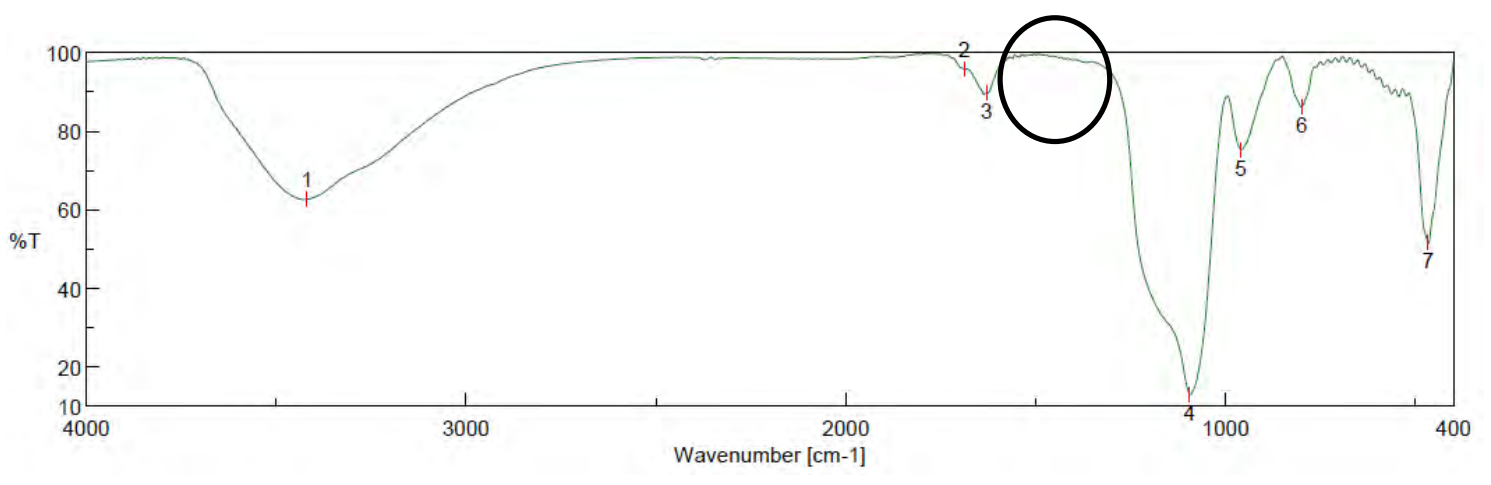

\begin{tabular}{|c|c|c|c|c|c|c|c|c|}
\hline \multicolumn{9}{|c|}{ [ Result of Peak Picking ] } \\
\hline No. & Position & Intensity & No. & Position & Intensity & No. & Position & Intensity \\
\hline 1 & 3419.17 & 62.6403 & 2 & 1688.37 & 95.7274 & 3 & 1629.55 & 89.5736 \\
\hline 4 & 1094.4 & 12.9905 & 5 & 960.377 & 75.2428 & 6 & 799.35 & 86.176 \\
\hline 7 & 467.653 & 51.668 & & & & & & \\
\hline
\end{tabular}

Figure 43. Silica IR spectra and peak picking.

IR $\left(\mathrm{KBr}, \mathrm{cm}^{-1}\right): 3419(\mathrm{OH}), 1688,1630,1094,960,799,468$. 


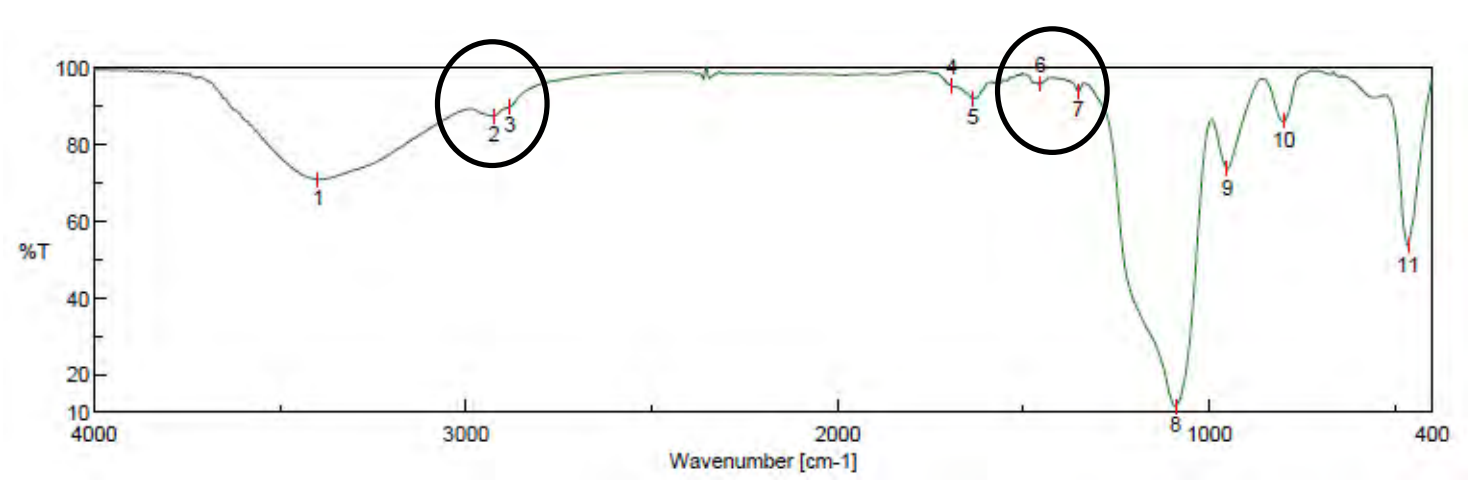

$\begin{array}{lllllllll}\text { [ Result of Peak Picking ] } & & & & & \\ \text { No. } & \text { Position } & \text { Intensity } & \text { No. } & \text { Position } & \text { Intensity } & \text { No. } & \text { Position } & \text { Intensity } \\ 1 & 3396.99 & 70.7155 & 2 & 2924.52 & 87.2351 & 3 & 2882.09 & 89.5077 \\ 4 & 1693.19 & 95.2412 & 5 & 1636.3 & 91.7327 & 6 & 1455.03 & 95.6008 \\ 7 & 1352.82 & 93.6417 & 8 & 1091.51 & 11.5569 & 9 & 951.698 & 73.4191 \\ 10 & 799.35 & 85.9126 & 11 & 465.725 & 53.3766 & & & \end{array}$

Figure 44. JG19 IR spectra and peak picking.

IR $\left(\mathrm{KBr}, \mathrm{cm}^{-1}\right): 3397(\mathrm{OH}), 2924(\mathrm{C}-\mathrm{H}), 2882(\mathrm{C}-\mathrm{H})$, 1693, 1636, 1455, 1352, 1092, 952, 799, 466.

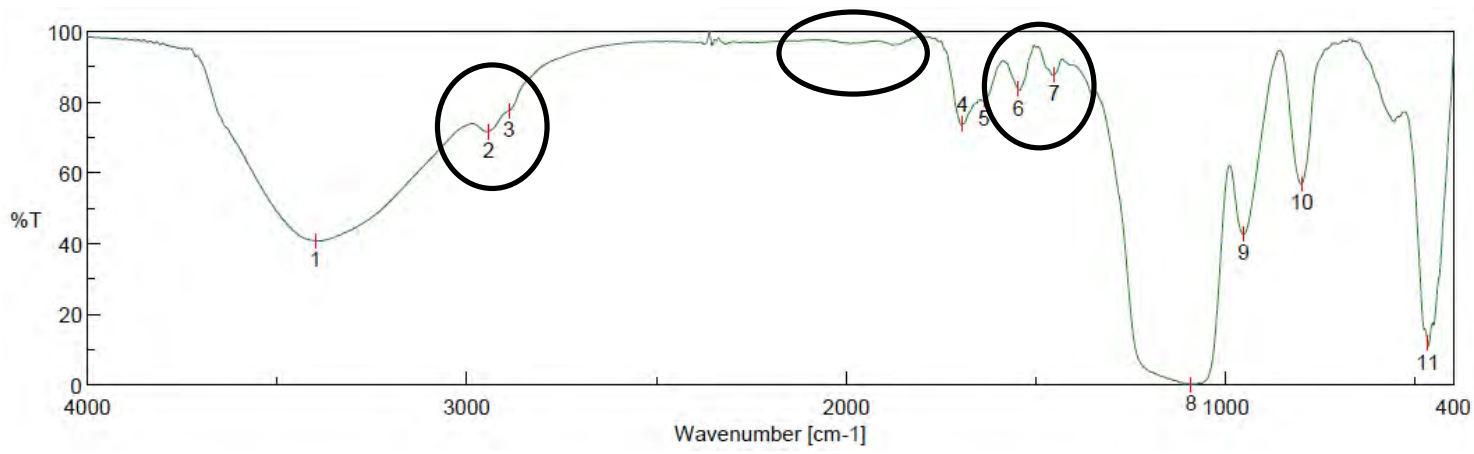

$\begin{array}{lllllllll}\text { [ Result of Peak Picking ] } & & & & & & \\ \text { No. } & \text { Position } & \text { Intensity } & \text { No. } & \text { Position } & \text { Intensity } & \text { No. } & \text { Position } & \text { Intensity } \\ 1 & 3398.92 & 40.8113 & 2 & 2942.84 & 71.6616 & 3 & 2888.84 & 77.6637 \\ 4 & 1693.19 & 73.853 & 5 & 1636.3 & 80.5831 & 6 & 1545.67 & 83.6045 \\ 7 & 1452.14 & 87.6839 & 8 & 1091.51 & 0.125121 & 9 & 953.627 & 42.5953 \\ 10 & 799.35 & 57.0033 & 11 & 467.653 & 11.8026 & & & \end{array}$

Figure 45. JG23 IR spectra and peak picking.

IR $\left(\mathrm{KBr}, \mathrm{cm}^{-1}\right): 3399(\mathrm{OH}), 2943(\mathrm{C}-\mathrm{H}), 2889(\mathrm{C}-\mathrm{H})$, 1693, 1636, $1546\left(\mathrm{C}_{\mathrm{Ar}}-\mathrm{C}_{\mathrm{Ar}}\right)$, 1452, 1092, 954, 799, 468.

The infrared characterization (Figures 43, 44 and 45) showed the presence of signals between $2950-2850 \mathrm{~cm}^{-1}$ indicated the existence of C-H groups and, around $1550 \mathrm{~cm}^{-1}$, the presence of aromatic compounds. It should be a characteristic signal from triple bonds/triazole groups at $2200-2000 \mathrm{~cm}^{-1}$, the low proportion of these groups may cause that it was not observed. 


\subsection{Polymer supported probes}

Starting from probe JG10, it was proposed the synthesis of a polymer capable to react by click chemistry with the probe. To achieve that purpose, there were a series of conditions to be fulfilled:

- The polymeric film had to have high water affinity.

- Depending on the its specific aim, the polymer had to be or soluble in water or remaining as a film maintaining, with good mechanical properties.

- There had to be a significant and selective increase of fluorescence in presence of mercury(II) derivatives.

In this regard, a series of monomers, specified in Figure 46, were selected.<smiles>C=CC(=O)OCCO</smiles>

2HEA<smiles>C#CCOC(=O)C(=C)C(=O)OC</smiles>

PGM<smiles>C=C(C)C(=O)OCCOC(=O)C(C)=CC</smiles><smiles>CCCCCCCCCCCC</smiles>
cross-linker<smiles>COC(OC)(C(=O)c1ccccc1)c1ccccc1</smiles>

photo-initiator DMPA

Figure 46. Monomers, crosslinker and photo-initiator used for the synthesis of polymers modified with mercury(II) sensitive probes.

2-Hydroxyethyl acrylate gave water affinity to the polymer. Propargyl methacrylate allowed the binding by click chemistry with the azide group of JG10. Finally, the proportions of ethylene glycol methacrylate, the crosslinker, gave the polymer its mechanical properties, not being soluble in water when there was enough of this component.

The process of polymerization consists of a mixture of the monomers, polymerized by photochemical radical polymerization with $\mathrm{DMPA}^{35}$. For the development of films, the polymerization was performed in a $100 \mu \mathrm{m}$ thick silanized glass hermetic mould upon irradiation with a UV mercury lamp, which was designed for that purpose by the Group of Polymers from Burgos University.

35 a) B. Redondo-Foj, M. Carsi, P. Ortiz-Serna, M. J. Sanchis, S. Vallejos, F. García, J. M. García, Macromolecules 2014, 47, 5334; b) B. Redondo-Foj, M. Carsi, P. Ortiz-Serna, M. J. Sanchis, F. García, J. M. García, J. Phys. D: Appl. Phys. 2013, 56, 295. 


\subsubsection{Synthesis, modification and characterization of the polymers:}

This kind of polymer was synthetized with a low percentage of probe, in order to not saturate the samples, which would have led to black-brown samples. If the polymers are very colourful it usually leads to no sensitivity to any cation. Therefore, and after testing with several percentages, the quantity that gave good results was having $5 \%$ of propargyl groups for the films and $1 \%$ for the soluble polymers.

\section{A) Water soluble polymer synthesis (JG32):}

As it is indicated in Figure 47, in order to introduce the fluorescent core JG10, it was proceeded as a normal catalytic reaction of triazole formation by click chemistry.

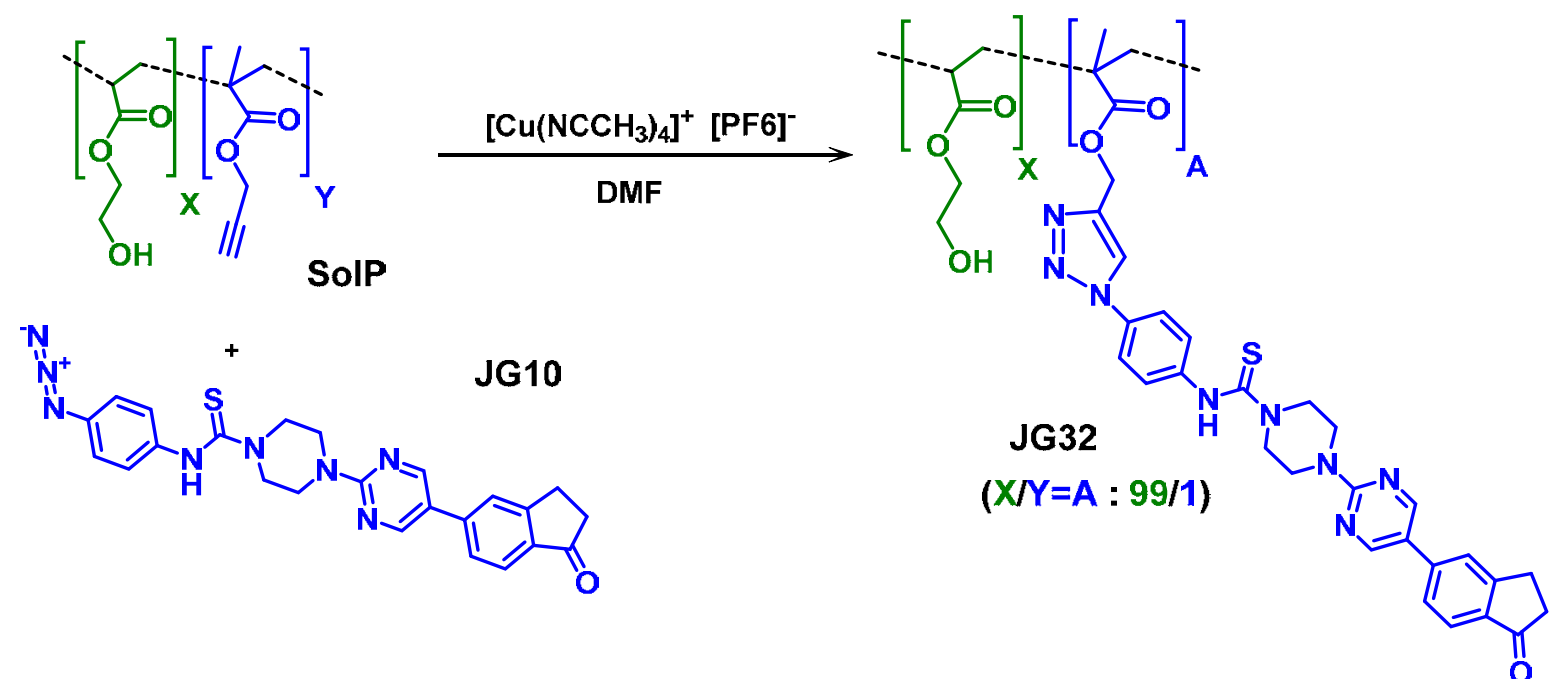

Figure 47. Synthesis of a mercury sensitive polymer derivative from 2-hydroxyethyl acrylate soluble in water (JG32)

The initial polymer was provided by the group of polymers of Burgos University. This polymer was a mixture of $99 \%$ molar of 2-hydroxyethyl acrylate and $1 \%$ molar of propargyl methacrylate, which resulted in a colourless slime. The polymeric slime was dissolved in dry DMF under nitrogen atmosphere, and JG10 was added to the mixture, in a proportion 1:1 with the amount of propargyl groups. Finally, once everything is dissolved, the $\mathrm{Cu}(\mathrm{I})$ catalyser was added and it was left under stirring at $30^{\circ} \mathrm{C}$ for 24 hours. For purification, the slime was precipitated by adding diethylether to the solution, and the slime was washed several times until obtaining a yellow slime with blue fluorescence under UV light. 
B) Synthesis of a fluorescent crosslinked polymer of 2-hydroxyethyl acrylate (JG25):

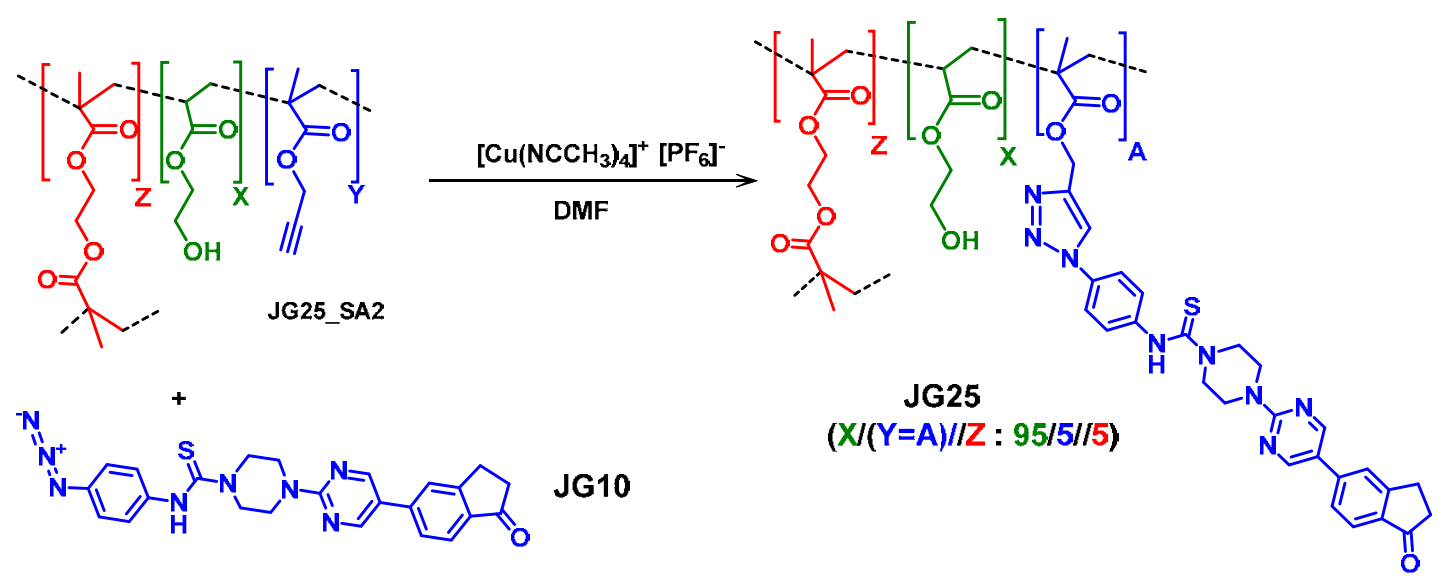

Figure 48. Synthesis of a fluorescent crosslinked polymer of 2-hydroxyethyl acrylate (JG25).

The initial polymer (JG25_SA2) was also provided by the group of polymers of Burgos University. This polymer was a mixture of $95 \%$ molar of 2-hydroxyethyl acrylate and $5 \%$ molar of propargyl methacrylate. In addition, it presented a $5 \%$ of ethylenglycolmetacrylate as crosslinker; resulting in a colourless film. The water-swelling percentage (WSP) of the membrane was $60 \%$ and the DMF swelling $300 \%$.

Following the scheme from Figure 48, a flask, adapted to contain the polymer, was put under nitrogen and JG10 was dissolved in dry DMF. After that, the film was put into the flask; once the polymer is swelled with $\mathrm{DMF}$, the catalyst containing $\mathrm{Cu}(\mathrm{I})$ was added. The polymer in solution was stirred with an orbital shaker for 72 hours, while it was observed that the yellow colour of the solution turned pale and the polymer yellowish (Figure 49). Finally, the film was cleaned by washing it with DMF, DMFwater and water, increasing the percentage of water little by little. This process must be performed carefully because of the different swelling depending on the solvent DMF $>$ Water $>$ dry; any drastic change would lead to a break of the polymer.
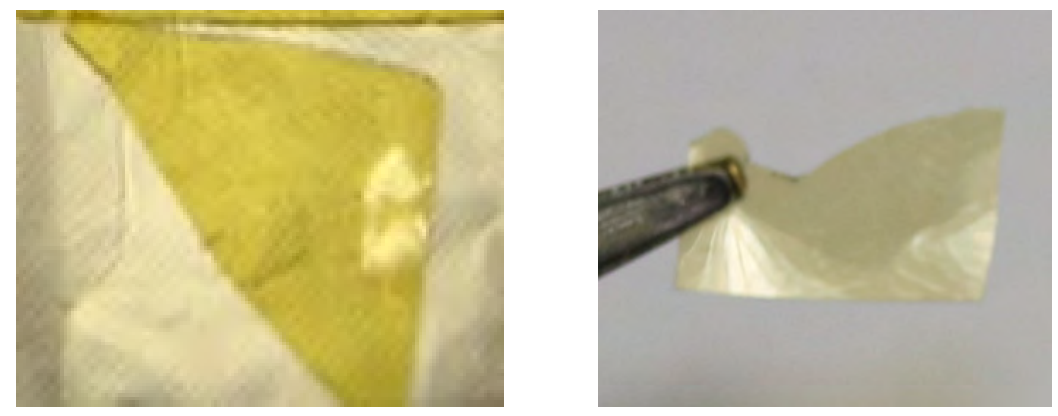

Figure 49. Picture of membranes JG25 once the reaction has finished.

C) Characterization of the polymers:

The synthesized polymers were characterized by IR (Figures 50 and 51), SEM (Figure 52A) + EDX (Figure 52B). 


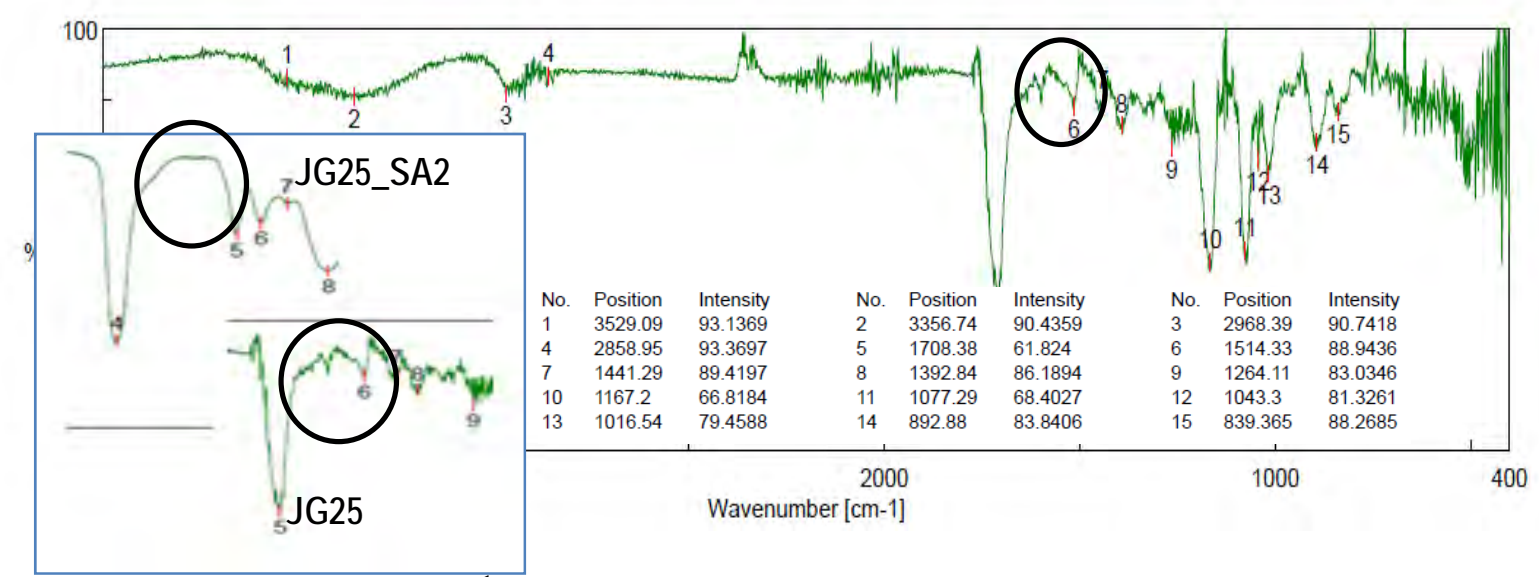

Figure 50. IR JG25 (ATR, $\left.\mathrm{cm}^{-1}\right)$ : 3529-3356 (O-H), 2968-2859 (C-H), $1708(\mathrm{C}=\mathrm{O}), 1514\left(\mathrm{C}_{\mathrm{Ar}}-\mathrm{C}_{\mathrm{Ar}}\right)$, 1441, 1393, 1264, 1167, 1077, 1043, 893, 839 (fingerprint zone).

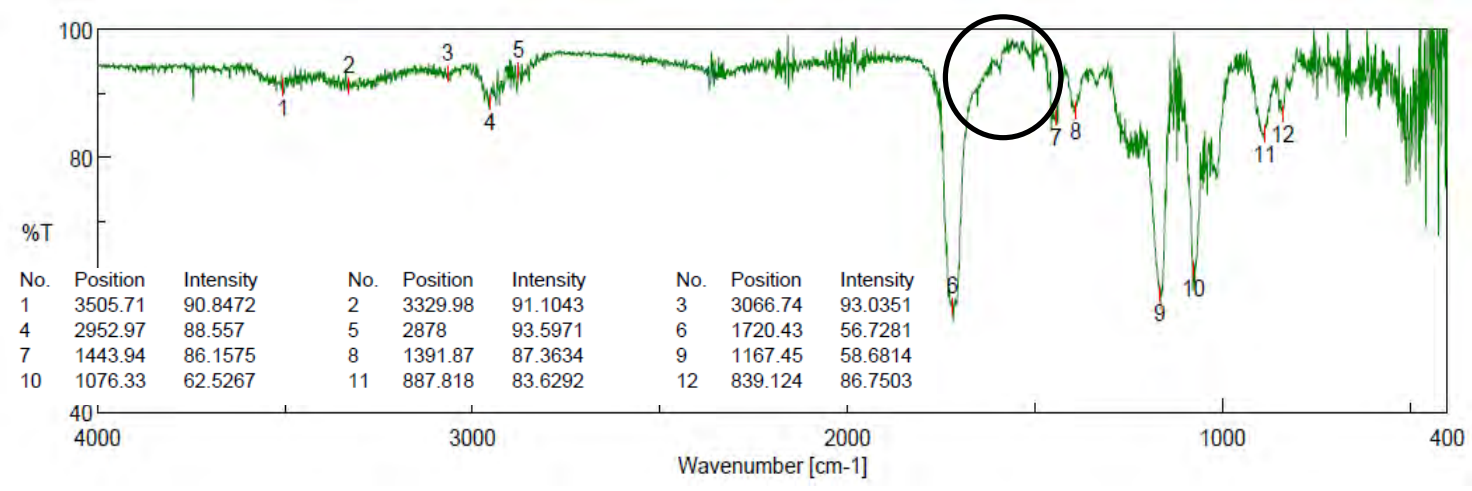

Figure 51. IR JG32 (ATR, $\left.\mathrm{cm}^{-1}\right)$ : 3506-3330 (O-H), 2953-2878 (C-H), $1720(\mathrm{C}=\mathrm{O}), 1444,1392$, 1167, 1076, 888, 839 (fingerprint zone).

In the case of JG25 (Figure 50) there were some signals at 1514 and $1598 \mathrm{~cm}^{-1}$ associated to the presence of the probe (aromatic groups). In contrast, there is no presence of these signals on the initial IR spectrum, their intensity was low due to the low percentage (5\%) (Inset in Figure 50). In comparison, JG32 (Figure 51) signals at $1600-1500 \mathrm{~cm}^{-1}$ were barely distinguished from the noise. It was because, for JG32, the percentage of the fluorogenic probe was too low (1\%), which agrees with previous results.
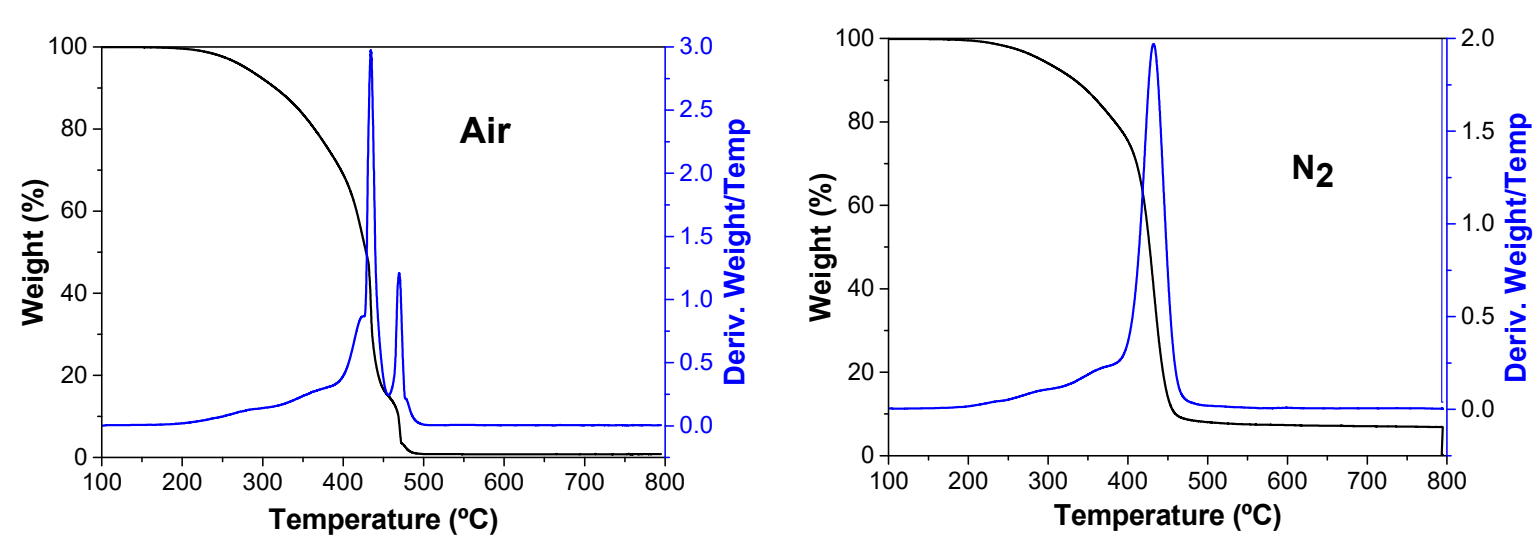

Figure 52. TGA of JG25, decomposition at $440^{\circ} \mathrm{C}$. 


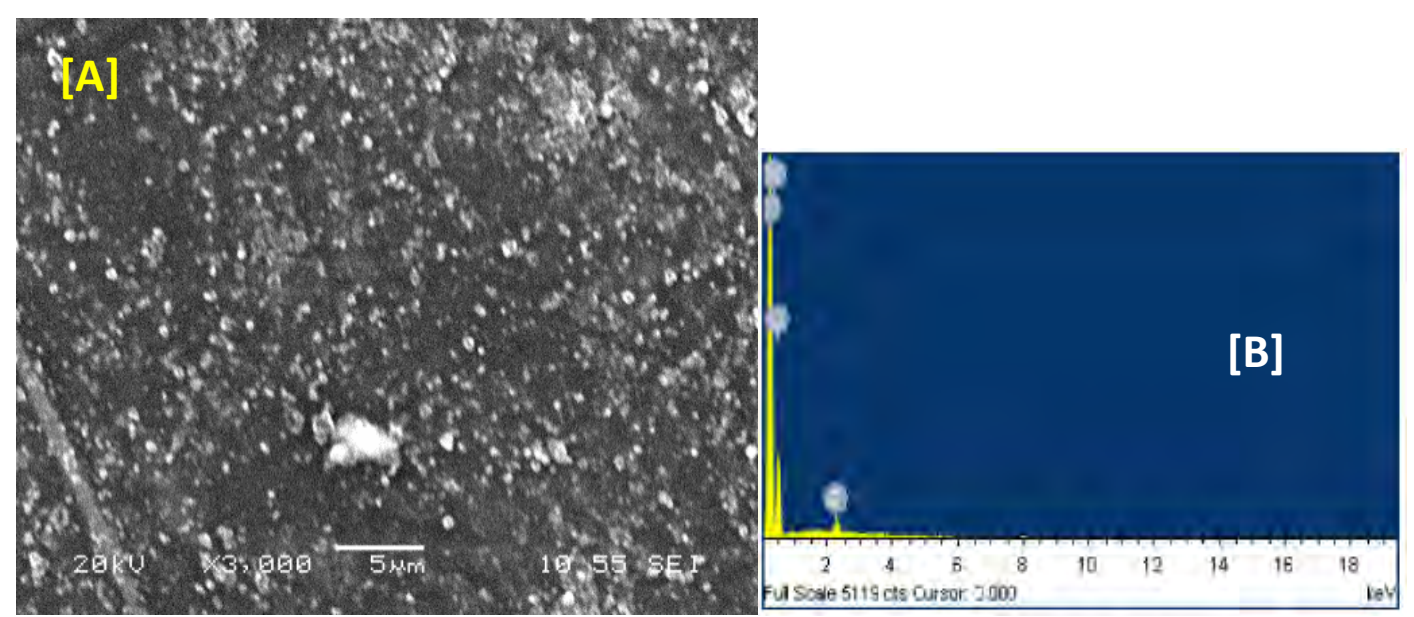

[C] $\quad$\begin{tabular}{|c|c|}
\hline Coating & $\mathbf{\%} \mathbf{S}_{\text {real }} / \mathbf{\% S}_{\text {teor }}$ \\
\hline Gold & 79.4 \\
\hline Gold & 100.7 \\
\hline Gold & 83.5 \\
\hline Carbon & 84.9 \\
\hline Carbon & 68.5 \\
\hline Carbon & 93.7 \\
\hline
\end{tabular}

Figure 53. [A] SEM image of JG25, [B] X-ray fluorescence analysis from JG25, [C] Comparison between theoretical proportions of sulfur atoms and amounts detected by EDX.

The SEM-EDX analysis were performed with gold and carbon recap. The atomic proportion was indicated by the EDX on different areas of the polymer. The proportion between oxygen or carbon/sulfur atoms was very similar to the theoretical results associated to a $100 \%$ stoichiometric reaction (60-100\% depending on the area). 


\section{QUALITATIVE TESTS OF MATERIAL SUPPORTED PROBES}

\subsection{Tests with substituted nanoparticles}

The nanoparticles were exposed to solutions of different cations. To do so, $15 \mathrm{mg}$ of silica nanoparticles JG23 were put in $0.5 \mathrm{~mL}$ water solutions of cations $(0.1 \mathrm{mM})$. Afterwards, the response was checked in visible and under UV-light (see Figure 54).

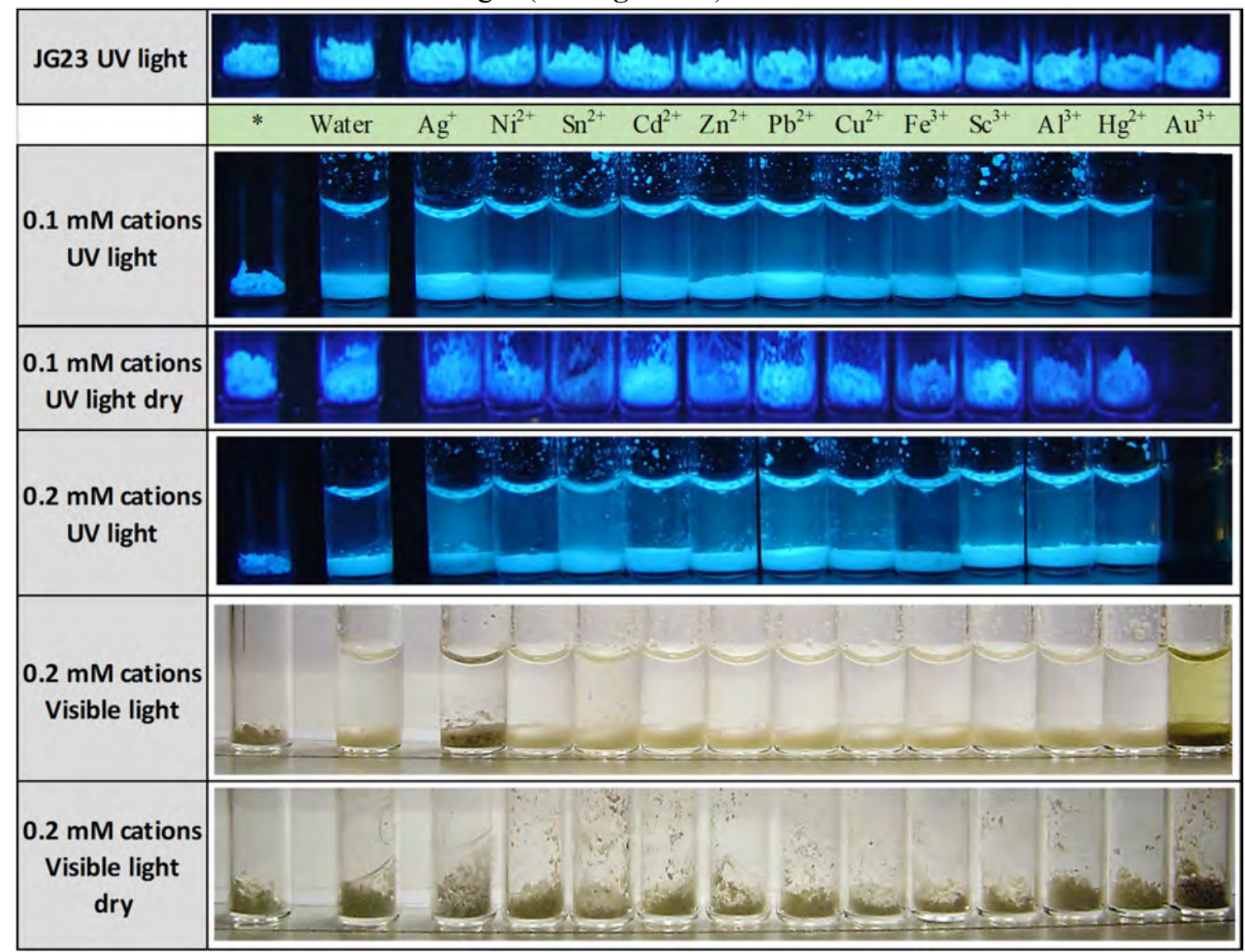

Figure 54. Pictures of vials adding cations in water solution over $15 \mathrm{mg}$ of silica nanoparticles modified (JG23). $366 \mathrm{~nm}$ light.

The pictures showed no remarkable changes in fluorescence. There was a disappearance of fluorescence in presence of $\mathrm{Au}(\mathrm{III})$ and a little increase for $\mathrm{Ag}(\mathrm{I})$. In addition, there was a change in colour for these two cations. For $\operatorname{Ag}(\mathrm{I})$ the silica nanoparticles became pink with low concentrations (Figure 55), and grey when no water or when the concentration was high. For Au(III) the silica nanoparticles become almost black (Au-NPs formation, see Chapter 4).

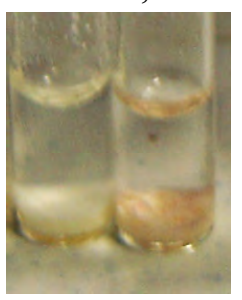

Figure 55. Pictures of $\mathrm{Ag}^{+}$addition zoom for modified JG23, $0.2 \mathrm{mM}$ under visible light, right. JG23 reference with no cations, left. 
In conclusion, the synthetized nanoparticles are not useful for detection of $\mathrm{Hg}(\mathrm{II})$ as they were synthetized, but they have other properties that are worthy to be studied, such as the formation of silver or gold nanoparticles in their surface (See Chapter 4).

\subsection{Tests with soluble polymer JG32}

In order to check the fluorescence of the soluble polymer JG32, it was compared to the azide probe JG10. The solution of the polymer was prepared and the concentration in a concentration of $0.012 \mathrm{~g} / \mathrm{L}$ of the polymer $\left(1 \%\right.$ of the probe, $10^{-6} \mathrm{~mol}$ probe $\left./ \mathrm{L}\right)$. Then, the cations were added, see Figure 56.

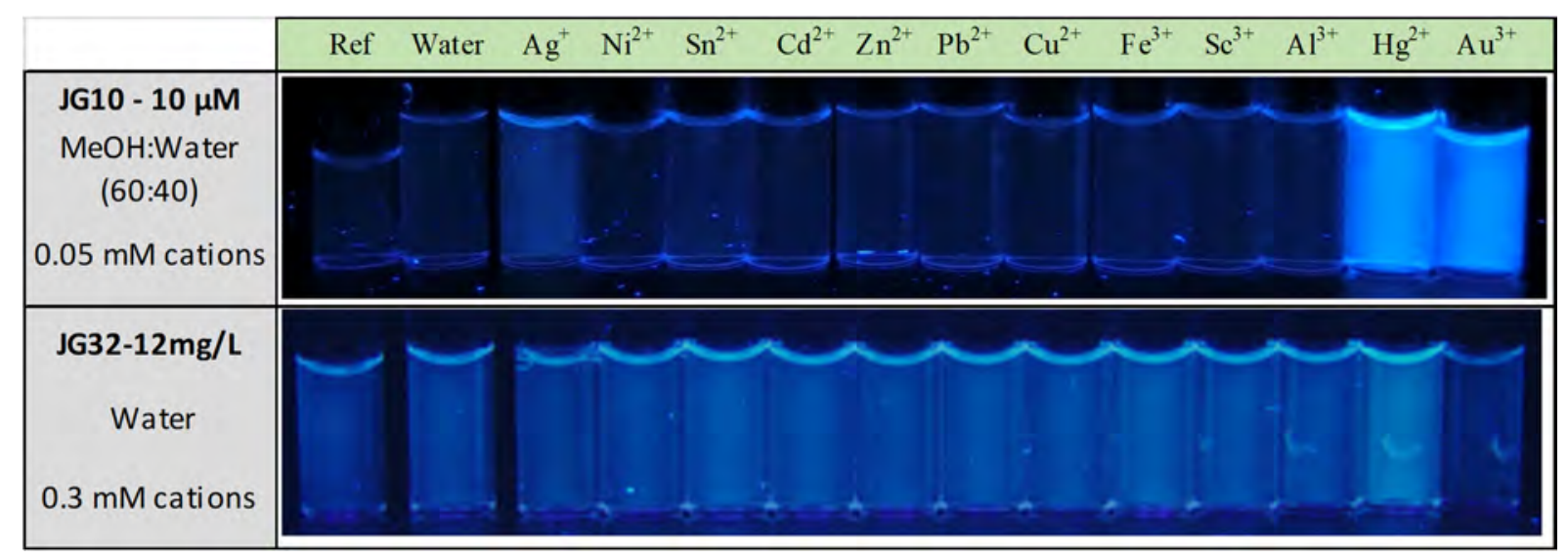

Figure 56. Fluorescence under UV light of JG10 and JG32 in solution with cations. $366 \mathrm{~nm}$ light.

In case of JG10 the fluorescence increased very little in presence of $\mathrm{Ag}(\mathrm{I})$ and increased highly in the presence of $\mathrm{Hg}(\mathrm{II})$ and $\mathrm{Au}(\mathrm{III})$. The polymer was in water and there were not any changes under visible light. The fluorescence under UV light $(366 \mathrm{~nm}$ ) increased for $\mathrm{Hg}(\mathrm{II})$ and decreased for $\mathrm{Au}(\mathrm{III})$.

The fluorescence of JG32 was also checked in the presence of other cations. Figure 57, sequence: Water, $\mathrm{Hg}(\mathrm{II}), \mathrm{MeHg}(\mathrm{II}), \mathrm{Au}(\mathrm{III}), \mathrm{Pd}(0), \mathrm{Rh}(\mathrm{III}), \mathrm{Ir}(\mathrm{III}), \mathrm{Pt}(\mathrm{II}), \mathrm{Co}(\mathrm{II}), \mathrm{Pd}(\mathrm{II})$. (As chlorides, except $\operatorname{Pd}(0)$ that was $\left.\operatorname{Pd}(\mathrm{dba})_{2}\right)$.

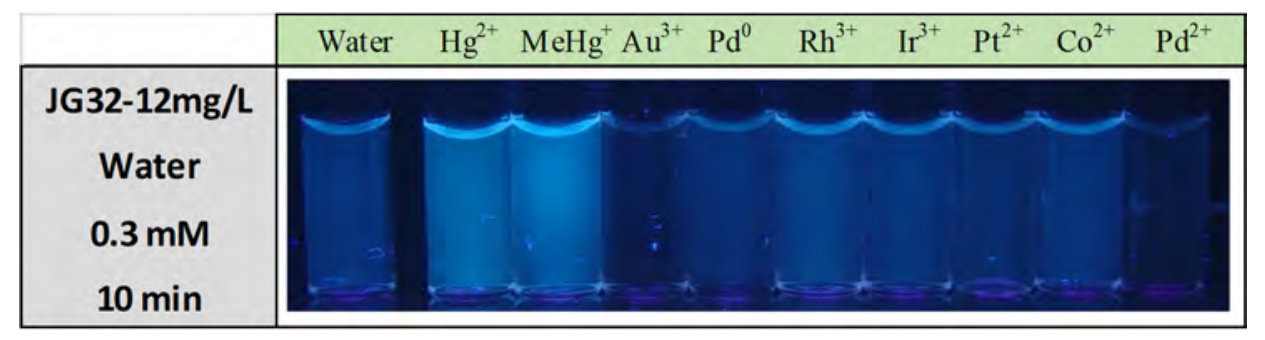

Figure 57. JG32 in water, fluorescence under UV light in presence of different cations. $366 \mathrm{~nm}$ light.

Two processes were observed, as it is shown in Figures 56 and 57, an increase of the fluorescence under UV light for $\mathrm{Hg}(\mathrm{II})$ and $\mathrm{MeHg}(\mathrm{II})$ and a decrease for $\mathrm{Au}(\mathrm{III})$ and $\mathrm{Pd}(\mathrm{II})$.

The increase in fluorescence in presence of different cations was also quantified in a fluorometer, as it is shown in Figures 58, 59 and 60: 


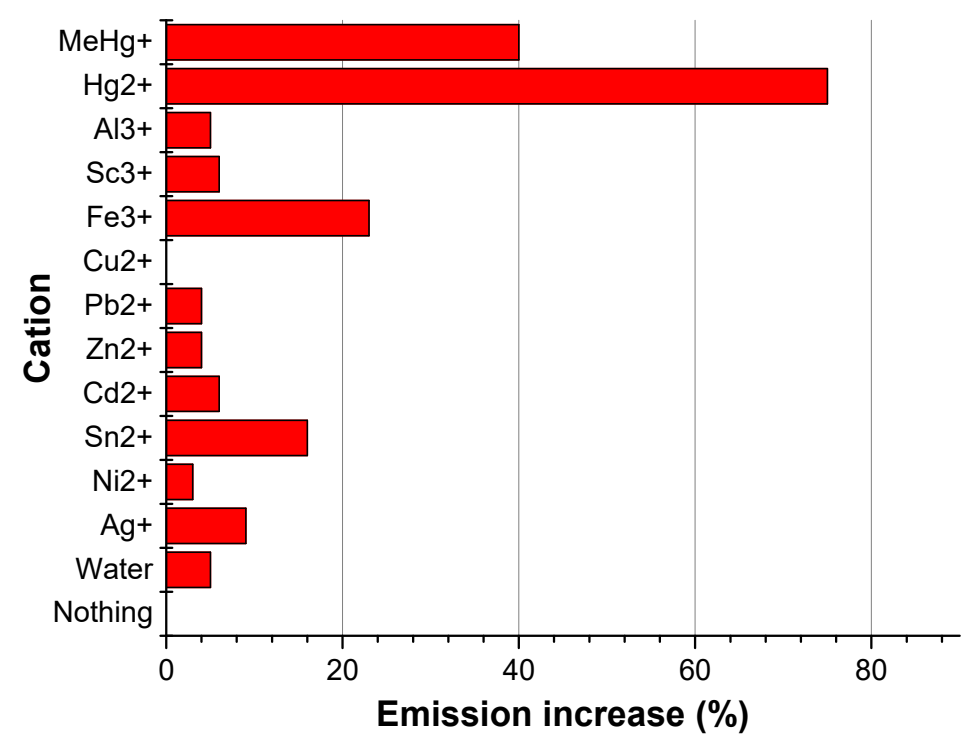

Figure 58. Variation in fluorescence of JG32 in water solution, $12 \mathrm{mg} / \mathrm{L}$. After adding cations, 0.2 $\mathrm{mM}$, and waiting 60 minutes. $\lambda_{\mathrm{exc}}=320 \mathrm{~nm}, \lambda_{\mathrm{em}}=455 \mathrm{~nm}$

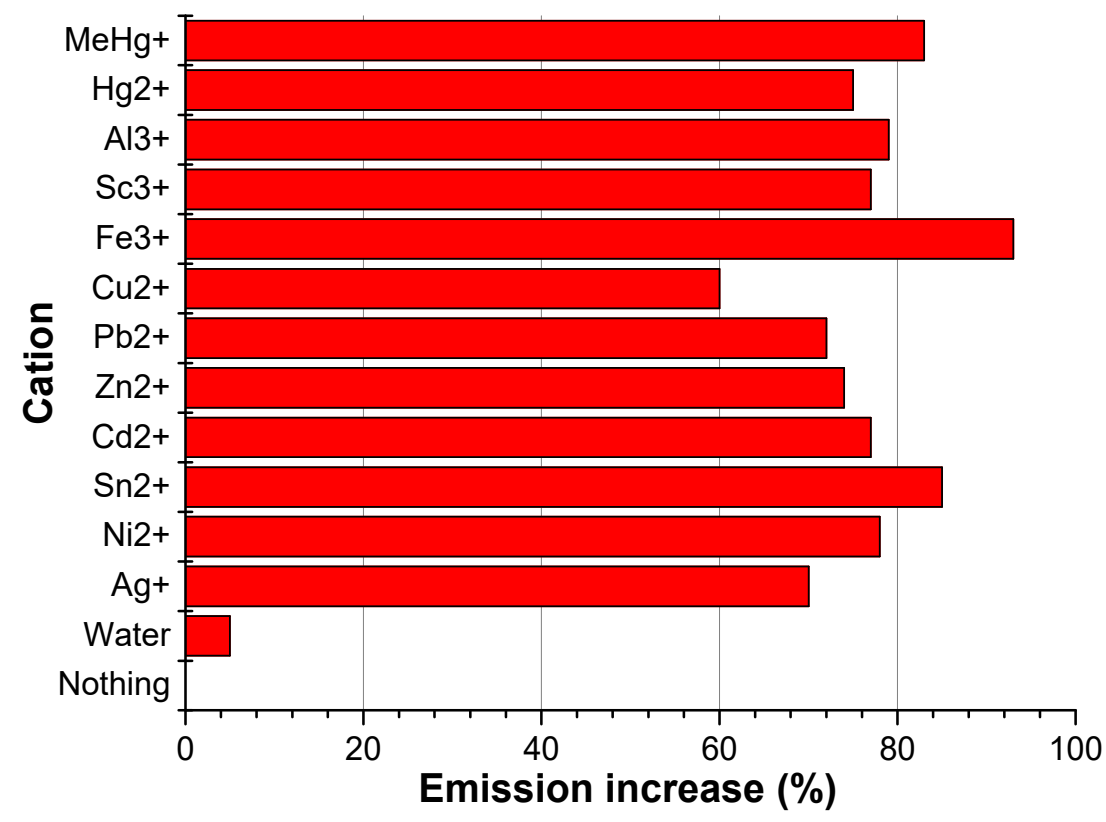

Figure 59. Variation in fluorescence of JG32 in water solution, $12 \mathrm{mg} / \mathrm{L}$. After adding cations, 0.2 $\mathrm{mM}$, waiting 60 minutes and increasing $0.2 \mathrm{mM}$ the concentration of $\mathrm{Hg}(\mathrm{II})$ in all of them. $\lambda_{\mathrm{exc}}=320$ $\mathrm{nm}, \lambda_{\mathrm{em}}=455 \mathrm{~nm}$ 


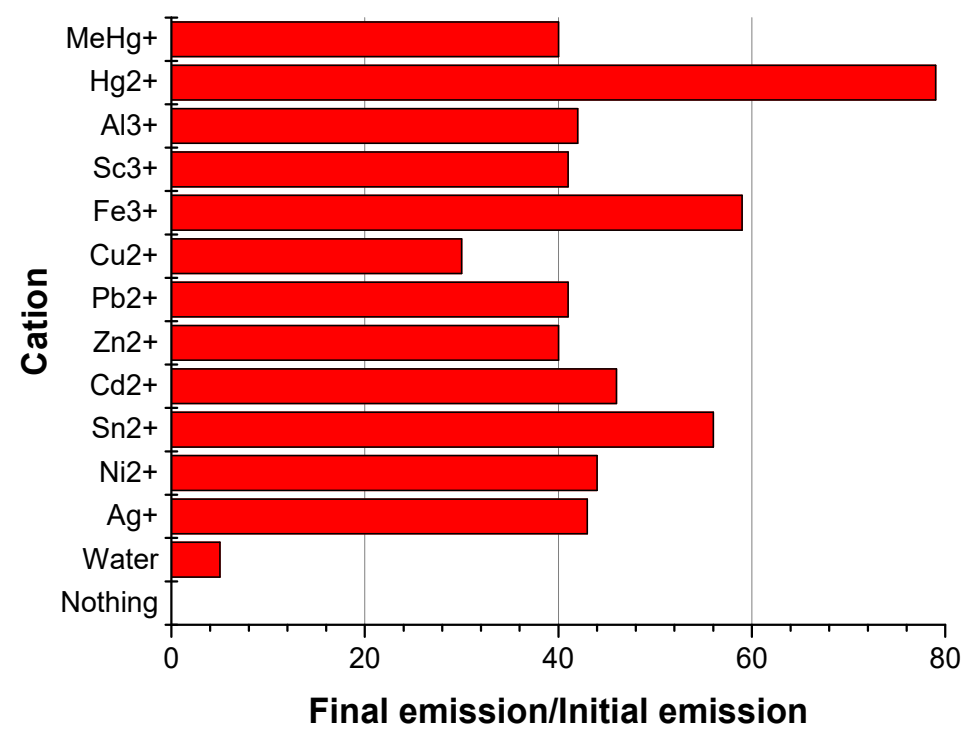

Figure 60. Variation of fluorescence of JG32 in water solution, $12 \mathrm{mg} / \mathrm{L}$. After adding cations, 0.2 $\mathrm{mM}$, waiting 60 minutes and increasing $0.2 \mathrm{mM}$ the concentration of $\mathrm{MeHg}(\mathrm{II})$ in all of them $\lambda_{\text {exc }}=$ $364 \mathrm{~nm}, \lambda_{\mathrm{em}}=445 \mathrm{~nm}$.

A major increase in fluorescence occurred in presence of $\mathrm{Hg}(\mathrm{II})$ and $\mathrm{MeHg}(\mathrm{II})$, moreover there were minor increases in the presence of $\mathrm{Sn}(\mathrm{II})$ and $\mathrm{Fe}(\mathrm{III})$, associated to the Lewis acidity of these cations. In addition, a total inhibition of the signal for $\mathrm{Au}(\mathrm{III})$ or partial inhibition of the signal for $\mathrm{Cu}(\mathrm{II})$ were also observed.

\subsection{Tests with polymeric film JG25}

Several pieces of polymer $0.3 \times 0.3 \mathrm{~cm}$ (approximately) were added to different vials and $60 \mu \mathrm{L}$ of the cations solutions $(5 \mathrm{mM})$ were added, enough to cover all the surface of the polymer. Then, the polymers were left to dry and a picture was taken under UV light, Figure 61, and the changes in fluorescence registered in the fluorometer, Figure 62.

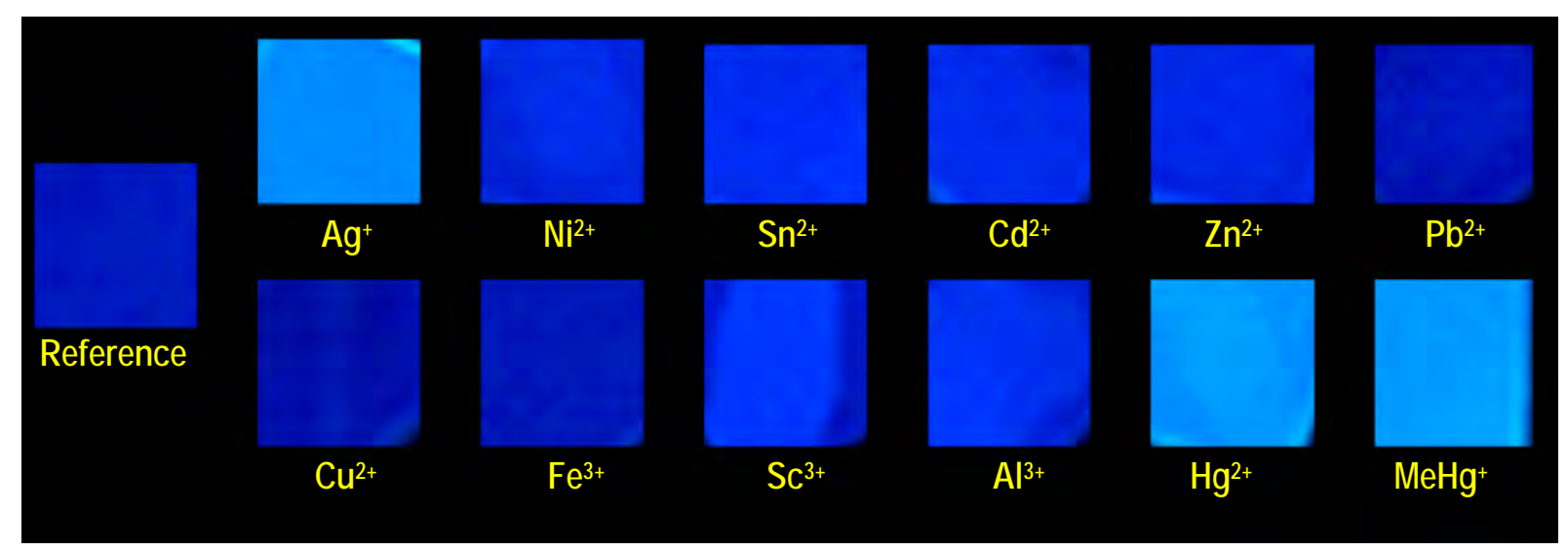

Figure 61. Fluorescent response of JG25 in presence of $60 \mu \mathrm{L}$ of cations (UV light). 


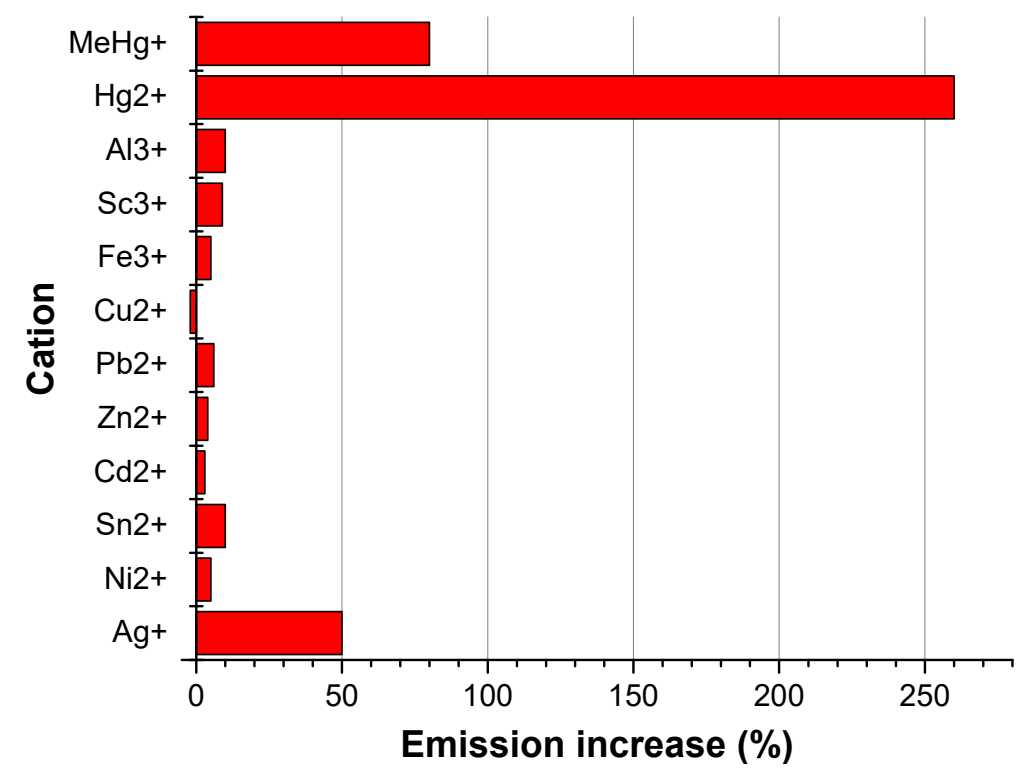

Figure 62. Fluorescent response of JG25 in the presence of $60 \mu \mathrm{L}$ of cations, $\lambda_{\mathrm{exc}}=364 \mathrm{~nm} . \lambda_{\mathrm{em}}=445$ $\mathrm{nm}$.

About the changes of the polymer in presence of cations, an increase in fluorescence can be seen for $\mathrm{Ag}(\mathrm{I})$, although $\mathrm{Hg}(\mathrm{II})$ and $\mathrm{MeHg}(\mathrm{II})$ produced a higher increase in fluorescence. This was easily quantified by measuring with a fluorometer, albeit it was hard to distinguish by the naked eye. 


\section{QUANTITATIVE TESTS OF MERCURY(II) SENSITIVE POLYMERS}

General conditions: Tests were performed for the two synthesized polymers, the soluble in water (JG32) and the film with water affinity (JG25). Because of that, there were two procedures to measure in the fluorometer:

- For the soluble polymer, a linear regression was necessary in order to find a concentration in which little variations change linearly the emission intensity and the absorbance.

- In the case of solid polymers in contact with a solution, the sample was put between two magnetic sheets with a hole in the middle and placed in an angle of 45 degrees between the lamp and the detector (Figure 63, see Annex). The concentration of analyte in the solution (in contact with the polymer) is successively increased in the cuvette and the changes in the fluorescence of the polymer were registered.

\subsection{Measurements with the soluble polymer JG32}

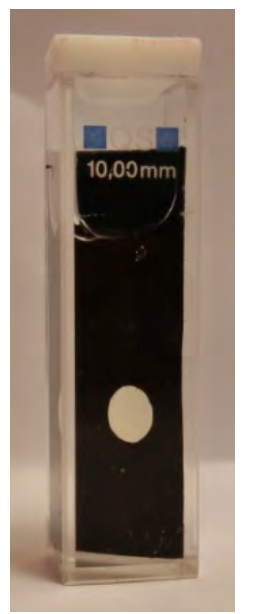

Figure 63. Cuvette adapted for measuring films.

\subsubsection{Work concentration calculation:}

In order to check that the fluorescence and absorbance change linearly with concentration the absorbance at $320 \mathrm{~nm}$ and emission at $453 \mathrm{~nm}\left(\lambda_{\mathrm{exc}}=320 \mathrm{~nm}\right)$ were measured for different concentrations, Figure 64.
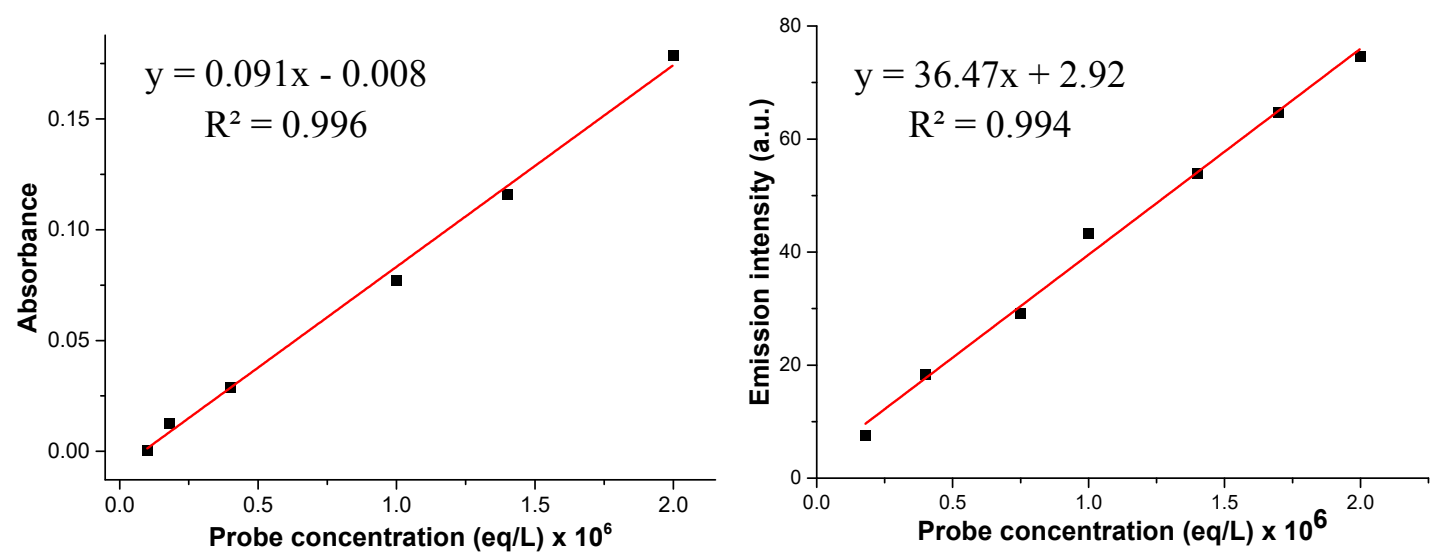

Figure 64. Absorbance (left) and emission intensity (right) of JG32 $\left(\lambda_{\mathrm{exc}}=320 \mathrm{~nm}\right)$ for different solutions.

The absorbance and fluorescence changes were linear from $2 \times 10^{-7}$ to $2 \times 10^{-6} \mathrm{eq} / \mathrm{L}$ of probe. Due to the low quantity of the sensor $(1 \%)$, the good work concentration is $\mathbf{0 . 0 1 2} \mathbf{g} / \mathbf{L}$ of polymer, or what is the same, $2 \times 10^{-6} \mathrm{eq} / \mathrm{L}$ of probe. The rest of the tests were performed in that concentration. 


\subsubsection{Kinetic response to $\mathrm{Hg}(\mathrm{II})$ :}

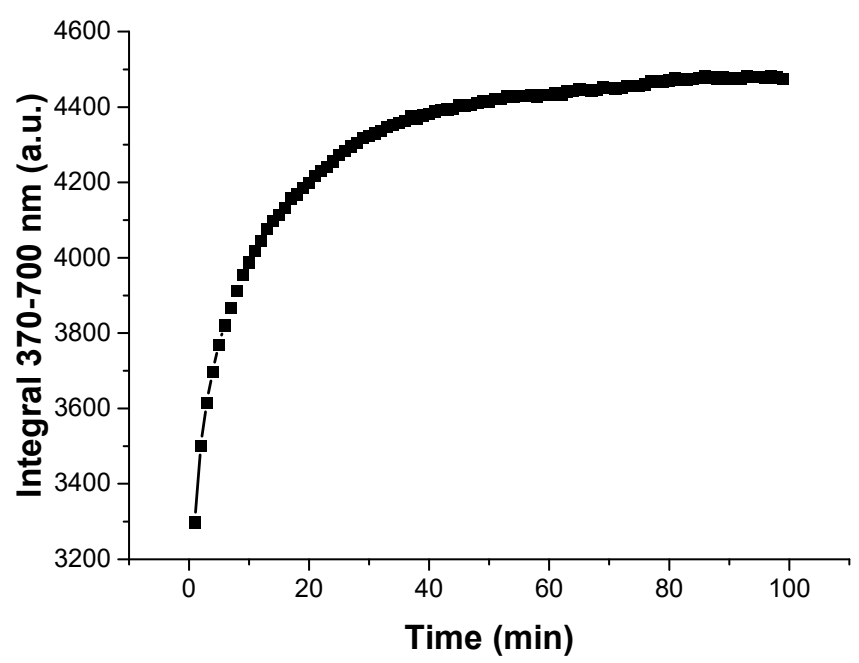

Figure 65. Kinetic response of JG32, $0.012 \mathrm{~g} / \mathrm{L}$, to aqueous solution of $\mathrm{Hg}\left(\mathrm{ClO}_{4}\right)_{2} 0.1 \mathrm{mM}, \lambda_{\text {exc }}=320$ $\mathrm{nm}, \lambda_{\mathrm{em}}=445 \mathrm{~nm}$. Measurements were taken every minute for 100 minutes.

Figure $\mathbf{6 5}$ shows that, for $\mathbf{J G 3 2}+\mathrm{Hg}\left(\mathrm{ClO}_{4}\right)_{2}$ the increase in fluorescence was instantaneous, but the equilibrium was reached after 50 minutes. These values depended on the polymer concentration and the $\mathrm{Hg}(\mathrm{II})$ concentration. Because of that, the measurements of the detection limit with successive additions of $\mathrm{Hg}(\mathrm{II})$ took this fact into account in order to give reliable results.

\subsubsection{Titration experiments by adding $\mathrm{Hg}\left(\mathrm{ClO}_{4}\right)_{2}$ :}
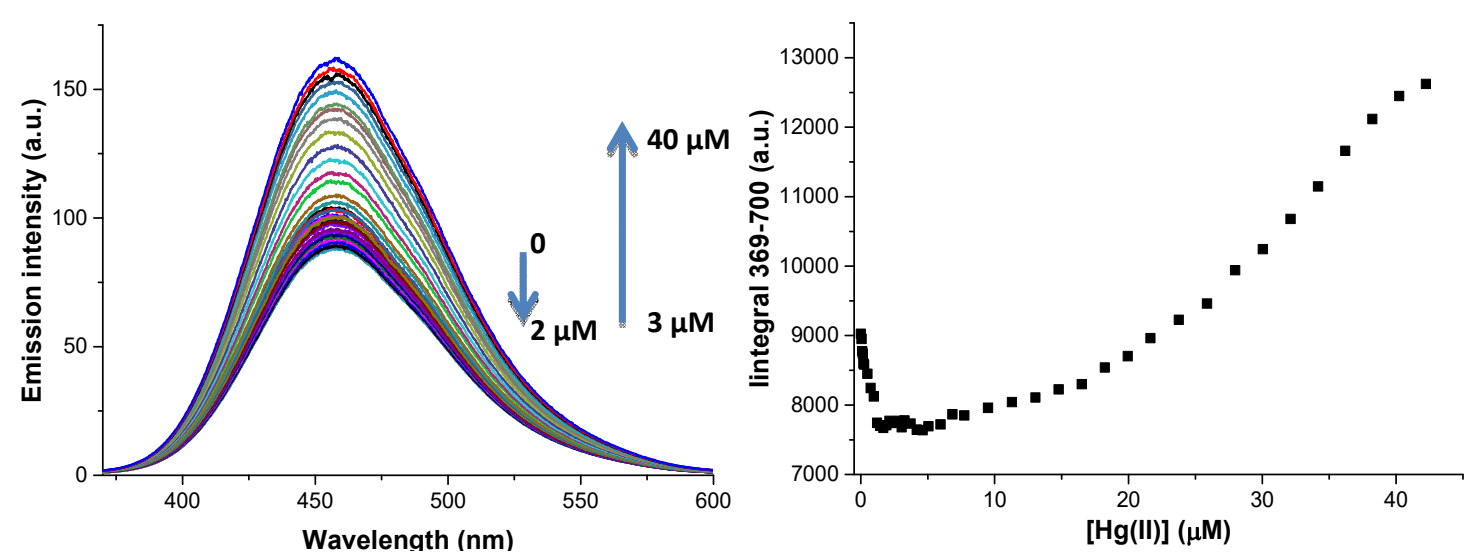

Figure 66. Left: Fluorescence curves of JG32, $0.012 \mathrm{~g} / \mathrm{L}$, by addition of increasing concentrations of $\mathrm{Hg}\left(\mathrm{ClO}_{4}\right)_{2}, \lambda_{\text {exc }}=320 \mathrm{~nm}$. Right: Titration plot by using integral surfaces of the fluorescence curves between 380-650 $\mathrm{nm}$ in response to increasing concentrations of $\mathrm{Hg}\left(\mathrm{ClO}_{4}\right)_{2}, \lambda_{\text {exc }}=320 \mathrm{~nm}$.

As it is indicated in Figure 66, JG32 solution fluorescence decreased linearly when concentration of $\mathrm{Hg}^{2+}$ was increased from 0 to $2 \mu \mathrm{M}$, then it remained constant until $3 \mu \mathrm{M}$ and, finally it increased. 


\subsubsection{Results of the $\mathrm{Hg}^{2+} \mathrm{LOD}$ calculation:}

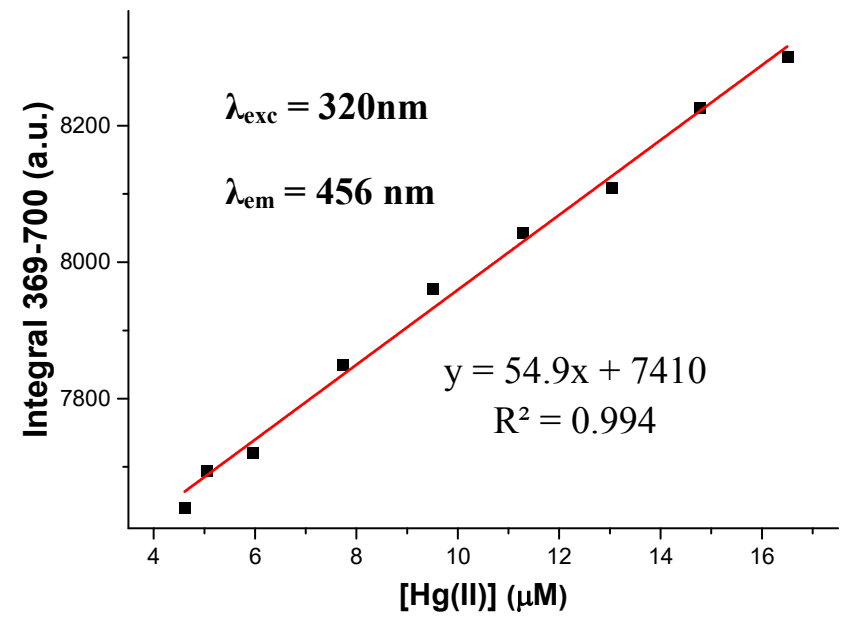

Figure 67. JG32, $0.012 \mathrm{~g} / \mathrm{L}$, in water titration regression plot by using integral surfaces of the fluorescence curves between $369-700 \mathrm{~nm}$, in response to increasing concentrations of $\mathrm{Hg}\left(\mathrm{ClO}_{4}\right)_{2}, \lambda_{\text {exc }}$ $=320 \mathrm{~nm}$. Linear regression for LOD calculation.

The data showed in Figure 67 allowed to calculate the LOD, being $7.6 \mu \mathrm{M}$ or $1.5 \mathrm{ppm}$ of $\mathbf{H g}^{2+}$. This limit was calculated increasing the concentration of $\mathrm{Hg}(\mathrm{II})$ every 2 minutes, the value of the limit was reached in 20 minutes. To use this limit with real samples, the fluorescence had to be superior to the initial value to be quantified, therefore there were two possible results: If the fluorescence is lower than the initial value, the quantity of $\mathrm{Hg}$ (II) stays between 0 to $22 \mu \mathrm{M}$. If the fluorescence was higher than the initial value, the quantity of $\mathrm{Hg}$ (II) was higher to $2.2 \mu \mathrm{M}$.

For this polymer there was a clear second effect of decreasing the fluorescence when the concentration was very low. Without more data this effect was associated to a kinetic effect. To check that effect, solutions near the LOD were prepared and the fluorescence was measured at different time periods; Figure 68 .

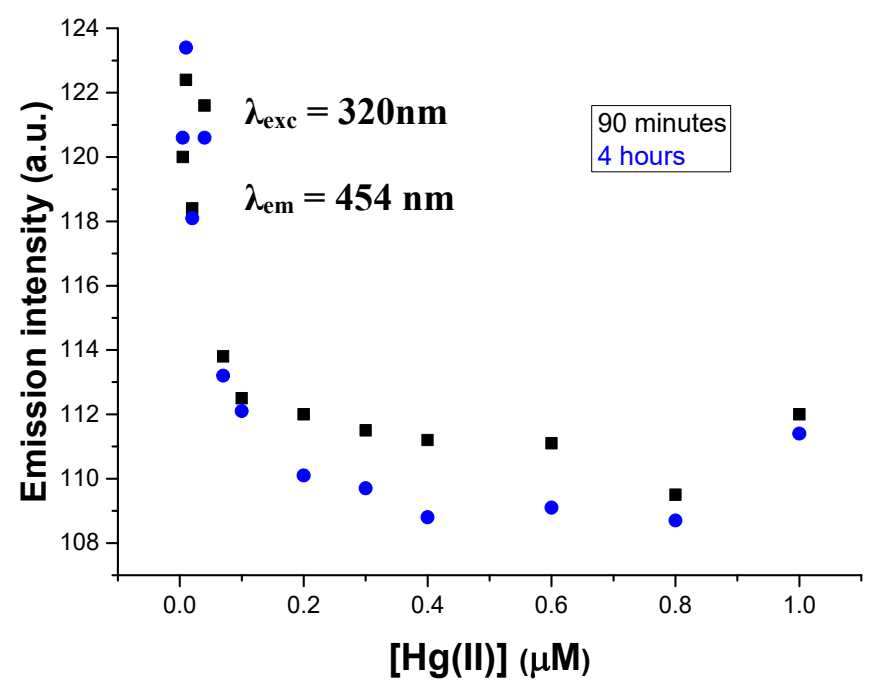

Figure 68. Emission intensity of JG32, $0.012 \mathrm{~g} / \mathrm{L}$, at low concentrations of $\mathrm{Hg}\left(\mathrm{ClO}_{4}\right)_{2}$ after different waiting times, $\lambda_{\mathrm{exc}}=320 \mathrm{~nm}$.

The analysis of the graph from Figure 68 led to the conclusion that the results under low concentrations barely change. This behaviour was checked three times giving similar results. 


\subsubsection{Kinetic response to $\mathrm{MeHg}(\mathrm{II})$ :}

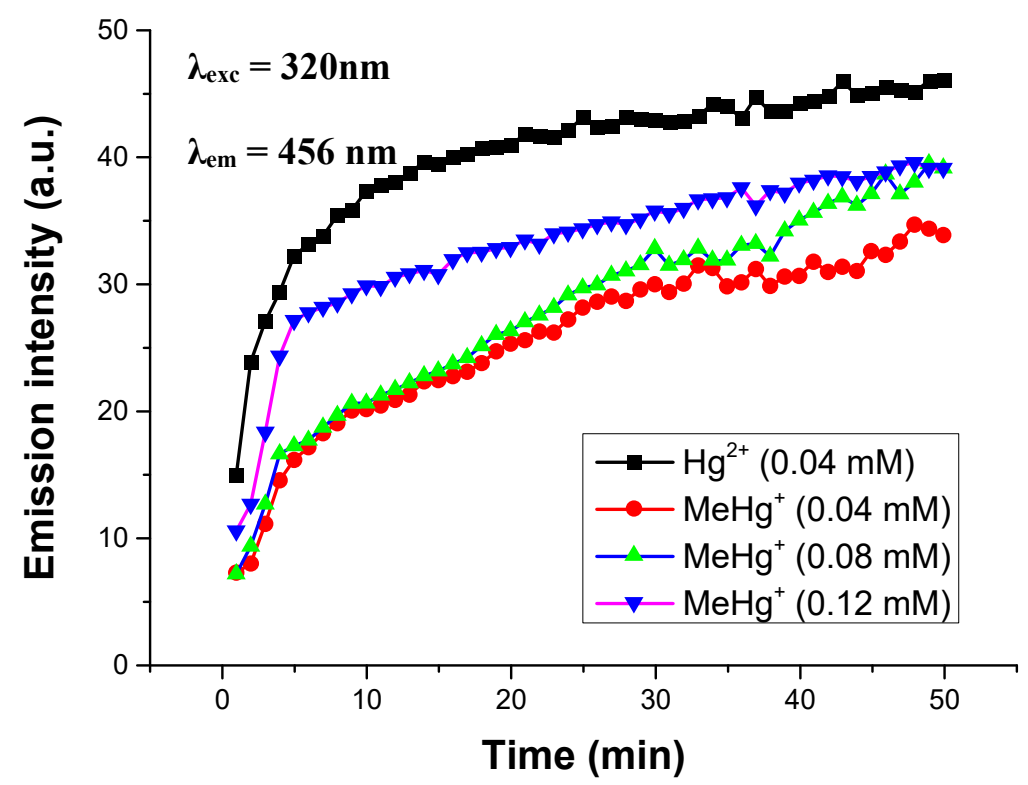

Figure 69. Kinetic response of JG32, $0.012 \mathrm{~g} / \mathrm{L}$, to aqueous solutions of $\mathrm{Hg}\left(\mathrm{ClO}_{4}\right)_{2}$ and $\mathrm{MeHgCl}$ at different concentrations, $\lambda_{\mathrm{exc}}=320 \mathrm{~nm}, \lambda_{\mathrm{em}}=456 \mathrm{~nm}$. Measurements were taken for 55 minutes.

Figure 69 shows that the increase in fluorescence was faster with $\mathrm{MeHg}(\mathrm{II})$ at the beginning, the first 5 minutes, but the maximum was reached more slowly and the final fluorescence was lower at the same concentration of the species.

\subsubsection{Titration experiments by adding $\mathrm{MeHg}(\mathrm{II})$ :}
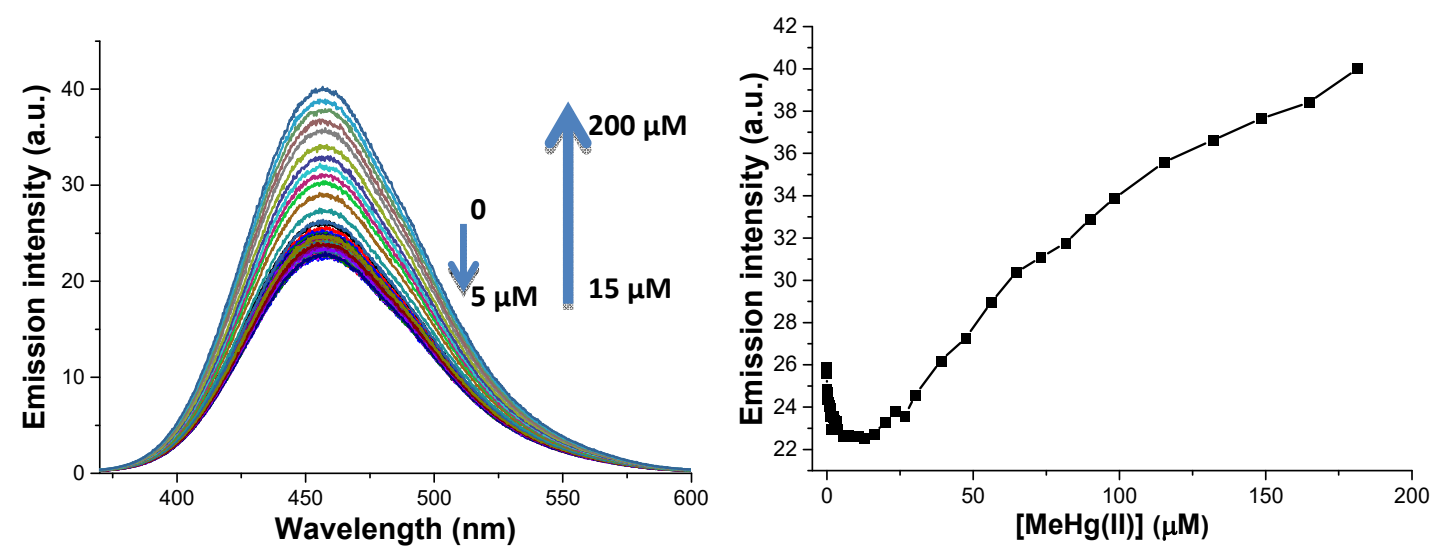

Figure 70. JG32 titration. Left: Fluorescence curves by addition of increasing concentrations of $\mathrm{MeHgCl}, \lambda_{\mathrm{exc}}=320 \mathrm{~nm}, \lambda_{\mathrm{em}}=458 \mathrm{~nm}$. Right: Titration plot by using the maximum of the fluorescence curves in response to increasing concentrations of $\mathrm{MeHgCl}, \lambda_{\mathrm{exc}}=320 \mathrm{~nm}, \lambda_{\mathrm{em}}=458 \mathrm{~nm}$. Additions every 3 minutes.

The titration profile represented in Figure $\mathbf{7 0}$ is very similar to the one obtained for experiments with $\mathrm{Hg}(\mathrm{II})$; Figure 66. However, for $\mathrm{MeHg}(\mathrm{II})$ the emission decreased until a concentration of $5 \mu \mathrm{M}$ of $\mathrm{Hg}$ (II) was reached and then it started to increase when a concentration of $15 \mu \mathrm{M}$ of $\mathrm{Hg}$ (II) was reached. 


\subsubsection{Results of the MeHg(II) LOD calculation:}

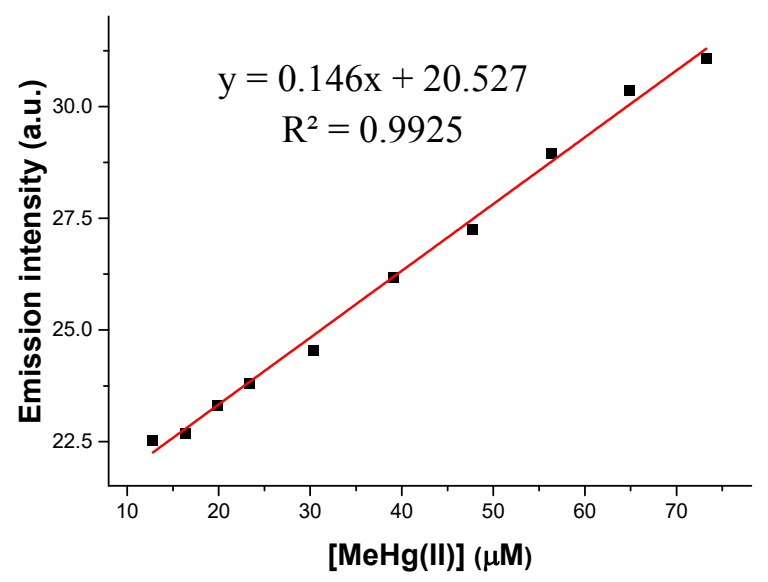

Figure 71. Regression plot by using the maximum of the fluorescence curves between $380-650 \mathrm{~nm}$ in response to increasing concentrations of $\mathrm{MeHgCl}, \lambda_{\mathrm{exc}}=320 \mathrm{~nm}, \lambda_{\mathrm{exc}}=458 \mathrm{~nm}$. LOD calculation.

The LOD calculated with the data from Figure 71 was $28 \mu \mathrm{M}$ or $6.5 \mathrm{ppm}$ of $\mathrm{MeHg}(\mathrm{II})$. The limit was reached in 40 minutes. To use this limit with real samples, the fluorescence should be higher than the initial value to be quantified, therefore there are two possible results: If the fluorescence was lower than the initial value, the quantity of $\mathrm{MeHg}$ (II) stays between $0-35 \mu \mathrm{M}$. If the fluorescence was higher than the initial value, the quantity of $\mathrm{MeHg}(\mathrm{II})$ was higher than $35 \mu \mathrm{M}$.

\subsubsection{Fluorescence Quantum Yield of JG32:}

The fluorescence quantum yield was calculated by using an integration sphere in water solution:

$$
\begin{gathered}
\Phi_{\mathrm{F}}(\mathbf{J G 3 2})=0.17 \pm 0.02 \\
\Phi_{\mathrm{F}}(\mathbf{J G 3 2}+\mathrm{Hg}(\mathrm{II}))=0.42 \pm 0.02
\end{gathered}
$$

\subsection{Measurements with the solid crosslinked polymer JG25}

\subsubsection{Kinetic response to $\mathrm{Hg}(\mathrm{II})$ :}

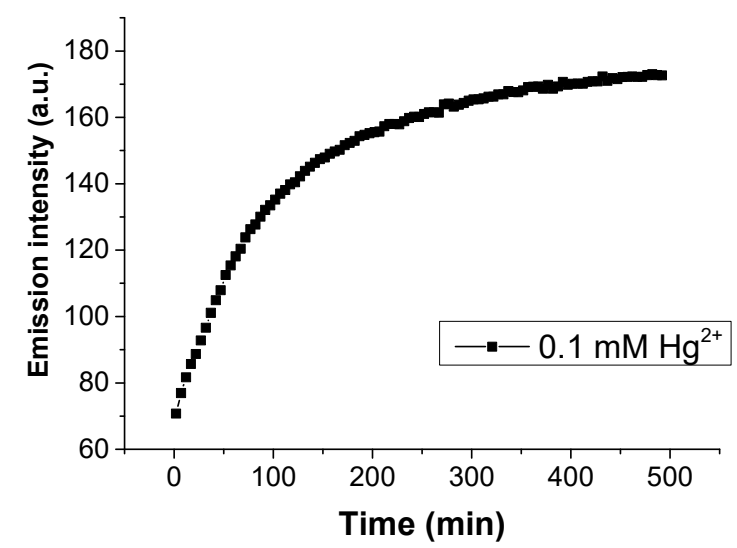

Figure 72. Kinetic response of JG25 to an aqueous solution of $\mathrm{Hg}\left(\mathrm{ClO}_{4}\right)_{2} 0.1 \mathrm{mM}$. $\lambda_{\text {exc }}=369 \mathrm{~nm}$. $\lambda_{\mathrm{em}}$ $=445 \mathrm{~nm}$. Measurements are taken every 5 minutes for 8 hours. 
After adding $\mathrm{Hg}$ (II) the fluorescent emission increased very quickly for the first five minutes, (Figure 72) then, increased linearly for 70 minutes (Figure 73A). The process was very slow and supposed a $54 \%$ increase in the global fluorescence; reaching $6 \%$ in the first 5 minutes and $33 \%$ in the first 90 minutes. Moreover, this process was dependent on the concentration of $\mathrm{Hg}(\mathrm{II})$ added, but the results were very similar when the time was very short, less than twenty minutes. (Figure 73B)
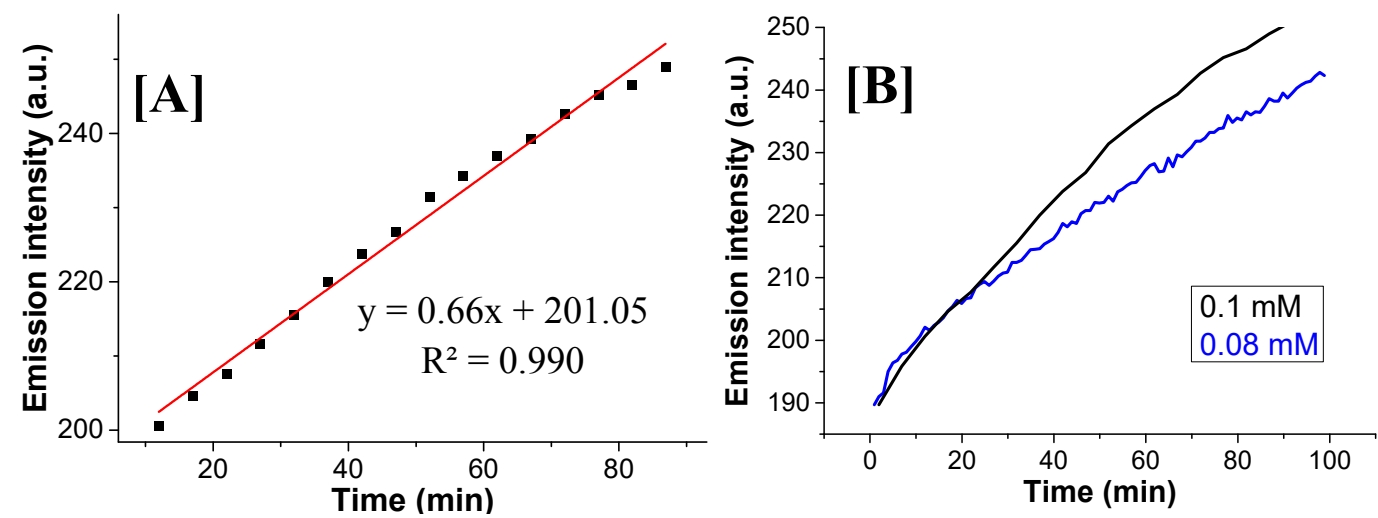

Figure 73. Kinetic response of $\mathbf{J G 2 5}$ in an aqueous solution of $\mathrm{Hg}\left(\mathrm{ClO}_{4}\right)_{2} 0.1 \mathrm{mM}$ [A] and comparison with $0.08 \mathrm{mM}[\mathrm{B}] \cdot \lambda_{\mathrm{exc}}=369 \mathrm{~nm} . \lambda_{\mathrm{em}}=445 \mathrm{~nm}, 100$ minutes.

\subsubsection{Titration experiments by adding $\mathrm{Hg}(\mathrm{II})$ :}

After the kinetic results, a deep study on the equilibrium was not performed because of the difficulty and low interest, the need of a long waiting time and the relation between concentration to it. In spite of this fact, there were some alternatives; it is noteworthy that the changes at the beginning of the additions were linear; as a consequence, adding quantities of $\mathrm{Hg}(\mathrm{II})$ every 5 minutes resulted in a graphic plot that was very similar to the typical plot for species studied in equilibrium, Figure $\mathbf{7 4 .}$
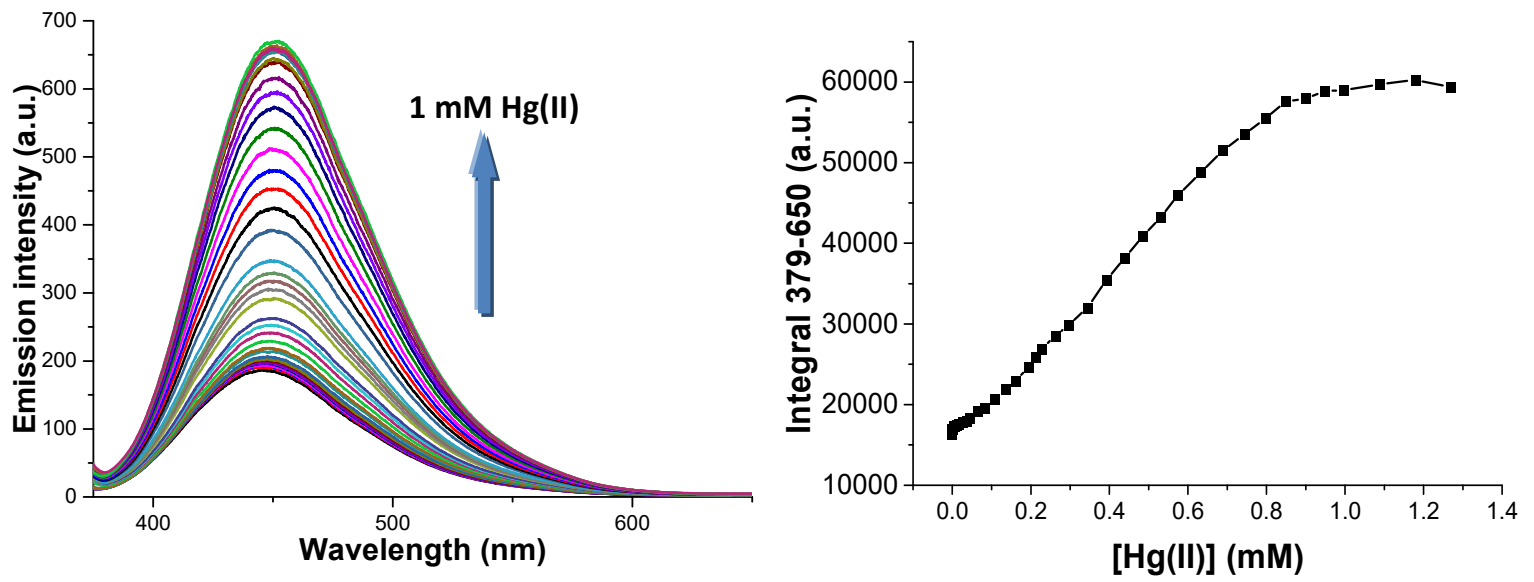

Figure 74. Left: Fluorescence curves by addition of increasing concentrations of $\mathrm{Hg}\left(\mathrm{ClO}_{4}\right)_{2}, \lambda_{\text {exc }}=369$ $\mathrm{nm}$. Right: Titration plot by using integral surfaces of the fluorescence curves between $379-650 \mathrm{~nm}$ in response to increasing concentrations of $\mathrm{Hg}\left(\mathrm{ClO}_{4}\right)_{2} \lambda_{\mathrm{exc}}=369 \mathrm{~nm}$.

In this way, the limit of detection obtained was a little higher than taking measurements when the interaction $\mathrm{Hg}$ (II)-probe reached the equilibrium. Nevertheless, the value was perfectly valid for an assigned concentration and time. The saturation of the signal was reached when the concentration of $\mathrm{Hg}$ (II) was near $1 \mathrm{mM}$ (two hours and a half after the first addition) and, in a concentration below 10 $\mu \mathrm{M}$, the variation of intensity between additions was linear. 


\subsubsection{Results of the LOD calculation:}

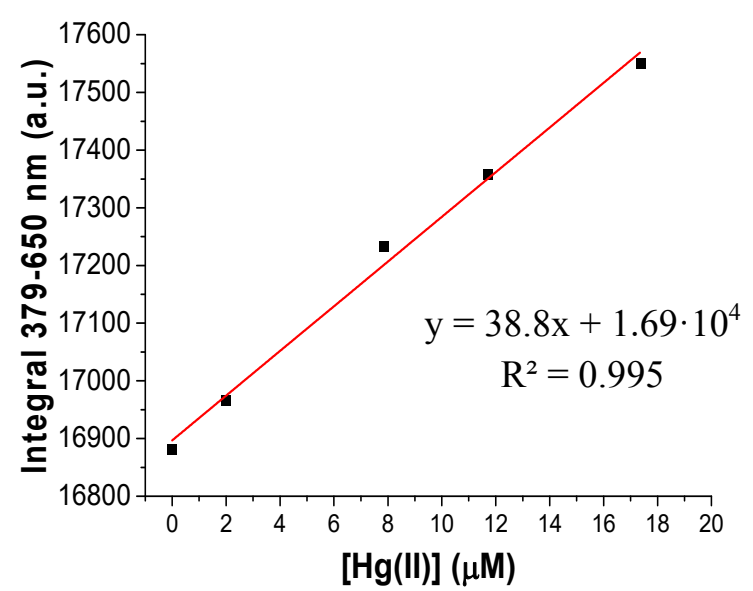

Figure 75. Linear regression by using integral surfaces of the fluorescence curves between 379-650 $\mathrm{nm}$ in response to increasing concentrations of $\mathrm{Hg}\left(\mathrm{ClO}_{4}\right)_{2}, \lambda_{\mathrm{exc}}=369 \mathrm{~nm}$, LOD calculation.

Therefore, with the data showed in Figure 75, the LOD was calculated to be $\mathbf{6 . 6} \boldsymbol{\mu M}$ or $\mathbf{1 . 3}$ ppm of $\mathbf{H g}(\mathbf{I I})$ in water. Value reached 15 minutes after the first measure, so this was the average time that was necessary to detect a noticeable increase of the fluorescence of the solution.

\subsubsection{Kinetic response to $\mathrm{MeHg}(\mathrm{II})$ :}

The behaviour of the probe in presence of $\mathrm{MeHg}(\mathrm{II})$ turned out to be different to the previous behaviour in presence of $\mathrm{Hg}(\mathrm{II})$. Figure 76 shows the changes in fluorescent emission on time by normalizing the spectra:

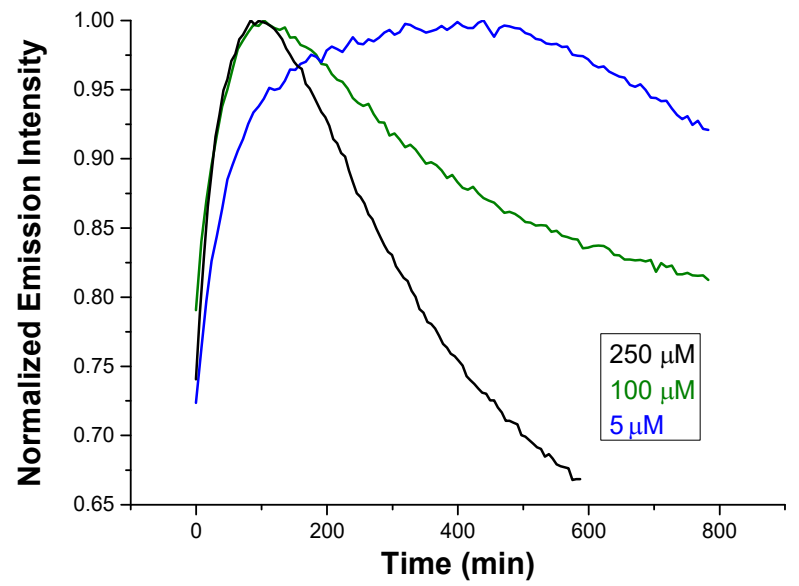

Figure 76. Normalized kinetic response of $\mathbf{J G 2 5}$ to $\mathrm{MeHgCl}$ aqueous solution. $\lambda_{\mathrm{exc}}=364 \mathrm{~nm}$. $\lambda_{\mathrm{em}}=$ $445 \mathrm{~nm}$

Several conclusions were obtained for the kinetic study. The increase on the emission was more or less the same independently of the $\mathrm{MeHg}$ (II) concentration. However, when the concentration was high, the maximum of emission was reached in 90 minutes, after that the concentration decreased. In contrast, when the concentration was low, the emission increase was slower, but the decrease started after 8 hours, which was observed for $\mathrm{MeHg}$ (II) $5 \mu \mathrm{M}$. 
The presence of two processes was a possible explanation for these facts. The first one was the complexation of the cation, which was very fast and needs a very low amount of $\mathrm{MeHg}$ (II) to reach the saturation. Then, there was another process in which the MeHg(II) modified the structure of the complex between the polymer and the $\mathrm{MeHg}$ (II) cation (possibly a reaction) which was slower and depended on the concentration.

\subsubsection{Titration experiments by adding $\mathrm{MeHg}(\mathrm{II})$ :}

By the same way than in the case of $\mathrm{Hg}(\mathrm{II})$, the titration experiments with the polymer where done by adding the solution of the cation every 5 minutes (Figure 77).
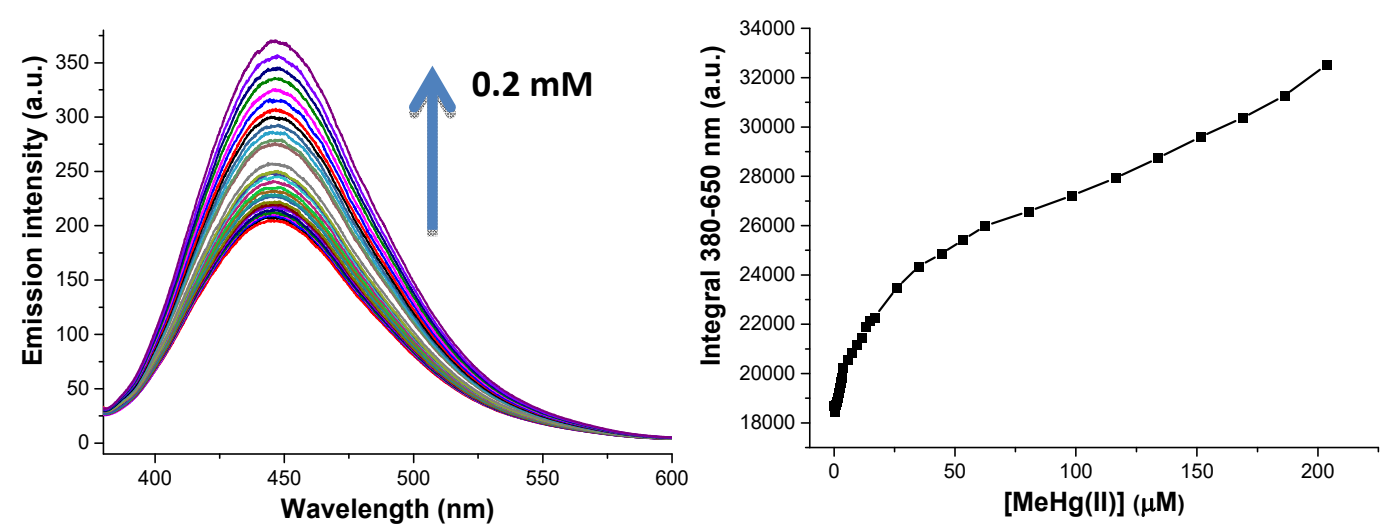

Figure 77. JG25 titration. Left: Fluorescence curves by addition of increasing concentrations of $\mathrm{MeHgCl}, \lambda_{\mathrm{exc}}=364 \mathrm{~nm}$. Right: Titration plot by using integral surfaces of the fluorescence curves between $380-650 \mathrm{~nm}$ in response to increasing concentrations of $\mathrm{MeHgCl}, \lambda_{\mathrm{exc}}=364 \mathrm{~nm}$.

During the measurements the emission intensity increased faster than in the case of $\mathrm{Hg}(\mathrm{II})$.

\subsubsection{Results of the LOD calculation:}

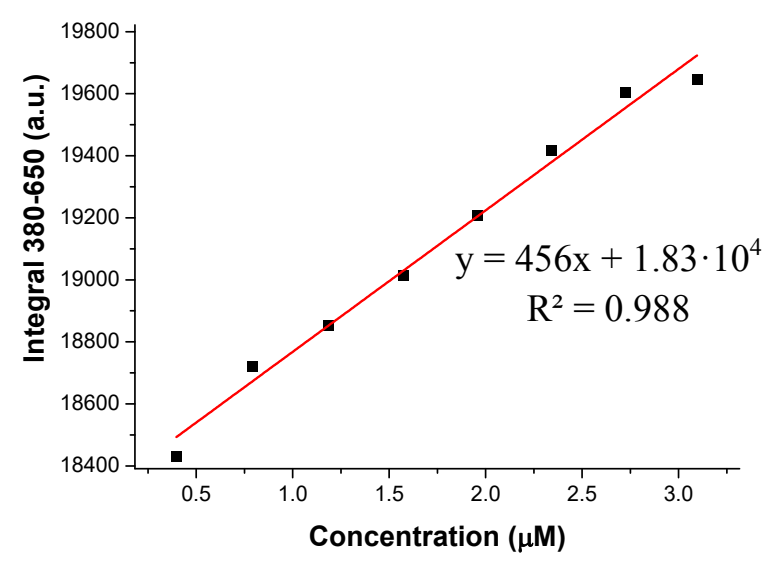

Figure 78. JG25 titration. Regression plot by using integral surfaces of the fluorescence curves between 380-650 $\mathrm{nm}$ in response to increasing concentrations of $\mathrm{MeHgCl}, \lambda_{\mathrm{exc}}=364 \mathrm{~nm}$. LOD calculation.

From Figure 78, the LOD calculated was $1.5 \mu \mathrm{M}$ or $0.3 \mathrm{ppm}$ of $\mathrm{MeHg}(\mathrm{II})$. This value was reached in less than 20 minutes. 


\subsubsection{Effect of pH on JG25:}

Although the quantitative measurements were performed in deionized water $(\mathrm{pH}=8$ approximately), it was important to study the $\mathrm{pH}$ effect, in order to do further studies, such as the measurements from fish samples, which were the final objective of the work.

In this regard, a solution buffered at $\mathrm{pH}=7.8$ (HEPES buffer, $5 \mathrm{mM}$ ) was acidified little by little with $\mathrm{HCl} 1 \mathrm{M}$ and changes in $\mathrm{pH}$ - fluorescence were evaluated (Table 3 and Figure 79). The results indicated that an increase of fluorescence occurred while lowering $\mathrm{pH}$. Then, by using the same process, the increase in fluorescence was measured in a sample that contained $10 \mu \mathrm{M}$ of $\mathrm{Hg}(\mathrm{II})$.

\begin{tabular}{|c|c|c|}
\hline $\boldsymbol{p H}$ & $\begin{array}{c}\text { Emission } \\
\text { intensity (a.u.) }\end{array}$ & $\begin{array}{c}\text { Emission intensity } \\
\mathbf{+} \mathbf{H g}(\mathbf{I I})(\boldsymbol{a} . \mathbf{u .})\end{array}$ \\
\hline 7.8 & 39 & 89.2 \\
\hline 7.5 & 43.5 & 100.6 \\
\hline 7.1 & 49.4 & 116.7 \\
\hline 6.6 & 60.9 & 148.4 \\
\hline 5.4 & 69.7 & 173 \\
\hline 4.6 & 96.4 & 243 \\
\hline 4 & 110.1 & 282.7 \\
\hline 3.5 & 121.9 & 318.7 \\
\hline
\end{tabular}

Table 3. Emission intensity, with and without $\mathrm{Hg}\left(\mathrm{ClO}_{4}\right)_{2}$, at different $\mathrm{pH}$, of JG25.

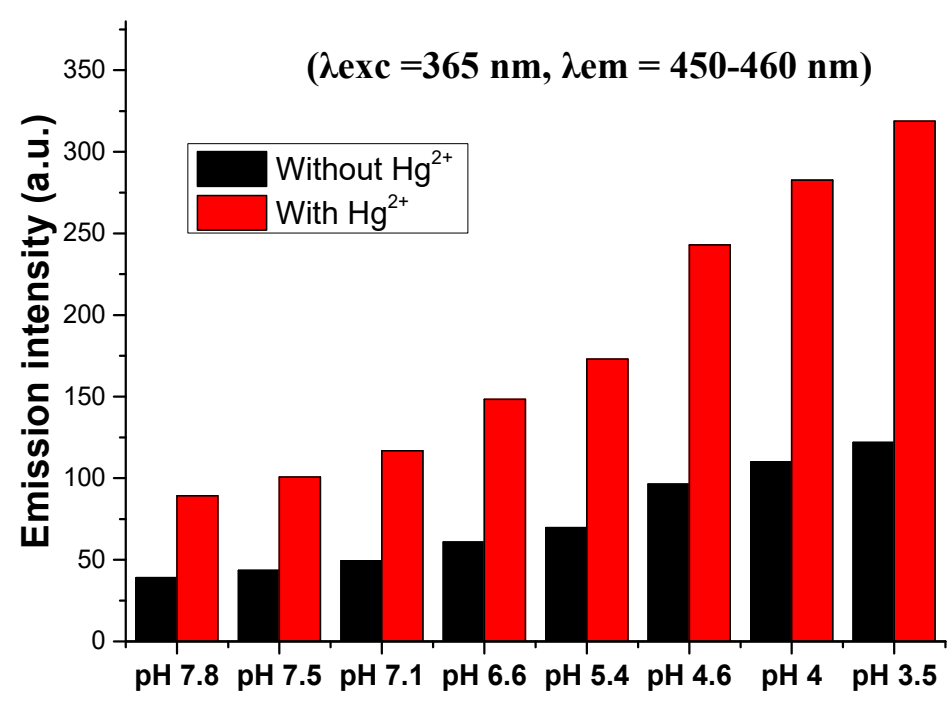

Figure 79. Emission intensity of JG25, with and without $\mathrm{Hg}\left(\mathrm{ClO}_{4}\right)_{2}$, at different $\mathrm{pH} ; \lambda_{\text {exc }}=365 \mathrm{~nm}$,

$$
\lambda_{\mathrm{em}}=455 \mathrm{~nm}
$$

The proportional increase in fluorescence with $\mathrm{Hg}(\mathrm{II})$ was higher in acidic $\mathrm{pH}$, being lower if the buffer was basic; see Figure 79. Therefore, it was determined that the best $\mathrm{pH}$ for evaluating $\mathrm{Hg}$ (II) presence was a controlled slightly acidic or neutral $\mathrm{pH}$. 


\section{ANALYSIS OF FISH SAMPLES}

In order to find the best conditions to have reproducible and reliable measurements of the presence of mercury in fish samples, some preliminary tests were necessary:

- Lyophilization of the fish; to avoid interference from the quantity of water in the sample.

- Extracting the samples; having homogeneous samples and with less interferents give reliability to the measurements.

- Qualitative measurements; so as to check if the fluorescence changes before any other analysis.

- ICP analysis, from the fish and the extracted samples.

- Fluorescent response of JG25 when in contact to fish and mercury extracts.

- Comparison ICP-JG25 results.

The scheme from Figure $\mathbf{8 0}$ represents a guide for the process.

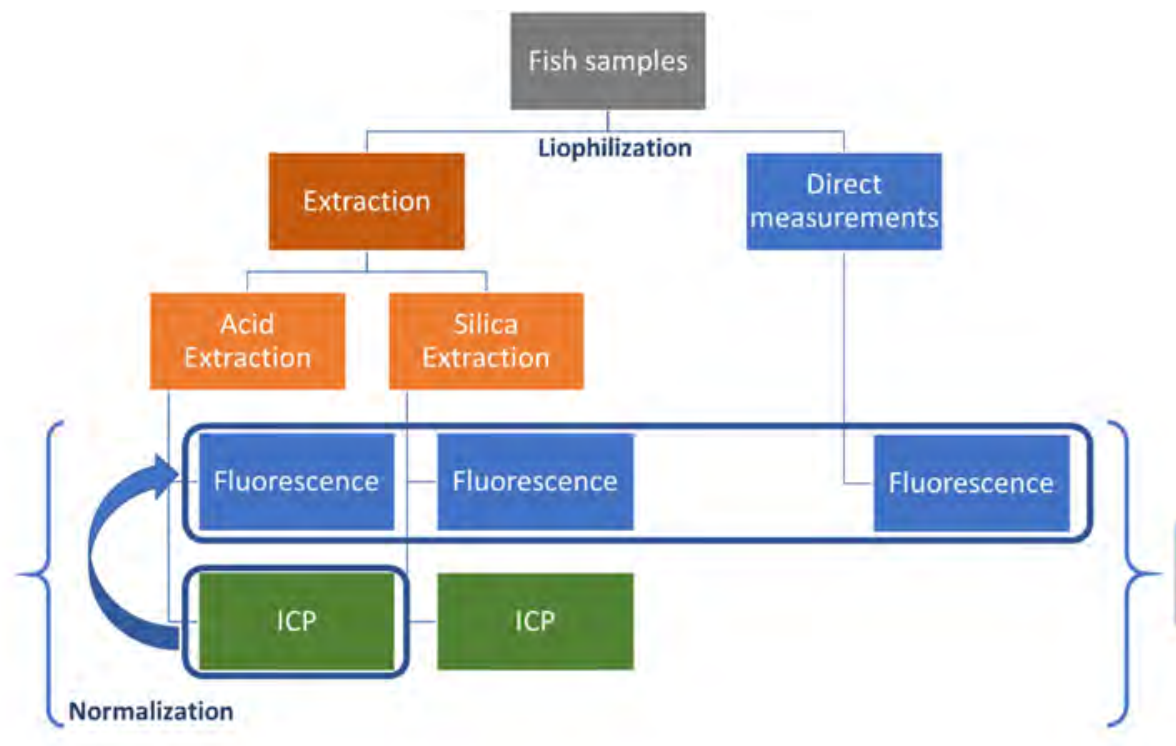

Comparison

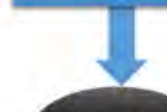

Figure 80. Scheme of treatment for analysis of fish samples.

\subsection{Extraction of mercury species from fish}

Two methods were tested: acidic extraction and silica extraction. It was also possible to perform a basic extraction, but it was discarded because of the high temperatures, the extracts presented an intense yellow colour and the probe had no good results at $\mathrm{pH}$ superior than 8 . In addition, all the measurements were done with lyophilized fish because the quantity of water is vital in the concentration calculation.

Acid extraction: $5 \mathrm{ml}$ of $\mathrm{HCl} 5 \mathrm{M}$ in water and $5 \mathrm{ml}$ of $\mathrm{NaCl} 0.25 \mathrm{M}$ in water were added to $0.5 \mathrm{~g}$ of lyophilized fish in a sealed vial. The mixture was sonicated for 10 minutes and heated for other 10 minutes at $60^{\circ} \mathrm{C}$. Then, the mixture was centrifuged at $4000 \mathrm{rpm}$ for 10 minutes and at $7000 \mathrm{rpm}$ for other additional 10 minutes. Afterwards, the liquid phase was filtered in a glass fibre filter of $0.22 \mu \mathrm{m}$ pore. Finally, the samples to be measured by fluorescence were concentrated to $1 \mathrm{ml}$ of water. 
Silica extraction: $0.5 \mathrm{~g}$ of fish and $2 \mathrm{ml}$ of water were mixed in a mortar and the mixture was grinded, $1 \mathrm{~g}$ of silica was then added and mixed. Next, the mixture was treated with $\mathrm{HCl} 5 \mathrm{M}$ in water following the same procedure used for the acid extraction. Finally, the samples to be measured by fluorescence were concentrated to $1 \mathrm{ml}$ of water.

\subsection{Determination of mercury by ICP-Mass analysis}

For determination of metals by ICP it was standardized a method in which the organic material was digested under highly concentrated $\mathrm{HNO}_{3}$ solutions. Afterwards, the samples are diluted and introduced in the system. The ICP was calibrated with standards ${ }^{36}$ and the concentration determined by adjusting the signal into a regression done with this calibration.

The samples were lyophilized, causing a loss in weigh of $70-80 \%$, which is shown so as to compare the real concentration in fish.

\begin{tabular}{|l|c|c|c|c|c|}
\cline { 3 - 4 } \multicolumn{1}{c|}{} & \multicolumn{2}{c|}{ Conc. $\mathbf{H g}(\mathbf{p p m})$ lyophilized } & & \multicolumn{2}{c|}{ Conc. $\mathbf{H g}(\mathbf{p p m})$ fresh } \\
\hline Sample & Acid extraction & $\begin{array}{c}\text { Silica } \\
\text { extraction }\end{array}$ & \% (Water) & $\begin{array}{c}\text { Acid } \\
\text { extraction }\end{array}$ & $\begin{array}{c}\text { Silica } \\
\text { extraction }\end{array}$ \\
\hline Swordfish & $5.1 \pm 0.1$ & $6.0 \pm 0.6$ & 73 & $1.4 \pm 0.1$ & $1.6 \pm 0.6$ \\
\hline Tuna & $3.1 \pm 0.1$ & $5.1 \pm 1.4$ & 71 & $0.9 \pm 0.1$ & $1.5 \pm 1.4$ \\
\hline Panga & $1.1 \pm 0.4$ & $1.3 \pm 0.6$ & 78 & $0.2 \pm 0.1$ & $0.3 \pm 0.1$ \\
\hline Salmon & $0.015 \pm 0.016$ & $0.07 \pm 0.01$ & 72 & 0 & 0 \\
\hline Conger eel & $2.4 \pm 0.1$ & $2.2 \pm 0.2$ & 80 & $0.5 \pm 0.1$ & $0.4 \pm 0.1$ \\
\hline Dogfish & $6.8 \pm 0.3$ & $3.9 \pm 0.1$ & 75 & $1.7 \pm 0.1$ & $1.0 \pm 0.1$ \\
\hline
\end{tabular}

Table 4. Amount of mercury detected by ICP-Mass analysis on fish samples, lyophilized fish (left), corresponding amount to fresh fish (right).
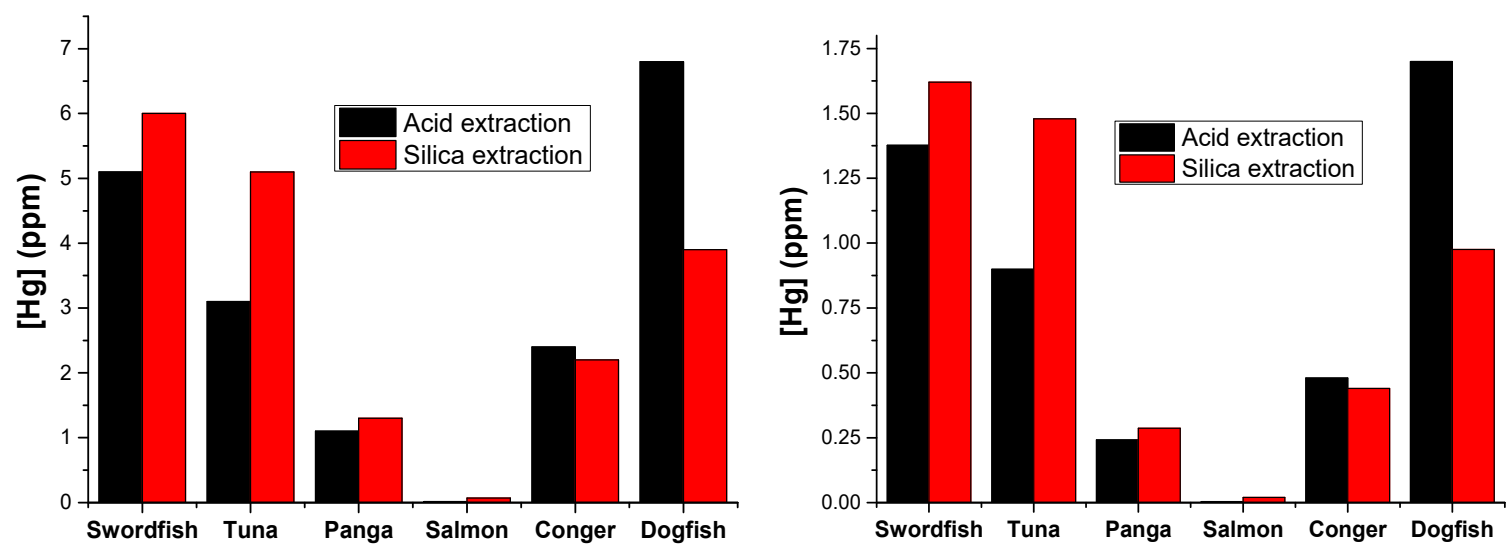

Figure 81. Representation of the amount of mercury detected by ICP-Mass analysis on fish samples, (left) lyophilized, (right) the corresponding amount to fresh fish.

The data from Table 4 and Figure 81 shows that the concentration was higher when the fish was bigger. Another important result was that the concentration of mercury in salmon could be

\footnotetext{
${ }^{36}$ The process was performed entirely by technicians at the Research building from University Burgos, given a pre-treated sample and the approximated concentration to expect.
} 
considered as 0 . This was normal because salmons were obtained from a fish farm, not from wild sea fish, so there was no possible bioaccumulation. The LOD of the polymer JG25 was $1.3 \mathrm{ppm}$ for $\mathrm{Hg}$ (II) and $0.3 \mathrm{ppm}$ for $\mathrm{MeHg}(\mathrm{II})$, therefore, a direct measurement would be theoretically possible, depending on the interferents and the proportion $\mathrm{g}$ (fish)/water volume. The order of magnitude in mercury concentration means that, in swordfish, tuna, conger eel and dogfish it could be over the LOD, especially in case of swordfish, which is known to have around the maximum amount of mercury ingestion per week recommended by the FDA, (1.3 ppm).

\subsection{Measurements of mercury extracts with JG25}

$1 \mathrm{~mL}$ of each extract was added to a cuvette with JG25. Then the fluorescence was checked. The variation of intensity was measured with water (blank) and then by adding the extract, the difference is the value given in Table 5 .

\begin{tabular}{|c|c|c|c|c|c|c|}
\cline { 2 - 7 } \multicolumn{1}{c|}{} & \multicolumn{6}{c|}{$\Delta$ Emission intensity, $\boldsymbol{\lambda}_{\text {exc }}=\mathbf{3 6 5} \mathbf{~ n m ~ ( a . u . ) , ~} \boldsymbol{\lambda}_{\text {em }}=\mathbf{4 5 5} \mathbf{~ n m ~ ( a . u . )}$} \\
\hline $\begin{array}{c}\text { Extraction } \\
\text { Method }\end{array}$ & Swordfish & Tuna & Panga & Salmon & Conger eel & Dogfish \\
\hline Acid & 301.6 & 229.6 & 0.91 & 5.5 & 152.1 & 377.3 \\
\hline Silica & 354.2 & 307.2 & 27.7 & 6.4 & 162.5 & 171.7 \\
\hline
\end{tabular}

Table 5: Emission intensity variation of JG25 in contact with fish samples.

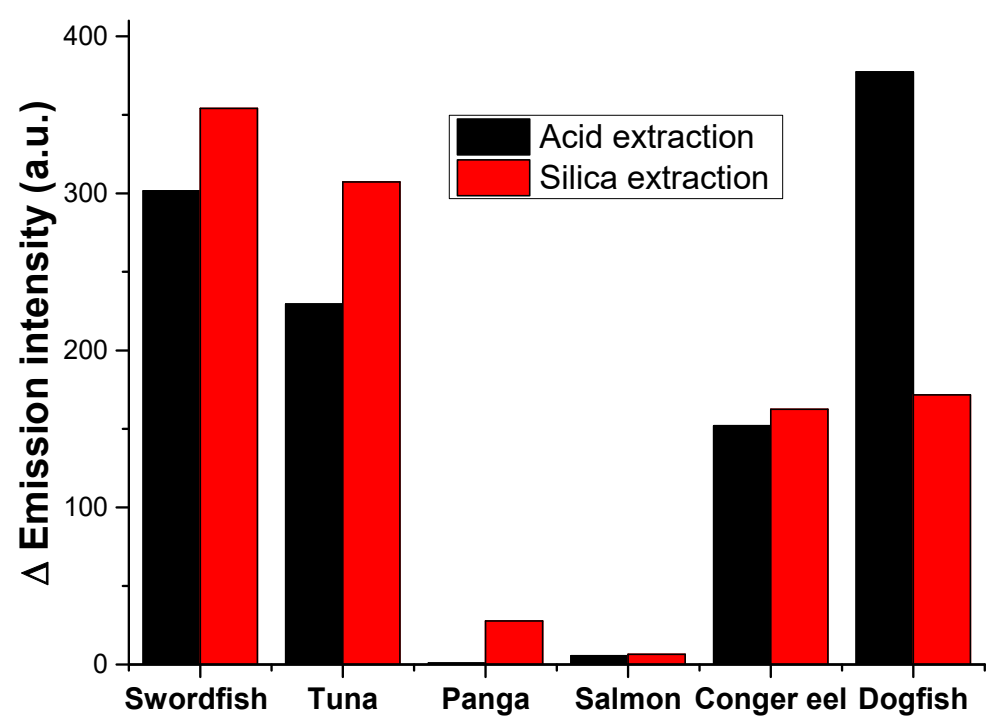

Figure 82. Emission intensity variation of $\mathbf{J G 2 5}$ in contact with fish samples, $\lambda_{\mathrm{exc}}=365 \mathrm{~nm}, \lambda_{\mathrm{em}}=455$ $\mathrm{nm}$.

From the representation in Figure 82 it is clear that the fluorescence was similar to the results of the ICP analysis, evidencing the relation between emission and ppm of $\mathrm{Hg}(\mathrm{II})+\mathrm{MeHg}(\mathrm{II})$. 


\subsection{Comparison ICP - JG25}

To compare the results, the process followed to elaborate Figure $\mathbf{8 3}$ was:

- Normalizing the graphs, taking as reference the ICP results of the acid extraction of dogfish.

- Considering the results of the ICP-Mass in ppm and converting the fluorescence values to ppm, from the ICP-Mass values.

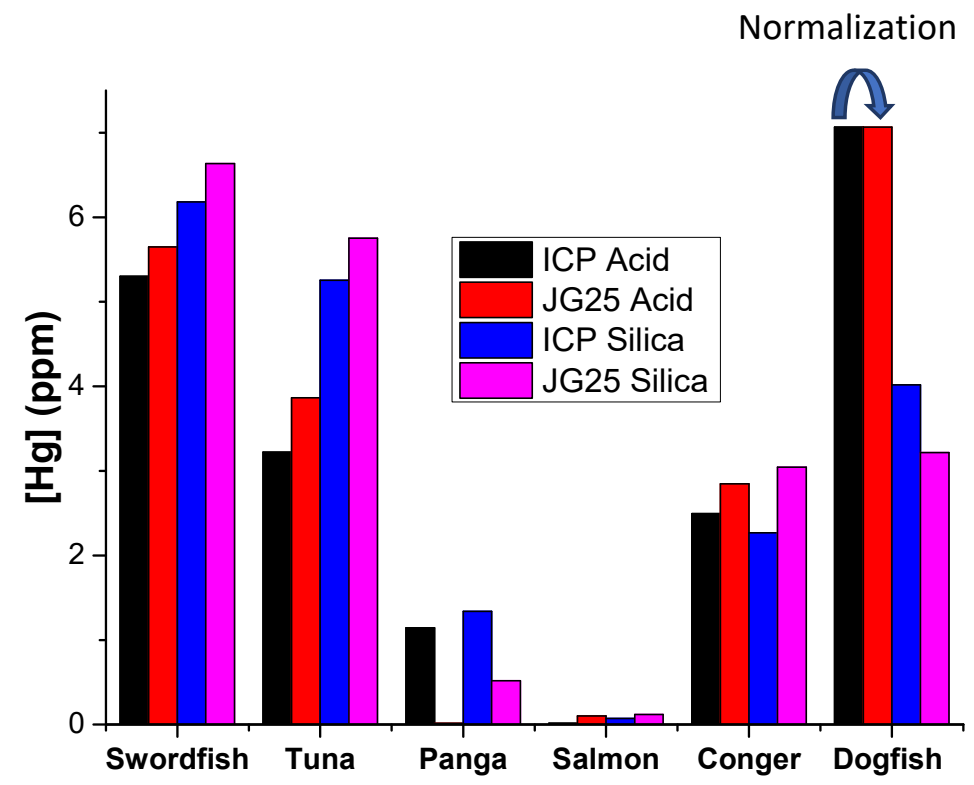

Figure 83. A comparison between the values of mercury in fish samples obtained by ICP-Mass analysis and fluorescent measurements.

The different results were a consequence of multiple factors such as the treatment of the samples or the fish matrix, although they were very close when comparing methods. The increase of fluorescence in solution was enough to see the signal of fluorescence for the samples with higher concentrations of mercury, namely swordfish, tuna and dogfish. None of the salmon samples showed an increase in fluorescence. For panga, the values were very close to the LOD, therefore, it was expected that there was no signal in fluorescence measurements. As a result, the method might be useful in order to measure quantitatively the mercury concentration when measuring above the LOD.

\subsection{Direct analysis of mercury on fish samples with JG25}

First, some preliminary tests were performed with fish samples that contained high quantities of mercury such as tuna and swordfish. After that, several samples of fish, tuna, swordfish, conger and panga were measured by homogenization with fish samples. As qualitative test, it gave good results but with low repeatability because of the different percentage of water, among other reasons.

Finally, the tests were performed by using directly the same samples originally used for extractions, $0.5 \mathrm{~g}$ of lyophilized fish were mixed with $2 \mathrm{ml}$ of water. Then, a piece of the polymeric sensor JG25 was added. To check the difference in fluorescence every polymer fragment in contact with fish samples was measured at different waiting times in the fluorometer, obtaining the results in Table 6 and Figure 84. The results may be compared with the corresponding results from the extraction by normalizing to one of them (dogfish in this case) (Figure 85). 


\begin{tabular}{|r|c|c|c|c|c|}
\cline { 2 - 6 } \multicolumn{1}{c|}{} & \multicolumn{5}{c|}{$\Delta$ Emission intensity 365 nm (a.u.) } \\
\hline $\begin{array}{l}\text { Sample/ } \\
\text { time (h) }\end{array}$ & Swordfish & Tuna & Panga & Conger eel & Dogfish \\
\hline $\mathbf{0 . 5}$ & 220.5 & 212.2 & 21.3 & 32 & 230.93 \\
\hline $\mathbf{1}$ & 260.8 & 237.7 & 21.97 & 65.79 & 272.2 \\
\hline $\mathbf{2 4}$ & 301.6 & 280 & 30.68 & 136.3 & 318.4 \\
\hline
\end{tabular}

Table 6. The relation between the concentration of mercury and the obtained values of fluorescence for fish samples and the polymeric sensor.

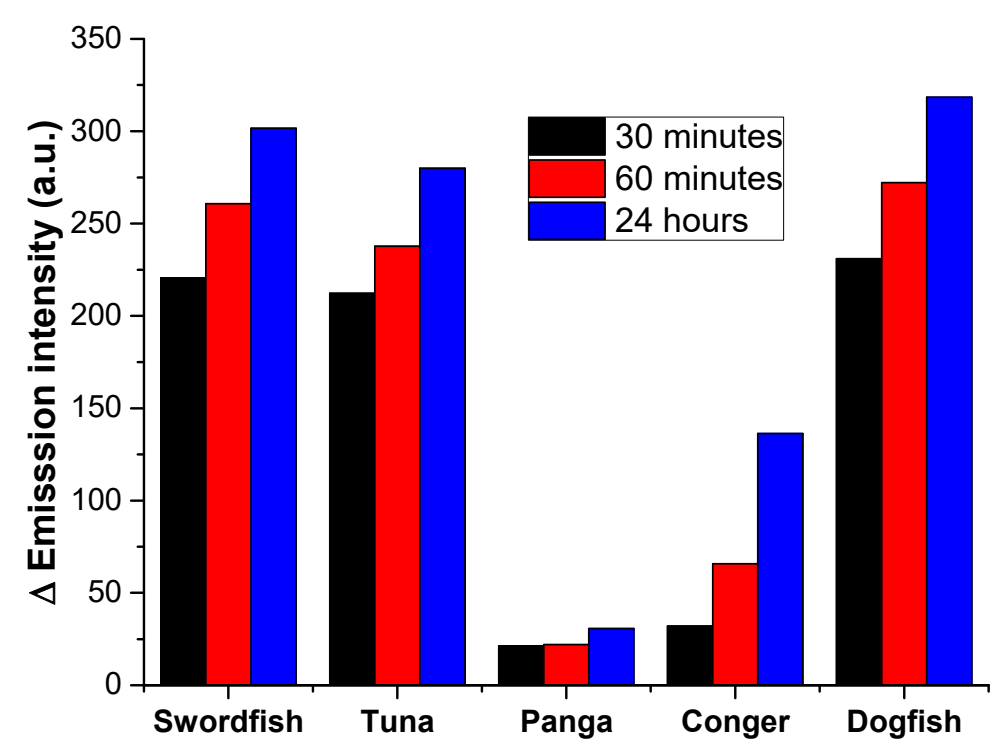

Figure 84. Emission intensity variation with JG25 in fresh fish samples $\left(\lambda_{\mathrm{exc}}=365 \mathrm{~nm}, \lambda_{\mathrm{em}}=455 \mathrm{~nm}\right)$ at different waiting times.

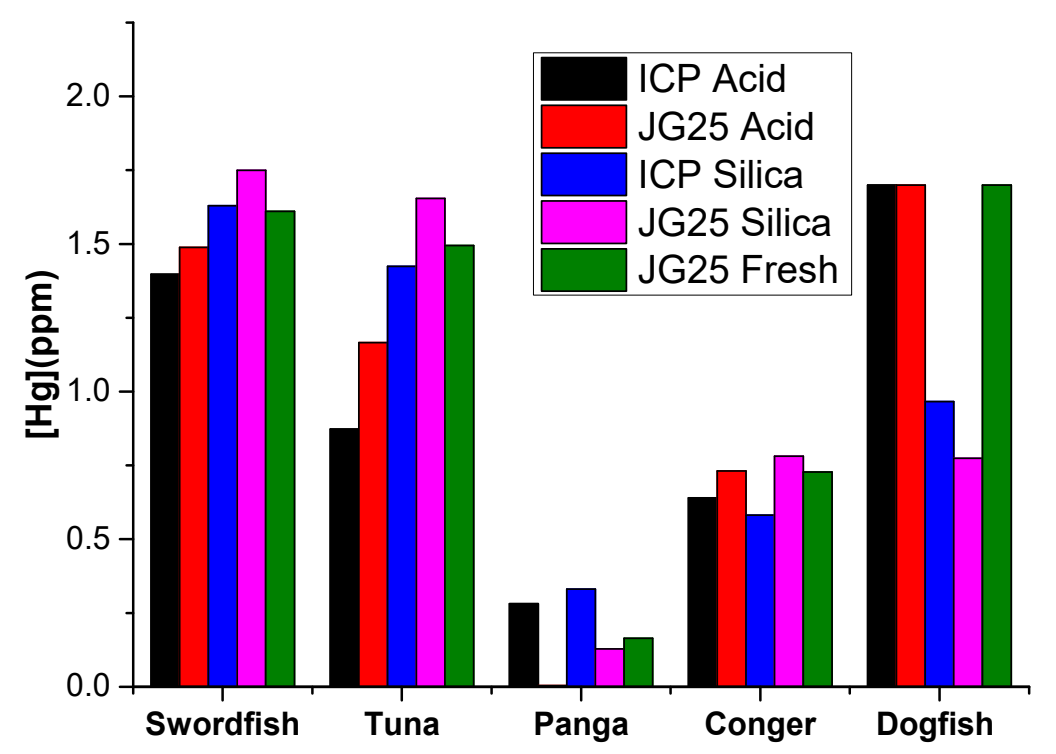

Figure 85. Emission intensity variation in experiments with fresh fish samples JG25 $\left(\lambda_{\text {exc }}=365 \mathrm{~nm}\right.$, $\lambda_{\mathrm{em}}=455 \mathrm{~nm}$ ) compared with the results from the extracts.

Therefore, a relation between the concentration of mercury and the obtained values of fluorescence was confirmed. 


\section{DATA SUMMARY}

\section{$\underline{\text { JG7 and JG30 }}$}

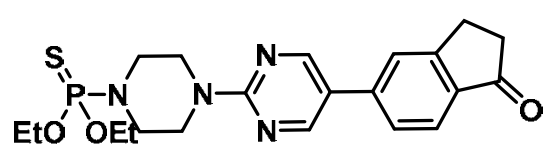

JG7

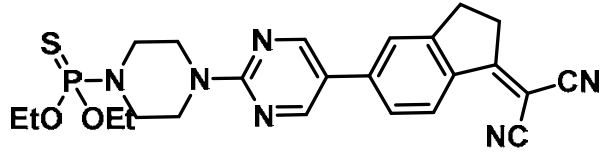

JG30

They were soluble in Methanol:Water 80:20.

JG7 was sensitive to $\mathrm{Hg}(\mathrm{II})$ and $\mathrm{MeHg}(\mathrm{II})$ whereas $\mathbf{J G 3 0}$ was sensitive only to $\mathrm{Hg}(\mathrm{II})$.

JG7 $\mathrm{MeOH} \lambda(\varepsilon)=318 \mathrm{~nm}\left(33000 \mathrm{M}^{-1} \mathrm{~cm}^{-1}\right)$

JG30 MeOH $\lambda(\varepsilon)=383 \mathrm{~nm}\left(38000 \mathrm{M}^{-1} \mathrm{~cm}^{-1}\right)$

\section{$\underline{\text { JG15 }}$}

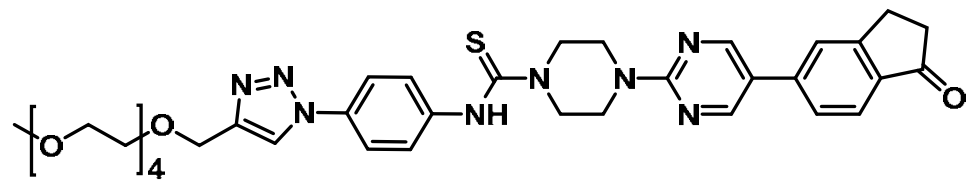

It was soluble in Methanol:Water 9:1.

JG15 MeOH $\lambda(\varepsilon)=320 \mathrm{~nm}\left(27000 \mathrm{M}^{-1} \mathrm{~cm}^{-1}\right)$.

Ф MeOH $(\mathbf{J G 1 5})=0.18 \pm 0.02$

Ф MeOH (JG15 $+\mathbf{H g}(\mathbf{I I}))=0.44 \pm 0.02(1$ equivalent no waiting time $)$

$\mathrm{Hg}(\mathrm{II})$ addition studies in $\mathrm{MeOH}$ were performed, the ${ }^{1} \mathrm{H}-\mathrm{NMR}$ evidenced complexation+reaction process.

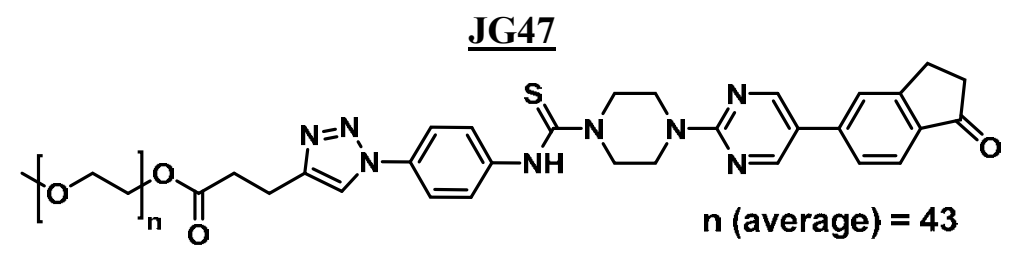

JG47 was soluble in $100 \%$ water.

JG47 MeOH $\lambda(\varepsilon)=315 \mathrm{~nm}\left(25000 \mathrm{M}^{-1} \mathrm{~cm}^{-1}\right)$

It was sensitive to $\mathrm{Hg}(\mathrm{II})$, but the response was highly dependent on the solvent.

Ф $\mathrm{MeOH}(\mathbf{J G 4 7})=0.08 \pm 0.02 / / \boldsymbol{\Phi} \mathrm{H}_{2} \mathrm{O}(\mathbf{J G 4 7})=0.1 \pm 0.02$

$\mathbf{\Phi} \mathrm{MeOH}(\mathbf{J G 4 7}+\mathbf{H g}(\mathbf{I I}))=0.64 \pm 0.02 / / \mathbf{\Phi ~} \mathrm{H}_{2} \mathrm{O}(\mathbf{J G 4 7}+\mathbf{H g}(\mathbf{I I}))=0.16 \pm 0.02$ 


\section{$\underline{\text { JG45 }}$}

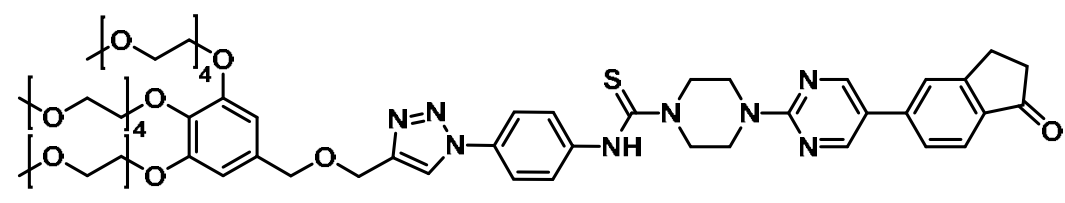

JG45 was soluble in $100 \%$ water.

JG45 MeOH $\lambda(\varepsilon)=310 \mathrm{~nm}\left(37000 \mathrm{M}^{-1} \mathrm{~cm}^{-1}\right)$

$\boldsymbol{\Phi} \mathrm{MeOH}(\mathbf{J G 4 5})=0.18 \pm 0.02 / / \boldsymbol{\Phi} \mathrm{H}_{2} \mathrm{O}(\mathbf{J G 4 5})=0.09 \pm 0.02$.

$\Phi \mathrm{MeOH}(\mathbf{J G 4 5}+\mathbf{H g}(\mathbf{I I}))=0.15 \pm 0.02 / / \mathbf{\Phi} \mathrm{H}_{2} \mathrm{O}(\mathbf{J G 4 5}+\mathbf{H g}(\mathbf{I I}))=0.11 \pm 0.02$.

It was sensitive to $\mathrm{Hg}(\mathrm{II})$ and $\mathrm{MeHg}(\mathrm{II})$, dependent on the media.

It provided selective detection of $\mathrm{MeHg}(\mathrm{II})$ over $\mathrm{Hg}(\mathrm{II})$ in $\mathrm{HEK}$ cells.

JG32 (polymer soluble in water) (1\% probe) $0.012 \mathrm{~g} / \mathrm{L}$ water solution

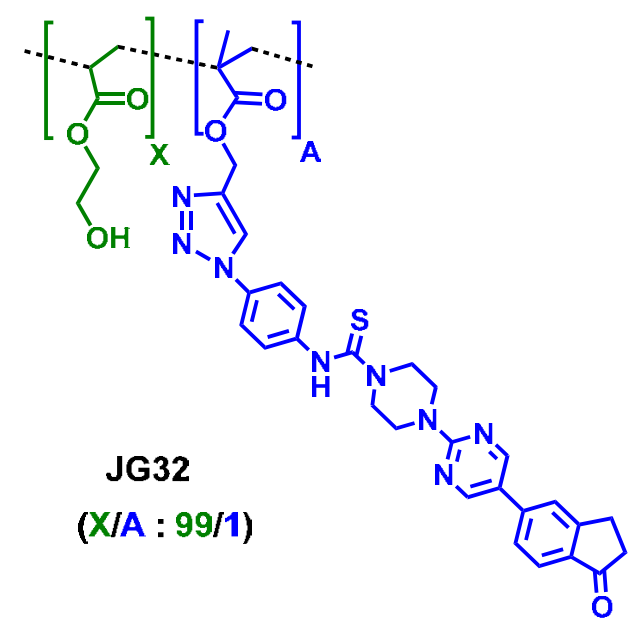

Ф $\mathrm{H}_{2} \mathrm{O}(\mathbf{J G 3 2})=0.17 \pm 0.02$

\section{${\underline{\mathrm{Hg}}\left(\mathrm{ClO}_{4}\right)_{2}}_{2}$}

Ф $\mathrm{H}_{2} \mathrm{O}(\mathbf{J G 3 2}+\mathbf{H g}(\mathbf{I I}))=0.42 \pm 0.02 \quad / / \quad$ LOD Hg(II) $=22 \mu \mathrm{M}$ or 4.4 ppm.

Time dependent results:

o The samples reached a maximum fluorescence / that did not decrease with time (at least within 100 minutes).

o A concentration of $\mathrm{Hg}$ (II) $0.1 \mathrm{mM}$ needed 50 minutes until maximum value of fluorescence was reached.

o To obtain a reliable LOD, the amount of time necessary for measurements was 20 minutes.

The fluorescence started to increase when the concentration was higher than $2 \mu \mathrm{M}$, and this process was independent of time. 


\section{$\underline{\mathrm{MeHgCl}}$}

LOD MeHg(II) $=39 \mu \mathrm{M}$ or $7.5 \mathrm{ppm}$.

Time dependent results:

o The fluorescence increased faster than with $\mathrm{Hg}(\mathrm{II})$, within the first 5 minutes.

o The final increase in fluorescence was lower than with $\mathrm{Hg}(\mathrm{II})$.

o 40 minutes was enough to have a good reproducibility of the LOD.

The fluorescence started to increase when the concentration was higher than $5 \mu \mathrm{M}$, and this process was not time-dependent.

\section{JG25 (Solid polymer with water affinity) (5\%probe)}

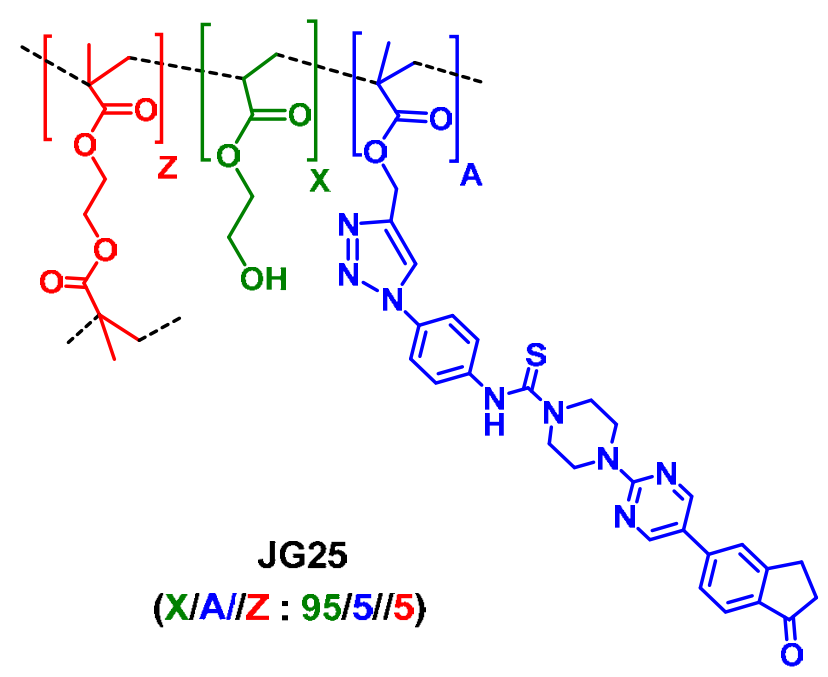

$\underline{\mathrm{Hg}\left(\mathrm{ClO}_{4}\right)_{2}}$

$\operatorname{LOD~} \mathrm{Hg}(\mathrm{II})=6.6 \mu \mathrm{M}$ or $1.3 \mathrm{ppm}$. (water).

Time dependent results:

o The samples reached a saturation point, and it did not decrease (at least in several hours).

o $\quad 0.1 \mathrm{mM}$ implied 8 hours until saturation.

o 15 minutes are the time necessary to have this LOD.

\section{$\underline{\mathrm{MeHgCl}}$}

LOD $\mathrm{MeHg}(\mathrm{II})=1.5 \mu \mathrm{M}$ or $0.3 \mathrm{ppm}$. (water)

Time dependent results: 
o The fluorescence increased with $\mathrm{MeHg}(\mathrm{II})$ and when the maximum was reached it started to decrease.

o Higher concentrations implied faster increase of the fluorescence, but once reached the maximum it decreased faster.

o The maximum of fluorescence was reached faster than with $\mathrm{Hg}(\mathrm{II}), 90$ minutes when in millimolar concentrations and less than 3 hours with $5 \mu \mathrm{M}$.

o The increase in fluorescence was lower than with $\mathrm{Hg}(\mathrm{II})$.

o 20 minutes was enough to have a good reproducibility of this LOD.

The $\mathrm{pH}$ changed the response to $\mathrm{Hg}(\mathrm{II})$ by increasing the emission intensity when the $\mathrm{pH}$ was lower, but the initial emission of the polymer was also higher.

The polymer worked for quantitative detection of mercury cationic species from fish extracts. 


\section{RESUMEN DEL CAPÍTULO}

El objetivo de este capítulo consistía en la modificación y mejora de sondas fluorescentes para la detección mercurio (II). Para ello se partió de los resultados de investigación anteriores en los cuales se habían elaborado un conjunto de sondas derivadas de la 5-bromoindanona que permitían la especiación entre $\mathrm{Hg}$ (II) y $\mathrm{MeHg}$ (II) mediante fluorescencia en medios orgánico-acuosos.

A partir de estos resultados, se trabajó en la síntesis de derivados solubles en agua que permitieran la detección de estos cationes. En primera instancia se modificó la sonda con cadenas de PEG. Esto permitió la solubilidad en medios $100 \%$ acuosos y además su utilización en medios celulares con buenos resultados, consiguiendo la detección selectiva en células $\mathrm{HEK}$ de $\mathrm{MeHg}$ (II) frente a $\mathrm{Hg}$ (II).

Posteriormente se modificaron matrices poliméricas con afinidad por el agua con similares objetivos. Por este procedimiento se creó un polímero soluble en agua y sensible a $\mathrm{Hg}$ (II) y $\mathrm{MeHg}$ (II) y un polímero en forma de film con afinidad por el agua para la detección en un material.

El material polimérico se utilizó en agua para la detección de mercurio (II) en matrices de pescados con resultados satisfactorios. Gracias a esto se elaboró una correlación entre los resultados de ICP masas de análisis de mercurio (II) y los resultados de aumento de fluorescencia del polímero; pudiendo llevar a cabo una detección semicuantitativa por fluorescencia y cuantitativa por comparación con resultados de ICP-masas. 



\section{CHAPTER 2}

PERYLENEMONOIMIDES.

\section{INTRODUCTION AND GENERAL \\ PROPERTIES}

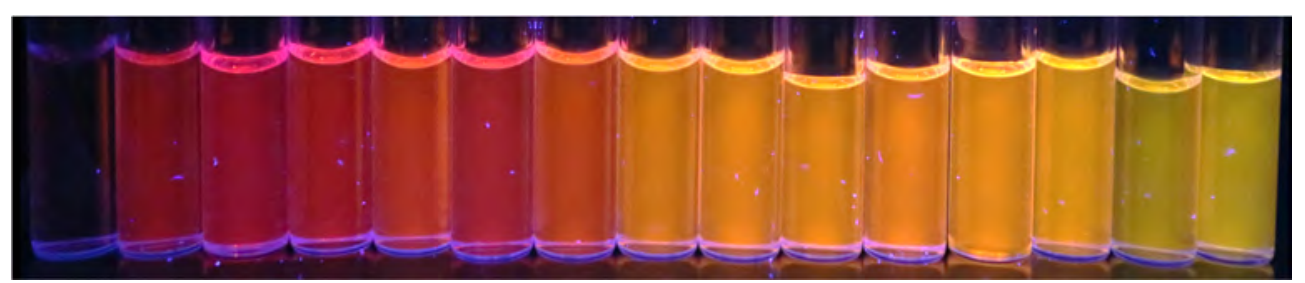

\section{ABSTRACT}

The aim of this chapter is to introduce the synthesis and properties of perylene derivatives. It starts by the reasons to choose perylenemonoimides as fluorescent backbones, followed by an explanation of why and how to use them in the development of new sensors. Finally, some of their potential applications, for detection and as biological markers, are introduced. 



\section{INTRODUCTION. PERYLENE DERIVATIVES, STRUCTURE AND PROPERTIES}

The word perylene comes from rylene. Rylenes are a family of dyes based on naftalene groups connected via peri-positions (Figure 4). As showed in Figure 1, the general structure of these compounds depends on the number of naftalene groups, when $n=0$ the dye is called perylene, $n=1$ terrylene and $\mathrm{n}=2$ quaterrylene. Being these 3 derivatives considered as the best options for organic synthesis.

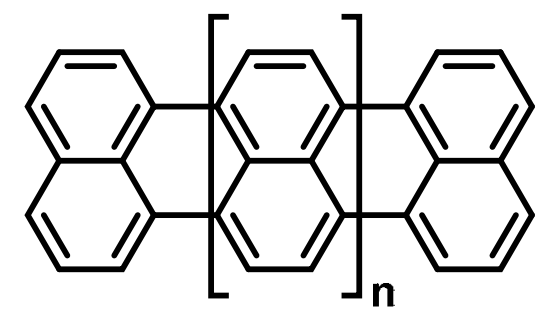

Figure 1. Rylene structure scheme.

Perylene derivatives are the most studied rylene dyes because of their characteristics. The highly conjugated structure gives great photoelectronic properties and diminishes the drawback of low solubility, a common issue when $n>1$. Perylene derivatives have been used in a wide range of applications within supramolecular chemistry, ${ }_{1}^{1}$ organic electronics ${ }^{2}$ or the development of chemical sensors. $^{3}$

Firstly, in regard to chemical sensors and the use of perylenes as colorants, it is important to distinguish between pigments and dyes. They are called pigments when their physical and chemical properties depend highly on aggregation; a fact directly associated to the way of synthesis, the presence of different solvents and temperature. In contrast, dyes are more independent from their environment; usually due to the inability to interact between them. These characteristics become of upmost importance in the case of perylene derivatives, that may behave as pigments or dyes depending on the structure of each specific derivative.

The starting materials when working with perylene derivatives are two, being both commercially available.

- Perylene, a yellow powder with strong blue fluorescence.

- Perylene dianhydride (PDA), the synthesis of which is standardized nowadays (Figure 2). In short, since the development by Kardos in 1912 from acenaphtene; ${ }^{4}$ the synthesis consisted of 4 steps to obtain the bisanhydride, which is deeply studied in literature. Furthermore, it is usually finished with an imidization process.

\footnotetext{
${ }^{1}$ F. Würthner, Chem. Commun. 2004, 14, 1564-1579.

${ }^{2}$ C. Huang, S. Barlow and S. R. Marder, J. Org. Chem. 2011, 76, 2386-2407.

${ }^{3}$ X. Zhang, S. Rehm, M. M. Safont-Sempere, F. Würthner, Nat. Chem. 2009, 1, 623-629.

${ }^{4}$ M. Kardos, D.R.P. 276357, 1913.
} 


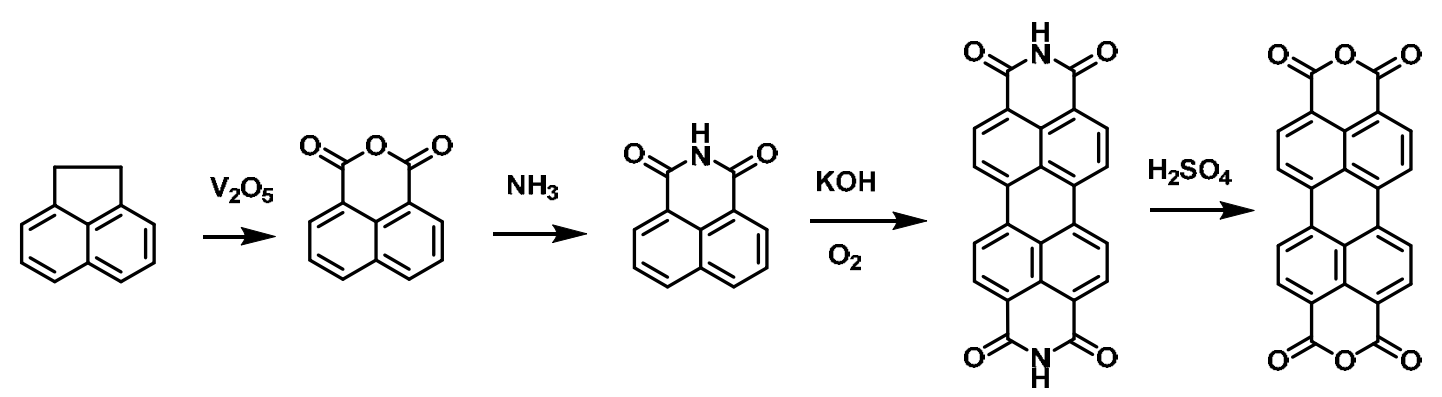

Figure 2. PDA synthetic scheme.

Between perylene and PDA, the second one has been the most interesting starting material for a variety of applications, due to its properties. The modification of the anhydride groups with amines and the substitution in peri, bay and ortho positions gives them a wide range of possibilities to modulate aggregation and/or electronical properties. Therefore, different substitution leads to changes in characteristics related with each other; such as colour, fluorescence or solubility. As a consequence, there is a huge quantity of possible variations and, due to the interesting applications, plenty of literature about the topic.

Starting from PDA, the most common way to proceed has been the introduction of imide groups, ${ }^{5}$ within the peri positions of the PDA. This reaction allows to alter the solubility/aggregation, minimizing changes in electronical properties. Concurrently, there are different possible perylene imide derivatives (PIs), depending on the substituents in the core. There are two positions susceptible to have the imide group, which is used as a method to classify PIs, Figure 3.

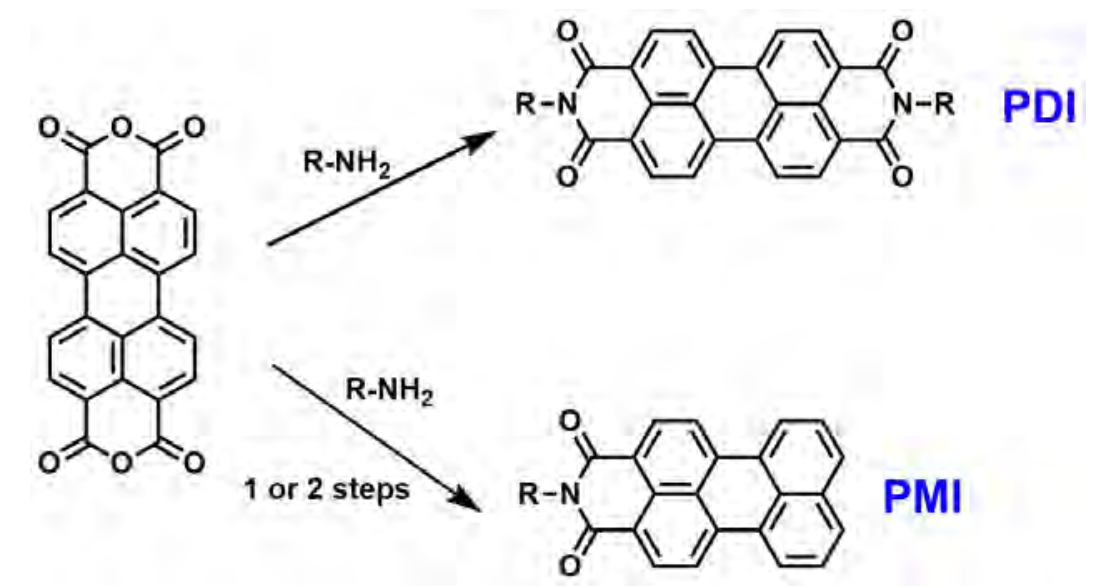

Figure 3. The two most common perylene imide derivatives (PIs): perylenediimide (PDI) and perylenemonoimide (PMI).

\subsection{Perylene imides (PIs) synthesis}

Physical and chemical properties of PIs rely on substitution. Then, the first step when using PIs is always to adapt the system to the objective to be fulfilled, which leads to introducing groups that are most likely to achieve particular goals. From the many variations, the usual positions to modify are divided into several groups (Figure 4):

${ }^{5}$ a) T. Maki, H. Hashimoto, J. Chem. Soc. Jap., 1951, 54, 544. b) Y. Nagao, Prog. Org. Coat., 1997, 31, 43-49. 

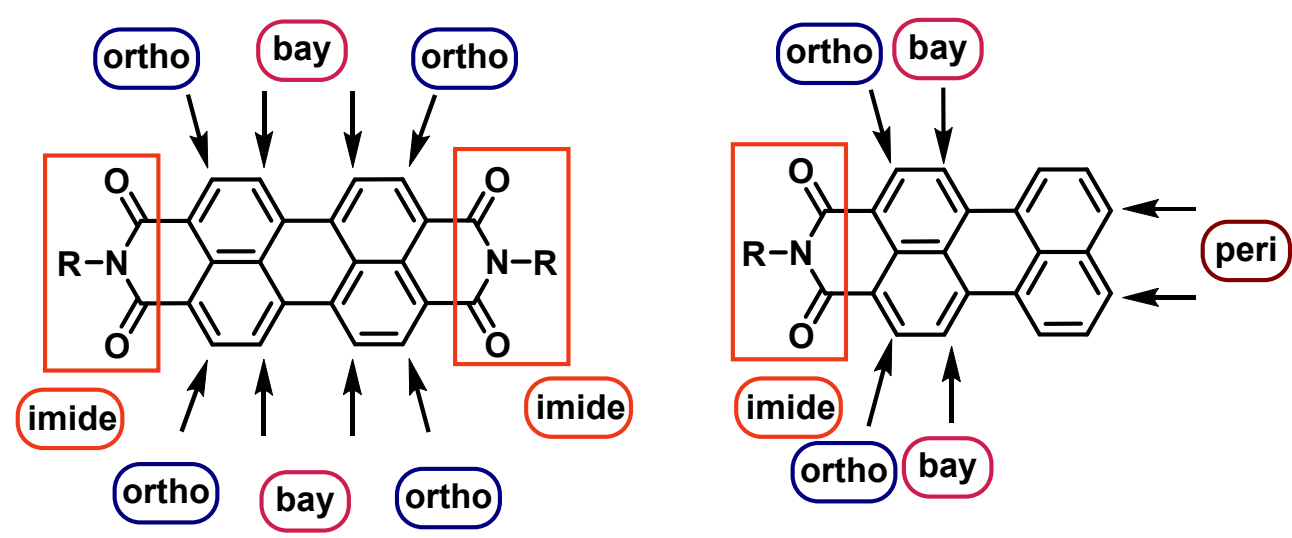

Figure 4. Possible substituted sensitive positions in PDIs and PMIs.

In this regard, there are several routes to functionalized PDIs and PMIs, the most common and remarkable are summarized in the Figures 5 and 6, respectively:

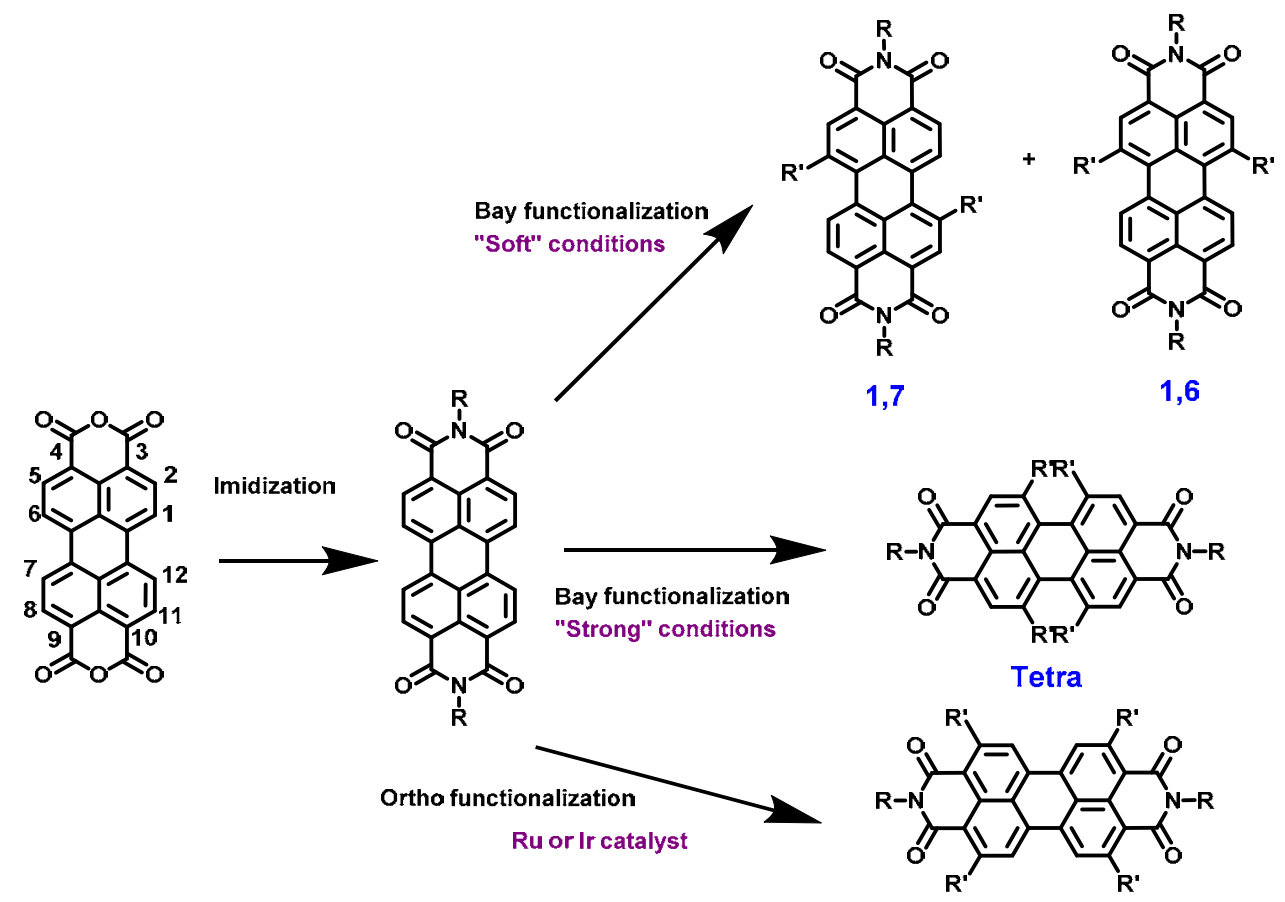

Figure 5. Some common synthetic routes for functionalization of PDIs.

Briefly summarized, PDIs are functionalized through several routes:

- Imide modification: The groups in the imide positions are not directly conjugated to the perylene core. Then, its modification alters solubility but does not affect the electronical properties to a large extent. However, it is possible to develop fluorogenic materials by introducing recognition units in those positions, by changing the fluorescence quantum yield and giving them a potential use as PET sensors. ${ }^{6}$

- Bay functionalization: Being the most common, it is directly related with their electronic properties. There are two main products, the bis-functionalization $(1,6$ and 1,7) and the tetrafunctionalization.

\footnotetext{
${ }^{6}$ I. Georgiev, A. R. Sakr, V. B. Bojinov, Dyes Pigm. 2011, 91, 332-339.
} 
- Ortho functionalization: ${ }^{7}$ The most "novel" procedure to modify PDIs, it may alter the electronical properties too, but distinctly to bay functionalization. It is performed by using iridium or ruthenium catalysts.

The scheme showed in Figure 5 gives an idea of the possibilities, although not the specific order of the reactions. Bay functionalization may start by bromination of the PDA and the imide groups may be changed after introducing different substituents in bay position. In addition, modification of groups alters reactivity and yields, making possible the modulation and optimization of a variety of PI derivatives.

PMIs functionalization ${ }^{8}$ is, in many occasions, similar to PDIs as showed in Figure 6.
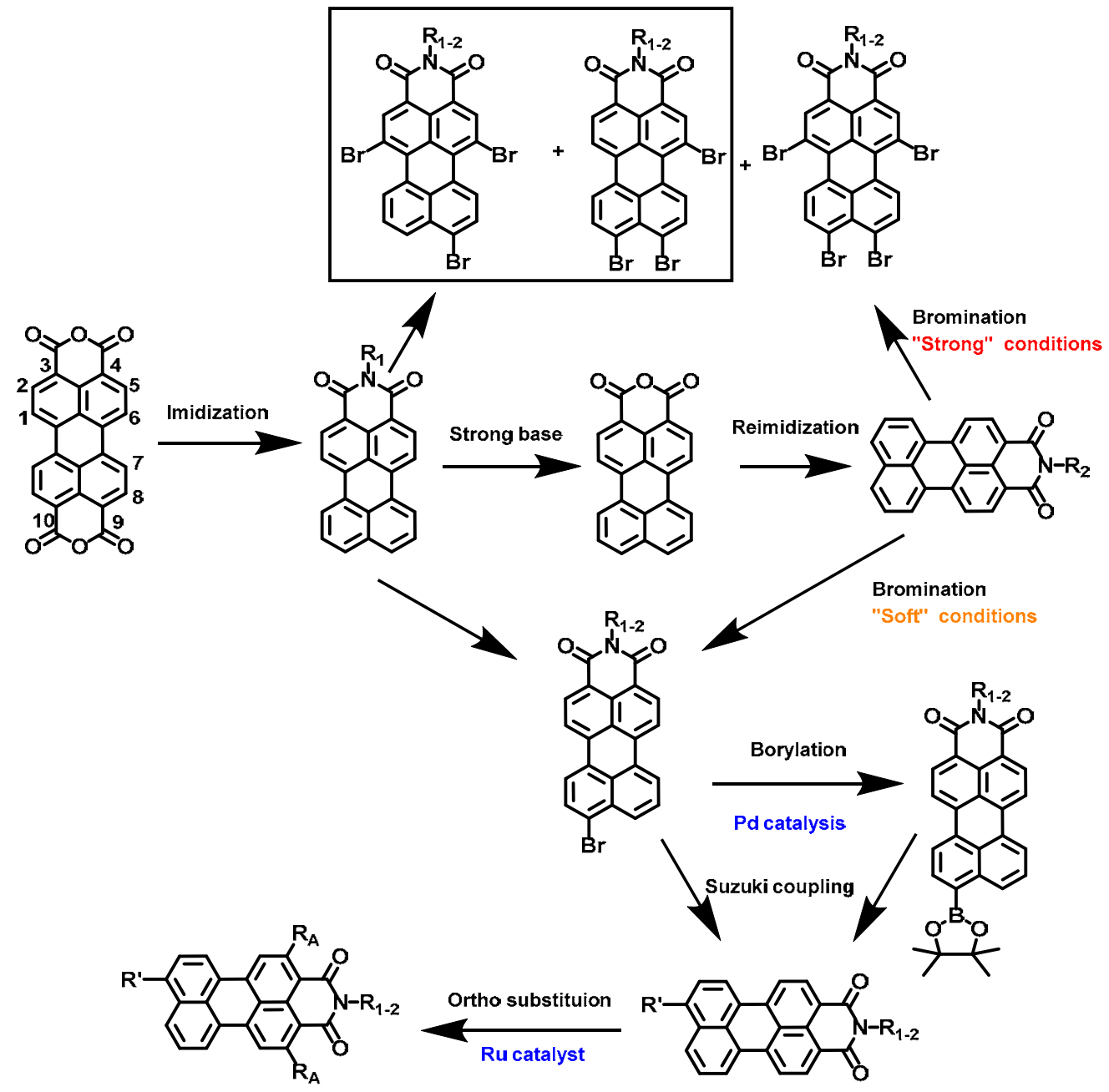

Figure 6. Some common synthetic routes for PMIs functionalization.

Being an extensive part of the work developed during the thesis, it is widely explained in section 3.3. in which the procedures from literature were optimized for the objectives of the research group. In general, the process starts with the imidization-decarbonylation of the PDA, in one or several steps. Then, depending on the purpose, it is substituted in peri, bay and/or ortho positions. In addition,

\footnotetext{
7 a) X. Li, H. Wang, J. A. Schneider, Z. Wei, W.-Y. Lai, W. Huang, F. Wudl, Y. Zheng, J. Mater. Chem. C. 2017, 5, 2781-2785. b) J. E. Bullock, M. T. Vagnini, C. Ramanan, D. T. Co, T. M. Wilson, J. W. Dicke, T. J. Marks, M. R. Wasielewski, J. Phys. Chem. B 2010, 114, 1794-1802.

${ }^{8}$ Y. Hu, S. Chen, L. Zhang, Y. Zhang, Z. Yuan, X. Zhao, Y. Chen, J. Org. Chem. 2017, 82, 5926-5931.
} 
it is possible to change the imide group, provided that the other substituents of the PMI are stable to the process.

- Imidization: starting from PDA there are two methods to obtain PMIs, by one-pot decarbonylation-imidization, ${ }^{9}$ or divided into three steps. ${ }^{10}$ First, the synthesis of the PDI; then, formation of the perylene monoanhydride-monoimide and, finally, decarbonylation of the monoanhydride.

- Peri substitution: once the PMI substrate is obtained, the most common step is monobromination in peri. The conditions are "soft" so as to obtain the mono-substituted product selectively.

- Bay + peri substitution: The process is usually performed starting by a bromination under "strong" conditions (high excess of bromine and high temperatures), which gives a mixture of two tri-brominated products with a low quantity of the tetra-brominated.

- Reimidization: When working with PMIs, different imide substituents provide several properties, so there could be many reasons to change them in later steps of the synthesis. Among the multiple possibilities, some examples are the groups that could not resist the conditions for the direct formation of the monoimide, from PDA (such as boc-protected amines) or some amines that are not bulky enough to make PMIs soluble in common solvents. For these or other reasons, it might be of interest changing the imide group. The process undergoes by the monoanhydride formation ${ }^{11}$ followed by the introduction of a primary amine.

- Ortho functionalization: It is the same procedure than when working with PDIs, which involves a Ruthenium catalyst in the process.

\subsection{Modulating PIs properties, the stacking of PIs}

Some interesting features of perylene imide derivatives (PIs) are the electronical properties, which led to exceptionally high fluorescence quantum yield, close to $100 \%$ in many occasions. Furthermore, the emission and absorbance are in the range of visible-NIR, and it is easily tuneable with the variation of substituents, ${ }^{12}$ increasing the applicability.

So as to fully understand the properties and applications of PIs, it is necessary to explain to some extent one of their most important sources, their ability to perform homo-stacking between molecules. The aggregates of PIs have been studied for decades, and yet, nowadays it is difficult to predict it before experimental testing. It is important not only because of the relation aggregationsolubility, but for the optoelectronical properties too, affecting directly the wavelength of absorbanceemission (energy levels) and the fluorescence quantum yields (more aggregation implies more possible non-radiative routes for relaxation).

The search on literature about the stacking of perylene derivatives usually leads to a massive amount of information because of the dependence on multiple factors. In this regard, Figure 7, which is based

\footnotetext{
${ }^{9}$ a) L. Feiler, H. Langhals, K. Polborn. Eur. J. Chem. 1995, 7, 1229-1244. b) L. Pleux, A. L. Smeigh, E. Gibson, Y. Pellegrin, E. Blart, G. Boschloo, A. Hagfeldt, L. Hammarström, F. Odobel, Energy Environ. Sci., 2011, 4, 2075-2084.

${ }^{10}$ Y. Geerts, H. Quante, H. Platz, R. Mahrt, M. Hopmeier, A. Böhm, K. Müllen; J. Mater. Chem. 1998, 8, $2357-$ 2369.

${ }^{11}$ T. Dentani, K. Funabiki, J.-Y Jin, T. Yoshida, H. Minoura, M. Matsui, Dyes and Pigments. 2007, 72, 303-307.

${ }^{12} \mathrm{C}$. Li, H. Wonneberger, Adv. Mater. 2012, 24, 613-636.
} 
on a review by Würthner et $\mathrm{al}^{13}{ }^{13}$ shows, schematically, the consequences of different ways of aggregation, and halfway situations. In addition, it is not only valid for perylene derivatives but for every molecule with capability to perform $\pi-\pi$ stacking.

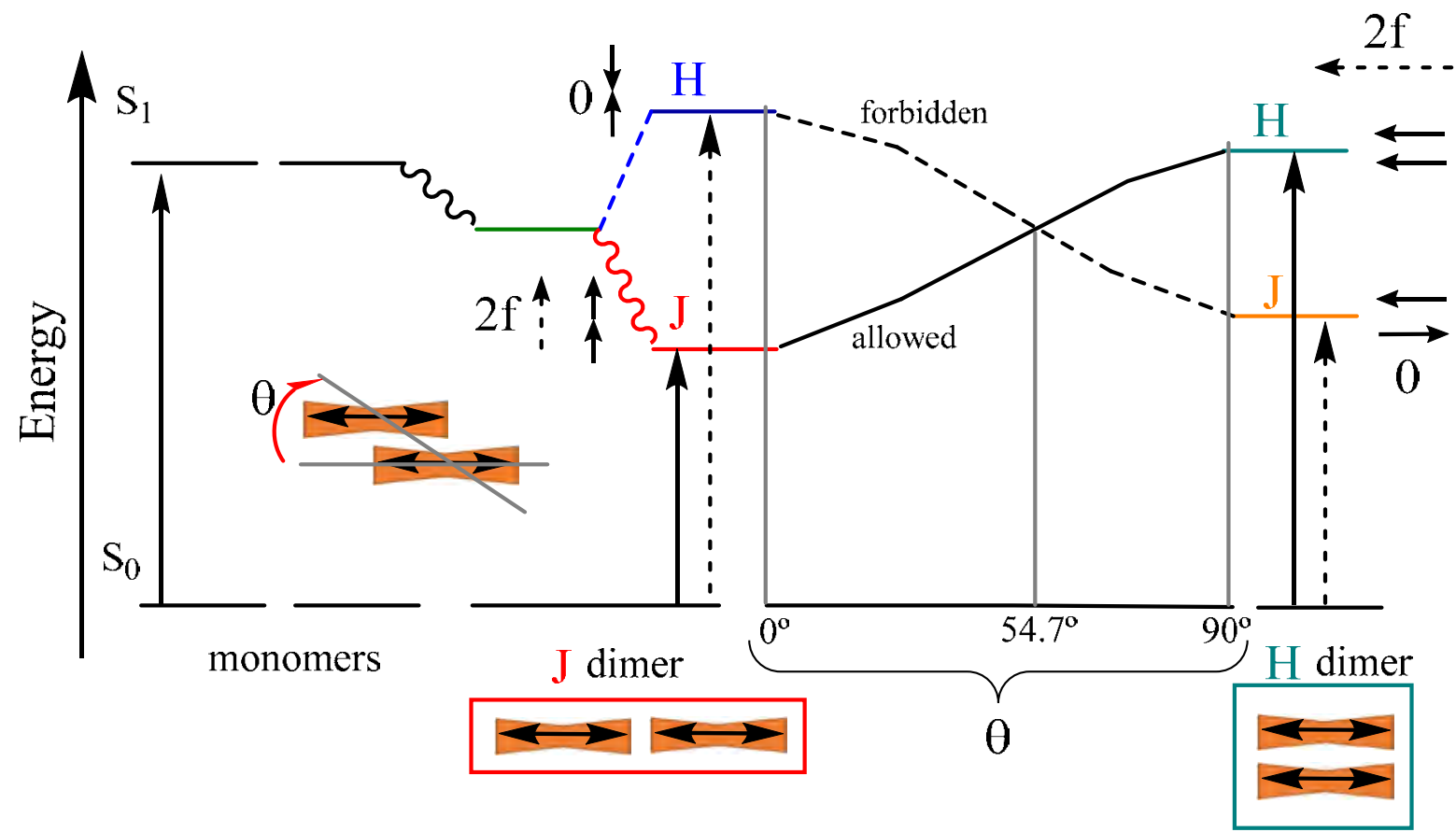

Figure 7. Energy diagram for 2D formation of dimer and the effect over the energy levels.

To simplify the situation, this section is explained from the point of view of perylene derivatives. Figure 7 considers the effect of having a monomer, and the different interaction between 2 molecules (dimers) in a 2D model. The relation aggregation-luminescent properties might be explained from the energy diagram in Figure 7:

- Monomers: The fluorescence is the one associated to the perylene derivatives.

- J-aggregates $\left(\boldsymbol{\theta}=\mathbf{0}^{\circ}\right)$ : when the perylene molecules are aggregated in the same plane. As a consequence, it creates a more accessible excited energy level for the electrons. It implies less energy for the fluorescent emission, giving a batochromic shift.

- H-aggregates $\left(\boldsymbol{\theta}=\mathbf{9 0}^{\circ}\right)$ : when perylene molecules are aggregated on top of each other. The more excited energy level for the electrons becomes less accessible, so the wavelength of emission increases, giving a hypsochromic shift.

Apart from what is shown, there are also many possible and more complex variations. Some of these properties are briefly summarized in this section, so as to understand the complexity of the topic. Nevertheless, most properties and interactions are widely explained in specific sources, focusing the topic on the situation and the molecule-system under study. This interpretation is based on Kasha's model, which is a qualitative explanation from the point of view of the interaction of transition dipole moments of chromophores, with respect to the spatial arrangement when the photoexcitation takes place. Halfway situations also exist depending on the angle $\theta$, in which it is aggregated. Situations between J-H aggregates are not only possible but the most common situation. In particular, it is possible to have very similar wavelength of emission, comparing with the monomer, if the right angle between molecules is achieved $\left(54.7^{\circ}\right)$.

${ }^{13}$ F. Würthner, C. R. Saha-Möller, B. Fimmel, S. Ogi, P. Leowanawat, D. Schmidt; Chem. Rev. 2016, 116, $962-1052$. 
To explain why the change in behaviour of perylene derivatives occurs when stacking, two opposite situations may be found (the examples were taken from the own research of our group):

- Substituents with low polarity (for example, aliphatic chains): the polarity of the core is higher than the polarity of the substituents. In consequence, when the solvent is highly polar (for instance, DMSO), the core would tend to be facing the solvent (J-aggregate), giving a batochromic shift. However, if the solvent is less polar (methylcyclohexane for example), the cores will tend to aggregate and the substituents will be facing the solvent, giving a hypsochromic shift. See example in Figure 8.

Aliphatic substituent
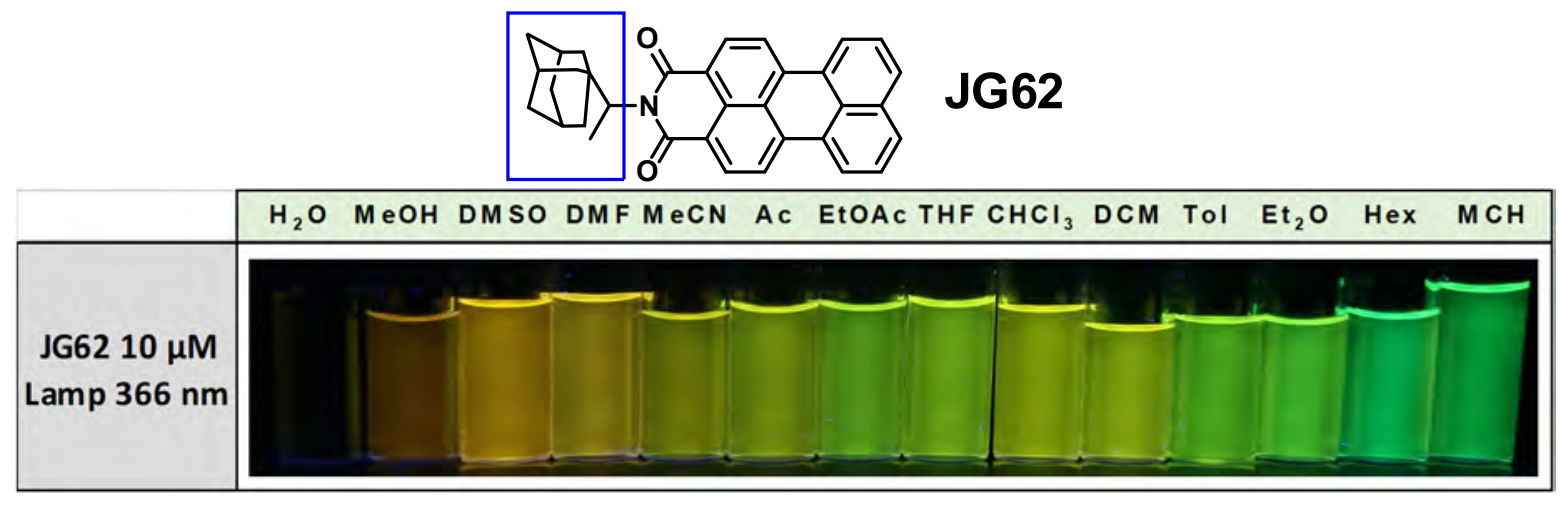

Figure 8. PMI JG62 solvatochromism in different solvents.

- Polar substituents (for example, hydroxyl or amino groups): when the polarity of the substituents is high enough, the behaviour may be the opposite, having H-aggregates when the solvent is more polar and J-aggregates in the opposite case. See example in Figure 9.

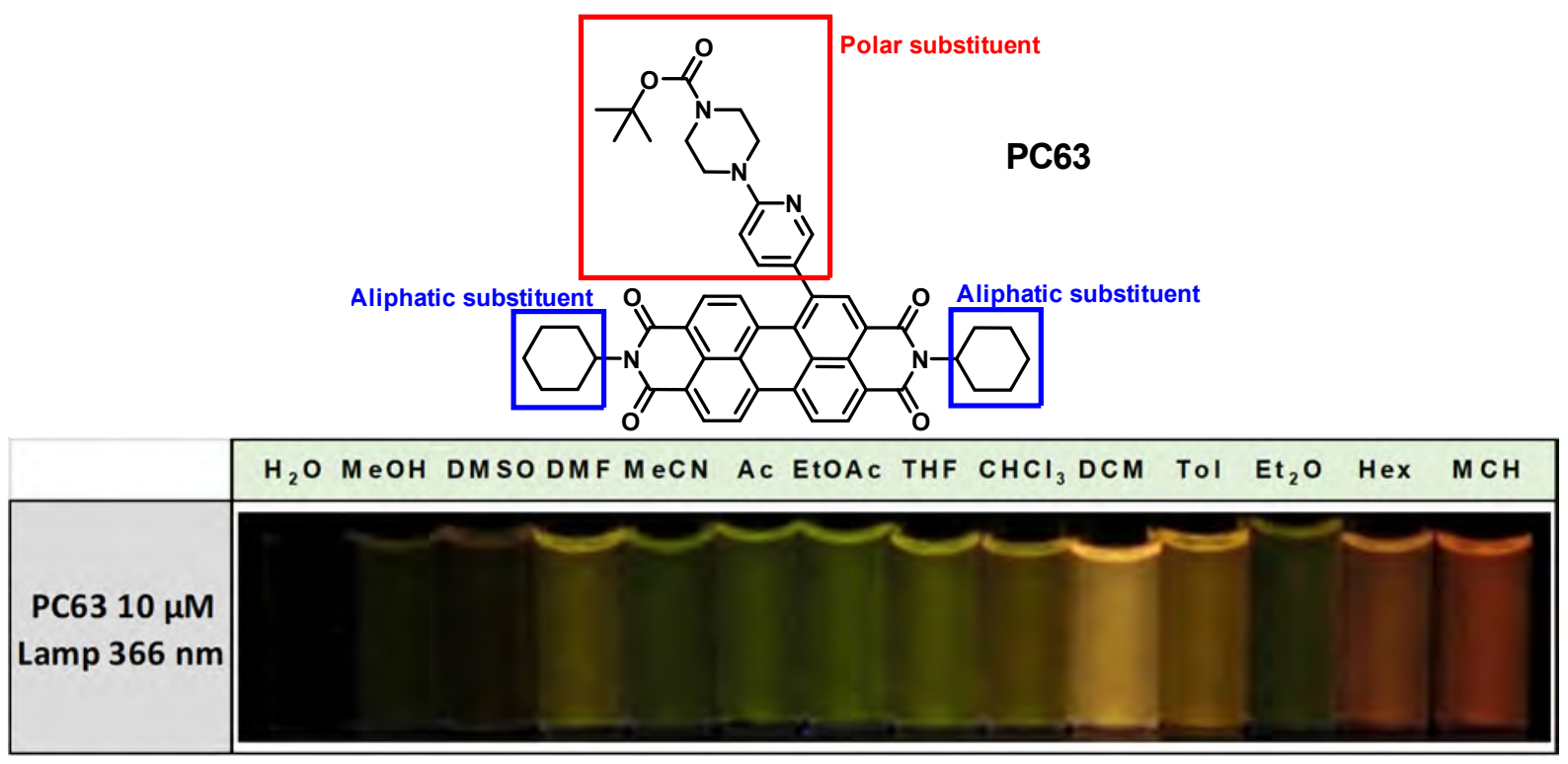

Figure 9. PDI PC63 solvatochromism in different solvents. ${ }^{14}$

Apart from this, it is important to remark that it is not only a matter of polarity of the coresubstituents. The position, conjugation and bulkiness of the substituents may have even a more important role; considering that, in reality, aggregation occurs in a $3 \mathrm{D}$ distribution. So as to fulfil what

${ }^{14}$ P. Calvo-Gredilla, J. García-Calvo, J. V. Cuevas, T. Torroba, J.-L. Pablos, F. C. García, J.-M. García, N. ZinkLorre, E. Font-Sanchis, Á. Sastre-Santos, F. Fernández-Lázaro, Chem. Eur. J. 2017, 23, 13973-13979. 
was explained above, it would be necessary to explore the possibility of stacking in a different position; having very polar substituents does not mean that it will tend to give $\mathrm{H}$-aggregates in polar solvents (Figure 10).

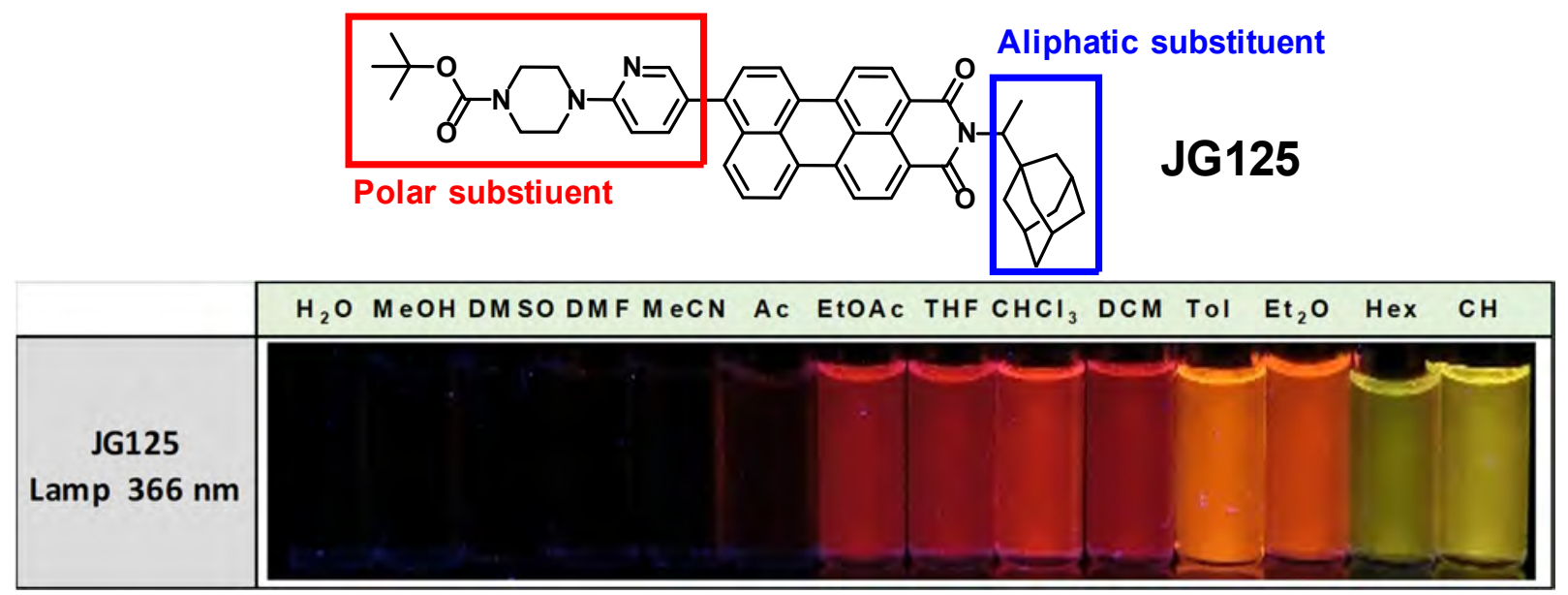

Figure 10. PMI JG125 $(10 \mu \mathrm{M})$ solvatochromism in different solvents

Additionally, talking about the aggregation, the size of the substituents introduced must be taken into account. Having bulky substituents reduces stacking and gives the products properties of a dye instead of a pigment. What is more, the presence of bulky substituents in the imide position increases dramatically the solubility of the PIs which, among other factors, leads to enhanced reactivity. Likewise, if it is not soluble enough, it might be impossible to perform new synthetic processes.

The introduction of substituents in other positions, besides the imide group, also alters the aggregation of the PIs but, in contrast, it changes deeply the optical properties. For PDIs, the introduction of different substituents in bay positions is the most common way to proceed and for PMIs, is the peri substitution. This technique allows to modulate, apart from the electronical properties, the possible ways of aggregation between them, which is directly related with solubility, absorbance and fluorescence. Moreover, there is a noteworthy distinction between PMI and PDI; although PMIs have more and easier synthetical variations, the lower symmetry may hinder getting a straightforward prediction-explanation for their stacking properties.

Besides, the great relation aggregation-concentration may be analysed not only by changes in luminescence. For example, applied to PMIs, the effect of having different concentrations was observed for some synthetized derivatives (JG2L from Chapter 3B), getting variations in the ${ }^{1} \mathrm{H}$ NMR signals depending on how concentrated is the sample, Figure 11. 


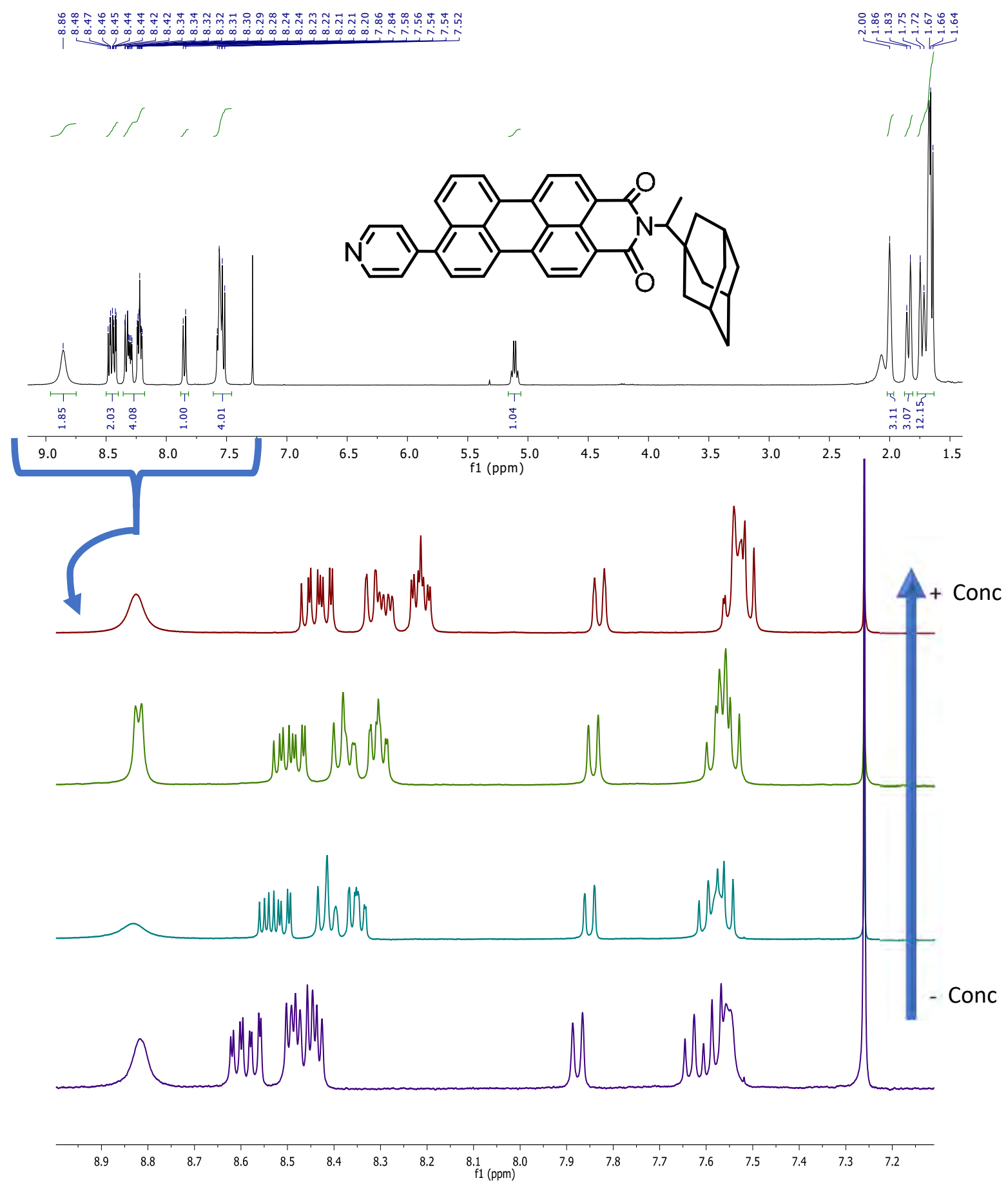

Figure 11. PMI JG2L, ${ }^{1} \mathrm{H}$ NMR in $\mathrm{CDCl}_{3}$, effect of dilution between $1-0.1 \mathrm{mM}$.

In Figure 11, the dependence on concentration of JG2L illustrates the aggregation properties. This reliance is not always so noticeable, but it evidences the importance of the substitution. In addition, increasing concentration leads to a decrease in fluorescence emission. The studies from Chapter 2 and 3 of this thesis are focused in using PMIs as chemical sensors, ligands and/or biological markers. Hence, the explanation of the stacking is usually complex, although there is plenty of literature applications related to the stacking. ${ }^{15}$

${ }^{15}$ a) L. Huang, V. J. Catalano, S.-W. Tam-Chang, Chem. Commun. 2007, 2016-2018. b) L. Huang, S.- W. TamChang, W. Seo, K. Rove, Adv. Mater. 2007, 19, 4149-4152. 


\subsection{Applications of PI derivatives}

From the point of view of research - future industrial applications, PIs are characterized by their outstanding electronical properties, having great tuneability for their fluorescence emission, photoinduced energy transfer or electron transfer processes. All these characteristics have many commercial and optoelectronical purposes, such as colorants, laser dyes, optical power limiters, fluorescent solar light collectors, xerographic photoreceptors, optical sensors, probes for biomacromolecules and, more recently, to singlet fission and artificial photosynthesis. ${ }^{16}$

PIs used as pigments are one of their most ancient implementations, due to their high absorptivity, absorption-emission tuneability properties (their colour depends on the aggregation-crystallization) and lack of metals. This characteristic is directly applied for the elaboration of paints and lackers. ${ }^{17}$

For light harvesting applications there is plenty of literature. ${ }^{18}$ The aim of the research is usually focused on improving the properties of existing systems, by combination of dyes with high absorption and good electronical properties. For instance, PIs with other more classic systems such as porphyrins. ${ }^{19}$ The most common objective of these PIs is using them for solar cells, because of their high absorption and capabilities as charge carriers, competing with fullerenes. Briefly commenting this topic, fullerenes have been more used until now because of their symmetry, which meant easier control over the solid packing structure. Nevertheless, the techniques have improved a lot and, nowadays, PIs may overcome some fullerene issues. Particularly, although most of the literature is about PDIs, PMIs have been also used as sensitizers in hybrid solar cells, ${ }^{20}$ demonstrating their high capabilities.

For biomedical applications there is also plenty of literature about cytotoxicity, interaction with different DNA strands and, specially, in biomedical imaging. ${ }^{21}$

From the point of view of the work of this thesis, PMIs were used as dyes in optical sensors/probes (introduced in Section 1.4) and, up to some extent and thank to some collaborations, for cellular imaging and DNA interaction, which summarized at the end of this chapter (Section 5).

\footnotetext{
${ }^{16}$ H.-C. Chen, C.-P. Hsu, J. N. H. Reek, R. M. Williams, A. M. Brouwer, ChemSusChem. 2015, 8, 3639.

${ }^{17}$ E. B. Faulkner, R. J. Schwartz, High Performance Pigments, Wiley-VCH, Weinheim, 2009.

${ }^{18}$ L. L. Pleux, A. L. Smeigh, E. Gibson, Y. Pellegrin, E. Blart, G. Boschloo, A. Hagfeldt, L. Hammarström, F. Odobel; Energy Environ. Sci., 2011, 4, 2075. b) A. Morandeira, J. Fortage, T Edvinsson, L. L. Pleux, E. Blart, G. Boschloo, A. Hagfeldt, L. Hammarström, F. Odobel; J. Phys. Chem. C. 2008, 112, 1721-1728. c) K. Tomizaki, P. Thamyongkit, R. S. Loewe, J. S. Lindsey, Tetrahedron 2003, 59, 1191-1207.

${ }^{19}$ M. A. Miller, R. K. Lammi, S. Prathapan, D. Holten, J. S. Lindsey; J. Org. Chem. 2000, 65, 6634-6649.

${ }^{20}$ C. Li, Z. Liu, J. Schöneboom, F. Eickemeyer, N. G. Pschirer, P. Erk, A. Herrmann, K. Müllen, J. Mater. Chem., 2009, 19, 5405-5415. b) J. Fortage, M. Séverac, C. Houarner-Rassin, Y. Pellegrin, E. Blart, F. Odobel, J. Photochem. Photobiol. A: Chem. 2008, 197, 156-169. c) J. Baffreau, L. Ordronneau, S. Leroy-Lhez, P. Hudhomme, J. Org. Chem. 2008, 73, 6142-6147.

${ }^{21}$ P. Shaoa, M. Bai, Chem. Commun. 2012, 48, 9498-9500.
} 


\subsection{PI derivatives as chemical sensors}

Literature about using PIs as sensors is wide and with many possible aims; starting from the more classic ion sensing, by introducing specific receptors as substituents. Usually, there is plenty of literature about the affinity of different ligands, that is taken as base to elaborate the synthesis, optimization and comparison with other pre-existing probes. In this regard, some systems are shown as examples for $\mathrm{Hg}^{2+}$ or $\mathrm{Fe}^{3+}$ detection.

$\mathbf{H g}^{2+}$ sensor: ${ }^{22}$ Fluorogenic perylene derivatives based on a PET mechanism are easy to develop by modifying the imide position PIs. As an example, the sensor developed by S. Malkondu and S. Erdemir (Figure 12 up) is soluble in water:DMF media giving a selective increase in fluorescence in yellow colour under $\mathrm{Hg}(\mathrm{II})$ presence.

$\mathrm{Fe}^{3+}$ sensor: ${ }^{23}$ In the same way than the previous $\mathrm{Hg}^{2+}$ sensor, the imide groups were substituted to develop selective fluorescent sensors. In this case, having triazole or several pyridine groups led to the selective detection of $\mathrm{Fe}^{3+}$ and/or $\mathrm{Ni}^{2+}$, also in DMF. (Figure 12 middle and down)

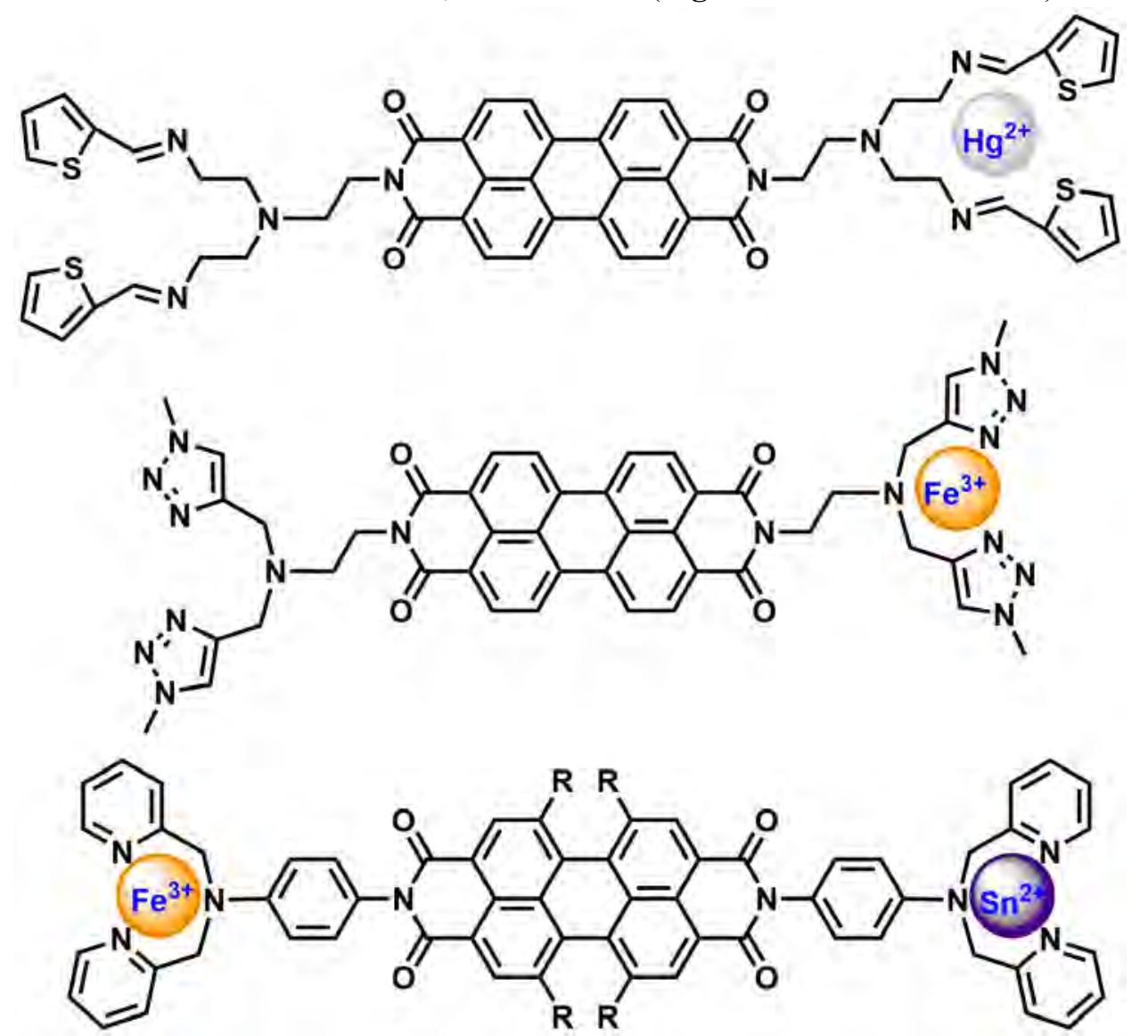

Figure 12. Synthetic probes sensitive to $\mathrm{Hg}^{2+}$ (up), $\mathrm{Fe}^{3+}$ (middle) and $\mathrm{Sn}^{2+}$ (down).

In case of PMIs, there are less literature using them as a chemical sensor backbone; probably due to their lower symmetry and more complicated synthesis. However, there are some backbones that have been extensively studied for multiple applications (Figure 13) without major changes.

\footnotetext{
22 S. Malkondu, S. Erdemir, Dyes Pigm. 2015, 113, 763-769.

${ }^{23}$ J. Lin, C. Zhu, X. Liu, B. Chen, Y. Zhang, J. Xue, J. Liu, Chin. J. Chem. 2014, 32, 1116-1120.
} 


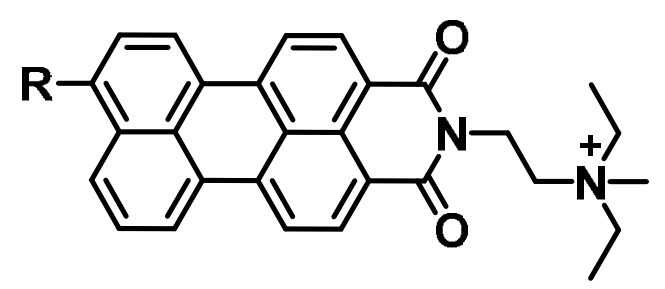

Figure 13. PMI with an ammonium group in the imide position, known as PIPER.

As it was stated, they have been used for multiple sensory purposes, going from $\mathrm{pH}$, temperature and solvent polarity detection to specific recognition of DNA.

Studying pH, temperature and solvent polarity with PMI derivatives. ${ }^{24}$ These properties are strictly related and using PMI derivatives to detect this kind of changes is quite straightforward and might be done with most PI derivatives, being the most important drawback its solubility.

PMI derivatives have been also used for specific recognition of DNA strands, ${ }^{25}$ such as Gquadruple ${ }^{26}$ or other biological targets of interest. They are based on the recognition in which amine groups were acting as PET quenchers; they are also used as $\mathrm{pH}$ sensors depending on the specifics of the molecules (see Figure 13 and literature about these derivatives).

${ }^{24}$ C. Kirmaier, H. Song, E. Yang, J. K. Schwartz, E. Hindin, J. R. Diers, R. S. Loewe, K. Tomizaki, F. Chevalier, L. Ramos, R. R. Birge, J. S. Lindsey, D. F. Bocian, D. Holten; J. Phys. Chem. B, 2010, 114, 14249-14264.

${ }^{25}$ L. Huang, S.-W. Tam-Chang; Chem. Commun., 2011, 47, 2291-2293.

${ }^{26}$ T. Taka, K. Joonlasak, L. Huang, T. R. Lee, S.-W. T. Chang, W. Tuntiwechapikul, Bioorg. Med. Chem. Lett., 2012, 22, 518-522. 


\section{OBJECTIVES OF THE CHAPTER}

Perylene derivatives have a wide field of applications. This chapter aims to introduce their strengths and weaknesses. First, the introduction has given an explanation about how they behave in different conditions, and how to modulate their properties. Additionally, due to the massive amount of possibilities when working with these derivatives; the first step was to decide which are the ones that fit better to the objectives. For the thesis, PMIs were chosen because of the different substitution possibilities and wide range of applications. Next, the synthesis was optimized (Section 3) so as to develop the starting materials. In this regard, the synthesis of brominated or boronated PMI derivatives allowed the binding with different recognition units. Finally, some general applications and properties are introduced.

The possibilities and properties are many depending on the different substituents. Therefore, the work is divided into several sections:

- General properties of the synthetized PMI derivatives are introduced in Section 4.1. Allowing a fast comparison between them.

- Section 4.2 aims to introduce a particular case of the use of a FRET system PMI-Bodipy, and the possibilities and advantages that could be reached in future research.

- The objective of Section 4.3 was to summarize different parameters related with absorbancefluorescence of the synthetized probes in the same solvent.

- Section 5 has the purpose of summarizing some properties of the synthetized PMI derivatives for biological applications, as cellular markers, cytotoxicity or DNA interaction. In order to introduce what results may be obtained for this kind of derivatives.

As a result, Section 4 is a comparison of the different products synthetized during the development of the thesis. Specific applications are discussed in Section 5 (some biological applications) and in the different parts of Chapter 3 (PMIs as sensors). 


\section{OPTIMIZED SYNTHESIS PROCEDURE FOR PERYLENEMONOIMIDES (PMIS)}

During the development of this thesis there have been some PMI derivatives that have been used as a starting material for the rest of the products. Because of that, the synthesis was optimized and adapted to subsequent needs. ${ }^{27}$

\subsection{Imidization of the PDA to Perylenemonoimide (PMI)}

Perylene-3,4-dicarboximide (PMIs) are usually synthesized from PDA and a bulky amine. As it was mentioned before, in Section 1.1., there are two methods; one of them is divided into three steps and the other is a one-pot reaction. Regarding that, the PDA is subjected to an imidization reaction with bulky amines $\left(\mathrm{R}_{2} \mathrm{C}-\mathrm{NH}_{2}\right.$ groups), during which, one of the two anhydride structures decarboxylates and the PMI is formed. The reaction conditions usually involve very high temperature and quinoline/imidazole as solvent, whereupon the thermally less stable imide-anhydride spontaneously loses the anhydride structure.

When the different sources are compared, the final yield is supposed to be a bit higher by performing the three-step synthesis (around $70 \%{ }^{10}$ against $50 \%{ }^{9 a}$ ) although the amount of solvent and time spent is also much higher. In any case, this is the first step when working with PMIs, and the reaction conditions are rarely detailed and; when it is done, usually, the reaction is not optimized.

As a consequence, the first step for producing it efficiently was the optimization of PMIs synthesis. Doing so, several bulky amine derivatives were tested so as to obtain PMIs in a quick, easy and highly efficient manner. Four of them are presented as example in Figure 14.
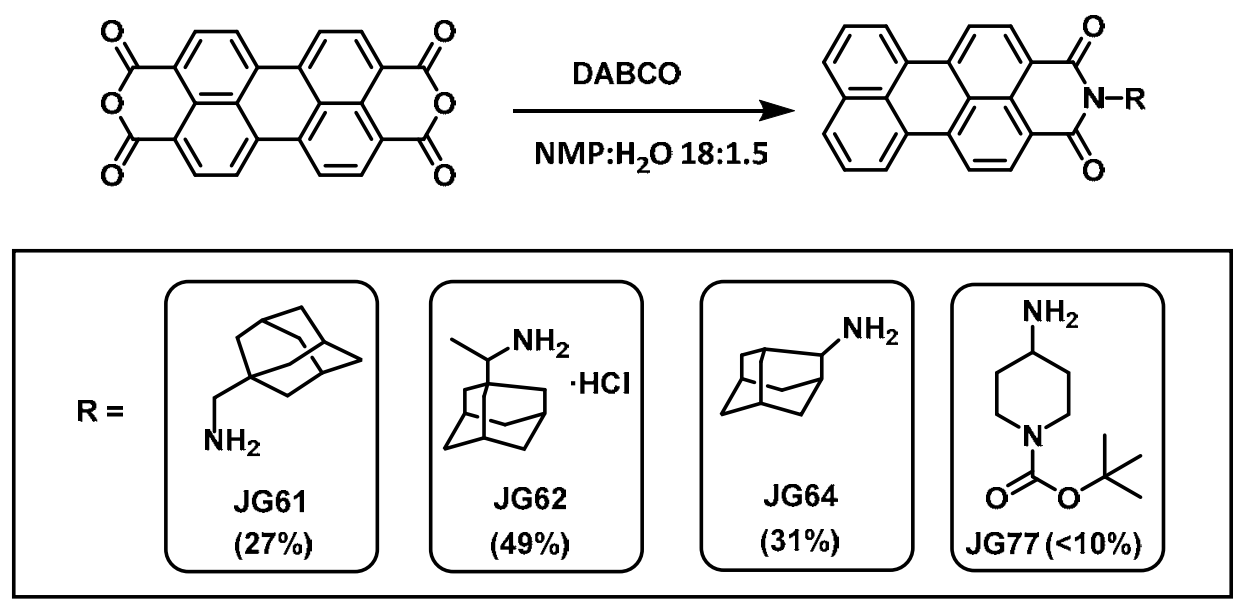

Figure 14. Scheme of a straightforward synthesis of PMIs from PDA.

From the reaction conditions it was concluded that, in order to obtain PMIs in one step and high yields, the primary amine must be chosen carefully, minimizing the number and quantity of byproducts. It is achieved by introducing bulky substituents, but:

\footnotetext{
${ }^{27}$ The specifics of each one of the performed reactions are detailed in Experimental Appendix 2.
} 
o If it is too bulky and close to the amine (JG64), the yield decreases because it does not react well and the conditions have to be more severe, leading to the formation of other products, prior to PMI.

o The boc protected groups (JG77) do not resist the conditions for imidizationdecarbonylation in one step, and the product becomes a mixture that would lead low yields and tedious purification processes.

o If the bulky substituent is not close enough to the primary amine the yield is lower, increasing the quantity of product totally decarboxylated and reducing the solubility of the final product (JG61).

Working with these different amine groups led to obtain the products in different yields. It was particularly interesting the yield that was achieved using the 1-adamantyl-ethylamine (JG62), being able to optimize it up to $49 \%$, once purified.

The specific conditions to get this yield were reached by using $250 \mathrm{mg}$ of PDA ( $0.64 \mathrm{mmol})$ each 10 $\mathrm{mL}$ of solvent (NMP: $\mathrm{H}_{2} \mathrm{O}$ 9:1); 1.1 equivalents of 1-adamantyl-ethylamine and 5 equivalents of DABCO. All the components were dissolved under sonication and then put to react at $190^{\circ} \mathrm{C}$ for 3 days. For purification of PMIs the method was adapted from literature. First, $1 \mathrm{M} \mathrm{HCl}$ solution $(25 \mathrm{ml}$ each $10 \mathrm{ml}$ of solvent) was poured into the flask and stirred for one hour. Then, the resulting mixture is filtered under reduced pressure. Finally, the solid was purified under column chromatography with DCM obtaining the product as a bright orange powder. It is also worth to remark that this reaction was moved up, working with the equivalent yields when starting with 250-3000 mg of PDA.

In literature, the most common PMI derivatives contain an ammonium group (as it was showed in Section 1.4), a 2,6-diisopropylphenyl substituent (or similar di-substituted phenyl groups) or dibranched alkyl amines (such as 1-pentylhexylamine), Figure 15. In contrast, the reported yields are variable depending on the source (usually between $30 \%-50 \%$ or less). The results may be compared by checking on literature having multiple examples of this kind of synthesis (see for example literature from Sections 2.2. and 2.4.).
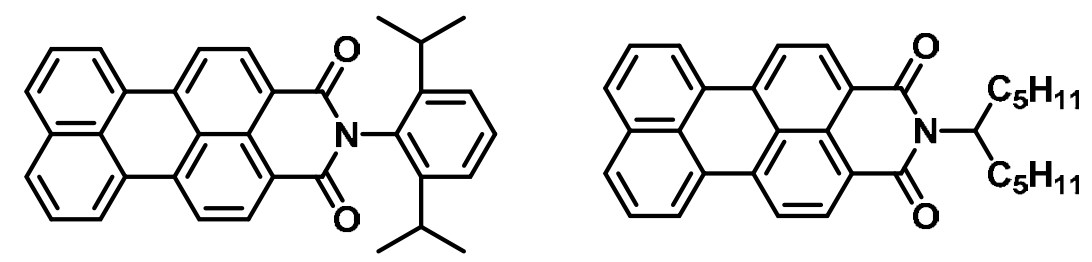

Figure 15. Common PMI derivatives used in literature, with diisopropyl imide substitution (left) and 2,6-diisopropylphenyl imide (right).

In addition, apart from choosing between one-pot or three steps synthesis, one way to optimise this synthesis would be reducing the necessary amount of time. Up until now, the standardized procedure requires a reactor and several days at $180-200^{\circ} \mathrm{C}$ to obtain the PMI products. However, there are some examples for synthesis of PDIs and PMIs by using a microwave system. ${ }^{28}$ During this thesis it was performed and optimized to get equivalent results after 30 min under microwave heating at $220{ }^{\circ} \mathrm{C}$, obtaining the products with the same yields.

${ }^{28}$ A) F. Rigodanza, E. Tenori, A. Bonasera, Z. Syrgiannis, M. Prato, Eur. J. Org. Chem., 2015, 23, 5060-5063.

B) Brian Guthrie, Zixing Wang, Jian Li, Mater. Res. Soc. Symp. Proc. 2008, 1091. 


\subsection{Bromination of PMIs}

Bromination reactions are extensively used and an easy way to modify PMIs, being usually the first step to tune their fluorescence and other properties. ${ }^{10}$ If the bromination reaction is performed under mild conditions, the monobrominated product is the only one obtained, in quantitative yields. However, using high temperatures and high excess of bromine lead to polybrominated products.

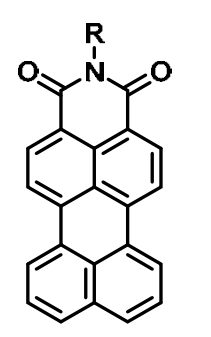

or

$\mathrm{CHCl}_{3}$ reflux

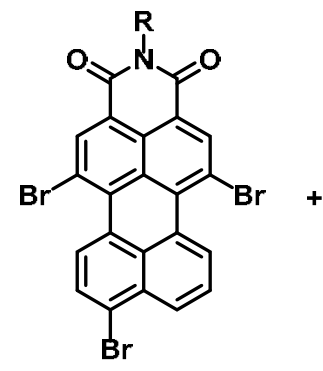

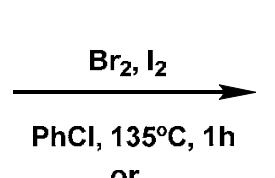
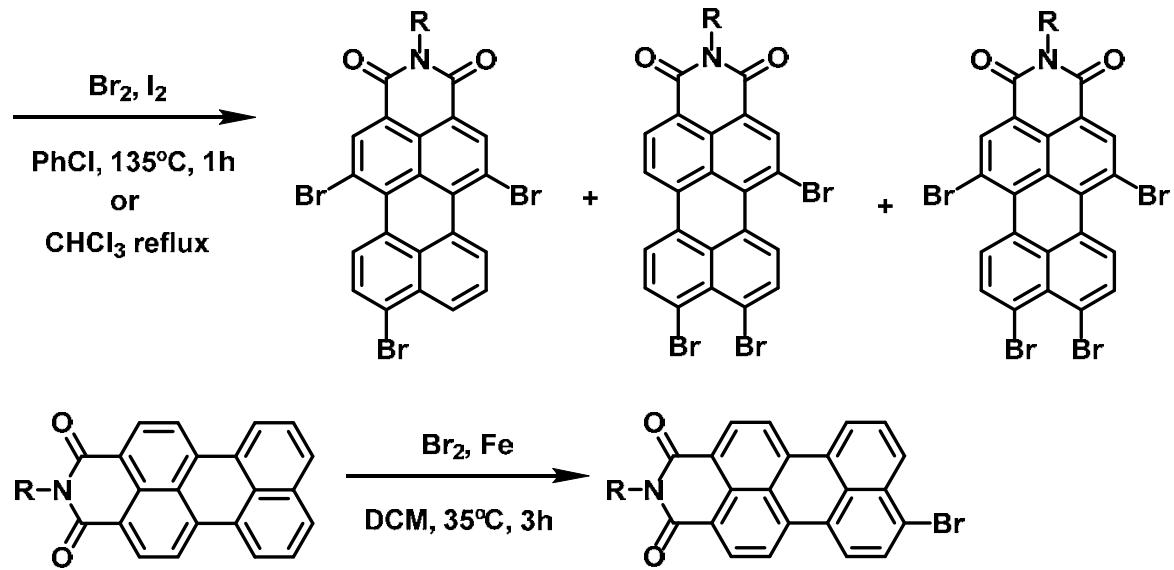

Figure 16. Synthetic route for bromination of PMI derivatives. Poly-bromination (up) and monobromination (down).

For some years, using the tri-brominated monoimide was the most common procedure. That was the case because bromines in bay position react easily with alcohol groups in basic media ${ }^{29}$ (sometimes by a copper(I) catalysed reaction) and the peri position bromine does not react. In contrast, $\mathrm{C}-\mathrm{C}$ coupling through Pd catalysed reactions or a reaction with an amine in basic media, ${ }^{30}$ occur in bay and peri position.

Despite the apparent advantages of the tri-brominated product, during the last years the brominated PMI derivatives that people work with have changed, from the tri-brominated to the mono-brominated product. For a while, tri-brominated PMIs were synthetized as they were a pure one-step major product from strong bromination conditions, until some authors reported the unspecificity of the reaction. ${ }^{31}$ When using strong conditions, like the ones specified in Figure 16 up, the PMI is not only tri-brominated but tetra-brominated too; what is more, the tri-bromination is not specific, but a mixture of isomers. In addition to this fact, the process of purification is tedious and very complex, needing several days, columns and crystallizations to separate the tri-brominated isomers. In fact, it is worth to remark that after the report about the existence of the isomers, the number of papers published working with tri-brominated PMIs decreased dramatically.

Nowadays, most researchers work with mono-brominated PMIs. However, it is true that there would be many possibilities for obtaining derivatives with groups in bay position. A possibility, would be leaving the purification until the bromine is substituted (Figure 17). Performing purification in later steps is likely to be a more efficient method, faster, with better yields and less waste (solvents and other purification material). In fact, it is likely to be the method that it has been followed by most authors, although not specified.

\footnotetext{
${ }^{29}$ P. Shao, N. Jia, S. Zhanga, M. Bai, Chem. Commun. 2014, 50, 5648-5651.

${ }^{30}$ T. Dentani, K. Funabiki, J.-Y. Jin, T. Yoshida, H. Minoura, M. Matsui, Dyes Pigm. 2007, 72, 303-307.

${ }^{31}$ A. Keerthi, Y. Liu, Q. Wang, S. Valiyaveettil, Chem. Eur. J. 2012, 18, 11669-11676.
} 


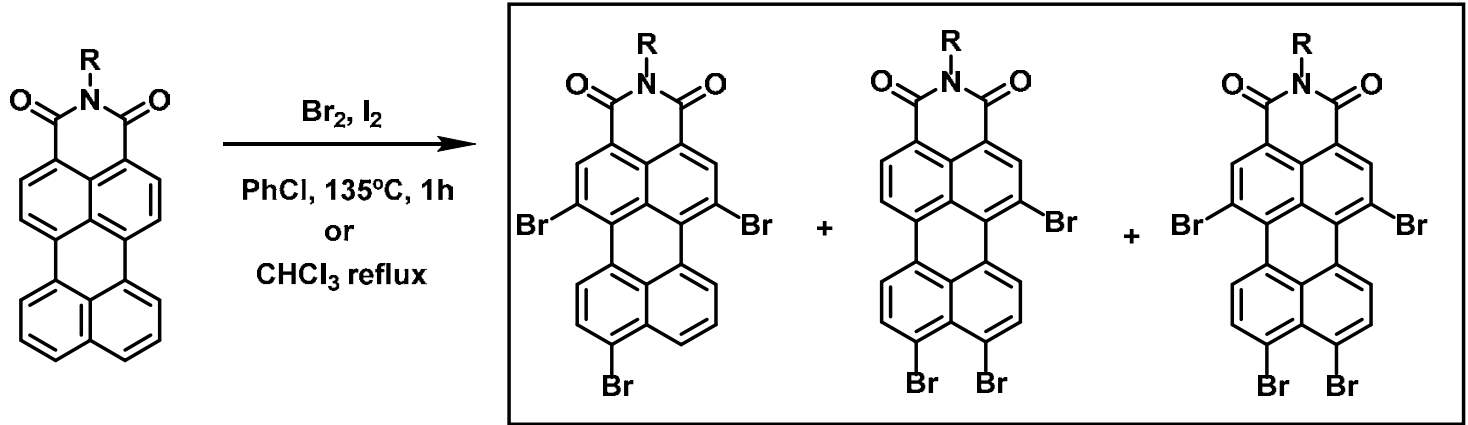

Hard to purify - Only viable for low amounts

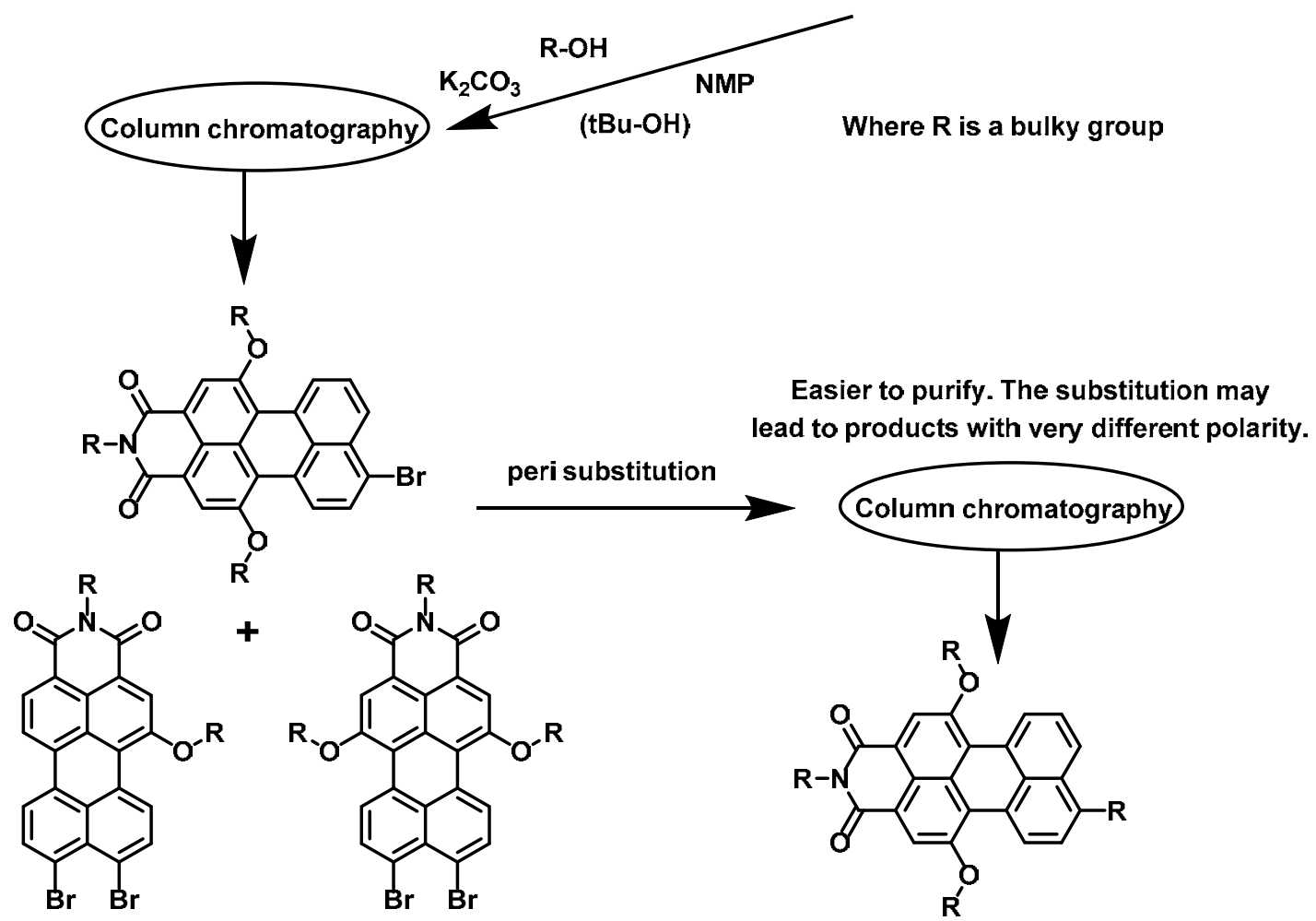

Figure 17. Schematic synthesis of a tri-substituted pure PMI. Example of the steps that may simplify the purification process.

During the development of the thesis only mono-brominated products were used (Figure $\mathbf{1 6}$ down), so as to simplify the synthetic routes. The synthesis only required 3 hours in DCM in a ten-fold excess of bromine; an extraction with saturated bisulfite solution and no further purification, obtaining the product as a red powder in a quantitative yield. This reaction was also scalable, tested between 50-500 mg. 


\subsection{Reimidization of PMIs}<smiles></smiles><smiles>[R]c1ccc2c3cccc4cccc(c5cccc1c52)c43</smiles>

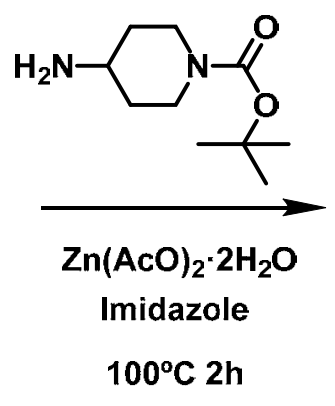<smiles></smiles>

Figure 18. Synthesis scheme of reimidization process in a PMI.

Reimidization procedure of PMIs ${ }^{32}$ and PDIs is widely studied in literature; it consists of a saponification treatment followed by an imidization process. For different reasons, the imide group may be substituted by a more appealing group. In a representative reaction, our starting material consisted of the N-1-adamantylethyl-PMI that was changed by a group containing a boc-protected amine (Figure 18). The free amine group was useful for the afterwards anchoring reaction to other species, such as a group with water affinity or a polymeric material (Chapter 3C).

On the other side, the presence of a group (R) in peri (Figure 18) is not casual. Having bulky groups in different positions (it could be also in ortho or bay) may increase the solubility of the monoanhydride intermediate and exclude the tendency of perylene derivatives to stack and precipitate. For instance, a bromine group in peri was enough to obtain the product in high yield. There are some limitations, for instance, the $\mathrm{R}$ group must resist the saponification conditions.

Everything considered, the synthesis is straightforward. The PMI is dissolved in $t-\mathrm{BuOH}$, next $\mathrm{NaOH}$ (or $\mathrm{KOH}$ ) was added in high excess to the solution (50 equivalents) and left under stirring at $80^{\circ} \mathrm{C}$ for 15 hours. After that, the mixture was quenched with high excess of glacial acetic acid and after one hour under stirring it was filtered and washed several times with methanol. It allowed to obtain the monoanhydride in quantitative manner. In the second step, the monoanhydride was transformed into monoimide by following a classical procedure for imidization. The monoanhydride was mixed with imidazole with a $5 \% \mathrm{~mol}$ of zinc acetate and heated at $100^{\circ} \mathrm{C}$. Once the imidazole became liquid and the monoanhydride was dissolved, the amine was added and the mixture was left under stirring for 2 hours at $100^{\circ} \mathrm{C}$. The residue was dissolved in DCM and washed with water (several times). After column chromatography, the product was obtained in high yield $>80 \%$, which could vary depending on the particular PMIs (starting reagent and product).

\footnotetext{
${ }^{32}$ A. Bolag, N.Sakai, S.Matile, Chem. Eur. J. 2016, 22, 1-10
} 


\subsection{Borylation of PMIs}

Suzuki-Miyaura couplings are one of the most common procedures in order to create aryl-aryl bonds in peri position. To perform the reaction it is necessary to have an aryl halide and an arylboronate (or boronic acid). Taking this into account, some reactions may require having the boronic acid/ester of the perylene either because of the impossibility to have it in the other reagent or to increase the general yield of the coupling reaction. The procedure is similar to the general preparation of aryl boronates, and there is plenty of literature about it. ${ }^{33}$ (Figure 19)

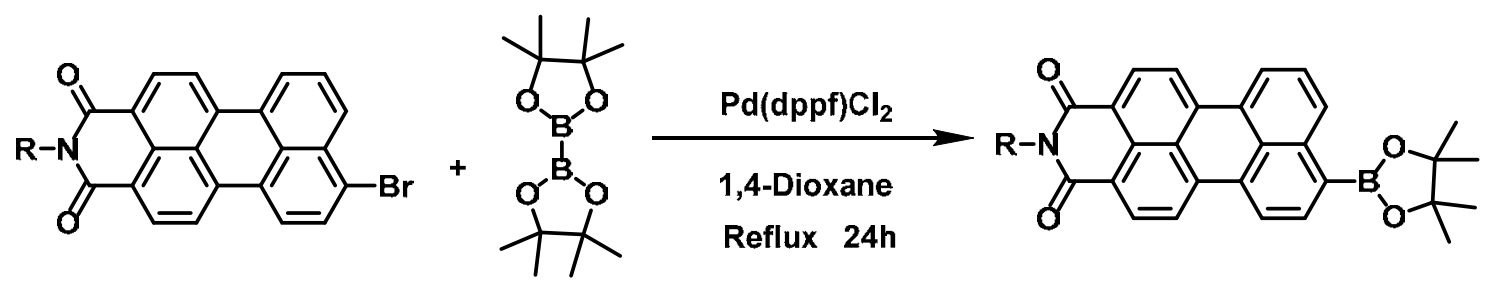

Figure 19. Synthesis scheme for borylation of PMIs.

As it is schematized in Figure 19, the brominated reagent was dissolved in 1,4-dioxane (around 10 $\mathrm{mM}$ ) under nitrogen atmosphere. After that, the palladium(0) catalyst was added to the solution (5\% mol) and the mixture was refluxed for 12-24 hours. The product obtained was purified (usually DCM:MeOH mixtures, 1-5\%) by column chromatography, obtaining yields around $50 \%$, being a process that competes with the homo-coupling.

\subsection{Introduction of groups in peri position by Suzuki reaction}

The Suzuki reactions have been performed following the classic conditions previously reported in literature. The most important issue when working with perylene derivatives is that the compound might not be soluble in some solvent mixtures because of the low solubility of PIs (especially when they have an imide group which is not bulky enough), which decreases the final yield. Several synthetic conditions were tested for different synthetized PMIs. As a general procedure, see Figure 20.
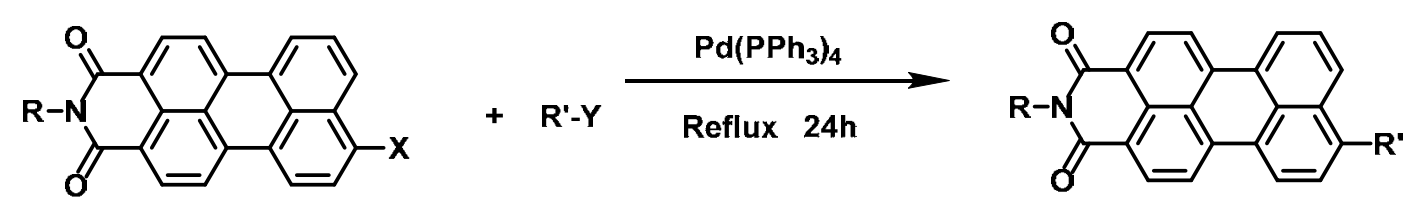

\section{$\mathbf{R}$ is a bulky group $\mathbf{R}^{\prime}-\mathbf{Y}$ is aryl halide or boronate \\ $X$ is an halide or boronate group (the opposite to " $Y$ ")}

Figure 20. General scheme for a Suzuki reaction in PMIs.

The brominated derivative, boronic acid derivative and the base $\left(\mathrm{Na}_{2} \mathrm{CO}_{3}, 5\right.$ equivalents) were added to a flask under nitrogen atmosphere, then they were dissolved in a mixture of solvents (see below) until a concentration 1-5 $\mathrm{mM}$ and left under reflux for 16-24 hours. The product was purified by column chromatography (by using mixtures DCM:MeOH from 0 to $5 \%$ ) obtaining products from red to purple, depending on the substituent.

\footnotetext{
33 See for example; P. Shao, M. Bai; Chem. Commun., 2012, 48, 9498-9500.
} 
From the multiple possibilities when choosing the solvents, three different mixtures were tested for PMIs.

- Using THF:water (8:1), is one of the most common mixtures used for performing Suzuki coupling reactions, such as the ones described Chapter 1. However, it does not work properly with most PMIs, probably because of the low solubility.

- Using a mixture Toluene:BuOH: $\mathbf{H}_{2} \mathrm{O}$ (4:1:0.4). This mixture has been used by our research group when working with many different products, including PMIs. It works for a wide variety of reagents (all that we have tested), however, the yield is usually lower than when optimized with other solvents.

- Mixture DME:water (2:1). It has been tested with some PMIs giving good yields, similar or better to the previous one.

There are plenty of conditions apart from the ones already explained. However, the performed reactions lead to yields equal or superior to $40 \%$ and they were not further optimized. ${ }^{27}$ 


\section{RELATION STRUCTURE-LUMINESCENCE OF THE SYNTHETIZED PMI DERIVATIVES}

Modification of peri and bay positions in PMIs are used for tuning their optical properties. ${ }^{34}$ Previous to the introduction of specific details and applications for particular compounds in Chapter 3, this section explains and compares some details related to the absorbance-fluorescence properties of the synthesizing PMI derivatives. The purpose of this study is to show how the solvent and the structure affects each other when changing them, which serves as an explanation for some of the possible and potential uses they may have, such as the fluorescent sensors that have been developed.

The study was divided into three sections. First, the influence of the solvent was studied for the different PMI derivatives.

- Starting materials: which includes PMIs JG62, JG73, JG75, JG2L and JG7L.

- PMI derivatives with phenyl, pyridine and pyrimidine piperazine substituents: JG125, JG116, JGphen, JG125d, JG117 and JGphend.

- Probes for K(I), Pb(II) detection and cellular imaging: JG76, JG121, JG119c1 and JG119c2.

- PMI-Ru(II) derivatives: JG10L and JG11L.

Secondly, there was an especial case that is worth mentioning, the synthesis and testing of a combination of Bodipy-PMI probe. Their properties are explained as introduction for future research in FRET systems giving the molecule special properties, mentioned in Section 4.2.

Finally, a table with a comparison between molar absorptivity, fluorescence quantum yields and fluorescence lifetime decay is presented and several conclusions are explained in this regard.

${ }^{34}$ C. Li, J. Schöneboom, Z. Liu, N. G. Pschirer, P. Erk, A. Herrmann, K. Müllen, Chem. Eur. J. 2009, 15, 878884 . 


\subsection{Influence of solvent on the synthesized PMIs}

4.1.1. Solvatochromism of starting materials (Figures 21-25):<smiles></smiles><smiles></smiles><smiles></smiles>

JG62 JG73 JG75<smiles></smiles>

JG2L<smiles></smiles>

JG7L

Figure 21. Molecular structure of PMIs JG62, JG73, JG75, JG2L and JG7L.

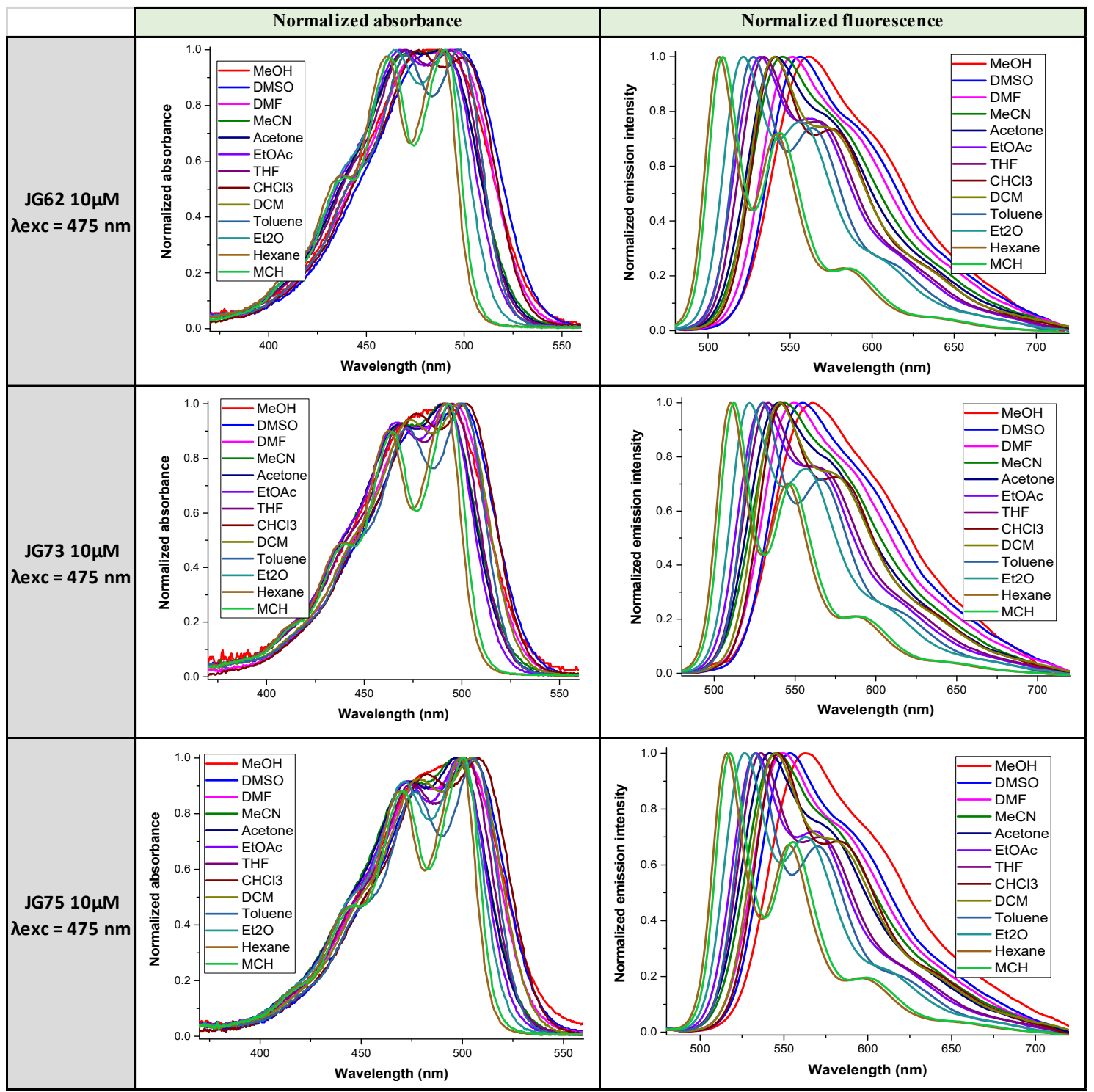

Figure 22. Normalized spectra of JG62, JG73 and JG75, $10 \mu \mathrm{M}$ in different solvents. 


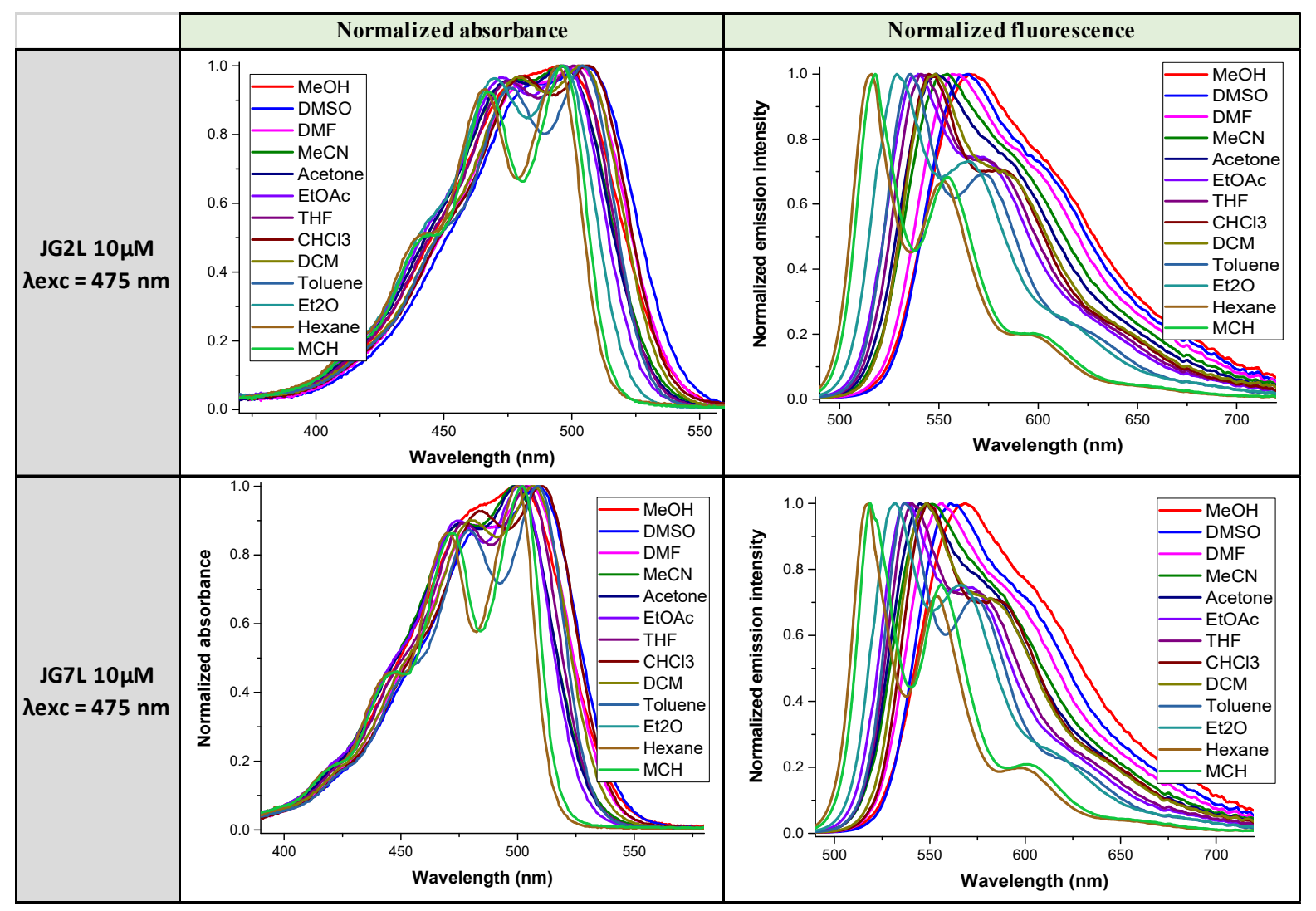

Figure 23. Normalized spectra of JG2L and JG7L, $10 \mu \mathrm{M}$ in different solvents.

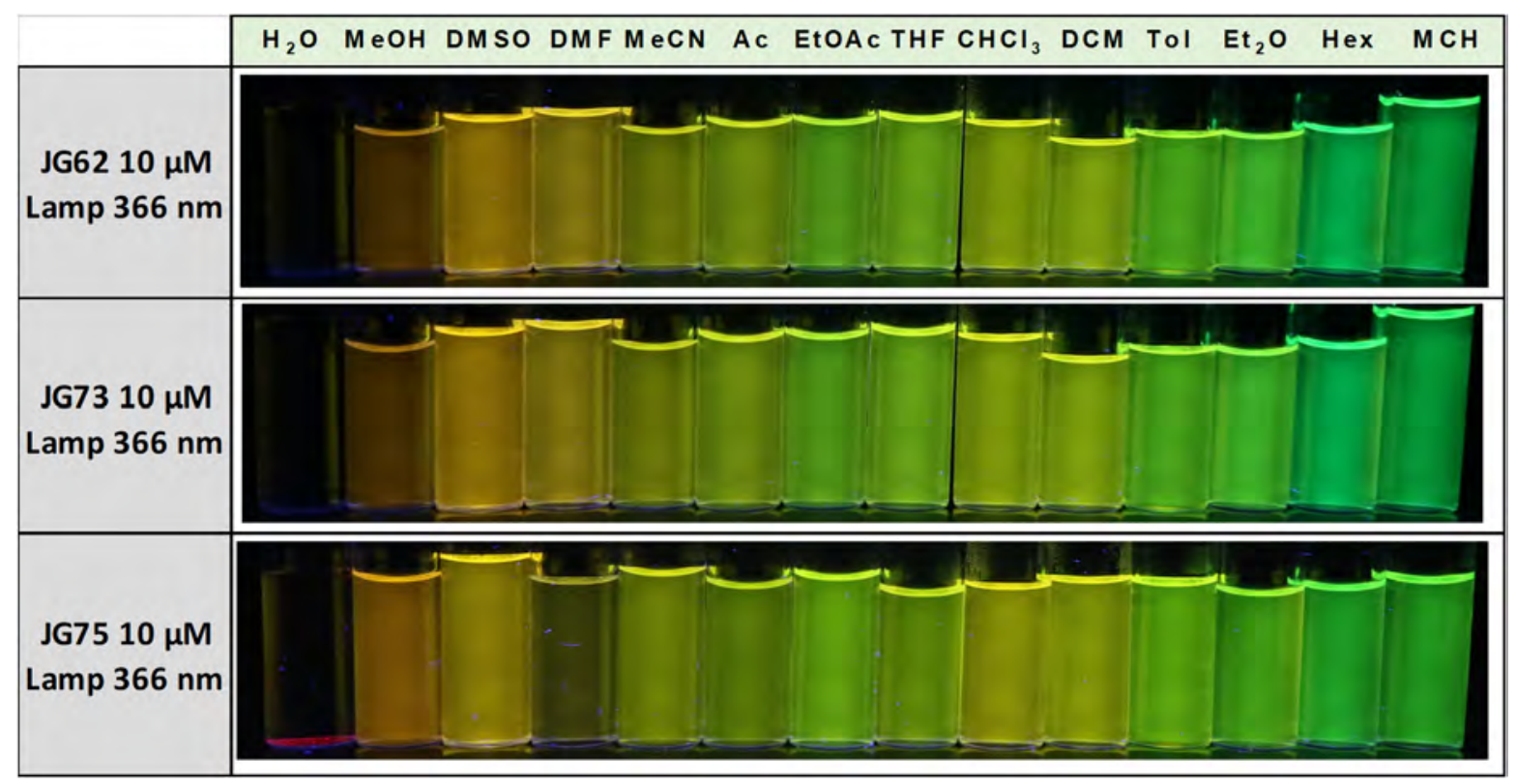

Figure 24. Picture under UV light of JG62, JG73 and JG75, $10 \mu \mathrm{M}$ in different solvents. 


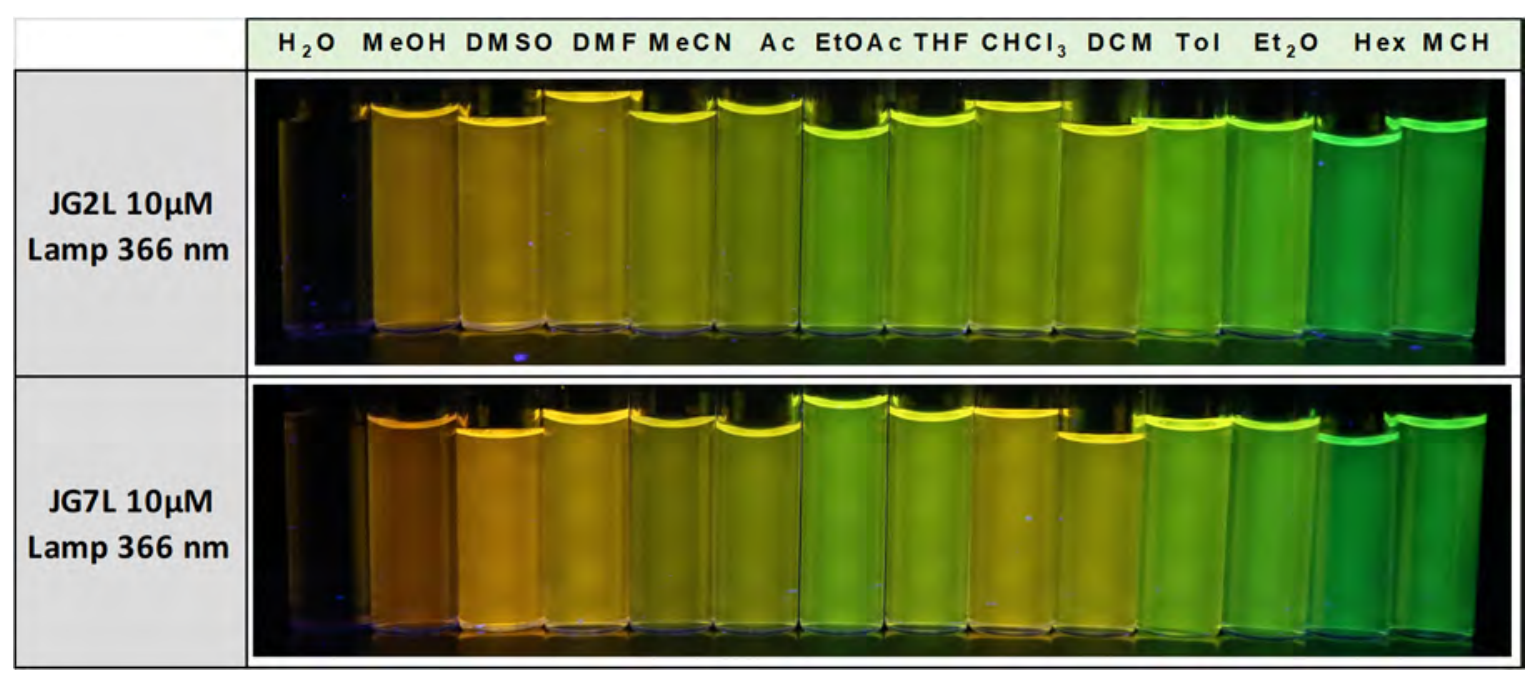

Figure 25. Pictures under UV light of JG2L and JG7L, $10 \mu \mathrm{M}$ in different solvents.

The properties and conclusions obtained may be summarized as follows:

- The effect of changing solvents on the absorbance was basically the same for all the probes, and not very remarkable for any of them. The maximum of absorbance showed a batochromic shift with polarity of around $20 \mathrm{~nm}$ from most polar solvents $(\mathrm{MeOH})$ to aliphatic solvents $(\mathrm{MCH})$.

- Fluorescent emission was more affected by changes in polarity than absorbance, it also has a batochromic shift with polarity, but higher, around 50-60 nm for the same range of solvents.

- None of them were soluble in water.

- JG75 had red fluorescence in solid state.

4.1.2. PMI derivatives with piperazine substituted phenyl, pyridine and pyrimidine substituents (Figures 26-30):
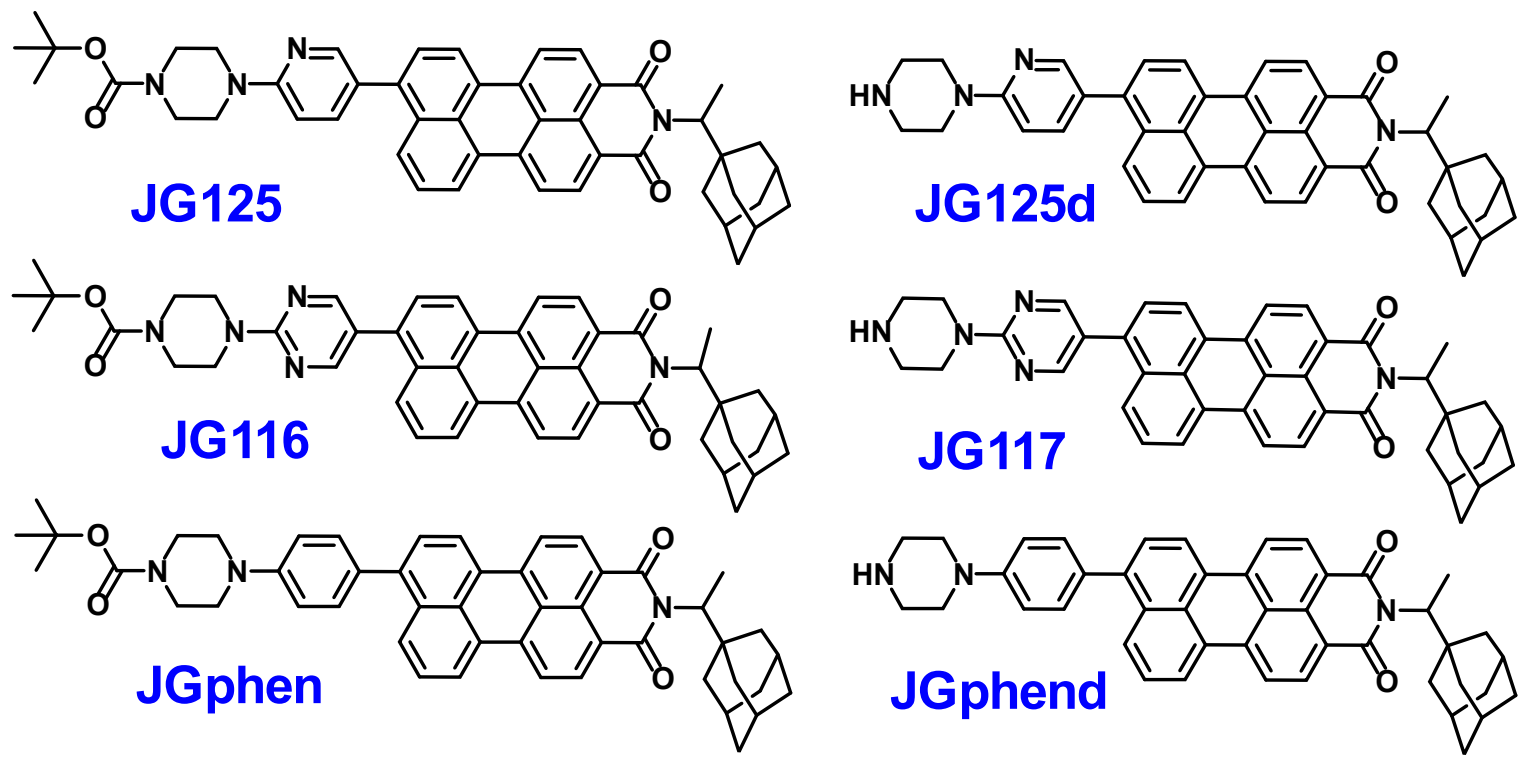

Figure 26. Molecular structure of PMIs JG125, JG116, JGphen, JG125d, JG117 and JGphend. 


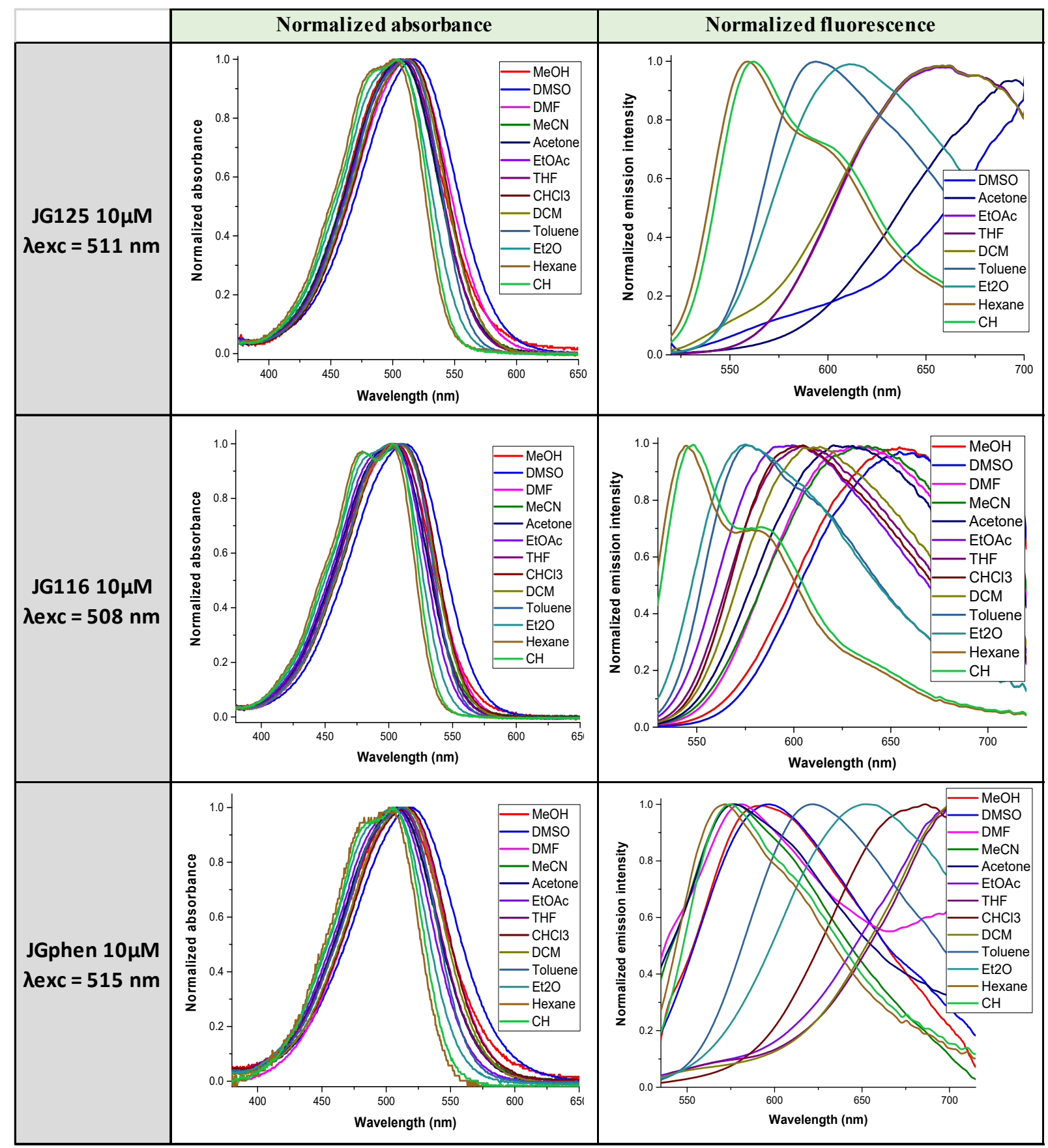

Figure 27. Normalized spectra of JG125, JG116 and JGphen, $10 \mu \mathrm{M}$ in different solvents. 


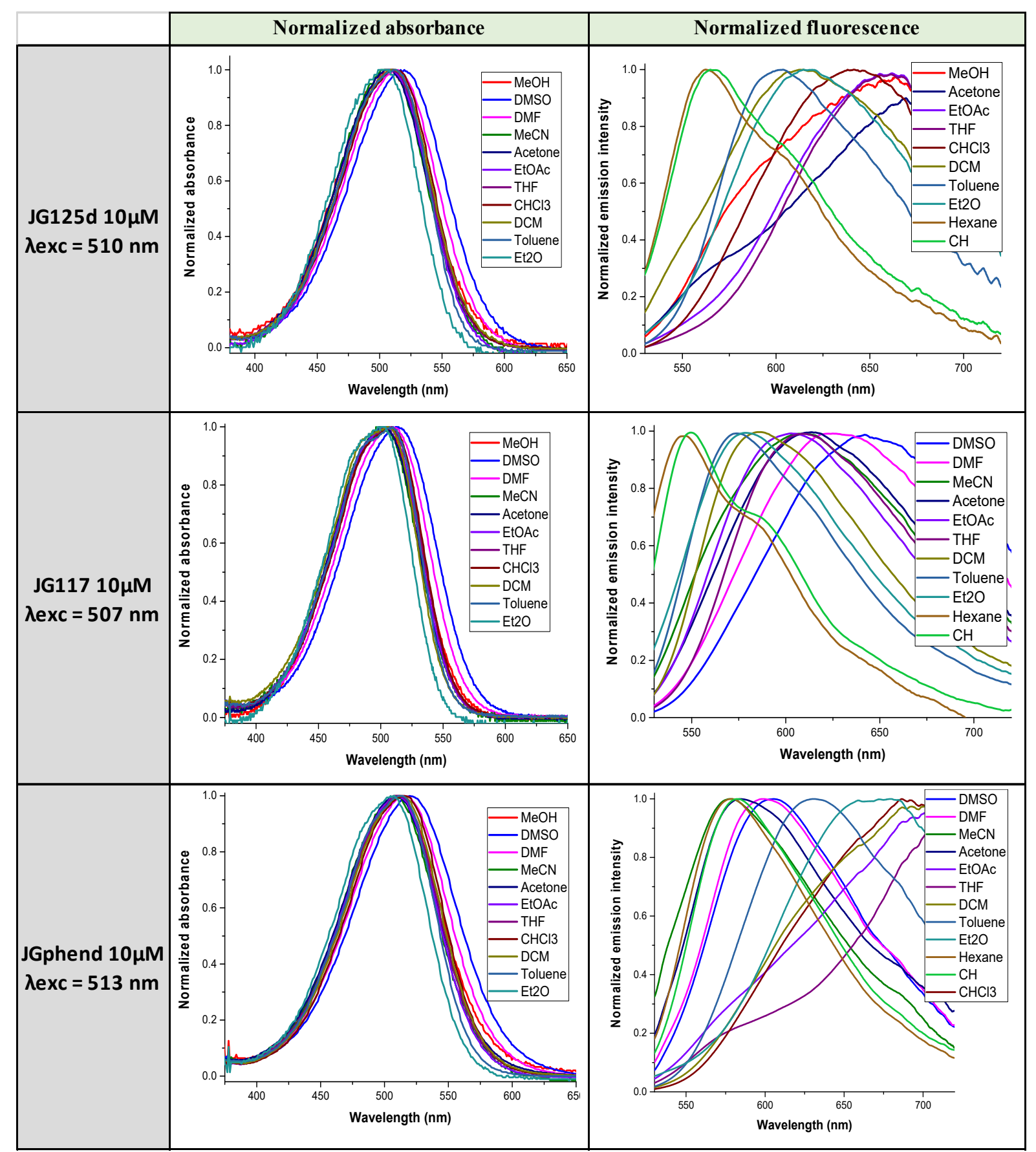

Figure 28. Normalized spectra of JG125d, JG117 and JGphend, $10 \mu \mathrm{M}$ in different solvents. 


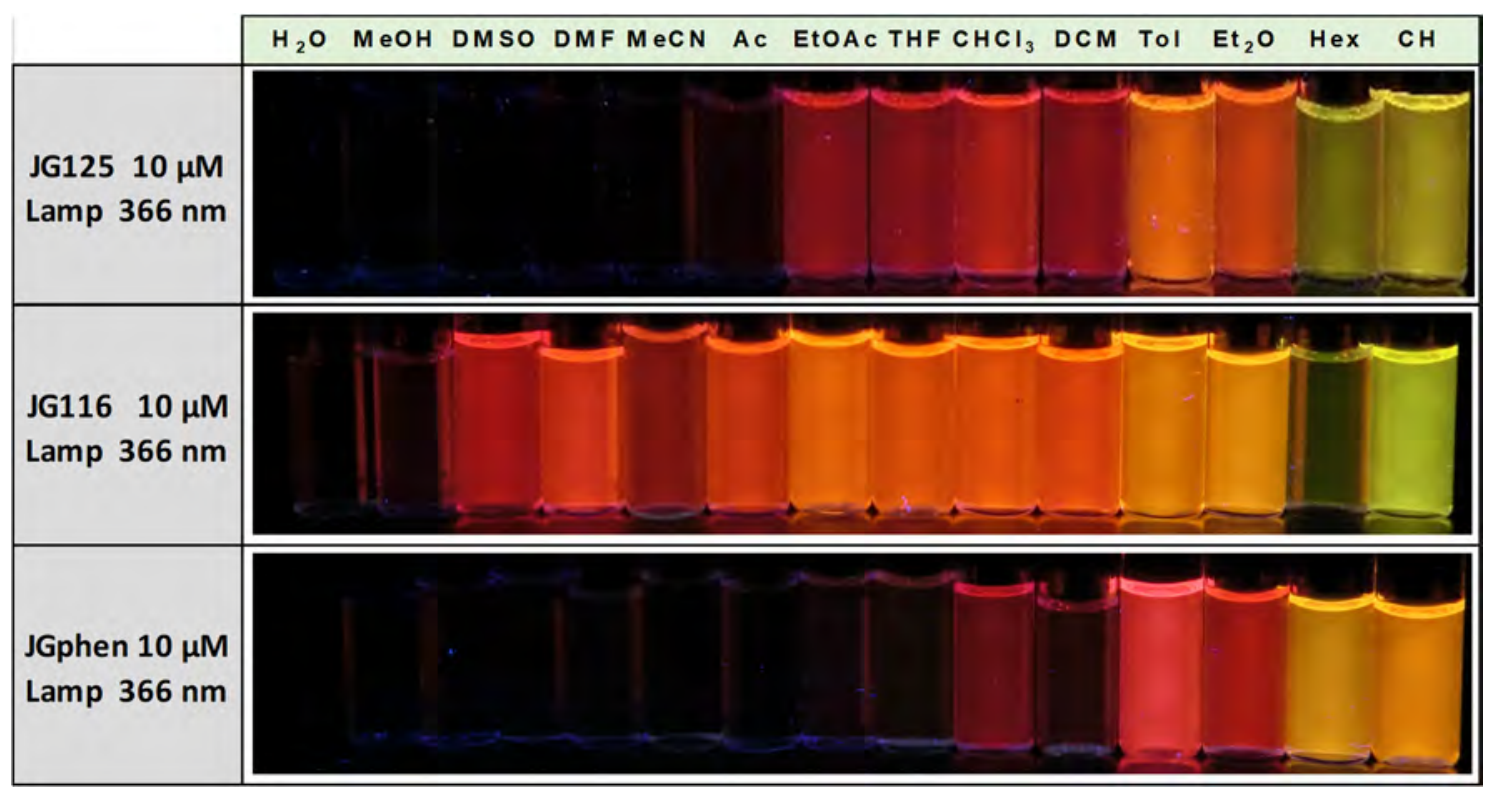

Figure 29. Pictures under UV light of JG125, JG116 and JGphen, $10 \mu \mathrm{M}$ in different solvents.

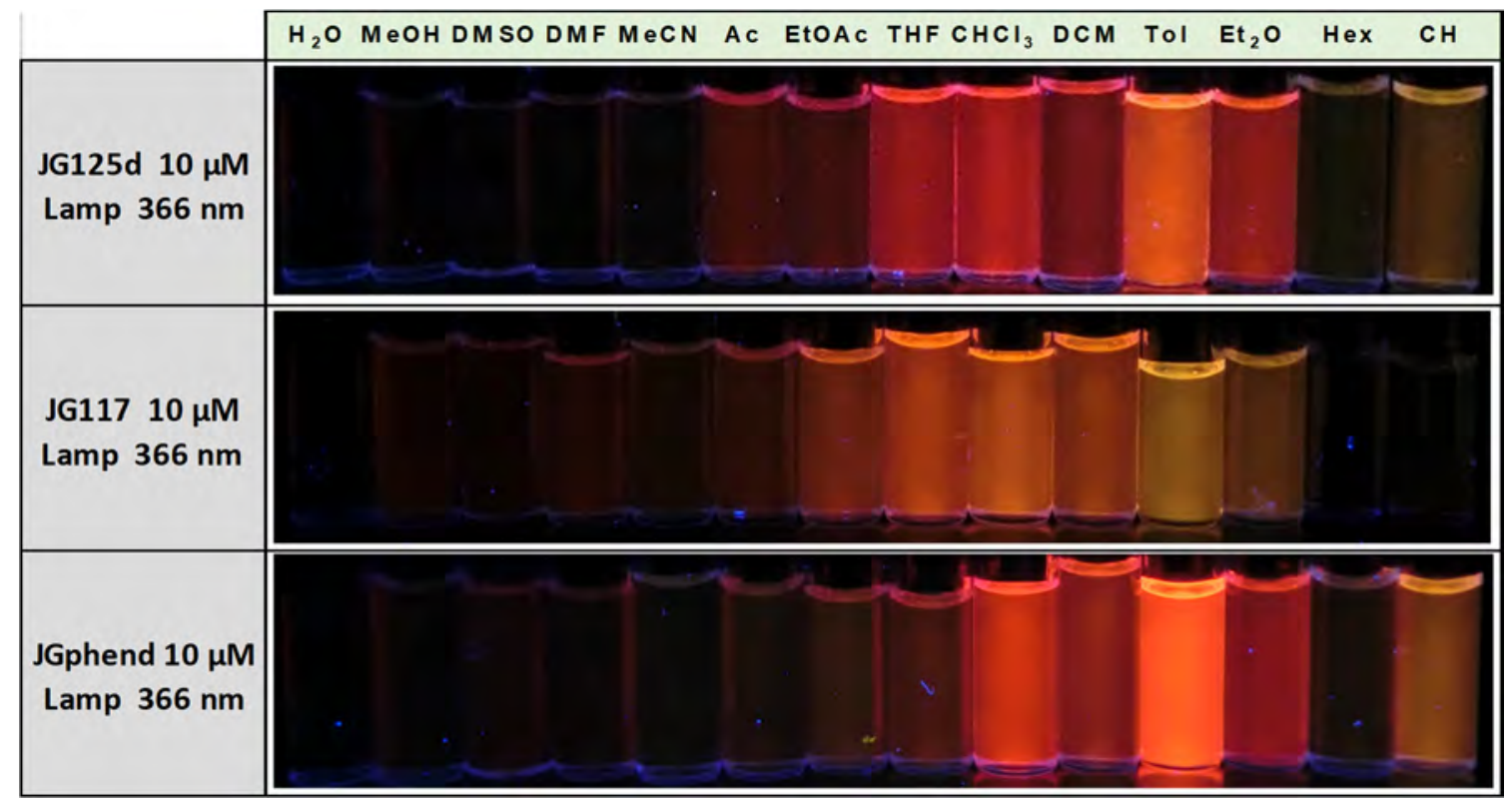

Figure 30. Pictures under UV light of JG125d, JG117 and JGphend, $10 \mu \mathrm{M}$ in different solvents.

The following facts were obtained from the data showed in Figures 26-30:

- The solubility decreased when the amine groups were deprotected.

- Fluorescence also decreased when deprotected, except for the one containing a phenyl group that increased in toluene and chloroform.

- Dissolved in the same solvent, the wavelength of emission followed the order Pyrimidine $>$ Pyridine $>$ Phenyl substituent.

- As a general rule, the fluorescence intensity was higher in the same order, Pyrimidine $>$ Pyridine $>$ Phenyl substituent.

- The batochromic shift with polarity was low in absorption $(<20 \mathrm{~nm})$ but high in fluorescence, even $150 \mathrm{~nm}$, reaching the infrared for some compounds, $\lambda_{\mathrm{em}}>700 \mathrm{~nm}$. 
4.1.3. Probes for studies with DNA, cellular imaging and detection of $\mathrm{K}^{+}$and $\mathrm{Pb}^{2+}$ (Figures 31-35):

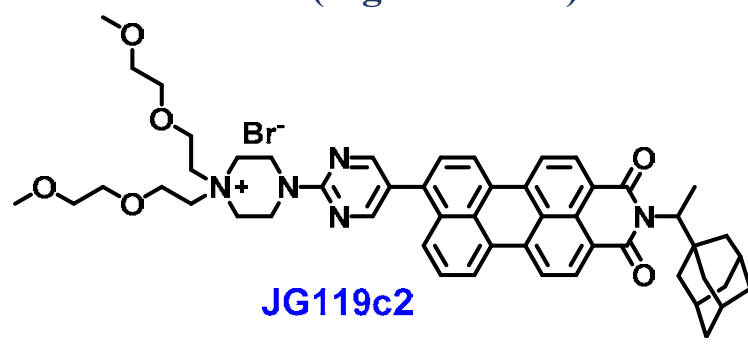

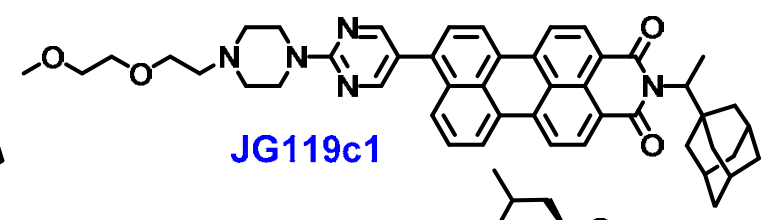<smiles>COCCOc1ccccc1N1COCCOCCOCCOCCOCCO1</smiles>

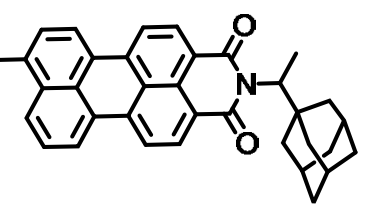
JG76<smiles>C1C2CC3CC1C3C2</smiles><smiles>CC(C)C1CCCCC1</smiles>

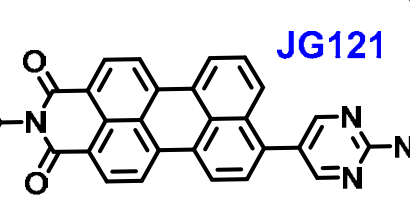
$O=$

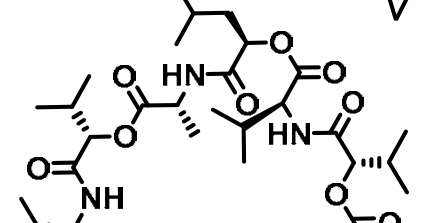<smiles>CCCNC(C(=O)OCCC)C(C)C</smiles><smiles>CCN[C@@H](C)C(C)=O</smiles>

Figure 31. Molecular structure of PMIs JG119c2, JG119c1, JG76 and JG121.

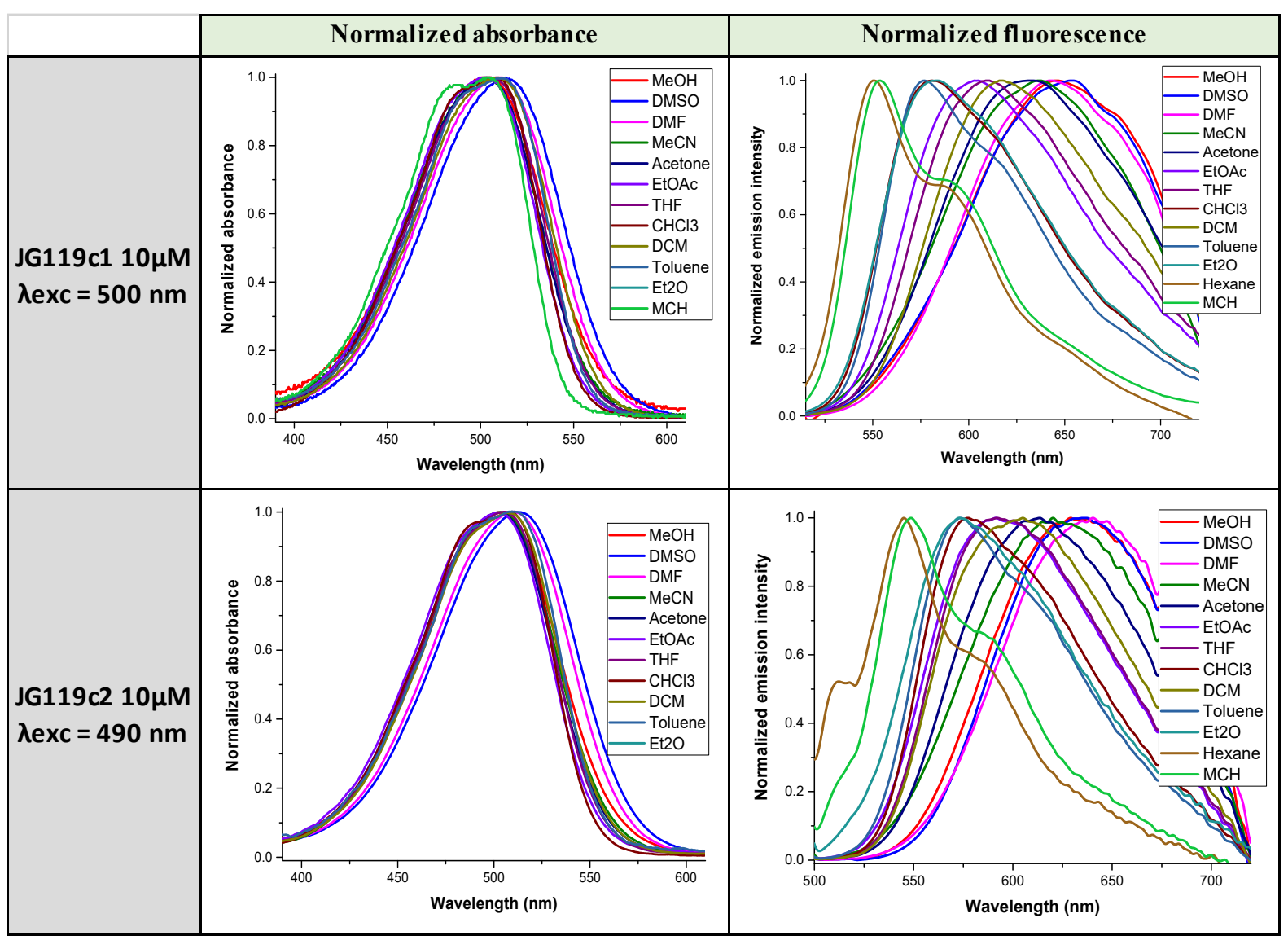

Figure 32. Normalized spectra of JG119c1 and JG119c2, $10 \mu \mathrm{M}$ in different solvents. 


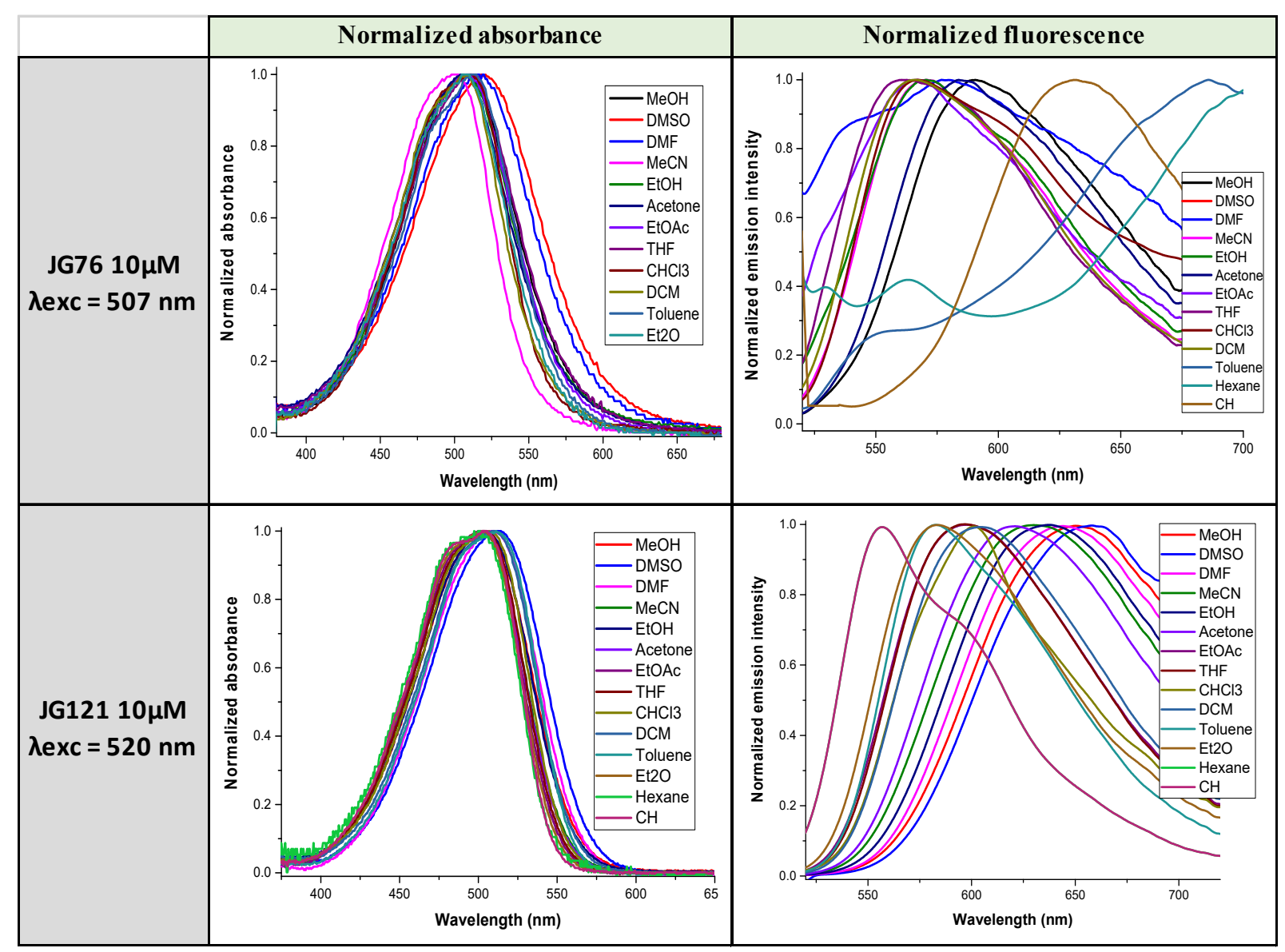

Figure 33. Normalized spectra of JG76 and JG121, $10 \mu \mathrm{M}$ in different solvents.

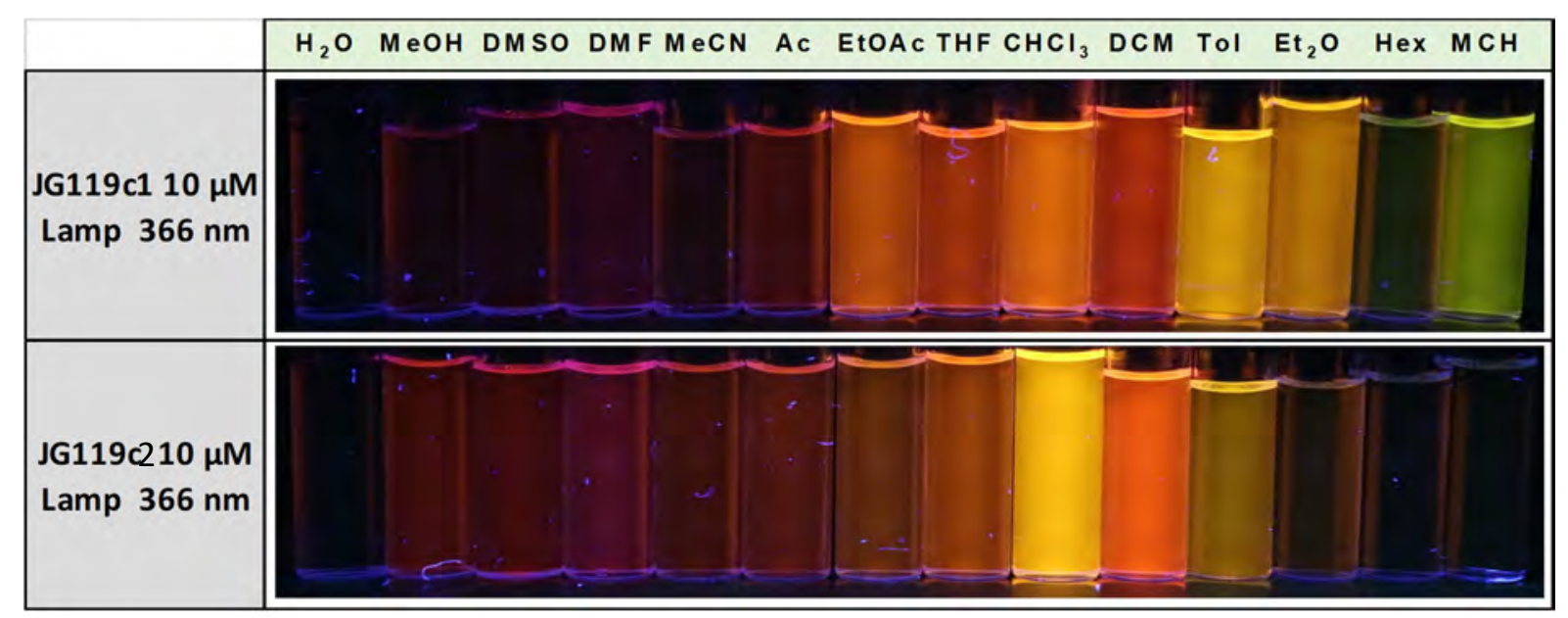

Figure 34. Pictures under UV light of JG119c1 and JG119c2, $10 \mu \mathrm{M}$ in different solvents. 


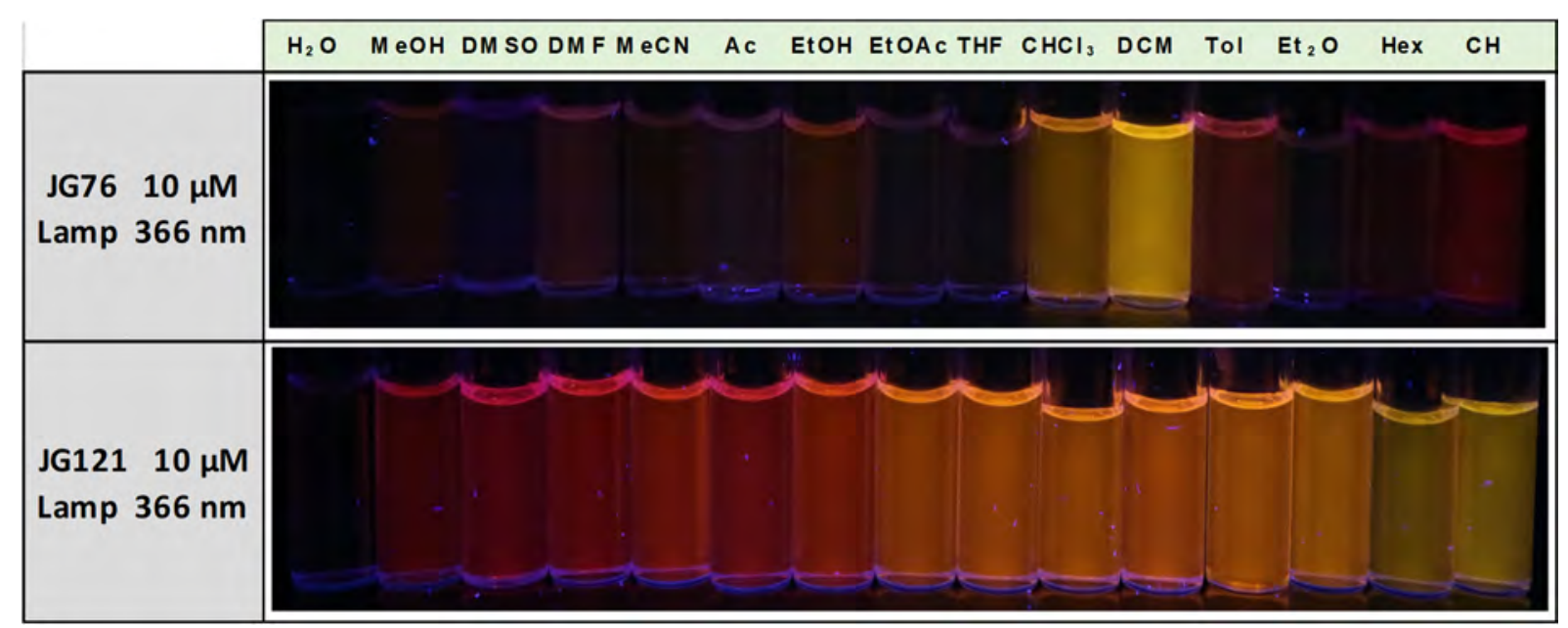

Figure 35. Pictures under UV light of JG76 and JG121, $10 \mu \mathrm{M}$ in different solvents.

For the compounds used as colorants for cells and DNA (JG119c1 and JG119c2):

- Both had similar absorption and emission spectra.

- Both had batochromic shifts with polarity of around $120 \mathrm{~nm}$ in emission (MeOH-Toluene).

- JG119c2 presented two PEGs and a total positive charge, which made it insoluble in aliphatic solvents.

For the potassium ligands PMI derivatives (JG76 and JG121):

- JG121 showed a polarity dependence that is directly related with the polarity index, with a regular change. This behaviour demonstrated to be useful for cell measurements (See Chapter 3C).

- JG76 had two different behaviours, one in polar solvents $\mathrm{MeOH}$ to DCM being fluorescent in yellow-orange and another with less polar solvents toluene to cyclohexane in which seemed to be fluorescent in deep red $(>600 \mathrm{~nm})$. However, the low solubility limited possible applications. 
4.1.4. PMI-Ru(II) complexes (Figures 36-41):

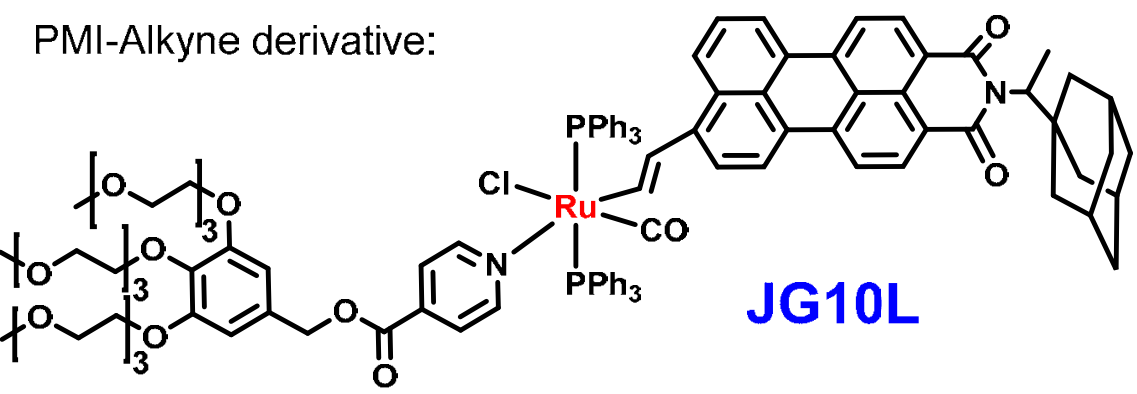

Figure 36. Molecular structure of Ru(II)-JG7L complex, JG10L.

PMI-p-pyridine derivative:

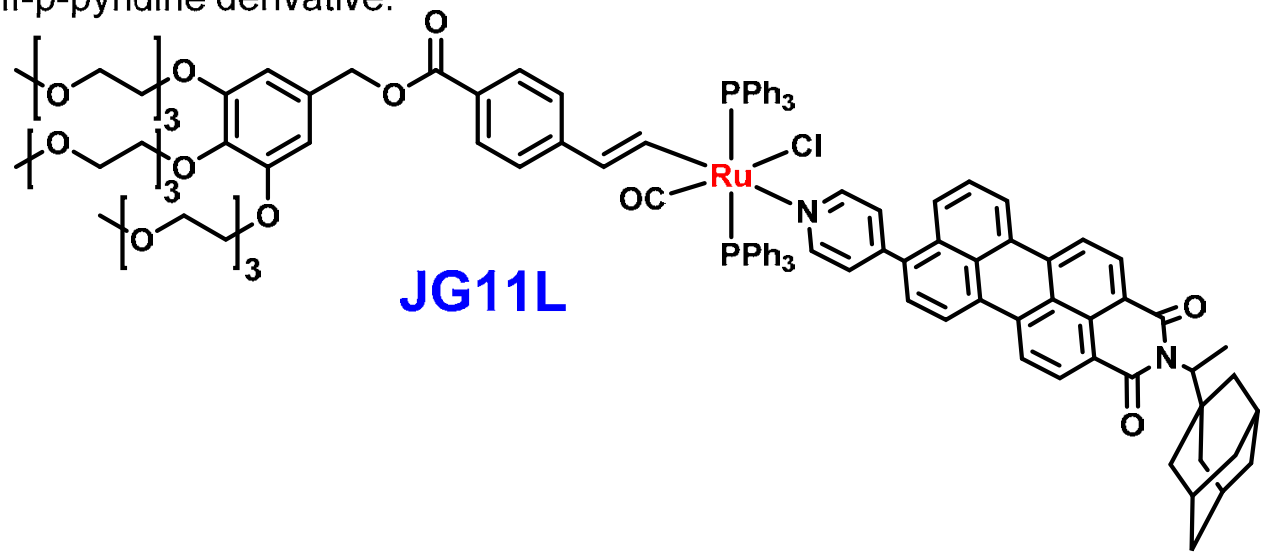

Figure 37. Molecular structure of Ru(II)-JG2L complex, JG11L.

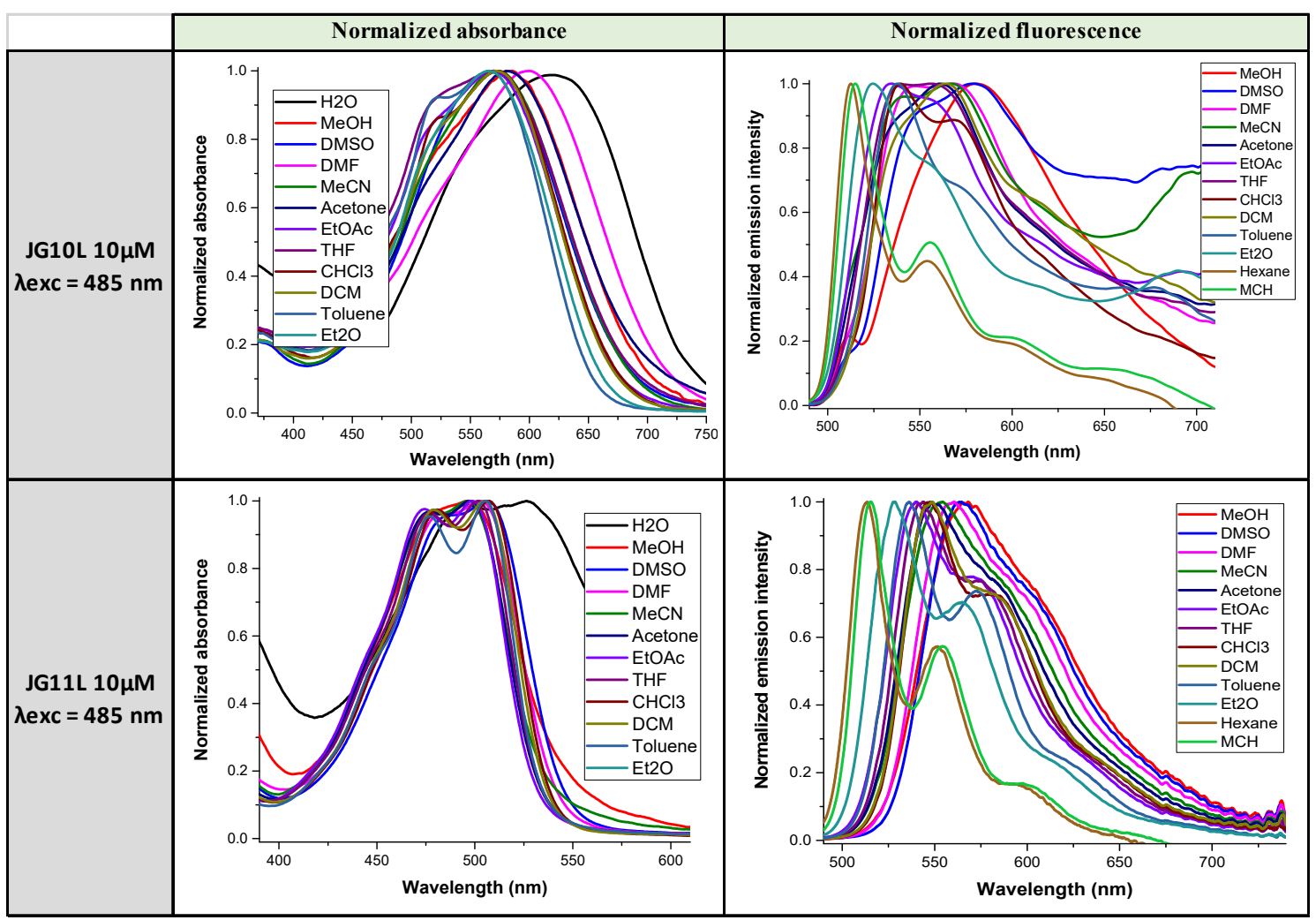

Figure 38. Normalized spectra of JG10L and JG11L, $10 \mu \mathrm{M}$ in different solvents. 


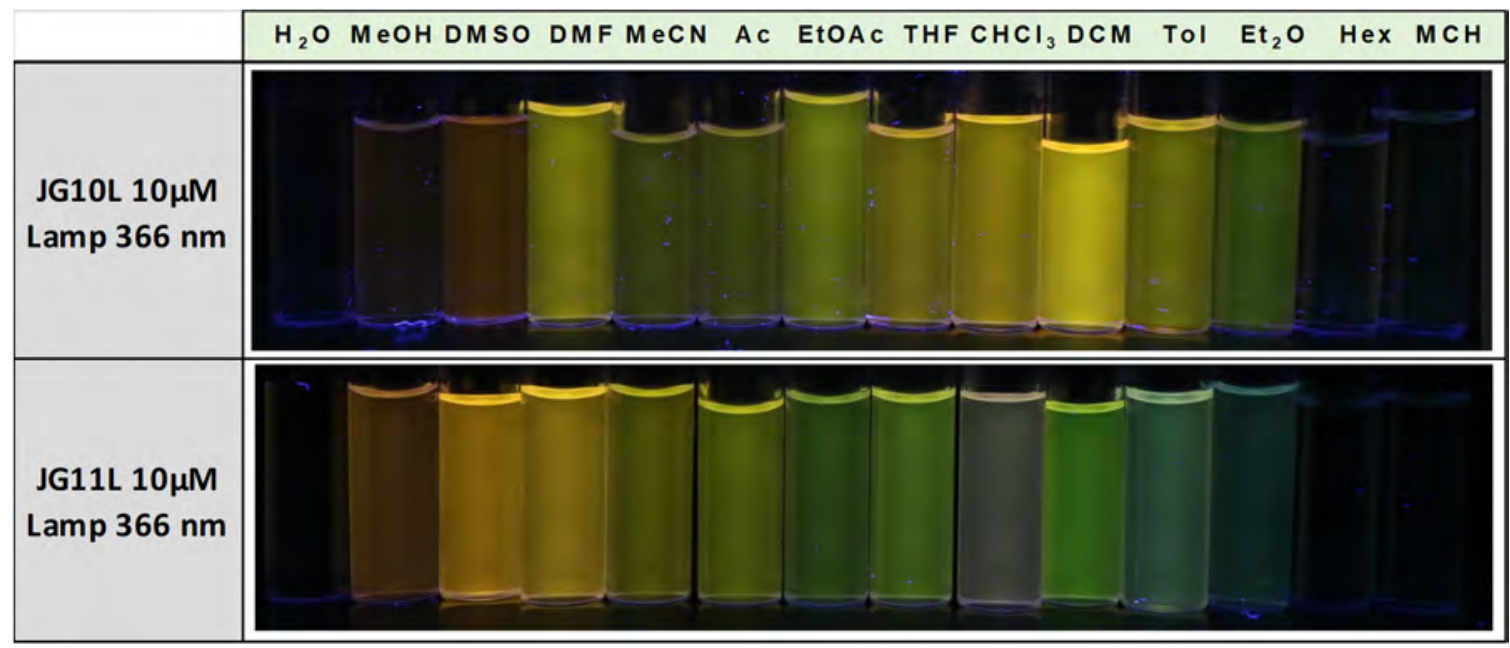

Figure 39. Pictures under UV light of JG10L and JG11L, $10 \mu \mathrm{M}$ in different solvents.

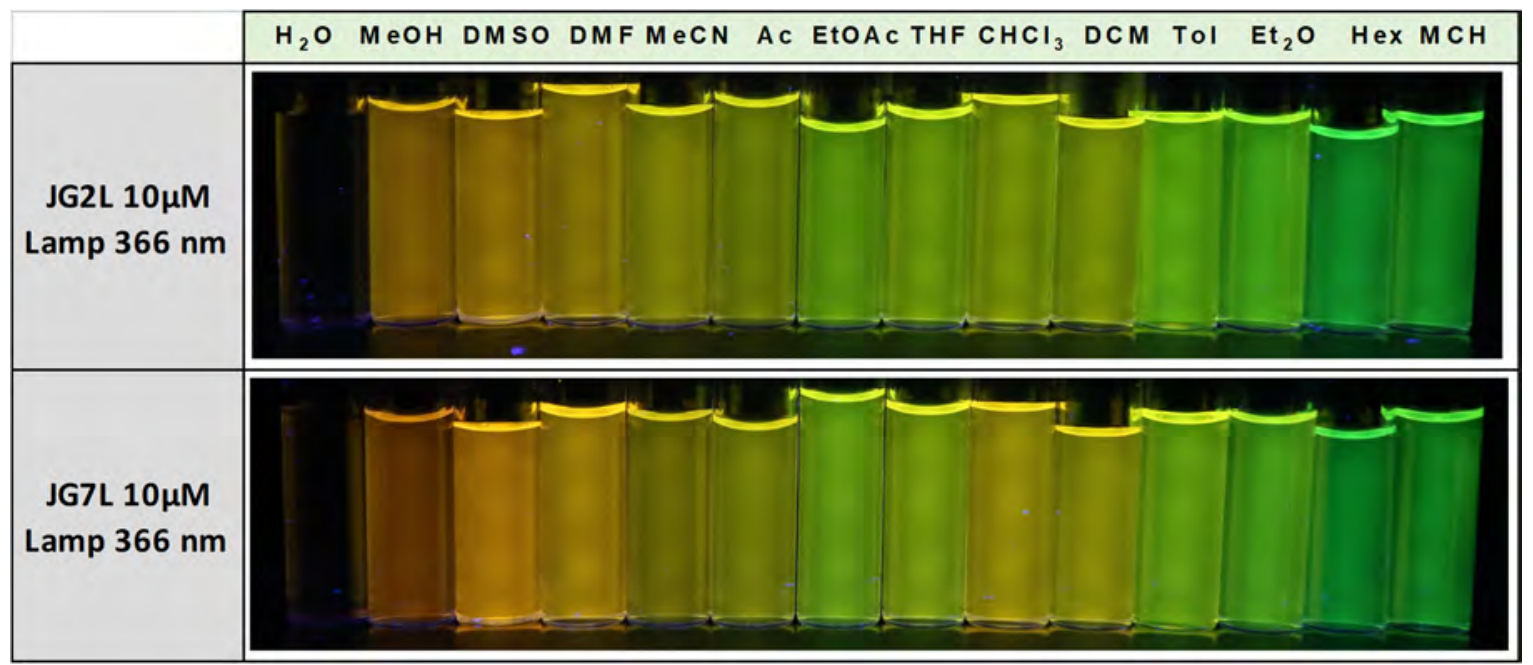

Figure 40. Pictures under UV light of JG2L and JG7L, $10 \mu \mathrm{M}$ in different solvents.

As it may be observed by comparing spectra in Figure 38, and between Figures 39 and 40, synthesizing a ruthenium (II) complex of a PMI affects directly its fluorescence. It was observed that, in some solvents such as DMSO or chloroform, the complexes JG10L and JG11L quickly degraded leading to their free PMI components (JG7L and JG2L respectively). JG10L was especially sensitive to solvents, freshly prepared solutions were barely fluorescent in any solvent (in region 500-600 nm), but it increased fast with time. It was also noteworthy that JG10L solutions possessed an emission band in IR region (around $700 \mathrm{~nm}$ or more), and the colour under visible light was very different from free PMI solutions in any solvent (Figure 41).

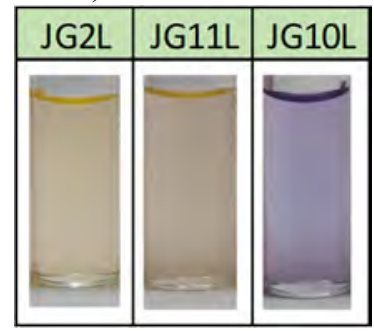

Figure 41. Picture under visible light of JG2L, JG11L and JG10L, $10 \mu \mathrm{M}$ in MeCN. 
These derivatives were studied in bioimaging, as cellular colorants, or for specific detection of analytes by changing the coordination sphere of the metal. These topics are more fully addressed in Chapter 3B.

\subsection{PMII-Bodipy combinations for creation of FRET systems}

As it was previously explained, PMIs are ideal for the creation of PET and FRET systems, being easy to modify from a bromine or boronate group. As it was explained in Chapter 0, FRET systems contain two or more fluorophores that may suffer from electronic changes depending on the conditions. For instance, they have potential applications as sensors or improved light sensitizers.

Besides perylene derivatives, there are many other fluorescent backbones commonly used for the synthesis of sensors. Among them, one of the most studied are bodipys. Because of that, it was proposed the study of a combination of Bodipy-PMI. Hence, the three different compounds from Figure 42 were evaluated.
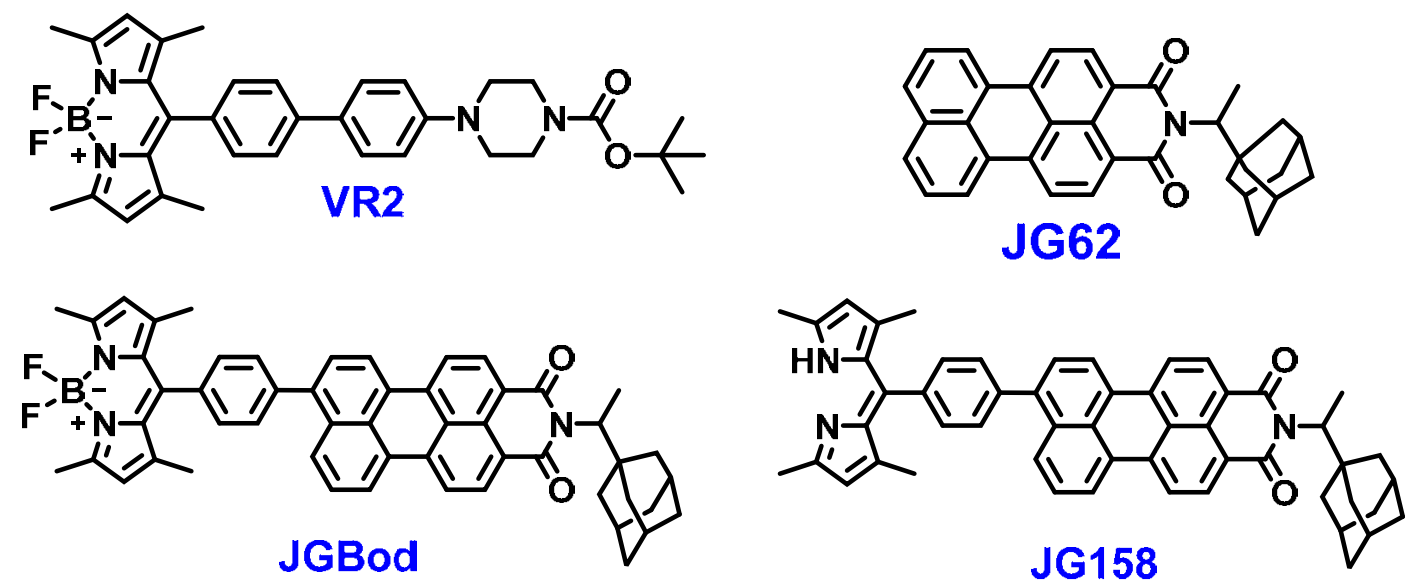

Figure 42. Molecular structure of VR2, JG62, JGBod and JG158.

The synthesis consisted of a Suzuki reaction from 4-iodophenyl bodipy with a PMI boronate in peri position (JG75). In case of VR2, a boc protected phenyl-piperazine group was used, instead of the PMI (JG75), because it was checked to have higher solubility and fluorescence than the iodinated derivative and no change in position or any remarkable effect in its solvatochromic response.

JG158 was a PMI-dipyrrin derivative synthetized from JGBod by elimination of the $-\mathrm{BF}_{2}$ group with methanesulfonic acid in DCM. ${ }^{35}$ The process led to obtaining the product quantitatively, what was quickly confirmed by the lack of fluorine signals on the ${ }^{19}$ F-NMR (see Experimental Appendix 2).

With these probes, their absorbance-fluorescence response was evaluated in comparison between the free PMI (JG62), free bodipy (VR2), combination Bodipy+PMI (JGBod) and PMI+dipyrrin (JG158). 35 J. Urieta, B. L. Maroto, F. Moreno, A. R. Agarrabeitia, M. J. Ortiza, S. de la Moya, RSC Adv., 2015, 5, 68676-
68680. 


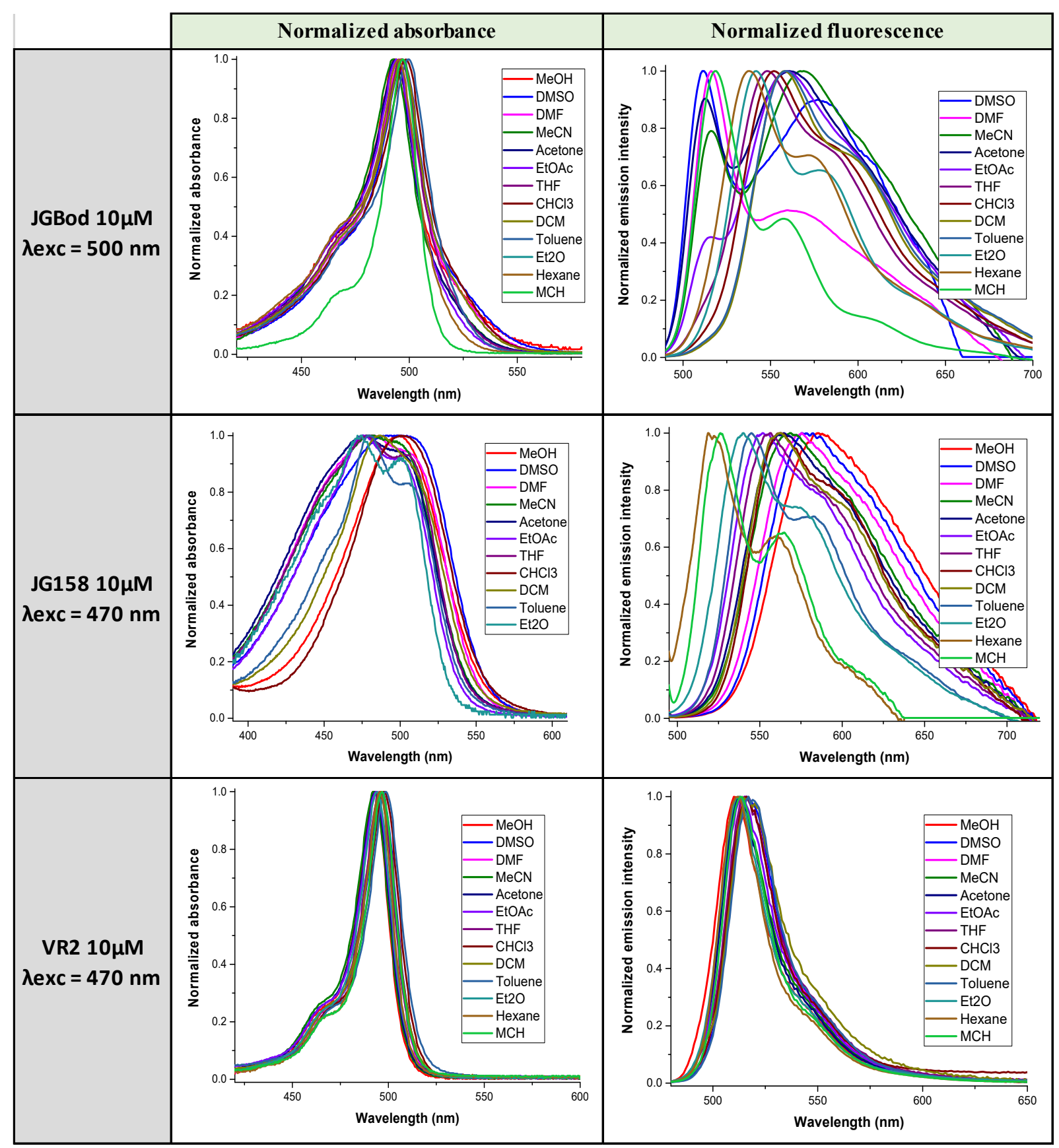

Figure 43. Normalized spectra of VR2, JGBod and JG158, $10 \mu \mathrm{M}$ in different solvents. 


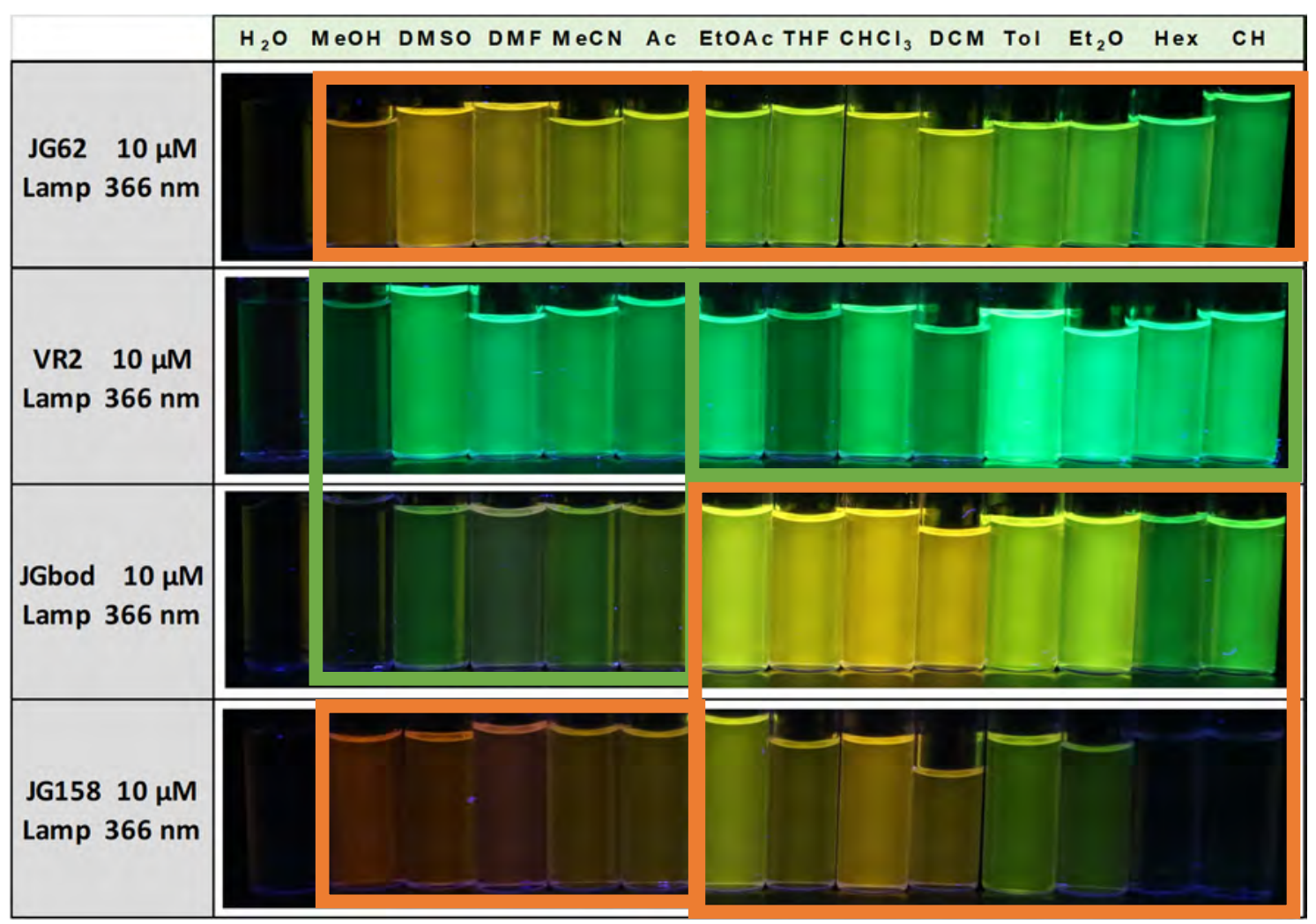

Figure 44. Pictures under UV light of JG62, VR2, JGBod and JG158, $10 \mu \mathrm{M}$ in different solvents.
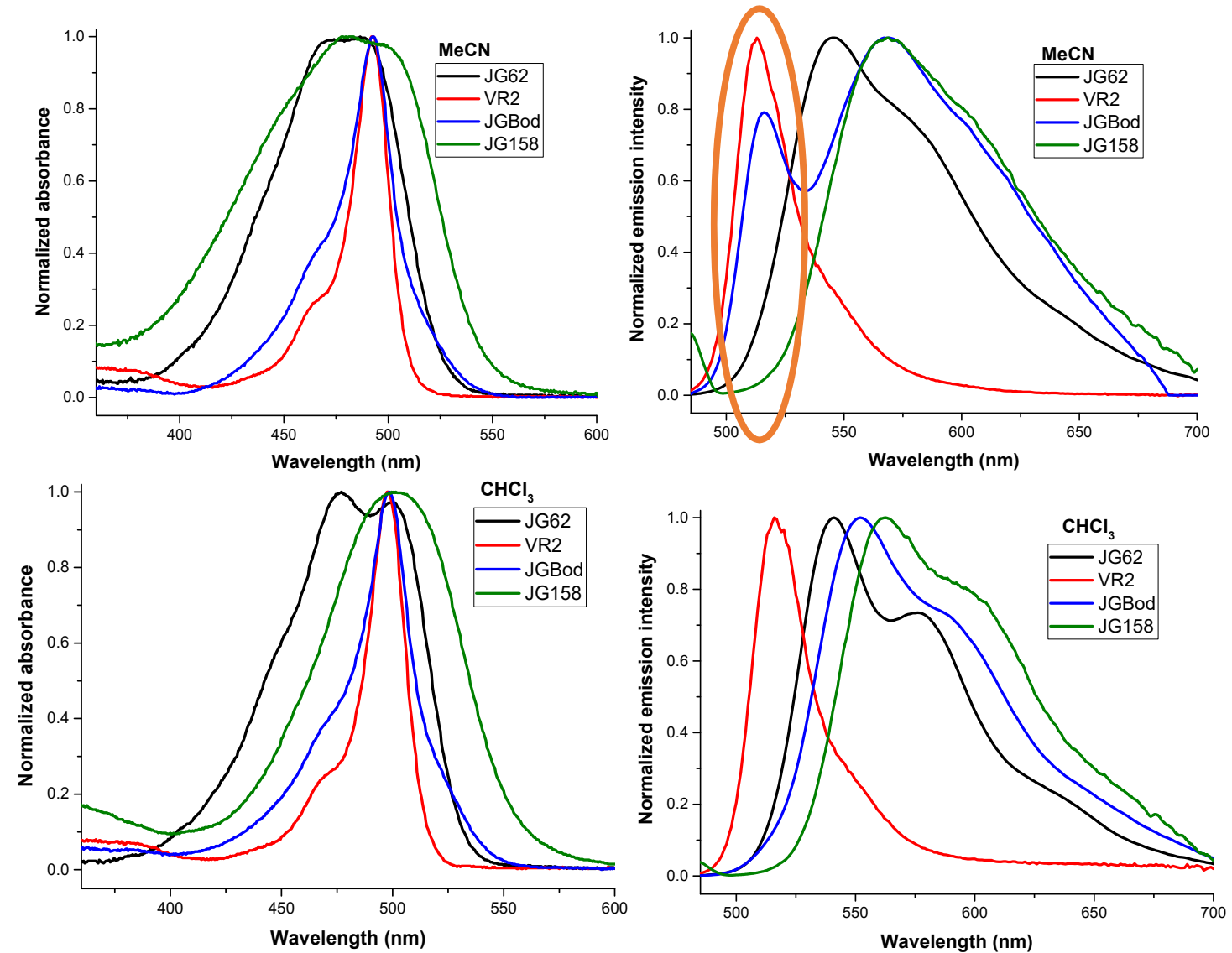

Figure 45. Normalized spectra of VR2, JGBod and JG158, $10 \mu \mathrm{M}$ in different acetonitrile (up) and in chloroform (down). 


\begin{tabular}{|c|c|c|c|c|c|c|}
\cline { 2 - 7 } \multicolumn{1}{c|}{} & $\begin{array}{c}\boldsymbol{\lambda}(\mathbf{m a x} \\
\mathbf{a b s})\end{array}$ & $\boldsymbol{\varepsilon}\left(\mathbf{c m}^{-\mathbf{1}} \mathbf{M}^{-\mathbf{1}}\right)$ & $\boldsymbol{\Phi}_{\mathbf{F}}(\mathbf{\%})$ & $\boldsymbol{\tau}(\mathbf{n s})$ & $\boldsymbol{\chi} \mathbf{2}$ & $\begin{array}{c}\text { Stokes Fl. } \\
\text { Shift }\end{array}$ \\
\hline JG62 & 500 & 34300 & 90 & 5.09 & 0.98 & $510-560$ \\
\hline VR2 & 499 & 42000 & 12 & 3.25 & 1.025 & $510-520$ \\
\hline JGBod & 499 & $\mathbf{5 4 0 0 0}$ & 97 & 4.22 & 1.075 & $510-575$ \\
\hline JG158 & 505 & 46300 & 8 & 4.14 & 1.02 & $520-585$ \\
\hline
\end{tabular}

Laser $(\tau) \quad 510 \mathrm{~nm}$

Table 1. Spectral data of VR2, JGBod and JG158 in chloroform solution.

From Figures 43, 44 and 45 and Table 1 the different behaviour of the colorants was studied. In Figure 44, it is marked in green when the fluorescence from bodipy is observed in the solution, and in orange when the fluorescence comes from the PMI. It seemed to be a tendency, which was confirmed by comparing the spectra in Figure 45. In summary several conclusions were obtained:

- The absorbance bands shape and position of bodipy (VR2) and PMI+Bodipy (JGBod) were very similar; and different from PMI (JG62) and PMI-Pyrrin (JG158), similar between them.

- The values of molar absorptivity are maximized for the molecule PMI+Bodipy (JGBod).

- When the polarity of the solvent was the one of the ethyl acetate or lower, the fluorescence of the molecules containing a PMI was quite similar in all cases; the emission of bodipy or pyrrin is completely overlapped being indistinguishable, to get an emission band similar to single PMI but with a batochromic shift.

- In case of having polarity of acetone or higher, the emission bands of PMI and bodipy were distinguishable between the two species. Process that does not occur for PMI-pyrrin (JG158) in which fluorescence was similar to a PMI derivative.

- The fluorescence quantum yield was maximized in the presence of the two fluorophores, reaching values very close to $100 \%$.

In conclusion, there are many possibilities for future applications of this kind of probes. The most straightforward one would be the creation of polarity probes, consequence of the aggregation properties, deeply studied by F. Würthner in many of the publications of his research group. ${ }^{13} \mathrm{In}$ addition, it is a first step for developing new probes working by FRET. For example, by binding a receptor between the PMI and the bodipy, a subject that is not widely studied yet, but with promising results.

Furthermore, the increase in molar absorptivity, combined with fluorescence quantum yields superior to $90 \%$ along with the possibility of tuning absorbance from one probe, and emission from the other, made them potential candidates for light harvesting applications. 


\subsection{Photophysical parameters}

This section summarizes the photophysical parameter of the PMI derivatives using for different applications during the development of this thesis (Table 2).

\begin{tabular}{|l|c|c|c|c|c|c|}
\cline { 2 - 7 } \multicolumn{1}{c|}{} & $\boldsymbol{\lambda}(\mathbf{m a x} \mathbf{A})$ & $\boldsymbol{\varepsilon}\left(\mathbf{c m}^{-\mathbf{1}} \mathbf{M}^{-\mathbf{1}}\right)$ & $\boldsymbol{\Phi}_{\mathbf{F}}(\mathbf{\%})$ & $\boldsymbol{\tau}(\mathbf{n s})$ & $\boldsymbol{\chi 2}$ & Stokes Fl. Shift \\
\hline JG62 & 500 & 34300 & 90 & 5.09 & 0.98 & $510-560$ \\
\hline JG73 & 502 & 33300 & 91 & 4.74 & 1.024 & $510-570$ \\
\hline JG75 & 508 & 22100 & 88 & 4.82 & 1.013 & $515-570$ \\
\hline JG2L & 507 & 34200 & 99 & 4.49 & 0.999 & $510-570$ \\
\hline JG7L & 511 & 48900 & 92 & 4.52 & 1.044 & $510-575$ \\
\hline JG125 & 513 & 40900 & 23 & 3.41 & 0.995 & $560->700$ \\
\hline JG116 & 511 & 40000 & 88 & 3.69 & 1.072 & $550-660$ \\
\hline JGphen & 516 & 32300 & $68^{*}$ & 4.23 & 1.123 & $540->700$ \\
\hline JG125d & 512 & 22700 & $78^{*}$ & 3.60 & 1.005 & $565-700$ \\
\hline JG117 & 509 & 28700 & 92 & 4.01 & 1.166 & $550-650$ \\
\hline JGphend & 515 & 17020 & 69 & 3.78 & 1.134 & $575->700$ \\
\hline JG119c1 & 508 & 37100 & 77 & 3.84 & 1.077 & $500-650$ \\
\hline JG119c2 & 508 & 32700 & 87 & 3.89 & 1.026 & $550-640$ \\
\hline JG76 & 511 & 19100 & 58 & 3.70 & 1.134 & $500-*$ \\
\hline JG121 & 507 & 33200 & 99 & 3.75 & 1.066 & $500-660$ \\
\hline JG10L & 576 & 46300 & 38 & 4.74 & 1.078 & $515-585$ \\
\hline JG11L & 505 & 46000 & 30 & 4.57 & 1.098 & $510-570$ \\
\hline VR2 & 499 & 42000 & 12 & 3.25 & 1.025 & $510-520$ \\
\hline JGBod & 499 & 54000 & 97 & 4.22 & 1.075 & $510-575$ \\
\hline JG158 & 505 & 46300 & 8 & 4.14 & 1.02 & $520-585$ \\
\hline Solvents & & CHCl 3 & DCM & & \\
Laser $(\tau)$ & 510 nm & & & & & \\
Shift & + & Red with polarity & From MeOH to MCH or CH \\
\hline & low solubility and deep red in solvents with low polarity. & \\
\hline
\end{tabular}

Table 2. Spectral data of the synthesized probes in similar conditions.

Table 2 shows how the different properties change depending on the substitution in the same solvent, except JG10L and JG11L that underwent degradation in chloroform.

General conclusions:

- When the conjugation of PMI was increased the wavelength of absorbance increased.

- The $\Phi_{\mathrm{F}}$ was high for PMIs in chloroform except for JG158. Donor groups decreased the final fluorescence (PET quenching) but, being chloroform a solvent with pseudo acid behaviour, the effect was minimized respect to others (See JG76 or JG125).

- The $\tau$ was always between 3.5-5 ns.

- The stokes shift in fluorescence between solvents (MeOH-MCH) was maximized for JG116, JG125 and their derivatives; the PMIs containing a pyrimidine-piperazine or pyridinepiperazine group in peri. This fact made them the best polarity sensitive sensors, which is very useful for cellular localization. 


\section{SPECIFIC APPLICATIONS OF THE SYNTHESIZED PMI DERIVATIVES}

\subsection{Sensors}

As other fluorescent backbones, PMIs can be used for the development of fluorescent sensors. Chapter 3 of the thesis is focused in some of the different uses of the synthesized fluorescent molecules for the detection of several species of interest.

The different modifications performed in the thesis were two, one was the introduction of a donor group, whose electrons were capable of quenching the fluorescence of the molecule in solution (PET mechanism). The donor group was surrounded at the same time by a recognition system (for instance a crown ether) capable of recognising species selectively (such as $\mathrm{K}^{+}$) and once it was complexed, the electrons from the donor group were no longer free and quenching the fluorescence that was detected and analysed. (Chapter 3C)

In contrast to Chapter 3C, sensors based on a reaction mechanism were also synthesised. In the end, the process is quite similar; the synthetized probe has a donor group that inhibits fluorescence, when it recognises a substrate, it reacts and the product no longer suffers from quenching. That is the case of the synthetized probes for explosives. (Chapter 3A)

Finally, there is a third case for probes having a metal core. A metal (such as Ru(II)) was complexed with different ligands. This complex interacted with the analyte of interest, usually changing the coordination sphere, which produced a change in its luminescence properties. (Chapter 3B)

In summary, different probes for analyte detection were synthetized, detection of explosives (triacetone triperoxide) (Chapter 3A), carbon monoxide (Chapter 3B), potassium cations (and some other ionophores of potassium, indirectly) and lead(II) cations (Chapter 3C).

\subsection{Interaction studies with DNA}

In the development of the thesis, several derivatives from PMI were synthetized with the specific purpose of studying their interaction with biological samples. In doing so, the group collaborated with other researchers ${ }^{36}$ to measure parameters like cytotoxicity, interaction with DNA (comparison G2-G4) and cellular location. All these parameters have many interests:

Cytotoxicity: having selective cytotoxicity between different cell cultures might be useful, selectivity to produce death over tumoral cells and not in the healthy ones is a common objective for drugs.

Interaction with DNA, in particular, selectivity to the DNA G-quadruplex. The selective detection has proven to be of great interest. Without going into greater detail, which may be found elsewhere, ${ }^{37}$ due to not being the fundamental purpose of the thesis, some of the possible functions of the Gquadruplex are in chromosome sites as promoters of genes or oncogenes ${ }^{38}$ minisatellites of the

\footnotetext{
${ }^{36}$ Collaborations performed with the group of Begoña García, at Burgos University, and Sebastian Pons, at Barcelona University. The measurements were performed by Natalia Busto and Antonio Herrera, respectively.

${ }^{37}$ G. Biffi, D. Tannahill, J. McCafferty, S. Balasubramanian, Nat. Chem. 2013, 5, 182-186.

${ }^{38}$ L. Yuan, T. Tian, Y. Chen, S. Yan, X. Xing, Z. Zhang, Q. Zhai, L. Xu, X. Weng, B. Yuan, Y. Feng, X. Zhou, Sci. Rep. 2013, 3, 1811-1820.
} 
chromosomes (associated to hypermutability) ${ }^{39}$ and genomic instability or telomeres, stabilizing their structure. $^{40}$

Cellular location: PMI derivatives are fluorescent in the region of visible-infrared what makes them ideal for studies of localization in cells as fluorescent markers. Cellular fluorescent markers have many applications, such as to know where a drug acts or what happens to a particular part of a cell after being under treatment.

There are antecedents of using perylene derivatives in biological research, such as for PIPER (Figure 13). Molecules like PIPER present a broad hydrophobic core and aggregation properties that interact with DNA-duplex or quadruplex selectively. In addition, the complexation constants resulted to be of high order $\left(10^{5}-10^{7} \mathrm{M}^{-1}\right)$. The five PMI derivatives from Figure 46 were studied in different cell cultures (such as tumoral HeLa cells and non-tumoral cells, IMR-9), looking for any change in the cells or their fluorescence.

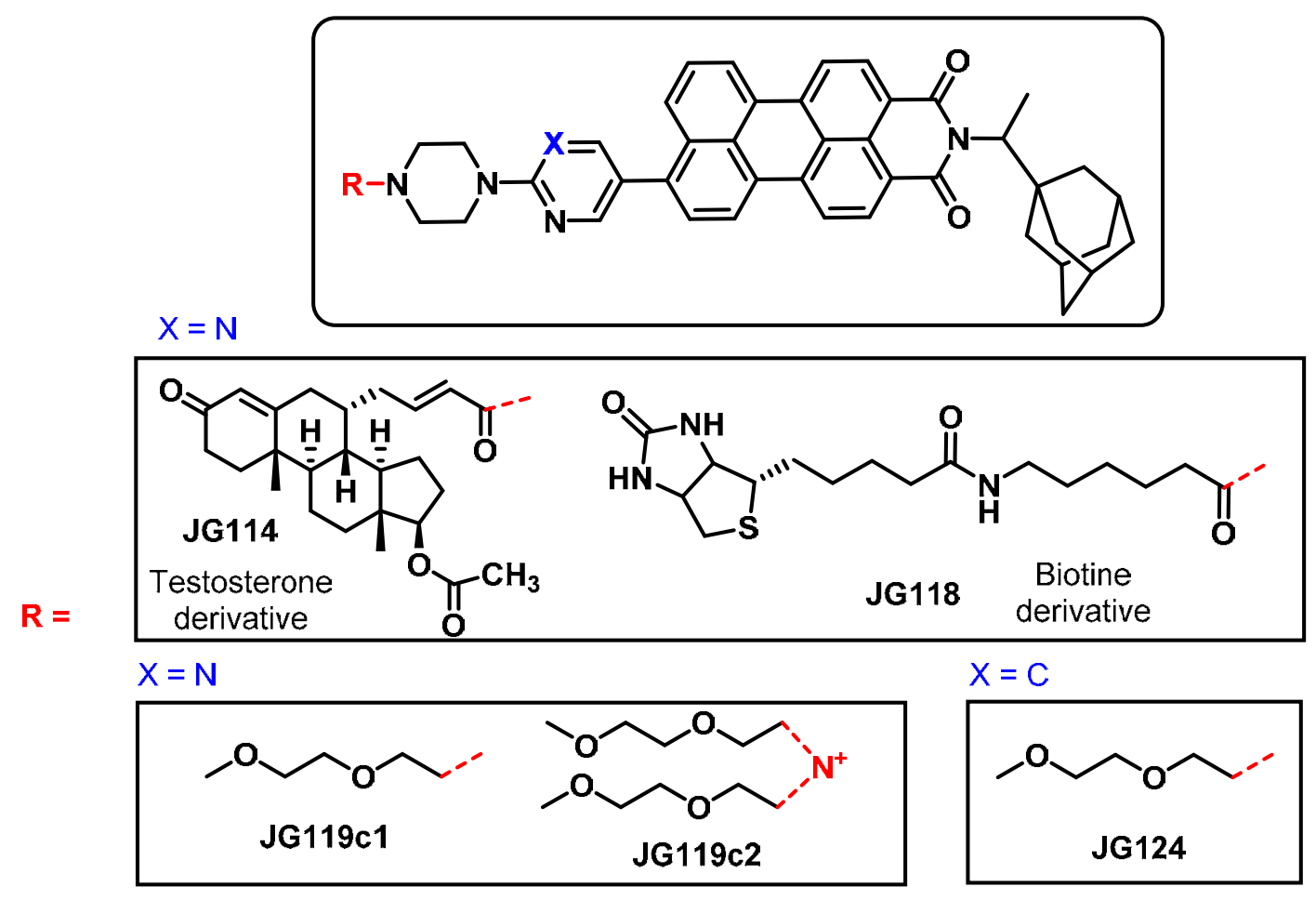

Figure 46. PMI structures synthetized for biological studies.

Unremarkable results were observed for JG114 and JG118, having both a slight decrease in fluorescence in presence of DNA-G4, with no selectivity. In case of JG118 a low level of cytotoxicity was also observed. For the PEG derivatives, JG119c1 and JG124 led to results of no particular interest (G4 interaction, cytotoxicity or cellular location). In contrast, preliminary results with JG119c2 showed that this molecule was capable of interacting selectively with DNA G4 (for instance in telomers, Figure 47), by an increase in fluorescence that was also registered by cellular microscopy (Figure 48).

\footnotetext{
${ }^{39}$ R. B. Alec, J. Jeffreys, P. Bois, J. Buard, Y. E. Dubrova, G. Grant, C. R. H. Hollies, C. A. May, R. Neumann, M. Panayi, A. E. Ritchie, A. C. Shone, E. Signer, J. D. H. Stead, K. Tamaki, Electrophor. 1999, 20, 1665-1675. ${ }^{40}$ L. L. Anne De Cian, C. Douarre, N. Temime-Smaali, C. Trentesaux, J.-F. Riou, J.-L. Mergny, Biochimie 2008, 90, 131-155
} 


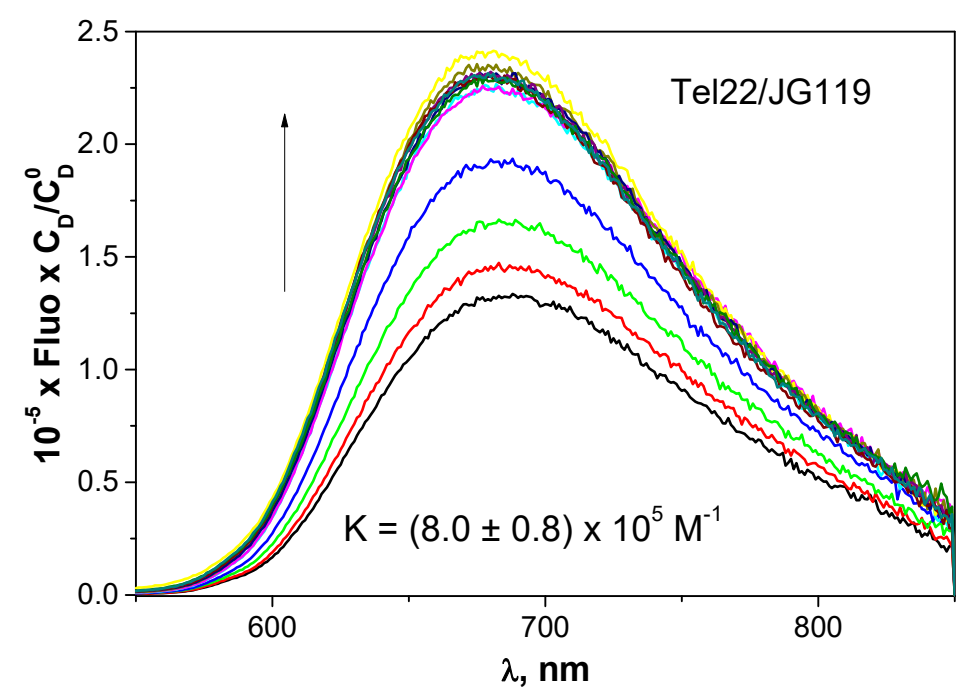

Figure 47. Fluorescence spectra recorded during Tel22/JG119c2 titration. $\lambda_{\text {exc }}=486 \mathrm{~nm}, C_{D}=14 \mu \mathrm{M}$, $\mathrm{C}_{\mathrm{P}} / \mathrm{C}_{\mathrm{D}}=0-5, \mathrm{I}=0.11 \mathrm{M}(90 \mathrm{mM} \mathrm{LiCl}, 10 \mathrm{mM} \mathrm{KCl}$ and $10 \mathrm{mM}$ lithium cacodylate $), \mathrm{pH}=7.2$ and $\mathrm{T}$ $=25{ }^{\circ} \mathrm{C}$.

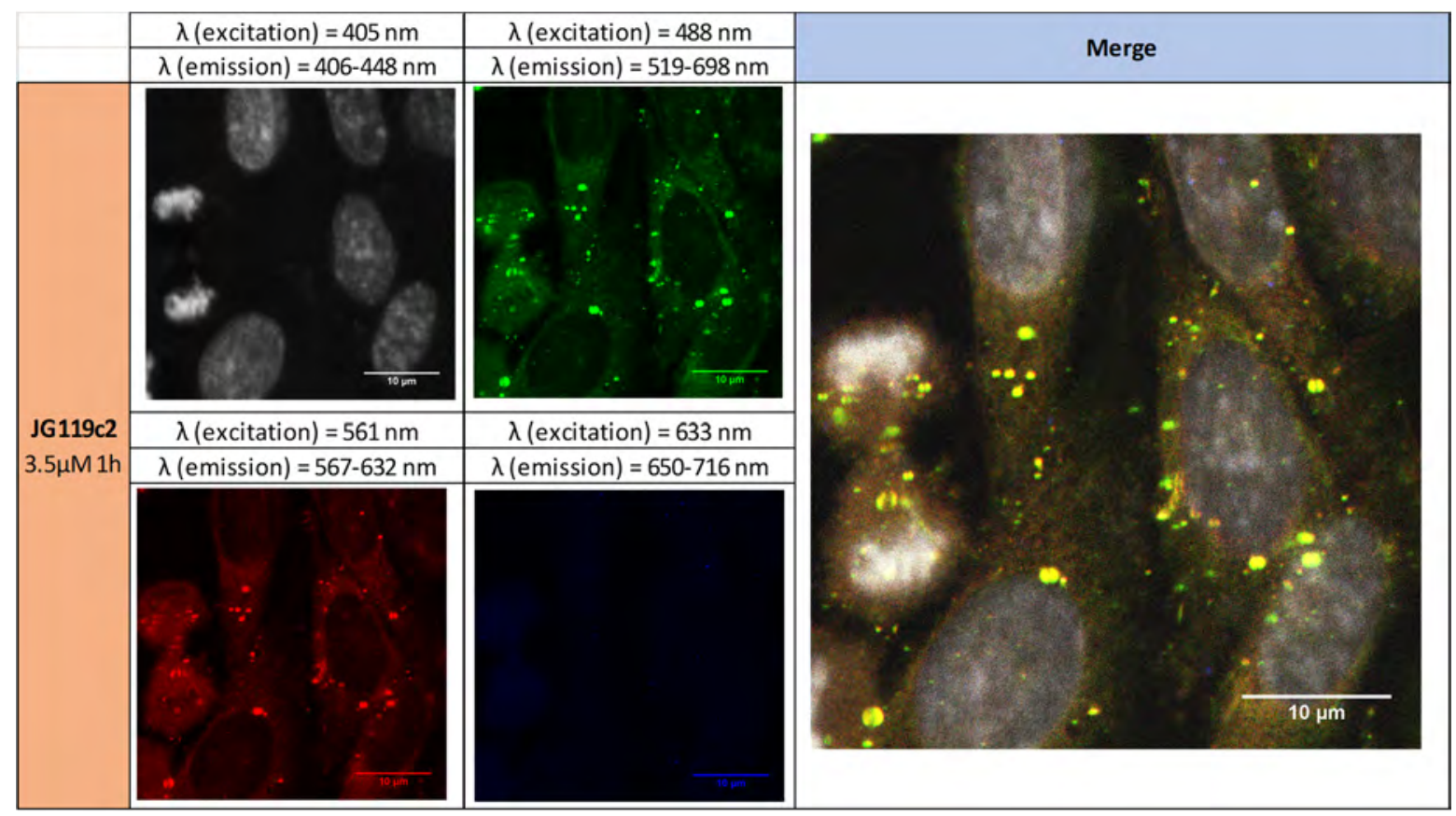

Figure 48. Cellular uptake of $50 \mu \mathrm{M}$ JG119c2 in HEK cells after 1 hour of incubation. The probe is located around the nuclei.

These results were promising, leading to future research in the field, especially for JG119c2 and derivatives. In addition to these probes, specifically designed for biological measurements and cellular imaging, some of the other PMI derivative probes synthetized as sensors were also tested in cells, such as the $\mathrm{CO}$ sensitive derivatives or the fluorescent potassium ionophores; the results are specified in Chapters 3B and 3C, respectively. 


\section{RESUMEN DEL CAPÍTULO}

Este capítulo sirve de introducción a las propiedades y características de los derivados de perileno, sustratos coloreados y fluorescentes con potencial aplicación en la creación de sensores de gran eficiencia y estabilidad, así como materiales con aplicaciones en fotoelectrónica y medicina.

En primera instancia, se explican los métodos de síntesis y modificación molecular para la creación de derivados de perileno de interés. De entre las múltiples opciones, los más comunes y más extensivamente utilizados, son las perilenodiimidas (PDI) y perilenomonoimidas (PMI). Esto es así debido a su color rojizo, su fluorescencia en la región del visible $(500-900 \mathrm{~nm})$ y la posibilidad de modificación de múltiples maneras para la modulación de sus propiedades físicas (como la solubilidad) y fotoquímicas (región de absorción-emisión fluorescente y su posible variación en condiciones controladas).

En este sentido es particularmente importante la capacidad para variar de absorción-fluorescencia según los sustituyentes, lo cual afecta indirectamente las posibilidades de empaquetamiento y, por tanto, la solubilidad.

Las aplicaciones de las PMI y PDI son variadas, desde tintes a sondas moleculares pasando por receptores de luz para células fotovoltaicas. Por todo ello, el campo a tratar es amplio, centrándose en la síntesis, optimización y obtención de determinadas variantes de PMIs que podrían resultar de interés. En este sentido, y como introducción al Capítulo 3, distintos derivados sintetizados se han comparado en distintos disolventes, así como los parámetros que presentan; tales como rendimiento cuántico de fluorescencia, tiempo de vida de fluorescencia o absortividad molar. Todo esto permite hacerse una idea de cuales podrían ser los más útiles y que funciones podrían llevar a cabo, como la interacción selectiva con ADN, optimización de células fotovoltaicas o sensores químicos. Todas estas propiedades se encuentran más ampliadas para casos concretos durante el desarrollo de las distintas partes del Capítulo 3. 


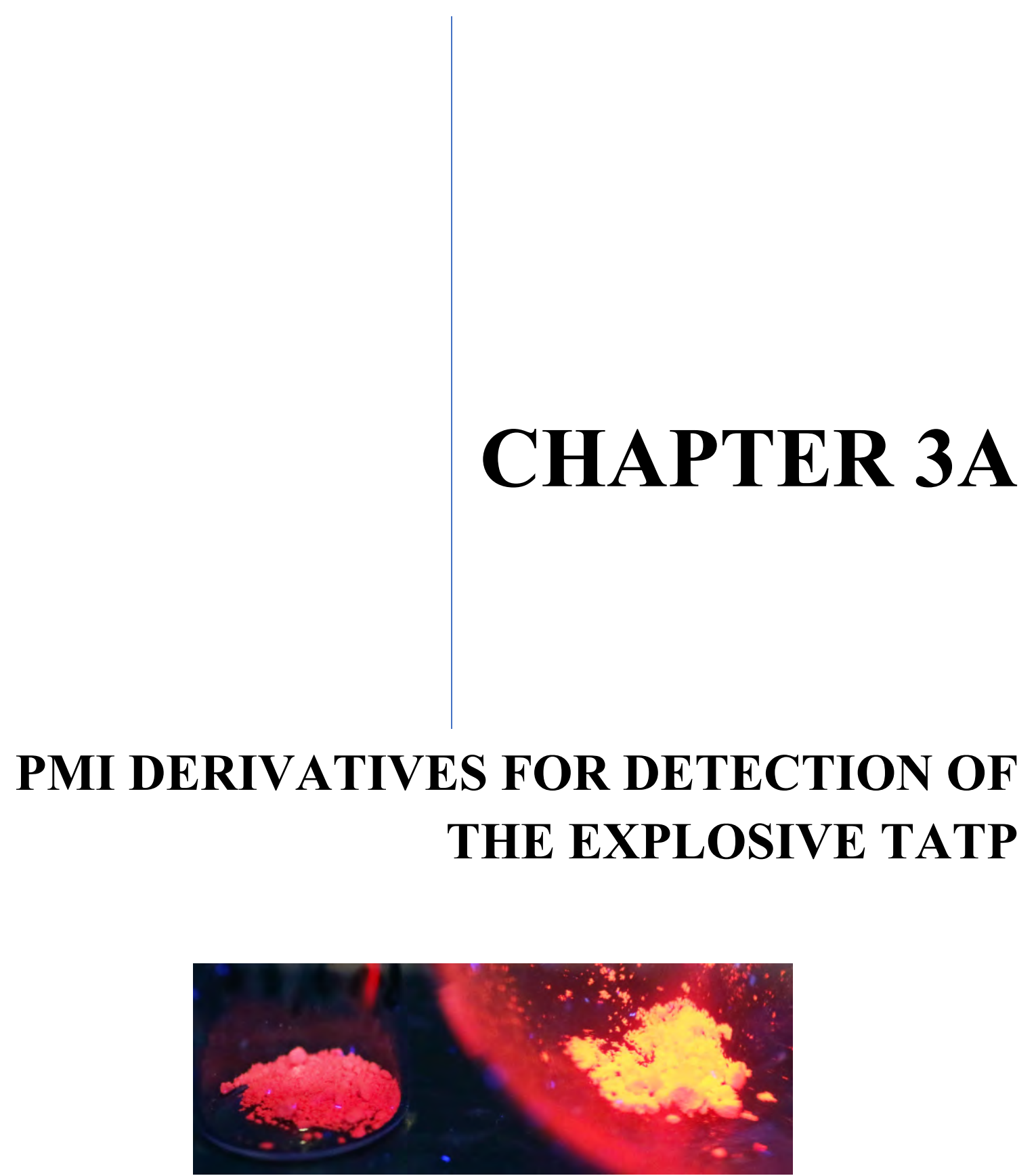

\section{ABSTRACT}

Within the development of the thesis, several PMI derivatives have been synthesised and modified as fluorescent backbones for the synthesis of fluorescent probes. Some PMI derivatives have proven to be sensitive and selectively oxidized by the explosive TATP giving a measurable increase in fluorescence. ${ }^{1}$

${ }^{1}$ J. García-Calvo, P. Calvo-Gredilla, M. Ibáñez-Llorente, D. C. Romero, José V. Cuevas, G. García-Herbosa, M. Avella, T. Torroba, J. Mater. Chem. A, 2018, 6, 4416-4423. 



\section{INTRODUCTION}

An explosive is defined as a substance that reacts violently in presence of another chemical reagent. This process is characterized for emitting gases at such high temperature, pressure and speed that affects the surroundings. ${ }^{2}$ They are usually classified by their use and the easiness of ignition (Figure 1).

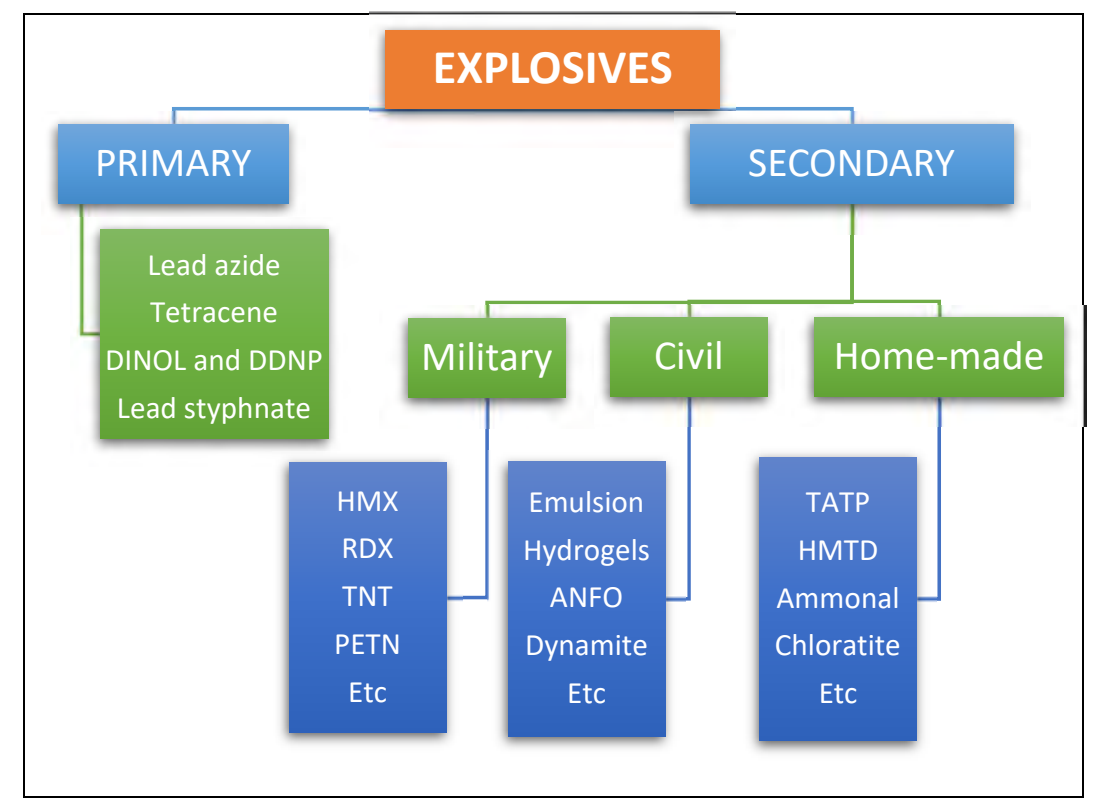

Figure 1. Types of explosives, classification scheme. ${ }^{3}$

Primary explosives are easily ignited, being what starts secondary ones. Secondary explosives are easier to handle and usually the main source of the explosion. ${ }^{4}$ Within this group, triacetone triperoxide is the one this work is centred of. As a chemical reagent, it belongs to the group of peroxide explosives, having oxidizing features. In addition, it has some analogue structures such as the diacetone diperoxide (DADP) or hexamethylene triperoxide amine (HMTD) (Figure 2).

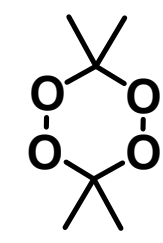

DADP

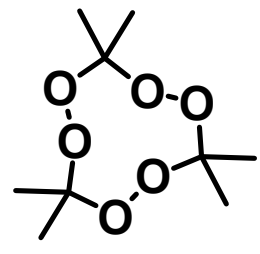

TATP

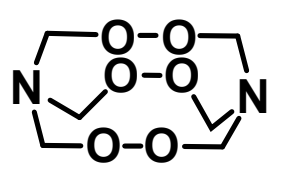

HMTD

Figure 2. Explosives based on peroxides.

TATP is $88 \%$ more powerful than TNT and with very low vapour pressure, $7 \mathrm{~Pa}$ (sublimation of $0.9 \%$ weight per hour), ${ }^{5}$ which makes it unsuitable for military uses.

\footnotetext{
${ }^{2}$ REAL DECRETO 230/1998, de 16 de febrero, por el que se aprueba el Reglamento de explosivos.

3 J. Yinon, S. Zitrin, Modern Methods and Applications in Analysis of Explosives, Wiley, West Sussex, Chinchester, 1993.

${ }^{4}$ S. Fordham. High Explosives and Propellants, Pergamon international library, Oxford, 1980.

${ }^{5}$ J. C. Oxley, H. Chen, Propellants Explos. Pyrotech. 2002, 27, 197-246.
} 


\subsection{The importance of TATP and its detection}

Triacetone triperoxide (TATP) is a powerful explosive with no military use due to its too high sensitivity to mechanical shock and difficulty to safe handling. ${ }^{6}$ In addition, TATP is easily prepared from acetone and hydrogen peroxide under acidic catalysis. ${ }^{7}$ and its appearance does not draw attention, being a white powder similar to sugar (Figure 3).

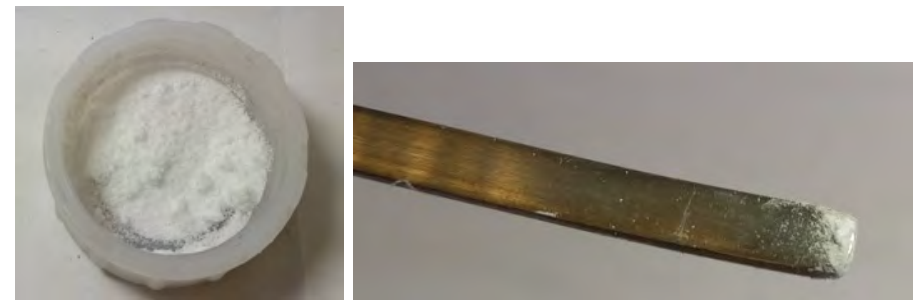

Figure 3. Pictures of TATP powder.

Due to these facts, TATP has been frequently used in suicide terrorist attacks, comprising an important threat in mass events and public transport. ${ }^{8}$

\subsection{Methods for detection of TATP}

TATP constitutes an improvised explosive almost untraceable by dogs or sniffer devices, usually trained for nitrogen-containing explosives ${ }^{9}$. The lack of nitro groups or aromatic moieties makes detection of this improvised explosive a hard task, ${ }^{10}$ although the number of studies in the field has increased in the last years. Until now, TATP is detected by mass spectrometry, ${ }^{11}$ ion mobility spectrometry, ${ }^{12}$ or multiphoton spectroscopy, ${ }^{13}$ although the signature of TATP is not clearly visible except by bulky mass spectrometers. ${ }^{14}$ As a consequence, portable and selective devices still need to be developed.

\footnotetext{
${ }^{6}$ a) M. A. C. Härtel, T. M. Klapötke, B. Stiasny, J. Stierstorfer, Propellants Explos. Pyrotech. 2017, 42, 623634; b) Homemade explosives, Indiana Intelligence Fusion Center, 2015, 302 W. Washington St., Indianapolis, http://www.arkiaai.com/files/handouts/2016/Spring/homemade_explosives.pdf, accessed June 2018.

${ }^{7}$ F. Dubnikova, R. Kosloff, J. Almog, Y. Zeiri, R. Boese, H. Itzhaky, A. Alt, E. Keinan, J. Am. Chem. Soc. 2005, 127, 1146-1159.

${ }^{8}$ M. Ranstorp, M. Normark, Eds.: Understanding Terrorism Innovation and Learning: Al-Qaeda and Beyond, Routledge, Taylor \& Francis Group LLC, 7625 Empire Drive, Florence, Kentucky 41042-2919, USA, Chapter 1, pp. 1-15, 2015.

9 a) K. Yeager, in: Trace Chemical Sensing of Explosives, (Ed.: R. L. Woodfin), Wiley, New Jersey, Chapter 3, pp. 43-67, 2007; b) T.-H. Ong, T. Mendum, G. Geurtsen, J. Kelley, A. Ostrinskaya, R. Kunz, Anal. Chem. 2017, 89, 6482-6490; c) S. Sheykhi, L. Mosca, P. Anzenbacher Jr., Chem. Commun. 2017, 53, 5196-5199.

${ }^{10}$ a) R. Schulte-Ladbeck, M. Vogel, U. Karst, Anal. Bioanal. Chem. 2006, 386, 559-565; b) M. J. Kangas, R. M. Burks, J. Atwater, R. M. Lukowicz, P. Williams, A. E. Holmes, Crit. Rev. Anal. Chem. 2017, 47, 138-153; c) S. Girotti, E. Ferri, E. Maiolini, L. Bolelli, M. D’Elia, D. Coppe, F. S. Romolo, Anal. Bioanal. Chem. 2011, 400, 313-320; d) G. E. Collins, M. P. Malito, C. R. Tamanaha, M. H. Hammond, B. C. Giordano, A. L. Lubrano, C. R. Field, D. A. Rogers, R. A. Jeffries, R. J. Colton, S. L. Rose-Pehrsson, Rev. Sci. Instrum. 2017, 88, 1-9.

${ }^{11}$ M. Makinen, M. Nousiainen, M. Sillanpaa, Mass Spectrom. Rev. 2011, 30, 940-973.

${ }^{12}$ D. Jiang, L. Peng, M. Wen, Q. Zhou, C. Chen, X. Wang, W. Chen, H. Li, Anal. Chem. 2016, 88, 4391-4399.

${ }^{13}$ S. Tang, N. Vinerot, D. Fisher, V. Bulatov, Y. Yavetz-Chen, I. Schechter, Talanta 2016, 155, 235-244.

${ }^{14}$ a) J. Tomlinson-Phillips, A. Wooten, J. Kozole, J. Deline, P. Beresford, J. Stairs, Talanta 2014, 127, 152-162;

b) D. N. Correa, J. J. Melendez-Perez, J. J. Zacca, R. Borges, E. M. Schmidt, M. N. Eberlin, E. C. Meurer, Propellants Explos. Pyrotech. 2017, 42, 370-375; c) S. Hagenhoff, J. Franzke, H. Hayen, Anal. Chem. 2017, 89, $4210-4215$
} 
In this regard, some research has been oriented to the field of chemically modified nanosensor arrays, ${ }^{15}$ and optical portable methods. Up to date, the detection has been performed by the indirect detection of $\mathrm{H}_{2} \mathrm{O}_{2}$ from TATP and linked to oxidative processes, ${ }^{16}$ what was successfully achieved by colorimetric $^{17,18}$ (Figure 4) or fluorimetric ${ }^{13 b, 19}$ sensing. However, acetone or water peroxide are not dangerous but very common substances, which may lead to false positives due to their presence in many products, such as cosmetics. In fact, it constitutes the main reason for not allowing to introduce liquids in mass events or planes. Although this kind of devices would give quick results, it is likely to be a false positive.

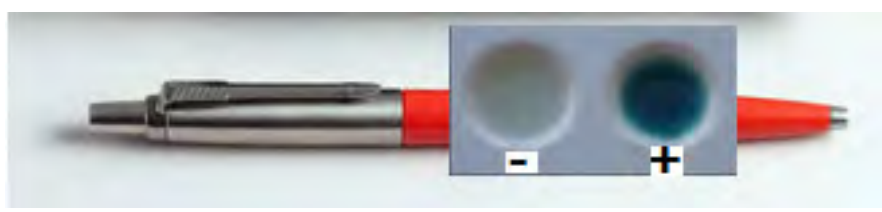

Figure 4. ACRO-P.E.T. - a simple to use pen like device for colorimetric detection of explosives, ${ }^{20}$ based on the degradation products of peroxides.

In contrast, TATP direct detection has only been achieved by fluorescence quenching ${ }^{21}$ or quartz crystal microbalances. ${ }^{22}$ Nevertheless, it has been widely demonstrated that turn-ON fluorogenic chemosensing systems are sensitive and selective tools for many analytes. ${ }^{17}$ Therefore, fluorogenic probes that are specific for TATP are highly valuable methods for detection of peroxide explosives. In this way, to find a substrate that is capable to undergo a turn-on fluorescence by a selective oxidation is the goal of many researchers.

\footnotetext{
${ }^{15}$ A. Lichtenstein, E. Havivi, R. Shacham, E. Hahamy, R. Leibovich, A. Pevzner, V. Krivitsky, G. Davivi, I. Presman, R. Elnathan, Y. Engel, E. Flaxer, F. Patolsky, Nat. Commun. 2014, 5, Art. 4195.

${ }^{16}$ a) S, Parajuli and W. Miao, Anal. Chem. 2013, 85, 8008-8015; b) S. Malashikhin and N. S. Finney, J. Am. Chem. Soc. 2008, 130, 12846-12847.

17 a) A. Üzer, S. Durmazel, E. Ercag, R. Apak, Sens. Actuators B 2017, 247, 98-107; b) Z. Can, A. Uzer, K. Turkekul, E. Ercag, R. Apak, Anal. Chem. 2015, 87, 9589-9594; c) M. Xu, J.-M. Han, C. Wang, X. Yang, J. Pei, L. Zang, ACS Appl. Mater. Interfaces 2014, 6, 8708-8714.

${ }^{18}$ a) H. Lin, K. S. Suslick, J. Am. Chem. Soc. 2010, 132, 15519-15521; b) Z. Li, W. P. Bassett, J. R. Askim, K. S. Suslick, Chem. Commun. 2015, 51, 15312-15315; c) J. R. Askim, Z. Li, M. K. LaGasse, J. M. Rankin, K. S. Suslick, Chem. Sci. 2016, 7, 199-206; d) V. Kumar, K.-H. Kim, P. Kumar, B.-H. Jeon, J.-C. Kim, Coord. Chem. Rev. 2017, 342, 80-105.

${ }^{19}$ a) E. Sella, D. Shabat, Chem. Commun. 2008, 5701-5703; b) W. Xu, Y. Fu, Y. Gao, J. Yao, T. Fan, D. Zhu, Q. He, H. Cao, J. Cheng, Chem. Commun. 2015, 51, 10868-10870; c) J. Chen, W. Wu, A. J. McNeil, Chem. Commun. 2012, 48, 7310-7312; d) Y. Salinas, R. Martínez-Máñez, M. D. Marcos, F. Sancenón, A. M. Costero, M. Parra, S. Gil, Chem. Soc. Rev. 2012, 41, 1261-1296.

${ }^{20}$ Z. C. Caron, D. J. Meekins, M. J. Platek, O. J. Gregory, Nanomat. Synth, 2016, 1, 1531-1537.

${ }^{21}$ a) M. R. Rao, Y. Fang, S. De Feyter, D. F. Perepichka, J. Am. Chem. Soc. 2017, 139, 2421-2427; b) H. Q. Zhang, W. B. Euler, Sens. Actuators B 2016, 225, 553-562.

22 a) D. Lubczyk, C. Siering, J. Lörgen, Z. B. Shifrina, K. Müllen, S. R. Waldvogel, Sens. Actuators B 2010, 143, 561-566; b) D. Lubczyk, M. Grill, M. Baumgarten, S. R. Waldvogel, K. Müllen, ChemPlusChem 2012, 77, $102-105 ;$ c) B. A. G. Hammer, K. Müllen, Chem. Rev. 2016, 116, 2103-2140.
} 


\section{OBJECTIVES}

In Chapter 2, it has been demonstrated that perylenemonoimides (PMIs) as well as perylene diimides (PDIs) are good options when searching for new fluorogenic reporters. PMIs/PDIs are suitable for multiple chemical modifications and optical sensing, ${ }^{23}$ that could be appropriate for the selective detection of many analytes; between them oxidizing agents and specifically, pristine TATP.

The aim was to modify the fluorescent PMI and PDI cores with donor groups to obtain oxidizable compounds suitable for anchoring to surfaces, which may lead to sensitive and selective detection of TATP. From the initial tests, it was discovered that a modification of PMI and PDI cores with different groups resulted in the modulation of the electron donor-acceptor effect on the fluorescent core (see solvatochromism studies, Chapter 2). With the purpose of detecting oxidative species, a group of PMI derivatives containing a piperazine was chosen because of the outstanding performance in terms of selectivity and sensitivity, along with the possibility to perform covalent anchoring to different matrixes, by one amino-group.

The first results of the research were published using a PDI derivative, being adapted for detection of TATP in solution and over a polymeric surface (Figures 5 and 6). ${ }^{24}$ After that, the research was oriented to detect TATP in vapour flow, a yet unresolved issue, ${ }^{25}$ using both PDI and PMI derivatives. The results explained in this chapter are focused in PMI derivatives, as part of the published paper. ${ }^{1}$
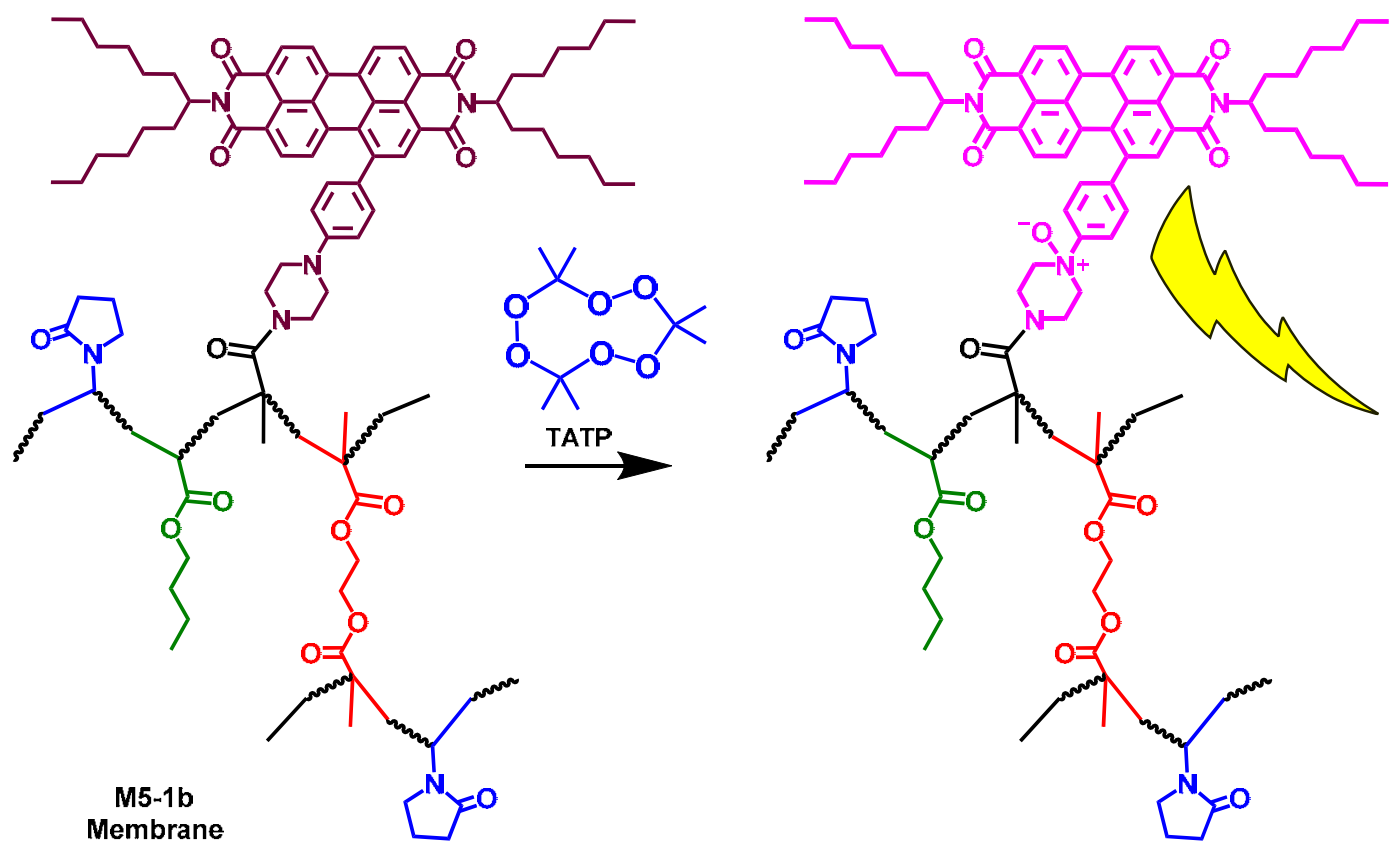

Figure 5. Film synthetized in previous research, ${ }^{23}$ sensitive to TATP.

\footnotetext{
${ }^{23}$ a) M. T. Vagnini, M. W. Mara, M. R. Harpham, J. Huang, M. L. Shelby, L. X. Chen, M. R. Wasielewski, Chem. Sci. 2013, 4, 3863-3873; b) Z. Liu, C. Tonnelé, G. Battagliarin, C. Li, R. A. Gropeanu, T. Weil, M. Surin, D. Beljonne, R. Lazzaroni, M. Debliquy, J.-M. Renoirt, K. Müllen, J. Phys. Chem. B 2014, 118, 309-314; g) A. Sanguineti, M. Sassi, R. Turrisi, R. Ruffo, G. Vaccaro, F. Meinardi, L. Beverina, Chem. Commun. 2013, 49, 1618-1620; c) P. Shao, N. Jia, S. Zhang, M. Bai, Chem. Commun. 2014, 50, 5648-5651.

${ }^{24}$ P. Calvo-Gredilla, J. García-Calvo, J. V. Cuevas, T. Torroba, J. L. Pablos, F. C. García, J. M. García, N. ZinkLorre, E. Font-Sanchis, A. Sastre-Santos, F. Fernández-Lázaro, Chem. Eur. J. 2017, 23,13973-13979.

${ }^{25}$ N. Gomes, Trace Detection of TATP Vapors Using a Low-Mass Thermodynamic Sensor. Open Access Master's Thesis, 2017, 1067. http://digitalcommons.uri.edu/theses/1067.
} 


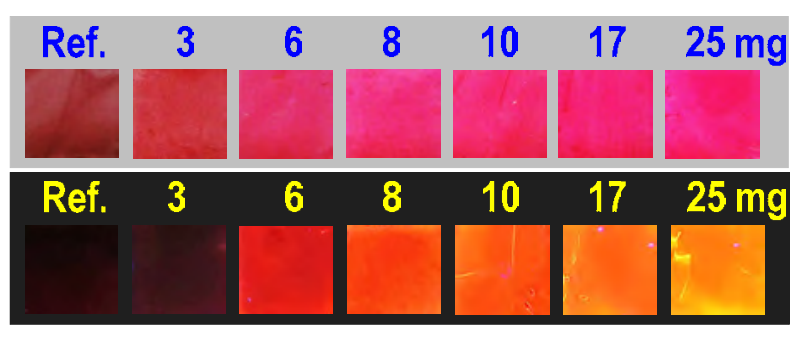

Figure 6. The qualitative effect of exposing the M5-1b membrane to increasing amounts of TATP vapor; under white light (up) and under UV light (down). 


\section{SYNTHESIS OF PMI DERIVATIVES SENSITIVE TO TATP}

\subsection{Synthesis of the PMI molecular derivatives and election of the probe}

In case of the synthesized PMI derivatives it was planned to develop them to work by selective oxidative reaction. In this regard, several molecules containing piperazine derivatives were tested to be susceptible to oxidation. Then, PMI containing this kind of substitution were potentially capable to perform these changes.

Starting from the brominated PMI JG73 (Figure 7), it was modified by Suzuki reaction with phenyl, pyridine and pyrimidine - piperazine derivatives.<smiles></smiles>

JG73

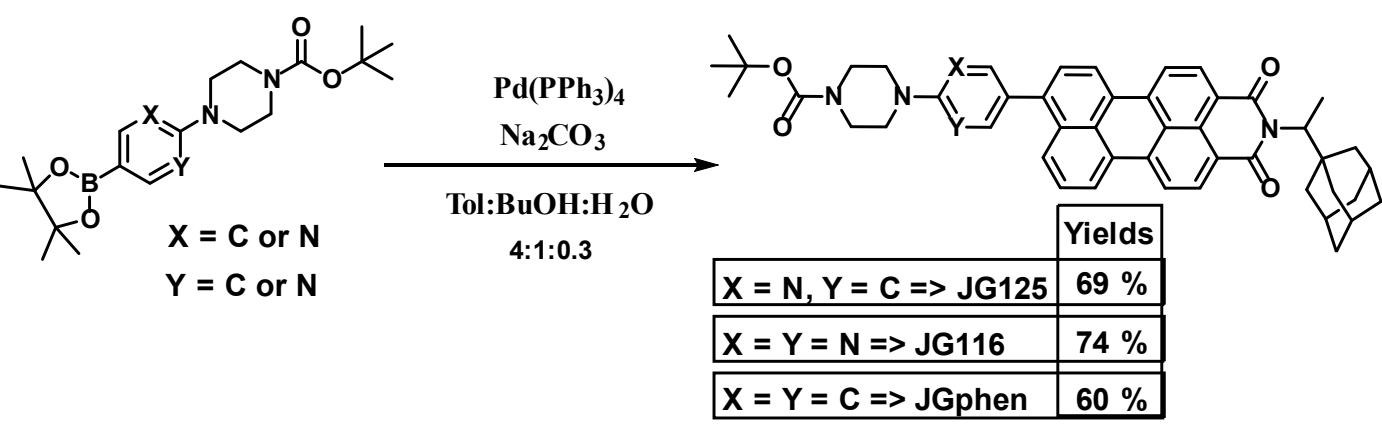

Figure 7. Synthesis of PMI-piperazine boc-protected derivatives, with different substitution; JG125 (pyridine), JG116 (pyrimidine) and JGphen (phenyl).

Although the three different compounds; containing a pyrimidin, pyridine and phenyl group, were synthetized, not all of them were good options for being used as sensors.

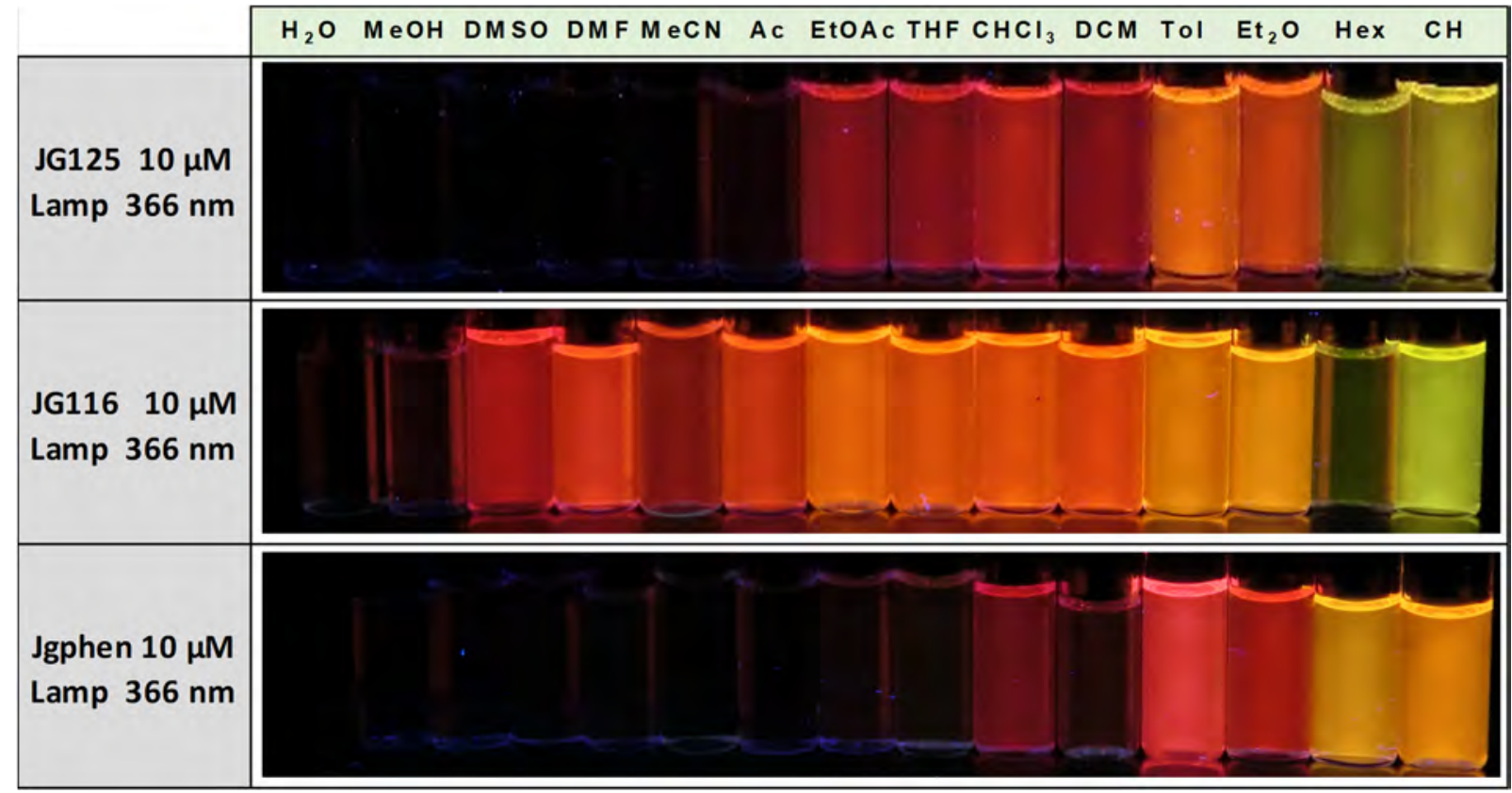

Figure 8. Pictures under UV light of $10 \mu \mathrm{M}$ solutions of different PMI-piperazine derivatives in different solvents ordered by polarity index. 
As it can be seen in Figure 8, the fluorescence of the pyrimidine derivative was very high $\left(\Phi_{\mathrm{F}}\left(\mathrm{CHCl}_{3}\right)\right.$ $=88 \%$ ), so it was not a good option for a turn-on probe. In contrast, pyridine and phenyl derivatives had lower fluorescence and were better options (Figure 9).
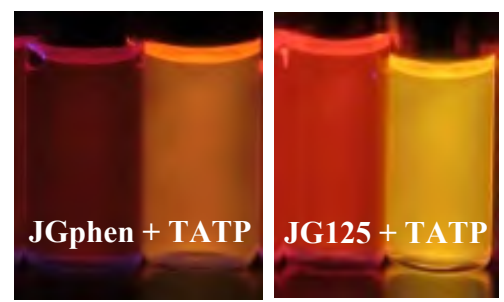

Figure 9. Picture under UV light of JGphen (left) and JG125 (right) $50 \mu \mathrm{M}, 0.5 \mathrm{~mL} \mathrm{CHCl} \mathrm{CH}_{3}$ solutions $+1 \mathrm{mg}$ of TATP

In spite of the increase in fluorescence, JGphen, the one containing the phenyl group, presented low solubility in most solvents, which was an important drawback when synthesizing and testing it. Furthermore, a greater dependence on concentration (fluorescence/concentration) was also observed. For those reasons, JG125 was the probe selected for TATP detection.

Afterwards, in order to be anchored to a surface, the amine group of the probe needed to be deprotected, the reaction was performed in strong acid environment, Figure 10.

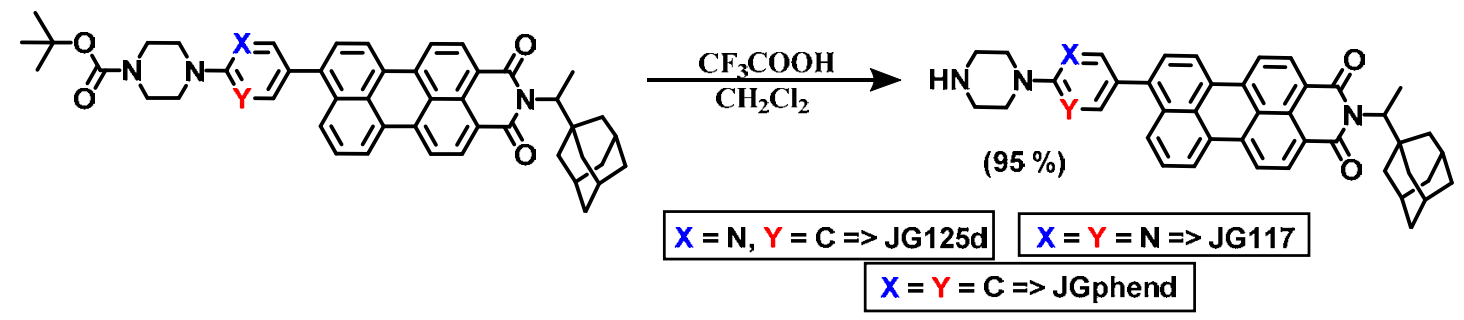

Figure 10. Synthesis of PMI-piperazine amine free derivatives with different substitution; JG125d,

\section{JG117 and JGphend.}

The deprotection proceeded with quantitative yields by addition of $\mathrm{CF}_{3} \mathrm{COOH}$ to a solution of the $\mathrm{N}$ boc derivative in DCM, followed by neutralization and being washed with water.

\subsection{Synthesis of triethoxysilyl perylene derivatives}

For covalently binding molecules to silica nanoparticles it was widely explained in literature the use of triethoxysilanes. ${ }^{26}$
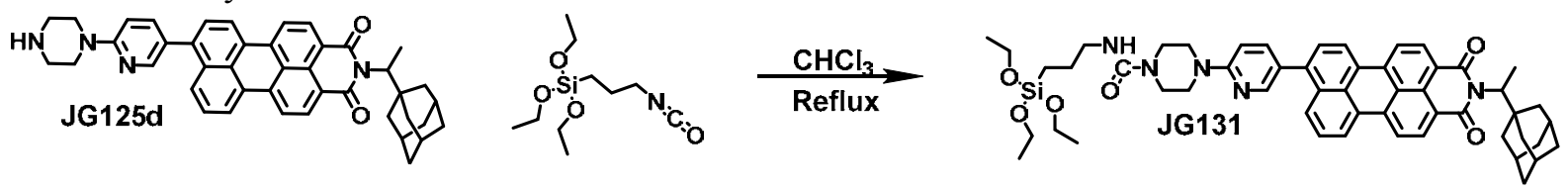

Figure 11. Synthesis of the triethoxysilyl derivative, JG131 from JG125d.

As it is shown in Figure 11, for the synthesized PMI, JG125d $(0.006 \mathrm{mmol} / \mathrm{mL})$ was dissolved in $\mathrm{CHCl}_{3}$, then triethoxy(3-isocyanatopropyl)silane was added to the solution and stirred under reflux for 24 hours. The resulting product was checked by ${ }^{1} \mathrm{HNMR}$ and introduced to the next reaction without further purification.

${ }^{26}$ M. Glad, O. Norrlöw, B. Sellergren, N. Siegbahn, K. Mosbach, J. Chromatogr. A 1985, 347, 11-23. 


\subsection{Synthesis of silica materials with supported perylene derivatives}

The triethoxysilil derivatives were mixed with silica materials: Silica 10-20 nm Sigma Aldrich $99.5 \%$ and TLC plates Merck Aluminium sheets $5 \times 10 \mathrm{~cm}$ Silicagel 60 .

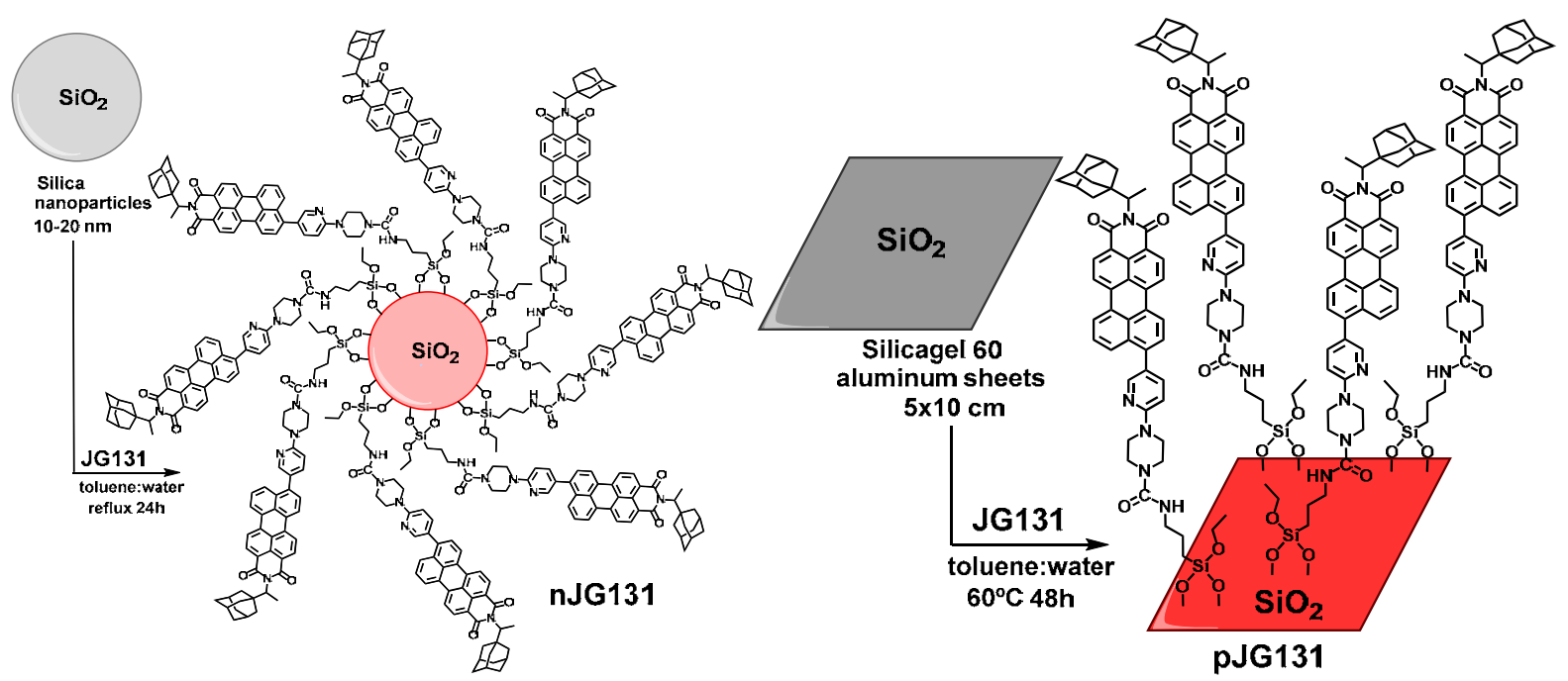

Figure 12. Silica derivatives of JG131, in silica nanoparticles (n) and in a TLC (p).

Functionalized silica nanoparticles (Figure 12 left) were prepared from $500 \mathrm{mg}$ of silica nanoparticles and $4 \mathrm{mg}$ of the triethoxysilyl perylene derivative. The mixture was refluxed at $112{ }^{\circ} \mathrm{C}$ in toluene:water $(50 \mathrm{~mL}: 10 \mu \mathrm{L})$ for 24 hours. Finally, the nanoparticles were washed with $2 \times$ toluene, 2 $\times \mathrm{DCM}$ and $2 \times \mathrm{Et}_{2} \mathrm{O}$. The product obtained was called $\mathbf{n J G 1 3 1}$.

By the same way, the silane derivatives were bonded to silica TLC plates (Figure 12 right), $0.5 \mathrm{mg}$ of JG131 every $5 \times 10 \mathrm{~cm}$ plate. Instead of treating it under reflux, it was heated at $60{ }^{\circ} \mathrm{C}$ for 48 hours in toluene (covering the plate), until the solutions had neither colour nor fluorescence. Then, the TLC plates were cleaned by the same procedure. The product obtained was called pJG131.

Additionally, JG125 was subjected to the same process to obtain pJG131. Albeit, in this case it was an adsorption process, obtaining the product adsorbed in silica. It was called pJG125.

\subsection{Characterization of the functional materials}

The materials were characterized with several techniques; registering fluorescent profiles, by EDX composition, TGA and TEM images.

nJG131, pJG131 and pJG125 gave colourless solutions when in a mixture with organic solvents (no leaching of probe from the material). Next, they were characterized by checking the fluorescent profiles, emission and excitation, Figure 13. 

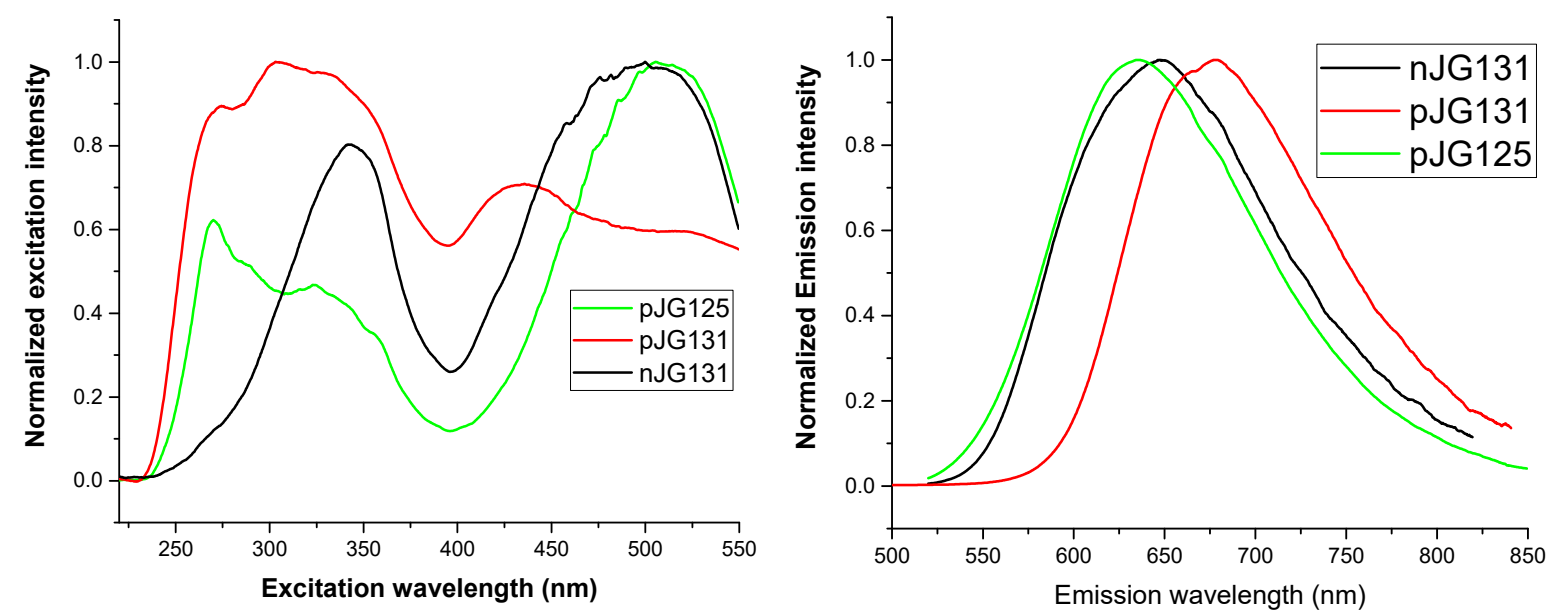

Figure 13. Normalized emission (right) and excitation (left) spectra of the supported probes in silica.

The proportions of carbon/silicon were also checked by EDX analysis (Energy-dispersive X-ray spectroscopy), shown in Figure 14.

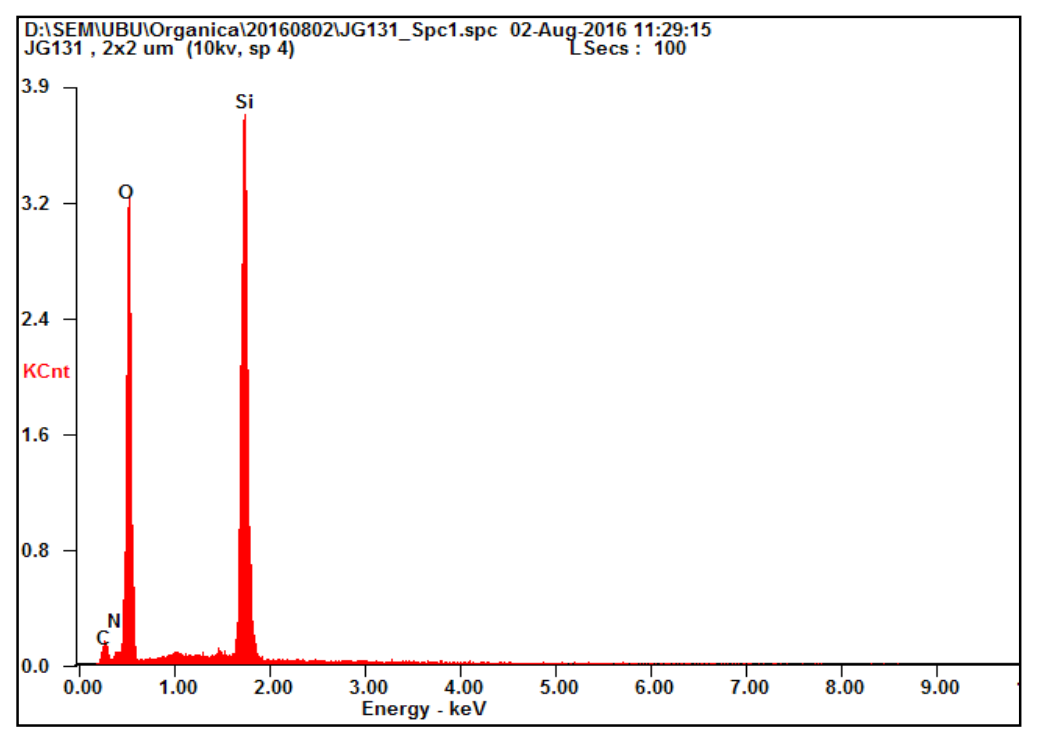

Figure 14. EDX profile of pJG131.

From the different profiles, the proportions $\mathrm{Si} / \mathrm{C}$ obtained were around 2-5\%(C) in case of the silica nanoparticles and $8 \%(C)$ for the TLC plates. These results were in agreement with the supposed proportions of carbon due to the quantity of perylene added, $2.8 \%$ for the silica nanoparticles. For the TLC plates, the supposed proportion could not be calculated, but the experimental results were quite close to the ones obtained for the nanoparticles.

TGA (Thermogravimetric analysis) was performed for the nanosilica substituted materials nJG131 (Figure 15). 


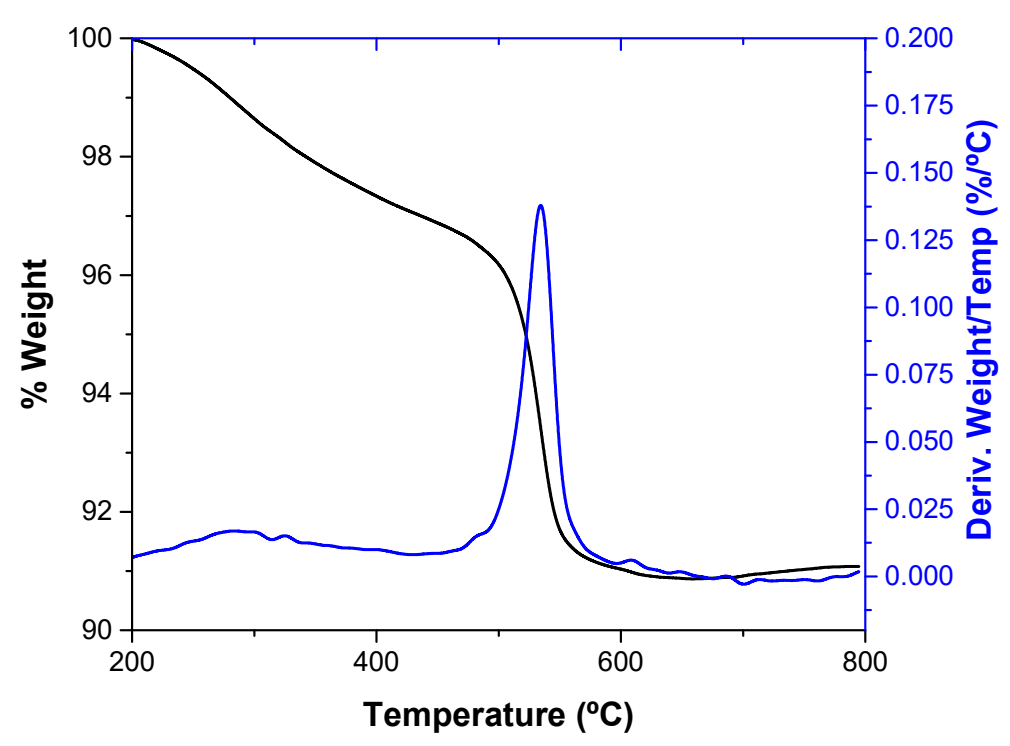

Figure 15. TGA in $\mathrm{N}_{2}$ of nJG131.

TGA from Figure 15 shows that nJG131 decreased $8 \%$ in weight when heated over $550{ }^{\circ} \mathrm{C}$, corresponding to the presence of organic matter, presumably PMI derivative in each case. Furthermore, a peak of weight loss was observed in the silica substituted with PMI at $300{ }^{\circ} \mathrm{C}$ temperature, which was in agreement with the degradation when calculating the melting point.

TEM and SEM images were also registered, Figure 16, to demonstrate the nanometric and micrometric structures.
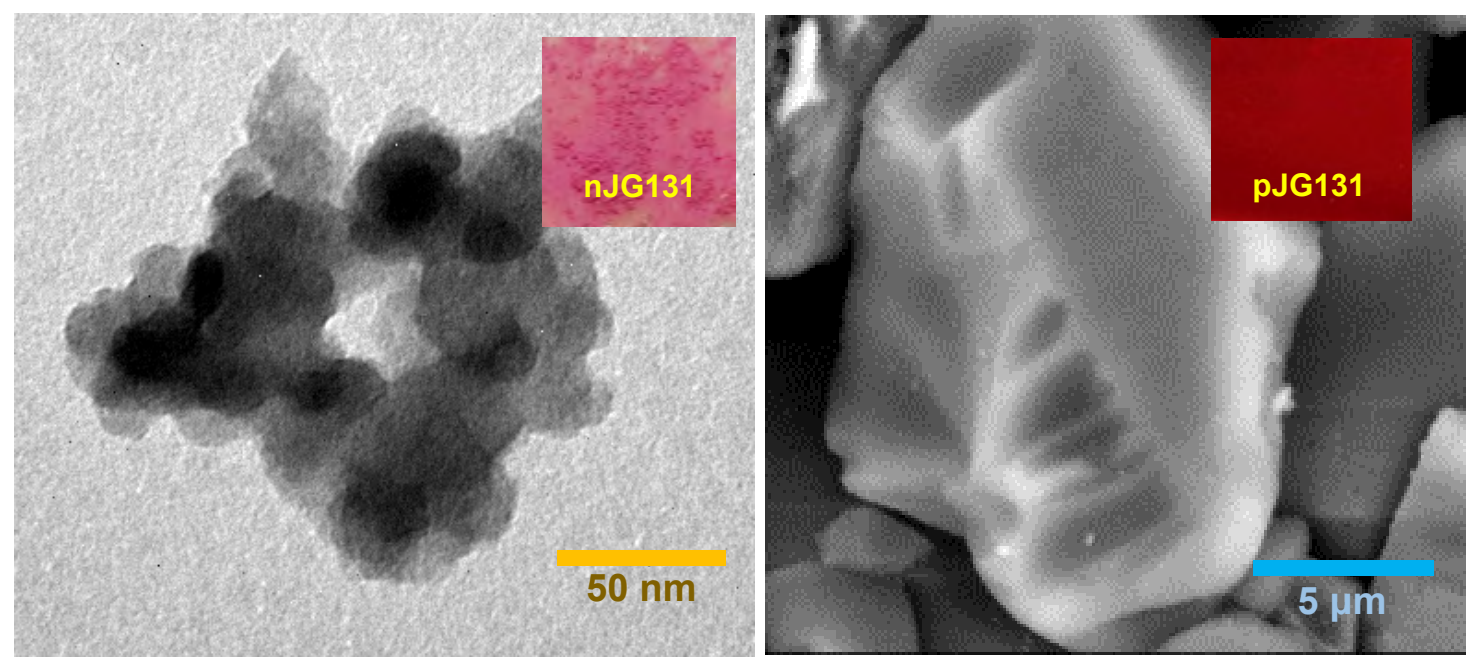

Figure 16. TEM image of nJG131 (left) and SEM of pJG131 (right). 


\section{QUALITATIVE AND QUANTITATIVE MEASUREMENTS OF JG125 WITH OXIDIZING REAGENTS}

\subsection{Probe in solution, election of the solvent}

The probes, JG125 (Boc-protected probe) and JG125d (deprotected) had properties as potential detectors for oxidizing agents, which was corroborated in preliminary testing. First, their general behaviour was studied in solution.

The solvatochromism of compounds JG125 and JG125d (10 $\mu \mathrm{M})$ was useful for studying the effect and the solubility of the probes in different solvents, because of that, it was the first step in the study of these compounds. As it is shown in Figure 17, they were highly soluble in chlorinated solvents, ethyl acetate, tetrahydrofuran and toluene.

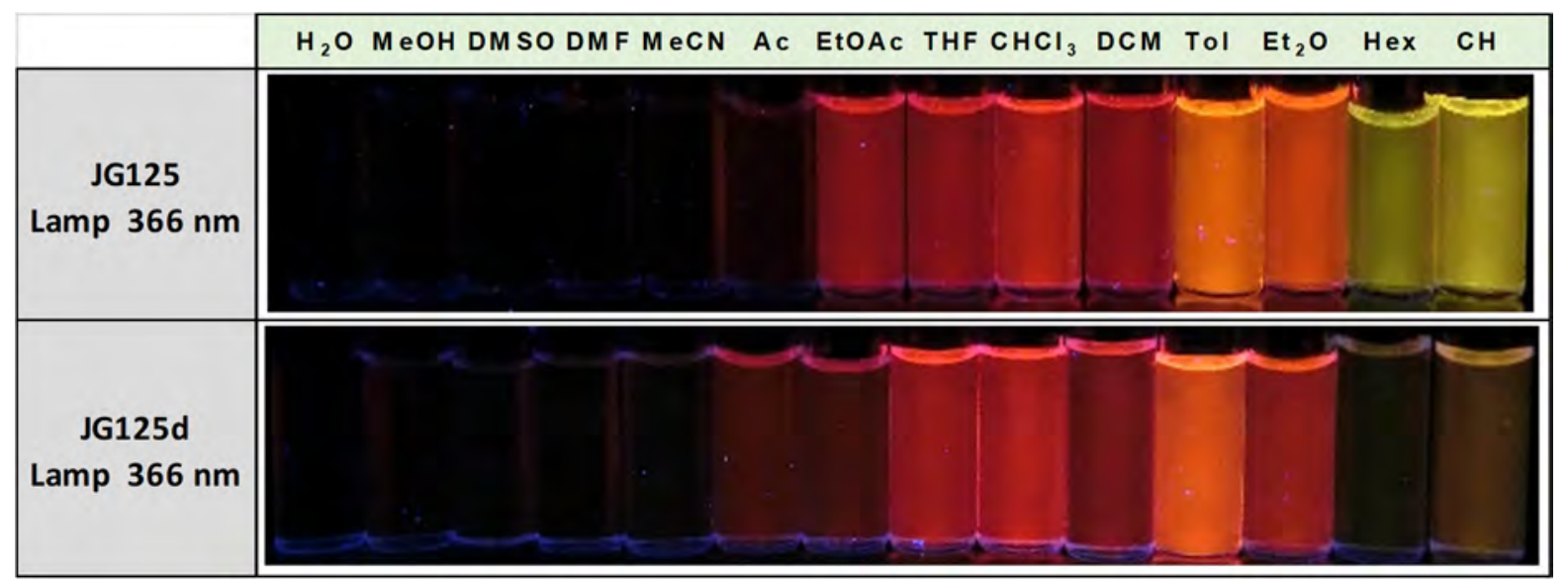

Figure 17. Picture under UV light of $10 \mu \mathrm{M}$ solutions of JG125 and JG125d in different solvents.

For these probes, there were no biological limitations about using one specific solvent, selecting the one more suitable for detection. Volatile solvents such as DCM were discarded; besides, THF is prone to radical secondary processes and toluene solutions presented high fluorescence from the beginning. Consequently, $\mathbf{C H C l}_{3}$ was selected as the best option to fulfil the objectives.

Deprotected probe JG125d had similar fluorescence in most solvents. However, its sensitivity to oxidizers became much lower, as it was registered in preliminary tests (Figure 18). The fact was afterwards associated to the different influence of molecular orbitals, when there was a free amino group (based on DFT calculations, Section 6).
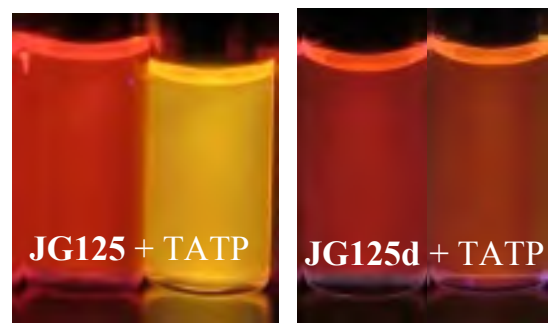

Figure 18. Picture under UV light of JG125 (left) and JG125d (right) $10 \mu \mathrm{M}, 0.5 \mathrm{~mL} \mathrm{CHCl}{ }_{3}$ solutions $+1 \mathrm{mg}$ of TATP. 
Therefore, the fluorescence of the probe in solution increased in the presence of TATP, although only slightly when having the amino group deprotected. To make materials sensitive to oxidizing reagents, the amine could be deprotected and bound to the material covalently by this amino group.

\subsection{Qualitative response of solutions to different oxidizing reagents}

Before performing quantitative measurements, the probes were tested with several oxidizing reagents. All the probes were dissolved in $\mathrm{CHCl}_{3}: \mathrm{MeOH}$ 9:1. The reason for using solvent mixtures was because of the minimal change respect $\mathrm{CHCl}_{3}$ pristine solutions and the increase of solubility of some oxidative reagents; insoluble in $100 \% \mathrm{CHCl}_{3}$. The change under visible and UV light was registered.

The experiment from Figure 19 shows $500 \mu \mathrm{L}$ solutions of JG125 that were prepared in $\mathrm{CHCl}_{3}: \mathrm{MeOH}$ 9:1, then, $10 \mu \mathrm{L}$ of the next solutions were added:
A) Reference (Nothing).
B) HEPES 0.5 $\mathrm{M}$ in water.
C) $\mathrm{HCl} 0.01 \mathrm{M}$ in water.
D) TATP $0.2 \mathrm{M}$ in $\mathrm{CHCl}_{3}$.
E) MCPBA $0.2 \mathrm{M}$ in $\mathrm{CHCl}_{3}$.
F) Oxone $0.2 \mathrm{M}$ in water.
G) TNB $1 \mathrm{mg}$.

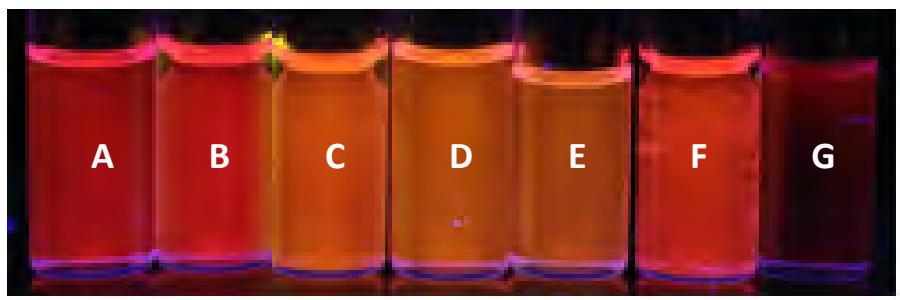

Figure 19. Response under UV light of JG125 against different oxidizing agents.

It was concluded that JG125 was very sensitive to oxidants such as TATP and MCPBA. In contrast, it was also sensitive to acid species and underwent fluorescence quenching at high concentrations of TNB.

\subsection{Work concentration}

In order to choose an optimum concentration for a quantitative studio, the absorbance and fluorescence of the probes were checked to be linear while concentration changes were small (Figures 20 and 21). Studied in $\mathrm{CHCl}_{3}: \mathrm{MeOH}(9: 1 \mathrm{v}: \mathrm{v})$ :
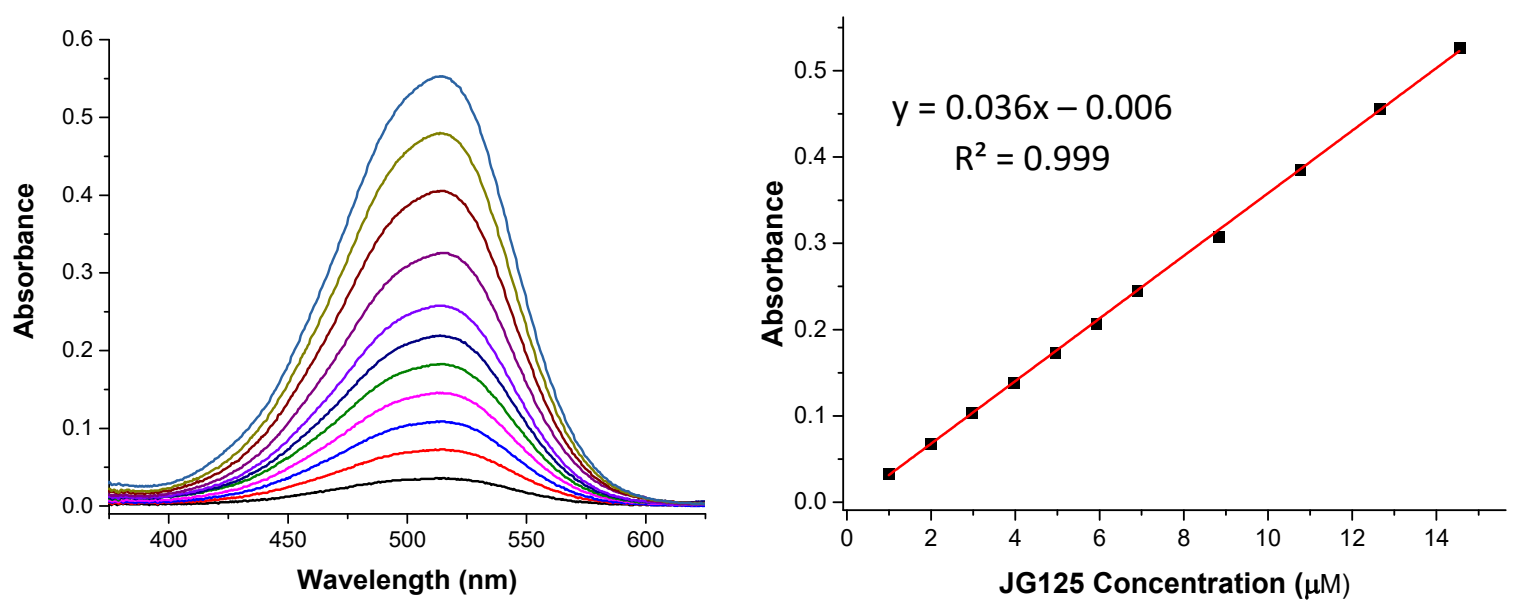

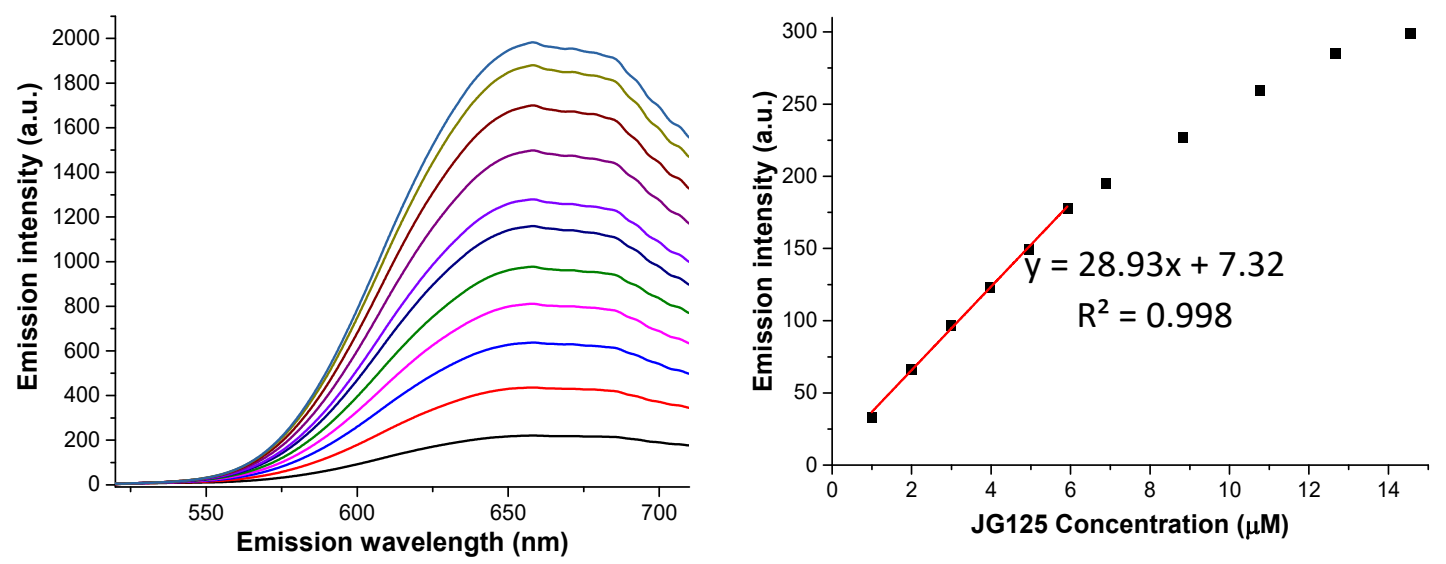

Figure 20. Absorbance (up) and fluorescence (down) of JG125 solution in $\mathrm{CHCl}_{3}: \mathrm{MeOH}$ 9:1, under increasing concentrations of JG125, $\lambda_{\text {exc }}=512 \mathrm{~nm}$ (down).
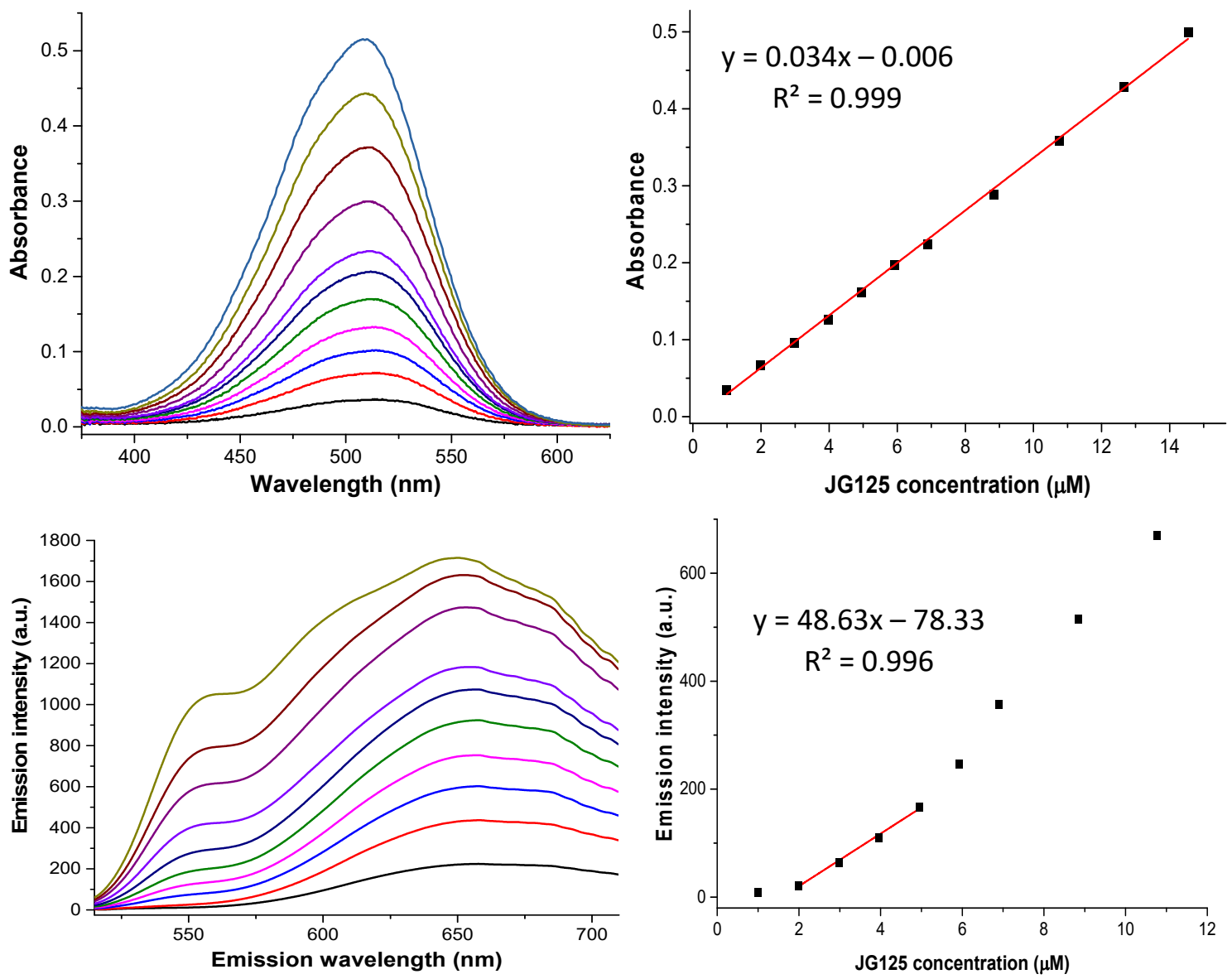

Figure 21. Absorbance (up) and fluorescence (down) of JG125 solution in $\mathrm{CHCl}_{3}: \mathrm{MeOH}$ 9:1, under increasing concentrations of JG125 and excess MCPBA. $\lambda_{\text {exc }}=562 \mathrm{~nm}$ (down).

The work concentration ideally should stay at 0.1 of absorbance or less, to avoid inner filter effects, possible dynamic quenching or stacking processes. It implied a concentration below $5 \mu \mathrm{M}$. The emission increased at $550 \mathrm{~nm}$ after adding the oxidant. Taking into account the results, the chosen concentration was $\mathbf{2 . 5} \boldsymbol{\mu M}$, value around which the Lambert-Beer law (or pseudo-Lamber-Beer linear behaviour for fluorescence) was fulfilled. 


\subsection{Quantitative measurements of JG125 vs TATP / MCPBA in solution}

To compare the results and the possibility of having different processes, not only the response to TATP was evaluated. The changes with MCPBA, other strong oxidant, were also measured, determining their optical changes and the limit of detection (LOD) for both analytes.

The LOD was calculated by the method explained in Chapter 0; the values of false positive and false negative were fixed as equal or inferior to $5 \%$.

Solutions of JG125 $(2.5 \mu \mathrm{M})$ in $\mathrm{CHCl}_{3}: \mathrm{MeOH}$ 9:1 were prepared.

The conditions were:

- $\quad$ TAATP] and [MCPBA] were increased by adding from a concentrated solution in the same solvent.

- $\lambda_{\mathrm{exc}}=500 \mathrm{~nm}, \lambda_{\mathrm{em}}=556 \mathrm{~nm}$.

- The absorbance and fluorescence changes were registered at $25{ }^{\circ} \mathrm{C}$.

\subsubsection{TATP titration:}
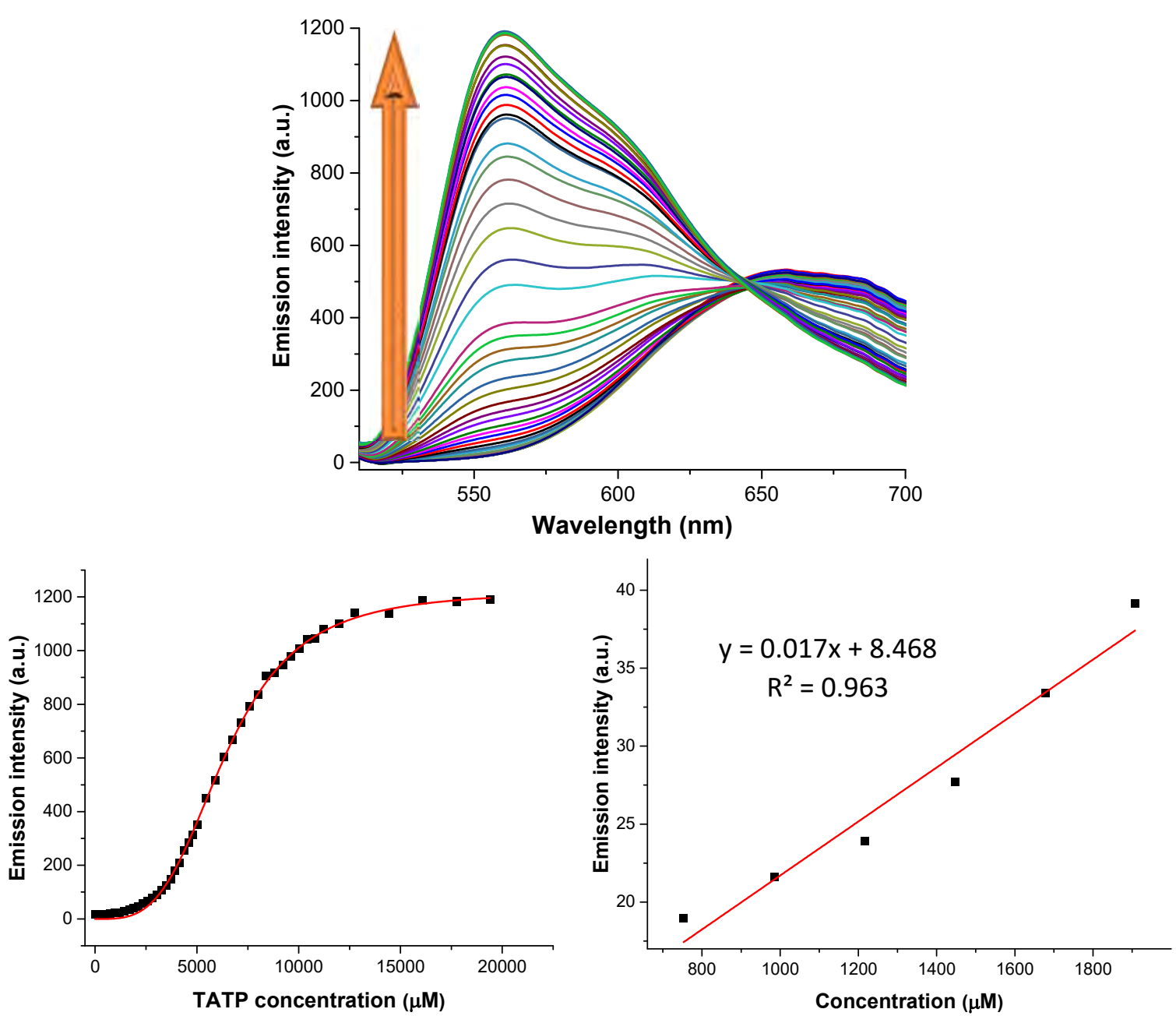

Figure 22. Titration (up), fluorescence profile at $556 \mathrm{~nm}$ (down-left) and calibration for the limit of detection (down-right) of $\mathbf{J G 1 2 5}$ solution $2.5 \mu \mathrm{M}$ in $\mathrm{CHCl}_{3}: \mathrm{MeOH} 9: 1$, under increasing concentrations of TATP.

The titration profile for TATP showed on Figure 22 was obtained, and the limit of detection was calculated to be $620 \mu \mathrm{M}$ or $0.27 \mathrm{mg}$ of TATP in $2.5 \mathrm{~mL}$, from the linear fitting at low concentrations. 


\subsubsection{MCPBA titration:}
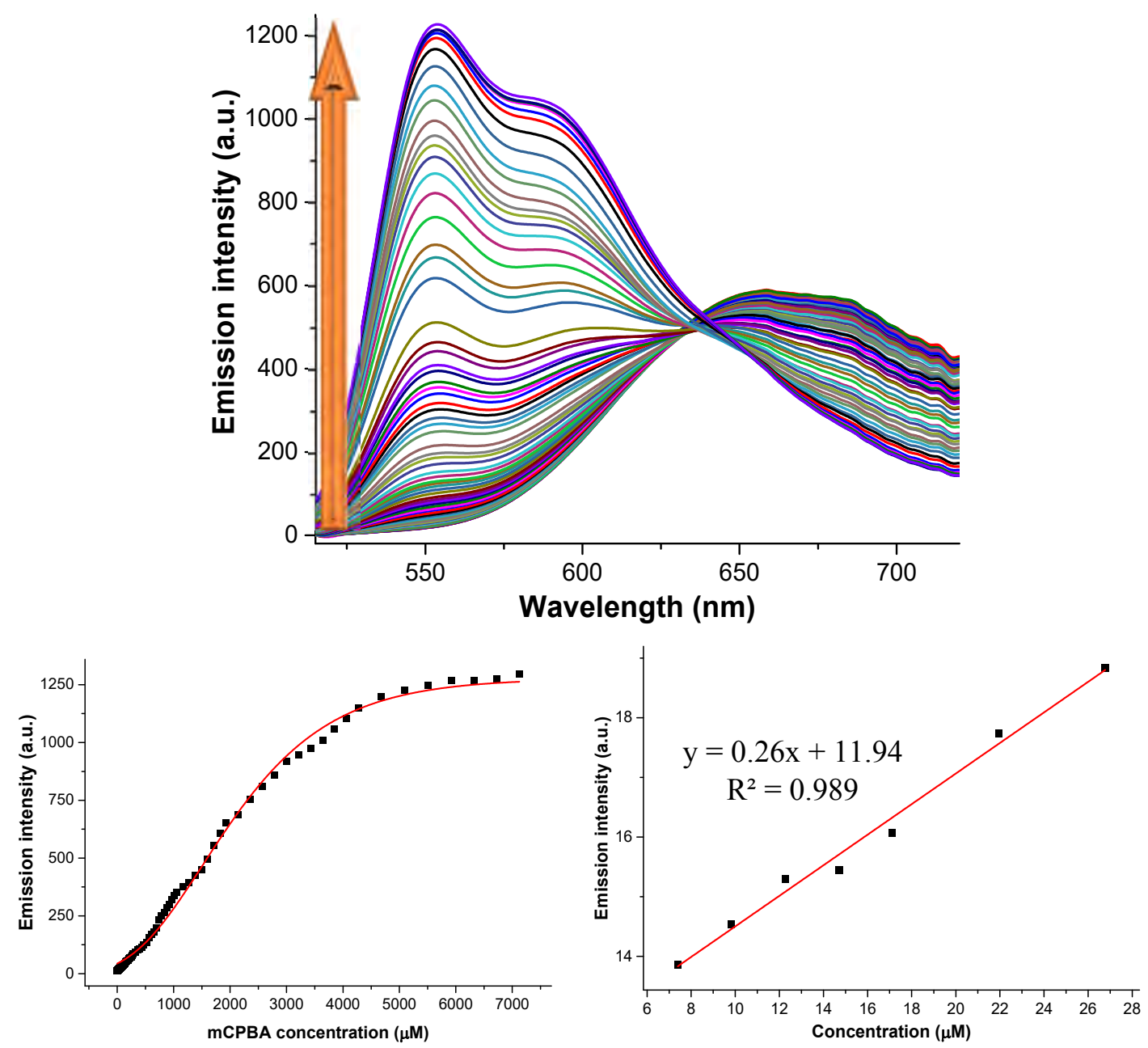

Figure 23. Titration (up), fluorescence profile at $556 \mathrm{~nm}$ (down-left) and calibration for the limit of detection (down-right) of JG125 solution $2.5 \mu \mathrm{M}$ in $\mathrm{CHCl}_{3}: \mathrm{MeOH} 9: 1$, under increasing concentrations of MCPBA.

For MCPBA the titration profile is showed on Figure 23, and the limit of detection was calculated to be $7.4 \mu \mathrm{M}$ or $\mathbf{0 . 0 0 3 2} \mathrm{mg}$ of MCPBA in $2.5 \mathrm{~mL}$ from the linear fitting at low concentrations.

In both cases an oxidation process of the fluorogenic probe JG125 occurred. In comparison, it happened faster in case of MCPBA, which was predictable due to the higher oxidative ability of MCPBA with respect to TATP.

\subsection{Quantum yield and lifetime measurements}

Quantum yields were determined by using an integration sphere system while keeping in mind that the error associated to the method is $1-2 \%$, higher than the experimental results when making replicas.

$$
\begin{aligned}
& \Phi_{\mathrm{F} \mathrm{JG125}}\left(\mathrm{CHCl}_{3}\right)=0.23 \pm 0.01 \\
& \Phi_{\mathrm{F} \mathrm{JG125+TATP}}\left(\mathrm{CHCl}_{3}\right)=0.65 \pm 0.01 \\
& \Phi_{\mathrm{F}} / \Phi_{\mathrm{F}, 0}=\mathbf{2 . 8}
\end{aligned}
$$




\section{TATP DETECTION IN MODIFIED SILICA}

Several silica modified samples were tested as potential TATP sensors from the PMI derivative (JG125), 3 kinds of PMI modified silica were synthesized:

- Modified silica nanoparticles (nJG131): $4 \mathrm{mg}$ of probe every $500 \mathrm{mg}$ of silica.

- Modified supported silica (pJG131): $0.5 \mathrm{mg}$ every $5 \times 10 \mathrm{~cm}$ layer.

- Silica adsorbed probe (pJG125) were studied: $0.5 \mathrm{mg}$ every $5 \times 10 \mathrm{~cm}$ layer.

The ratio probe/silica was altered obtaining different results. This ratio affected the colour and, especially, the fluorescence. Therefore, it was chosen the optimum ratio, that shown a clear increase in fluorescence.

\section{Experimental system to measure in gas:}

The objective was to measure the increase in fluorescence with TATP, in doing so, two flasks were connected as it is showed in Figures 24 and 25. A flux of $N_{2}$ was regulated to be $100 \mathrm{~cm}^{3} / \mathrm{min}$ and the temperature in the analyte flask around $55^{\circ} \mathrm{C}$, going through a flask with $2 \mathrm{mg}$ of TATP. The changes in the fluorescence of the probes were detected after a few minutes.

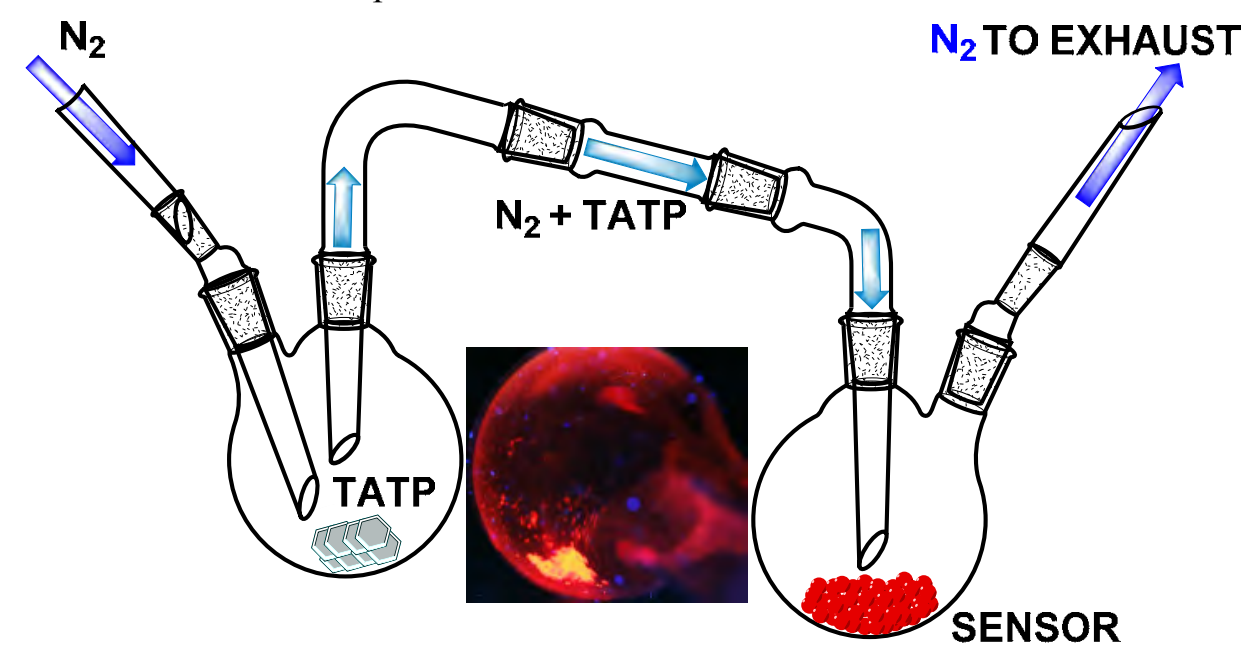

Figure 24. Scheme of the system to measure TATP current with the supported sensor.

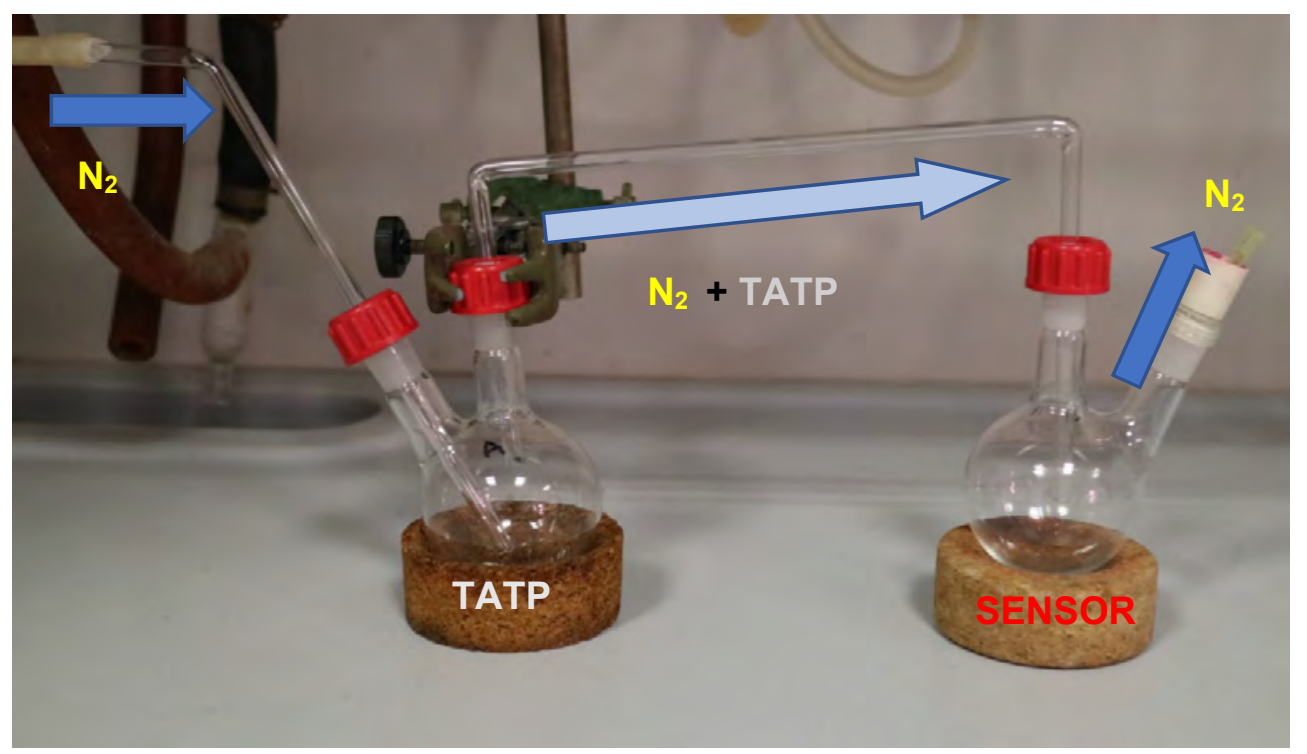

Figure 25. Picture of the system to measure TATP in a gas stream with the supported sensor. 
The silica supported probes were evaluated by several methods that gave complementary information about the influence of different analytes. In a first instance, the detection process was studied as a of code of colour under UV light. Afterwards, the detection capacity was evaluated by a more quantitative approach by registering changes in fluorescence intensity (by comparing the fluorescence quantum yield for higher precision) and in the position of the maximum of emission. Finally, a quantitative study for the specific detection of TATP vapours was also performed. All the results are summarized schematically in Section 5.5.

\subsection{Study of change in fluorescence as a code of colours}

To detect qualitatively the presence of TATP in a quick-straightforward way, it was useful to compare any variation with the initial colour, Figures 26, 27 and 28.

The test consisted of taking pictures to distinguish how a series of vapour affect the samples.

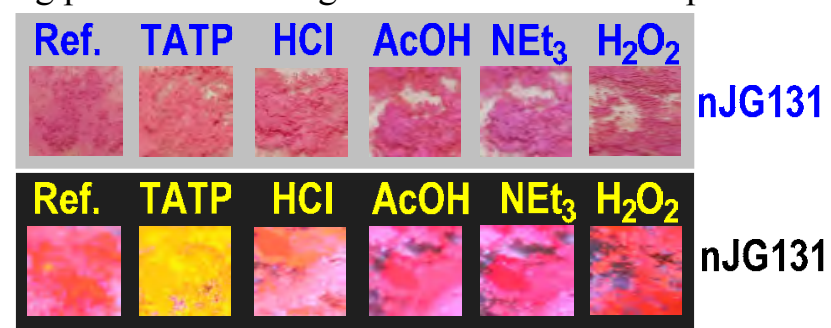

Figure 26. Picture of nJG131 glued into glass plates and put in presence of different vapours. Visible light (up) and $366 \mathrm{~nm}$-light (down).
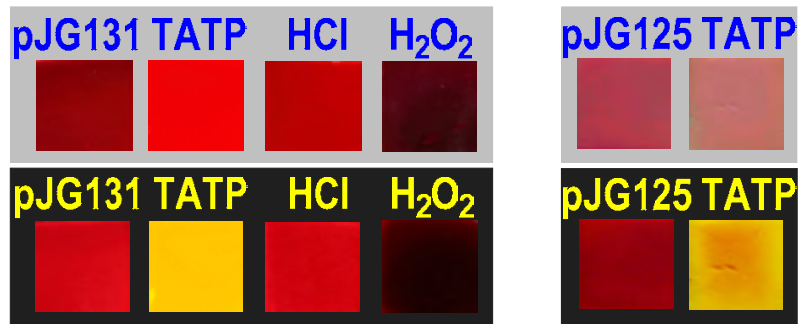

Figure 27. Picture of pJG131 (left) and pJG125 (right) in presence of different vapours. Visible light (up) and $366 \mathrm{~nm}$-light (down).

In addition, $\mathbf{n J G 1 3 1}$ was tested in presence of other solvents, such as acetone or $\mathrm{Et}_{2} \mathrm{O}$ :

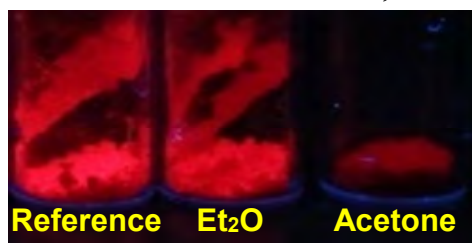

Figure 28. nJG131 powder after being in presence of $\mathrm{Et}_{2} \mathrm{O}$ and acetone, $15 \mathrm{mg}$ under $366 \mathrm{~nm}$-light.

- TATP increased the fluorescence of silica in yellow.

- Acetic acid and triethyl amine vapours did not have effect over the initial colour-fluorescence.

- Hydrogen peroxide vapours decreased the fluorescent emission.

- Diethylether vapours had no effect neither on fluorescence nor colour.

- Acetone vapours decreased the final fluorescence.

- Strong acid vapours $(\mathrm{HCl})$ increased the fluorescence in yellow. Notwithstanding, the process was reversible with weak basis vapours (triethyl amine). In contrast, the increase of fluorescence in presence of TATP was not reversible. 


\subsection{Quantitative study of fluorescence}

The increase in fluorescence of solids was recorded by measuring the solids and determining the increase in fluorescent quantum yield $\left(\Phi_{\mathbf{F}}\right)$, Figure 32. As it was explained, the fluorescence of the solids is directly associated to the ratio [probe]/silica. Therefore, providing that the quantity of probe was not too high or too low, the increase was proportional to the analyte detected.

The most straightforward way to detect the presence of the analyte was the increase in fluorescence of the modified material. However, the measurements were sometimes reliant on the position of the solid in the fluorometer (especially in powder samples) and these factors were difficult to control without designing a specific device for doing so. As a consequence, fluorescence quantum yields were the most trustworthy way to measure it quantitatively. The results of acquiring the excitation and emission spectra are shown in Figures 29, 30 and 31 for nJG131, pJG131 and pJG125 respectively.
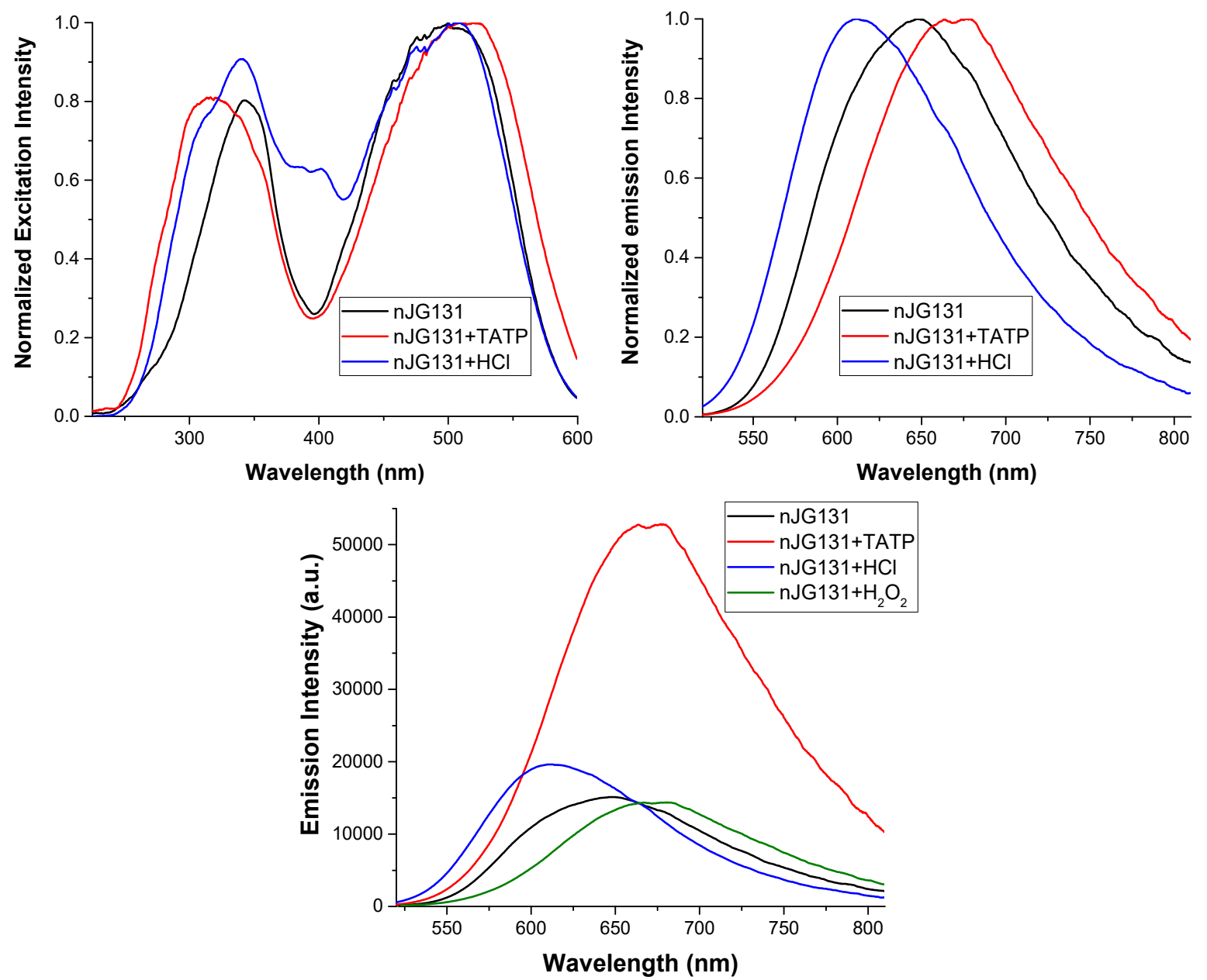

Figure 29. Response in excitation and fluorescence of nJG131. $\lambda_{\text {exc }}=492 \mathrm{~nm}, \lambda_{\text {em }}=614 \mathrm{~nm}$. 

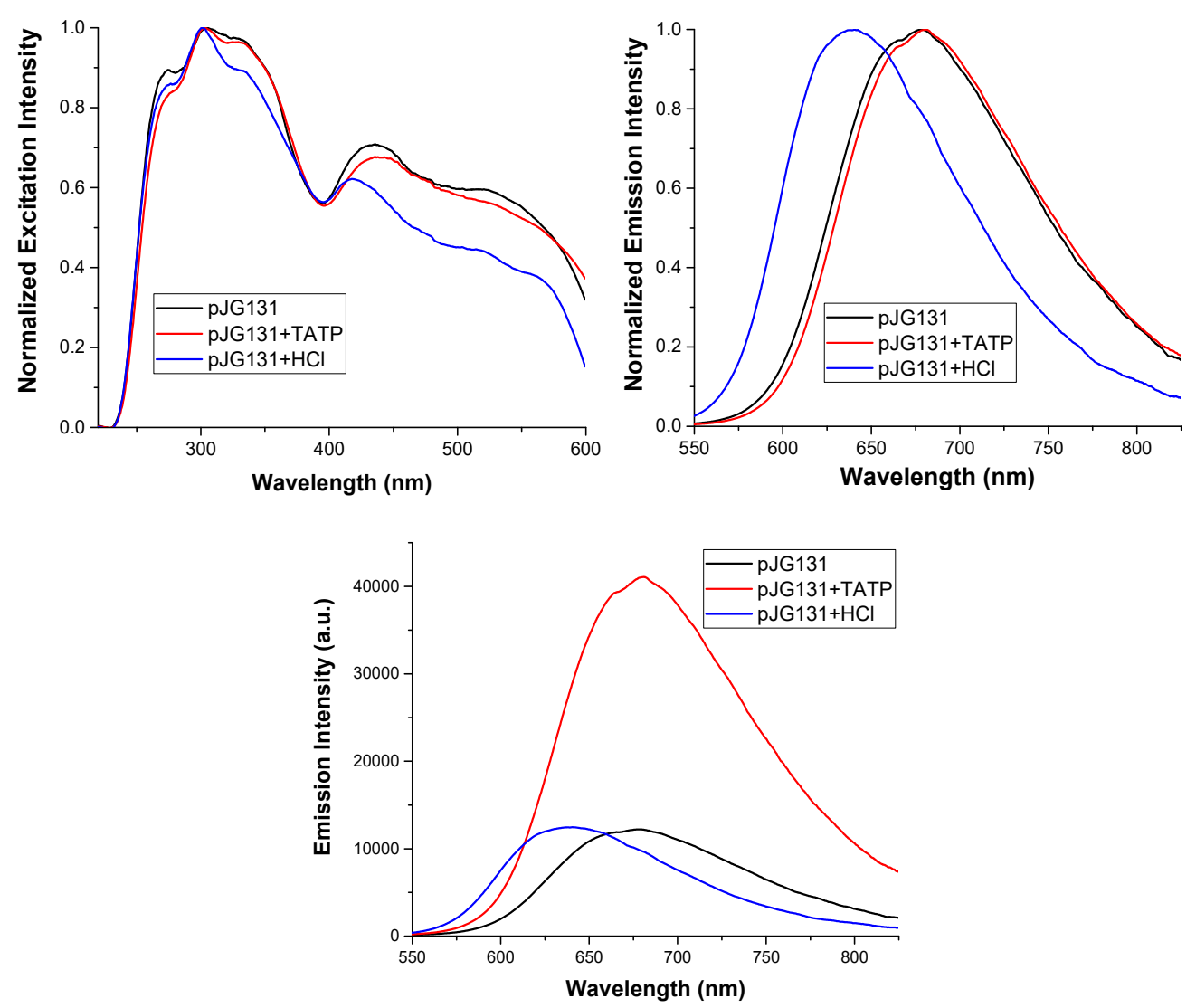

Figure 30. Response in excitation and fluorescence of pJG131. $\lambda_{\mathrm{exc}}=438 \mathrm{~nm}, \lambda_{\mathrm{em}}=630 \mathrm{~nm}$.
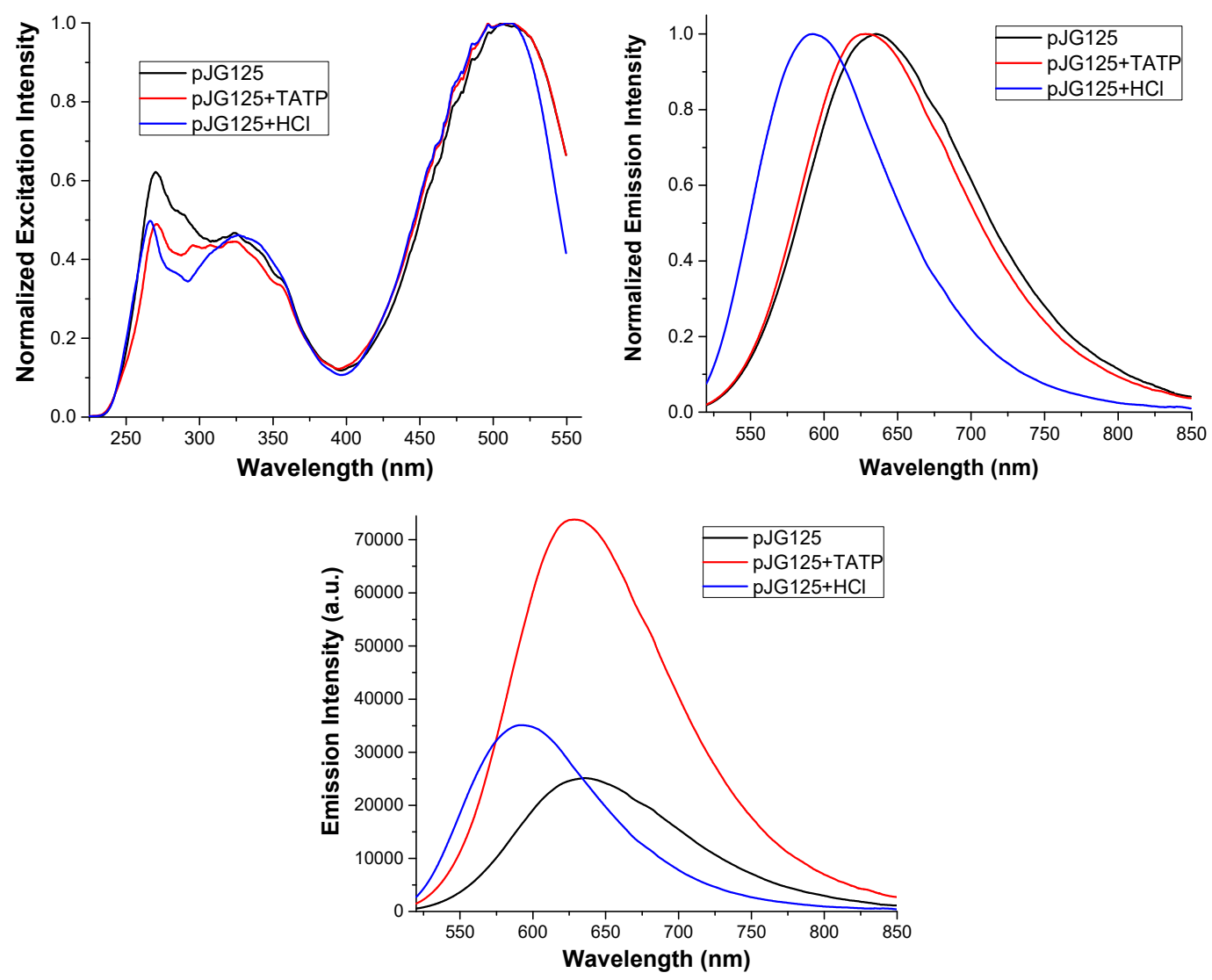

Figure 31. Response in excitation and fluorescence of absorbed pJG125. $\lambda_{\mathrm{exc}}=500 \mathrm{~nm}, \lambda_{\mathrm{em}}=605 \mathrm{~nm}$. 
These spectra from Figures 29, 30 and 31 allow to distinguish between acid and TATP in several ways. They had a maximum of emission shift to lower wavelengths in presence of acids and a much higher increase in presence of TATP than $\mathrm{HCl}$; after neutralizing with $\mathrm{Et}_{3} \mathrm{~N}$ vapours.

\subsection{Study of increment on the emission}

In doing so, it was necessary to have a fluorometer provided with an integration sphere. ${ }^{27}$ The accuracy of this method was checked by measuring several replicas, obtaining errors of less than $2 \%$.

\begin{tabular}{|c|c|c|}
\cline { 2 - 3 } \multicolumn{1}{c|}{} & $\boldsymbol{\Phi}_{\mathrm{F}, \mathrm{TATP}} / \boldsymbol{\Phi}_{\mathrm{F}, \mathbf{0}}$ & $\boldsymbol{\Phi}_{\mathrm{F}, \mathrm{HCl}} / \boldsymbol{\Phi}_{\mathrm{F}, \mathbf{0}}$ \\
\hline nJG131 & 3.5 & 1.6 \\
\hline pJG131 & 3.4 & 1.0 \\
\hline pJG125 & 3.0 & 1.4 \\
\hline
\end{tabular}

Figure 32. Increase in fluorescence between the initial and after being under excess of TATP.

In all cases, the increase in fluorescence went from 2-4 \% to 7-15\%, depending on each specific ratio [probe]/silica. As a general rule, as it is shown in Figure 32, the increase in fluorescence with the probes was situated between 3 to 3.5 times in all samples for TATP and less than 1.7 times with $\mathrm{HCl}$ samples, after being under $\mathrm{Et}_{3} \mathrm{~N}$ vapours.

\subsection{Titration under increasing concentration of TATP}

Previous testing conditions were performed under a flux of TATP and retired within a few minutes. Additionally, the increase in emission was checked by measuring the fluorescence quantum yield, after acquiring the emission spectra, in order to improve accuracy in determinations. However, the potential of the probe for TATP quantification would had not been fully evaluated without an estimation of the minimum amount in gas that produces a detectable response.

The calibration was performed in steady state, fixed quantities of TATP were vaporized in the presence of a sample containing $15 \mathrm{mg}$ of silica nanoparticles and the fluorescence increase was measured; after the complete vaporization of each TATP sample. The results are displayed in Figure 33 (qualitative results) and in Figure 34, fluorescent emission measurements.

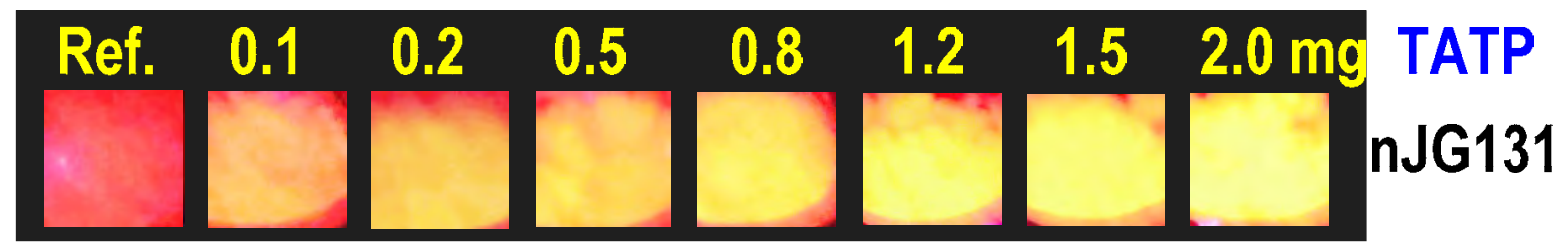

Figure 33. Picture of the response of nJG131 in different quantities of TATP.

\footnotetext{
${ }^{27}$ Edinburgh Instruments FLS980 with adapted integration sphere (See Annex).
} 

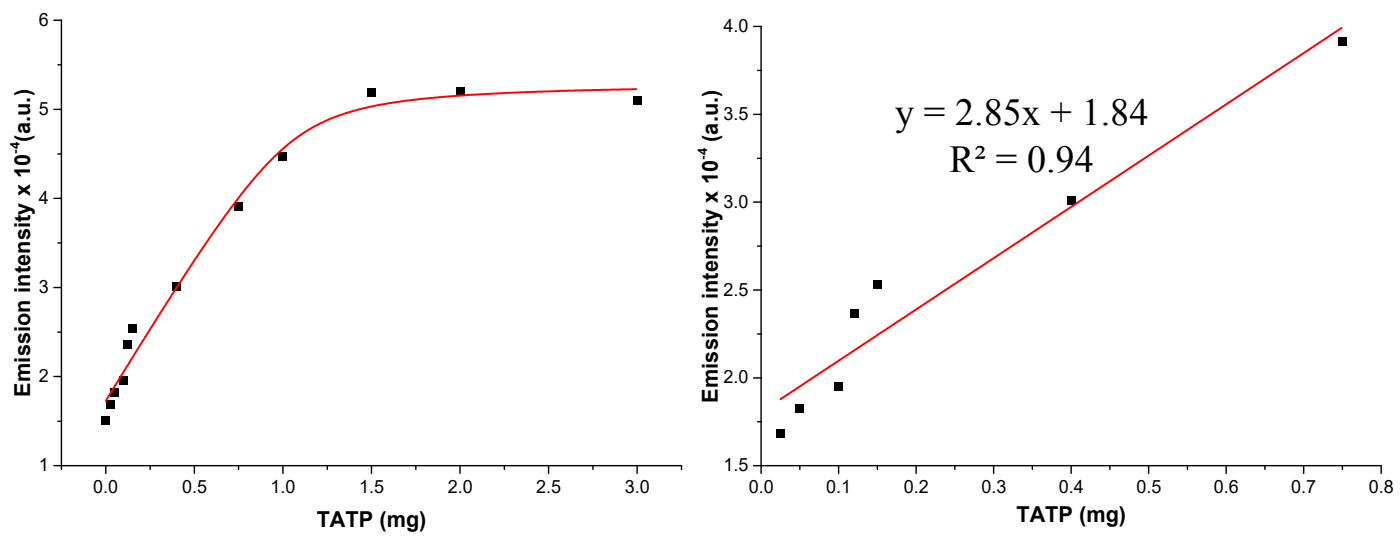

Figure 34. Calibration, adding increasing concentrations of TATP. over nJG131. $\lambda_{\mathrm{exc}}=495 \mathrm{~nm}, \lambda_{\mathrm{em}}=$ $620 \mathrm{~nm}$.

By making a linear regression at low quantities of TATP (Figure 34 right) the LOD was calculated when detecting TATP. The calculations led to a limit of detection of $\mathbf{0 . 1 2} \mathbf{~ m g}$. (Same method than previous LODs).

The limitations of the procedure had the consequence of low repeatability if lower quantities were tried to be measured, because of the difficult of handling TATP powder. Therefore, $0.12 \mathrm{mg}$ represents the minimum amount reliably detected by the technique developed.

\subsection{Summary of fluorescence measurements}

- The presence of TATP was detected easily and qualitatively with any of the silica systems, by a code of colours or registering the increase in fluorescence.

- TATP was distinguished from acid interferents by the change in the wavelength of emission. Moreover, triethylamine vapours (weak base) produced a decrease in fluorescence when the response was due to acid vapours.

- The LOD for TATP was calculated to be $0.12 \mathrm{mg}$ with $15 \mathrm{mg}$ of silica and as a vapour flow.

- There was no response against diethyl ether vapours and a decrease in emission with possible interferents such as hydrogen peroxide or acetone.

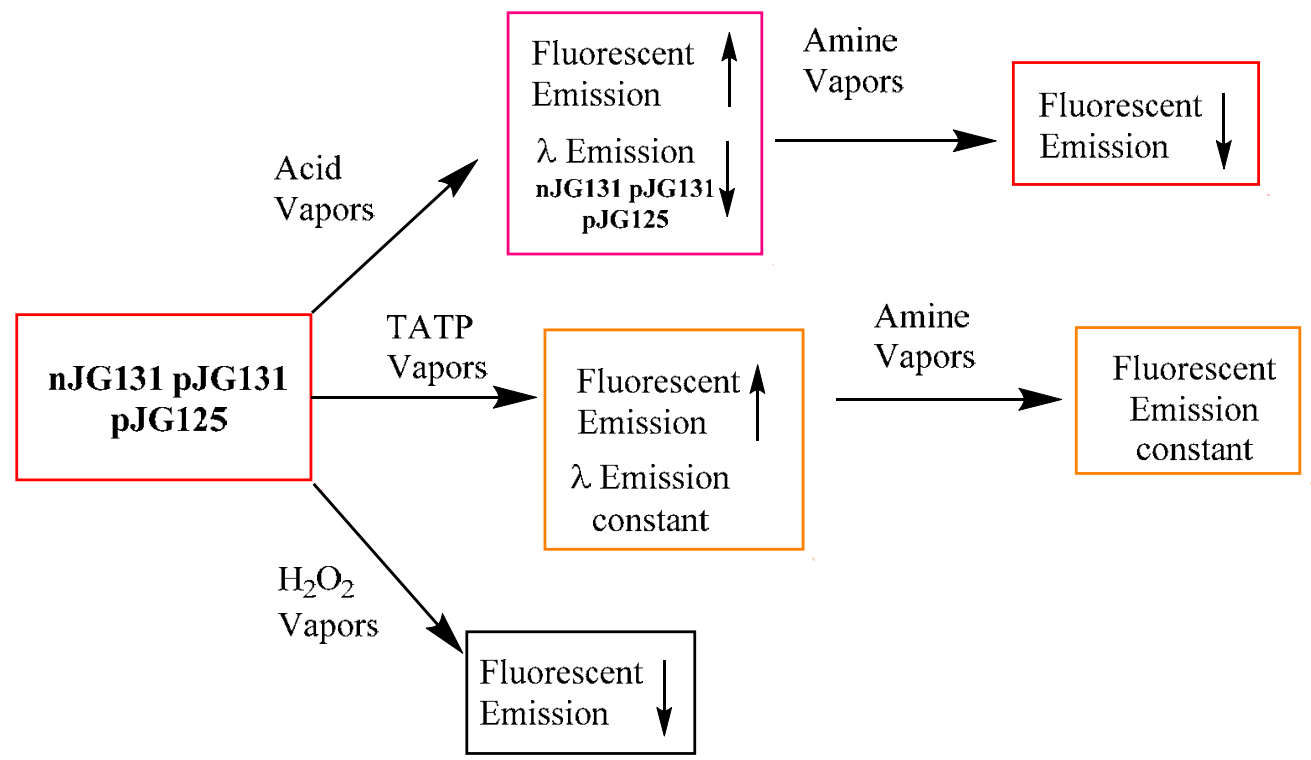

Figure 35. Scheme showing the interpretation of the results and the application of the different probes. 


\section{DETECTION MECHANISM}

The proposed mechanism to justify the increase in fluorescence of the PMI derivatives was an oxidation over one nitrogen of the piperazine group. This process was supported by several techniques, such as electrochemistry, DFT calculations and the isolation of the oxidation peak in mass spectra.

\section{1. $\quad$ Electrochemistry measurements}

Cyclic voltammetry results of compounds JG125 and JG125d are shown in Figures 36 and 37; the internal reference used was Ferrocene.

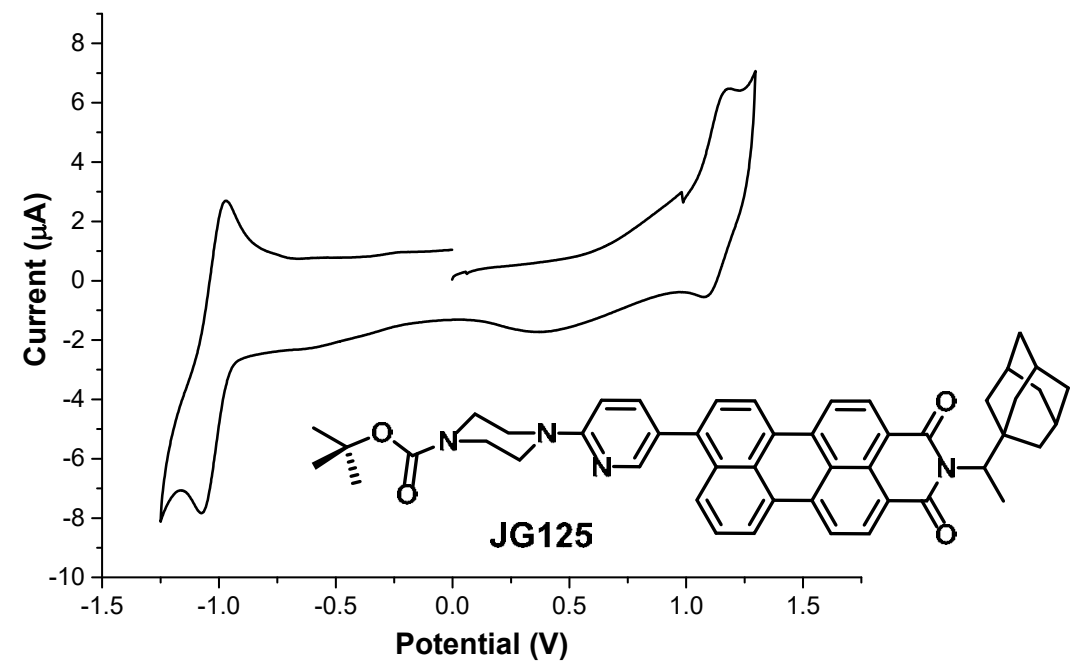

Figure 36. Cyclic voltammogram of compound JG125.

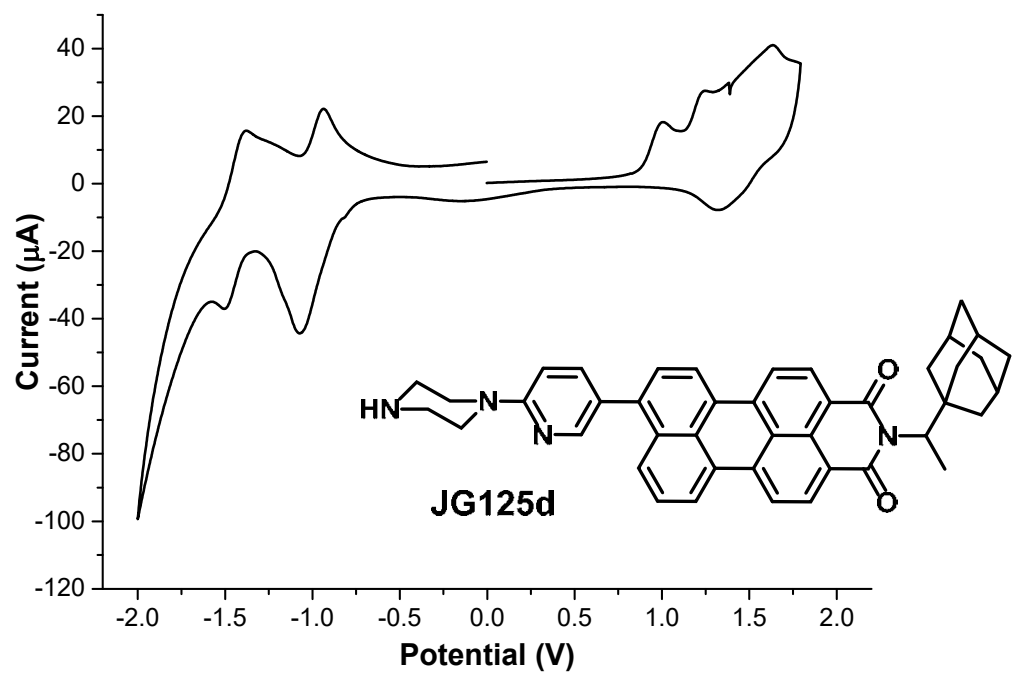

Figure 37. Cyclic voltammograms of compound JG125d

The cyclic voltammograms from Figures 36 and 37 show important differences between JG125 and JG125d. While JG125 underwent a reversible oxidation at $\mathrm{E}^{\mathbf{o}}=+1.13 \mathrm{~V}$, JG125d showed an irreversible oxidation wave with a value of $\mathrm{E}_{\mathrm{pk}}=+1.01 \mathrm{~V}$. This different behaviour was assigned to the presence or absence of the N-H bond. 
The radical cation formed by the one-electron oxidation of the secondary amine in JG125d should be very reactive leading to the observed electrochemical irreversibility and, therefore, the $\mathrm{E}_{\mathrm{pk}}$ value could not be related directly to thermodynamic values. These values indicated an estimated difference between the oxidation potential of both compounds of $0.12 \mathrm{~V}$. A fact that explained the change in fluorescence (change in oxidation) but not the difference between them. This other fact was attributed to the influence of molecular orbitals which were studied by quantum mechanical computer calculations.

\subsection{Quantum chemical calculations ${ }^{28}$}

The optimization of compounds was carried out separately as neutral and radical cation in DFT calculations at the $\mathrm{B}^{2} \mathrm{LYP}^{23} / 6-31(\mathrm{~d}, \mathrm{p})$ level with simultaneous computation of $\mathrm{PCM}$ solvation ${ }^{29}$ energies in DCM, by using Gaussian 09, Revision D.01. ${ }^{30}$ Each structure was verified to be a true minimum by the absence of imaginary frequencies in the vibrational analysis. Figure $\mathbf{3 8}$ displays the HOMO and the SOMO for JG125 and Figure 39 for JG125d.

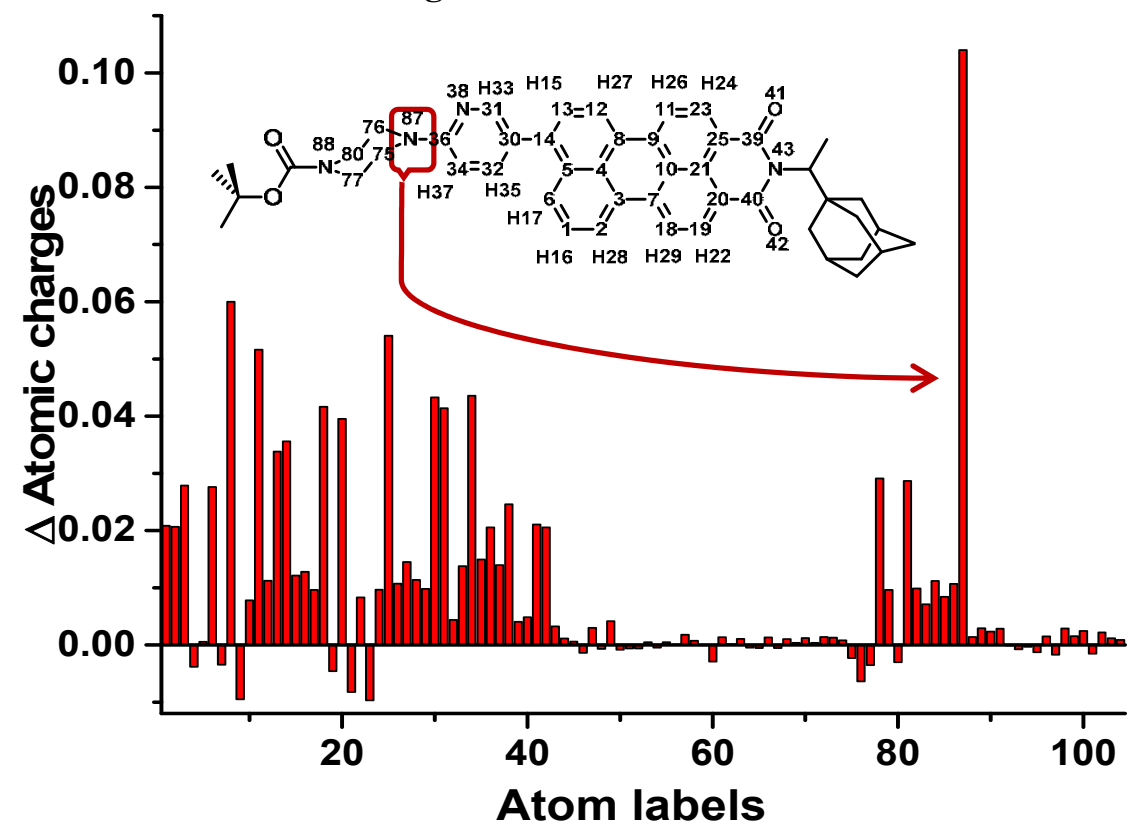

Figure 38. DFT calculated HOMO and SOMO plots for compound $\mathbf{J G 1 2 5} / \mathbf{J G 1 2 5}{ }^{+\cdot}$ and HOMO plot of JG125[O]. NBO plot of differences between radical and neutral charges for every atom in $\mathbf{J G 1 2 5} / \mathbf{J G 1 2 5}^{+\cdot}$. The higher variation was observed for the nitrogen atom labelled as 87 . Outer groups are not labelled.

\footnotetext{
${ }^{28}$ The calculations were performed by Jose Vicente Cuevas Vicario, from Burgos University.

${ }^{29}$ A. Capobianco. A. Velardo. A. Peluso. Comput. Theor. Chem. 2015, 1070, 68-75.

${ }^{30}$ M. J. Frisch, G. W. Trucks, H. B. Schlegel, G. E. Scuseria, M. A. Robb, J. R. Cheeseman, G. Scalmani, V. Barone, B. Mennucci, G. A. Petersson, H. Nakatsuji, M. Caricato, X. Li, H. P. Hratchian, A. F. Izmaylov, J. Bloino, G. Zheng, J. L. Sonnenberg, M. Hada, M. Ehara, K. Toyota, R. Fukuda, J. Hasegawa, M. Ishida, T. Nakajima, Y. Honda, O. Kitao, H. Nakai, T. Vreven, J. A. Montgomery, Jr., J. E. Peralta, F. Ogliaro, M. Bearpark, J. J. Heyd, E. Brothers, K. Raghavachari, A. Rendell, J. C. Burant, S. S. Iyengar, J. Tomasi, M. Cossi, N. Rega, J. M. Millam, M. Klene, J. E. Knox, J. B. Cross, V. Bakken, C. Adamo, J. Jaramillo, R. Gomperts, R. E. Stratmann, O. Yazyev, A. J. Austin, R. Cammi, C. Pomelli, J. W. Ochterski, R. L. Martin, K. Morokuma, V. G. Zakrzewski, G. A. Voth, P. Salvador, J. J. Dannenberg, S. Dapprich, A. D. Daniels, O. Farkas, J. B. Foresman, J. V. Ortiz, J. Cioslowski, D. J. Fox, Gaussian 09, Revision D.01, Gaussian, Inc. Wallingford CT, 2013.
} 


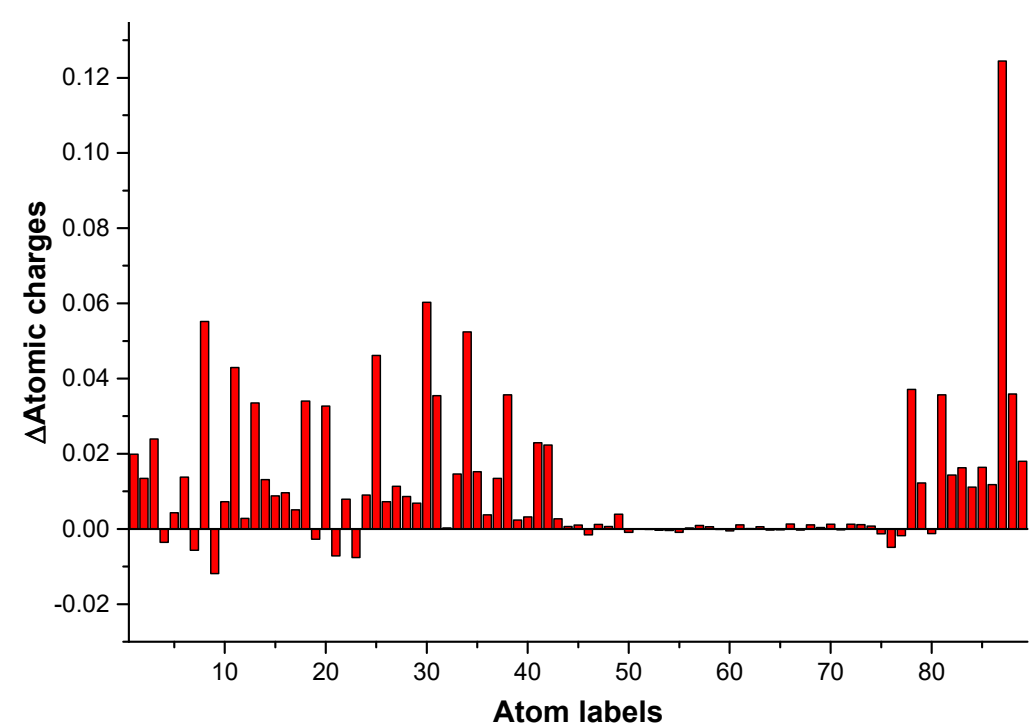

Figure 39. Difference between the charges of the atoms in JG125d and the cationic radical JG125d $\mathrm{d}^{+}$. The higher variation was observed for the nitrogen atom labelled as 87 .

The results agreed with the oxidation in the N(87), the amine group of piperazine position. The NBO analysis ${ }^{31}$ showed the highest increase in the charge, after the removal of one electron, located on the piperazine nitrogen atom directly bonded to the position 2 of the pyridine ring (See N87 position in Figure 38). This atom showed an increase in charge of 0.104 for JG125 (its natural charge value changed from -0.458 in the neutral compound to -0.354 in the oxidized radical cation) and 0.124 in compound JG125d (variation from -0.454 in the neutral compound to -0.330 in the oxidized radical cation). It was expected that the oxidation by TATP started on this nitrogen atom (Figure 40).

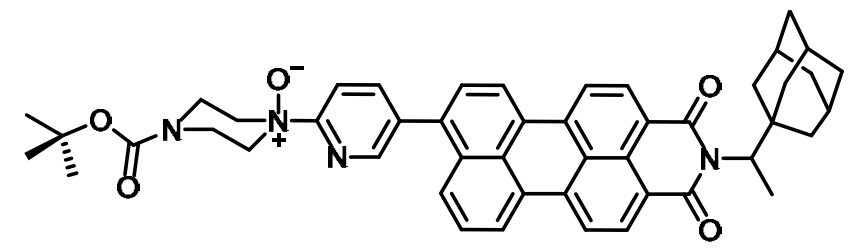

Figure 40. JG125[O], oxidized molecule.

From the representation of the orbitals (Figures 41 and 42), as expected, these two molecular orbitals displayed a similar topology. Both orbitals were centred on the PMI core and the pyridine ring with important participation of the piperazine nitrogen atom bonded to the position 2 of the pyridine ring. There was, as well, a small participation of orbitals belonging to the oxygen atoms of the PMI fragment.

\section{HOMO JG125}

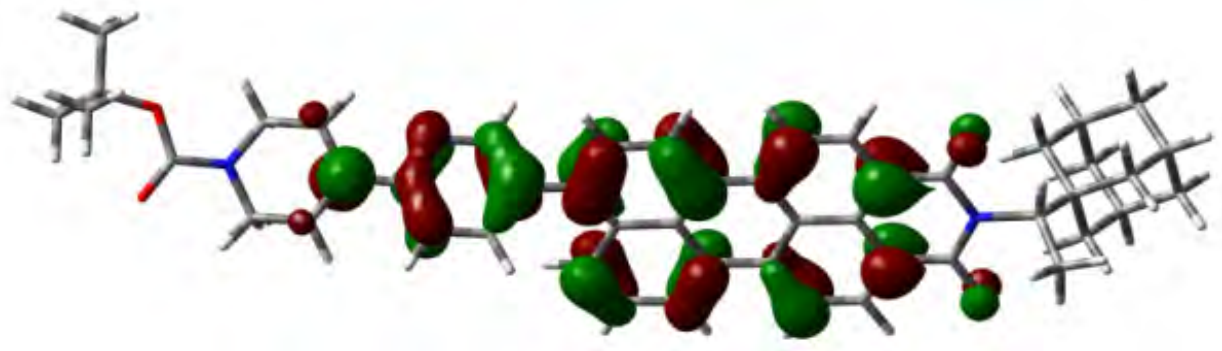

${ }^{31}$ E. D. Glendening, A. E. Reed, J. E. Carpenter, F. Weinhold, NBO Version 3.1. 


\section{SOMO JG125}

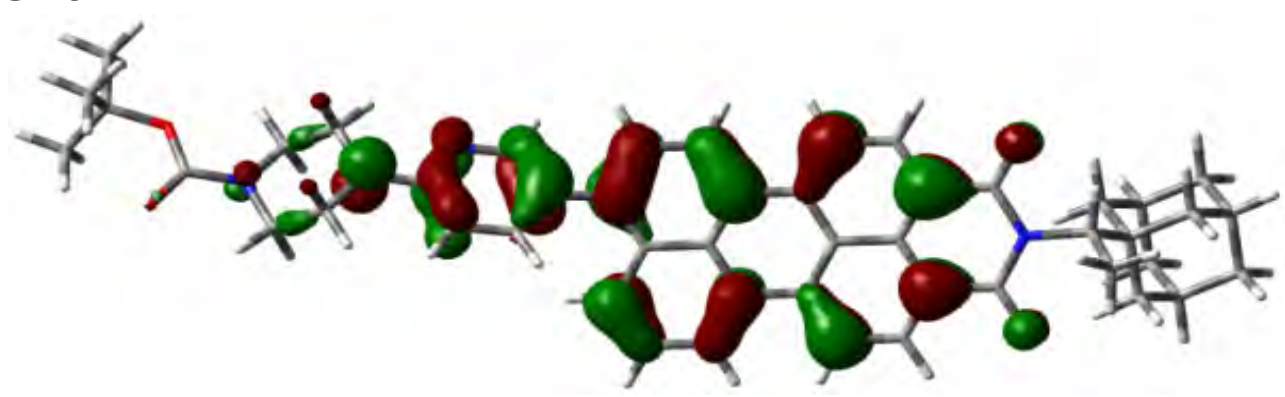

Figure 41. HOMO and SOMO orbital diagram representation of JG125.

HOMO

JG125d

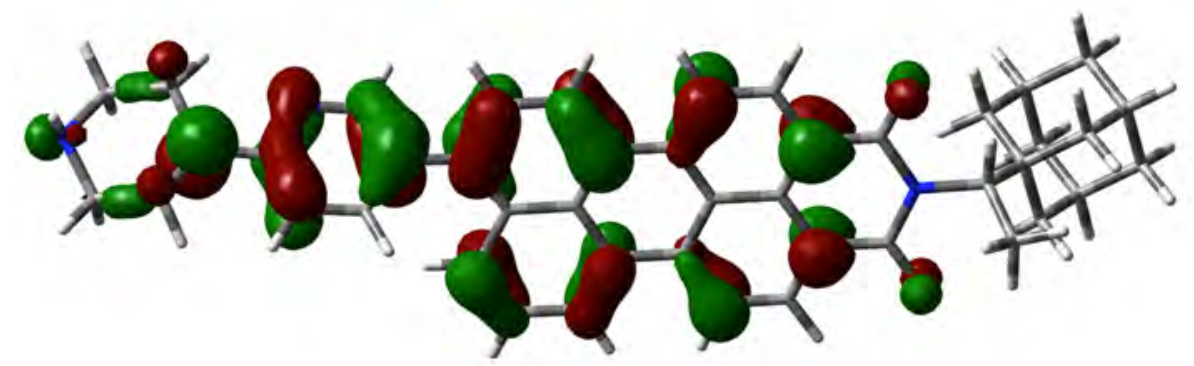

SOMO

$[\mathrm{JG125d}]^{+}$

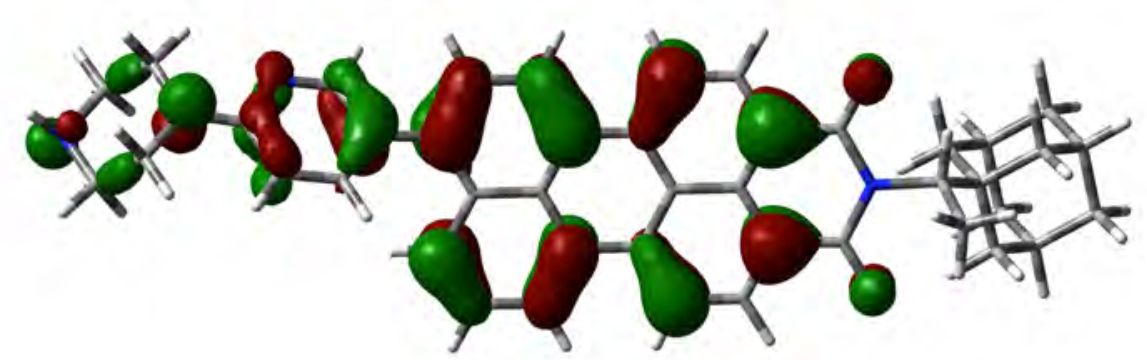

Figure 42. HOMO and SOMO orbital diagram representation of JG125d.

The most important difference observed between orbitals calculation was the small participation of the orbitals of the N-H bond of the piperazine in the deprotected compound JG125d while the protection with Boc of the amine in JG125 cancelled this contribution to the HOMO. The difference in the structure of HOMOs between both compounds stabilized the HOMO of JG125 in $0.04 \mathrm{eV}$. The difference in the reversibility observed in the electrochemistry can be related to the observed differences in the HOMO of JG125/JG125d as well. The oxidation process affected the charge of the atoms with orbitals participating in the HOMO, displaying an increase of the positive electric charge of these atoms (Figure 43).

HOMO

JG125[O]

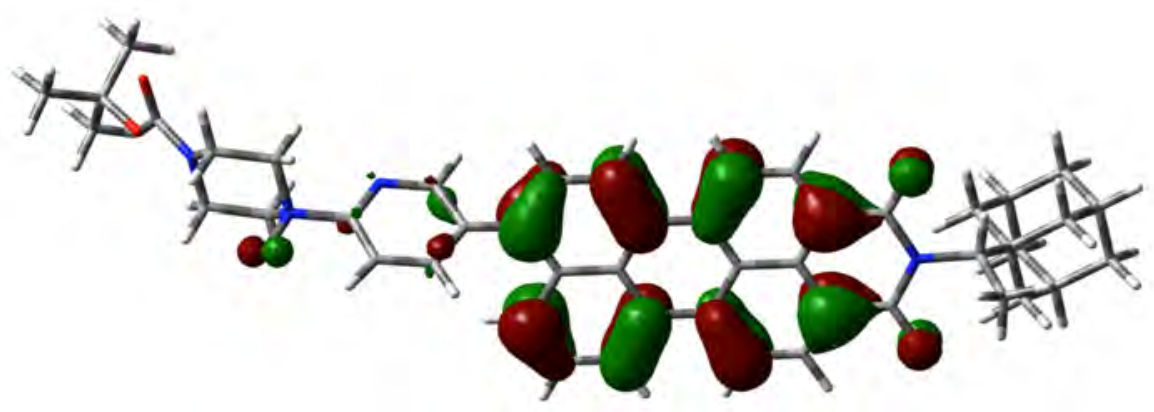


LUMO JG125[O]

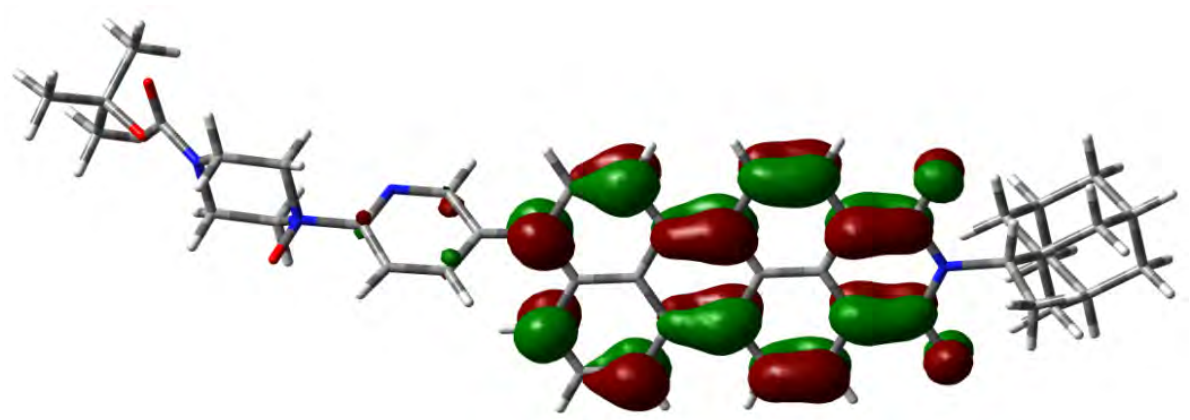

Figure 43. DFT calculated HOMO/LUMO plots for compound JG125[O].

Furthermore, the study of the oxidized molecule gave information that could be associated to the change in fluorescence. As it can be seen in Figure 43, the contribution of the orbitals from the pyridine to the PMI structure was diminished when oxidized, stopping the charge transfer. Then, the fluorescence should be higher and more similar to a non-substituted PMI, which was in agreement with the experimental result, in which a shift to lower wavelength was observed.

\subsection{Mass spectrometry of JG125 after detecting TATP}

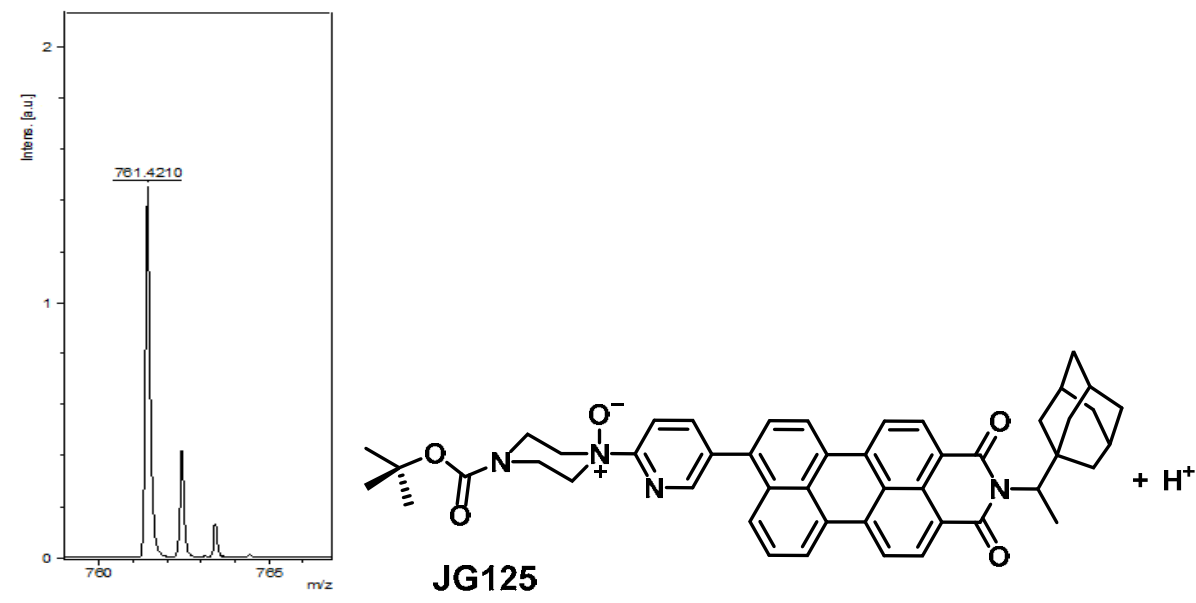

Figure 44. Mass spectrometry of compound JG125[O]. The highest peak corresponded to JG125 + Oxygen $+\mathrm{H}^{+}$, detected by Mass Spectrometry in the MALDI MS spectrum. HRMS (MALDI+, DCTB) $\mathrm{m} / \mathrm{z}$ calcd. for $\mathrm{C}_{48} \mathrm{H}_{49} \mathrm{~N}_{4} \mathrm{O}_{5}: 761.3703\left(\mathrm{M}^{+}+1\right)$; found: 761.4210 .

MALDI-TOF mass spectrometry (Figure 44), showed a peak at $\mathrm{m} / \mathrm{z} 761.42$, corresponding to the protonated $\mathrm{N}$-oxide of $\mathrm{JG} 125+\mathrm{O}+\mathrm{H}^{+}(\mathrm{m} / \mathrm{z}$ calcd. 761.37$)$, validating the oxidative process. 


\section{CONCLUSIONS}

A series of fluorogenic compounds and silica-based materials were synthetized so as to generate fluorescence in the presence of vapours of the explosive triacetone triperoxide, TATP

Compound JG125, a PMI derivative, was synthetized. This compound was capable of detecting TATP selectively from acids. The LOD was $0.27 \mathrm{mg}$ in a $2.5 \mathrm{~mL}$ chloroform solution.

Additionally, JG125 was used to modify silica materials. The fluorescence of these materials increased highly and selectively in presence of TATP vapours (Figure 35). Specifically, it was possible to detect up to $0.12 \mathrm{mg}$ in vapour; in a few minutes after exposition to a TATP stream. Furthermore, the materials were highly selective to TATP, being a reversible process if it was an effect of acid vapours and not giving response to $\mathrm{H}_{2} \mathrm{O}_{2}$, acetone or $\mathrm{Et}_{2} \mathrm{O}$ vapours.

The explanation for the detection mechanism consisted of the oxidation of the amino-substituted piperazine group. When the oxidation occurred, the organization of the HOMO-LUMO orbitals of the molecule changed, causing the end of the quenching in fluorescence. When it was oxidized the distribution of the orbitals became similar to pristine PMI derivatives; which was in agreement to the fluorescence observed.

In conclusion, JG125, and the materials derived from it, were suitable to detect TATP, minimizing the risk of a false positive detection, which was a common issue in literature. 


\section{RESUMEN DEL CAPÍTULO}

El objetivo de este capítulo es el desarrollo de una sonda fluorescente capaz de detectar el explosivo conocido como TATP, "la madre de Satán", mediante un aumento de fluorescencia selectivo frente a posibles interferentes.

El TATP es un explosivo de fabricación casera utilizado comúnmente en ataques terroristas. La extrema volatilidad y dificultad de manejo hacen imposible cualquier aplicación que consista en producir una explosión controlada. Químicamente, el TATP es un peróxido producido a partir de componentes de fácil acceso, principalmente acetona, y su propia estructura hace muy difícil su detección debido a que solo contiene tres tipos de átomos, carbono, hidrógeno y oxígeno, y ningún anillo aromático.

Hasta ahora, se han publicado varias investigaciones concernientes a su detección, no obstante, los métodos más fiables como el ICP masas son de difícil acceso, lentos, muy costosos y difíciles de manejar. Por otro lado, las técnicas basadas en detección con sensores estaban basadas o bien en detectar productos de degradación, o una detección de apagado de fluorescencia; técnicas con gran tendencia a dar falsos positivos.

El trabajo de este capítulo de la tesis consistió en la elaboración de un derivado fluorescente de PMI capaz de oxidarse en presencia de TATP vapor selectivamente, frente a ácidos o agua oxigenada. En primer lugar, se elaboró una sonda soluble en disolventes orgánicos que en presencia de TATP aumentaba de fluorescencia. Una vez comprobado su funcionamiento, esta sonda se unió a materiales modificados de sílica, que permitieron la detección fluorescente en un material. El método de detección, se comprobó que ocurría mediante la oxidación del grupo piperazina de la molécula, obteniendo un cambio en la fluorescencia, lo cual coincidía entre cálculo teórico y resultados experimentales.

En resumen, en este capítulo se explica la síntesis y el funcionamiento de sondas de TATP fluorescentes, a partir de PMIs potencialmente oxidables. Esta sonda era capaz de detectar mediante un aumento en la fluorescencia la presencia de este explosivo frente a sus productos de degradación, como agua oxigenada o acetona; lo cual se presenta como una gran ventaja en comparación con la mayor parte de dispositivos comerciales actuales. 


\section{CHAPTER 3B}

\section{PMI-Ru(II) COMPLEXES FOR CO}

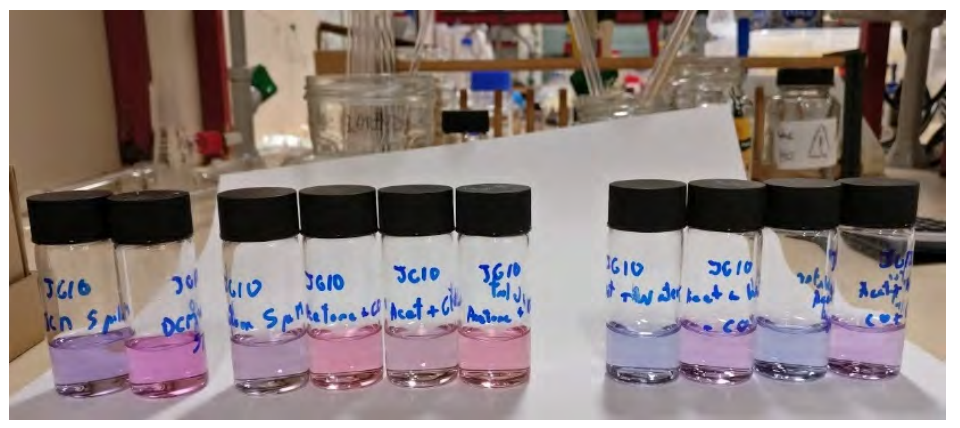

DETECTION

\section{ABSTRACT}

The combination between PMI luminescent properties and the possibilities of metallic $\mathrm{Ru}(\mathrm{II})$ complexes leads to new possibilities for the synthesis of fluorogenic probes. One potential use might be the creation of new CO sensitive systems. In addition, it opens new ways to tune fluorescence of PMIs with applications in different areas of chemistry and biochemistry. ${ }^{1}$

\footnotetext{
${ }^{1}$ The research of this Chapter was performed in the Imperial College (London) in collaboration with the group of James D. Wilton-Ely.
} 



\section{THE ROLE OF CARBON MONOXIDE}

\subsection{The toxicity of $\mathrm{CO}$ gas for living beings}

Carbon monoxide (CO) is a gas well known because of its toxicity for mammals. This gas acts by reducing the oxygen transport capacity of haemoglobin, causing asphyxia and other effects, depending on its concentration and the time of exposure (Figure 1). In case of long-term exposure to $\mathrm{CO}$, even in low quantities (around $35 \mathrm{ppm}$ ), it produces migraine, dizziness and chronic damage on the nervous system. ${ }^{2}$ While quantities superior to $2000 \mathrm{ppm}$ cause instant death. However, what makes it more dangerous, and the main reason to search for new detection methods, is its lack of colour, odour and taste. ${ }^{3}$

Time of exposure (hours)

\begin{tabular}{|c|c|c|c|c|c|c|c|}
\hline ppm Co & $\mathbf{1}$ & $\mathbf{2}$ & $\mathbf{4}$ & $\mathbf{8}$ & $\mathbf{1 2}$ & $\mathbf{1 6}$ & $\mathbf{2 4}$ \\
\hline $\mathbf{3 5}$ & - & - & - & - & Headache & Nausea & Dizziness \\
\hline $\mathbf{5 0}$ & - & - & - & Headache & Nausea & Dizziness & Fatigue \\
\hline $\mathbf{7 5}$ & - & - & Headache & Nausea & Dizziness & Fatigue & Collapse \\
\hline $\mathbf{1 0 0}$ & - & - & Nausea & Dizziness & Fatigue & Collapse & Unconsciousness \\
\hline $\mathbf{2 0 0}$ & - & Nausea & Dizziness & Fatigue & Collapse & Unconsciousness & Unconsciousness \\
\hline $\mathbf{4 0 0}$ & Nausea & Dizziness & Fatigue & Collapse & Unconsciousness & Unconsciousness & Unconsciousness \\
\hline $\mathbf{2 0 0 0}$ & Death & Death & Death & Death & Death & Death & Death \\
\hline
\end{tabular}

Figure 1. Consequences of different times of exposure to different concentrations of $\mathrm{CO}$.

Typically, the presence of $\mathrm{CO}$ has been associated to underground mines. In those situations, it was common the use of canary birds for detection of this kind of gases, along with carbon dioxide and methane. Canary birds are very sensitive to any change in the concentration of components in air, stopping singing and dying whenever long-term breathing is dangerous. Nevertheless, $\mathrm{CO}$ importance is not limited to mines. $\mathrm{CO}$ is a pollutant from combustion of vehicles or fuel powered engines, which could perform incomplete combustion leading to $\mathrm{CO}$ emission. In consequence, the sources of this gas are many, being a cause for concern in our nowadays society.

\subsection{Biological applications of $\mathrm{CO}$}

Apart from the $\mathrm{CO}$ toxicity by inhalation, in the last years the knowledge about its role in living cells and tissues has been discovered to be of great importance; even being a key element for some biological processes. For instance, in small quantities, it seems to act as anti-inflammatory or anticoagulant, and it has been discovered that it is an important component in the activity of some enzymes, with direct application for avoiding some cardiovascular disorders. ${ }^{4}$

\footnotetext{
${ }^{2}$ I. Blumenthal, J. R. Soc. Med. 2001, 94, 270-272.

3 a) L. J. Wilkinson, R. H. Waring, G. B. Steventon, S. C. Mitchell, Molecules of Death. Carbon Monoxide: The Silent Killer, 2nd ed.; Imperial College Press: London, 2007, 37. b) K.Rajiah, E. M. Mathew, Afr. J. Pharm. Pharmacol. 2011, 5, 259. c) J. C. Normand, C. Durand, B. Delafosse, Arch. Mal. Prof. Environ. 2011, 72, 240.

${ }^{4}$ R. Motterlini, L. E. Otterbein, Nat. Rev. Drug Discov. 2010, 9, 728-743.
} 
From these discoveries, it was concluded that there would be many possible therapeutic applications in a wide variety of biological environments. ${ }^{5}$ Taking into account therapeutic applications, a series of chemical metallic complexes for controlled CO releasing were synthesised (Figure 2). These molecules are known as CORMs (CO releasing molecules) and photoCORMs, when the release is produced by light irradiation. In those cases, and for future research in the topic, it is especially relevant the use of CO detectors, which will allow to understand which kind of biological systems contain $\mathrm{CO}$, where it is located and where it goes if released.
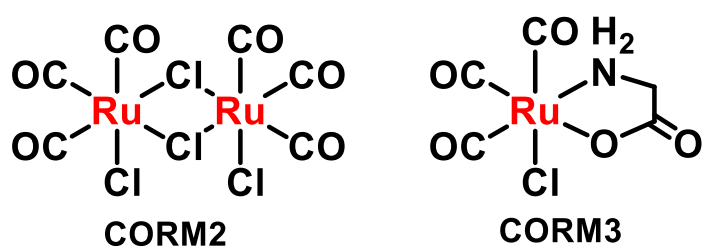

Figure 2. Two derivatives used for the controlled release of $\mathrm{CO}$ within cellular environments.

\subsection{Detection of $\mathrm{CO}$}

The first technology developed for CO detection was based on electrochemical - semiconductor sensors; ${ }^{6}$ however, this kind of detectors are likely to give false positives. The lack of specific interactions makes them more sensitive to the presence of particles in the air and/or any changes in the proportions of different gases than, to the presence of $\mathrm{CO}$ itself.

In the last years, researchers have focused their efforts in using methods that work through optical detection, which has been revealed as a potential solution for having sensitive, cheap, fast and repeatable detection. First positive results were achieved by the use molecular probes of triruthenium complexes, ${ }^{7}$ rhodium complexes, ${ }^{8}$ porphyrins ${ }^{9}$ and iron complexes. ${ }^{10}$ All of them responded to CO presence, but with important limitations, such as low sensitivity, high toxicity or very complicated synthetic procedures.

Additionally, as it was previously mentioned, it was discovered that $\mathrm{CO}$ detection within cells was also of high interest. The first contributions in this regard came from several groups who synthetized CO fluorescent probes, mostly based on palladium. ${ }^{11}$ Nevertheless, they presented very important drawbacks, such as the use of cytotoxic heavy metal salts, the needing of organic solvents (which are also toxic for biological environments) or the requirement long times for cellular uptake of the probes. That combination of disadvantages made those probes not viable for being used in living cells, considering that they usually died in the process.

Finally, in the last years, the work has been oriented to the use of ruthenium complexes instead of palladium, which have given good and promising results, as it is detailed in Section 2.

\footnotetext{
5 a) C. Szabo, Nat. Rev. Drug Discov. 2016, 15, 185-203. b) S. H. Heinemann, T. Hoshi, M. Westerhausen, A. Schiller, Chem. Commun. 2014, 50, 3644-3660.

${ }^{6} \mathrm{~N}$ Barsan, U Weimar, J. Phys.: Condens. Matter. 2003, 15, 813-839.

${ }^{7}$ M. Itou, Y. Araki, O. Ito, H. Kido, Inorg. Chem. 2006, 45, 6114.

${ }^{8}$ A. Gulino, T. Gupta, M. Altman, S. Lo Schiavo, P. G. Mineo, I. L. Fragalá, G. Evmenenko, P. Dutta, M. E. Van der Boom, Chem. Commun. 2008, 0, 2900-2902.

${ }^{9}$ S. Paul, F. Amalraj, S. Radhakrishnana, Synth. Met. 2009, 159, 1019.

${ }^{10}$ D. Benito-Garagorri, M. Puchberger, K. Mereiter, K. Kirchner, Angew. Chem. Int. Ed. 2008, 47, 9142-9145.

${ }^{11}$ a) J. Wang, J. Karpus, B. S. Zhao, Z. Luo, P. R. Chen, C. He, Angew. Chem. Int. Ed. 2012, 51, 9652-9656. b)

B. W. Michel, A. R. Lippert, C. J. Chang, J. Am. Chem. Soc. 2012, 134, 15668-15671.
} 


\section{Ru(II) COMPLEXES FOR CO DETECTION, ANTECEDENTS AND POSSIBLE IMPROVEMENTS}

The initial objective for creating $\mathrm{Ru}(\mathrm{II})$ PMI complexes was to develop cheap, selective and sensitive CO-detectors. The first publications in this regard, such as the one by Itou et al., ${ }^{7}$ were promising, but not optimized for $\mathrm{CO}$ detection, needing from previous reduction of the species.

To solve the different issues, the group of Wilton-Ely (London), in collaboration with the group of Martínez-Máñez (Valencia) published several papers in which they optimized the use of $\mathrm{Ru}(\mathrm{II})$ complexes for the purpose $\mathrm{CO}$ detection. ${ }^{12}$ First, they synthetized a Ru(II) complex in which a BTD ligand was substituted by $\mathrm{CO}$, what produced a massive change in colour-fluorescence in the process. Then, several variations were also tested (Figure 3).

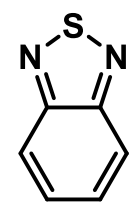

BTD

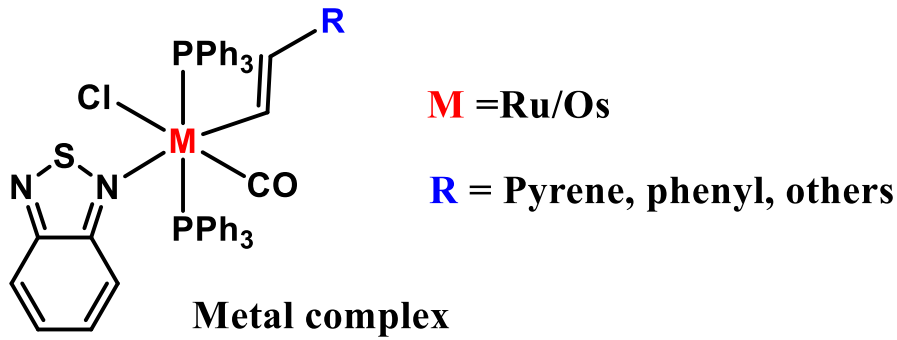

Metal complex

Figure 3. Molecular structure of metallic complexes with BTD.

These synthetized molecules were embedded in silica gel (Figure 4) and cellulose paper to create supported materials. The materials also changed its fluorescence and/or colour, presenting very low LODs. The best advantage was the possibility to create a sensitive to naked-eye detection mechanism, and cheaper than commercial devices.

a)

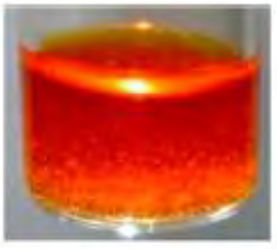

b)

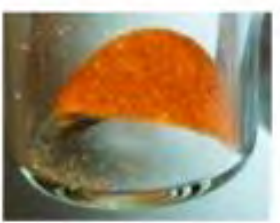

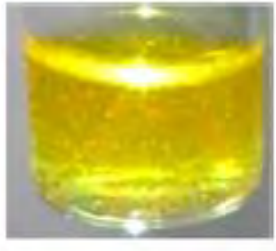

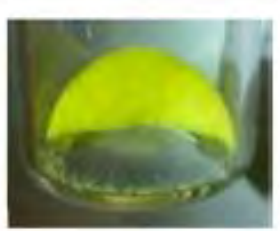

Figure 4. Pictures of the changes of DCM solution (a) and dry (b) silica supported probe containing BTD, Ruthenium and pyrene substituents. Before bubbling CO (left) and after in presence of CO

(right).

\footnotetext{
12 a) C. de la Torre, A. Toscani, C. Marín-Hernández, J. A. Robson, M. C. Terencio, A. J. P. White, M. J. Alcaraz, J. D. E. T. Wilton-Ely, R. Martínez-Máñez, F. Sancenón. J.Am. Chem. Soc. 2017, 139, 18484-18487 b) A. Toscani, C. Marìn-Hernández, M. E. Moragues, F. Sancenón, P. Dingwall, N. J. Brown, R. Martínez-Máñez, A. J. P. White J. D. E. T. Wilton-Ely, Chem. Eur. J., 2015, 21, 14529-14538. c) C. Marín-Hernández, A. Toscani, F. Sancenón, J. D. E. T. Wilton-Ely, R. Martínez-Máñez, Chem. Commun. 2016, 52, 5902-5911 d) M. E. Moragues, A. Toscani, F. Sancenón, R Martínez-Máñez, A. J. P. White, J. D. E. T. Wilton-Ely, J. Am. Chem. Soc. 2014, 136, 11930-11933.
} 
In the last years, the work with $\mathrm{CO}$ ruthenium complexes was oriented to tracking its presence in living cells. In doing so, two aims had to be addressed, the water affinity of the complex and the excitation wavelength. In this way, water soluble probes would lead to faster cellular uptake and, in addition, combined with the use of excitation wavelengths superior to $400 \mathrm{~nm}$, it would make the cells less likely to die.

Therefore, a new complex was created by changing BTD for TBTD and using a PEG in the vinyl group to increase water affinity (Figure 5). ${ }^{12 a}$<smiles></smiles>

Figure 5. Molecular structure of a $\mathrm{Ru}(\mathrm{II})$ complex used for detection of $\mathrm{CO}$ in cells. ${ }^{12 \mathrm{a}}$

This complex had the fluorescence of TBTD quenched by $\mathrm{Ru}(\mathrm{II})$, which was increased when freed. Besides, this probe was tested in "RAW 264.7" cells. The results showed a selective increase to CO presence against other interferents, such as tryptophan or cysteine; with low incubation times, and not being toxic for cells after freeing the ligand. Additionally, it gave positive results when used for ex vivo detection in a mouse model. The issue of avoiding photodegradation was overcome using double photon excitation with a $715 \mathrm{~nm}$ laser.

In spite of the results, the characteristics of fluorescent probes for $\mathrm{CO}$ detection could be significantly improved. Some possibilities are:

- Having emission at higher wavelengths. The use of double photon excitation experiments is only an improvement to UV excitation. This is because it requires from high intensity energy sources so as to the excitation takes place, which may also have adverse effects over cells or even degrade the samples.

- Increasing water solubility, to reduce the requirement for organic solvents when testing in cellular environments.

- Achieving higher fluorescent quantum yields, to provide an easier and more accurate detection. 


\section{OBJECTIVES}

The aim of this chapter was to show some potential applications of PMIs as fluorescent ligands for creation of stable metallic complexes, modifying their photoelectronic properties; which may provide them with straightforward applications as sensors. Additionally, they also might be interesting for possible light harvesting devices or biological applications, such as photodynamic therapy (PDT) or cellular markers.

Taking into account all the antecedents, the aim of the work was the improvement of $\mathrm{Ru}(\mathrm{II})-\mathrm{CO}$ probes by developing water soluble derivatives, with no excitation at low wavelength or double photon experiments, and with a high drastic change in colour/fluorescence. Besides, it was posed the study of other possible fields of applications, apart from sensing.

The idea was to develop a complex with $\mathrm{Ru}(\mathrm{II})$ in which fluorescence would change in presence of $\mathrm{CO}$ when the coordination sphere changes. The task was addressed by using PMI derivatives, as fluorescent ligands in the visible region. PMIs were modified in peri position by two kinds of substituents, so as to make PMI probes suitable ligands, a pyridine group and a triple bond. Besides, to complete the research, other possible applications as sensors were evaluated, including the detection of cyanide, $\mathrm{Cu}(\mathrm{II})$ or isonitriles.

Regarding biological applications, water solubility was proposed to be improved by introducing ligands with high water affinity, such as highly branched PEG derivatives. Additionally, when PEG chains were introduced in some particular PMI derivatives it was found that they presented affinity for some DNA strands, specifically, G-quadruplex (Chapter 2). This piece of information was of great importance for the development of new biological markers or, for example, to modulate the cytotoxic of these derivatives. 


\section{DESIGN OF THE PMI-Ru(II) COMPLEXES}

Taking into account the results of previous research, the starting point was the change of two parts of the complexes (Figure 6). First, using a branched PEG that gave more water affinity (Figure 7). Additionally, and at the same time, using fluorescent molecules with excitation wavelength in the visible region $(>450 \mathrm{~nm}$ ) and high fluorescence quantum yield, such as PMI derivatives (Figure 8). In this way, it was possible to synthesize three new PMI-Ru(II) derivatives (Figure 9)

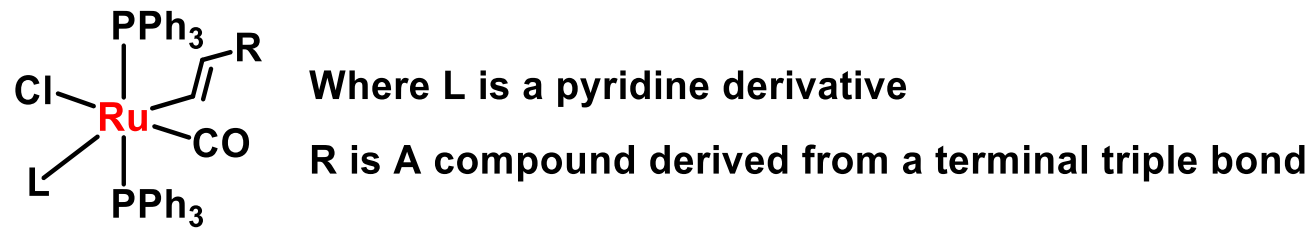

Figure 6. $\mathrm{Ru}(\mathrm{II})$ structure and positions for variation, $\mathrm{R}$ and $\mathrm{L}$.

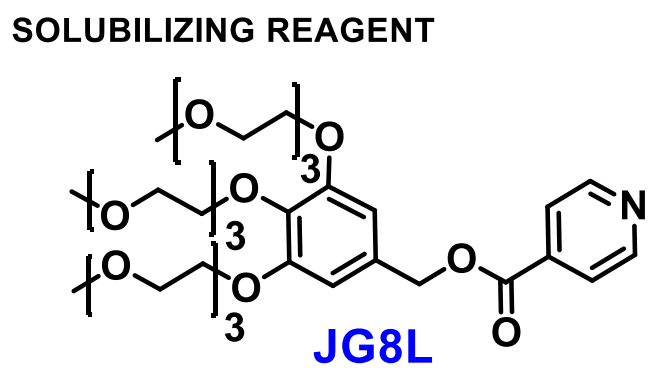<smiles>c1csc(-c2ccc3nsnc3c2)c1</smiles>
TBTD

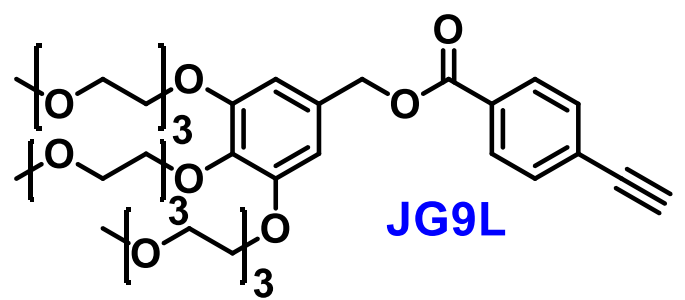

Figure 7. Complementary reagent to the PMI, PEG derivatives JG8L and JG9L, in order to increase solubility and TBTD for comparisons.

\section{PERYLENEMONOIMIDE DERIVATIVES:}
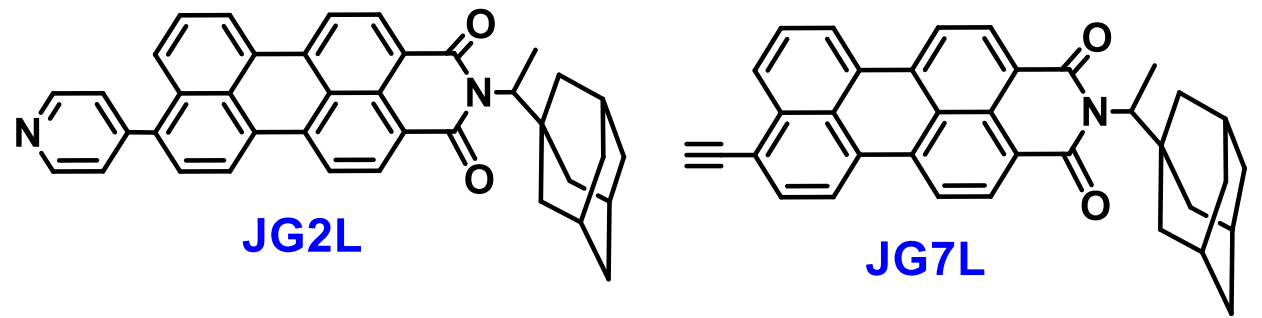

Figure 8. PMI chosen for the $\mathrm{Ru}$ (II) complexes, with a pyridine terminal group (JG2L) and with terminal triple bond (JG7L). 


\section{RUTHENIUM COMPLEXES:}

PMI-Alkyne derivatives:
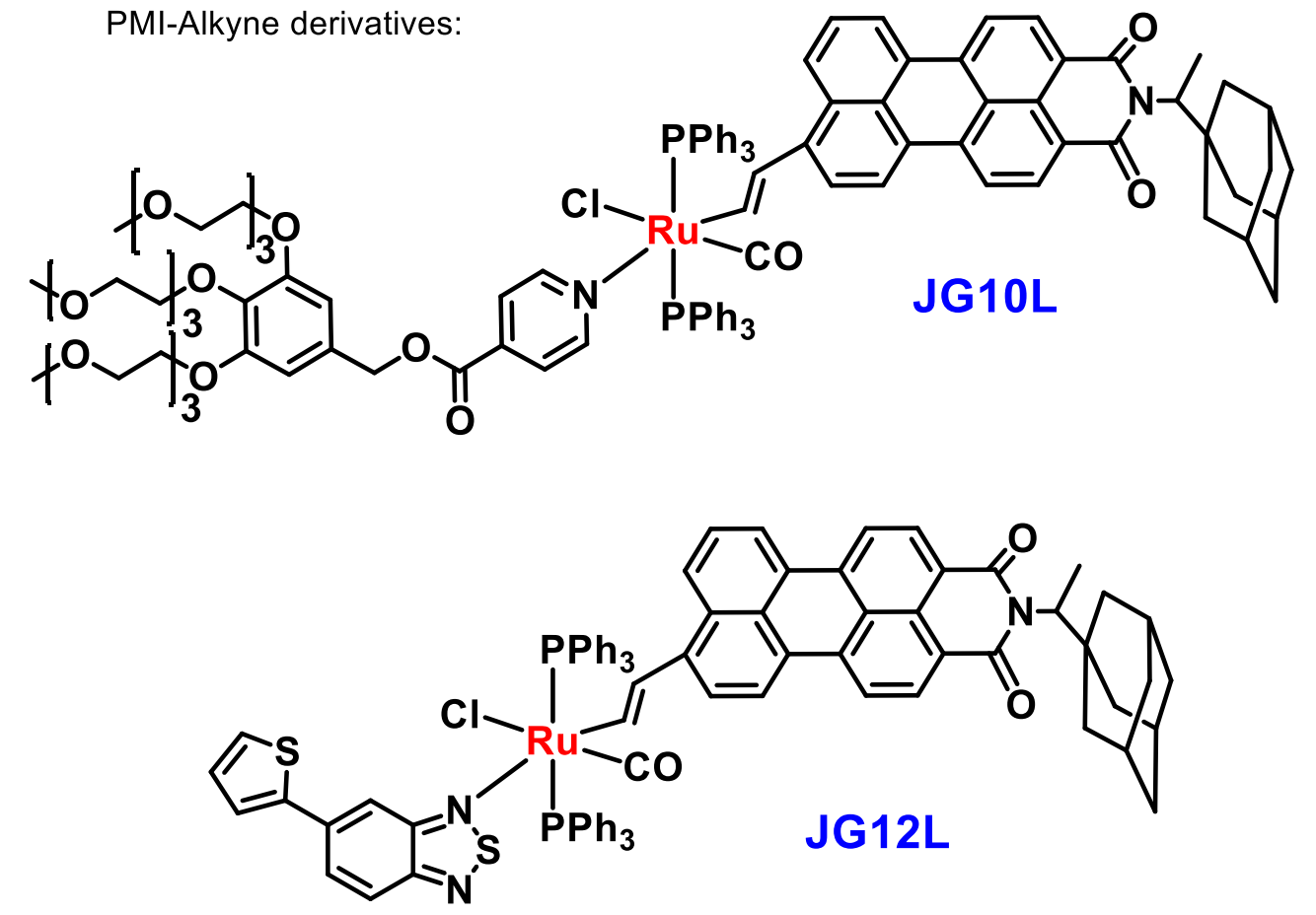

PMI-pyridine derivative:

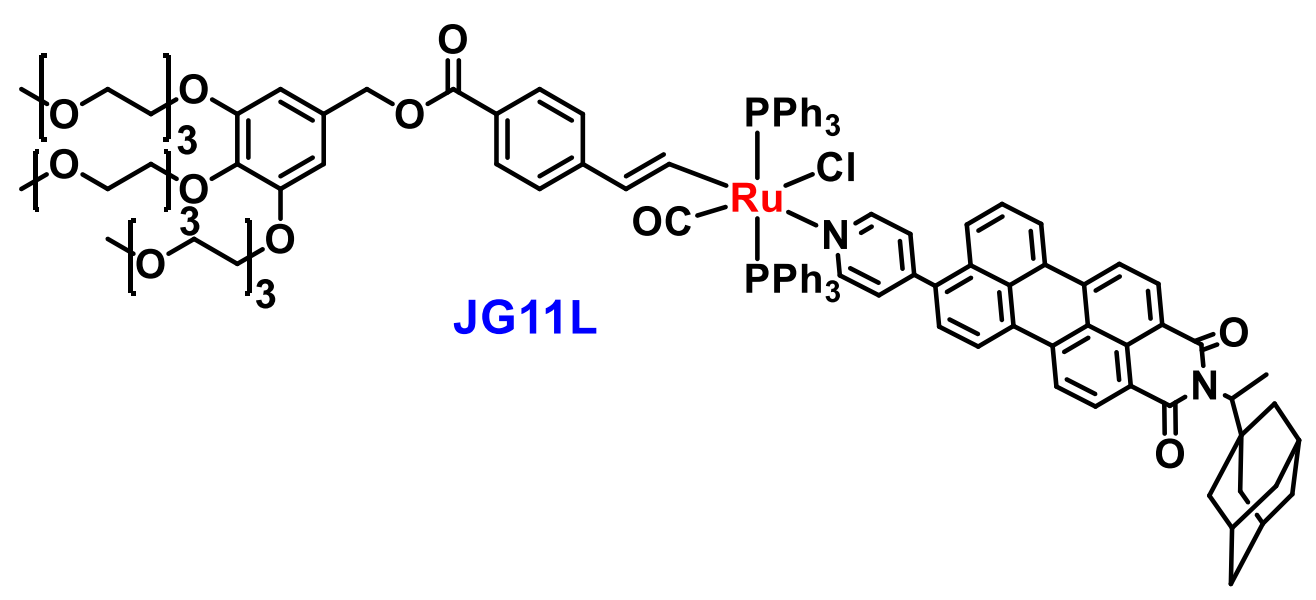

Figure 9. Molecular structures of $\mathrm{Ru}(\mathrm{II})$ complexes synthetized. 


\section{SYNTHESIS OF Ru(II) COMPLEXES ${ }^{13}$}

The conditions for the synthesis of the different intermediates and final products were adapted to several grams of the PEG derivatives, $100-300 \mathrm{mg}$ of PMI derivatives and 50-100 $\mathrm{mg}$ of Ru(II) complexes. A summary of the synthesis steps is detailed in this section.

\subsection{PMI derivatives}

The compounds JG7L and JG2L were synthetized from the peri-monobrominated PMI (JG73) throughout a catalysed Suzuki-Miyaura coupling reaction.

\subsubsection{Pyridine derivative:}
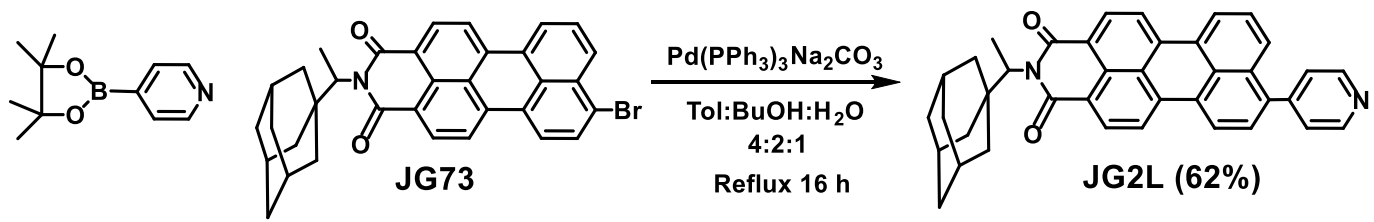

Figure 10. Synthesis scheme of PMI-Pyridine derivative JG2L by Suzuki reaction.

The reaction performed was a Suzuki coupling between PMI-Br (JG73) and p-pinacolboranepyridine, under nitrogen, reflux and overnight. The reaction was purified by silica gel column chromatography with pristine DCM, to obtain the product as a red powder. No particular difficulty was observed; although some eluents, such as methanol, led to no separation of the products in the column.

\subsubsection{Triple bond derivative:}

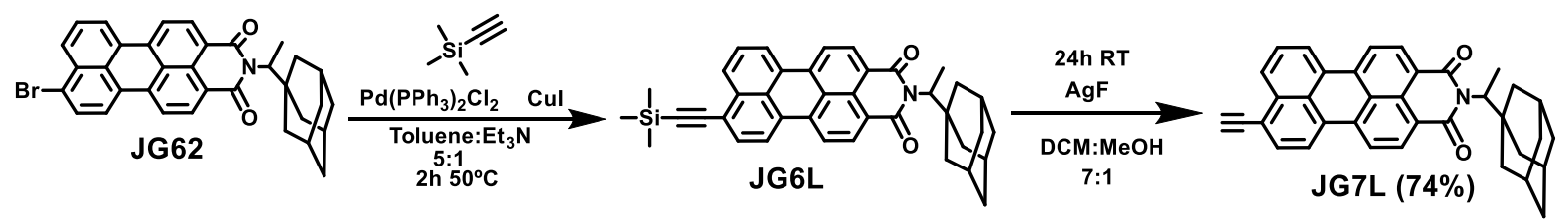

Figure 11. Synthesis scheme of PMI-triple bond derivative JG7L.

The synthesis was performed in two steps. First, the introduction of the silane derivative in toluene, under nitrogen and soft conditions. After that, the silanized PMI was purified by silica gel column chromatography in DCM:Hexane (1:2). The product was checked by ${ }^{1}$ HNMR. Next, the silane was removed by stirring overnight at room temperature the compound in the presence of AgF dissolved in DCM:. $\mathrm{MeOH}, 7: 1$. The solution was quenched with a solution of conc. $\mathrm{HCl} /$ water $1: 1$, then a second silica gel column chromatography (DCM:Hexane $(8: 2)$ ) was performed obtaining the product as a red solid powder.

For this process, it was followed the procedure from Hutchison et al. ${ }^{14}$ However, the fluoride deprotection reagent was changed, due to no results with tetrabutylammonium fluoride, to silver

\footnotetext{
13 The specific recipes for each molecule are specified in Experimental Appendix 2 and 3.

${ }^{14}$ J. A. Hutchison, H. Uji-i, A. Deres, T. Vosch, S. Rocha, S. Müller, A. A. Bastian, J. Enderlein, H. Nourouzi, C. Li, A. Herrmann, K. Müllen, F. De Schryver, J. Hofkens Nat. Nanotech. 2014, 9, 131-136.
} 
fluoride. While they obtained a global yield of $52 \%$, the optimization of their process allowed to increase the final yield to $74 \%$, considering all purification processes.

\subsection{PEG derivatives synthesis}

The procedures for the synthesis of gallic acid PEG derivatives were mostly the same than the followed in Chapter 1, getting equivalent results (Figure 12). The procedure started from a nucleophilic substitution of the tosylated PEG with the hydroxyl groups from the gallic ester and finishing with the reduction of the ester group to alcohol.
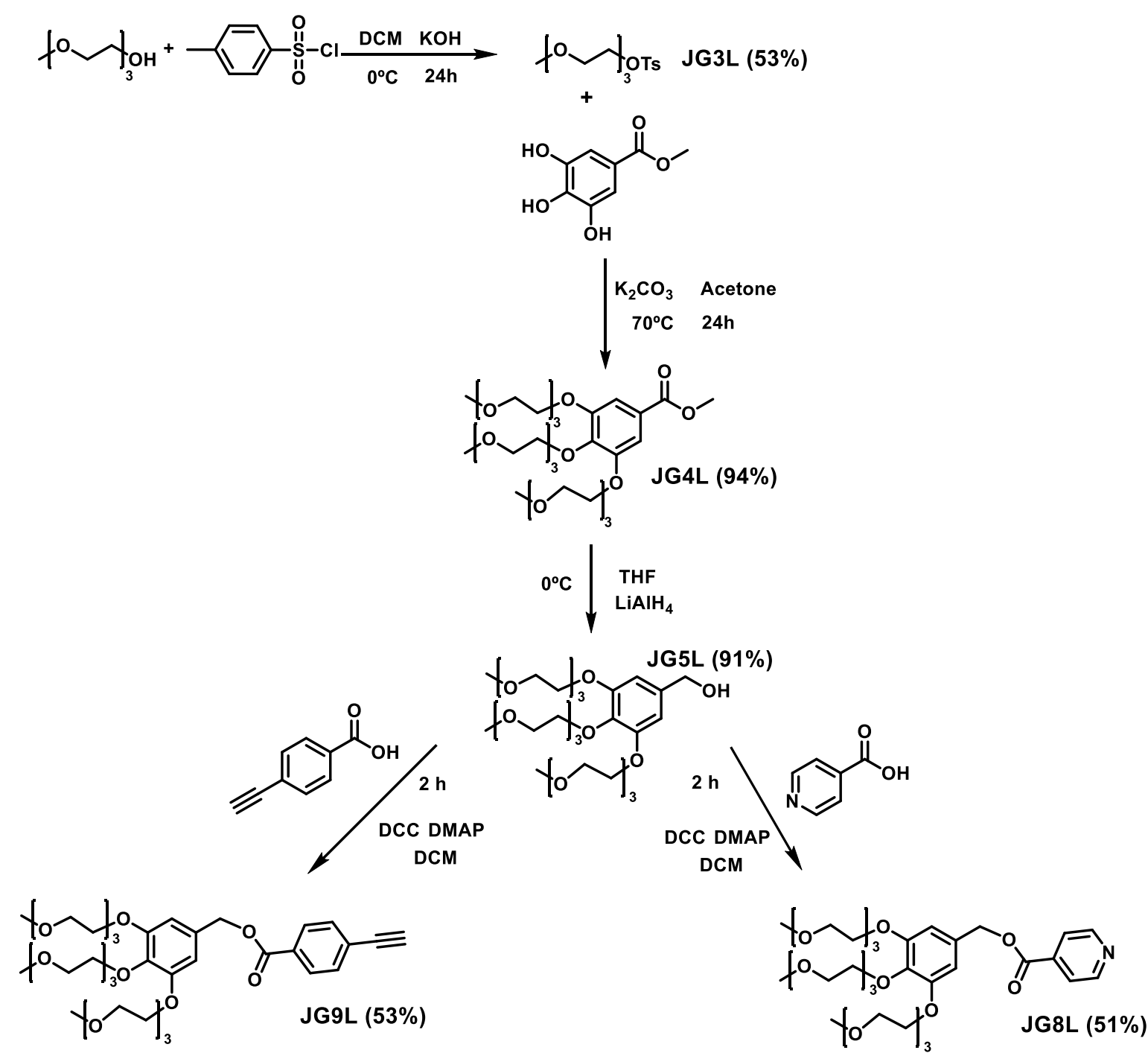

Figure 12. Synthesis scheme for PEG derivatives to increase Ru(II) complexes solubility.

The only difference, with respect to the synthesis in Chapter 1 was the final step from which it was performed an esterification in the presence of DCC and DMAP. After 2 hours under stirring, they were purified by silica gel column chromatography (DCM:MeOH), obtaining the product as viscous yellow oil. 


\subsection{Ru(II) complexes synthesis}

As it is represented in Figure 13, Ru(II) starting complex and JG7L were dissolved in DCM. The solution slowly turned from orange to purple. After one hour, the pyridine-PEG (JG8L) was added. One hour later, the product was precipitated with hexane and washed several times with hexane. The complex was isolated as a dark blue solid in $84 \%$ yield.
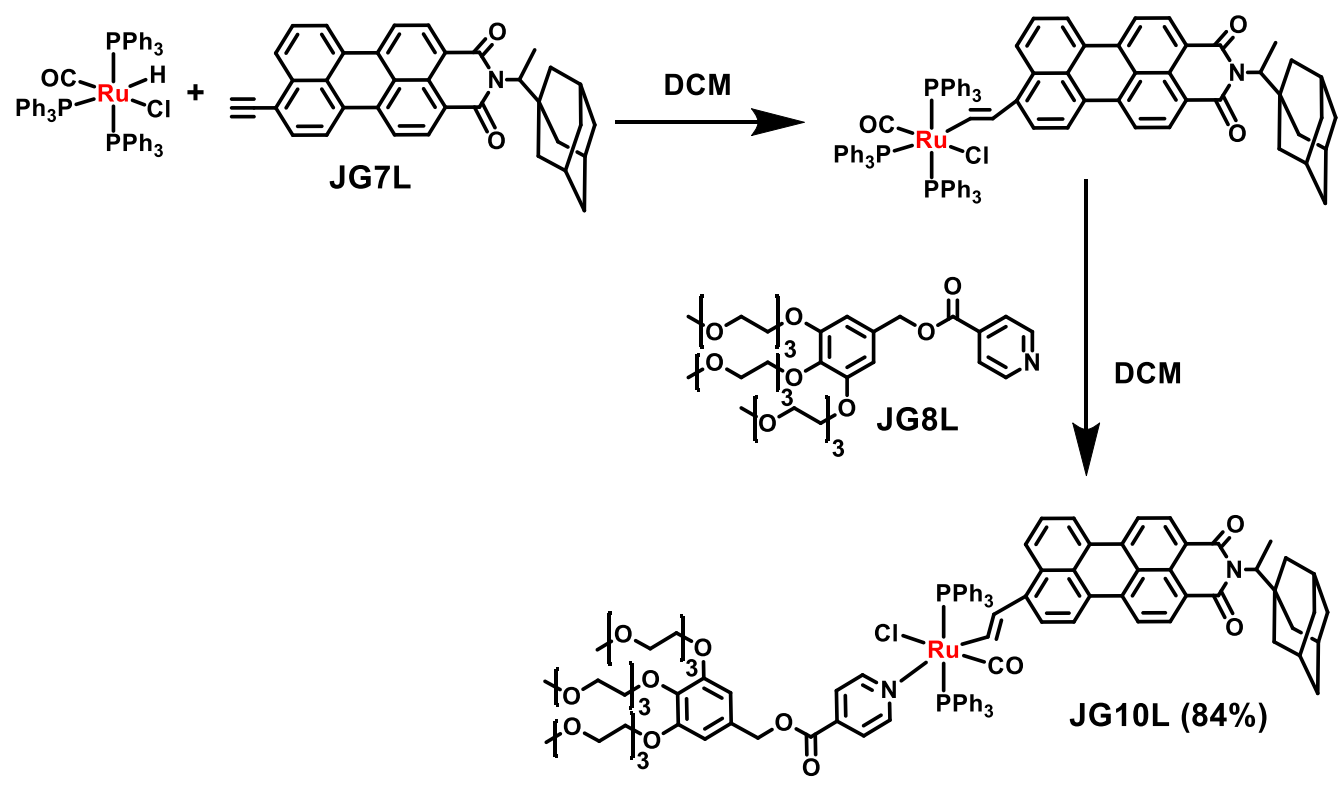

Figure 13. Synthesis of $\mathrm{Ru}(\mathrm{II})$ complexes JG10L.

The synthesis of JG12L was similar to JG10L but instead of using the PEG derivative JG8L, it was introduced a molecule of TBTD.

The synthesis of JG11L (Figure 14) was a bit different from JG10L, starting with the metal complex with two MeCN molecules, to improve reactivity. The $\mathrm{Ru}(\mathrm{II})$ complex and JG6L were dissolved in DCM and, after 30 minutes, tetraethylamonium chloride was added. Finally, one hour later, JG2L was added, and the reaction remained under stirring for another hour. The product JG11L was precipitated and washed with DCM, isolating the pure fraction as a red powder.
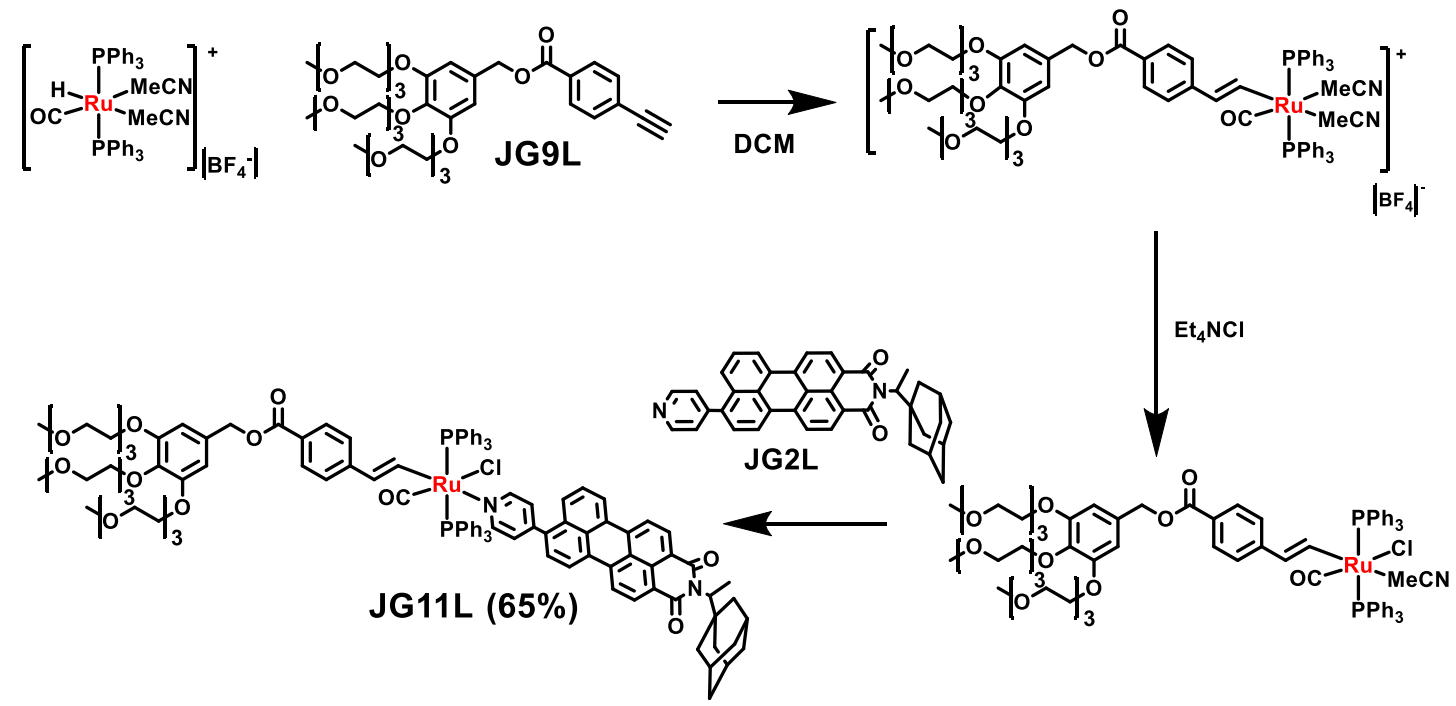

Figure 14. Synthesis of Ru(II) complexes JG11L. 


\section{ABSORBANCE AND FLUORESCENCE INTERPRETATION}

As it was previously showed in Chapter 2, the differences on the parameters of the synthetized compounds (Table 1) were evaluated along with the results of solvatochromism (Figures 15 and 16). It gave some of the properties so as to find the proper applications for the probes.

\begin{tabular}{|c|c|c|c|c|c|c|}
\cline { 2 - 7 } \multicolumn{1}{c|}{} & $\boldsymbol{\lambda}(\mathbf{m a x} \mathbf{a b s})$ & $\boldsymbol{\varepsilon}\left(\mathbf{c m}^{-\mathbf{1}} \mathbf{M}^{-\mathbf{1}}\right)$ & $\boldsymbol{\Phi}_{\mathbf{F}}(\mathbf{\%})$ & $\boldsymbol{\tau}(\mathbf{n s})$ & $\boldsymbol{\chi}^{\mathbf{2}}$ & $\begin{array}{c}\text { Stokes } \\
\text { Fl. Shift }\end{array}$ \\
\hline JG2L & 504 & 37200 & 91 & 4.45 & 1.116 & $510-570$ \\
\hline JG7L & 507 & 49000 & 90 & 4.62 & 1.085 & $510-575$ \\
\hline JG10L & 576 & 46300 & 37.5 & 4.74 & 1.078 & $515-585$ \\
\hline JG11L & 505 & 46000 & 29.5 & 4.57 & 1.098 & $510-570$ \\
\hline
\end{tabular}

* "Stokes Shift" refers to the difference of the position of max $\lambda_{\text {em }}$ between the most polar $(\mathrm{MeOH})$ and the less polar solvent $(\mathrm{MCH})$.

Table 1. Parameters for comparison of compounds in DCM.

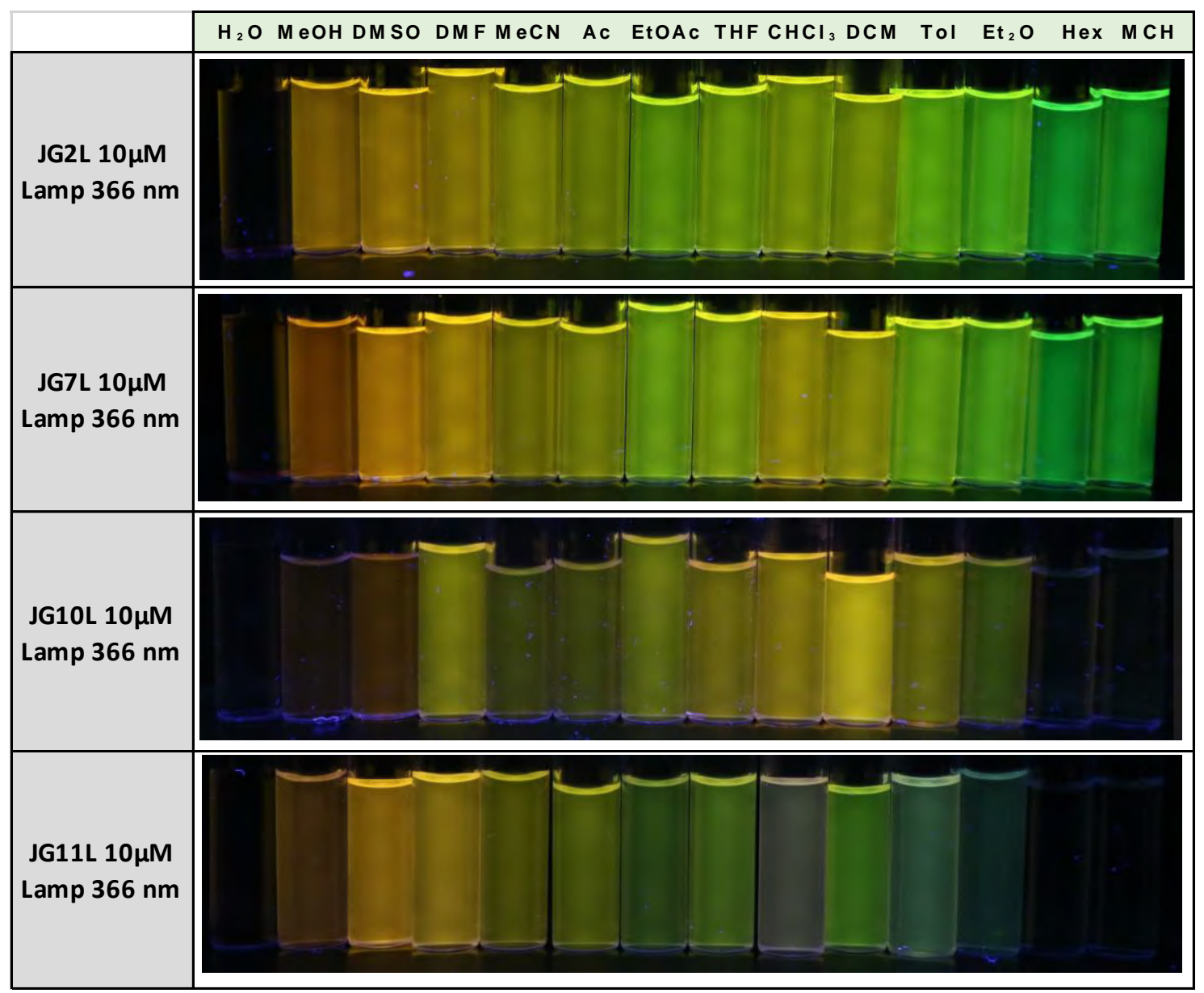

Figure 15. Solvatochromism of JG2L, JG7L, JG10L and JG11L under $366 \mathrm{~nm}$ light. 


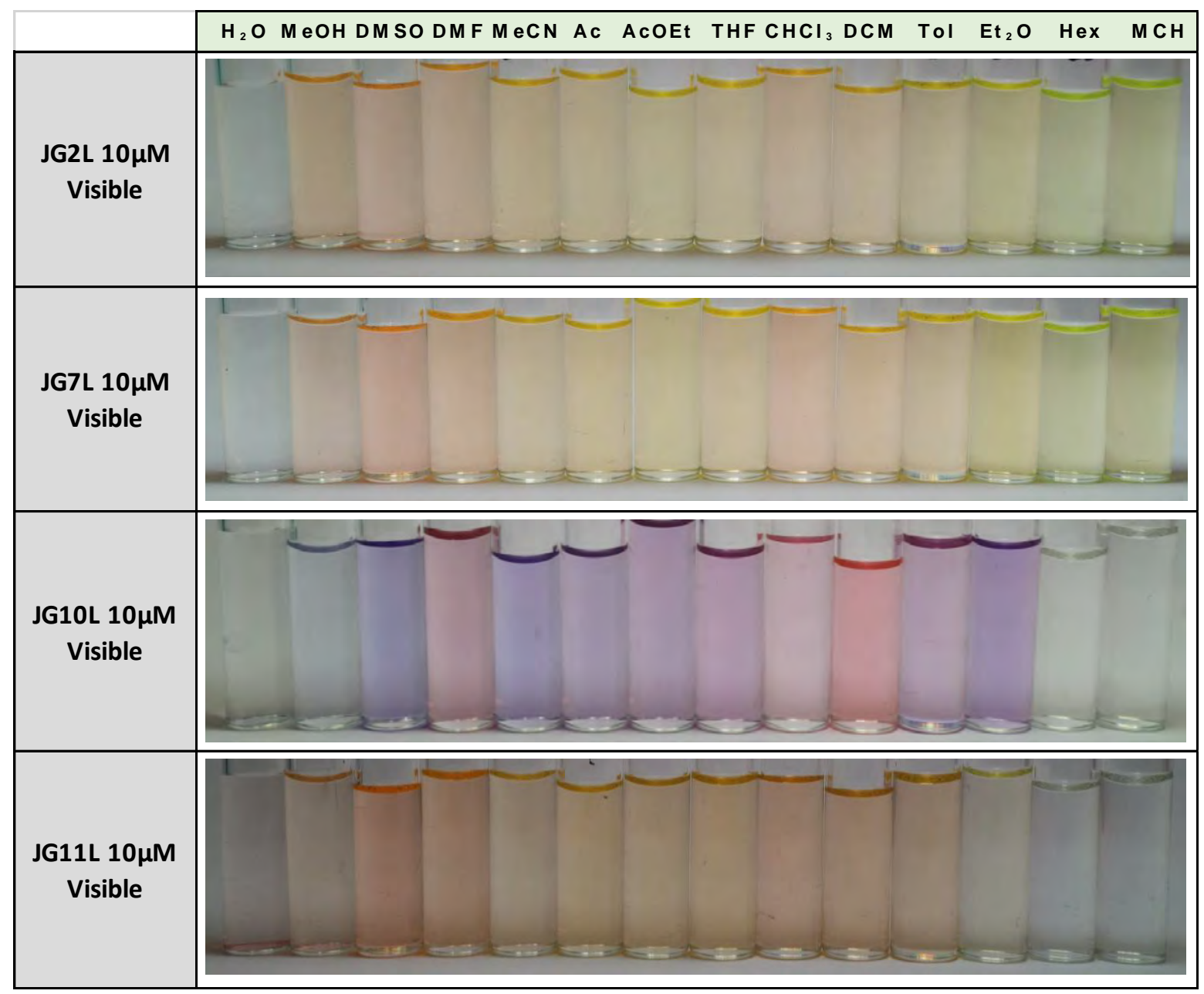

Figure 16. Solvatochromism of JG2L, JG7L, JG10L and JG11L under visible light.

- The behaviour of JG2L and JG7L in solution was very similar between them and to pristine PMI.

- JG10L and JG11L were unstable in some solvents such as chloroform, changing their fluorescence and/or precipitating with time.

- JG10L presented a secondary emission band between 600-750 nm, low but wide.

- None of them were soluble in $\mathbf{1 0 0 \%}$ water solution.

- JG10L was unstable in solution when in presence of light, increasing fluorescence with time.

- The fluorescence quantum yield of JG7L decreased from 90 to $38 \%$ when in Ru(II) complex (JG10L) and the fluorescence lifetime decay associated to the perylene core increased in $0.12 \mathrm{~ns}$.

- JG11L was slightly soluble in organic:water mixtures, although no fluorescence was observed. In addition, it was checked that the $\Phi_{\mathrm{F}}$ decreased highly, from $91 \%$ to $30 \%$ in DCM compared to JG2L. The fluorescence lifetime decay associated to the perylene core increased in $0.12 \mathrm{~ns}$ compared to JG2L. 


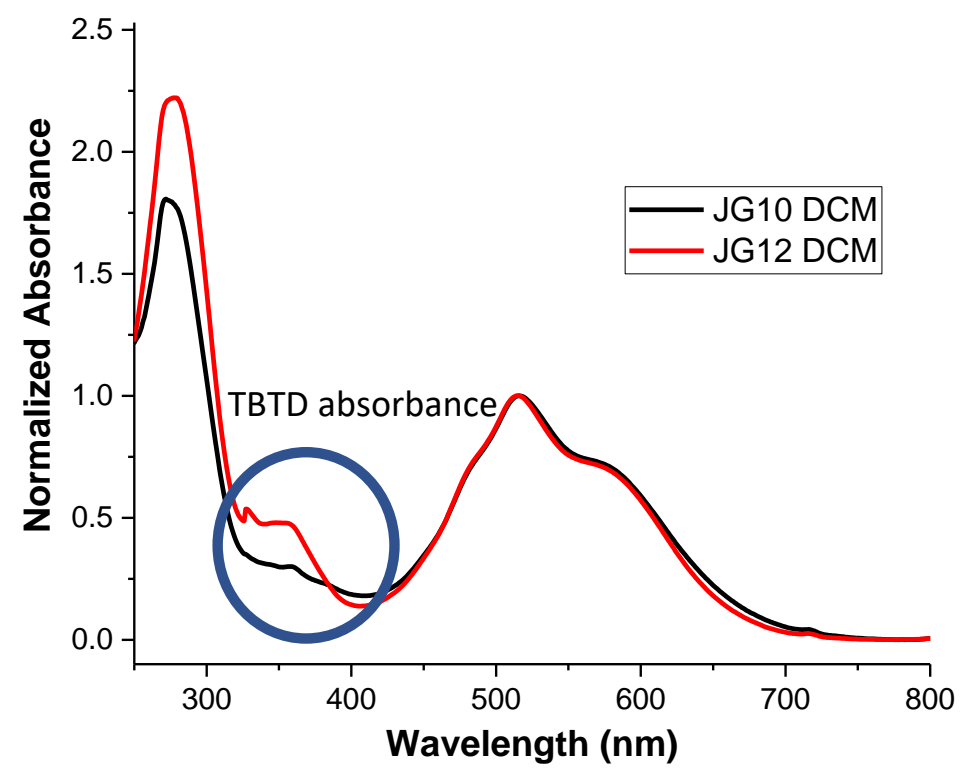

Figure 17. Absorbance comparison of JG10L and JG12L in DCM.

- JG10L and JG12L have only small differences, such as the absorbance of TBTD (Figure 17). This lack of photoelectronic advantages from JG12L against JG10L led to focus the studies in JG10L, which was a bit more soluble in water containing solvents, increasing its applicability.

In conclusion, the synthesized $\mathrm{Ru}(\mathrm{II})$ complexes were not soluble in $100 \%$ water, although they may accept some percentage of water in organic solvents. JG12L was similar to JG10L but less soluble in water. Finally, it was determined that the best solvents to measure were acetone and DCM, although JG10L underwent degradation with light/time. 


\section{TESTS WITH PMI-Ru(II) PROBES}

\subsection{CO detection}

Following the results of previous researchers, the consequences of bubbling CO to JG10L and JG11L solutions in DCM are shown in the Figures 18 and 19. The substitution of the ligands had been already reported in previous papers from Wilton-Ely and coworkers, a change of the pyridine group with a $\mathrm{CO}$ molecule led to a change in the properties of the complex, affecting directly their absorbance-fluorescence and solubility.
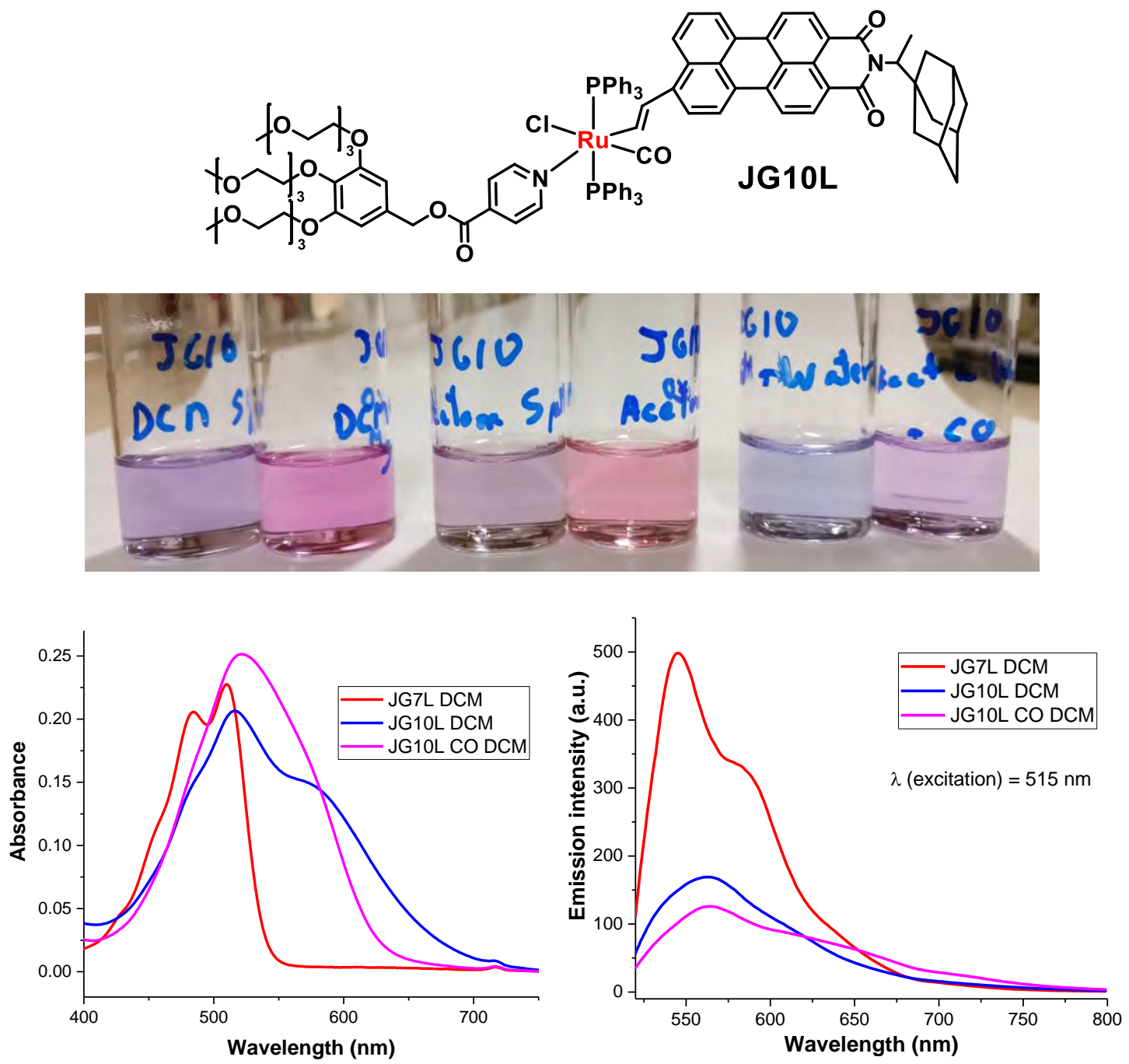

Figure 18. Visible appearance of $5 \mu \mathrm{M}$ solutions, DCM, acetone and acetone:water 9:1; of JG10L (up). Solvatochromic study $10 \mu \mathrm{M}$ of JG10L, absorbance (down left) and fluorescence (down right) in different solvents.

From the results that shows Figure 18, it was concluded that the emission increased at higher wavelengths (region of $620-720 \mathrm{~nm}$ ) and decreased at its initial maximum $(570 \mathrm{~nm})$. The absorbance peaks stretched from the initial band. 

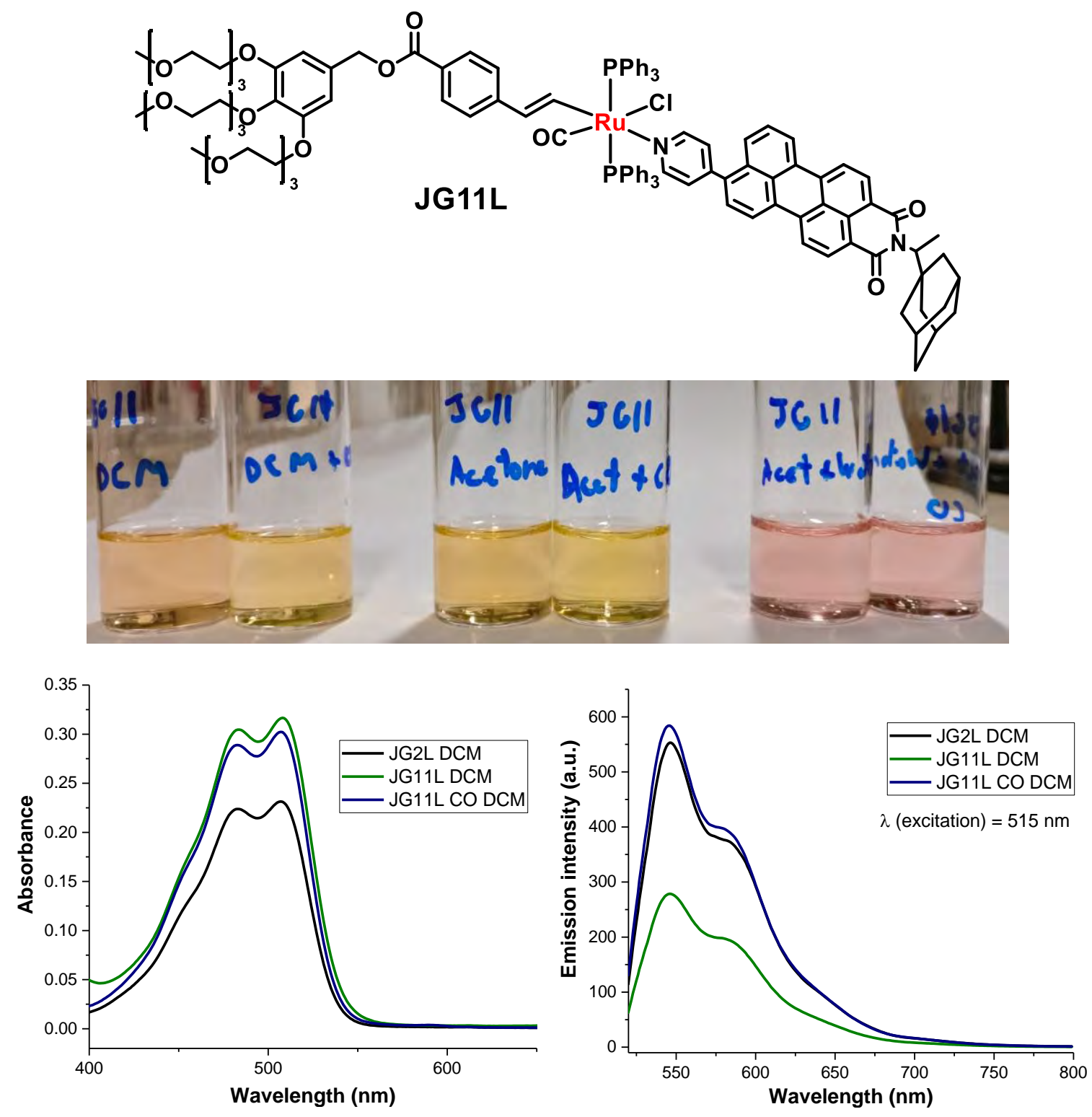

Figure 19. Visible appearance of $5 \mu \mathrm{M}$ solutions, DCM, acetone and acetone:water 9:1; of JG11L (up). Solvatochromic study $10 \mu \mathrm{M}$ of JG11L, absorbance (down left) and fluorescence (down right) in different solvents.

In contrast with JG10L, the results obtained for JG11L (Figure 19), showed that the fluorescence of JG11L doubles in presence of $\mathbf{C O}$, and the absorbance increased too. It seemed that the PMI was substituted by $\mathrm{CO}$ in the complex, which led to an increase in fluorescence, as it was checked by comparing with JG2L 


\subsection{Effect of $\mathrm{CO}$ and Glutathione}

Glutathione (Glut), Figure 20, is a tripeptide presented in cells in millimolar concentrations, it possesses a thiol group and acts as an antioxidant. For the purpose of detecting $\mathrm{CO}$ within cells, it would be of upmost importance to evaluate the possible interference of this species.<smiles>N[C@@H](CCC(=O)N[C@@H](CS)C(=O)NCC(=O)O)C(=O)O</smiles>

Figure 20. Glutathione chemical structure.

Because of that, several solutions were prepared in which glutathione was also presents in solution.

\subsubsection{JG10L + Glut tests:}

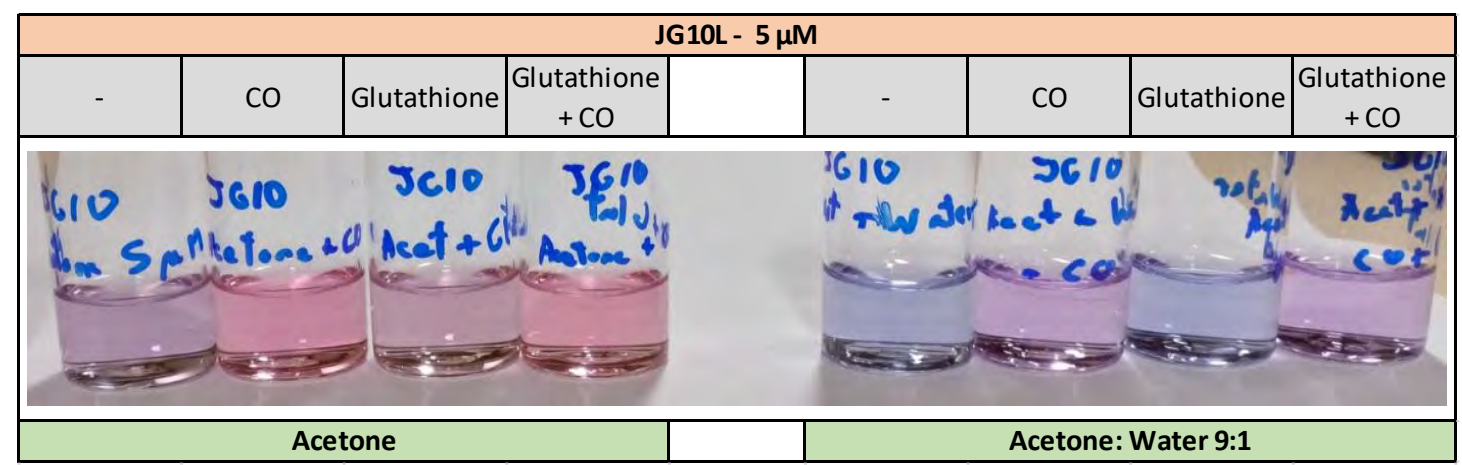

Figure 21. Visible appearance of $5 \mu \mathrm{M}$ solutions of JG10L in Acetone (left) and Acetone:Water 9:1 (right). Reference and vials with bubbled CO, Glutathione $0.1 \mathrm{mM}$ and Glutathione $0.1 \mathrm{mM}+\mathrm{CO}$ bubbled.
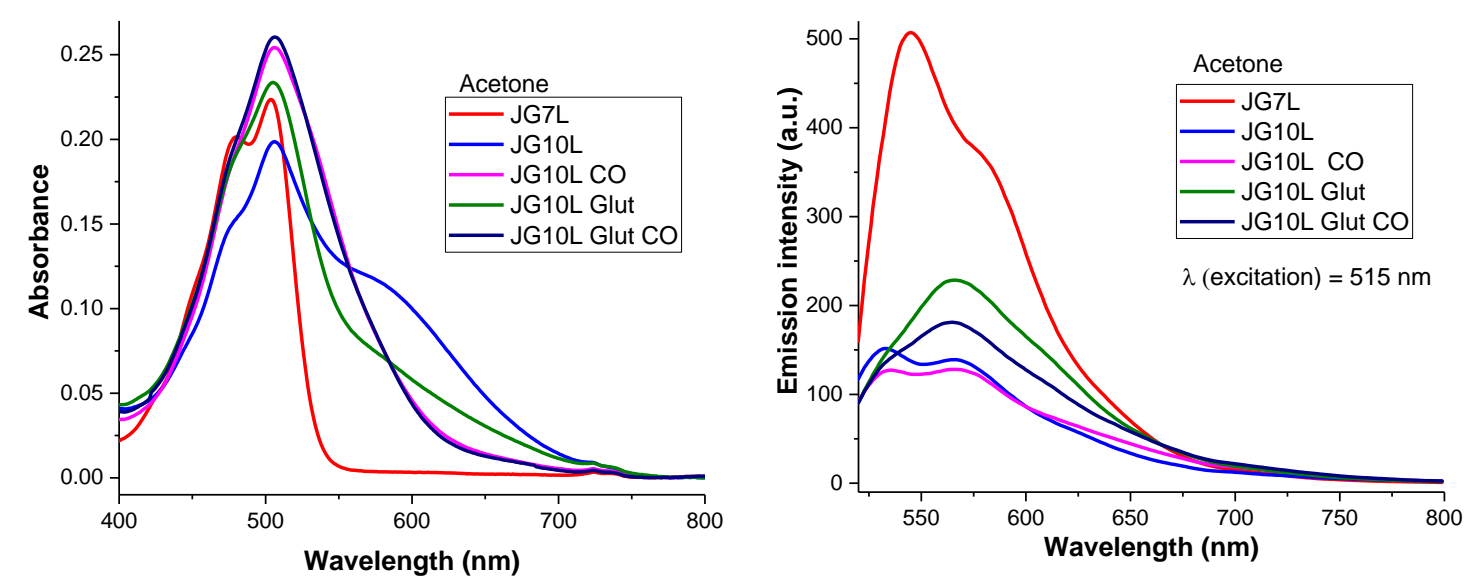

Figure 22. Absorbance (left) and fluorescence (right) spectra of $5 \mu \mathrm{M}$ solutions in acetone of JG7L and JG10L. Reference, bubbled CO, Glutathione $0.1 \mathrm{mM}$ and Glutathione $0.1 \mathrm{mM}+\mathrm{CO}$ bubbled.

The presence of CO increased the fluorescence of JG10L solutions at $650 \mathbf{~ n m}$ or more, but only slightly. The absorption at $600 \mathrm{~nm}$ disappeared in presence of CO. In contrast, the presence of glutathione increased the fluorescence of the solution at $575 \mathrm{~nm}$. It was a slow process that reached the emission of JG7L with time. In the experiment it was determined that Glutathione response process competed with the effect of bubbling $\mathrm{CO}$, and it could be stopped by bubbling it. (Figures 21 and 22) 

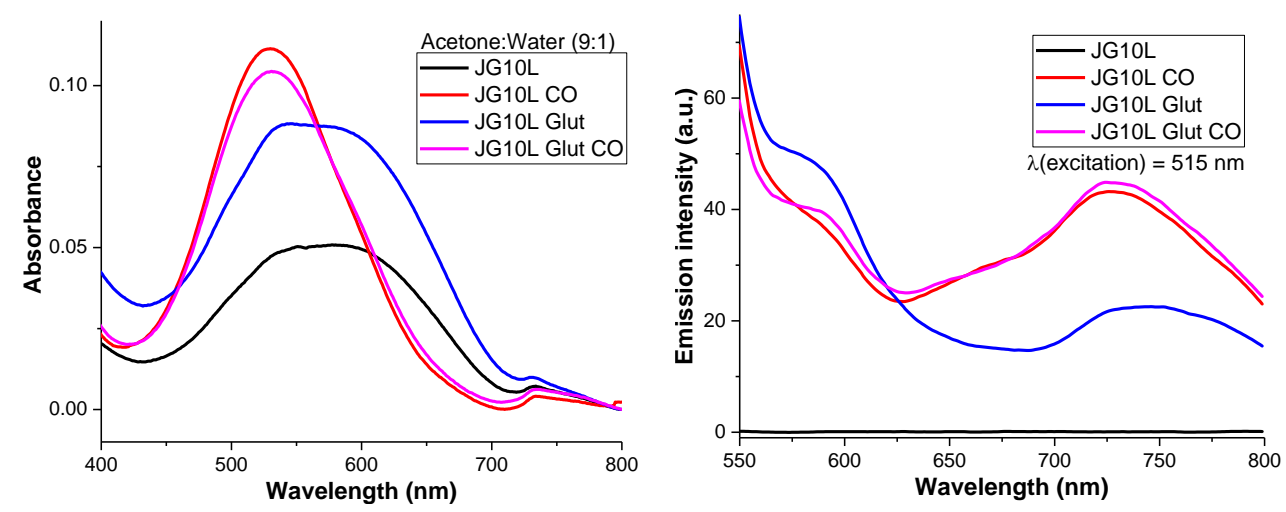

Figure 23. Absorbance (left) and fluorescence (right) spectra of $5 \mu \mathrm{M}$ solutions in acetone:water 9:1 of JG10L. Nothing, bubbled CO, Glutathione $0.1 \mathrm{mM}$ and Glutathione $0.1 \mathrm{mM}+\mathrm{CO}$ bubbled.

In case of acetone:water 9:1 mixtures (Figure 23), the compound JG10L precipitated slowly. In the presence of $\mathrm{CO}$, the absorbance broadened and the fluorescence seemed low and in the red region, the growth was also higher when in the presence of $\mathrm{CO}$.

\subsubsection{JG11L + Glut tests:}

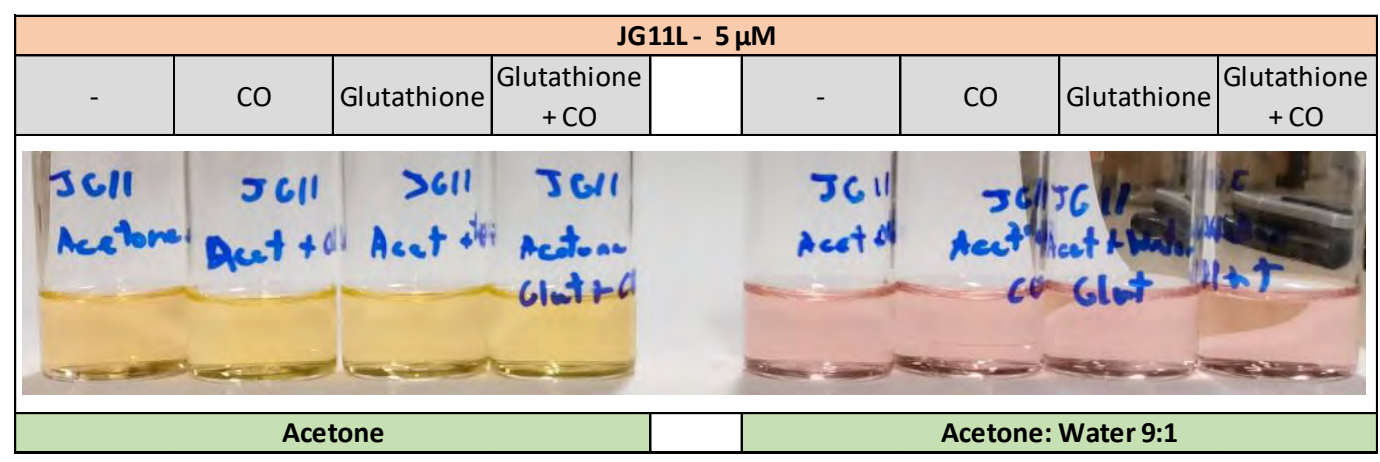

Figure 24. Visible appearance of $5 \mu \mathrm{M}$ solutions of JG11L in Acetone (left) and Acetone:water 9:1 (right). Reference, bubbled CO, Glutathione $0.1 \mathrm{mM}$ and Glutathione $0.1 \mathrm{mM}+\mathrm{CO}$ bubbled.
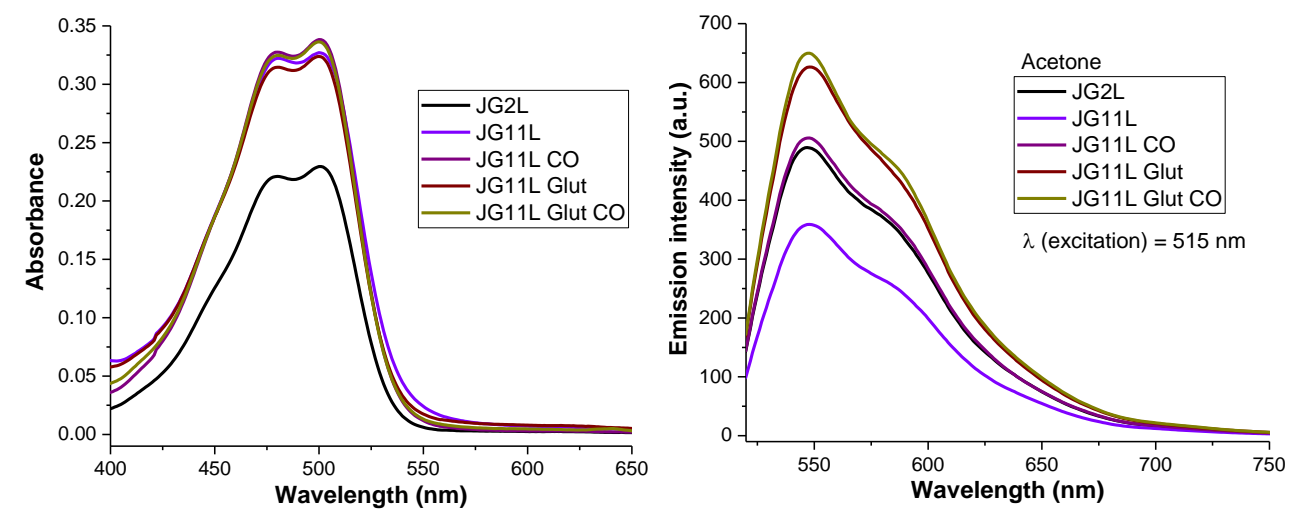

Figure 25. Absorbance (left) and fluorescence (right) spectra of $5 \mu \mathrm{M}$ solutions in acetone of JG2L and JG11L. Reference, bubbled CO, Glutathione $0.1 \mathrm{mM}$ and Glutathione $0.1 \mathrm{mM}+\mathrm{CO}$ bubbled.

When CO was bubbled, the solution of compound JG11L increased its fluorescence until it had the same than the corresponding to the JG2L solution. However, the fluorescence seemed to increase more when there was glutathione in the media; the explanation is probably a pH-Lewis acid effect with the pyridine group. (Figures 24 and 25) 

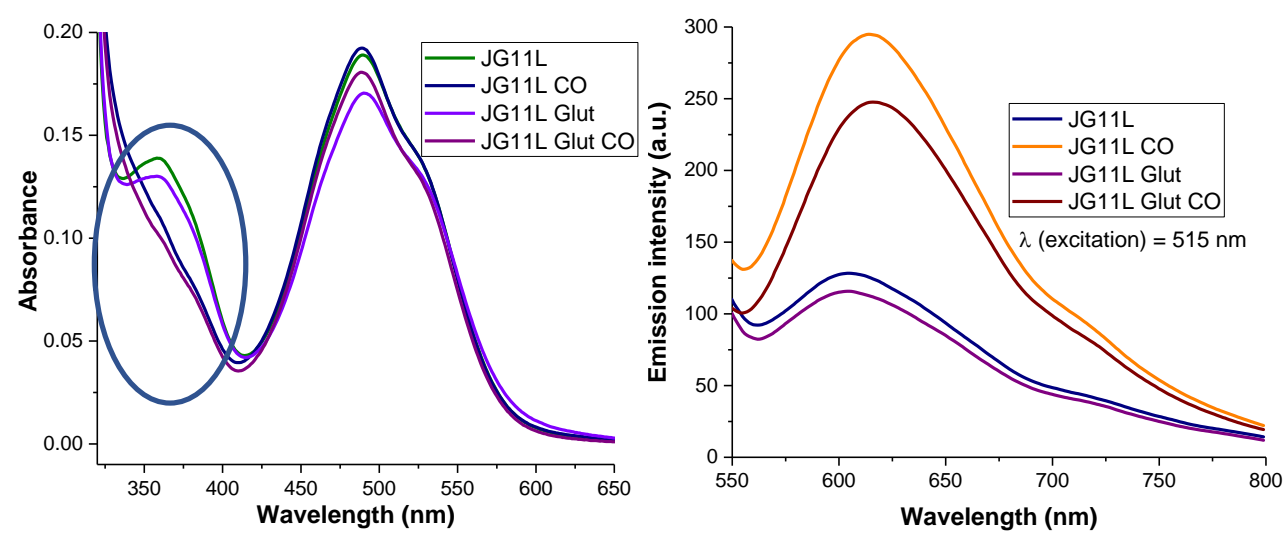

Figure 26. Absorbance (left) and fluorescence (right) spectra of $5 \mu \mathrm{M}$ solutions in acetone:water 9:1 of JG11L. Nothing, bubbled CO, Glutathione $0.1 \mathrm{mM}$ and Glutathione $0.1 \mathrm{mM}+\mathrm{CO}$ bubbled.

For acetone:water 9:1 mixtures (Figure 26), the compound became red and precipitated except when CO was bubbled through the solution, this seemed to be a way of aggregation, since it changed colour instantly. The absorbance band at $350 \mathrm{~nm}$ did not appear in presence of $\mathrm{CO}$, and the fluorescence increased selectively with $\mathrm{CO}$, due to the higher solubility.

\subsection{Tests with other species}

\section{Analytes effect over the complexes JG10L and JG11L (Figures 27-29)}

$5 \mu \mathrm{M}$ solutions in acetone of JG10L and JG11Lwere studied in presence of different analytes (Figure 27) which were added from concentrated acetone solutions. Reference, $\mathrm{Cu}\left(\mathrm{ClO}_{4}\right)_{2}, \mathrm{AgClO}_{4}$, $\mathrm{NBu}_{4} \mathrm{CN}, \mathrm{HAuCl}_{4}, t$-BuNC.

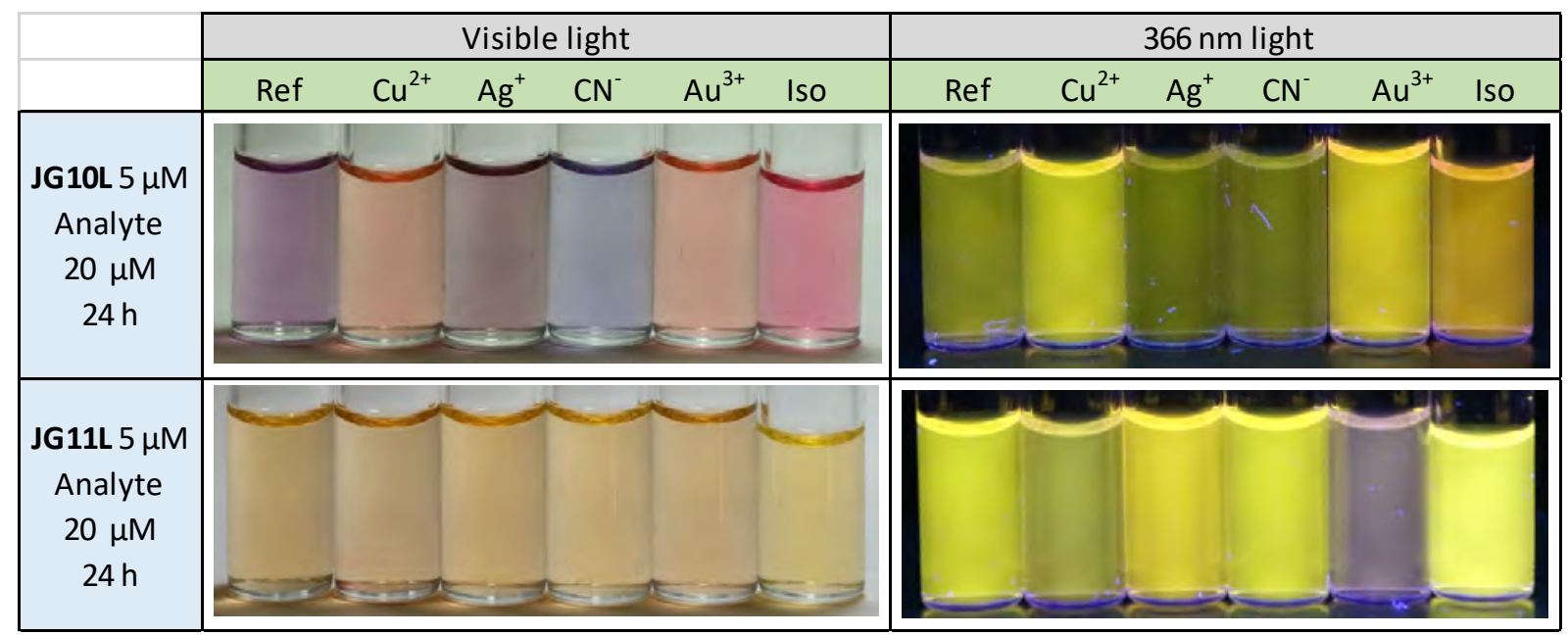

Figure 27. Visible and UV-light appearance of $5 \mu \mathrm{M}$ solutions of JG10L and JG11L in Acetone. Vials with different analytes $20 \mu \mathrm{M}$ under visible-UV light, at different waiting times. 

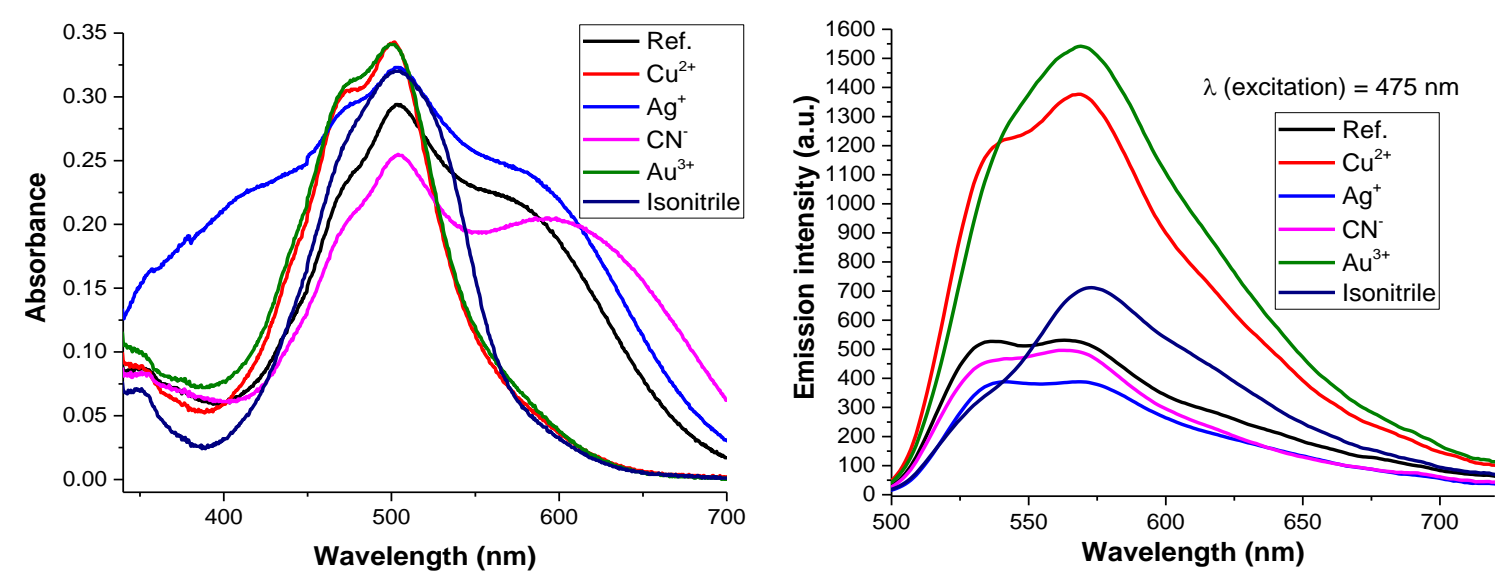

Figure 28. Absorbance and emission spectra of JG10L $(5 \mu \mathrm{M})$ with different analytes $(20 \mu \mathrm{M})$ after 24 hours.
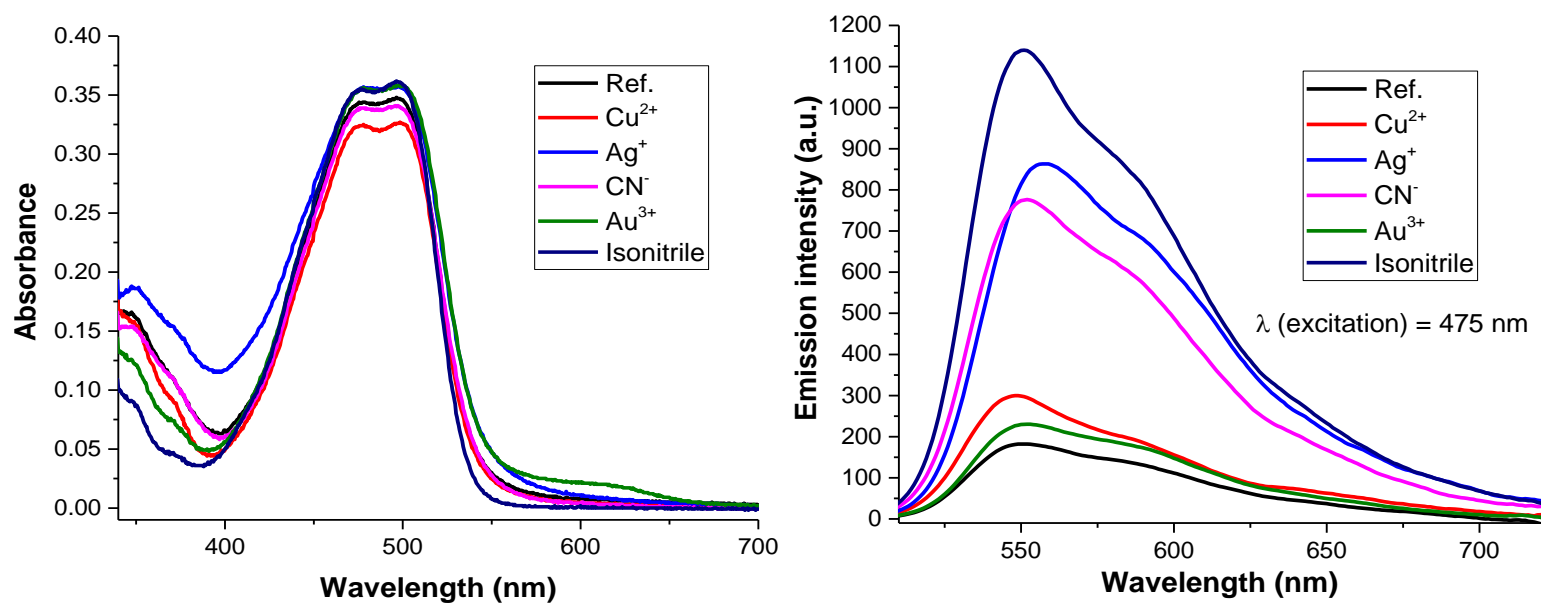

Figure 29. Absorbance and emission spectra of JG11L $(5 \mu \mathrm{M})$ with different analytes $(20 \mu \mathrm{M})$ after 24 hours.

\section{Effect of light (Figure 30)}

When the solution in acetone was left under a high intensity lamp for 5 minutes, the process was very similar to the addition of $\mathrm{Cu}(\mathrm{II})$, Figure 30 .

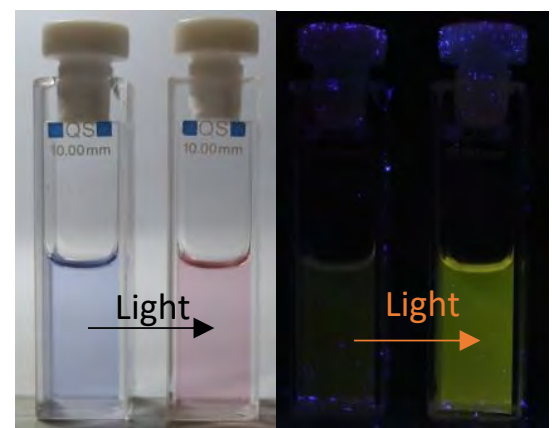

Figure 30. Visible appearance of $5 \mu \mathrm{M}$ solutions of JG10L in acetone. Before and after light irradiation. Under visible light (left) and UV light (right). 
Furthermore, additional tests were performed with different cations, anions and oxidizing agents, previous pictures (Figures 27-30) summarize the different responses. ${ }^{15}$

\section{Interpretation of the results:}

Three different changes were observed.

- One behaviour was observed in presence of $\mathrm{Cu}^{2+}$, acid cations and/or light. This change was associated to acids and/or redox responses. ${ }^{16}$ As it was checked in previous tests with gluthatione and in the additional experiments, ${ }^{15}$ it produced an increase in fluorescence and a stretch in the absorption for JG10L.

- $\mathrm{Au}^{3+}$ and especially $\mathrm{Ag}^{+}$, change the colour and absorption of the solutions which was in agreement with a redox mechanism.

- $\quad \mathrm{CN}^{-}, \mathrm{CO}$ and $t$-butylisonitrile seemed to lead to a change in the coordination sphere increasing fluorescence for JG11L but not for JG10L; which is in agreement with the substitution of the pyridine group. In addition, the response to cyanide of JG10L produce a remarkable change in absorption (the solution became blue).

\footnotetext{
${ }^{15}$ For more information see Experimental Appendix 5.

${ }^{16}$ E. R. dos Santos, J. P. T. Venancio, C. Serpa, J. M. G. Martinho, R. M. Carlo, J. Phys. Chem. C, 2016, 120, $22831-22843$.
} 


\section{SUMMARY}

\section{Solvatochromism and solubility}

JG10L was light sensitive and quickly degraded in some solvents.

was insoluble in water, hexane and $\mathrm{MCH}$.

had a fluorescence quantum yield of $38 \%$, in comparison with $90 \%$ of JG7L. (DCM)

had a fluorescence lifetime decay of $4.74 \mathrm{~ns}$ in comparison with $4.62 \mathrm{~ns}$ of JG7L. (DCM)

precipitated when water percentage was increased. (Acetone)

JG11L was insoluble in water, hexane and $\mathrm{MCH}$.

had a fluorescence quantum yield of $30 \%$, in comparison with $91 \%$ of the JG2L. (DCM)

had a fluorescence lifetime decay of $4.57 \mathrm{~ns}$ in comparison with $4.45 \mathrm{~ns}$ of JG2L. (DCM)

precipitated with time in several solvents.

\section{CO interaction}

JG10L stretched and increased absorbance. (Acetone and DCM)

increased it emission at $650 \mathrm{~nm}$, decreasing at $565 \mathrm{~nm}$. (Acetone and DCM)

$\underline{\mathrm{JG11L}}$ increased its absorbance and emission. (Acetone and DCM)

A deeper evaluation of JG10L and JG11L, led to the conclusion that it was likely to have two kinds of interactions, and acid/oxidizing agents response and a change in the coordination sphere.

Its sensitivity makes these products of great interest in photodynamic therapy, a fact that was found to happen in perylene-ruthenium complexes. ${ }^{17}$ However, the main drawback of the probe was the low solubility in water. In fact, some tests were attempted to be carried out in cell cultures, but the low solubility hindered the cellular uptake and it could not be observed in the microscope. The next step would be to increase the solubility of the complexes and running some test with this kind of derivatives. Changing the triphenylphosphine groups would be the straightforward way to proceed.

In summary, the synthetized probes were similar to previously published results. Two CO probes with sensitivity for CO in DCM or acetone solutions and with different signal when in presence of glutathione/acids. For future research, these probes are potential candidates to be further modified to increase their water affinity and to improve the uptake in cells, which would increase and improve their applications.

${ }^{17}$ a) P. J. S. Maia, I. de Aguiar, M. d. S. Velloso, D. Zhang, E. R. dos Santos, J. R. de Oliveira, J. C. Junqueira, M. Selke, R. M. Carlos, J. Photochem. Photobiol. A: Chem. 2018, 353, 536-545. b) C. Mari, H. Huang, R. Rubbiani, M. Schulze, F. Würthner, H. Chao, G. Gasser, Eur. J. Inorg. Chem. 2017, 12, 1745-1752. 


\section{RESUMEN DEL CAPÍTULO}

El monóxido de carbono es un gas que se produce por semicombustión de materia orgánica. Bajas concentraciones de este gas ya son altamente tóxicas para los mamíferos, lo cual adquiere especial relevancia ya que es totalmente incoloro, inodoro e insípido. Por todo ello, la elaboración de sensores rápidos, baratos y fiables es de gran importancia. En contraste con su toxicidad, en los últimos años, se ha descubierto que el $\mathrm{CO}$ forma parte de ciclos enzimáticos y equilibrios intracelulares. Gracias a esto, y la elaboración de moléculas liberadoras de CO (CORMS), actualmente se trabaja en la síntesis de antiinflamatorios y el tratamiento de problemas cardiovasculares relacionados con su presencia.

Con el propósito de detectar $\mathrm{CO}$, se ha pasado de la utilización de sistemas electroquímicos a la investigación en sistemas ópticos, siguiendo cambios de color y fluorescencia. Basándose en los resultados de investigaciones previas, las sondas derivadas de complejos de $\mathrm{Ru}(\mathrm{II})$ tienen gran potencial en este sentido.

Así, en colaboración con el grupo del profesor Wilton-Ely del Imperial College (Londres), se han elaborado un grupo de sondas que combinan el color-fluorescencia de las PMIs con un núcleo metálico de rutenio. Las moléculas sintetizadas fueron sensibles a $\mathrm{CO}$ en disolventes orgánicos, como acetona y diclorometano.

Por otra parte, se observó que algunos de los complejos de rutenio eran altamente sensibles a la presencia de luz y determinados analitos, ocurriendo procesos de redox y cambios en la esfera de coordinación que alteraban su color y fluorescencia. Estos procesos serían muy interesantes para ser aplicados en detección de analitos en fase gas ( $\mathrm{CO}$, cianuro o isonitrilos), estudios intracelulares y la detección de oxígeno singlete (terapia fotodinámica). Sin embargo, esto no fue posible debido a la baja solubilidad en agua del compuesto, lo cual impedía la entrada al interior celular en cultivos. En consecuencia, para el futuro, se propuso la elaboración de nuevas sondas intercambiando los ligandos trifenilfosfina por otros grupos menos voluminosos y con mayor solubilidad en medios acuosos y profundizar en la posible aplicación para la detección de otros analitos en fase gas a parte del CO, como la presencia de isonitrilos. 


\section{CHAPTER 3C}

PMI DERIVATIVES FOR $\mathrm{K}^{+}$AND $\mathrm{Pb}^{2+}$

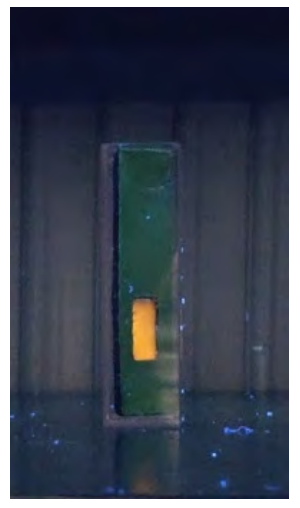
SENSING

\section{ABSTRACT}

Potassium cations are of upmost importance at a biological level, therefore, the modulation of the capacity of some molecules to complex $\mathrm{K}^{+}$and the synthesis of fluorescent probes for their detection might lead to the elaboration of drugs with improved pharmacological properties and the understanding about how some biological processes work. Moreover, being cereulide a toxic potassium ionophore, its detection by competition with an artificial complex ${ }^{1}$ and the consequences of using derivatives were studied. Additionally, a related PMI derivative demonstrated selectivity to $\mathrm{Pb}^{2+}$, a toxic metallic cation that is presented as an environmental contaminant, needing for a quick and cheap detection system, which was achieved by the use of the PMI derivative as a fluorogenic probe.

${ }^{1}$ J. García-Calvo, S. Ibeas, E. C. Antón-García, T. Torroba, G. González-Aguilar, W. Antunes, E. GonzálezLavado and M. L. Fanarraga. ChemistryOpen 2017, 12, 562-570. 



\section{INTRODUCTION, PMI-MODIFIED RECEPTORS FOR FLUORESCENT DETECTION}

Perylenemonoimides (PMIs) have demonstrated to be a useful and effective tool for the development of selective fluorescent sensors by reaction (Chapter 3A) and an interesting ligand/substituent for metallic complexes (Chapter 3B). Besides, as it was studied in Chapter 1, the synthesis of a fluorescent derivative for sensing by complexation may be performed by modification with the proper receptor, which was the objective of this chapter.

One of the most common procedures for synthesizing complexation receptors (ligands), specifically for cations, is the use of crown ether or cryptand derivatives. They are characterized by their tuneable affinity when changing the opening of the rings. In regard to the selectivity of the crown ethers/cryptands, most of them are sensitive to more than one cation. However, this selectivity depends on many factors, such as the different substitution around the receptor or the solvent. Essentially, having less than $100 \%$ specificity is a drawback but, when the cations are not interferents between them in the particular conditions for their detection, it may become an advantage, increasing the applicability of the probe. An example might be one of the probes treated on this chapter, a complexation probe that gives selective response to both $\mathrm{K}^{+}$and $\mathrm{Pb}^{2+}$ cations in solution and selectively to $\mathrm{Pb}^{2+}$ as material.

Additionally, probes with high selectivity for some cations may be used for the indirect detection of non-fluorescent species that compete in complexation equilibria. For instance, in this chapter it is studied the detection of natural potassium ionophores, such as cereulide or valinomycin, by a fluorescent probe selective to $\mathrm{K}^{+}$in solution.

Apart from crown ethers or cryptands, the fluorescent detection of cations, such as lead or potassium, may be studied from fluorescent derivatives of natural potassium ionophores, such as cereulide, modifications of high interest in chemistry and biochemistry. Because of that, this chapter starts with an introduction about some of the properties that make interesting the research in $\mathrm{K}^{+}$, natural potassium ionophores (cereulide and derivatives) and $\mathrm{Pb}^{2+}$ recognition. 


\section{DETECTION OF POTASSIUM CATIONS}

The role of potassium is of upmost importance in a wide range of biological processes such as nerve transmission, cardiac excitability, epithelial fluid transport or cell proliferation. ${ }^{2}$ In addition, an abnormal potassium level is a symptom of many diseases such as food poisoning (cereulide), heart diseases, cancer or AIDS, among others. ${ }^{3}$

Due to its multiple roles, the scope of $\mathrm{K}^{+}$detection is mainly oriented in 2 ways:

- Selective detection against other cations (specially $\mathbf{N a}^{+}$) in solution. For example, to determine its right concentration in a serum.

- Measurements in cellular media. The detection of different $\mathrm{K}^{+}$concentration in different parts of the cell is of upmost importance, changes in concentration are related with cellular permeation or other intracellular equilibria.

To address potassium detection issues, the most classical way has been atomic flame spectrophotometry (FAAS). This method allows measuring potassium concentration selectively, although having limitations, such as needing concentrations higher to $5 \mathrm{mM}$ and the destruction of the sample in the process.

To achieve a more sensitive detection it is necessary to apply absorbance-fluorescence techniques. Fluorescence has special importance due to the possibility to measure very low concentrations without destroying the sample. It is not invasive, very sensitive and potentially selective; what is more, it is the ideal method to perform cell measurements. Apart from all these advantages, with a fluorescent selective probe, the presence of free potassium could be quantified and, indirectly, it could give information about other species with affinity for potassium cations, which would interfere in the final signal.

In consequence, the ideal $\mathrm{K}^{+}$sensor must fulfil some particular conditions:

- Working in extracellular and intracellular environments.

- Good selectivity, especially against sodium cations.

- No pH interference in physiological range.

- Large fluorescent response, to distinguish it from the associated biological fluids and the residual fluorescence of some biological components (such as the blue fluorescence of tryptophan).

In relation with detection of the cation in different solvents, in the last years the importance of fluorescent methods has grown in interest. First results in potassium sensitive fluorescent molecules

\footnotetext{
2 a) S. Bonnet, S. L. Archer, J. Allalunis-Turner, A. Haromy, C. Beaulieu, R. Thompson, C. T. Lee, G. D. Lopaschuk, L. Puttagunta, S. Bonnet, G. Harry, K. Hashimoto, C. J. Porter, M. A. Andrade, B. Thebaud, E. D. Michelakis, Cancer Cell 2007, 11, 37-51; b) P. M. Hudgins, G. B. Weiss, J. Pharmacol. Exp. Ther. 1968, 159, 91-97; c) P. Kofuji, E. A. Newman, Neuroscience 2004, 129, 1043-1054; d) C.-C. Shieh, M. Coghlan, J. P. Sulli1192 Eur. J. Org. Chem. 2015, 1189-1192 van, M. Gopalakrishnan, Pharmacol. Rev. 2000, 52, 557-594; e) A. D. Wickenden, Pharmacol. Ther. 2002, 94, 157-182.

3 a) C. H. Brennan, A. Lewis, J. M. Littleton, Neuropharmacol. 1989, 28, 1303-1307; b) A. R. Gonçalves, A. M. Nahas, Diabetologia 2011, 54, 2963-2964; c) R. H. Grimm, J. D. Neaton, P. J. Elmer, K. H. Svendsen, J. Levin, M. Segal, L. Holland, L. J. Witte, D. R. Clearman, P. Kofron, R. K. LaBounty, R. Crow, R. J. Prineas, New Engl. J. Med. 1990, 322, 569-574; d) H. Kager, W. J. Wadman, G. G. Somjen, J. Neurophysiol. 2000, 84, 495-512; e) K. Kunzelmann, J. Membr. Biol. 2005, 205, 159-173; f) L. P. E. Laurent, W. W. Walther, Lancet 1935, 225, 1434-1435; g) L. C. MacGregor; F. M. Matschinsky, J. Biol. Chem. 1986, 261, 4052-4058; h) J. E. Mitchell, R. L. Pyle, E. D. Eckert, D. Hatsukami; R. Lentz, Psychol. Med. 1983, 13, 273-278; i) G. G. Somjen, J. L. Giacchino, J. Neurophysiol. 1985, 53, 1098-1108.
} 
were based on the use of a potassium-binding benzofuran isophthalate, PBFI (Figure 1), which is nowadays a commercially available compound. Nevertheless, it has some major drawbacks such as its low selectivity between $\mathrm{K}^{+}$and $\mathrm{Na}^{+},{ }^{4}$ when both are presented in high concentrations.

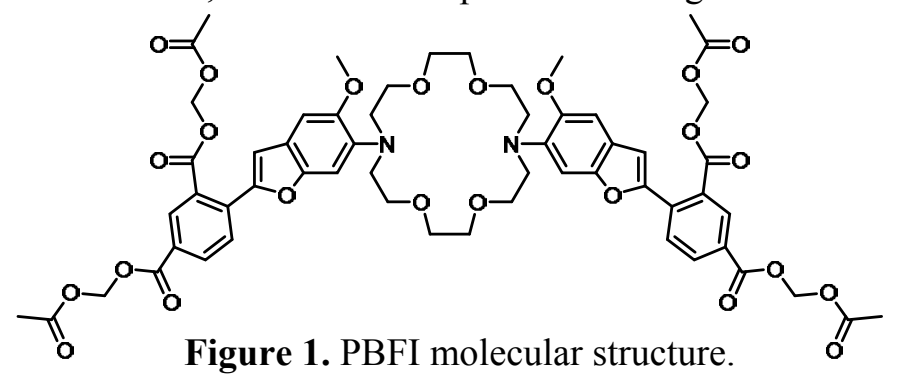

After that, new derivatives have been developed on the basis of more suitable crown ethers and cryptand derivatives, Figure 2.

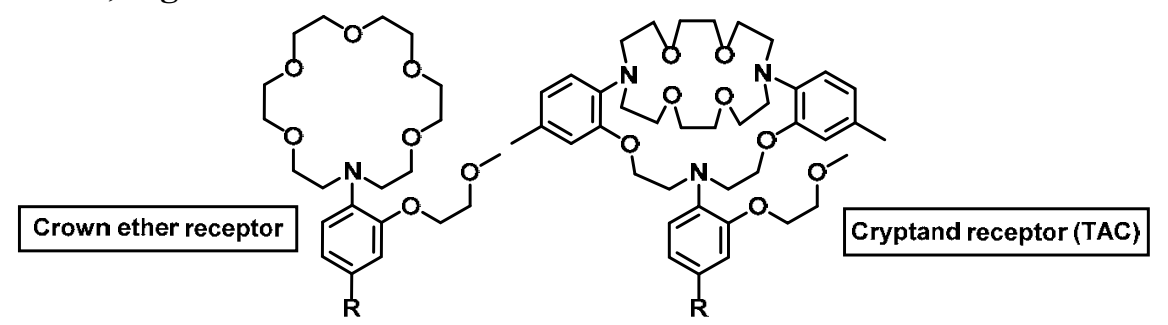

Figure 2. Two of the most common receptors when developing new fluorescent probes, "R" group represents the different fluorescent signalling units.

A lot of research has been performed about derivatives from these two receptors. On one hand, for the triazacryptand, the research started by optimizing different synthetic processes, ${ }^{5}$ next, the fluorescent receptor was changed to more suitable molecular structures with a bodipy derivative (TAC-BODIPY $)^{6}$ and finally, its selectivity and LOD were measured and improved by working either in water buffers - organic mixtures. ${ }^{7}$ On the other hand, apart from TAC derivatives, there are also more simple potassium sensitive receptors, such as $\mathrm{K}^{+}$-selective crown ether (Figure 2). ${ }^{8}$ They also have high selectivity $\mathrm{K}^{+} / \mathrm{Na}^{+}$and, in addition, they are easier to synthesize having similar results in terms of selectivity, solubility or limits of detection.

The ability of these kind of receptors for complexation of potassium cation makes them suitable for its detection. What is more, following this idea, it is possible to design a fluorescent probe for potassium that act by displacement assays with natural potassium ionophores, such as cereulide or valinomycin, in solution and in cell cultures.

\footnotetext{
${ }^{4}$ P. Jezek, F. Mahdi, K. D. Garlid, J. Bio. Chem. 1990, 265, 10522-10526.

${ }^{5}$ H. He, M. A. Mortellaro, M. J. P. Leiner, R. J. Fraatz, J. K. Tusa, J. Am. Chem. Soc. 2003, 125, 1468-1469.

${ }^{6}$ X. Zhou, F. Su, Y. Tian, C. Youngbull, R. H. Johnson, D. R. Meldrum, J. Am. Chem. Soc. 2011, 133, 1853018533; d) W. Namkung, P. Padmawar, A. D. Mills, A. S. Verkman, J. Am. Chem. Soc. 2008, 130, 7794-7795; e) X. Zhou, F. Su, W. Gao, Y. Tian, C. Youngbull, R. H. Johnson, D. R. Meldrum, Biomaterials 2011, 32, 85748583; f) R. D. Carpenter, A. S. Verkman, Org. Lett. 2010, 12, 1160-1163; g) X. Li, X. Gao, W. Shi, H. Ma, Chem. Rev. 2014, 114, 590-659; h) T. Hirata, T. Terai, H. Yamamura, M. Shimonishi, T. Komatsu, K. Hanaoka, T. Ueno, Y. Imaizumi, T. Nagano, Y. Urano, Anal. Chem. 2016, 88, 2693-2700.

${ }^{7}$ B. Sui, X. Yue, M. G. Tichy, T. Liu, K. D. Belfield. Eur. J. Org. Chem. 2015, 6, 1189-1192.

8 a) T. Schwarze, R. Schneider, J. Riemer, H.-J. Holdt, Chem. Asian J. 2016, 11, 241-247; b) T. Schwarze, J. Riemer, S. Eidner, H.-J. Holdt, Chem. Eur. J. 2015, 21, 11306-11310; c) S. Ast, T. Schwarze, H. Müller, A. Sukhanov, S. Michaelis, J. Wegener, O. S. Wolfbeis, T. Körzdörfer, A. Dürkop, H.-J. Holdt, Chem. Eur. J. 2013, 19, 14911-14917.
} 


\section{THE IMPORTANCE OF CEREULIDE}

\subsection{The role of Cereulide}

Cereulide is a cyclic depsipeptide produced by specific strains of the bacteria called Bacillus cereus, ${ }^{9}$ that acts as potassium ionophore and toxin. The importance of this bacteria comes from its growth in badly preserved food, causing two different types of disease, diarrheal and emetic syndrome. ${ }^{10}$

- The diarrheal syndrome is developed after the ingestion of food contaminated with spores or vegetative cells (8-16 hours after consumption). It is caused by vegetative growth of the enterotoxins in the small intestine. These endospores are capable of surviving to cooking temperatures and can rapidly germinate and produce their toxins, especially during food chilling or food heating. ${ }^{11}$ These spores may be found as a natural contaminant in several food products, especially meat, vegetables and milk. ${ }^{12}$

- The emetic syndrome (1-5 hours after consumption) is usually associated with the ingestion of foodstuff contaminated with the preformed toxin, cereulide, produced during the growth of bacteria in food, ${ }^{13}$ and associated with rice or other starch rich products, such as pasta and potato-based products. ${ }^{14}$

The similarities of their symptoms to contamination with other bacteria makes difficult the identification of $B$. cereus as the source. Usually, the symptoms are underestimated and misled with the ones caused by other more common bacteria, such as the Staphylococcus aureus. Only when the outbreak is reported for many people and the source is found, it has been associated to cereulide. ${ }^{15}$ The main difference with other kind of bacteria is that once cereulide is produced in food it can not be removed; only extreme conditions are enough (long periods at $\mathrm{pH}$ superior to 9.5 and more than $120^{\circ} \mathrm{C}$ ) to degrade the product, and only in small quantities. Therefore, the only viable way to prevent intoxication with cereulide is by its early detection in food.

In addition to the widely studied and short-term consequences of $B$. cereus ingestion, several authors have started to study some possible relation with diseases without a clear culprit. That is the case of the studies with some types of diabetes ${ }^{16}$ which proved that exposure of pancreatic beta cells to low quantities of cereulide $(1 \mathrm{ng} / \mathrm{mL})$ led to cellular death. This effect is supported by the high stability of this cyclic peptide and the likeness of having such low quantities in food, that it wouldn't be possible to detect with the existent techniques.

\footnotetext{
9 a) L. P. S. Arnesen, A. Fagerlund, P. E. Granum, FEMS Microbiol. Rev. 2008, 32, 579-606; b) E. Granum, T. Lund, FEMS Microbiol. Lett. 1997, 157, 223-228.

${ }^{10}$ G. Lücking, M. K. Dommel, S. Scherer, A. Fouet, M. Ehling-Schulz, Microbiology 2009, 155, 922-931.

${ }^{11}$ M. Kranzler, K. Stollewerk, K. Rouzeau-Szynalski, L. Blayo, M. Sulyok, M. Ehling-Schulz, Front. Microbiol. 2016, 7, 1640.

12 Y. Cui, Y. Liu, X. Liu, X. Xia, S. Ding, K. Zhu, Toxins 2016, 8, 156.

${ }^{13}$ S. Ceuppens, N. Boon, M. Uyttendaele, FEMS Microbiol. Ecol. 2013, 84, 433-450.

${ }^{14}$ N. A. Logan, J. Appl. Microbiol. 2011, 112, 417-429.

${ }^{15}$ Bennett, R. Bacilus cereus in Guide to Foodborne Pathogens, Labbé 2001, 51-60.

${ }^{16}$ R. Vangoitsenhoven, D. Rondas, I. Crévecoeur, W. D’Hertog, P. Baatsen, M. Masini, M. Andjelkovic, J. Van Loco, C. Matthys, C. Mathieu, L. Overbergh, B. Van der Schueren, PLoS ONE 2014, 9, e104866.
} 


\subsection{Cereulide chemical structure}

Cereulide, the emetic toxin of B. cereus, is a cyclic depsipeptide ${ }^{17}$ with structure cyclo[-(D-OLeu-D-Ala- $\left.\left.-\mathrm{L}-\mathrm{O}-\mathrm{Val} \mathrm{-}_{\mathrm{L}}-\mathrm{Val}\right)\right]_{3}$ closely related to the structure of valinomycin, cyclo[-(D-O-Val ${ }_{-\mathrm{D}}-\mathrm{Val}-\mathrm{L}_{\mathrm{L}}-$ $O-A l a-L-V a l)]_{3}$, being both potassium cation ionophores (Figure 3). ${ }^{18}$
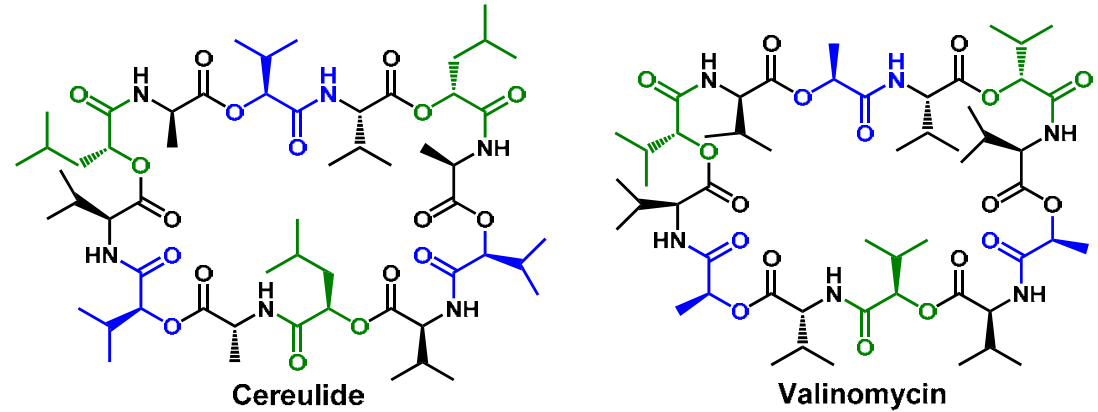

Figure 3. Chemical structures of cereulide and valinomycin.

The characterization and isolation of these two compounds was widely studied and documented by the group of Isobe et al. ${ }^{19} \mathrm{X}$-ray crystal structures of cereulide and some complexes of valinomycin with $\mathrm{K}^{+}, \mathrm{H}^{+}$or even $\mathrm{NH}_{4}^{+}$are known. Stability studies by NMR for the stability complexes are also known, being $\mathrm{K}^{+}>\mathrm{H}^{+}>\mathrm{NH}_{4}^{+}$(methanol solution).

\subsection{Toxicity comparison valinomycin-cereulide and structural variations}

The structural differences between cereulide and valinomycin are minor. Both of them are cyclic depsipeptides formed by 12 aminoacids/hydroxyacids that act as potassium ionophores by reequilibrating the transmembrane potential (Figure 4). However, while valinomycin has been used as a drug to maintain the potassium equilibria, some studies with cereulide showed that it disrupts this transmembrane potential in mitochondria of eukaryotic cells (studied for Hep-2 cells, human larynx carcinoma), leading to mitochondrial degeneration and, subsequently, to cell death; ${ }^{20}$ being fifteen times more toxic than valinomycin. ${ }^{21}$

When the studies were focused in the toxicity issue, it has been determined that toxicity was related to potassium concentration in plasma. ${ }^{22}$ Particularly, it was registered that the uptake of $\mathrm{K}^{+}$was more potent for valinomycin when $\left[\mathrm{K}^{+}\right]>80 \mathrm{mM}$, whereas cereulide is active at concentrations lower to 1 $\mathrm{mM}$ and has higher accumulation inside cells.

\footnotetext{
${ }^{17}$ a) N. A. Magarvey, M. Ehling-Schulz, C. T. Walsh, J. Am. Chem. Soc. 2006, 128, 10698-10699; b) M. EhlingSchulz, E. Frenzel, M. Gohar, Front. Microbiol. 2015, 6, 704; c) G. Lücking, E. Frenzel, A. Rütschle, S. Marxen, T. D. Stark, T. Hofmann, S. Scherer, M. Ehling-Schulz, Front. Microbiol. 2015, 6, 1101.

${ }^{18}$ A. Makarasen, T. Nishikawa, M. Isobe, Synthesis 2009, 2184-2204.

${ }^{19}$ A. Makarasen, K. Yoza, M. Isobe. Chem. Asian J. 2009, 4, 688-698.

20 a) M. A. Andersson, P. Hakulinen, U. Honkalampi-Hamalainen, D. Hoornstra, J.-C. Lhuguenot, J. MakiPaakkanen, M. Savolainen, I. Severin, A.-L. Stammati, L. Turco, A. Weber, A. von Wright, F. Zucco, M. Salkinoja-Salonen, Toxicon 2007, 49, 351-367; b) E. L. Jaaskelainen, V. Teplova, M. A. Andersson, L. C. Andersson, P. Tammela, M. C. Andersson, T. I. Pirhonen, N.-E. L. Saris, P. Vuorela, M. S. Salkinoja-Salonen, Toxicol. in Vitro 2003, 17, 737-744.

${ }^{21}$ E. G. Biesta-Peters, M. W. Reij, R. H. Blaauw, P. H. In't Veld, A. Rajkovic, M. Ehling- Schulz, T. Abee, Appl. Environ. Microbiol. 2010, 76, 7466-7472.

${ }^{22}$ V. V. Teplovaa, R. Mikkolaa, A. A. Tonshina, N.-E. L. Sarisa, M. S. Salkinoja-Salonen, Tox. Appl. Pharm. 2006, 210, 39-46.
} 


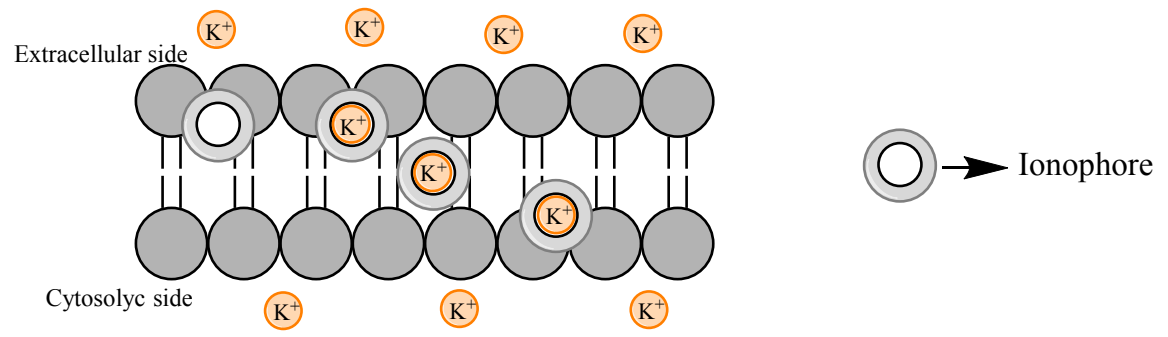

Figure 4. Model of the transport across biological membrane with a potassium ionophore.

Regarding the specific structure of cereulide, it is important to point out that several authors have reported the existence of more than one derivative from the standard cereulide structure. Latest studies have revealed that cereulide is not a single cyclic depsipeptide from $B$. cereus, but the major product of a family of, at least, 18 cereulide variations, ${ }^{23}$ with 1 or 2 different amino/hydroxyacids. However, they are not easy to identify, even by MS analysis; requiring a deep analysis by UPLC-TOF, ion-trap MS and ${ }^{13} \mathrm{C}$ experiments. Furthermore, the changes in the amino/hydroxy acids lead to new cyclic depsipeptides that may have different activities and involvement within potassium transport in comparison to cereulide exposure, especially the long-term consequences.

In spite of these studies, until now it remains unknown why some 12-ring cyclic depsipeptides act as a $\mathrm{K}^{+}$-controlling drug while others are toxic and if this behaviour could be artificially modulated, especially at low concentrations. Therefore, an interesting step forward is the study of the effect of changing specific amino/hydroxy acids of the ionophore, following the effects in potassium transport, complex stability and the experimental consequences in cell cultures, by comparing with previous research and by performing studies on pancreatic cells. ${ }^{16}$

\subsection{Detection of cereulide and derivatives}

Due to cereulide toxicity, rapid and portable detection methods to screen the presence of preformed toxin in foodstuffs are required to prevent food-borne outbreaks. With this aim in mind, there are two ways to deal with it, selective detection of the B. cereus or detection of the emetic toxin.

- Detection of the B. cereus: ${ }^{24}$ The selective detection of the different components of the family of $B$. cereus has been addressed by biologists. It is known that the B. cereus family includes 5 important species which have $99 \%$ similarities in the tRNA sequence. Then, PCR methods have been used to detect it. In spite of these existent methods, until now there is no standardized way to distinguish between closely related species. In addition, there is no method to distinguish between pathogenic or not pathogenic strains and even if it were possible, it would not prove that the emetic toxin was to be produced. ${ }^{25}$

- Detection of the emetic toxin, cereulide: There are several methods to detect this analyte, being a molecule that acts as potassium cation ionophore.

\footnotetext{
${ }^{23}$ S. Marxen, T. D. Stark, E. Frenzel, A. Rütschle, G. Lücking, G. Pürstinger, E. E. Pohl, S. Scherer, M. EhlingSchulz, T. Hofmann, Anal. Bioanal. Chem. 2015, 407, 2439-2453.

${ }^{24}$ ISO 7932, ISO 21871.

25 a) P. F. Horwood, G. W. Burgess, H. J. Oakey, FEMS Microbiol. Lett. 2004, 236, 319-324; b) M. EhlingSchulz, N. Vukov, A. Schulz, R. Shaheen, M. Andersson, E. Märtlbauer, S. Scherer, Appl. Environ. Microbiol. 2005, 71, 105-113.
} 
o Mass spectrometry analysis (MS) is the standardized method to detect depsipeptides and other derivatives of cereulide from cell cultures. This method allows to identify different components of a sample based on the molecular mass. Cereulide molecular weight is already known $(1153.42 \mathrm{~g} / \mathrm{mol})$, detecting it by MS is the best way to have a reliable proof of its presence in a sample.

o Liquid chromatography (HPLC) also allows to identify the presence of cereulide, by comparison with a standard. In contrast to MS, compounds with similar structure would give signal in the same position (valinomycin or other depsipeptide), having lower selectivity but being cheaper and easier to be performed. It is usually performed in combination with mass spectrometry (HPLC-MS).

o In vitro assays ${ }^{26}$ have proven to be sensitive to cereulide, by studying parameters such as cellular death.

o Bioassay detect, by staining, changes in transmembrane potential. The studies have been performed with boar spermatozoa. ${ }^{27}$

Although HPLC, MS and in vitro assays are the most reliable techniques, they have the drawback of requiring dedicated facilities. ${ }^{28}$ In contrast, bioassays are quicker and cheaper. In addition, depending on the required selectivity, new and more simple techniques could be addressed. Regarding that, it was proposed a simple competition process between a natural potassium ionophore and an artificial potassium ionophore that would give a measurable signal (Figure 5).

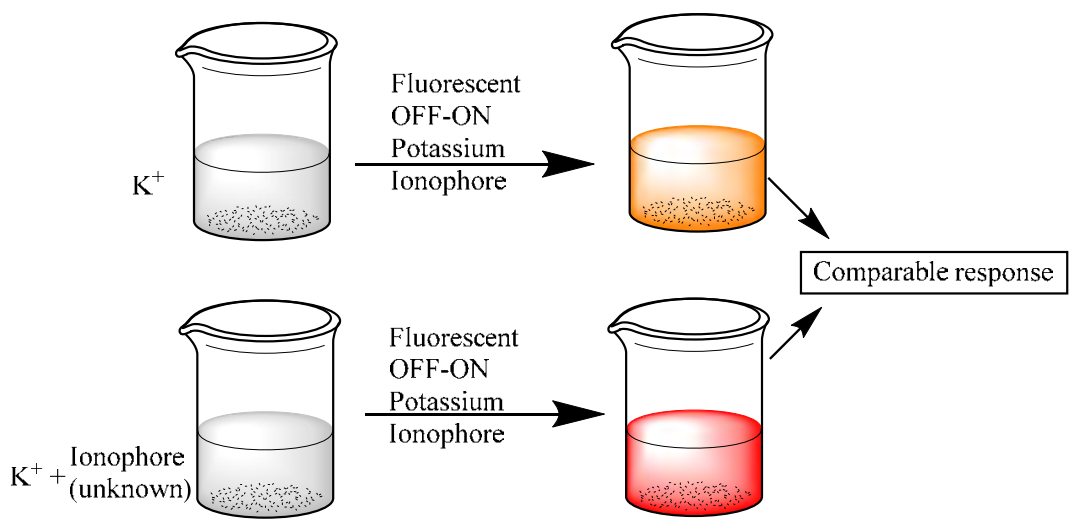

Figure 5. Schematic representation of a possible way to measure the presence of potassium ionophores.

In conclusion, the only way to detect unequivocally the presence of cereulide and/or specific isomers would be by difficult and expensive analysis. Nevertheless, there is the possibility of detecting species that interact in the potassium equilibria in the solvent. Because of that, the objective was to create a system that competed with natural potassium ionophores, in a way in which a specific treatment (by adding a colorant and/or measuring a particular property, such as conductivity or fluorescence) may allow detecting potassium ionophores in a sample.

\footnotetext{
${ }^{26}$ W. J. J. Finlay, N. A. Logan, A. D. Sutherland, Appl. Environ. Microbiol. 1999, 65, 1811-1812.

${ }^{27}$ M. A. Andersson, E. L. Jääskeläinen, R. Shaheen, T. Pirhonen, L. M. Wijnands, M. S. Salkinoja-Salonen, Internat. J. Food Microbiol. 2004, 94, 175-183.

28 a) M. M. Häggblom, C. Apetroaie, M. A. Andersson, M. S. Salkinoja-Salonen, Appl. Environ. Microbiol. 2002, 68, 2479-2483; b) T. Bauer, T. Stark, T. Hofmann, M. Ehling-Schulz, J. Agric. Food Chem. 2010, 58, 1420-1428; c) V. Fricker, U. Messelhaüßer, U. Busch, S. Scherer, M. Ehling-Schulz, Appl. Environ. Microbiol. 2007, 73, 1892-1898; d) H. T. Rønning, T. N. Asp, P. E. Granum, Food Addit. Contam. Part A 2015, 32, 911921; e) L. Delbrassinne, M. Andjelkovic, A. Rajkovic, P. Dubois, E. Nguessan, J. Mahillon, J. Van Loco, Food Anal. Methods 2012, 5, 969-979.
} 


\section{DETECTION OF LEAD(II)}

Lead(II) is the most stable and common cationic form of the heavy metal. The toxicity of this cation is well-known for humans since the $60 \mathrm{~s}$. At that time, different scientific research started to show the consequences of the exposure to even low concentrations of this cation. Its presence in human body could cause kidney diseases, damage to the nervous system, diminished intellectual capacity, heart disease, gastrointestinal diseases, bone fracture, cancer or even death. As a consequence, many environmental agencies, ${ }^{29}$ from different countries have displayed information about the different risks of lead(II) contamination. ${ }^{30}$

Summarizing some specific data, while pre-industrial blood lead levels (BLL) were estimated to be $0.016 \mu \mathrm{g} / \mathrm{dL}$, in the late $20^{\text {th }}$ century it ranged from 0.8 to 3.2 , reaching $25 \mu \mathrm{g} / \mathrm{dL}$ for some children in industrial centres. ${ }^{31}$ Before 2012, safety and environment agencies had reached an agreement in identifying $10 \mu \mathrm{g} / \mathrm{dL}$ in blood as a level of concern. ${ }^{32}$ But, nowadays, it has been demonstrated that lower quantities, as $5 \mu \mathrm{g} / \mathrm{dL}$ in blood, ${ }^{33}$ increase the risk of cancer in adults and can cause neurological damage, ${ }^{34}$ especially among children; in fact, the limits were asked to be decreased again. Furthermore, it has been legislated that the ingestion of more than $0.5 \mu \mathrm{g}$ per day may be dangerous ${ }^{35}$ due to the accumulation properties of the metal in bones, kidneys and liver. ${ }^{36}$ Nevertheless, the most common source of lead contamination is tap water. The limit of lead in potable water fixed by the world health organization is $10 \mu \mathrm{g} / \mathrm{L}$ (ppb), ${ }^{37}$ and for beverages $6 \mu \mathrm{g} / \mathrm{L}$, being the limit values given by the environmental protection agency $15 \mathrm{ppb}$ as the "action level".

In our daily life the sources of lead could be many, for instance, a kid could ingest lead by chewing on a toy painted with lead-containing paint. Other known sources could be lead-acid batteries (especially important for the workers of this kind of industry), vehicle exhaust, dust in the air, paint and soil and its incorporation to the diet by contaminated food. Lead concentrations has been found in products from some animals, as it has occurred for milk, ${ }^{38}$ or in products with great capacity to dissolve this metal, such as vinegar; ${ }^{39}$ although the major source continues to be tap water.

Lead(II) in tap water comes from two sources, whether from environmental pollution from factories or from aging pipes. The most recent example, and with high impact, is the case called "Flint water

29 United States Environmental Protection Agency, https://www.epa.gov/lead/national-lead-poisoningprevention-week, accessed $23^{\text {th }}$ June 2018.

${ }^{30}$ M. J. Kosnett, R. P. Wedeen, S. J. Rothenberg, K. L. Hipkins, B. L. Materna, B. S. Schwartz BS, H. Hu, A. Woolf, Environ. Health Perspect. 2007, 115, 463-471.

${ }^{31}$ a) R. Jones, D. Homa, P. Meyer, D. Brody, K. Caldwell, J. Pirkle, M.- J. Brown, Pediatrics. 2009, 123, 376385. b) D. C. Bellinger, A. M. Bellinger, J. Clin. Investig. 2006, 116, 853-857.

${ }^{32}$ H. R. Pohl, S. Z. Ingber, H. G. Abadin, "Chapter 13. Historical View on Lead: Guidelines and Regulations". In S. Astrid, S. Helmut, R. K. O. Sigel, Lead: Its Effects on Environment and Health. Metal Ions in Life Sciences, Gruyter, Berlin, pp. 435-470, 2017. b) S. Tong, Y. von Schimding, T. Prapamontol, Bullet. World Health Org. 2000, 78.

${ }^{33}$ Centers for Disease Control and Prevention of USA, https://www.cdc.gov/nceh/lead/, accessed $23^{\text {th }}$ June 2018.

${ }^{34}$ K. Klotz, T. Göen. "Chapter 6. Human Biomonitoring of Lead Exposure". In S. Astrid, S. Helmut, R. K. O. Sigel, Lead: Its Effects on Environment and Health, Metal Ions in Life Sciences, Gruyter, Berlin, 99-122, 2017.

${ }^{35}$ S. G. Gilbert, B. Weiss, Neurotox. 2006, 27, 693-701.

${ }^{36}$ a) P. B. Tchounwou, C. G. Yedjou, A. K. Patlolla, D. J. Sutton, Molecular, Clin. Environ.Tox., 2012, 101, 133-164. b) J. Chen, S. Xiao, X. Wu, K. Fang, W. Liu, Talanta, 2005, 67, 5, 992-996.

${ }^{37}$ C. M. George, L. Sima, M.H. Jahuira Arias et al., Bull. W. H. Org. 2014, 92, 8, 565-572.

38 a) A. Oskarsson, L. Jorhem, J. Sundberg, N.-G. Nilsson, L. Albanus, Sci. Tot. Environ., 1992, 111, 83-94. b)

G. Ayumi K. Koyashiki, M. M. Bastos-Paoliello, P. B. Tchounwou, Rev. Environ. H. 2010, 25, 243-253.

${ }^{39}$ K. Ndungú, S. Hibdona, A. Russell-Flegal, Talanta, 2004, 64, 258-263. 
crisis", in Michigan (United States). ${ }^{40}$ The issue begun in 2014 and it is still nowadays an unsolved problem. In 2014 they started to take the water from Flint river and use it for human consumption, without any pre-treatment. The composition of the water from the river caused the corrosion of pipes which led to get water with metallic salts and oxides, being lead(II) the most dangerous component in suspension and in solution.

In order to solve this issue two measures have been taken in the last years. The first, and temporary solution, was the treatment of water with phosphates, which would reduce corrosion. However, in any case, this is temporary and with a very limited effect because it does not work whenever the pipes are already corroded. Therefore, the only real solution is the replacement of the pipes, which is still a work in progress because of the high costs, monetary and in the amount of time required.

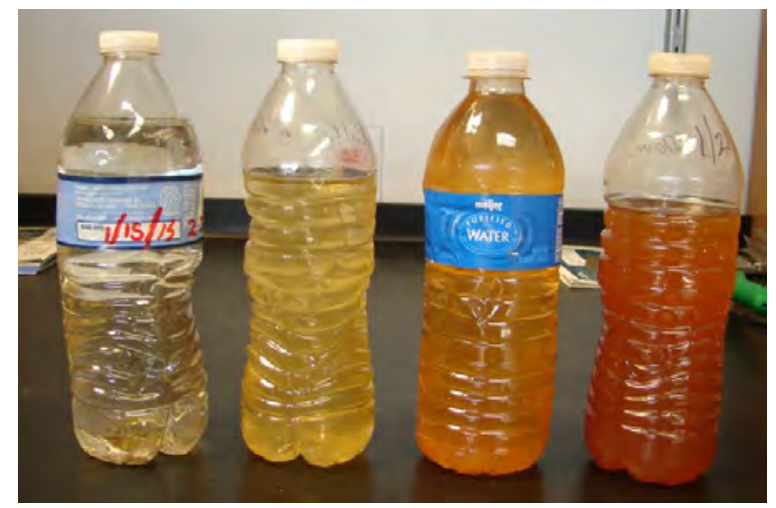

Figure 6. Picture of water samples from Flint, from left to right January $15^{\text {th }} 2015$ ( 2 samples),

$$
\text { January } 16^{\text {th }} \text { and January } 21^{\text {st }} \text {. }
$$

When observing the lead contaminated samples from Flint (Figure 6), there are some facts that characterize them. First, it does contain lead(II) in solution but it is mostly a dispersion of lead and metallic oxides and salts. In consequence, in order to decrease the concentration of lead(II), it was usually filtered. Secondly, the colour of the solution would complicate having a fast and quantitative response to determine lead concentration. Therefore, a quick detection is an unsolved issue because the process of measuring lead(II) in coloured solutions needs for expensive and slow analytical methods, such as HPLC and/or MS, and a previous filtration step.

\subsection{Methods for detection of Lead(II)}

Before describing any detection method for $\mathrm{Pb}(\mathrm{II})$ it is important to know some concerning aspects about it. Regarding that, $\mathrm{Pb}$ (II) salts solubility in water has been deeply studied in literature, ${ }^{41}$ being highly dependent in factors such as $\mathrm{pH}$, the counterions or temperature. Moreover, although a counterion could form an insoluble salt, in many cases the complex may be found in solution, with high stability constants. Some important data about water solubility:

o Acetate, nitrate and perchlorate lead salts are highly soluble in deionized water.

o Carbonate lead salt is very insoluble (at most $50 \mu \mathrm{M}$ ).

\footnotetext{
${ }^{40}$ B. Venkataraman, "The Paradox of Water and the Flint Crisis", Environment (online magazine), March 2018.

${ }^{41}$ a) IUPAC solubility database. Updated in February 2015. b) H. Lawrence Clever, F. J. Johnson, J. Phys. Chem, 1980, 9, 752-784.
} 
o Phosphate lead salts are highly insoluble, and $\mathrm{pH}$ dependent. Having a controlled $\mathrm{pH}$ between 6 and 7 leads to $\mathrm{Pb}(\mathrm{II})$ concentration in solution lower to $5.5 \mu \mathrm{M}$ and $\mathrm{pH}$ higher than 7 implies less than $1 \mu \mathrm{M}$.

o Sulfate lead salts are more soluble, but they also precipitate at concentrations around $0.1 \mathrm{mM}$ or higher.

In consequence, solubility is an important issue, especially when dealing with samples without pre-treatment and/or non-ionization processes.

\section{Classic analytical methods:}

Some analytical methods are standardized for detection of lead containing samples, flame atomic absorption spectrometry (FAAS), electrothermal atomic absorption spectrometry (ETAAS), inductively coupled plasma optical emission spectrometry (ICP-OES) and inductively coupled plasma-mass spectrometry (ICP-MS). All of them consist of the evaluation of the concentration by comparison with the signal of $\mathrm{Pb}(\mathrm{II})$ with a known calibration. The main drawbacks for all of them are the high costs and the need of special facilities and specialized people for handling and interpreting the data.

Apart from the measuring technique, another common issue for all detection methods is the interference of the matrix, being necessary a process of digestion, a preconcentration step and the removal of the matrix, in most cases.

- Digestion of the sample, to degrade the organic material in solution. It is common the use of high temperatures, such as microwave, and/or highly concentrated acid solutions.

- Extraction of the lead, whose necessity depends on the measuring technique. Then, there are several ways in which might be performed. For instance, coprecipitation, solid phase microextraction (SPM), single drop microextraction (SDME) or solidified floating organic drop microextraction (SFODME) are some of the possible methods. They are performed by different combinations of specific purification methods. These are some of the possible steps:

o Adding a specie with affinity to lead cations.

0 Adding an organic solvent.

o Liquid-liquid extraction.

o Retention column.

o Using a metal-organic frameworks (MOF) or metal nanoparticles. ${ }^{42}$

o Extractions in solid phase using sonication systems. ${ }^{43}$

o Filtration.

Different combinations achieve either the precipitation of the lead derivatives, the precipitation of the interferents or give to the lead cations solubility in organic media, using a $\mathrm{Pb}$ (II) complex. In regard to the optimization to better, faster and cheaper results, there are many papers that use different extraction methods and compare between them. ${ }^{44}$

\footnotetext{
42 a) M. Salarian, A. Ghanbarpour, M. Behbahani, S. Bagheri, A. Bagheri, Microchim. Acta 2014, 181, 9991007. b) M. Ghaedi, M. Rezakhani, S. Khodadoust, K. Niknam, M. Soylak. Sci. W. J. 2012, 2012, 1-9.

${ }^{43}$ M. Sadeghi, E. Rostami, D. Kordestani, H. Veisi, M. Shamsipura, RSC Adv. 2017, 7, 27656-27667.

${ }^{44}$ O. A. Urucu, F. Dönmez, E. K. Yetimoglu, J. Anal. M. Chem. 2017, 1-7.
} 


\subsection{Colorimetric and fluorometric lead(II) sensors in literature}

Most of the research into detecting $\mathrm{Pb}$ (II) is focused on the development and improvement of different extraction processes, but not so much in the use of cheap and portable methods. This fact is quite surprising because, being $\mathrm{Pb}(\mathrm{II})$ what is considered in chemistry as a big and soft cation, there are many possibilities for developing specific molecular receptors. In consequence, the synthesis of a receptor bound to a chromogenic or fluorogenic backbone is an aspect to be considered.

In literature, many colorimetric molecular sensors and materials have been prepared. However, they have very important issues that need for improvements, such as the detection limits and the interferents. For colorimetric measurements, limits of detection are in the range of $0.01 \mathrm{mM}$ or higher, which would be around $5000 \mathrm{ppb}$ of $\mathrm{Pb}(\mathrm{II})$, being 500 times higher than the quantity considered as toxic. However, it is not the case for fluorescent probes, they reach much lower LODs and could be used in cellular environments. Among them, most fluorescent probes are based in using peptides ${ }^{45}$ or proteins ${ }^{46}$ but their non-specific interactions and the lack of simplicity limit their use. On the other hand, there are several molecular derivatives that have proven to be useful and with high specificity respect to other cations, although rarely working in water media. ${ }^{47}$

There are many publications referring to fluorescent molecules of $\mathrm{Pb}(\mathrm{II}){ }^{48}$ One of the first and most cited fluorescent probes for detection of $\mathrm{Pb}$ (II) was the synthetized by the group of Miller. ${ }^{49}$ They called the probe leadfluor-1 (Figure 7), and it was described as a lead sensitive probe with an easy synthesis protocol and with satisfactory results for detection within cells, although its fluorescence quantum yield was very low. In comparison, other authors such as the group of Marbella, ${ }^{50}$ synthetized a new probe, called leadglow (Figure 7), to improve some of the previous flaws. The new probe worked in MeOH:water $2.5 \% \mathrm{v} / \mathrm{v}$ with much higher fluorescence and high selectivity.
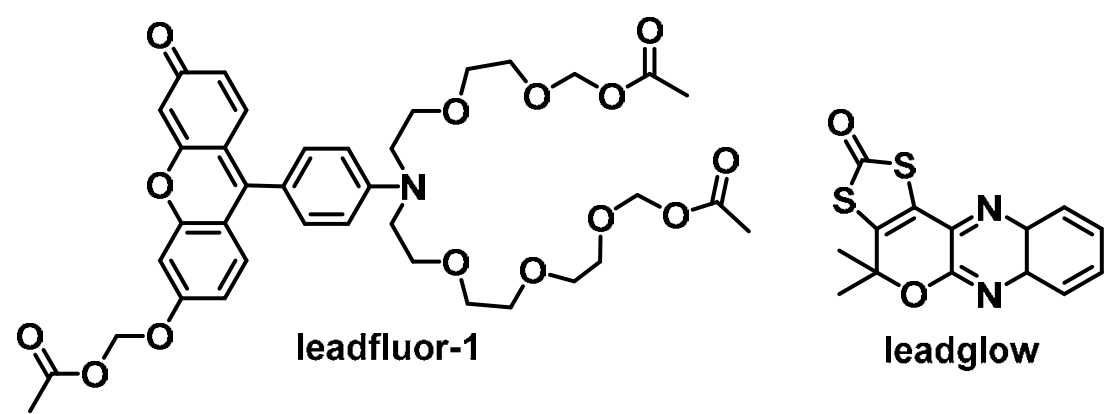

Figure 7. Chemical structure of leadfluor- $1^{49}$ (left) and leadglow ${ }^{50}$ (right).

In addition, there are also other species that have been used for the modification of materials for $\mathrm{Pb}$ (II) detection, such as fluorescent calixarenes and some fluorescent ligands. ${ }^{51}$ However, there are not many examples and they are usually very complicated to synthesize and work with; additionally, in most cases, the change in fluorescence is not very remarkable, leaving wide room for improvements.

\footnotetext{
${ }^{45}$ S. Deo, H. A. Godwin, J. Am. Chem. Soc. 2000, 122, 174-175.

${ }^{46}$ P. Chen, B. Greenberg, S. Taghavi, C. Romano, D. van der Lelie, C. He. Angew. Chem. Int. Ed. 2005, 44, 2715-2719.

${ }^{47}$ C. -T. Chen, W. -P. Huang. J. Am. Chem. Soc. 2002, 124, 6246-6247.

${ }^{48}$ T.-C. Zheng, F.-Y. Yan, D.-C. Shi, Y. Zou, S.-1. Zhang, L. Chen, Fluorescent Probes for Detection of Lead Ion Recent Innovations in Chemical Engineering, Bentham Science, Vol. 7, 2014.

${ }^{49}$ E. W. Miller, Q. He, C. J. Chang, Nat. Prot. 2008, 3, 777-783.

${ }^{50}$ L. Marbella, B Serli-Mitasev, P. Basu, Angew. Chem. Int. Ed. 2009, 48, 3996 -3998.

${ }^{51}$ D. Faye, J.-P. Lefevre, J. A. Delaire, I. Leray, J. Photochem. Photobiol. A, 2012, 234, 115- 122.
} 
In spite of the high quantity of research in the field, the main issue when searching literature about fluorescent probes for detection of $\mathrm{Pb}(\mathrm{II})$ is the surprising lack of information about the solvent. Notwithstanding, when selective detection of $\mathrm{Pb}$ (II) is performed, it should be taken into account that it might be tampered by many counterions. As it was shown, the quantity of species that complex $\mathrm{Pb}(\mathrm{II})$ is wide (sulfates, phosphates, carbonates...), many of them have high affinity constants and are prone to precipitation as insoluble solids. In fact, when looking for new $\mathrm{Pb}$ (II) probes they should be checked to work under some conditions:

- $\quad$ HH not higher to 7.8 (precipitation of the hydroxide). It was found that, in some papers, it is studied the response to $\mathrm{Pb}^{2+}$ at $\mathrm{pH}$ close to $10 .^{49}$

- Not using buffers such as PBS; it would produce the precipitation of $\mathrm{Pb}(\mathrm{II})$ in solution, having a maximum lead concentration in the range of $\mu \mathrm{M}$, less than $500 \mathrm{ppb}$. This limit is higher than the LODs of most colorimetric probes and some fluorescent ones. Although in some papers, some probes (Figure 8) are supposed to work in PBS media. ${ }^{52}$

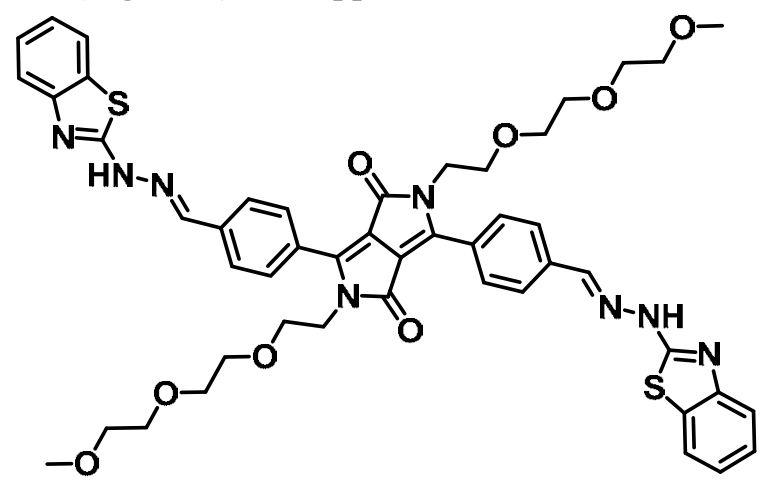

Figure 8. Diketopyrrolopyrrole-based fluorescence turn-on probe for the detection of $\mathrm{Pb}(\mathrm{II})$ in aqueous solution and living cells. ${ }^{52}$

- HEPES, and other sulfate containing buffers, precipitate when the concentration of $\mathrm{Pb}$ (II) is higher than $0.1 \mathrm{mM}$.

Using other buffers such as acetate might seem a good option; however, if the concentration of the buffer is very high respect to the receptor, the competition between complexes could interfere in the measurements. For example, the complex $(\mathrm{AcO})_{1-4} \mathrm{~Pb}(\mathrm{II})$ in water have successive association constants around $200-4000\left(\mathrm{M}^{-1}\right.$ to $\left.\mathrm{M}^{-4}\right),{ }^{53}$ which may compete with the formation of a fluorescent complex. In fact, this kind of complexation could, in many occasions, be used for displacing the equilibria and restoring the free probe (See Section 12.6).

Other unsolved issue, in regard of $\mathrm{Pb}$ (II) fluorometric and colorimetric probes, is how to work in dark coloured samples. Nowadays there is not much research about it in spite of being the usual way in which lead contaminated samples are presented (see Figure 6). Thus, the elaboration of materials that change colour/fluorescence in the presence of $\mathrm{Pb}(\mathrm{II})$ that can be used in original solutions with no sample manipulation is a highly desirable task to overcome.

\footnotetext{
${ }^{52}$ X. Yang, Y. Zhang, Y. Li, X. Liu, J. Mao, Y. Yuan, Y. Cui, G. Suna, G. Zhang, RSC Adv., 2016, 6, 5200452008 .

${ }^{53}$ S. Gobom, Nature 1963, 197, 283-284.
} 


\section{OBJECTIVES OF THE CHAPTER}

The initial objective of this part of the Thesis was based on the aims of the European Project "SNIFFER". This name was an acronym of "Sensory devices network for food supply chain security" in which the research group was involved. The project was focused on the development of new tools for detection and prevention of environmental contamination with chemical, biological, radiological or nuclear agents (CBRN). In this regard, it was determined that an important source of risk for human health came from food poisoning, specifically from the products that some bacteria produce when they grow in badly preserved food, the contaminant known as cereulide.

The importance of cereulide and how it works was extensively explained in the introduction, in short it is related with potassium equilibria within cells. As a consequence, while working in the development of molecular probes, it was of particular interest the development of a probe sensitive to cereulide. This task was oriented by creating receptors that may compete with this natural potassium ionophore, leading to its detection.

Simultaneously to these studies, it was discovered that the receptor that was sensitive to potassium cations, was selective to three species $\mathrm{K}(\mathrm{I}), \mathrm{Ba}(\mathrm{II})$ and $\mathrm{Pb}(\mathrm{II})$. Being $\mathrm{Pb}$ (II) of special importance, as a very toxic cation, the work took interest in creating a material for its detection.

In summary, several objectives were proposed:

- Synthesis of a potassium receptor. Two possibilities were considered, a crown ether derivative and a cryptand.

- Modifying the receptor with a fluorescent backbone, a PMI.

- Synthesis of artificial cereulide, from the amino/hydroxyacids, due to the low quantities in contaminated natural samples and because of the price of acquiring it commercially.

- Synthesis of some modified cereulide derivatives and study of the consequences of variations in its structure (potassium affinity, potassium transport in vesicles, cytotoxicity...).

- Fluorescent detection of K(I) in solution.

- Fluorescent detection of cereulide in solution and in cellular environments.

Additionally, when the initial evaluation gave positive results for $\mathrm{Pb}(\mathrm{II})$ detection the objectives were extended to:

- Fluorescent detection of $\mathrm{Pb}(\mathrm{II})$ in solution.

- Preparation and study of a fluorescent material sensitive to $\mathrm{Pb}(\mathrm{II})$ in solution.

- Testing the $\mathrm{Pb}(\mathrm{II})$ sensitive material in coloured solutions. 


\section{SYNTHESIS OF FLUORESCENT MOLECULAR SENSORS WITH CRYPTAND AND CROWN ETHER RECEPTORS}

The synthesis of potassium sensitive molecules started by choosing the receptors, Figure 9. Two kinds of derivatives with high affinity and selectivity for $\mathrm{K}^{+}$were found, a crown ether and a triazacryptand (TAC) (Figure 9). There were others but, as it was explained in the Section 3, the selectivity $\mathrm{K}^{+} / \mathrm{Na}^{+}$was lower.

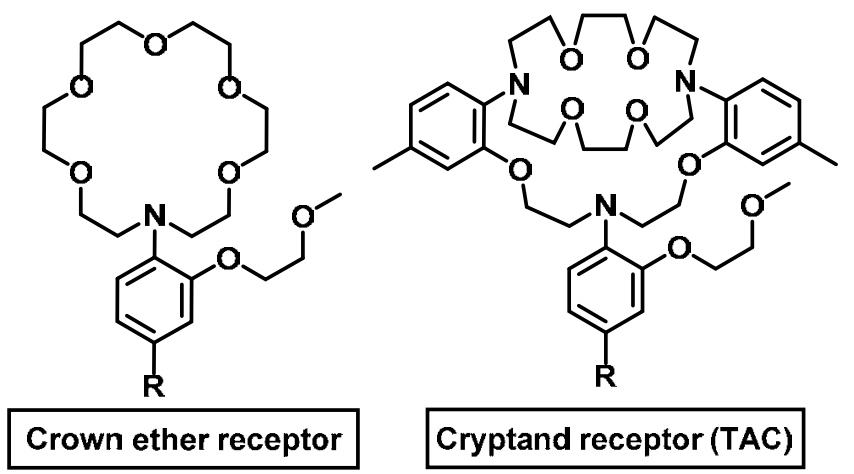

Figure 9. (2) Molecular structure of a crown ether and a cryptand derivative that have characteristics as $\mathrm{K}^{+}$receptors.

They were proposed to be modified with a fluorescent backbone in R position, in this case a PMI, due to its outstanding fluorescent properties.

Both compounds shared the two first steps of synthesis (Figure 10).

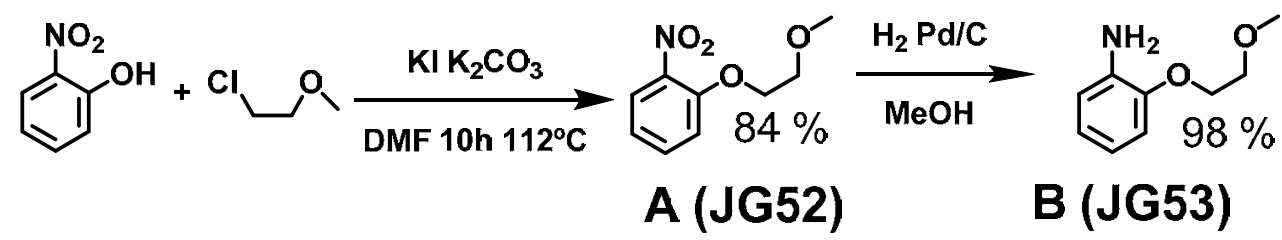

Figure 10. Synthesis of 2-(2-methoxyethoxy)aniline.

Starting from ortho-nitrophenol, an ethylene monomethyl ether was introduced in the alcohol position, by nucleophilic substitution in DMF. In literature, the presence of this group is a common characteristic in potassium cations to the receptors. In the second step, the nitro group was reduced to amino group by hydrogenation on $\mathrm{Pd} / \mathrm{C}$. It was the previous step to the synthesis of the crown ether or cryptand.

\subsection{Synthesis of the crown ether derivative (JG76) $)^{54}$}

Following the procedure from literature (Figure 11), the first step was a nucleophilic substitution in water, in the presence of an excess of 2-chloroethanol. For closing the crown ether ring,

\footnotetext{
${ }^{54}$ a) H. He, M. A. Mortellaro, M. J. P. Leiner, R. J. Fraatz, J. K. Tusa, J. Am. Chem. Soc. 2003, 125, 1468-1469. b) S. Ast, T. Schwarze, H. Mller, A. Sukhanov, S. Michaelis, J. Wegener, O. S. Wolfbeis, T. Körzdörfer, A. Dürkop, H.-J. Holdt, Chem. Eur. J. 2013, 19, 14911 - 14917
} 
the second part was performed at high dilution of JG54 and adding the ditosylate slowly and under reflux.

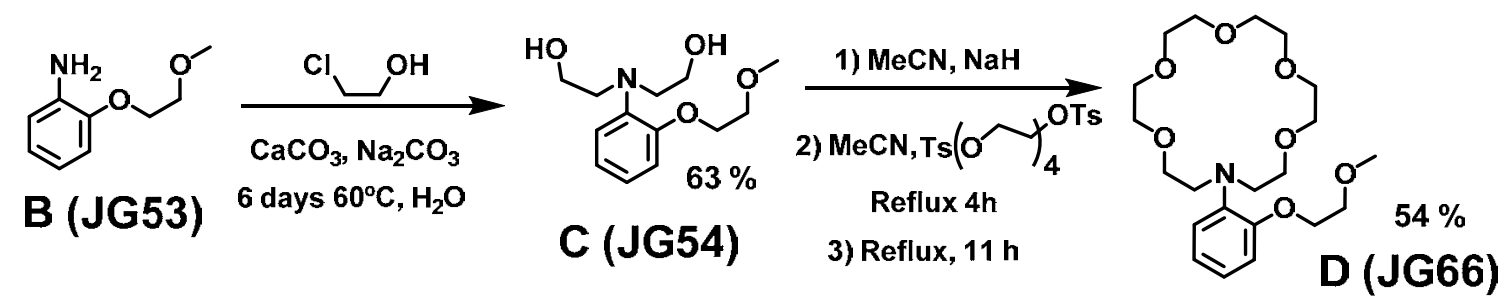

Figure 11. Synthesis of the crown ether JG66.

In contrast with carbonylation processes, commonly performed in literature, a bromination was carried out (Figure 12), to adapt the product to the objectives of our project.

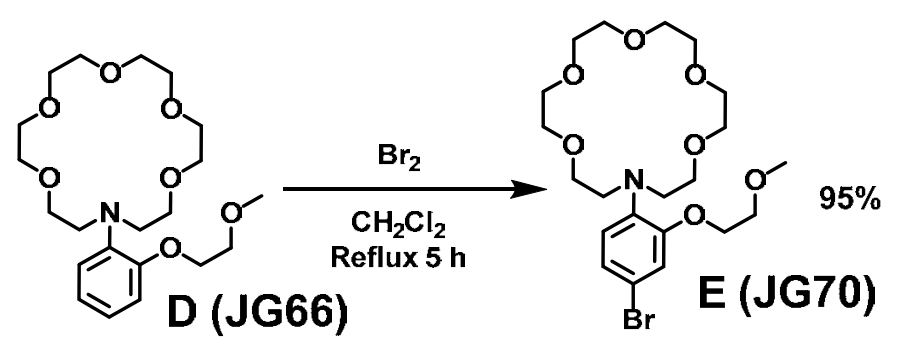

Figure 12. Synthesis of the crown ether JG70.

With the brominated product the fluorescent backbone was synthetized by Suzuki reaction between the brominated crown ether and the boronic ester of the PMI (Figure 13).

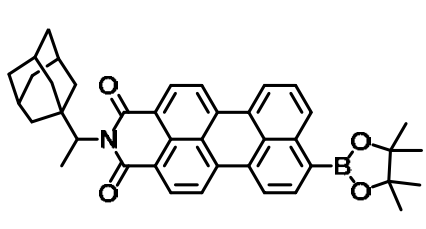

JG75

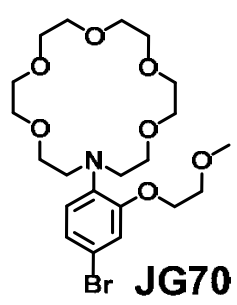

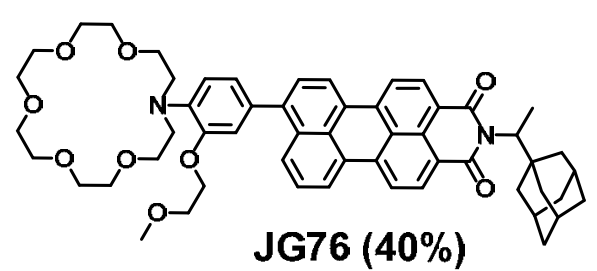

Figure 13. Synthesis of PMI-crown ether, JG76.

\subsection{Synthesis of the cryptand derivative, TAC-PMI (JG103) 55}

The detailed process of synthesis for TAC (JG93) was already published. ${ }^{55}$ The first part of the synthesis was performed by a substitution reaction and finished by the hydrogenation of the nitro groups to amine, with no particular difficulties (Figure 14).

\footnotetext{
${ }^{55}$ a) B. Sui, X. Yue, M. G. Tichy, T. Liu, D. D. Belfield, Eur. J. Org. Chem. 2015, 1189-1192. b) H. He, A. M. Mortellaro, M. J. P. Leiner, R. J. Fraatz, J. K. Tusa, J. Am. Chem. Soc. 2003, 125, 1468-1469.
} 
${ }_{4 \mathrm{~h} 60^{\circ} \mathrm{C}}^{\mathrm{O}} \underset{\mathrm{DMF} \mathrm{K}_{2} \mathrm{CO}_{3}}{\mathrm{Br}}$

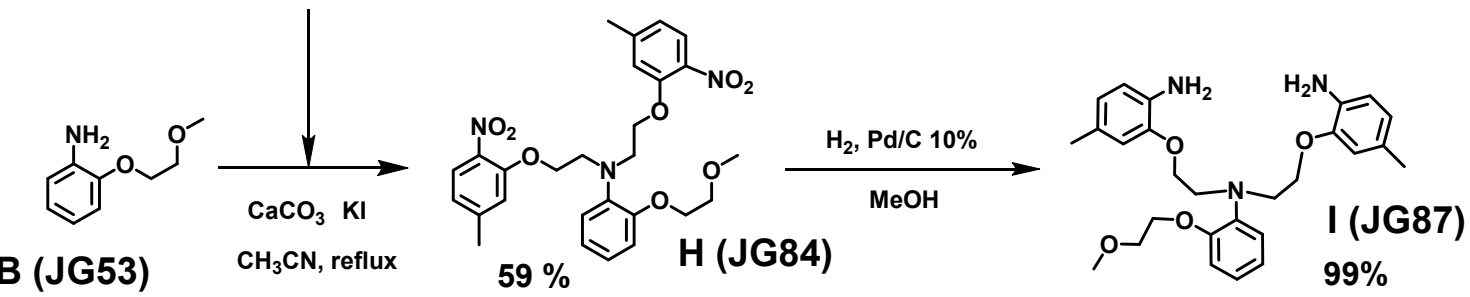

Figure 14. Synthesis of starting diethoxyaniline derivative.

The second part was divided into two steps, closing the ring and the cryptand respectively (Figure 15). The reaction was a nucleophilic substitution, slowly adding the diiodinated derivative on the diluted probe, to favour the closing of the ring over the coupling between different molecules.

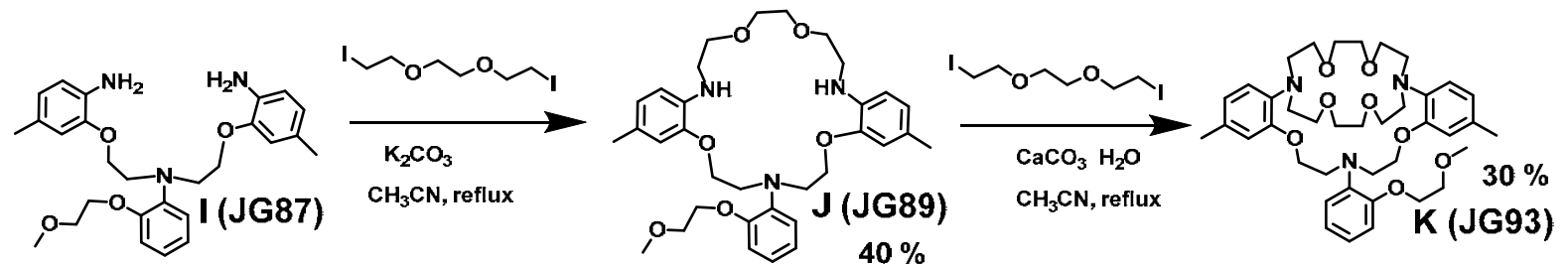

Figure 15. Synthesis of TAC JG93.

In the same way used for the crown ether derivative, TAC was modified by introducing a bromine atom in the aniline group (Figure 16).

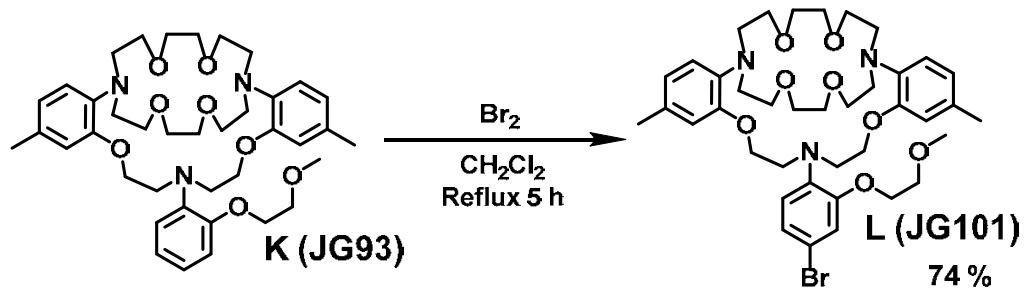

Figure 16. Synthesis of TAC-Br JG101.

With the brominated product, the bromine was substituted with PMI by Suzuki coupling (Figure 17).
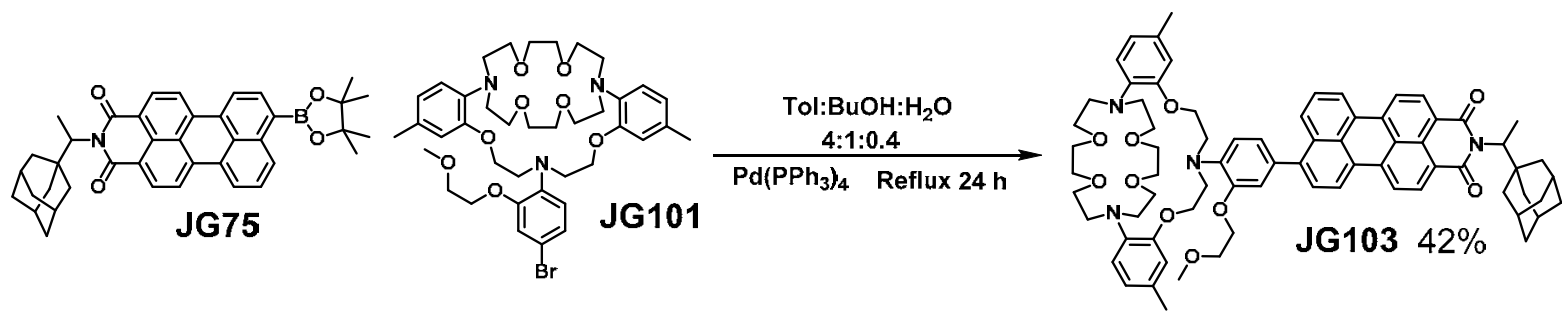

Figure 17. Synthesis of PMI-TAC JG103. 


\section{SYNTHESIS OF CEREULIDE AND DERIVATIVES}

The research in preparation of peptides is extensive and with many possible routes to achieve different reactions. Cereulide chemical structure is a cyclic reiteration, cyclo[-(D-O-Leu- $-\mathrm{D}-\mathrm{Ala}-\mathrm{L}-\mathrm{O}-$ Val-L-Val)] 3 . In consequence, the obvious way to orient the synthesis was by iterating the substructure and cycling the product. This synthesis was possible starting from four commercial monomeric structures, Figure 18.<smiles>CC(C)C(O)C(=O)O</smiles>

(S)-2-hydroxy-3-methylbutanoic acid<smiles>CC(C)CC(O)C(=O)O</smiles><smiles>C[C@H](NC(=O)OC(C)(C)C)C(=O)O</smiles>

(tert-butoxycarbonyl)-L-valine

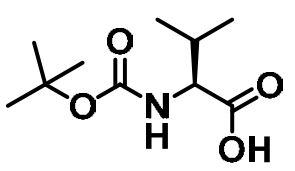

(S)-2-hydroxy-4-methylpentanoic acid

Figure 18. Monomeric structures for the synthesis of cereulide.

The procedure to obtain cereulide is based on a convergent mechanism for prolonging an ester-amide chain. It was divided into 5 steps, that may be applied for every work addressing the elongation of a steric-peptidic chain.

1. Selective protection of specific terminal groups.

2. Elongation of the chain.

3. Selective deprotection of the product.

4. Repetition of steps 2 and 3.

5. Capping the chain.

For the synthesis of cereulide two protecting groups were chosen:

- Carboxylic acid protected by a benzyl ring. The synthesis was performed by esterification in anhydrous DMF at room temperature, overnight, Figure $19 .{ }^{56}$ Quantitative yields were obtained.
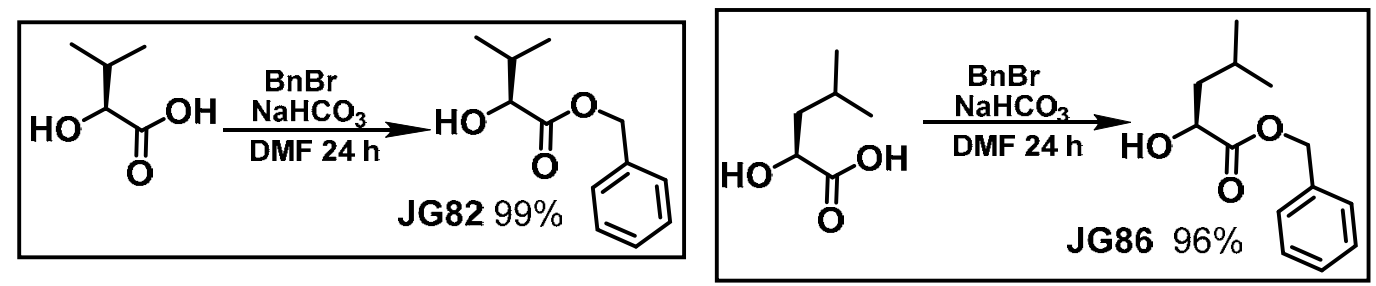

Figure 19. Synthesis reaction for protection of (S)-2-hydroxy-3-methylbutanoic acid and (S)-2hydroxy-4-methylpentanoic acid.

- Boc-protected amino group. Synthetized from tert-butyl carbonic anhydride. In THF and in an ice bath overnight. ${ }^{57}$ Alternatively, they are commercially available.

Next step was the elongation of the chain. In doing so, two kinds of reactions were performed:

- Esterification. The chosen method was selected depending on reagents and products, and their respective stereochemistry.

\footnotetext{
${ }^{56}$ Y. Peng, H. W. Pang, T. Ye, Org. Lett., 2004, 6, 3781-3784.

${ }^{57}$ D. M. Shendage, R. Froehlich, G. Haufe, Org. Lett., 2004, 6, 3675-3678.
} 
o Procedure 1: Steglich reaction. Preserving the stereochemistry of the carbon bonded to the hydroxyl group. It presented mild conditions and high yield. In DCM solution. DCC was used to favour the formation of an intermediate with high reactivity in presence of alcohols. DMAP was used as base and nucleophile, catalysing the process.

o Procedure 2, Mitsunobu reaction. The reaction changes the stereochemistry of the carbon from the alcohol group. It was performed by using $\mathrm{DEAD}, \mathrm{PPh}_{3}$ in $\mathrm{THF}$ solution.

- Amide formation. There are many methods to perform the reaction between the amine and the carboxylic acid. Two methods were carried out. On one hand, the most commonly used, was the one catalysed by the use of PyBop, for the formation of an intermediate more reactive against amines, using DIPEA as base. The solvents tested were DCM and dry DMF.

On the other hand, the combination between EDCI and subsequent addition of HOBt was another possibility to the synthesis of amides. It was performed in dry DMF.

Additionally, it was necessary to deprotect selectively the protected groups. Because of that, the chosen groups for protection had two different deprotection mechanisms:

- Benzyl group deprotection. It proceeds by hydrogenation in presence of Pd supported on carbon. The reagent was dissolved in methanol and hydrogen was introduced in the reaction, at room temperature. The product was obtained quantitatively just by filtration of the $\mathrm{Pd} / \mathrm{C}$ and evaporation of the solvent.

- Boc-deprotection. In doing so, the reaction needed for highly concentrated acid. In this case, it was performed by bubbling $\mathrm{HCl}$ gas to an EtOAc solution of the compounds. The $\mathrm{HCl}$ was formed in situ, by reaction between $\mathrm{H}_{2} \mathrm{SO}_{4}$ and $\mathrm{NaCl}$. The products were obtained after evaporation of the solvent, as the hydrochloride salt.

All these reactions were adapted from literature to synthetize cereulide by a 15 steps mechanism, Figure 19. ${ }^{1,21}$ The product of each step is extensively explained and characterized in Experimental Appendix 3, including characterization by ${ }^{1} \mathrm{H}-\mathrm{NMR},{ }^{13} \mathrm{C}-\mathrm{NMR}$ and mass spectrometry analysis. 


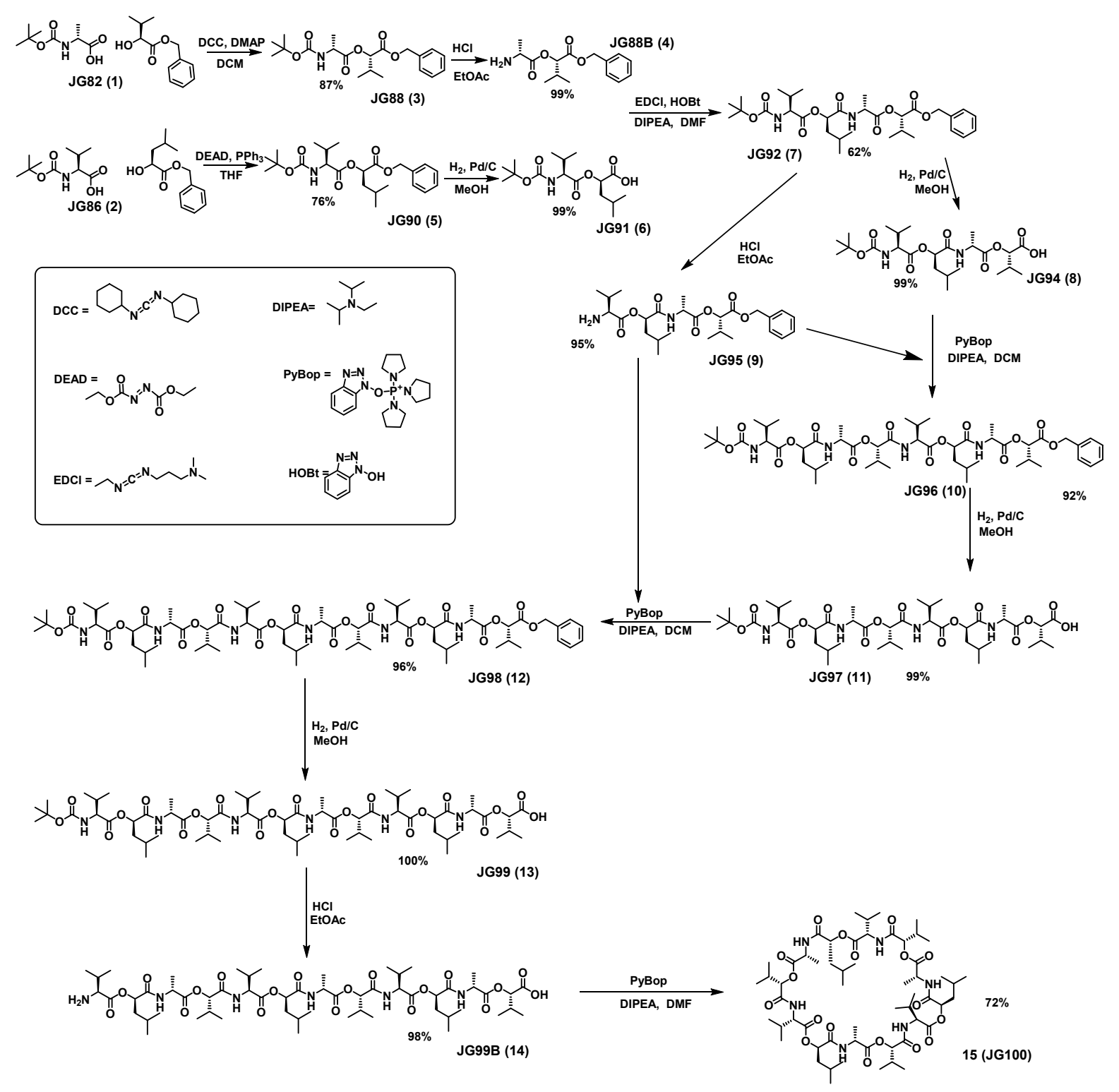

Figure 19. Synthesis scheme of cereulide.

Due to the interest of cereulide as potassium ionophore, obtaining a derivative with only slight changes in composition may lead to a better understanding on how this ionophore works and will expand the possibilities of the research, by understanding how it may affect its properties. In doing so, one of the components from the cyclic depsipeptide was changed by a group possessing a terminal carboxylic ester that was deprotected in order to introduce derivatives in that position, Figure 20.

Instead of performing a reaction between "9" (JG95) and "11" (JG97), JG95 was shortened by one unit (JG82). In a later step, before cyclization, it was introduced a new group containing a carboxylic ester (sensitive to acid media), instead of the isopropyl group. 


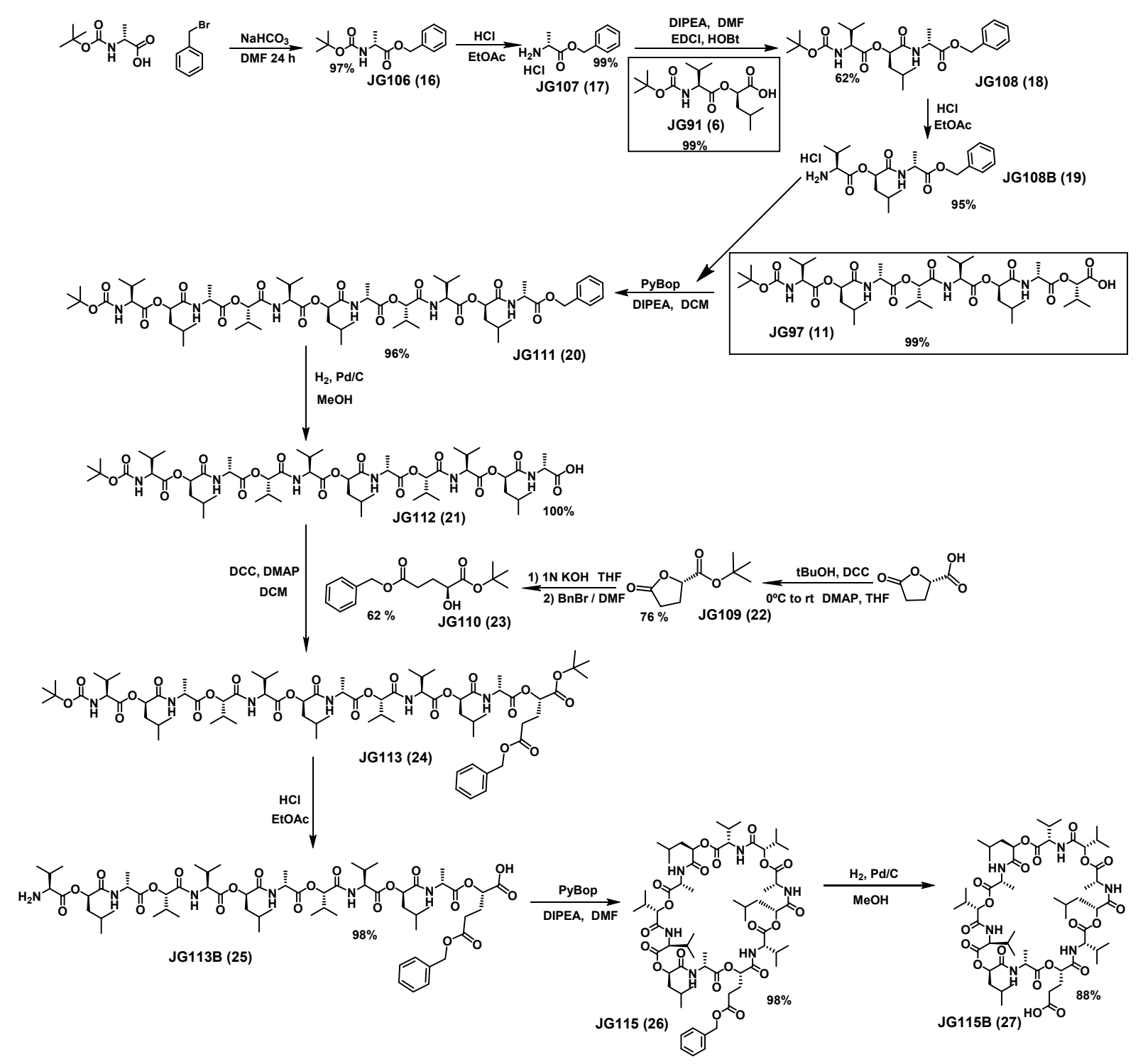

Figure 20. Synthesis of modified cereulide JG115 and JG115B.

The synthesis of "23" (JG110), which was the modification of the chain, was performed by following the procedure from the group of Koppetsch, Figure 21, explained in literature. ${ }^{58} \mathrm{~A}$ two steps process to obtain two protected ester groups, one with a tert-butyl group (acid deprotection) and one with a benzyl group (deprotection by hydrogenation).

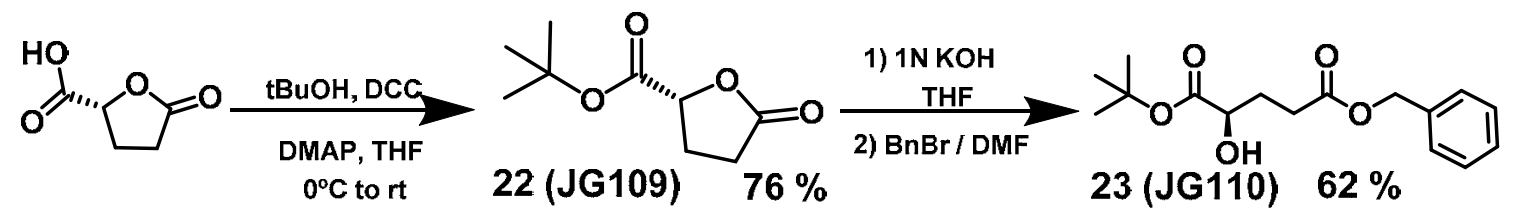

Figure 21. Synthesis scheme of a monomer for modifying cereulide, 5-benzyl 1-(tert-butyl) (R)-2hydroxypentanedioate (JG110).

In a final step, the carboxylic acid was modified with a fluorescent group, to act as cellular marker and to check the effect on the behaviour of cereulide by the different groups, Figure 22.

\footnotetext{
${ }^{58}$ S. G. Levy, V. Jacques, K. L. Zhou, S. Kalogeropoulos, K. Schumacher, J. C. Amedio, J. E. Scherer, S R. Witowski, R. Lombardy, K. Koppetsch, Org. Proc. Res. Dev. 2009, 13, 535-542
} 


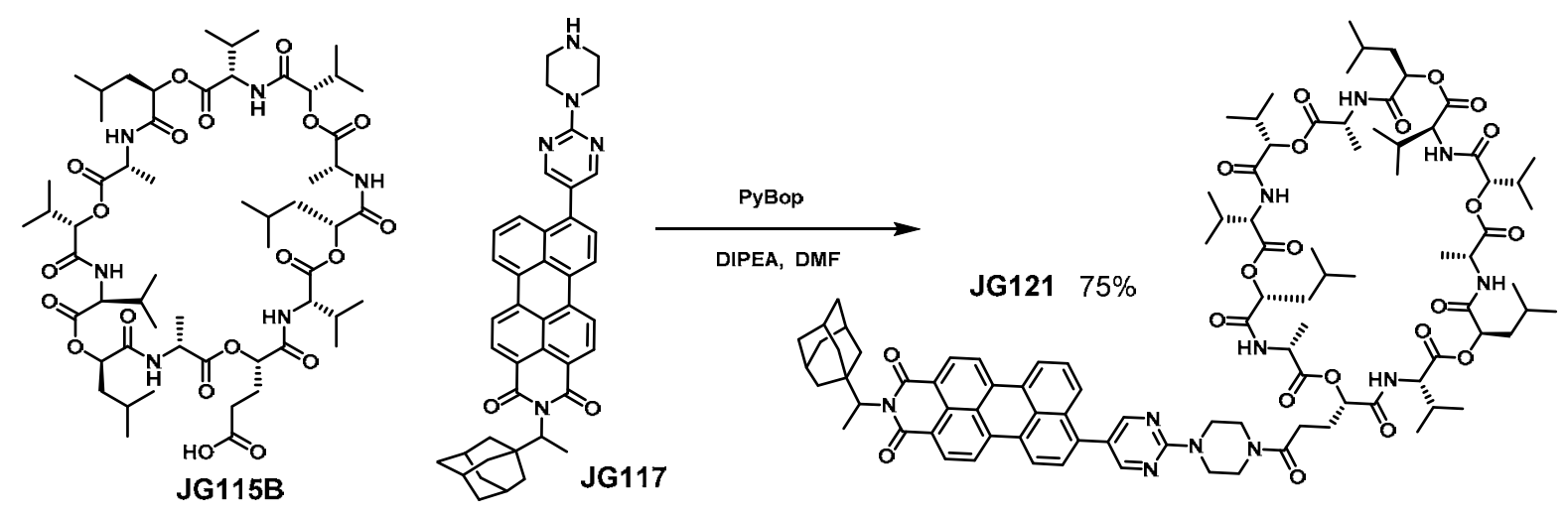

Figure 22. Synthesis of the PMI-cereulide derivative JG121 from JG115B.

In conclusion, the three cereulide derivatives shown in Figure $\mathbf{2 3}$ were obtained:
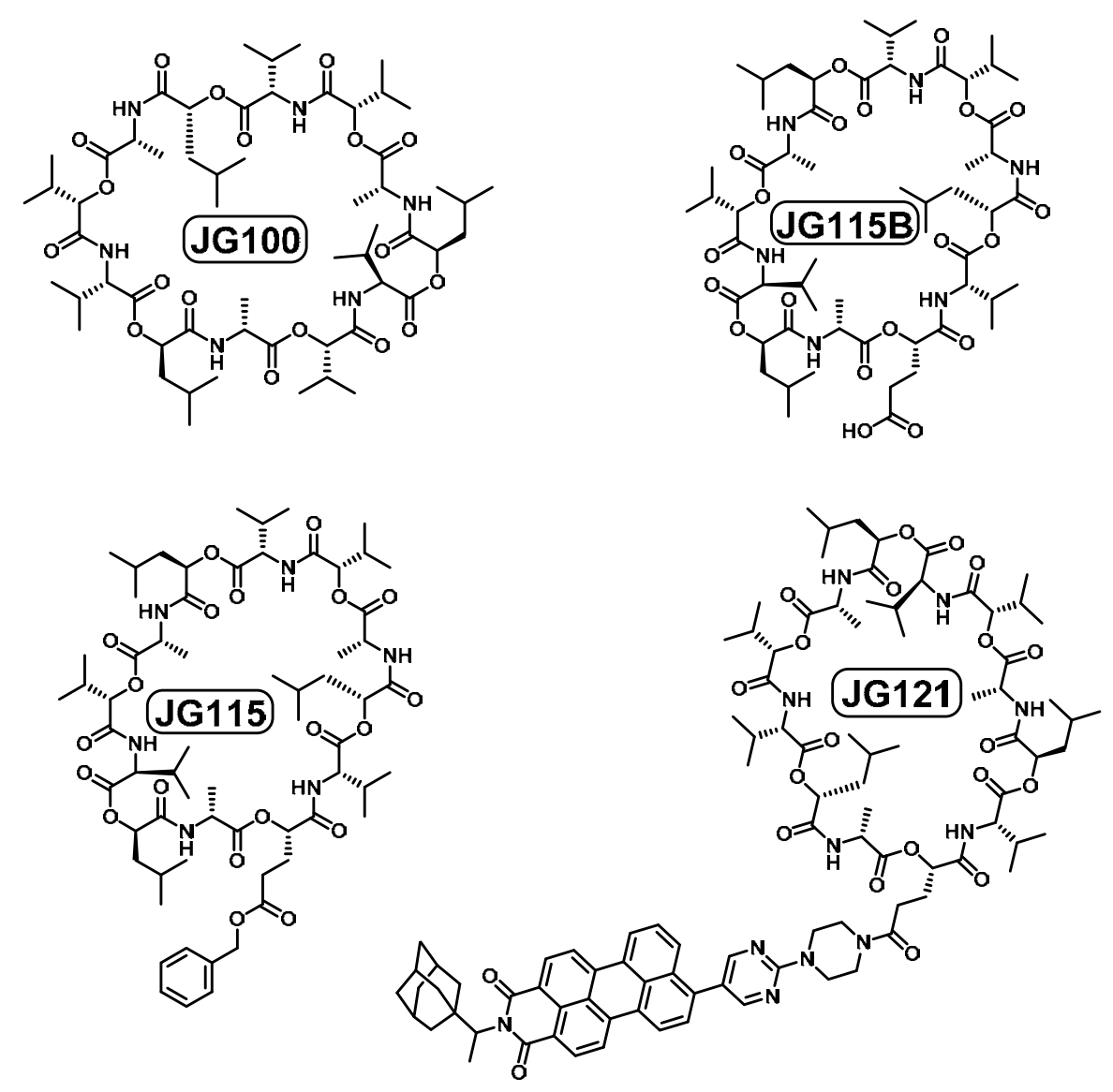

Figure 23. Molecular structure of synthesized cereulide derivatives JG100 (cereulide), JG115B, JG115 and JG121. 


\section{SOLVATOCHROMIC STUDIES OF CROWN ETHER (JG76) AND TAC (JG103) PROBES}

The experimental conditions were the general ones explained in Chapter $\mathbf{0}$. They were chosen in order to find the best solvent and with the aim of using the probe in water and biological environments; fulfilling the next conditions:

- The solubility of the probe had to be high. To prepare a solution at least $0.1 \mathrm{mM}$.

- The solvent had to be miscible with water.

- It had to be sensitive and selective to the analytes, with high final fluorescence and a measurable increase.

\subsection{Solvatochromism of JG76}

Pictures of Figure 24 were acquired for the samples in different solvents, under visible light and UV light, $366 \mathrm{~nm}$. In addition, to check the response of the solvents to $\mathrm{Pb}^{2+}$ and $\mathrm{K}^{+}$, concentrated solutions of $\mathrm{Pb}\left(\mathrm{ClO}_{4}\right)$ and $\mathrm{KCF}_{3} \mathrm{SO}_{3}$ were added, from $\mathrm{EtOH}$ solutions. The increase in fluorescence was higher with $\mathrm{Pb}(\mathrm{II})$, and a picture was taken, the response with $\mathrm{K}^{+}$was also measured.

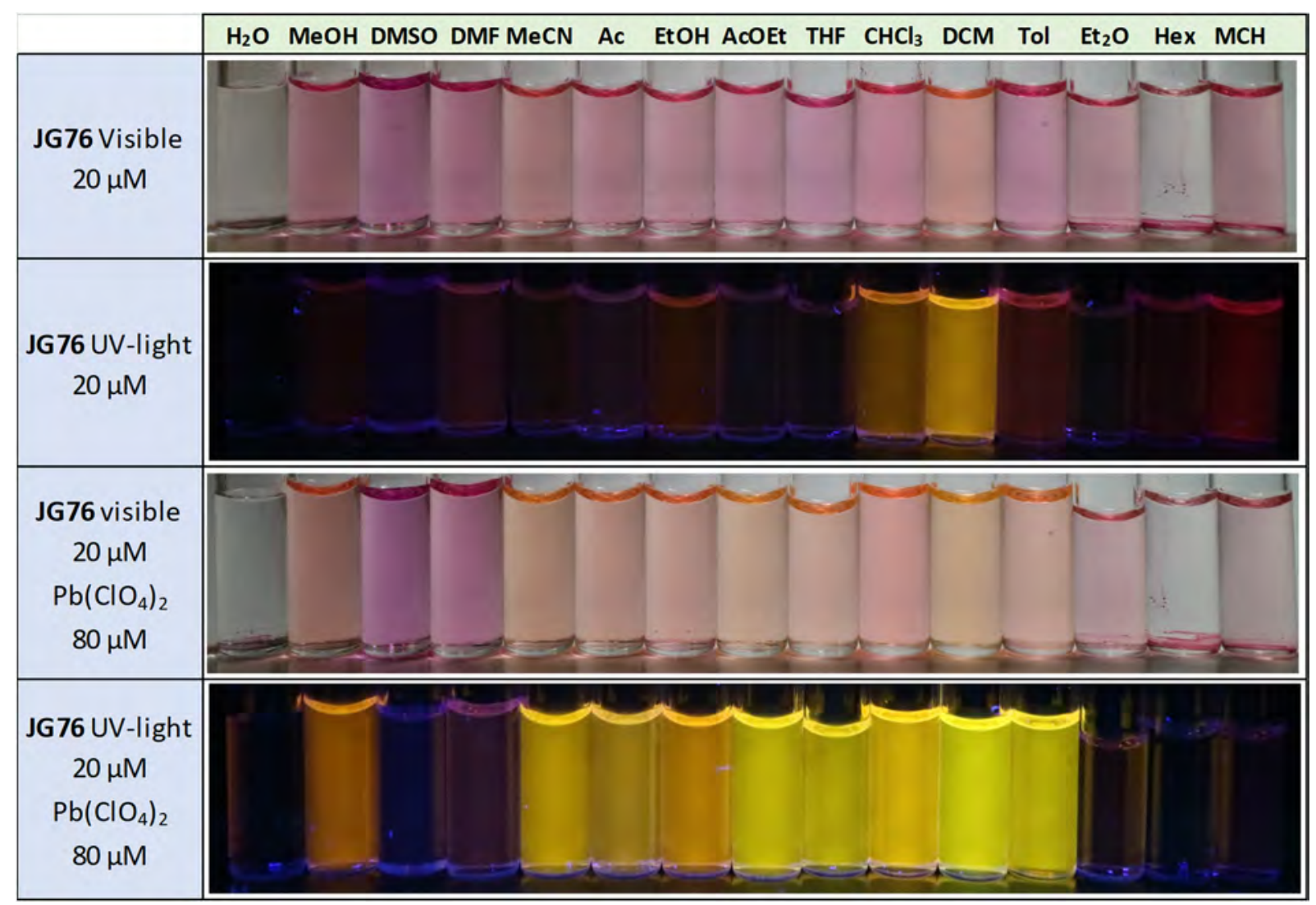

Figure 24. JG76 solutions ( $20 \mu \mathrm{M})$ in different solvents under visible light and under UV light; up, and response after the addition of $\mathrm{Pb}(\mathrm{II})$ dissolved in ethanol $(80 \mu \mathrm{M})$; down. 
Absorbance measurements: (Figure 25)
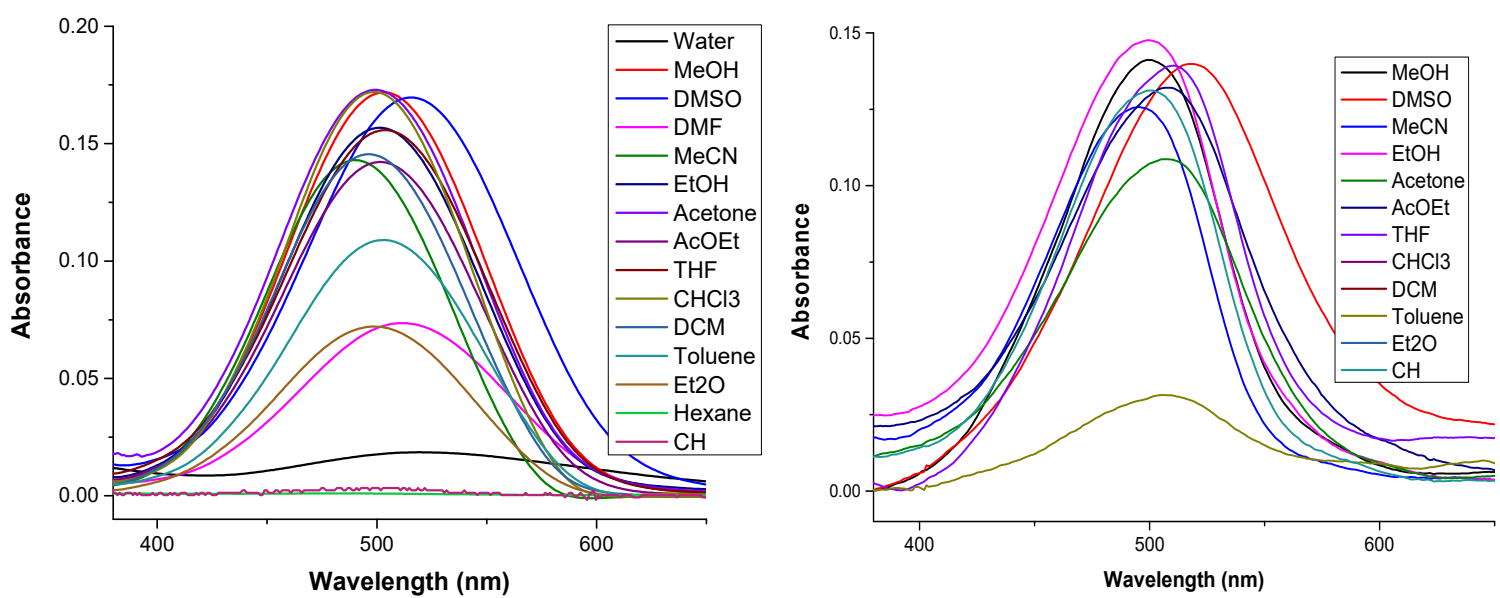

Figure 25. Absorption spectra in different solvents of JG76 solutions (5 $\mu \mathrm{M})$; left, and JG76 + $\mathbf{K}\left(\mathrm{CF}_{3} \mathrm{SO}_{3}\right)(50 \mu \mathrm{M})$; right.

The variation on the maximum of absorption, which is shown in Figure 25, was not significant, it was centred around $500 \mathrm{~nm}$. Additionally, there was no significant change after adding $\mathrm{K}\left(\mathrm{CF}_{3} \mathrm{SO}_{3}\right)$, since the increase in absorption for some solvents was due to an increase in solubility, when in presence of the cation.

Fluorescence results:
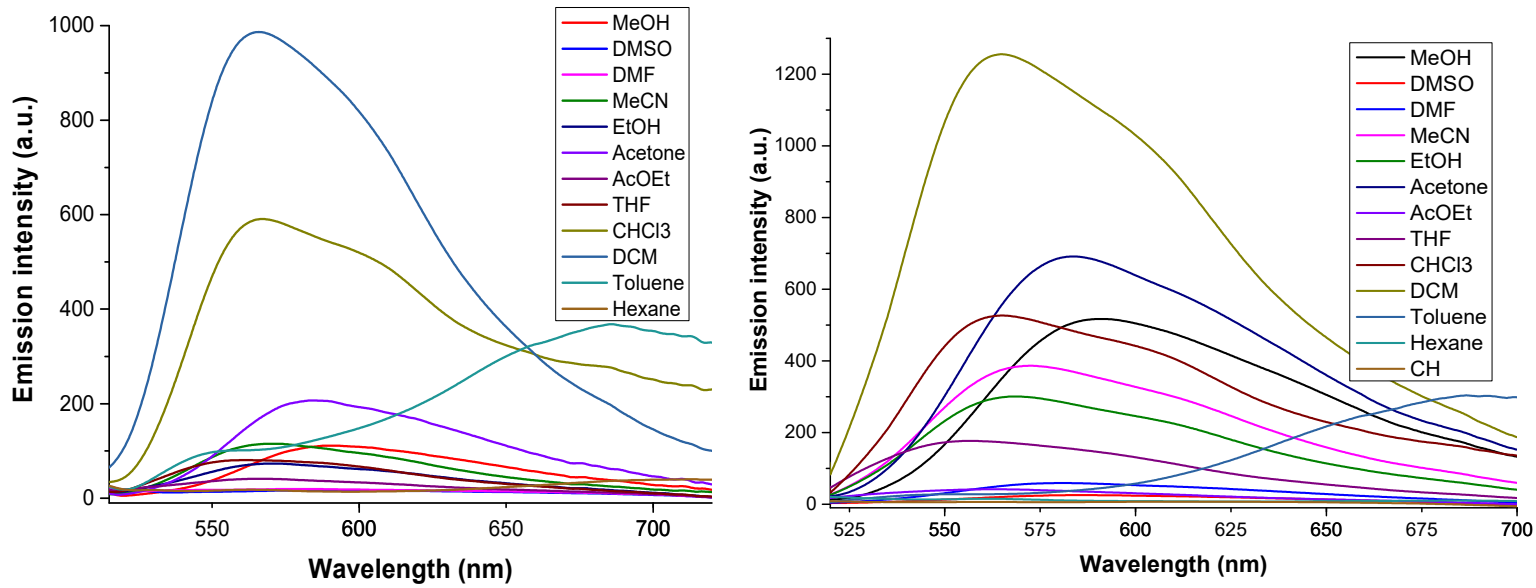

Figure 26. Fluorescence spectra in different solvents of JG76 solutions $(5 \mu \mathrm{M})$; left, and JG76 + $\mathbf{K}\left(\mathbf{C F}_{3} \mathbf{S O}_{3}\right)(50 \mu \mathrm{M})$; right. $\left(\lambda_{\mathrm{exc}}=507 \mathrm{~nm}\right)$

As it is shown in Figure 26, fluorescent emission intensity was high for dichloromethane and chloroform, low for toluene, ethanol and cyclohexane and very low for the rest of the solvents. They also presented wide bands of emission and an extra emission band in the red region $(>650 \mathrm{~nm})$, for aliphatic solvents. 
The emission intensity $\left(\lambda_{\mathrm{exc}}=500 \mathrm{~nm}\right)$ was studied at the maximum of emission, Figure 27.

\begin{tabular}{|c|c|c|c|}
\hline & $\mathbf{I} / \mathbf{I}_{0}$ & & $\mathbf{I} / \mathbf{I}_{\mathbf{0}}$ \\
\hline $\mathrm{MeOH}$ & 4.7 & $\mathrm{AcOEt}$ & 1.2 \\
\hline \begin{tabular}{|l|} 
DMSO \\
\end{tabular} & 2.3 & THF & 2.3 \\
\hline DMF & 8.1 & $\mathrm{CHCl}_{3}$ & 0.9 \\
\hline $\mathrm{MeCN}$ & 0.7 & DCM & 1.3 \\
\hline Acetone & 3.4 & Toluene & 0.3 \\
\hline EtOH & 4.5 & & \\
\hline
\end{tabular}

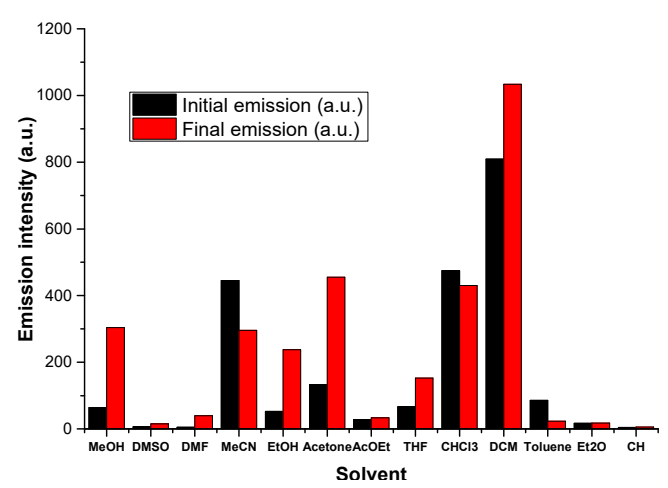

Figure 27. JG76 solutions (5 $\mu \mathrm{M})$ fluorescent increase after adding $+\mathbf{K}\left(\mathbf{C F}_{3} \mathbf{S O}_{3}\right)(50 \mu \mathrm{M})$, table (left). Graphic representation of the increase in fluorescent emission of the maximum (right).

The interpretation of the data from JG76 solvatochromism led to the following conclusions:

- The fluorescence decreased in toluene and acetonitrile.

- In DMSO, there was an increase in fluorescence, but the initial fluorescence was very low, almost zero, and the final fluorescence was also low. Therefore, in spite of the increase in fluorescence, it wasn't a good solvent.

- In DMF, the increase in emission seemed to be very high but it was because of the increase in solubility in presence of potassium cations; moreover, the fluorescence was too low.

- The fluorescence increase was very high for methanol, ethanol and acetone. These were the best options to make the study of the potential applications as $\mathrm{K}^{+}$or $\mathrm{Pb}^{2+}$ fluorogenic sensors.

\subsection{Solvatochromism of JG103:}

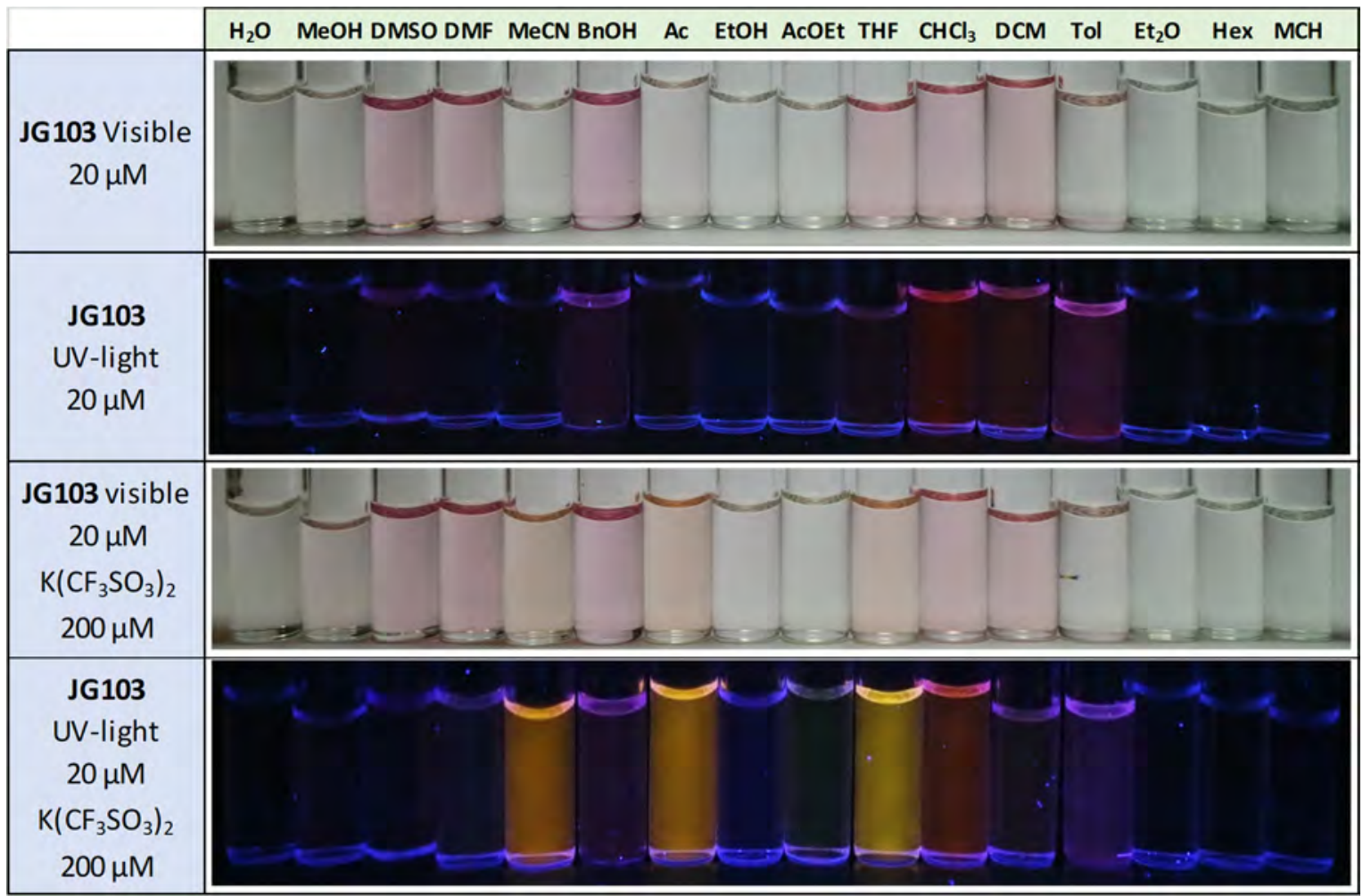

Figure 28. JG103 $(20 \mu \mathrm{M})+\mathrm{K}\left(\mathrm{CF}_{3} \mathrm{SO}_{3}\right)(200 \mu \mathrm{M})$ solutions in different solvents under visible light and under UV light, $366 \mathrm{~nm}$. 
In Figure 28 it can be seen an emission increased in $\mathrm{MeCN}$, acetone and THF. The main issue of the test was the solubility. The probe was highly soluble only in DMSO, DMF, $\mathrm{BnOH}, \mathrm{THF}, \mathrm{CHCl}_{3}$ and DCM. However, after the addition of $\mathrm{K}^{+}$, the solubility in acetonitrile and acetone increased, increasing also the fluorescence.

The absorption and fluorescent emission of the samples were measured, obtaining the results shown in Figures 29 and 30.
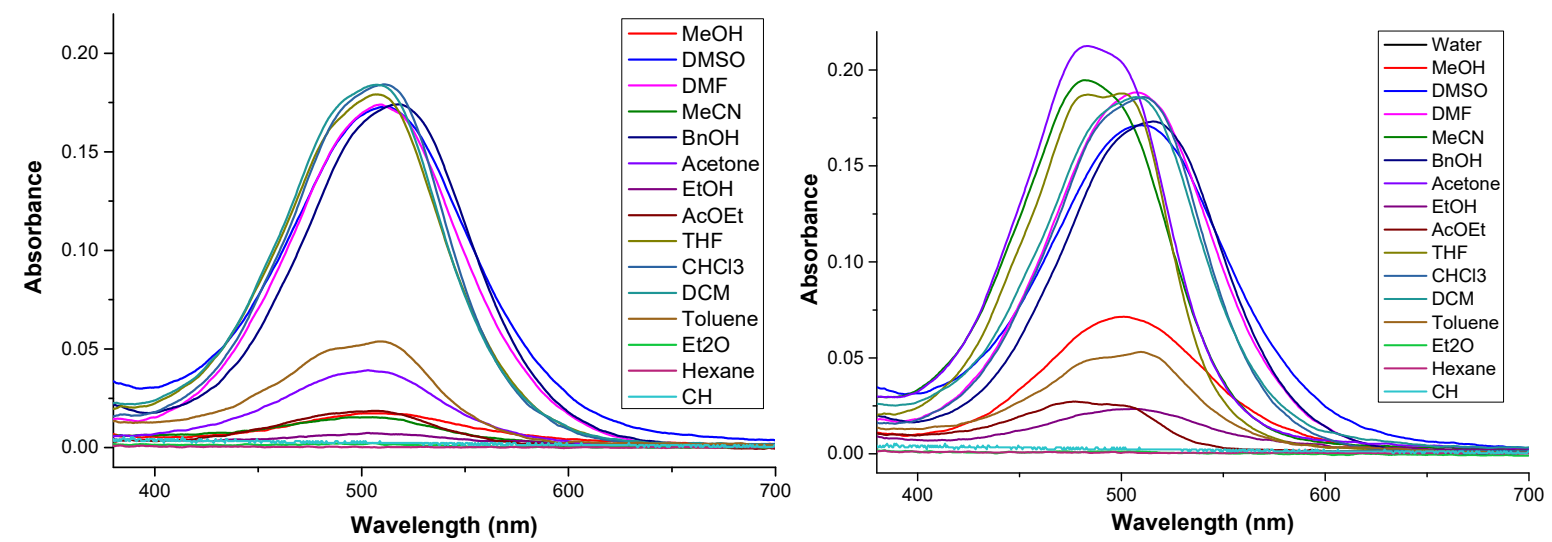

Figure 29. Absorption spectra in different solvents of JG103 $(20 \mu \mathrm{M})$; left, and JG103 $(20 \mu \mathrm{M})+$ $\mathrm{K}\left(\mathrm{CF}_{3} \mathrm{SO}_{3}\right)(200 \mu \mathrm{M})$; right.

From these spectra it can be stated:

- Before adding $\mathrm{K}\left(\mathrm{CF}_{3} \mathrm{SO}_{3}\right)$, the maximum of absorption was around $510 \mathrm{~nm}$, for all solvents in which it was soluble.

- After adding 10-fold excess $\mathrm{K}\left(\mathrm{CF}_{3} \mathrm{SO}_{3}\right)$, the maximum of absorption in acetone and acetonitrile was found around $485 \mathrm{~nm}$.
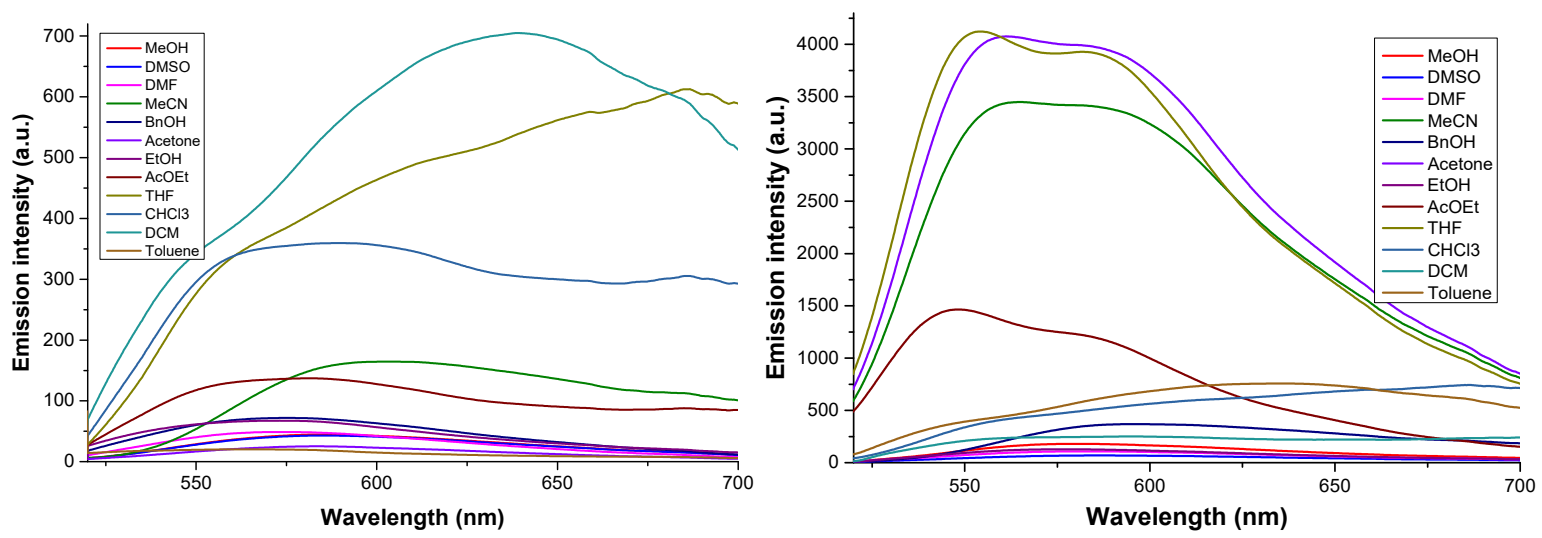

Figure 30. Fluorescence spectra in different solvents of JG103 $(20 \mu \mathrm{M})$; left, and JG103 $(20 \mu \mathrm{M})+$ $\mathrm{K}\left(\mathrm{CF}_{3} \mathrm{SO}_{3}\right)(200 \mu \mathrm{M})$; right. $\left(\lambda_{\mathrm{exc}}=500 \mathrm{~nm}\right)$ 
The emission intensity and the wavelength were studied $\left(\lambda_{\mathrm{exc}}=500 \mathrm{~nm}\right)$ at the maximum of emission, Figure 31.

\begin{tabular}{|c|c|c|c|}
\multicolumn{1}{c|}{} & I/I $_{\mathbf{0}}$ & & $\mathbf{I}_{\mathbf{1}}$ \\
\hline Water & ---- & AcOEt & 24.6 \\
\hline $\mathrm{MeOH}$ & 6.1 & $\mathrm{THF}$ & 33.1 \\
\hline $\mathrm{DMSO}$ & 1.5 & $\mathrm{CHCl}_{3}$ & 1.2 \\
\hline $\mathrm{DMF}$ & 2.5 & $\mathrm{DCM}$ & 0.7 \\
\hline $\mathrm{MeCN}$ & 76.3 & Toluene & 1.1 \\
\hline $\mathrm{BnOH}$ & 2.3 & $\mathrm{Et}_{2} \mathrm{O}$ & 1.9 \\
\hline Acetone & 38.4 & Hexane & 1 \\
\hline EtOH & 5.2 & Cyclohexane & 1.1 \\
\hline
\end{tabular}

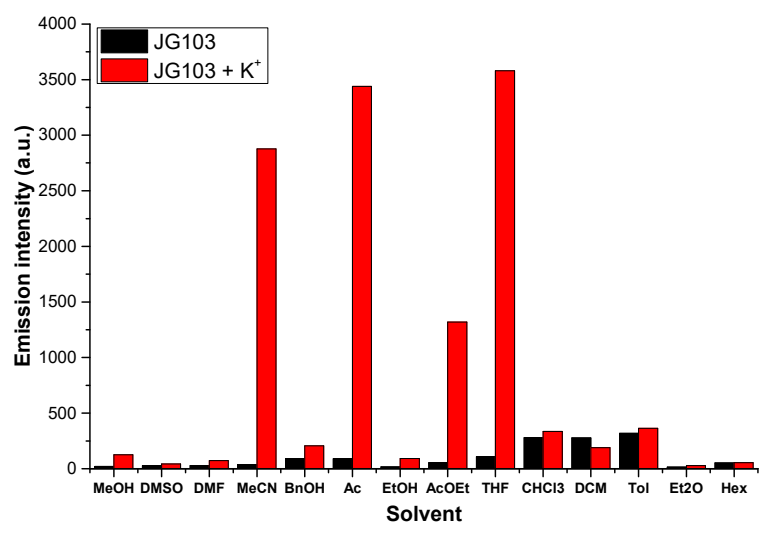

Figure 31. JG103 solutions $20 \mu \mathrm{M}$, emission increase of the different solvents, table (left) and graph (right) $\left(\lambda_{\text {exc }}=500 \mathrm{~nm}\right)$

The interpretation of the data from JG103 solvatochromism led to the next conclusions:

- The maximum of emission was found between 550-650 $\mathrm{nm}$ after adding $\mathrm{K}^{+}$; being broad bands before the analyte addition.

- The increase in emission was very high in $\mathrm{MeCN}$, acetone, EtOAc and THF but it was not soluble before adding $\mathrm{K}^{+}$.

- In DMSO and DMF the emission was too low.

- In water, $\mathrm{MeOH}, \mathrm{EtOH}, \mathrm{Et}_{2} \mathrm{O}$, hexane and cyclohexane the probe was insoluble.

Although there were several possibilities with high response to potassium cations, quantitatively the only option was measuring in $\mathrm{BnOH}$ with and without potassium cations; because of the solubility issues. Qualitatively, it may be studied also in acetonitrile and acetone, having the best results in final fluorescence.

\subsection{Concluding remarks}

The probes JG76 and JG103 were not soluble in $100 \%$ water media. On one hand, JG76 had a clear and remarkable response dissolved in acetone and alcoholic solvents such as ethanol or methanol, that are miscible with water. Because of that, it was deeply studied qualitatively and quantitatively.

On the other hand, JG103 was highly insoluble in most solvents and increased its solubility when in presence of potassium cations. The response was studied in acetonitrile, and in benzylic alcohol, however, it was not highly soluble in the first one and with low fluorescence in the second. Therefore, the studies with this probe were more limited and are included in the Experimental Appendix 5. 


\section{EFFECT OF IONS, pH AND WATER PERCENTAGE ON CROWN ETHER PROBE (JG76)}

\subsection{General conditions}

In the pictures, when adding some analyte, it appeared to be a change of colour. Actually, it was an increase in fluorescence. The maximum of absorption was around $500-507 \mathrm{~nm}$ and constant, so what was seen are the changes of the fluorescence in the visible region, in which it was not necessary a UV lamp; but it allowed to see the effect more clearly.

- The ions were dissolved in water, $5 \mathrm{mM}$ and added to the probe solution.

- Water mixtures had the same concentration than pure organic solvents

\subsection{Tests with JG76}

The counterions were non-coordinative species, specified in the Annex.

\subsubsection{Cations test 1:}

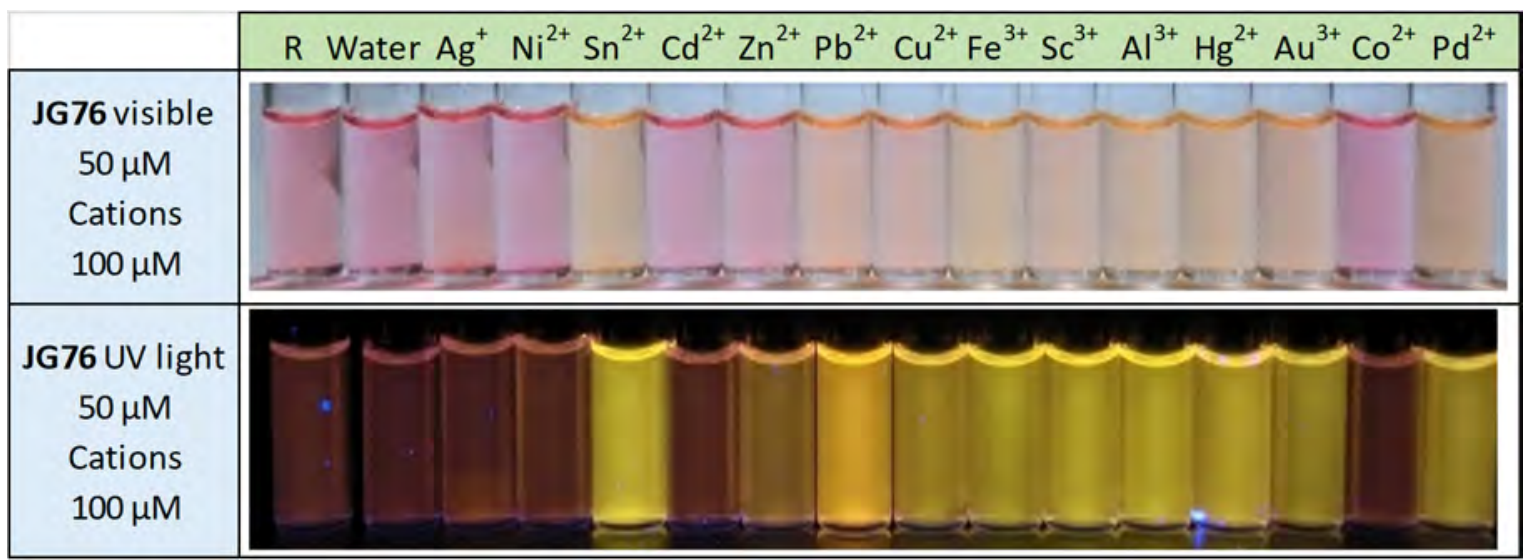

Figure 32. JG76 in ethanol $(50 \mu \mathrm{M})$, in presence of different cations $(100 \mu \mathrm{M})$ under visible light and under $366 \mathrm{~nm}$ light.

From the pictures of the solvatochromism in Figure 32, it was deduced that:

- The cations $\mathrm{Ag}^{+} \mathrm{Ni}^{2+} \mathrm{Cd}^{2+} \mathrm{Co}^{2+}$ induced no change in fluorescence.

- The cations $\mathrm{Sn}^{2+} \mathrm{Zn}^{2+} \mathrm{Cu}^{2+} \mathrm{Fe}^{3+} \mathrm{Sc}^{3+} \mathrm{Al}^{3+} \mathrm{Hg}^{2+} \mathrm{Au}^{3+} \mathrm{Pd}^{2+}$ induced an increase in the yellow fluorescence.

- The cation $\mathrm{Pb}^{2+}$ induced an increase in fluorescence in orange.

\subsubsection{Cations test 2:}

The following test (Figure 33) was done due to the interest of studying the fluorescence in presence of alkaline and alkaline earth cations. 


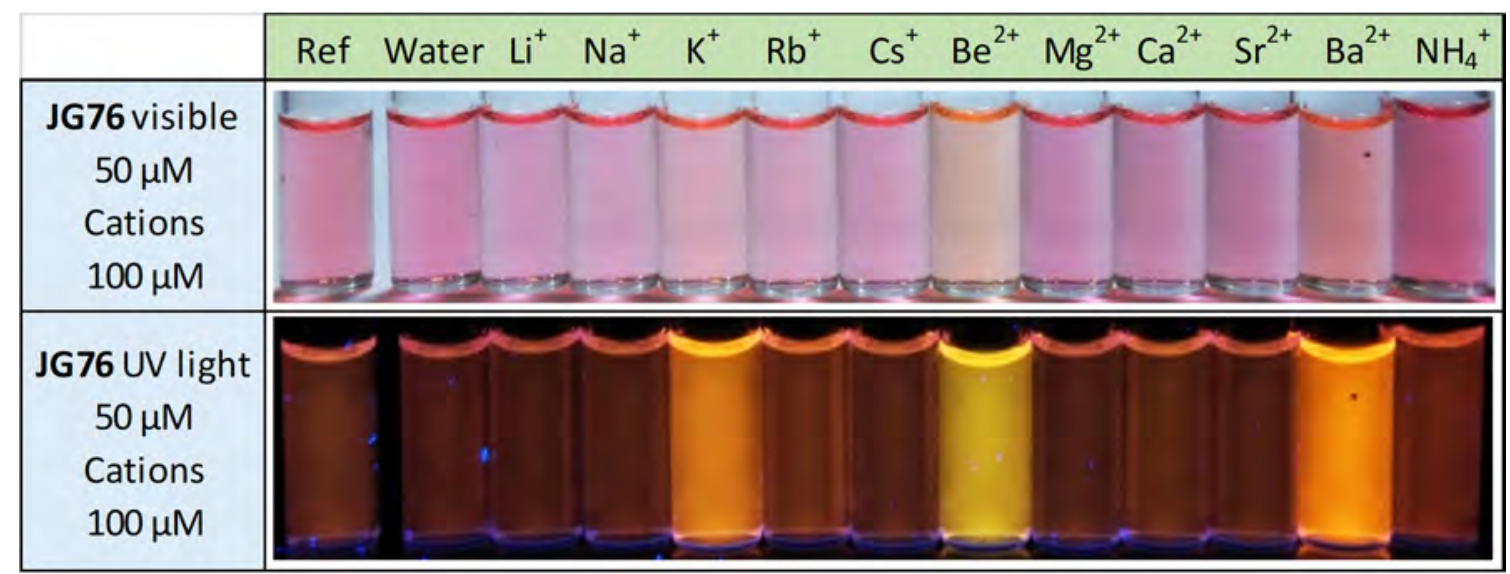

Figure 33. JG76 in ethanol $(50 \mu \mathrm{M})$, in the presence of different cations $(100 \mu \mathrm{M})$ under visible light and under UV light.

From Figure 33, it may be observed that the cations $\mathrm{K}^{+}, \mathrm{Be}^{2+}$ and $\mathrm{Ba}^{2+}$ induced an increase in fluorescence in orange.

\subsubsection{Anions test:}

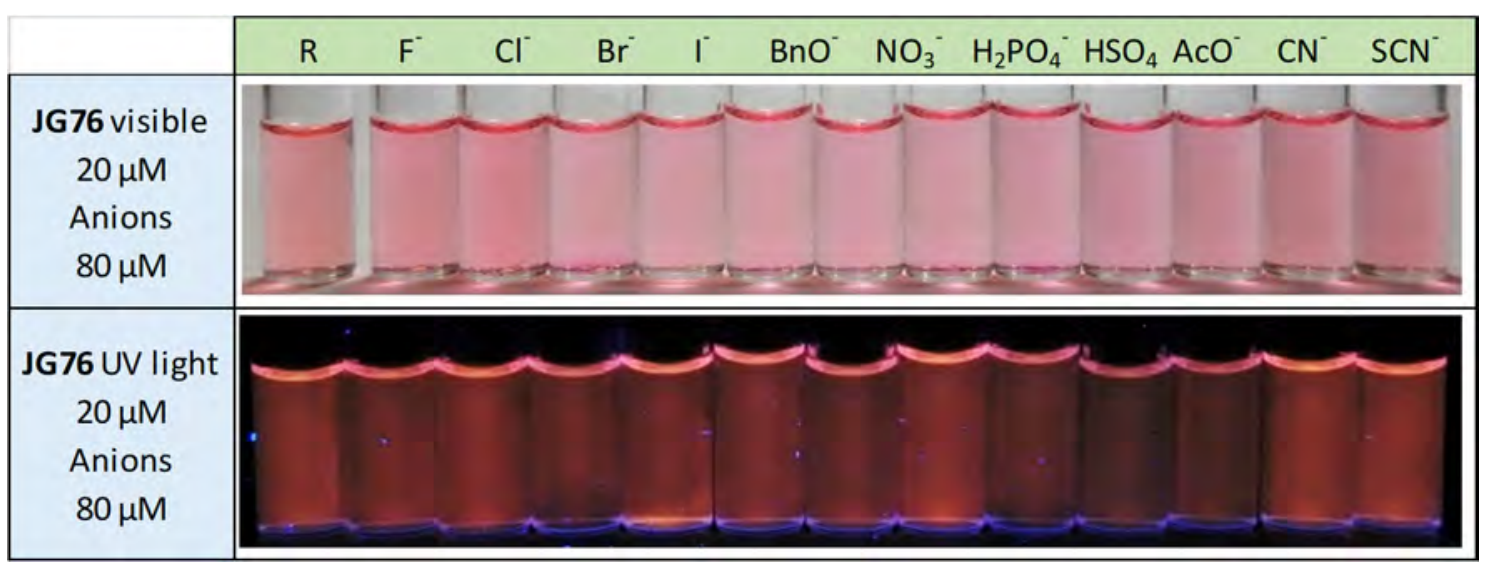

Figure 34. JG76 in ethanol $(50 \mu \mathrm{M})$, in the presence of different anions $(100 \mu \mathrm{M})$ under visible light and under UV light.

As it is shown in Figure 34, the anions did not produce significant changes in the JG76 solutions.

\subsubsection{Quantitative fluorescent response}

Some of the species with special behaviour were selected, and the absorbance and fluorescence were measured (Figure 35). The variation in absorbance was not significant, changes were most likely due to the dilution of the probe. 

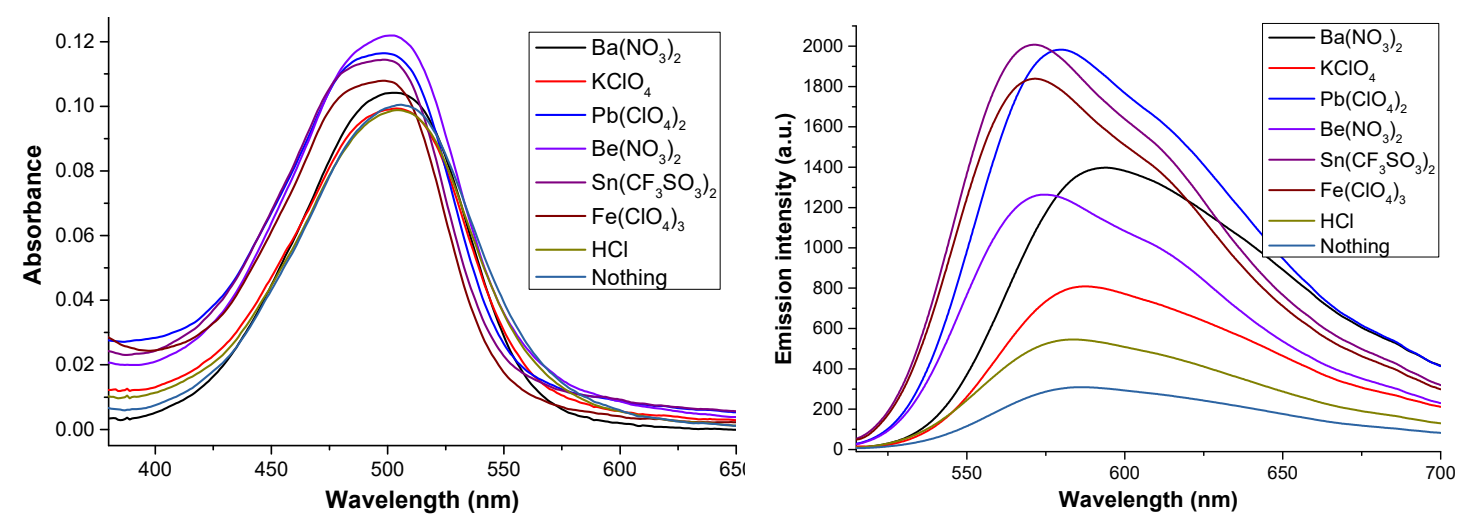

Figure 35. JG76 in ethanol $(50 \mu \mathrm{M})$, in presence of different cations $(100 \mu \mathrm{M})$ absorbance and fluorescence. $\left(\lambda_{\mathrm{exc}}=500 \mathrm{~nm}\right)$

In the table below, (Figure 36) the maximum of emission of cations and the wavelength shifts are shown, being a high increase in presence on most cations, but with different wavelength of emission.

\begin{tabular}{|c|c|c|c|c|c|}
\cline { 2 - 5 } \multicolumn{1}{c|}{} & $\begin{array}{c}\text { Max. Em. } \\
\text { Wavelength } \\
\text { (nm) }\end{array}$ & $\mathbf{I} / \mathbf{I}_{\mathbf{0}}$ & & $\begin{array}{c}\text { Max. Em. } \\
\text { Wavelength } \\
\text { (nm) }\end{array}$ & $\mathbf{I} / \mathbf{I}_{\mathbf{0}}$ \\
\hline $\mathrm{HCl}$ & 582 & 1.9 & $\mathrm{KCF}_{3} \mathrm{SO}_{3}$ & 587 & 6 \\
\hline $\mathrm{KClO}_{4}$ & 587 & 2.6 & $\mathrm{~Pb}\left(\mathrm{ClO}_{4}\right)_{2}$ & 580 & 7.1 \\
\hline $\mathrm{Ba}\left(\mathrm{NO}_{3}\right)_{2}$ & 592 & 4.1 & $\mathrm{Fe}\left(\mathrm{ClO}_{4}\right)_{3}$ & 572 & 7.9 \\
\hline $\mathrm{Be}\left(\mathrm{NO}_{3}\right)_{2}$ & 575 & 4.9 & $\mathrm{Sn}\left(\mathrm{CF}_{3} \mathrm{SO}_{3}\right)_{2}$ & 572 & 8.1 \\
\hline
\end{tabular}
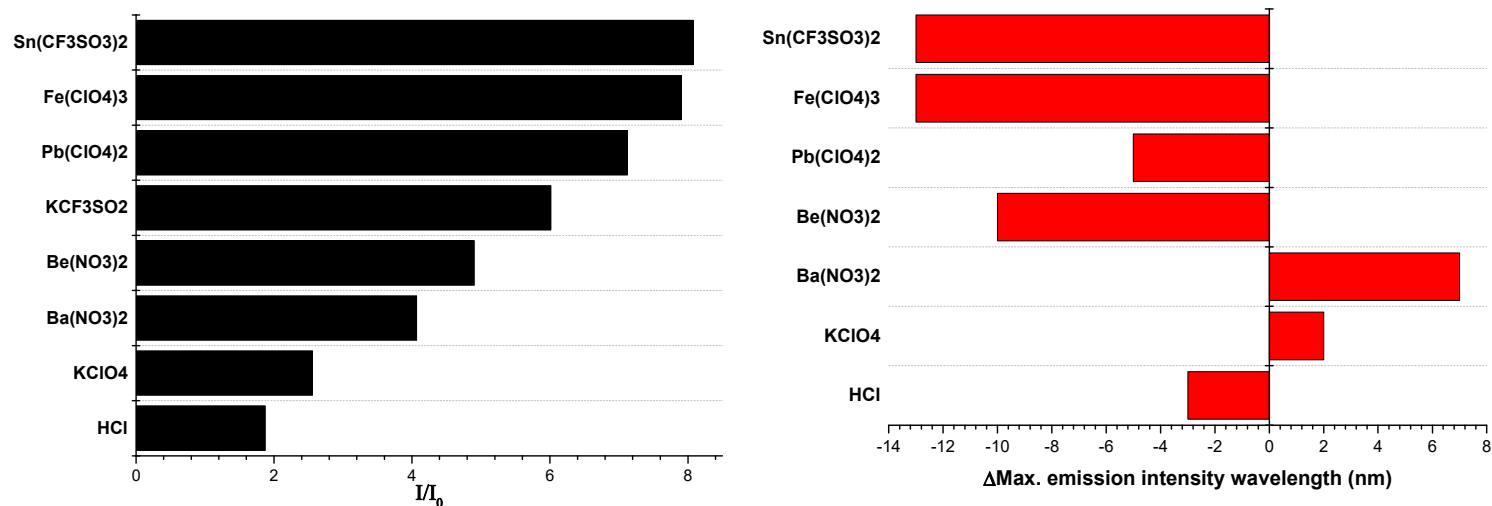

Figure 36. JG76 in ethanol $(50 \mu \mathrm{M})$, in the presence of different cations $(100 \mu \mathrm{M})$, increase in maximum emission intensity, left; and a change in its wavelength of emission, right. $\left(\lambda_{\mathrm{exc}}=500 \mathrm{~nm}\right)$

\subsection{5. $\mathrm{pH}$ response of JG76:}

For JG76 $(20 \mu \mathrm{M})$ the maximum proportion water/ethanol to prevent precipitation was $30 \%$ of water. The compound JG76 was dissolved in EtOH, $25 \mu \mathrm{M}$ and the solvent was $70 \% \mathrm{EtOH}, 30 \%$ buffer solution in water, $20 \mathrm{mM}$ of HEPES, Figure 37. (The region of HEPES buffer for $\mathrm{pH}$ regulation is 6.8 to 8.2 ). 


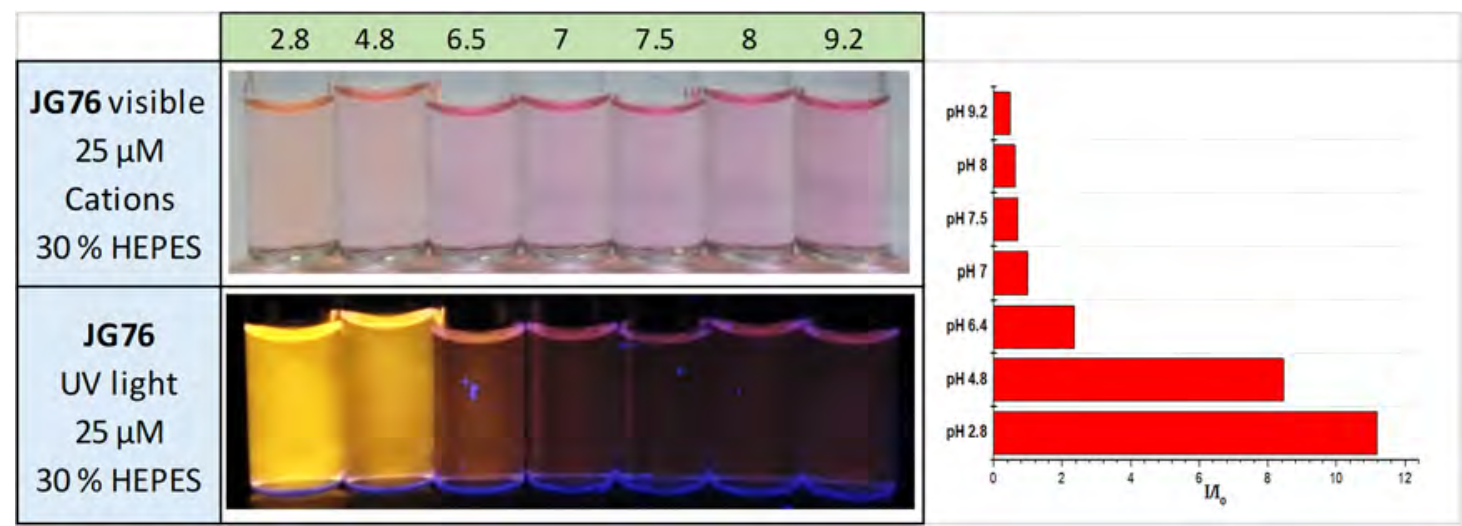

Figure 37. JG76 in ethanol, in a mixture with a buffer solution of different $\mathrm{pH}(20 \mu \mathrm{M})$, buffer 20 $\mathrm{mM}$ HEPES. Under visible and $366 \mathrm{~nm}$ UV light. $\left(\lambda_{\mathrm{exc}}=500 \mathrm{~nm}\right)$.

In conclusion, the probe was very sensitive to the $\mathbf{p H}$ of the solution, especially when far from buffer regulation. A buffer solution, with $\mathbf{p H}$ higher than 7 , was enough to avoid the $\mathbf{p H}$ effect in the fluorescence.

\subsubsection{JG76 with ions at controlled pH}

Due to the previous results, it was necessary to check the behaviour of the probe in buffer solution and in the presence of cations.

- The compound JG76 was first dissolved in EtOH, from which the final solution was [JG76] = $20 \mu \mathrm{M}$ in $70 \% \mathrm{EtOH}-30 \% \mathrm{H}_{2} \mathrm{O}(\mathrm{v} / \mathrm{v})$ buffer solution, $20 \mathrm{mM}$ of HEPES.

- $\quad$ The buffer $\mathrm{pH}$ was 7.

A picture was taken by adding 5 equivalents of different species $(100 \mu \mathrm{M})$, Figure 38. Additionally, the increase in emission was also registered (Figure 39).

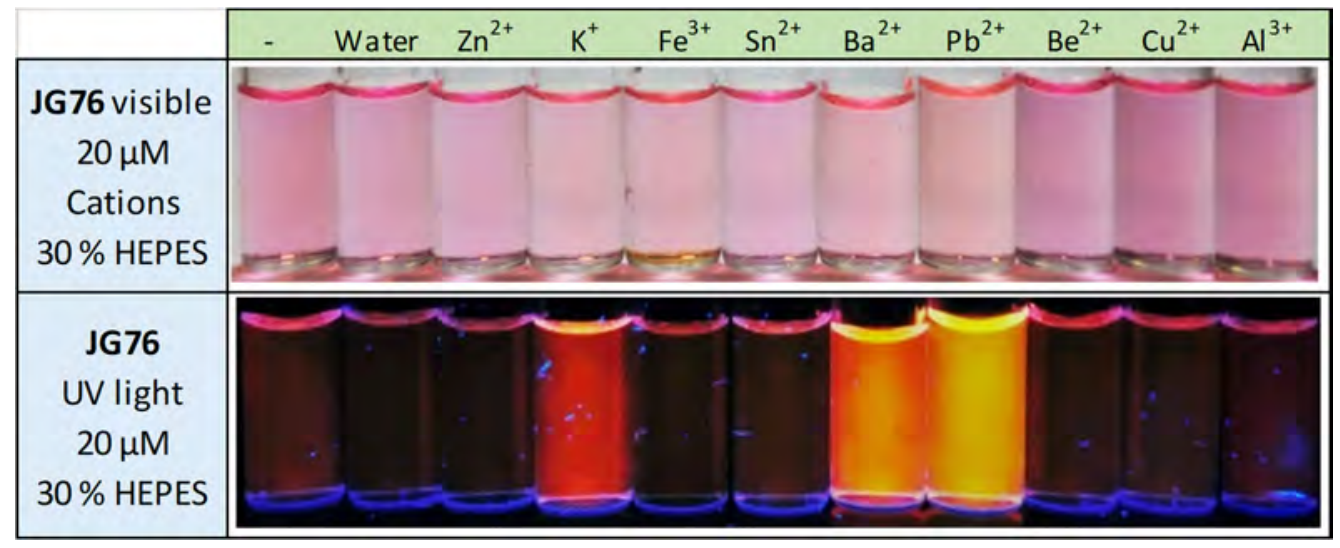

Figure 38. JG76 in ethanol-water mixture (30\% water, $20 \mu \mathrm{M})$, buffer solution of $\mathrm{pH}$ 7, $20 \mathrm{mM}$ of HEPES. Addition of different cations $(0.1 \mathrm{mM})$, under visible and $366 \mathrm{~nm}$ UV light. 

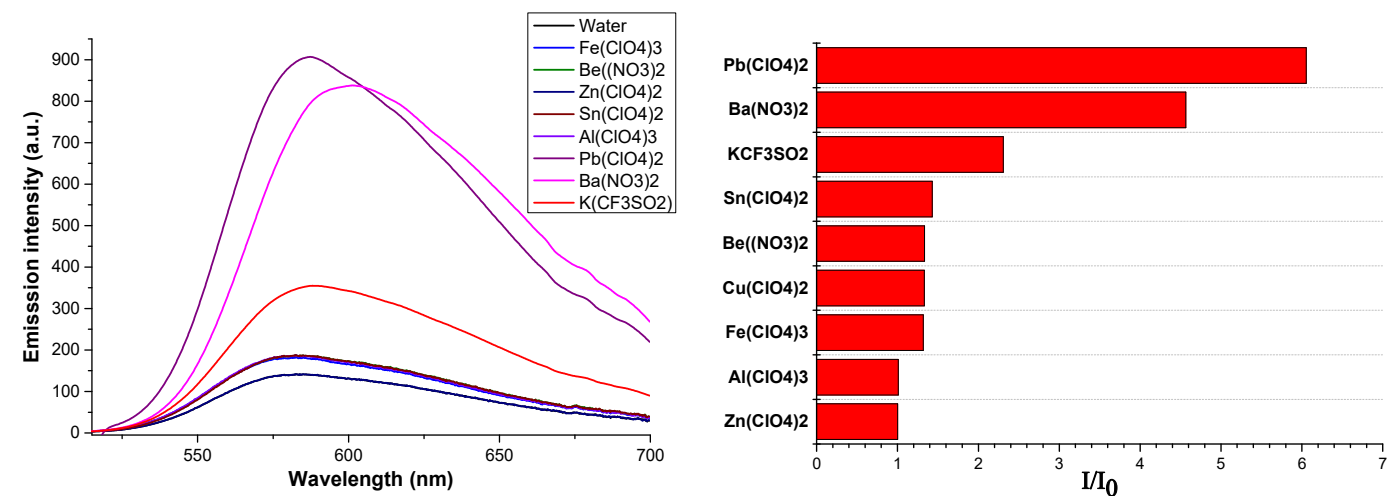

Figure 39. JG76 in ethanol-water mixture (30\% water, $20 \mu \mathrm{M})$, buffer solution of $\mathrm{pH}$ 7, $20 \mathrm{mM}$ of HEPES. Addition of different cations, $0.1 \mathrm{mM}$. Fluorescence spectra (left) and increase in total emission (right). $\lambda_{\mathrm{exc}}=500 \mathrm{~nm} \cdot \lambda_{\mathrm{em}}=571 \mathrm{~nm}$.

Fluorescence of JG76 in $70 \% \mathrm{EtOH}-30 \% \mathrm{H}_{2} \mathrm{O}(\mathrm{v} / \mathrm{v})$ buffer solution increased selectively for $\mathrm{K}^{+}$, $\mathrm{Ba}^{2+}$ and $\mathrm{Pb}^{2+}$ :

- Around $30 \%$ in the presence of Lewis acid cations.

- Around $130 \%$ in the presence of $\mathrm{K}^{+}$.

- Around $350 \%$ in the presence of $\mathrm{Ba}^{2+}$.

- Around $500 \%$ in the presence of $\mathrm{Pb}^{2+}$.

\subsection{Analyte detection comparison, JG76 against JG103}

JG76 working conditions were optimized in ethanol, qualitatively and quantitatively. Moreover, to improve selectivity, $30 \%$ of HEPES buffer in water at $\mathrm{pH} 7$ lead to no response when in presence of acidic cations. The solution is selective to $\mathrm{K}^{+}, \mathrm{Pb}^{2+}$ and $\mathrm{Ba}^{2+}$.

JG103 was not good for quantitative measurements. It possessed low solubility in water miscible solvents and the selectivity was not better than for JG76, (See Experimental Appendix 5)

In consequence, quantitative measurements, such as thermodynamic equilibrium constants, stoichiometry calculation or limits of detections were only performed for probe JG76. 


\section{DETECTION TESTS FOR $\mathrm{K}^{+}, \mathrm{Pb}^{2+}$ AND CEREULIDE IN SOLUTION}

First, some solutions of the probe were prepared in ethanol and the absorbance (Figure 40) and fluorescence (Figure 41) were checked at high dilution.

Absorbance at $500 \mathrm{~nm}$ :

\begin{tabular}{|c|c|c|c|}
\hline $\begin{array}{c}{[\mathbf{J G 7 6}]} \\
(\boldsymbol{\mu M})\end{array}$ & Absorbance & $\begin{array}{c}{[\mathbf{J G 7 6}]} \\
(\boldsymbol{\mu M})\end{array}$ & Absorbance \\
\hline 6.5 & 0.182 & 2 & 0.046 \\
\hline 4.8 & 0.126 & 1.6 & 0.044 \\
\hline 3.6 & 0.09 & 1.2 & 0.026 \\
\hline 2.8 & 0.06 & 0.8 & 0.006 \\
\hline
\end{tabular}

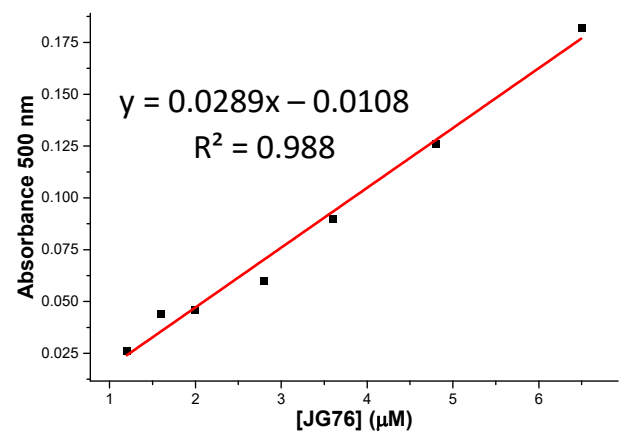

Figure 40. Absorbance at different concentrations of JG76.

Fluorescence $\left(\lambda_{\mathrm{exc}}=500 \mathrm{~nm}, \lambda_{\mathrm{em}}=573 \mathrm{~nm}\right)$ :

\begin{tabular}{|c|c|}
\hline $\begin{array}{c}\text { [JG76] } \\
(\boldsymbol{\mu M})\end{array}$ & $\begin{array}{c}\text { Em. Int. 573 } \\
\text { nm (a.u.) }\end{array}$ \\
\hline 6.5 & 140.7 \\
\hline 4.8 & 98.5 \\
\hline 3.6 & 66.3 \\
\hline 2.8 & 53.0 \\
\hline 2 & 34.8 \\
\hline 1.6 & 28.2 \\
\hline 1.2 & 34.5 \\
\hline 0.8 & 33.1 \\
\hline
\end{tabular}

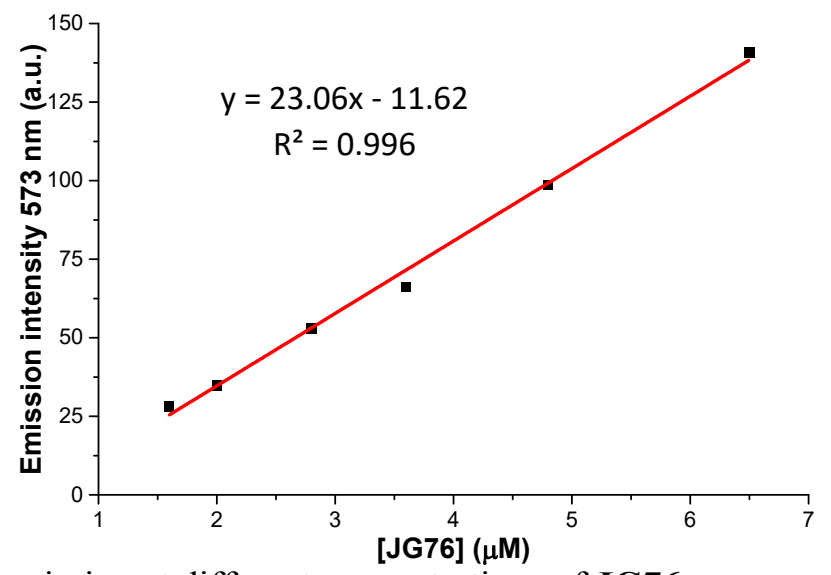

Figure 41. Fluorescent emission at different concentrations of JG76.

In conclusion, the absorbance and fluorescence change linearly between 1.6 to $6.5 \mu \mathrm{M}$.

The chosen concentration for the tests was between $2 \boldsymbol{\mu M}$ to $6 \boldsymbol{\mu M}$.

\subsection{JG76 Job's Plot, stoichiometric determination of the complex}

A group of solutions was measured with a molar fraction between $0-1$ of cations/JG76.

- The fluorescence was measured with $\lambda_{\mathrm{exc}}=500 \mathrm{~nm}$ and $\lambda_{\mathrm{em}}=571 \mathrm{~nm}$.

- The molar fraction of cations $(\mathrm{Xc})$ was represented versus the peak of emission $(\mathrm{F})$ minus the emission when $X_{\text {Analyte }}=0,\left(F_{0}\right)$ multiplied per the molar fraction $(X c)$. $X_{c}$ vs $X_{c}\left(F_{0}-F\right)$.

The Job's Plot analysis was performed to estimate the stoichiometry of the complex; taking into account the limited range of application of the technique explained in Chapter 0. The most likely

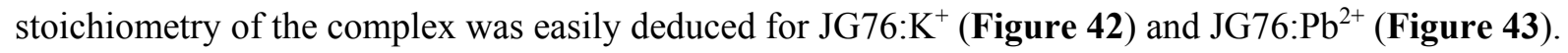



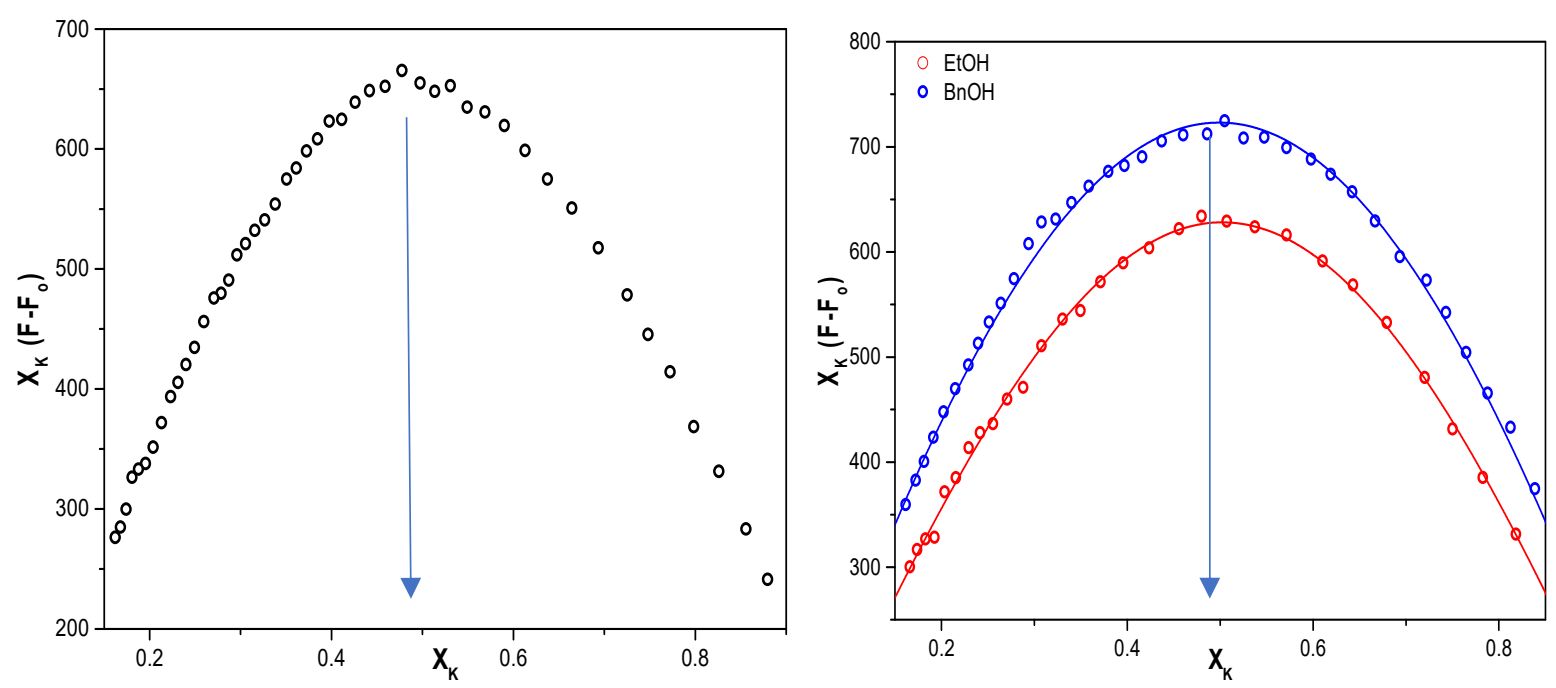

Figure 42. Job's Plot of JG76: $\mathrm{K}^{+}$complex, fluorescence analysis in EtOH (left) and $\mathrm{EtOH}$ and $\mathrm{BnOH}$ comparison (right)

The plot was represented several times as it is shown in Figure 42, obtaining always the maximum centred in 0.5 , which meant that the complex JG76: $\mathrm{K}^{+}$was $1: 1$.

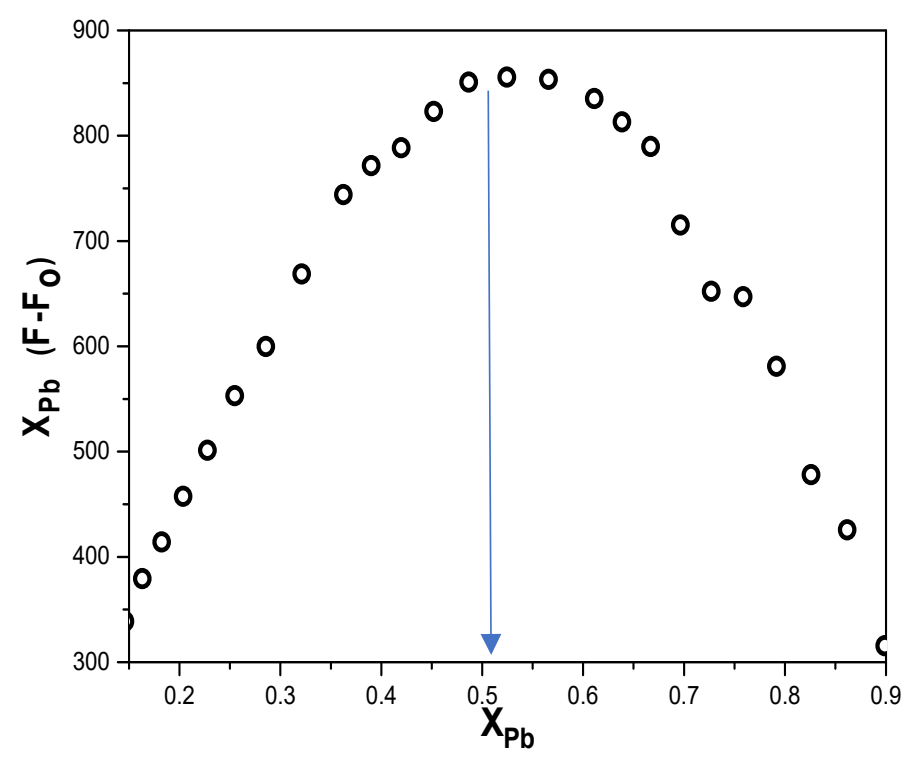

Figure 43. Job's Plot of $\mathbf{J G 7 6} / \mathrm{Pb}^{2+}$ complex, fluorescence analysis in $\mathrm{EtOH}$.

In the case of the complex JG76: $\mathrm{Pb}^{2+}$, which was represented in Figure43, the stoichiometry was also 1:1.

\subsection{Fluorescence Quantum Yields $\left(\Phi_{\mathrm{F}}\right)$}

Fluorescent quantum yields were determined by using an integration sphere (see example in Figure 44), this is an absolute method, as it was explained in Chapter 0. 


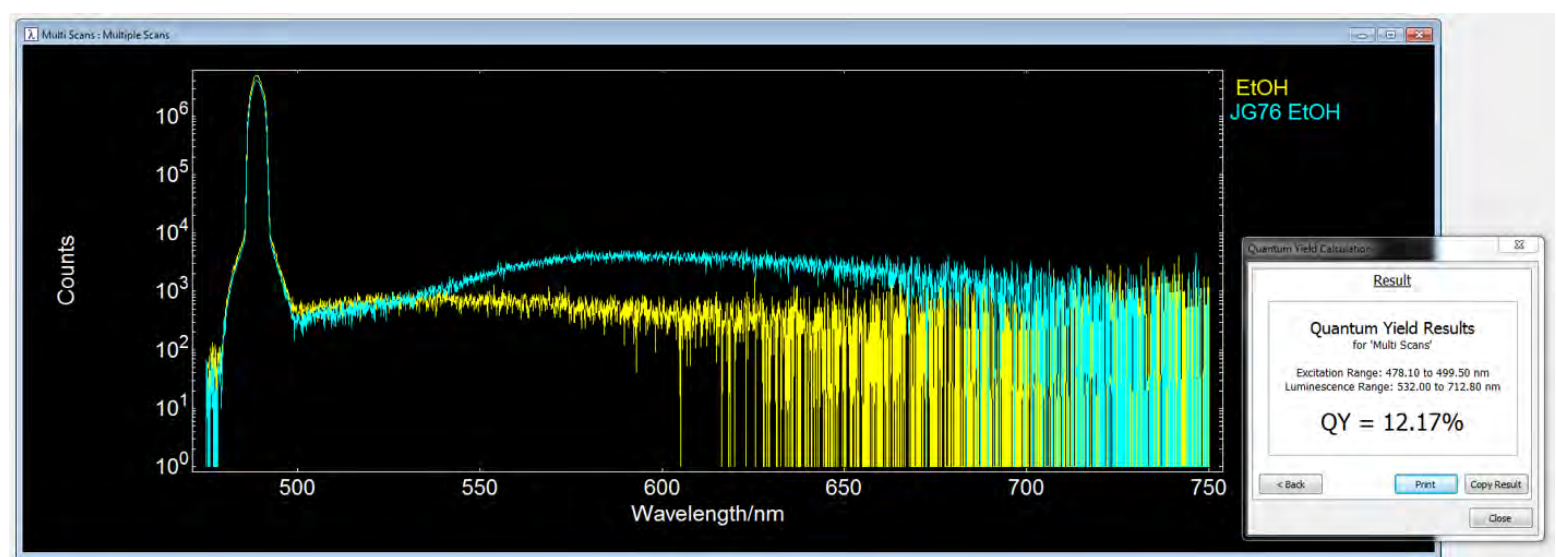

Figure 44. Example of the measurement of JG76 in EtOH solution with the software from FLS980 Edinburgh instrument to measure a quantum yield.

Keeping in mind that the error associated to the method is at least $1 \%$ the samples were repeated several times obtaining variations in the results around this number as it is shown in Figure 45.

\begin{tabular}{|c|c|c|c|c|c|}
\hline \multicolumn{5}{|c|}{ EtOH } \\
\hline $\boldsymbol{\Phi}_{\mathbf{F ~ J G 7 6 ~}}$ & $\mathbf{K}^{+}$ & $\mathbf{P b}^{2+}$ & error & $\boldsymbol{\Phi}_{\mathbf{F}, \mathbf{K}+} / \boldsymbol{\Phi}_{\mathbf{F}, \mathbf{0}}$ & $\boldsymbol{\Phi}_{\mathbf{F}, \mathbf{P b} 2+} / \boldsymbol{\Phi}_{\mathbf{F}, \mathbf{0}}$ \\
\hline 0.12 & 0.48 & 0.84 & 0.02 & 4 & 7 \\
\hline
\end{tabular}

\begin{tabular}{|c|c|c|c|c|}
\hline \multicolumn{5}{|c|}{$\mathrm{BnOH}$} \\
\hline$\Phi_{\mathrm{F} \text { JG103 }}$ & $\mathbf{K}^{+}$ & Oxone* & error & $\boldsymbol{\Phi}_{\mathbf{F}, \mathbf{K}+} / \boldsymbol{\Phi}_{\mathbf{F}, \mathbf{0}}$ \\
\hline \multirow[t]{4}{*}{0.01} & 0.05 & 0.13 & 0.01 & 5.2 \\
\hline & \multicolumn{3}{|c|}{ MeCN } & \\
\hline & $\Phi_{\mathrm{F} \text { JG103 }}$ & $\mathbf{K}^{+}$ & error & \\
\hline & --- & 0.14 & 0.01 & \\
\hline
\end{tabular}

* Oxone (Potassium peroxymonosulfate) behaves as acid and contains $\mathrm{K}^{+}$at the same time.

Figure 45. Table of quantum yields of JG76 and JG103 in different solvents and in presence of different species.

\subsection{Fluorescence decay lifetimes $(\tau)$}

Fluorescence decay lifetimes were measured using a time-correlated single photon counting instrument (FLS980 Series, Edinburgh instruments) with a $510 \mathrm{~nm}$ pulsed LED (Edinburgh instruments, EPL-510) light source having a 177.4 ps. Decays were recorded at $510 \mathrm{~nm}$ for each probe.

The probes JG76 and JG103 were tested in the studied solvents and in presence of lead and potassium cations. Calculating the lifetime decays from the graphs in Figure 46 and represented in Figure 47. 

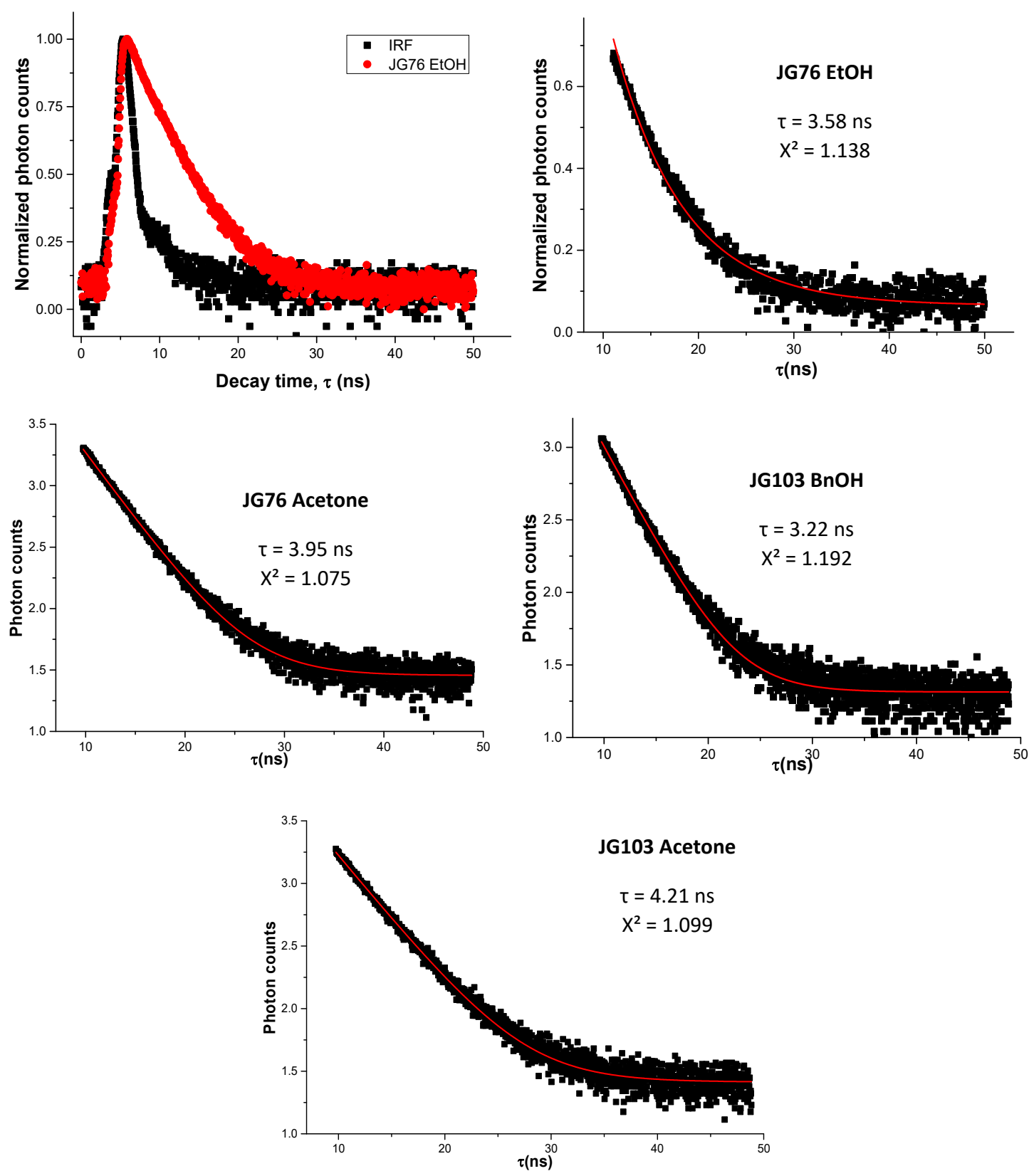

Figure 46. Lifetime decay fitting of JG76 dissolved in EtOH, IRF and fitting (up); JG76 in Acetone (middle left), JG103 BnOH (middle right) and JG103 acetone (down).

\begin{tabular}{|c|c|c|c|}
\hline Probe & Solvent & $\boldsymbol{\tau}(\mathbf{n s})$ & $\boldsymbol{\chi}^{\mathbf{2}}$ \\
\hline JG76 & EtOH & 3.58 & 1.138 \\
\hline JG103 & BnOH & 3.22 & 1.192 \\
\hline JG76 & Acetone & 3.95 & 1.075 \\
\hline JG103 & Acetone & 4.22 & 1.099 \\
\hline
\end{tabular}

Figure 47. Lifetime decay, JG76 and JG103 in different solvents.

- Both JG76 and JG103 had lifetime decays between 3-4.5 ns.

- In the same solvents, the fluorescence decay lifetime was very similar comparing JG103 and JG76, due to the perylene structure, responsible of the fluorescence.

- Measuring with and without potassium/lead cations no change in $\tau$ was observed. 


\subsection{Thermodynamic equilibrium constants}

\subsubsection{Measuring parameters and method:}

Equilibrium constants were measured for the complexes with JG76. By preparing a solution 2-5 $\mu \mathrm{M}$ of JG76 and increasing the concentration of $\mathrm{K}\left(\mathrm{CF}_{3} \mathrm{SO}_{3}\right)$ or $\mathrm{Pb}\left(\mathrm{ClO}_{4}\right)$, without changing the concentration of JG76 in solution.

\subsubsection{Equilibrium constant of $\mathrm{K}^{+}$and $\mathrm{Pb}^{2+}$ with JG76:}

JG76- $\mathrm{K}^{+}$complex was 1:1, in agreement with literature and the Job's Plot results. Additionally, the experimental results for lead cations were similar. In consequence, the equation to calculate thermodynamic equilibrium constants was the one explained in Chapter $\mathbf{0 .}$

$$
I_{F}=f_{P} C_{P}+\frac{f_{P A}-f_{P}}{2}\left[C_{P}+C_{A}+\frac{1}{K_{1}}-\sqrt{\left(C_{p}+C_{A}+\frac{1}{K_{1}}\right)^{2}-4 C_{P} C_{A}}\right] \cdot \text { Equation [1] }
$$

Being $C_{p}$ the concentration of JG76, $C_{A}$ the concentration of the analyte and $f_{P} / f_{P A}$ the proportional fluorescent factors of JG76 and the complex JG76-analyte, respectively. The fluorescence titration was performed under a constant concentration of probe and the fluorescence values fitted by nonlinear least squares regression, ${ }^{59}$ starting in an initial value of $\mathrm{K}_{1}, \mathrm{f}_{\mathrm{P}}$ and $\mathrm{f}_{\mathrm{PA}}$ and calculating the value of $\mathrm{K}_{1}$ and the error associated by iteration, Figure 48 for JG76 $+\mathrm{K}^{+}$and Figure 49 for $\mathrm{JG} 76+\mathrm{Pb}^{2+}$.
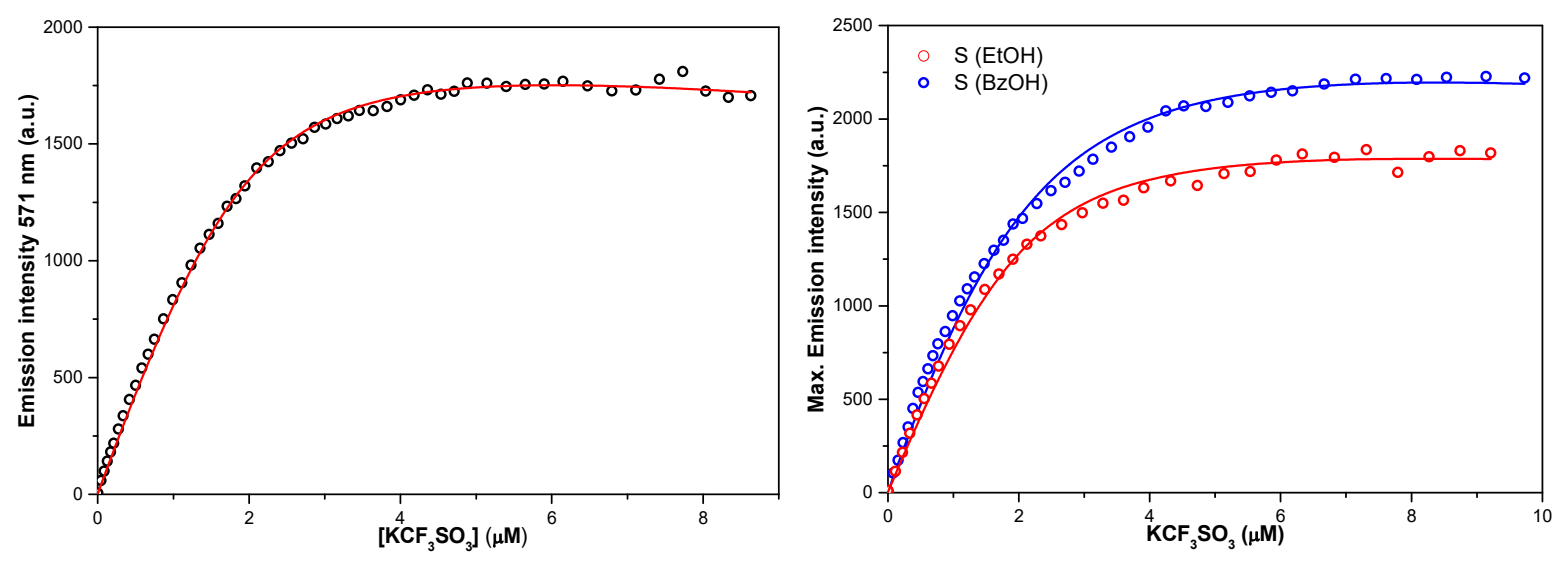

Figure 48. Fitted fluorescent emission of a titration with $\mathrm{K}\left(\mathrm{CF}_{3} \mathrm{SO}_{3}\right)$ of a $2 \mu \mathrm{M}$ solution of $\mathbf{J G 7 6}$ in $\mathrm{EtOH}(\mathrm{left})$ and $\mathrm{EtOH}$ and $\mathrm{BnOH}$ compared (right).

The calculation of the complexation constants was repeated 3 times for each solvent. Besides, the results were compared by doing a titration of $\mathrm{K}^{+}$with $\mathbf{J G 7 6}$ and JG76 with $\mathrm{K}^{+}$. The same results were obtained.

Ethanol solution:

- $\mathrm{K}\left(\mathrm{JG} 76+\mathrm{K}^{+}\right)=(2.2 \pm 0.1) \times 10^{6} \mathrm{M}^{-1}$

- $\quad \log K\left(J G 76+K^{+}\right)=6.34 \pm 0.03$

Benzylic alcohol solution:

- $\mathrm{K}\left(\mathrm{JG} 76+\mathrm{K}^{+}\right)=(1.3 \pm 0.1) \times 10^{6} \mathrm{M}^{-1}$

\footnotetext{
${ }^{59}$ The software for the calculation was Origin v2016.
} 
- $\quad \log \mathrm{K}\left(\mathrm{JG76}+\mathrm{K}^{+}\right)=6.11 \pm 0.03$

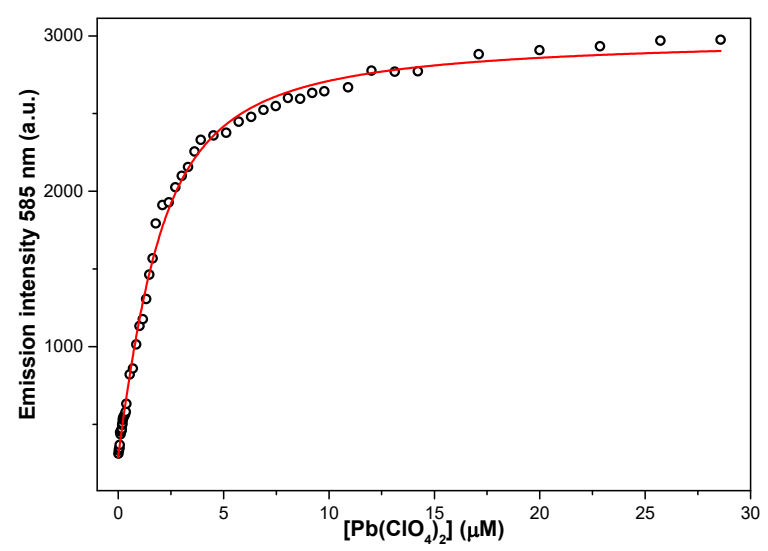

Figure 49. Fitted fluorescent emission of a $2 \mu \mathrm{M}$ JG76 solution in EtOH titrated with $\mathrm{Pb}\left(\mathrm{ClO}_{4}\right)_{2}$.

The fitting calculation of the complexation constants was repeated 3 times:

- $\mathrm{K}\left(\mathrm{JG} 76+\mathrm{Pb}^{2+}\right)=(1.55 \pm 0.1) \times 10^{6} \mathrm{M}^{-1}$

- $\log \mathrm{K}\left(\mathrm{JG76}+\mathrm{Pb}^{2+}\right)=6.19 \pm 0.02$

\subsubsection{Equilibrium constant Valinomycin- $\mathrm{K}^{+}$calculated by circular dichroism:}

There are some procedures described in literature to measure the thermodynamic equilibrium constant of natural potassium ionophores, such as valinomycin. ${ }^{60}$ Due to the chiral properties of these cyclic depsipeptides, circular dichroism is the one taken as standard for the thermodynamic constant calculation of these cyclic depsipeptides. Once a complex between the peptide and a cation is formed, the deviation of polar light may be measured.

In a reference experiment, the measurements were performed starting from a concentration of $0.3 \mathrm{mM}$ of valinomycin; then, the equivalents of potassium were gradually increased until 3.75 equivalents (1.13 mM), see Figure 50.

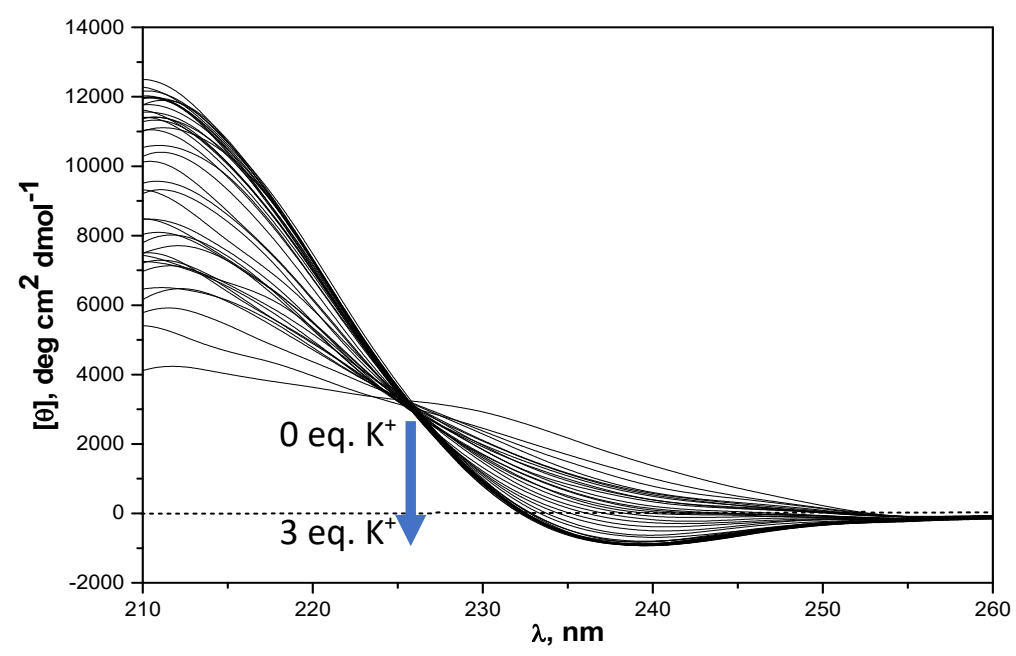

Figure 50. Circular dichroism of a valinomycin $0.3 \mathrm{mM}$ solution in ethanol, increasing the concentration of potassium cations from 0 to $1.13 \mathrm{mM}$.

${ }^{60}$ M. C. Rose, R. W. Henkens, Biochim. Biophys. Acta 1974, 372, 426-435 
The concentration of valinomycin was chosen because of the optimal concentration to work with the dichroism signal. Although the results were, apparently, the same explained in literature, it turned out that, checking the fitting at different wavelengths, the constant seemed to change depending on the wavelength.

Adjusting between $230 \mathrm{~nm}-250 \mathrm{~nm}$ the graphs in Figure 51 were obtained:
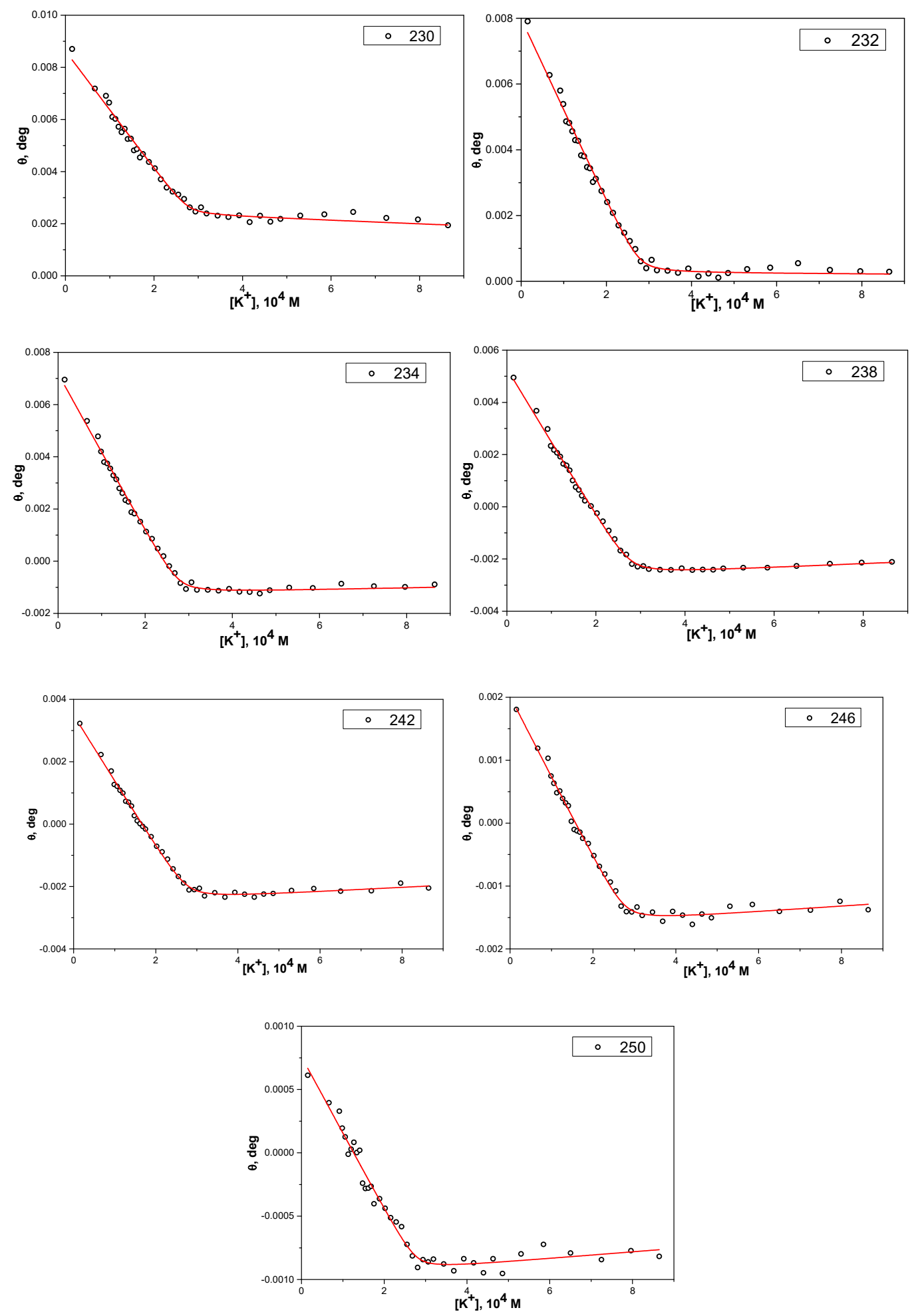

Figure 51. Molar ellipticity of a $0.3 \mathrm{mM}$ solution in ethanol of valinomycin, increasing the concentration of potassium cations from 0 to $1.13 \mathrm{mM}$ at different wavelengths $(230-250 \mathrm{~nm})$. 
The results could be represented by the same equation used for fluorescence which allowed to calculate the thermodynamic constant exposed in Figure 52.

\begin{tabular}{|c|c|c|}
\hline $\boldsymbol{\lambda .} \mathbf{( n m})$ & $\left.\mathbf{K} \times \mathbf{1 0}^{-\mathbf{5}} \mathbf{( M}^{\mathbf{- 1}}\right)$ & $\mathbf{L o g} \mathbf{~}$ \\
\hline 230 & 10.3 & 6.01 \\
\hline 232 & 8.62 & 5.94 \\
\hline 234 & 8.07 & 5.91 \\
\hline 238 & 6.55 & 5.82 \\
\hline 242 & 5.88 & 5.77 \\
\hline 246 & 6.33 & 5.8 \\
\hline 250 & 8.51 & 5.93 \\
\hline
\end{tabular}

Figure 52. Table of equilibrium constant valinomycin- $\mathrm{K}^{+}$calculated at different wavelengths.

Although in literature the given equilibrium constant was calculated at $238 \mathrm{~nm}$, and taken as independent from the wavelength, it turned out to be slightly dependent on it. This characteristic was observed in literature when the complexation occurs in DNA structures (different binding sites), ${ }^{61}$ but it was not the case. Therefore, for valinomycin, it was understood as a consequence of the limitations of the method.

In conclusion, the equilibrium constant in pristine ethanol was between $(0.6$ to 1.0$) \times 10^{6} \mathbf{M}^{-1}$ or (LogK) between 5.8 to 6.0; calculated with a $0.3 \mathrm{mM}$ of valinomycin.

\subsubsection{Valinomycin and Cereulide derivatives, equilibrium constant calculation by fluorescence:}

By using the indirect method explained in Chapter 0, the equilibrium constants were calculated by fluorescence. The procedure is schematized in Figure $\mathbf{5 3}$.
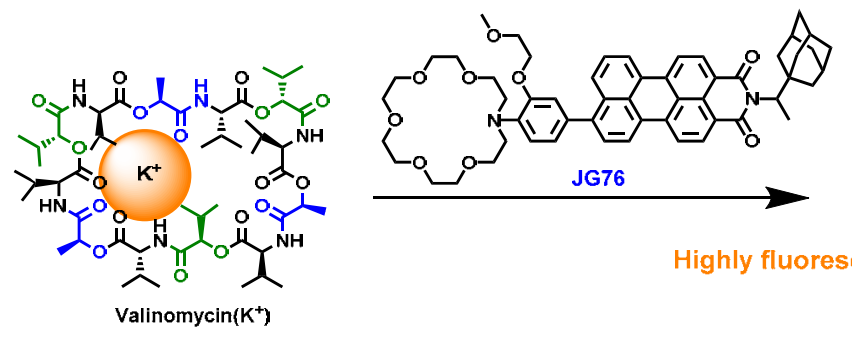

Highly fluorescent
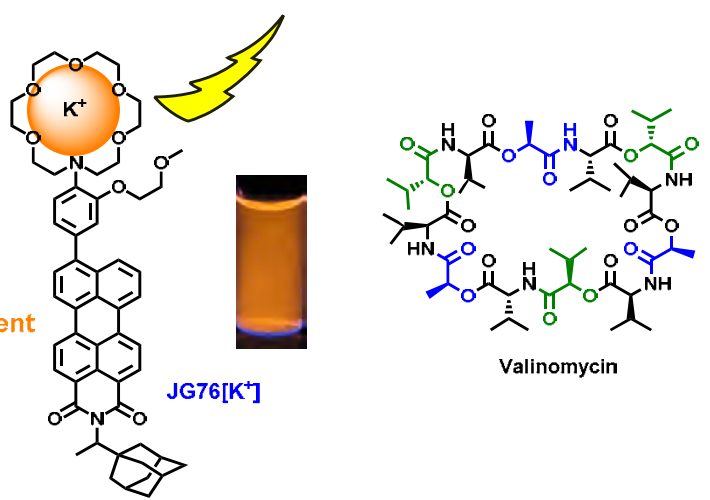

Figure 53. Scheme for the titration of the complex Valinomycin-K ${ }^{+}$with JG76 to calculate the complexation constant Valinomycin- $\mathrm{K}^{+}$.

The experiment started with a solution in ethanol of Valinomycin or Cereulide (V) and Potassium cations $(\mathrm{K})$, giving a complex between them $(\mathrm{VK})$ in equilibrium. When the probe $(\mathrm{S})$ was added, it formed a complex with free $\mathrm{K}^{+}$creating the new complex (SK) and replacing the previous complex (VK). In conclusion, the concentration of VK decreased, whereas the concentration of V increased. Then, the approximation used was:

$$
\mathrm{C}_{\mathrm{V}}-[\mathrm{VK}] \approx \mathrm{C}_{\mathrm{V}}
$$

${ }^{61}$ P. Kumar, R. Barthwal, Biochimie, 2018, 147, 153-169. 
And this simplification was more realistic when the initial proportion $\mathrm{V} / \mathrm{K}$ was as high as possible.

The equation used was Equation 2:

$$
[S K]=\frac{\left(C_{S}+C_{K}+\frac{1+K_{2} C_{V}}{K_{1}}\right)-\sqrt{\left(C_{S}+C_{K}+\frac{1+K_{2} C_{V}}{K_{1}}\right)^{2}-4 C_{S} C_{K}}}{2} \cdot \text { Equation [2] }
$$

The test was repeated several times with different initial proportions of $\mathrm{V} / \mathrm{K}$, a proportion $1 / 1,1 / 0.25$ and $1 / 0.1$. It was checked that the results were slightly different, but having the best fitting results when the proportions are 1:0.1 in which the approximation was more valid.
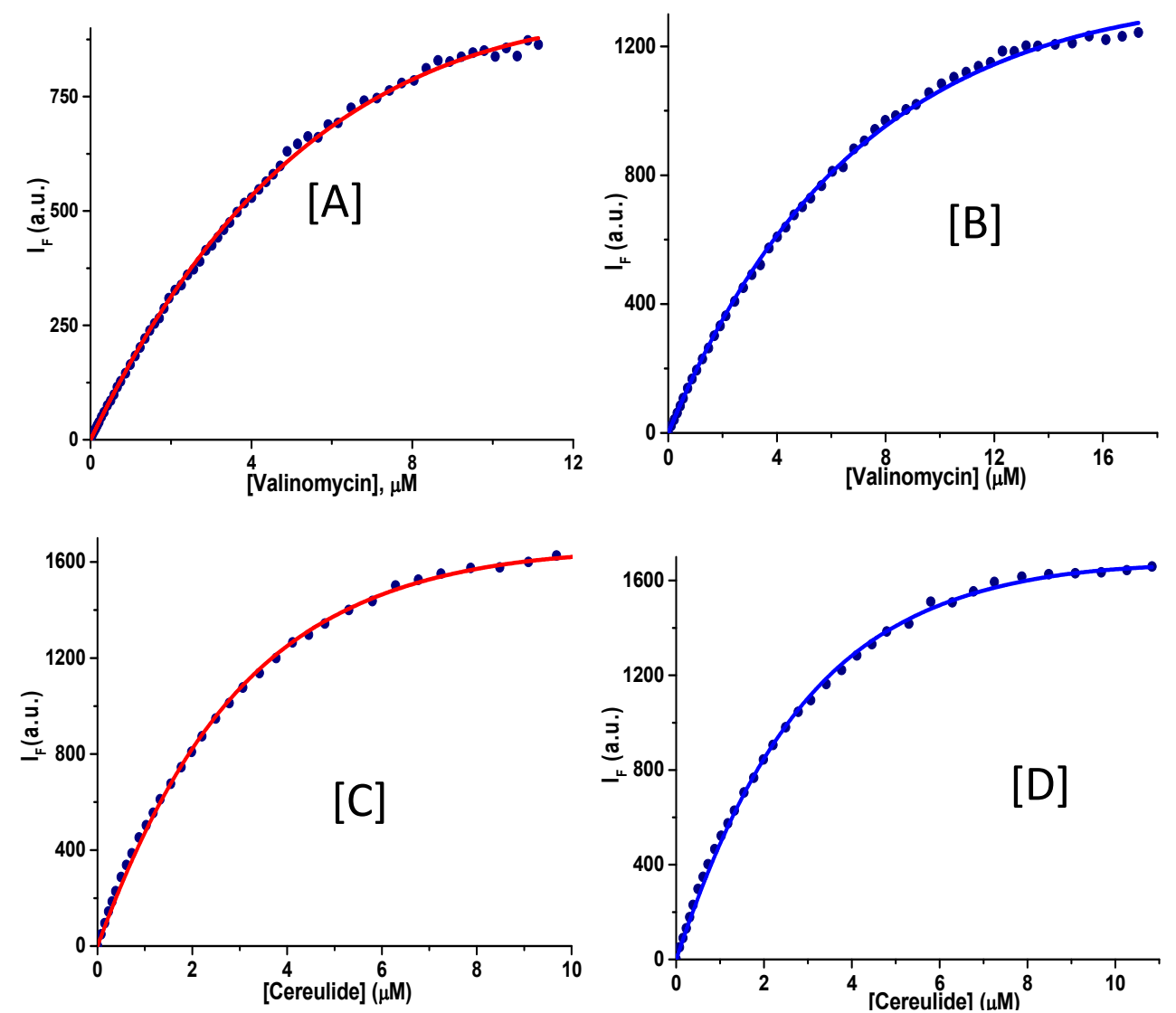

Figure 54. Fitted fluorescent titration emissions with JG76 of a $2 \mu \mathrm{M}$ solution of $\mathrm{K}\left(\mathrm{CF}_{3} \mathrm{SO}_{3}\right)$ and 20 $\mu \mathrm{M}$ of valinomycin and cereulide solution in $\mathrm{EtOH}(\mathrm{A}$ and $\mathrm{C}$ ) and $\mathrm{BnOH}(\mathrm{B}$ and $\mathrm{D})$, respectively.

The complexation equilibrium constants, calculated by this method, showed in Figure 54, after doing the average of three titrations, turned out to be:

- In EtOH solution:
o $\quad \mathrm{K}(\mathrm{Val} \mathrm{EtOH})=(9.4 \pm 0.2) \times 10^{5} \mathrm{M}^{-1}, \log \mathrm{K}(\mathrm{Val} \mathrm{EtOH})=5.97 \pm 0.01$
o $\mathrm{K}(\mathrm{Cer} \mathrm{EtOH})=(9.7 \pm 0.2) \times 10^{5} \mathrm{M}^{-1}, \log \mathrm{K}(\mathrm{Cer} \mathrm{EtOH})=5.99 \pm 0.01$

- In BnOH solutions:

o $\quad \mathrm{K}($ Val BnOH $)=(9.6 \pm 0.2) \times 10^{4} \mathrm{M}^{-1}, \log \mathrm{K}($ Val EtOH $)=4.98 \pm 0.01$

o $\quad \mathrm{K}(\mathrm{Cer} \mathrm{BnOH})=(10.3 \pm 0.2) \times 10^{4} \mathrm{M}^{-1}, \log \mathrm{K}(\mathrm{Val} \mathrm{EtOH})=5.01 \pm 0.01$ 
Moreover, with the data obtained, it can be represented, in the same graph (Figure 55), the amount of the reagents and the proportion of complex that we have during the titration:

- With the values of $\mathrm{K}_{2}$ and $\mathrm{K}_{1}$, the concentration of [SK $]_{\text {eq }}$ may be determined.

- With these data, the concentration of the species was calculated by these equations:

$$
\begin{gathered}
{[S]_{e q}=C_{S}-[S K]_{e q}} \\
{[V K]_{e q}=\frac{C_{V}+C_{K}-[S K]_{e q}+\frac{1}{K_{2}}-\sqrt{\left(C_{V}+C_{K}-[S K]_{e q}+\frac{1}{K_{2}}\right)^{2}-4 C_{v}\left(C_{K}-[S K]_{e q}\right)}}{2}} \\
{[V]_{e q}=C_{V}-[V K]_{e q}} \\
{[K]_{e q}=C_{K}-[S K]_{e q}-[V K]_{e q}}
\end{gathered}
$$
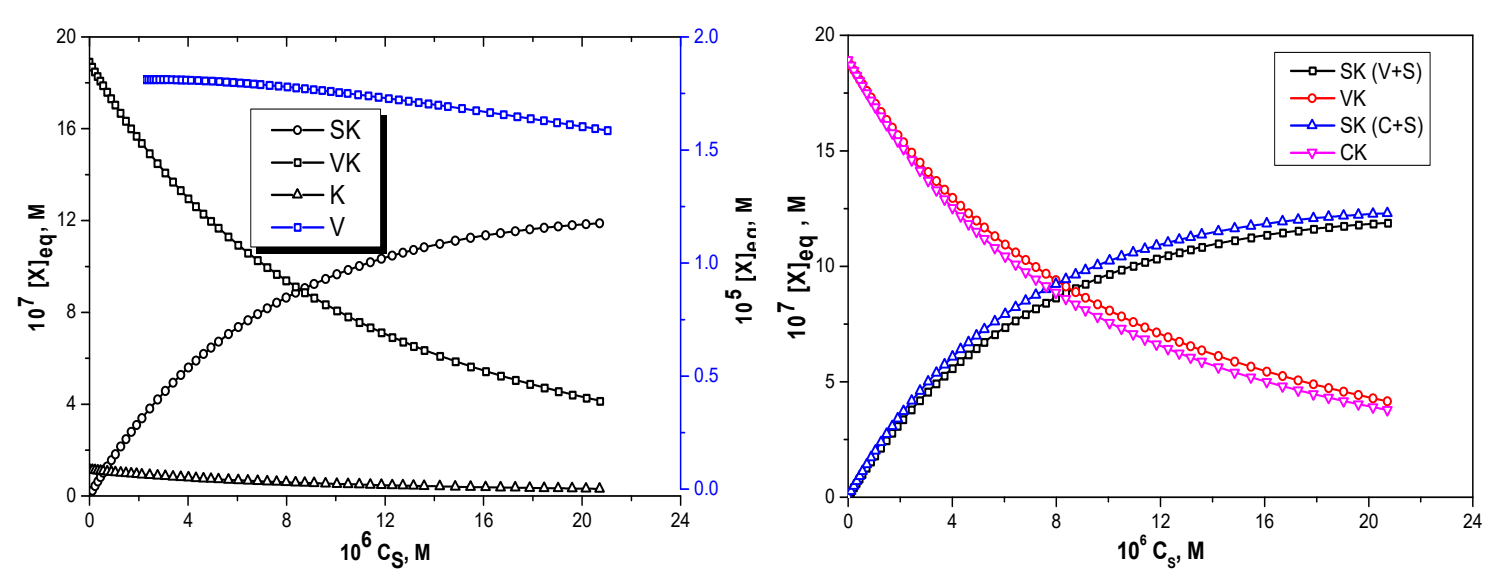

Figure 55. Concentration in the equilibrium in EtOH solution. Left, V-VK-K-SK equilibrium. Right, comparison of the concentration in equilibrium of the complexes of cereulide and valinomycin.

Apart from the experiments with valinomycin and synthetic cereulide (JG100), other 3 cereulide derivatives were synthetized; JG115, JG115B and JG121 (see Figure 23). They were also tested by the same method and in the same conditions, Figure 56.
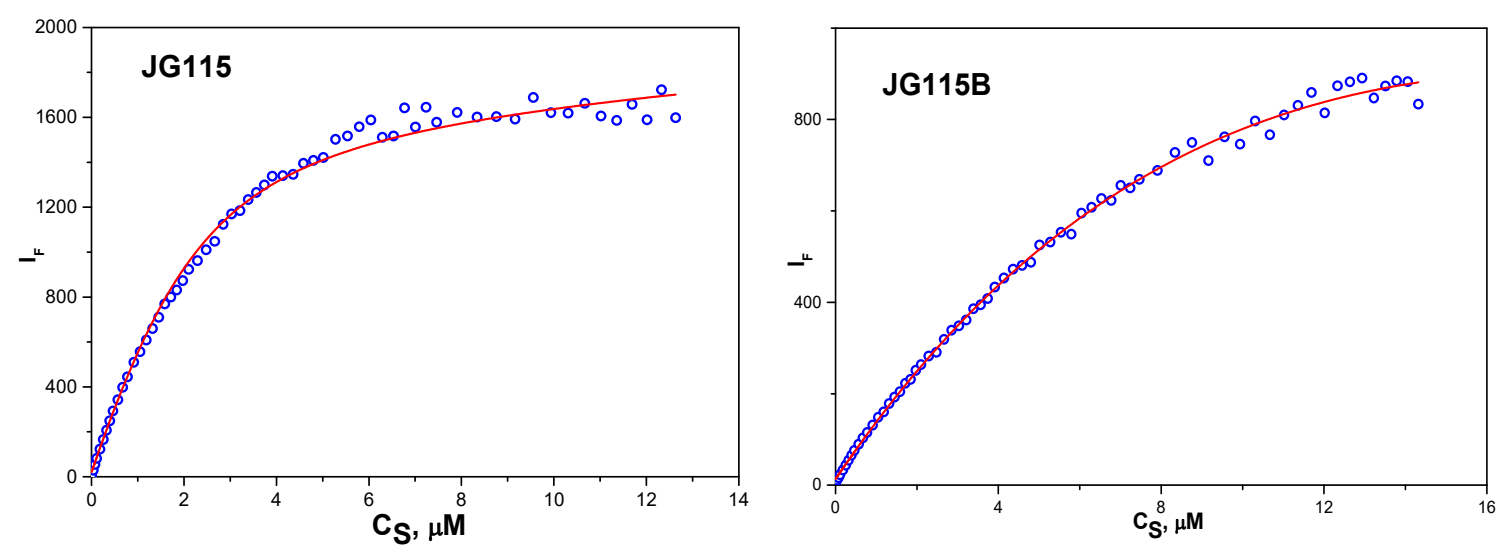

Figure 56. Fitted fluorescent titration emissions with $\mathbf{J G 7 6}$ of a $2 \mu \mathrm{M}$ solution of $\mathrm{K}\left(\mathrm{CF}_{3} \mathrm{SO}_{3}\right)$ and 20 $\mu \mathrm{M}$ of JG115/JG115B solutions in EtOH.

- In EtOH solution:

o $\mathrm{K}(\mathrm{JG1} 15 \mathrm{EtOH})=(3.9 \pm 0.1) \times 10^{4} \mathrm{M}^{-1}, \log \mathrm{K}(\mathrm{Val} \mathrm{EtOH})=4.59 \pm 0.01$

o $\quad \mathrm{K}(\mathrm{JG1} 15 \mathrm{~B} \mathrm{EtOH})=(9.3 \pm 0.2) \times 10^{5} \mathrm{M}^{-1}, \log \mathrm{K}(\mathrm{Cer} \mathrm{EtOH})=5.97 \pm 0.01$ 
These results should be carefully evaluated, as the calculations of the constants were performed taking into account previous results of the constant $\mathrm{JG} 76-\mathrm{K}^{+}$. Apart from being an approximation, the fitting process relies on this value; as a consequence, if the constants $K_{1}$ and $K_{2}$ were very different the method itself becomes unreliable, along with the results given.

It was taken as reference the error for the determination of $\mathrm{K}_{1}\left(\mathrm{JG} 76-\mathrm{K}^{+}\right)$. The error associated to the calculation was, approximately, $10 \%$ of the value of the constant. Therefore, if $\mathrm{K}_{2}$ were much lower than 10 times $K_{1}$, the error could be higher than the value of $K_{2}$ itself. What is more, the quantity that would be necessary to add of the cyclic depsipeptide should be higher in order to make the approximation valid $\left(\mathrm{C}_{\mathrm{V}}-[\mathrm{VK}] \approx \mathrm{C}_{\mathrm{V}}\right)$, which makes the approximation less realistic. As a consequence, in those cases, the values should be interpreted as an approximation, which was the case of JG115.

Thermodynamic equilibrium association constants

\begin{tabular}{|c|c|c|c|c|c|}
\hline & EtOH & $\mathbf{M}^{-1} \times \mathbf{1 0}^{-5}$ & Error & $\log (\mathbf{K})$ & Error \\
\hline $\mathrm{K}_{1}$ & $\mathbf{J G 7 6 - P b}^{2+}$ & 15.5 & 1 & 6.19 & 0.03 \\
\hline $\mathrm{K}_{1}$ & JG76-K $^{+}$ & 22.0 & 1 & 6.34 & 0.03 \\
\hline $\mathrm{K}_{2}$ & Valinomycin - K $^{+}$ & 9.4 & $0.2^{*}$ & 5.97 & $0.02^{*}$ \\
\hline $\mathrm{K}_{2}$ & Cereulide-K $^{+}$ & 9.7 & $0.2^{*}$ & 5.99 & $0.02^{*}$ \\
\hline $\mathrm{K}_{2}$ & JG115B-K $^{+}$ & 9.3 & $0.2^{*}$ & 5.97 & $0.01^{*}$ \\
\hline $\mathrm{K}_{2}$ & JG115-K $^{+}$ & 0.39 & $0.01^{*}$ & 4.59 & $0.01^{*}$ \\
\hline $\mathrm{K}_{2}$ & JG121-K $^{+}$ & $<\mathrm{JG} 115-\mathrm{K}^{+}$ & - & $<{\mathrm{JG} 115-\mathrm{K}^{+}}^{-}$ \\
\hline
\end{tabular}

*The error was given by the fitting curve

Figure 57. Equilibrium constants of different potassium ionophores calculated by fluorescence and the error associated.

The results are summarized in Figure 57, from them the following interpretation was elaborated:

- Valinomycin and cereulide had similar thermodynamic constant in pristine ethanol $\mathrm{K}(\mathrm{EtOH})$.

- For JG115B, K(EtOH) was calculated as $(9.3 \pm 0.2) \times 10^{5} \mathrm{M}^{-1}$. Mostly the same than for cereulide or valinomycin.

- For JG115, K(EtOH), was around the limit of the approximation, the estimation throughout this method gave the value around $(3.9 \pm 0.1) \times 10^{4} \mathrm{M}^{-1}$, although the repeatability was low.

- For JG121, K(EtOH) was tried to be calculated too. However, it did not led to any result detectable by the technique. It was likely that $\mathrm{K}\left(\mathrm{JG} 121-\mathrm{K}^{+}\right)<<\mathrm{K}\left(\right.$ Cereulide- $\left.\mathrm{K}^{+}\right)$.

Despite the lack of accuracy in the determination of some of the constants, the strength of the complexes was clear. It was observed a relation between their values and the specific compound:

$$
\mathrm{K}(\text { Cereulide }) \approx \mathrm{K}(\text { Valinomycin }) \approx \mathrm{K}(\mathrm{JG} 115 \mathrm{~B})>\mathrm{K}(\mathrm{JG} 115)>\mathrm{K}(\mathrm{JG} 121)
$$

The strength of the complex in ethanol seemed directly related with the size of the substituent when modifying the cereulide. In conclusion, it opened the possibility of tuning the value of the constant by introducing different modifications. The results were also in agreement with subsequent experiments, such as potassium transport (Section 11). 


\subsection{Limits of Detection (LODs)}

JG76 was the probe used for the LODs calculation of $\mathrm{K}^{+}, \mathrm{Pb}^{2+}$, valinomycin and cereulide. The tests were performed in ethanol solution, and the calculations were performed by using the method explained in Chapter 0, with the program " $R$ " by calculating when the possibility of false positive and false negative are $5 \%$ or less, giving a limit that was really measured.

\subsubsection{Limit of detection for $\mathrm{K}^{+}$}

In a solution $5 \mu \mathrm{M}$ of $\mathbf{J G 7 6}$, the concentration of $\mathrm{K}\left(\mathrm{CF}_{3} \mathrm{SO}_{3}\right)$ was gradually increased and the fluorescence spectra were registered (Figure 58).
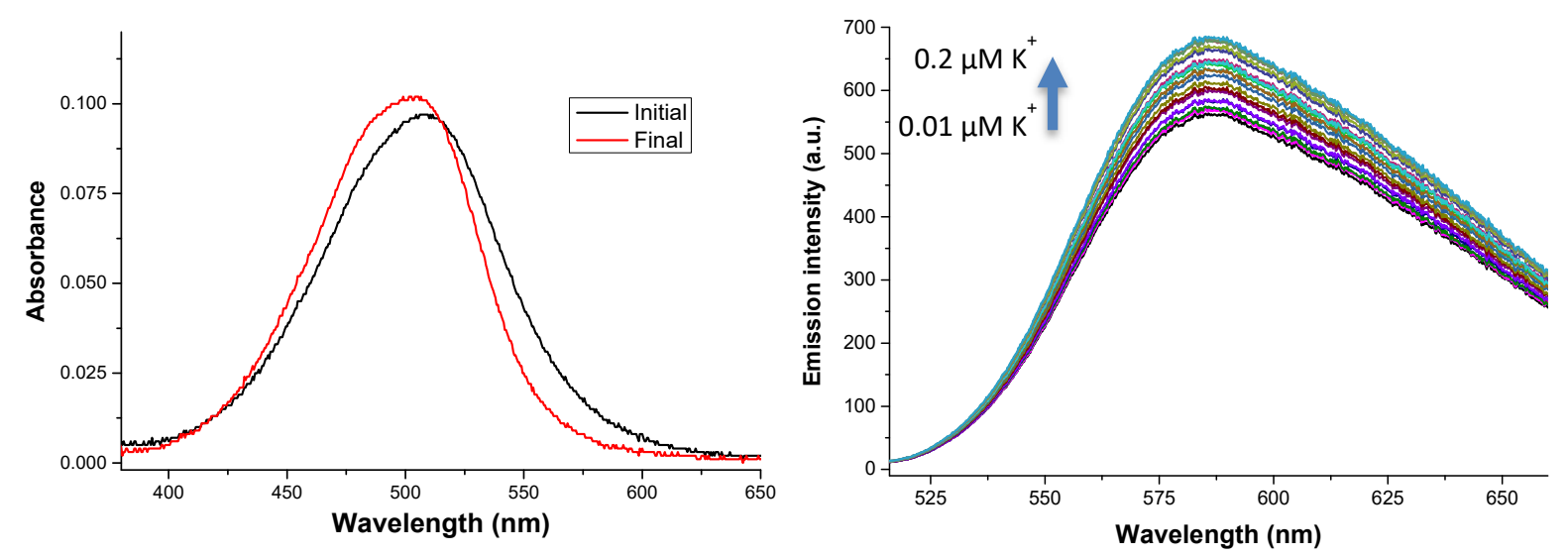

Figure 58. Absorbance and fluorescence spectra of a titration with JG76 $5 \mu \mathrm{M}$ in EtOH, increasing concentration from 0.01 to $0.2 \mu \mathrm{M}$.

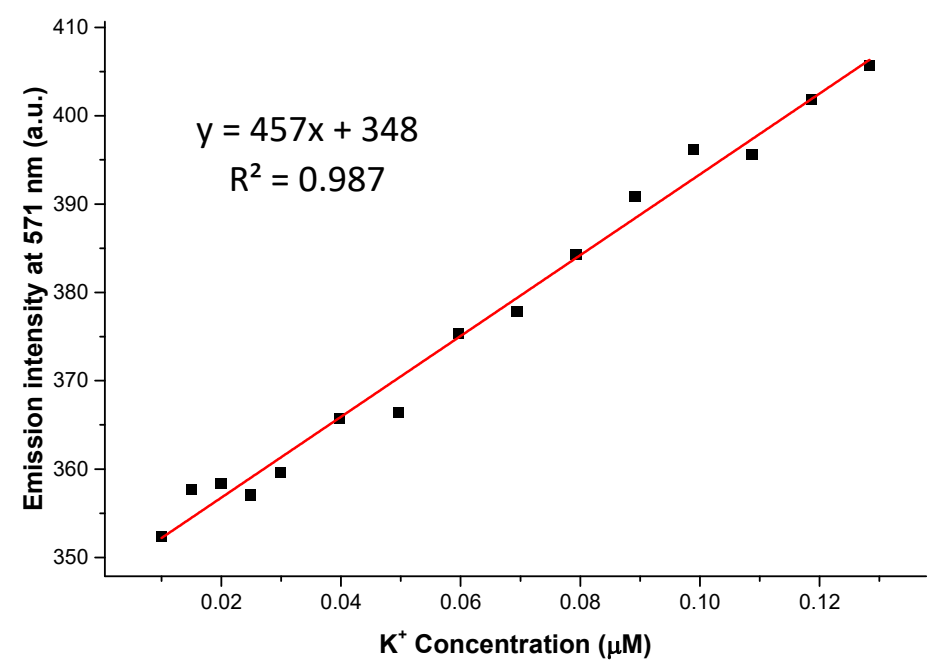

Figure 59. Linear regression of a titration of JG76 $5 \mu \mathrm{M}$ with $\mathrm{K}^{+}$in EtOH, increasing concentration from 0.01 to $0.2 \mu \mathrm{M}$ and studying the increase in the fluorescent emission.

The obtained limit of detection of $\mathrm{K}^{+}$with $\mathbf{J G 7 6}$ in EtOH solution was calculated from the linear regression represented in Figure 59, being $60 \mathrm{nM}$ or 2.3 ppb. 


\subsubsection{Limit of detection of $\mathrm{Pb}^{2+}$ with JG76}

In a solution $5 \mu \mathrm{M}$ of $\mathbf{J G 7 6}$ in ethanol, the concentration of $\mathrm{Pb}\left(\mathrm{ClO}_{4}\right)_{2}$ was gradually increased and the fluorescence spectra was registered (Figure 60).

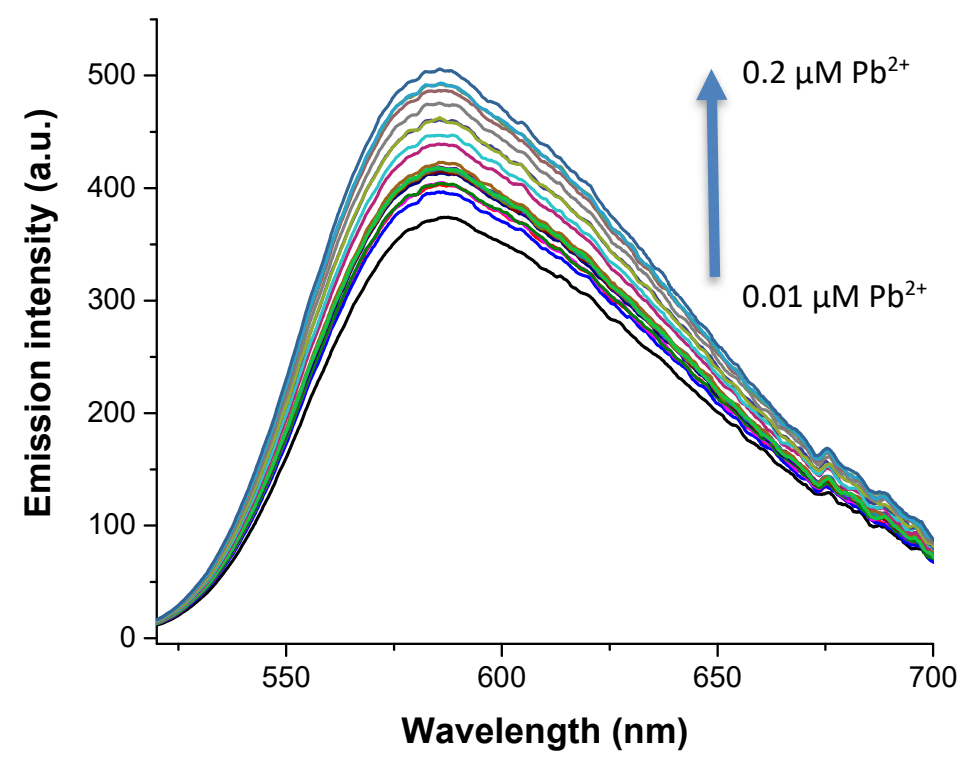

Figure 60. Fluorescent spectra of a titration with JG76, $5 \mu \mathrm{M}$ in EtOH, increasing concentration from 0.01 to $0.2 \mu \mathrm{M}$ of $\mathrm{Pb}^{2+}\left(\lambda_{\text {exc }}=500 \mathrm{~nm}\right)$.

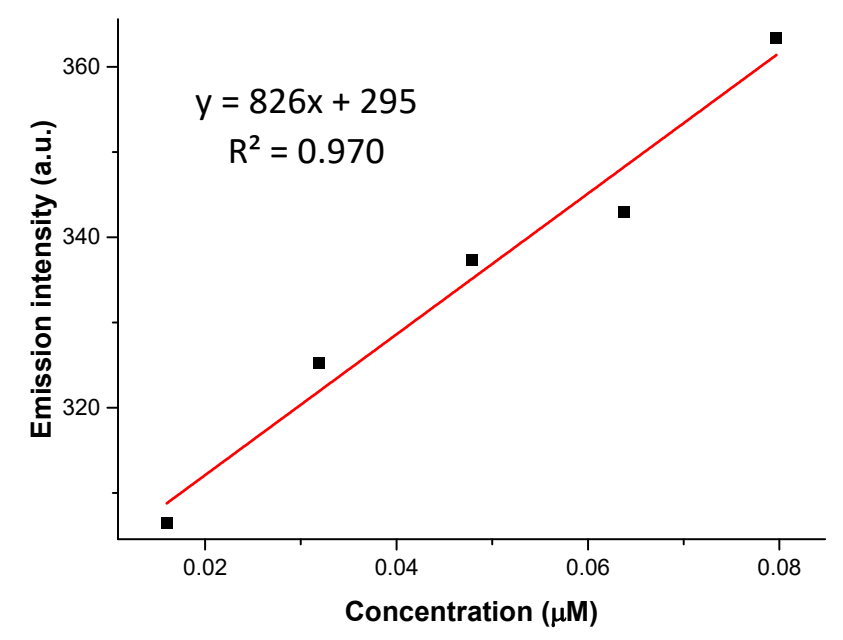

Figure 61. Linear regression of a titration of JG76 $5 \mu \mathrm{M}$ with $\mathrm{Pb}^{2+}$ in $\mathrm{EtOH}$, studying the increase in the fluorescent emission $\left(\lambda_{\mathrm{exc}}=500 \mathrm{~nm}, \lambda_{\mathrm{em}}=585 \mathrm{~nm}\right)$.

From the data in Figure 61, the obtained limit of detection for $\mathbf{P b}^{2+}$ was $30 \mathbf{n M}$ or $6 \mathbf{p p b}$. 


\subsubsection{Limit of detection of Valinomycin}

The LODs of valinomycin and cereulide were studied in a solution of probe and potassium, by adding valinomycin or cereulide (Figure 62). These results are only valid when the proportion of probe and potassium is within the range studied, otherwise it could change.

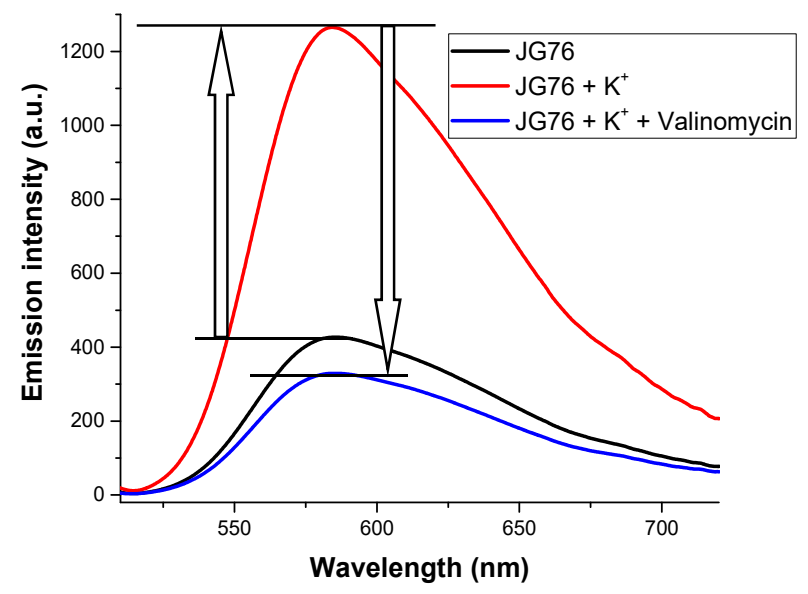

Figure 62. Fluorescent displacement assays of JG76, potassium cation and valinomycin. $\lambda_{\mathrm{exc}}=500$

$\mathrm{nm}$

The conditions for calculating the LOD were optimized:

- If $[\mathrm{JG} 76] /\left[\mathrm{K}^{+}\right]$increased the necessary concentration of valinomycin or cereulide was higher in order to be detected.

- If $[\mathrm{JG} 76] /\left[\mathrm{K}^{+}\right]$was too low the concentration of cereulide or valinomycin detected was higher too, because it was not detected until the free potassium cation was very low.

In a $5 \mu \mathrm{M}$ solution of $\mathbf{J G 7 6}$, with the concentration of $\mathrm{K}\left(\mathrm{CF}_{3} \mathrm{SO}_{3}\right)$ following the proportions $[\mathrm{JG} 76] /\left[\mathrm{K}^{+}\right]=1.25$, the concentration of valinomycin was increased in several additions, and the fluorescence spectra were registered (Figure 63).

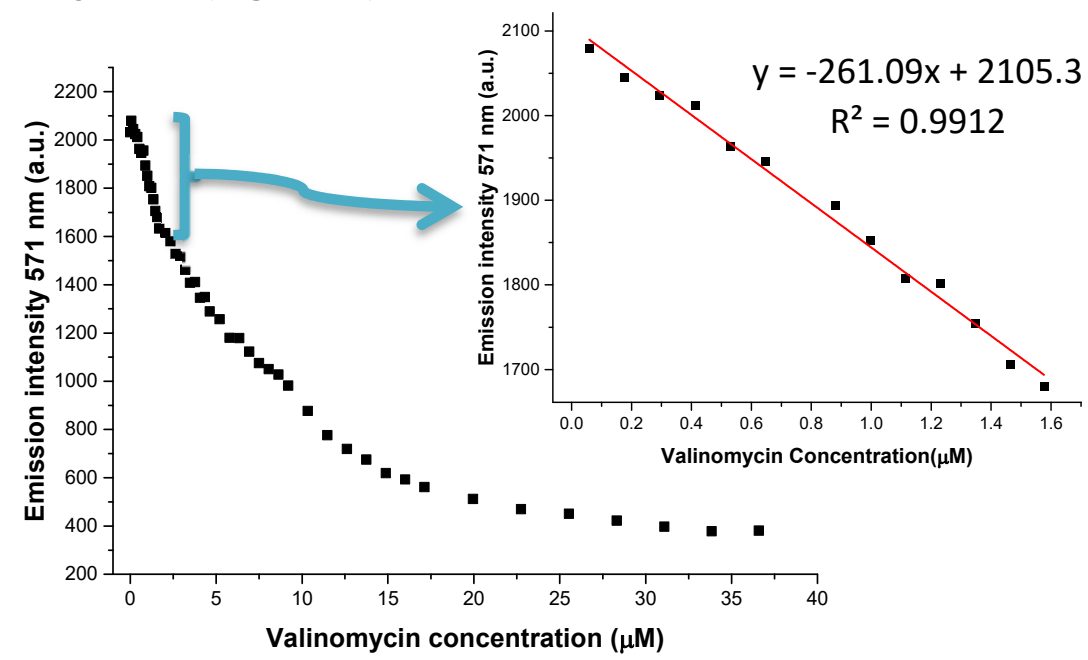

Figure 63. Regression of a titration with valinomycin of JG76 $5 \mu \mathrm{M}$ and $\mathrm{K}^{+} 3.75 \mu \mathrm{M}$ in EtOH studying the decreasing fluorescent emission. $\lambda_{\text {exc }}=500 \mathrm{~nm}$.

The detection limit calculated for valinomycin with JG76 in EtOH was $0.54 \boldsymbol{\mu M}$ or $600 \mathbf{p p b}$. 


\subsubsection{Limit of detection of Cereulide:}

In a solution $2 \mu \mathrm{M}$ of $\mathbf{J G 7 6}$, the concentration of $\mathrm{K}\left(\mathrm{CF}_{3} \mathrm{SO}_{3}\right)$ followed the proportions $[\mathrm{JG} 76] /\left[\mathrm{K}^{+}\right]=1.25$. The concentration of cereulide was increased in several additions, and the fluorescence spectra were registered (Figure 64).

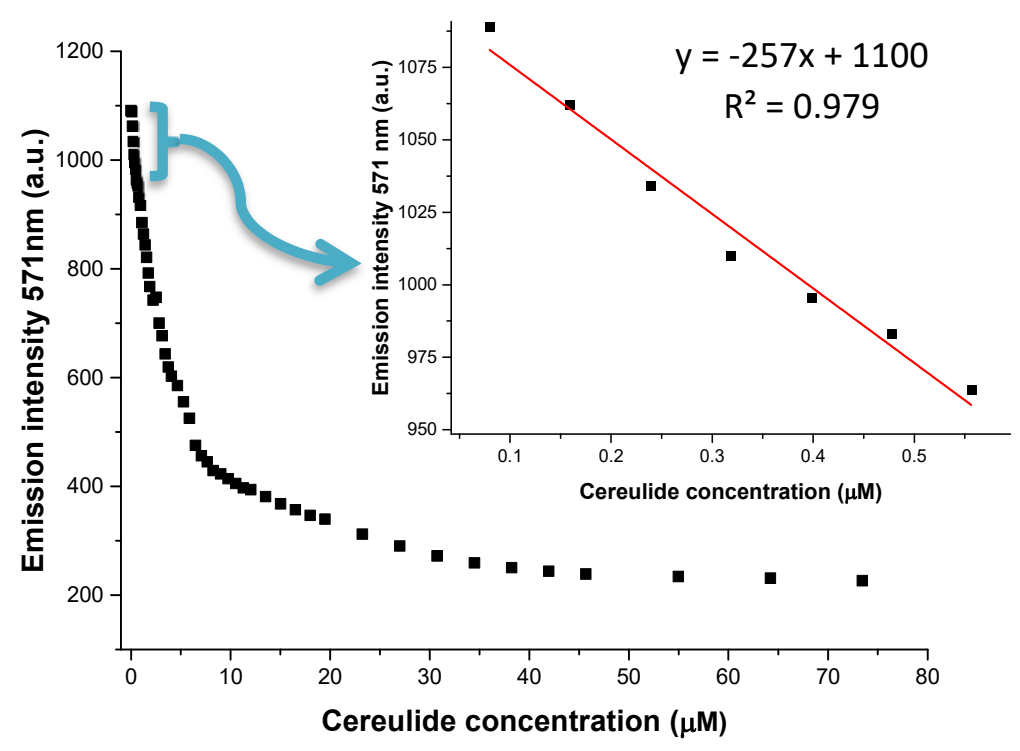

Figure 64. Regression of a titration with cereulide of JG76 $2 \mu \mathrm{M}$ and $\mathrm{K}^{+} 1.5 \mu \mathrm{M}$ in EtOH studying the decreasing fluorescent emission. $\lambda_{\mathrm{exc}}=500 \mathrm{~nm}$

The detection limit calculated for cereulide was $0.21 \mu \mathbf{M}$ or $240 \mathrm{ppb}$.

\subsubsection{Summary, LODs using JG76 in EtOH:}

\begin{tabular}{|c|c|c|c|}
\hline EtOH & LOD $(\mu \mathrm{M})$ & LOD (ppb) & {$[\mathrm{JG} 76] /\left[\mathrm{K}^{+}\right]$} \\
\hline $\mathbf{K}^{+}$ & 0.06 & 2.3 & - \\
\hline $\mathbf{P b}^{2+}$ & 0.03 & 6 & - \\
\hline Valinomycin* & 0.54 & 600 & 1.25 \\
\hline Cereulide* & 0.21 & 240 & 1.25 \\
\hline
\end{tabular}

Figure 65. LODs of $\mathrm{K}^{+}, \mathrm{Pb}^{2+}$, valinomycin and cereulide calculated in EtOH solution with probe JG76, quantities experimentally measured with $5 \%$ of false positive/negative. 


\section{MEASUREMENTS OF EXTRACTED CEREULIDE SAMPLES BY FLUORESCENCE}

JG76 was very sensitive and selective to potassium cations, with a complexation constant even higher than cereulide (in EtOH solution), $2.2 \times 10^{6} \mathrm{M}^{-1}$ against $0.9 \times 10^{6} \mathrm{M}^{-1}$. Therefore, it was chosen as fluorescent probe for detection of cereulide extracted from rice extracts.

\subsection{Culture and extraction of natural cereulide ${ }^{62}$}

The extraction of the natural cereulide was performed from cultures of B. cereus F4810/72 strains (see Figure 66), following the methodology developed for cooked rice. ${ }^{63}$ Briefly summarized, it consisted of the inoculation of rice with $300 \mathrm{CFU}$ (Colony forming units), average value found in rice dishes. The CFU was determined at several time points, along with cereulide production, by UPLC-TOF-MS. The extraction proceeded by using acetonitrile and several purifications, involving heating, extraction and centrifugation to obtain cereulide in a concentration around 0.2 to $3.5 \mu \mathrm{M}$ in acetonitrile.
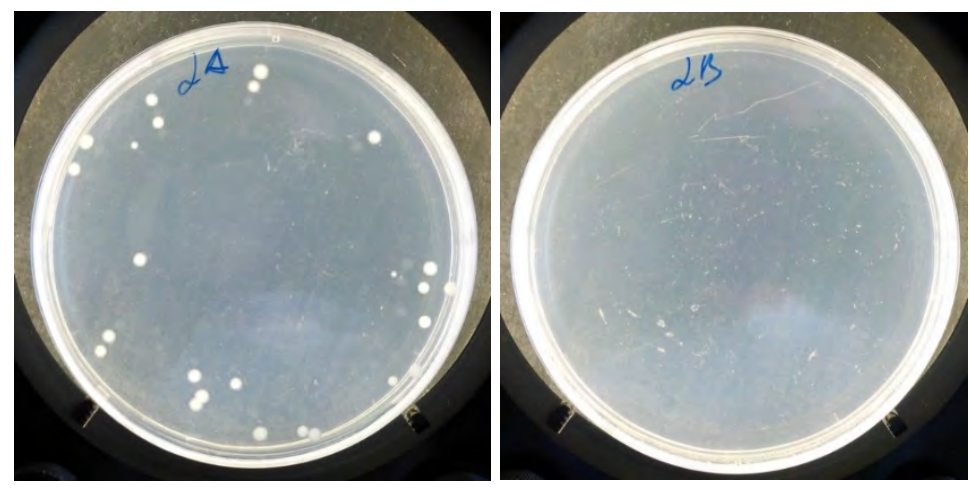

Figure 66. Cereulide extracts were placed in PCA culture (Plate count agar) before (left) and after (right) centrifugation to evaluate the presence of bacterial spores. No spores were detected after centrifugation.

\subsection{Fluorescence measurements of natural cereulide extracts}

\section{a) Conditions:}

Cereulide extracts were provided from rice cultures and extracted in acetonitrile with a concentration calculated by HPLC analysis. The idea was to measure the change in fluorescence in presence of:

- A constant concentration of JG76.

- A constant concentration of potassium cation.

\footnotetext{
${ }^{62}$ The procedure was performed by Wilson Antunes at Laboratório de Bromatologia e de Defesa Biológica (LBDB) do Exército, Lisboa, Portugal.

${ }_{63}$ a) A. Z. Muratovic, R. Tröger, K. Granelli, K.-E. Hellenäs, Toxins 2014, 6, 3326-3335; b) M. Yamaguchi, T. Kawai, M. Kitagawa, Y. Kumeda, Food Microbiol. 2013, 34, 29-37; c) M. Decleer, A. Rajkovic, B. Sas, A. Madder, S. De Saeger, J. Chromatogr. A 2016, 1472, 35-43.
} 
- $\quad$ The sample of cereulide, concentration unknown, was titrated by adding synthesized cereulide or valinomycin. These samples were provided in acetonitrile, which had concentrations between $0.2-3.5 \mu \mathrm{M}$.

\section{b) Measuring directly from extracted samples:}

First of all, the easiest way to perform the measurements would have been by making a solution of the samples and studying the effect of increasing cereulide concentration. This straightforward method led to high fluorescent results without response to increasing potassium cations or cereulide. To explain this fact, several reasons were suggested:

- The cereulide samples contained an unknown concentration of potassium or species that acted as Lewis acid. Because of that, high initial concentrations of potassium or some Lewis acids led to inaccuracy in the determination of cereulide.

- The cereulide samples had matrix contribution, that interfered in the measurements; therefore, there was a background fluorescence from the matrix, which may be easily eliminated by subtraction of the fluorescence when there was no probe JG76.

It can be other ways to interfere in the measurements. If that was the case, the matrix would need to be completely removed before measuring. All these issues were tested and solved when possible.

c) Eliminating the excess of potassium in solution:

Due to the higher solubility of cereulide in organic solvents, the solution was extracted by liquid-liquid extraction (DCM-Water); due to it, the effect of water-soluble interferents was prevented. Finally, the sample was evaporated and redissolved in $\mathrm{EtOH}$, avoiding the interference of any watersoluble ions before the titration. To check if the cereulide remained dissolved after the extraction, the final solution in EtOH solution was measured by UPLC to compare results, verifying its presence, Figure 67.

The UPLC method of calculation was:

- Studying the elution time of synthetic cereulide.

- Measuring the intensity of a sample of extracted cereulide.

- Making a calibration with synthetic cereulide at different concentrations.
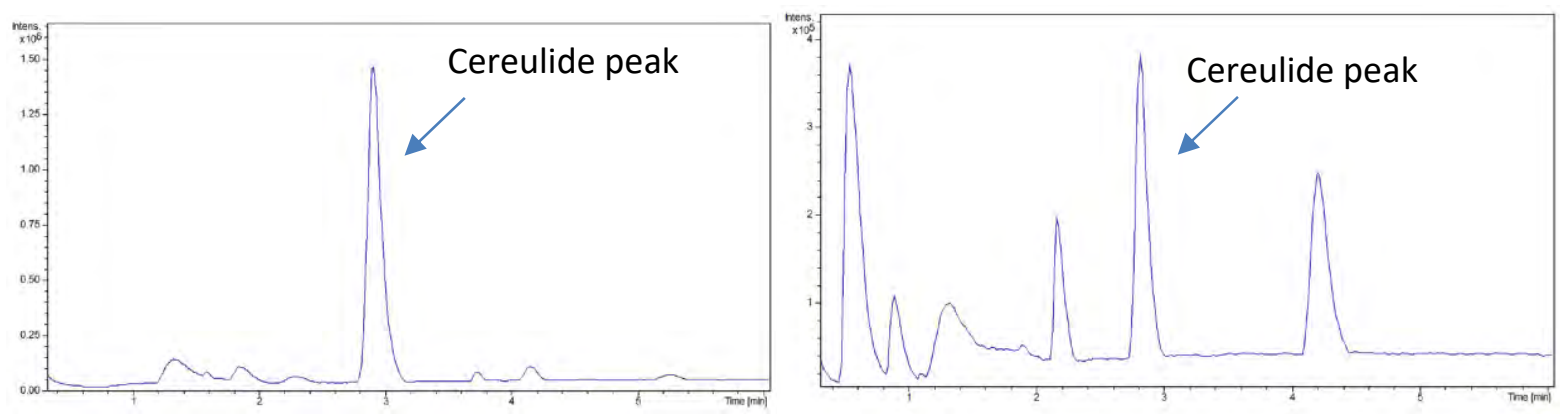

Figure 67. UPLC chromatogram of cereulide samples; Synthetic cereulide (left) and extracted sample (right).

The extracted cereulide samples were purified and concentrated in $\mathrm{EtOH}$ to a final solution containing around $1.2 \mu \mathrm{M}$ of cereulide. 


\section{d) Measuring the fluorescence of extracted samples:}

It must be taken into account that, obtaining cereulide from cultures was a difficult task that not always ends up with a growing culture of the bacteria. In consequence, it usually finished by having solutions of a few milliliters, and many times with concentrations around $1 \mu \mathrm{M}$ or less. Due to this fact, the stock of solution was very low.

To perform the measurements, after the extraction the variation in the signal of emission was measured, similarly to a regular standard addition analysis. Cereulide was added to a solution of $[\mathrm{JG76}]=2 \mu \mathrm{M}$ and $\left[\mathrm{K}^{+}\right]=0.75 \mu \mathrm{M}$ and the fluorescent response was compared between pure ethanol solution and extract solution.

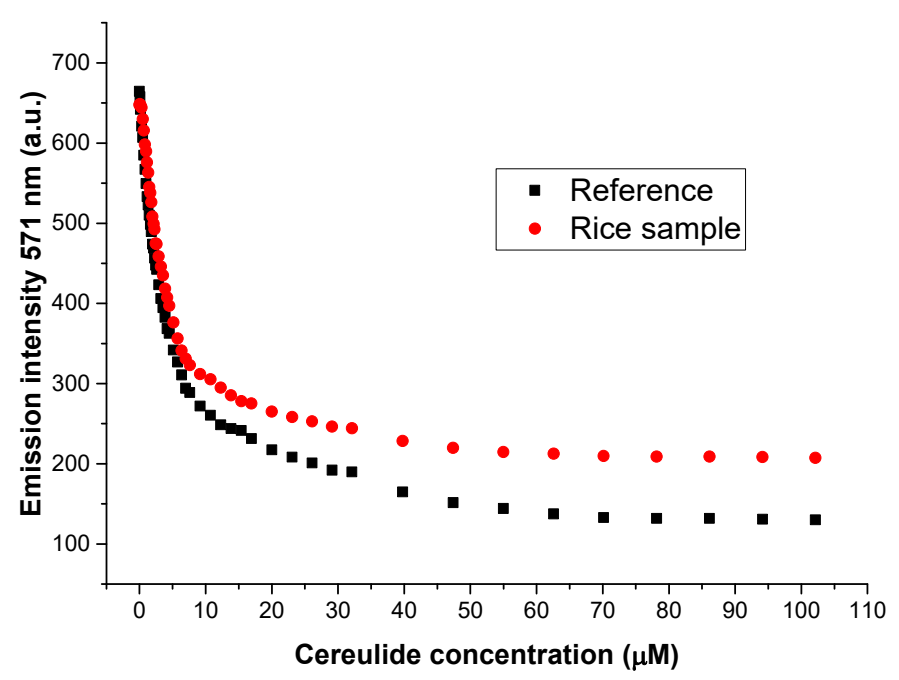

Figure 68. Emission intensity of rice sample (red) and reference (black) with increasing quantities of cereulide. The concentrations were $[\mathrm{JG} 76]=2 \mu \mathrm{M}$ and $\left[\mathrm{K}^{+}\right]=0.75 \mu \mathrm{M} . \lambda_{\mathrm{exc}}=500 \mathrm{~nm}$ and a $\lambda_{\mathrm{em}}=571$ $\mathrm{nm}$.

Figure 68 shows a comparison and results with cereulide samples and EtOH in which synthetic cereulide was added. Sample and reference were done several times the same day in order to minimize the experimental error.

\section{e) Interpretation of the results for a representative experiment:}

Before obtaining any conclusions from fluorescence, one of the most important factors that had influence over the results was the matrix of the rice sample rice. This matrix was supposed to be a mixture of proteins that may affect the measurements.

The only possibility to make reliable fluorescence quantification, or at least an approximation of the presence of cereulide in the samples, was to check how the emission of the probe was affected by the matrix, when the concentration of probe $\left(\mathrm{K}^{+}\right.$constant) and the concentration of potassium (probe constant) changed. To do so, the fluorescence of the matrix was measured without adding JG76 and after adding different concentrations of JG76, to discard interferences matrix-probe, Figure 69. 


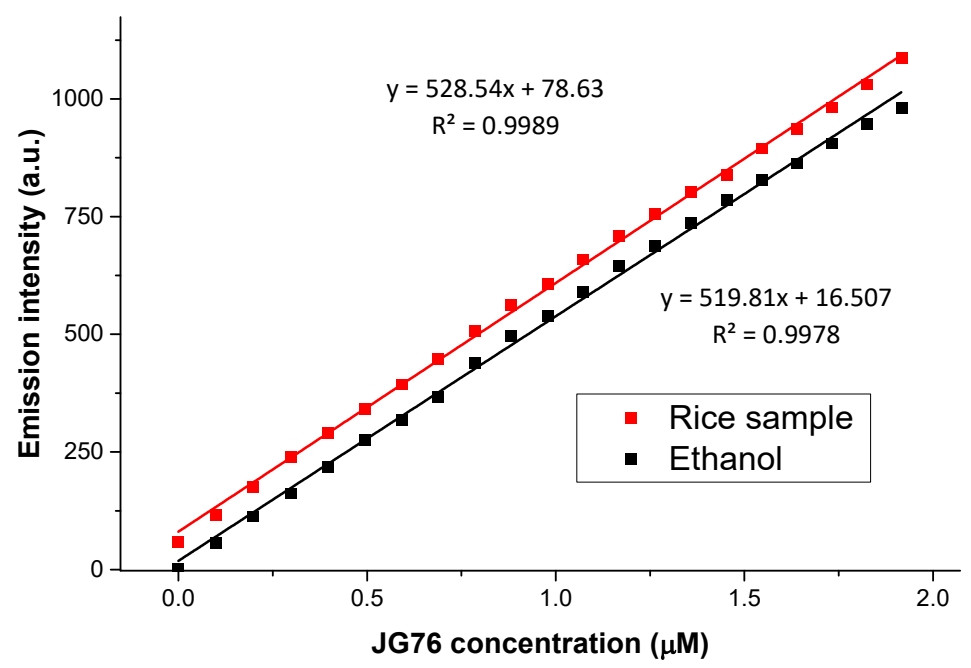

Figure 69. Comparison between ethanol and matrix solutions increasing JG76 concentration in ethanol rice samples and pure ethanol solution. $\lambda_{\mathrm{exc}}=500 \mathrm{~nm}, \lambda \mathrm{m}=571 \mathrm{~nm}$.

In conclusion, the changes in fluorescence were because there was a background fluorescence, which can be calculated when [JG76] $=0$, and it's barely affected by [JG76] at the work concentration.

f) Influence of the matrix correction for the determination of cereulide concentration

The background was subtracted from the titration. Then, due to the quantity of cereulide, the initial value of fluorescence, showed an approximation of the cereulide quantity in the sample. (See examples in Figures 70, 71 and 72)

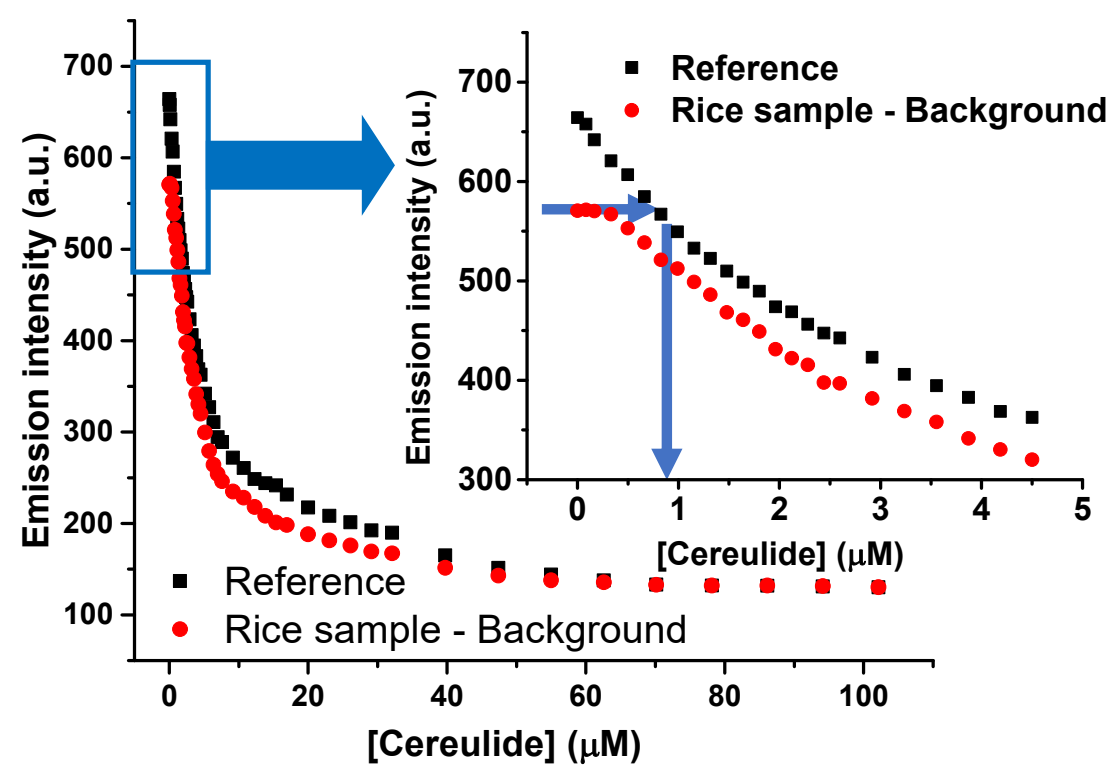

Figure 70. Fluorescent emission of a rice sample versus the reference by titration with increasing quantities of cereulide. The concentration of JG76 was $2 \mu \mathrm{M}$, and concentration of $\mathrm{K}^{+}$was $0.75 \mu \mathrm{M}$.

Cereulide in the rice sample was $1 \boldsymbol{\mu M} . \lambda_{\mathrm{exc}}=500 \mathrm{~nm}, \lambda \mathrm{m}=571 \mathrm{~nm}$. 


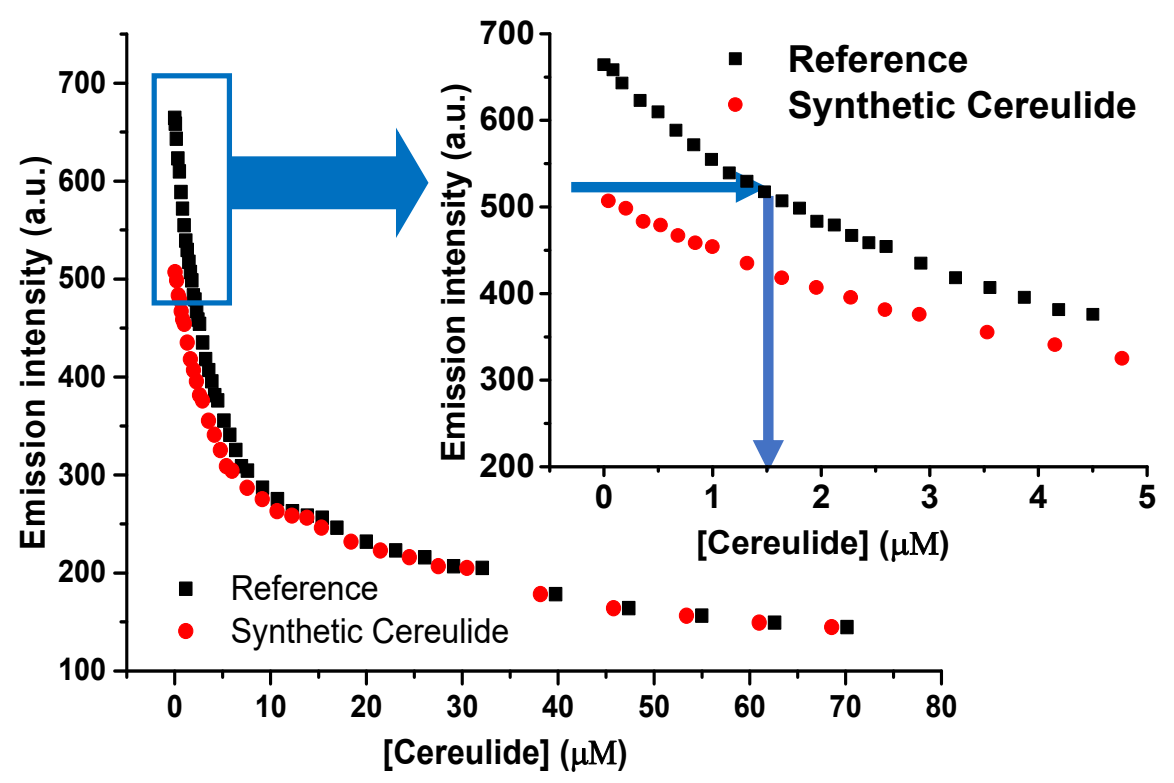

Figure 71. Fluorescent emission of a rice sample spiked with cereulide $1.75 \mu \mathrm{M}$, versus the reference by titration with increasing quantities of cereulide. The concentration of JG76 was $2 \mu \mathrm{M}$, and concentration of $\mathrm{K}^{+}$was $0.75 \mu \mathrm{M}$. $\lambda_{\mathrm{exc}}=500 \mathrm{~nm}, \lambda \mathrm{m}=571 \mathrm{~nm}$.

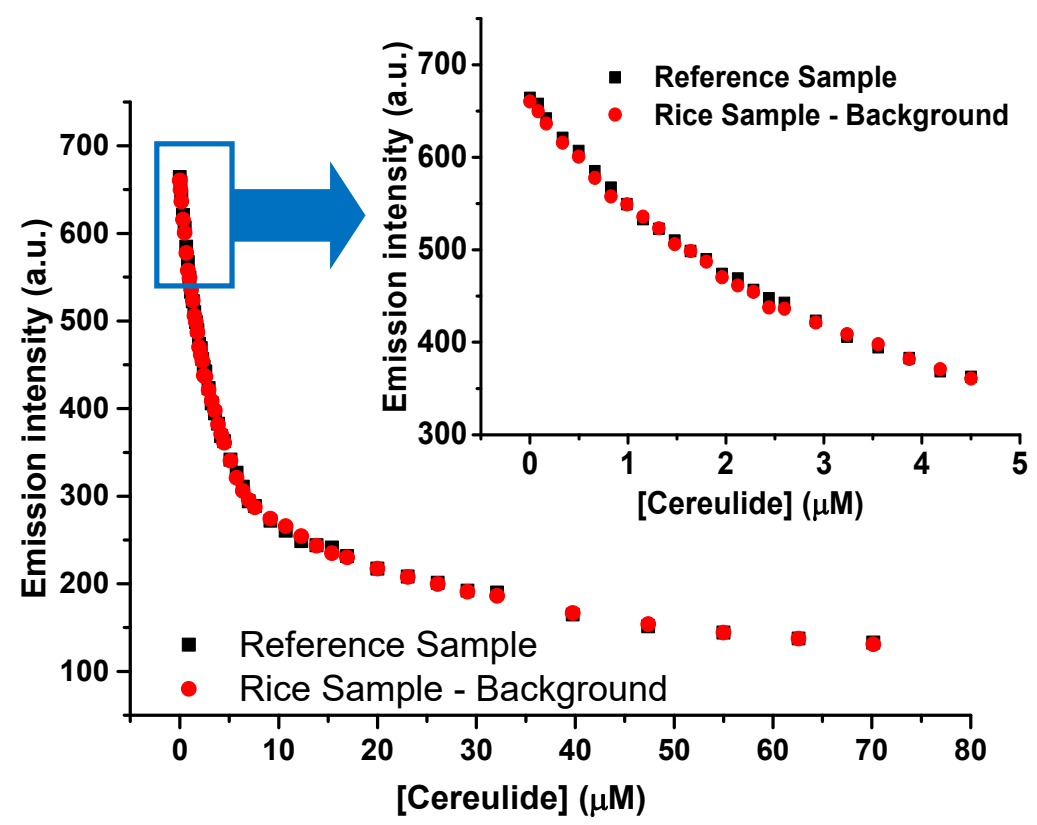

Figure 72. Fluorescent emission of a rice sample with no cereulide versus the reference by titration with increasing quantities of cereulide. The concentration of JG76 was $2 \mu \mathrm{M}$, and concentration of $\mathrm{K}^{+}$ was $0.75 \mu \mathrm{M}$. $\lambda_{\text {exc }}=500 \mathrm{~nm}, \lambda \mathrm{m}=571 \mathrm{~nm}$.

Three cases summarize the possibilities:

- Spiked samples (Figure 71) showed results in agreement with the cereulide added.

- The extracts gave an average of $\mathbf{1 . 0} \pm \mathbf{0 . 2} \boldsymbol{\mu M}$ compared to a sample that was checked to be $1.2 \mu \mathrm{M}$ by mass spectrometry (Figure 70).

- A rice sample, without containing cereulide, gave no difference in the titration (Figure 72). 
For the calculation of cereulide concentration it would not be necessary to perform the whole titration curve to make the calibration, however, it was advisable to ensure that the procedure was reliable. Additionally, the process had to be repeated several times to be reliable.

g) Summary about how to process data:

Once the method was validated it was simplified to 5 steps:

- The cereulide is extracted from the culture by the optimized procedure. ${ }^{62}$

- Acetonitrile solutions are evaporated, extracted in DCM:water and redissolved in ethanol solution. The fluorescence of the background is registered.

- The probe JG76 and $\mathrm{K}\left(\mathrm{CF}_{3} \mathrm{SO}_{3}\right)$ are added (prefixed concentration) to the sample, and the fluorescence intensity is measured.

- The intensity of the background is subtracted to the intensity of JG76 sample.

- If the value obtained was not significantly different from a blank, the quantity of cereulide is considered as 0 . If it is different, further analysis is necessary.

o To obtain a concentration value from fluorescence, it is calculated a linear regression around the result by adding synthetic cereulide or valinomycin.

o Mass spectrometry analysis is required for a higher precision in the determination.

In this case, cereulide was measured indirectly and by fluorescence. The method was more sensitive than mass spectrometry analysis to interferents, so the conditions were controlled and the results carefully evaluated. In any case, measuring by fluorescence is likely to give different results than UPLC-MS. The elution by UPLC gives peaks not only associated to cereulide but for its many possible derivatives. In contrast, the results from fluorescence were associated to their different potassium affinities; therefore, it introduces uncertainty in the measurements and it would not give the same results than pure cereulide. 


\section{BIOLOGICAL DETECTION OF POTASSIUM, COMPARISON BETWEEN IONOPHORES}

\subsection{Potassium transport of cyclic depsipeptides and their comparison}

In Section 9.4 it was calculated that the different potassium ionophores had different affinity for potassium cations. It is also well known from literature that natural ionophores, such as valinomycin or cereulide, have a direct response involving intra-extracellular equilibrium of potassium (See Section 2). For instance, it was studied that whereas valinomycin acts regulating the equilibria, independently of potassium global concentration, in case of cereulide it destabilizes the cells when the concentration of potassium is low. ${ }^{21}$ One of the causes of the different behaviours may be associated to small changes in the structure, which affects the potassium transport. In consequence, the possibility of influencing the potassium transport by changing the structure is worthy of being studied.

The fluorescent assays were performed by the group of Barboiu at Montpellier (France),${ }^{64}$ they used what is called the Fast Filter method, in order to read quasi instantaneously the emission at $510 \mathrm{~nm}$ under alternate excitation at 403 and $460 \mathrm{~nm}^{65}$ The experiments were performed in unilamellar vesicles (LUV):

- Inside the LUV: aqueous solution of $10 \mathrm{mM}$ sodium phosphate, $\mathrm{pH} 6.4,100 \mathrm{mM} \mathrm{NaCl}$.

- Outside the LUV: aqueous solution $(1.85 \mathrm{~mL})$ of $10 \mathrm{mM}$ sodium phosphate, $\mathrm{pH} 6.4,100 \mathrm{mM}$ $\mathrm{KCl}$.

Procedure: Upon initiating the experiment, $0.02 \mathrm{~mL}$ of $1 \mathrm{mM}$ compound in DMSO was added to the measurement cell containing HPTS-loaded LUV (8-hydroxypyrene-1,3,6-trisulfonic acid) after $50 \mathrm{~s}$. Then, $29 \mu \mathrm{L}$ of $0.5 \mathrm{M}$ aqueous $\mathrm{NaOH}$ were added after $100 \mathrm{~s}$ bringing external $\mathrm{pH}$ to 7.4. Finally, maximal changes in dye emission were obtained at the end of each experiment by lysis of the liposomes with detergent $(0.04 \mathrm{~mL}$ of $5 \%$ aqueous Triton $\mathrm{x} 100)$.

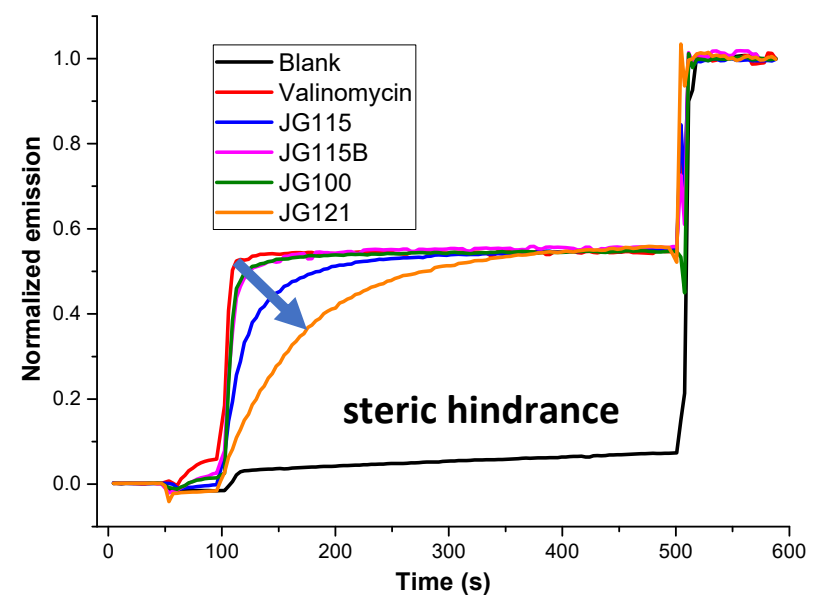

Figure 73. Intensity ratio $\left(\mathrm{I}_{460} / \mathrm{I}_{403}\right)$ over time of solution containing HPTS-loaded LUV followed by a) compound addition (@50 sec.) of valinomycin (for reference), DMSO (blank), JG115, JG115B, JG100 or JG121; b) base addition (@100 sec.) and finally c) Triton for lysis (@100 sec.). All spectra are normalized (max/initial emission)

\footnotetext{
${ }^{64}$ Institut Européen des Membranes, Montpellier, France. The measurements were performed by Yves-Marie Legrand and Li Yuhao.

${ }^{65}$ Using a Perkin Elmer fluorometer and measuring at $20^{\circ} \mathrm{C}$.
} 
JG115 and JG115B, JG100 (Cereulide) and JG121were tested and compared to the reference compound, Valinomycin, Figure 73. The results in terms of potassium transport had the same order than the stability constants:

$$
\mathrm{K}(\text { Valinomycin }) \approx \mathrm{K}(\text { Cereulide }) \approx \mathrm{K}(\mathrm{JG} 115 \mathrm{~B})>\mathrm{K}(\mathrm{JG} 115)>\mathrm{K}(\mathrm{JG} 121)
$$

This fact was really interesting due to the implications in biomedicine, valinomycin is a drug used for controlling processes related with potassium equilibria. Then, a deep study of this kind of ionophores may lead to understand how to modulate transport with different derivatives, which may open a new field for the development of new drugs with straightforward pharmaceutical applications.

\subsection{Intracellular measurements}

Another interesting aspect of the cereulide detection is the visualization of the action of cereulide in living cells. For this purpose, cellular location studies were performed by the group of López-Fanarraga $^{66}$ in HeLa cells (human cervical carcinoma cells), cultured under standard conditions. ${ }^{67}$

- With the fluorogenic probe JG76.

- In a combination of JG76 and Cereulide (JG100).

- With the fluorescent cereulide derivative JG121.

Procedure and data:

* Hela cells were incubated with the probe in the culture medium.

* Cells were fixed with $4 \%$ paraformaldehyde before taking images.

* The nuclei of fixed cells were stained with Hoechst dye (bisbenzimide), fluorescent in blue, before performing high-resolution confocal microscopy imagining.

* All confocal cell images were pseudo-coloured. excitation at $488 \mathrm{~nm}$, and emission in green/red/near red.

For the images of JG76: (18 $\mu \mathrm{M}$ in 1\% DMSO/culture medium (v/v)) - Figure 74

$>$ After 12 hours exposure, the probe stained intracellular vesicular structures that resembled endo-lysosomes (Figure 74 upper).

$>$ After 24 hours exposure, the probe JG76 displayed a pattern clearly localized within cytoplasmic and endosomal membranes (arrows) (Figure 74 middle).

$>$ After $120 \mathrm{~h}$ of staining the probe, JG76 was also localized in the cytoplasmic membrane (green arrow) (Figure 74 lower).

HeLa cells did not display detectable toxicity signs when grown in the presence of the potassium ions fluorescent probe JG76 for up to $120 \mathrm{~h}$.

${ }^{66}$ M. López-Fanarraga and Eloisa López Lavado from Universidad de Cantabria, Santander (Spain).

${ }^{67}$ a) L. Rodriguez-Fernandez, R. Valiente, J. Gonzalez, J. C. Villegas, M. L. Fanarraga, ACS Nano 2012, 6, 6614-6625; b) L. García-Hevia, R. Valiente, R. Martín-Rodríguez, C. Renero-Lecuna, J. González, L. Rodríguez-Fernández, F. Aguado, J. C. Villegasa, M. L. Fanarraga, Nanoscale 2016, 8, 10963-10973; c) B. Sanz, M. P. Calatayud, T. E. Torres, M. L. Fanarraga, M. R. Ibarra, G. F. Goya, Biomaterials 2017, 114, 62-70. 
nucleus/JG76

$12 \mathrm{~h}$ exposure

nucleus/JG76

$24 \mathrm{~h}$ exposure

nucleus/JG76

$120 \mathrm{~h}$ exposure
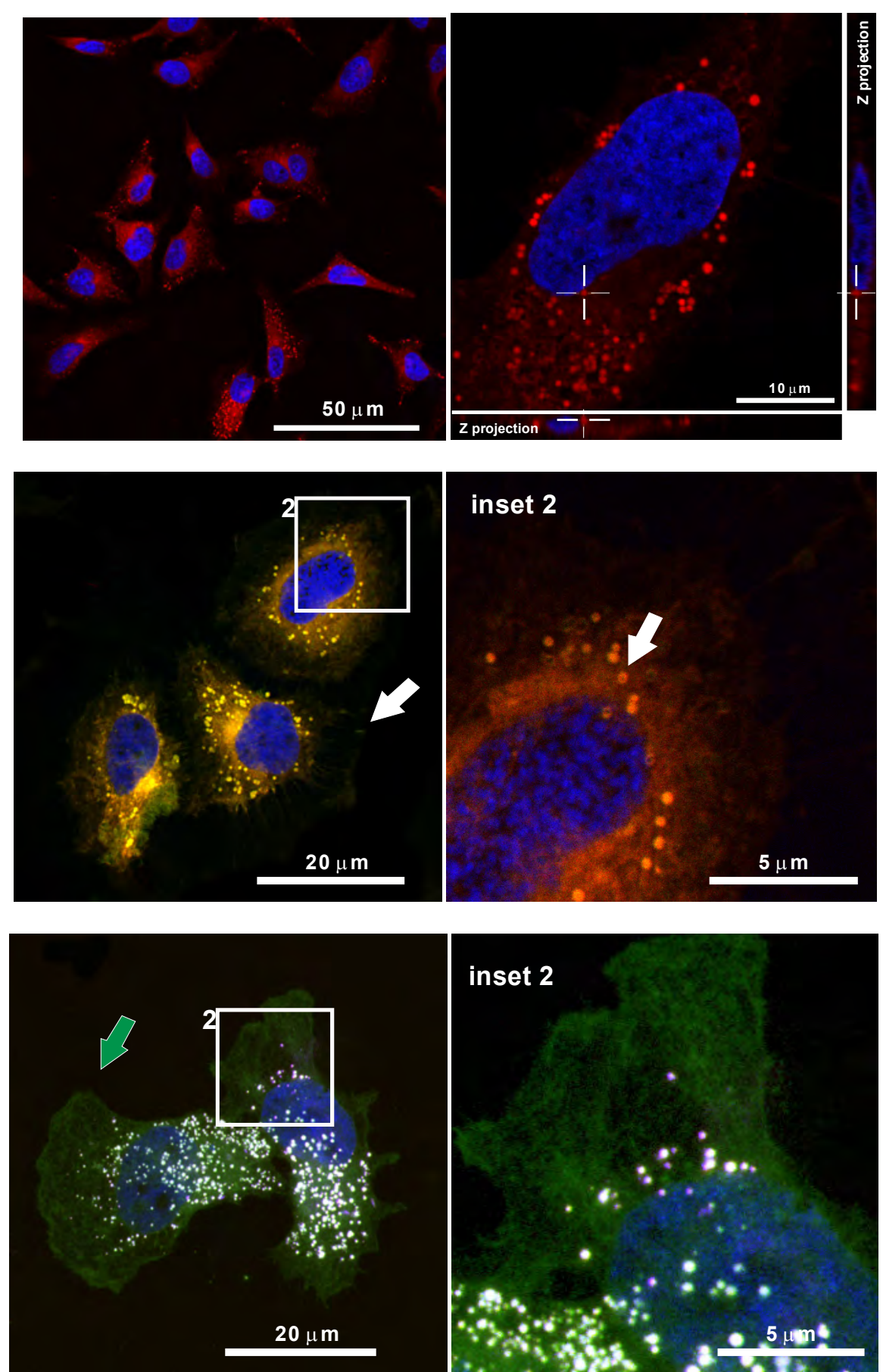

Figure 74. Confocal microscopy projection images of probe JG76 in HeLa cells $12 \mathrm{~h}$ after staining. (Upper). $24 \mathrm{~h}$ after staining (Middle), $120 \mathrm{~h}$ after staining (Lower). Nuclei were stained with Hoechst dye (blue channel). The different emissions were obtained by exciting the probe sequentially with the 488, 562 and $638 \mathrm{~nm}$ lasers. Different fluorophore emissions are pseudo-coloured in their respective wavelengths $($ green $=500-550 ;$ red $=570-620 ;$ purple $=662-737 \mathrm{~nm})$. 


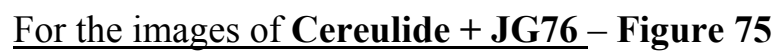

HeLa cells, cultured under standard conditions, were incubated with the probe $(18 \mu \mathrm{M})$ for two hours.

$>$ After 2 hours of exposure to JG76, the cells were exposed to synthetic cereulide ( $35 \mu \mathrm{M})$ for other 2 hours (Figure 75 upper left).

$>$ After 2 hours exposure to JG76 and cereulide, the near red emission of endosomes diminished (less green in pseudo-colour) and the staining of the cytoplasmic membrane also diminished. The cytosol appeared more stained with the probe. (Figure 75 upper right)

$>24$ hours after the addition of cereulide to the cells, the cellular viability decreased significantly and several cells appeared wrinkled, showing membrane blebbing and cytosol vacuolization.

nucleus/JG76

$2 \mathrm{~h}$ exposure JG76

(left)

$+2 \mathrm{~h}$ exposure cereulide

(right)
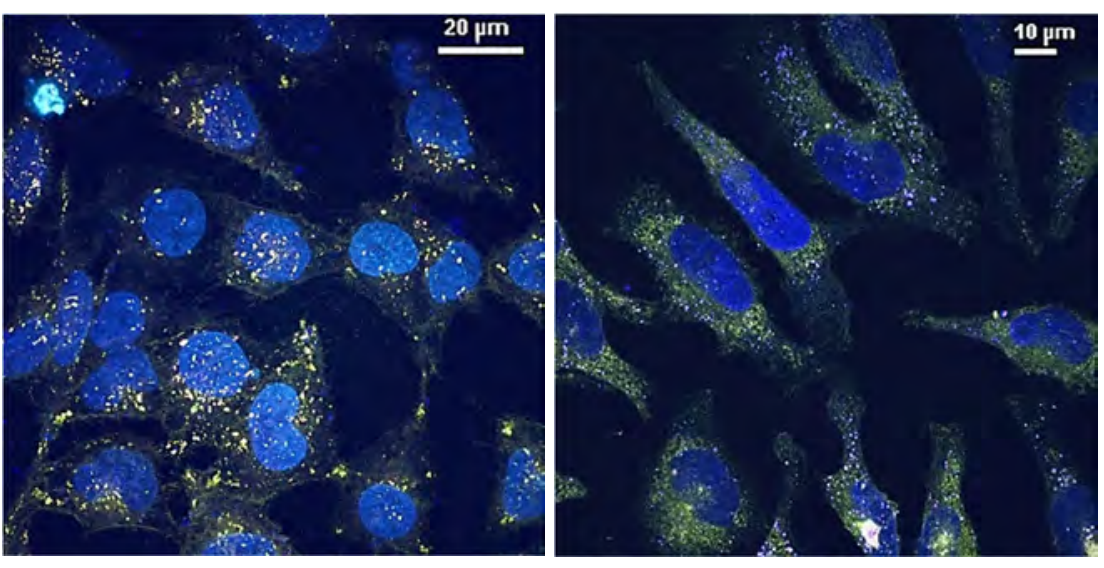

nucleus/JG76

$24 \mathrm{~h}$ exposure
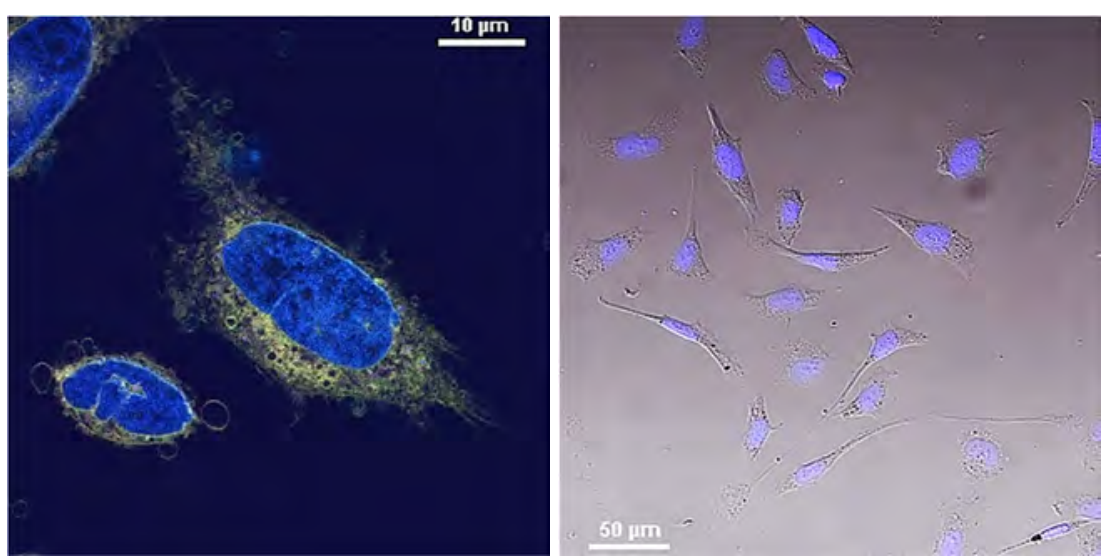

Figure 75. Confocal microscopy projection images obtained by exciting the probe sequentially with the $488 \mathrm{~nm}$ laser. Nuclei are stained with Hoechst dye (blue channel). Probe JG76 in HeLa cells. $2 \mathrm{~h}$ after staining (Upper left), $2 \mathrm{~h}$ after addition of cereulide on previous sample (Upper right). $24 \mathrm{~h}$ after

addition of cereulide on first sample (Lower left), showing large dark vacuoles. Fluorescence microscopy image of HeLa cells $12 \mathrm{~h}$ after addition of cereulide, used as reference (Lower right).

From the images, it was clear that the initial fluorescence of JG76, complexed with potassium ions in potassium rich structures of the cells, was quenched when there was cereulide presence in HeLa cells, leaving only the residual fluorescence of JG76 in the membranes of the cells, evidencing the action of the cereulide. Even more, the morphological changes of the HeLa cells, experienced by the cereulide action on time were easily followed by the residual fluorescence of JG76 on membranes; changes such as the formation of large vacuoles and membrane blebbing, thus, proving the efficiency of the probe JG76, as a cereulide chemical sensor by potassium ions complexation displacement. 


\section{For the images of JG121:- Figure 76}

In Figure 76, it may be observed that probe JG121 displayed an apparent pH sensitive metachromasy, where late lysosomes displayed excitation and emission preferences more in the far red, compared to endosomes, that are shown displaying a green emission. Early to late endosome maturation was accompanied by a rainbow colour change (white arrow).

nucleus/JG121

$120 \mathrm{~h}$ exposure
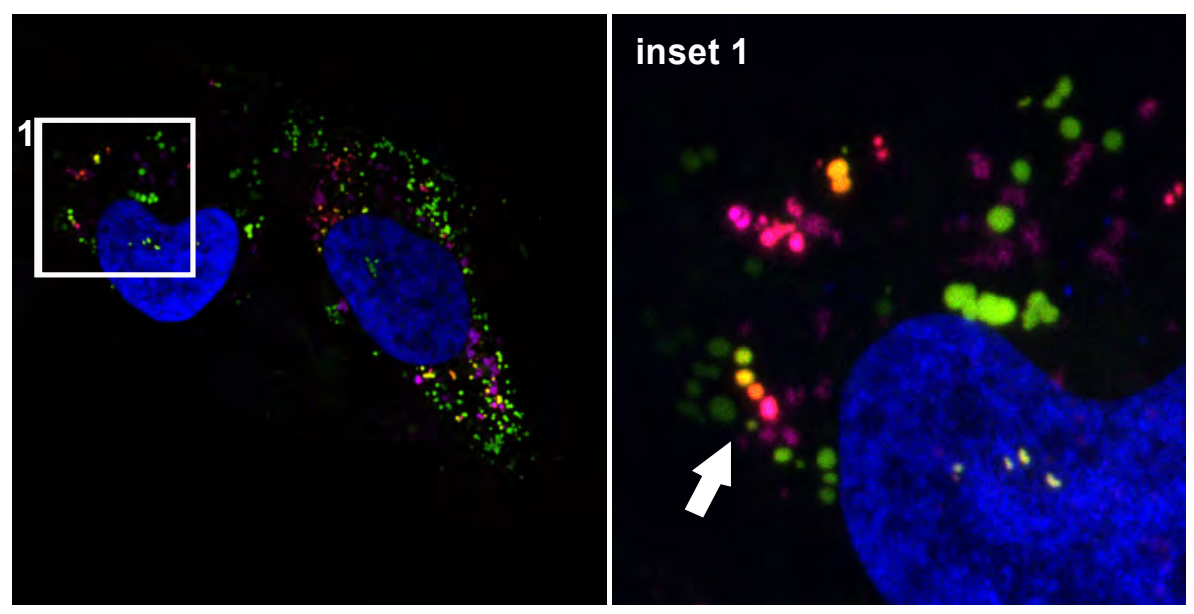

Figure 76. Confocal microscopy projection images of probes JG121 obtained exciting the probe sequentially with the 488, 562 and $638 \mathrm{~nm}$ lasers. Different fluorophore emissions were pseudocoloured in their respective wavelengths (green $=500-550 ;$ red $=570-620 ;$ purple $=662-737 \mathrm{~nm}$ ).

Nuclei were stained with Hoechst dye (blue channel).

\section{Summary:}

Despite the toxicity shown by cereulide in other types of cells, HeLa cells did not display detectable toxicity signs when grown in the presence of either the fluorescent cereulide, JG121, or the potassium fluorescent probe JG76 for up to $120 \mathrm{~h}$. Therefore, the fluorescent or fluorogenic probes can be considered as useful tools for the visualization of cereulide in live cells as well as the localization of highly polar potassium rich structures, in comparison to low polarity membrane structures, from live cells. ${ }^{68}$

In addition to these experiments, further research is being performed for cereulide and its derivatives, using cell cultures more sensitive to changes in potassium equilibria (pancreatic cells). Two objectives are aimed, comparing the effect of using different potassium ionophores (toxicity and specificity) and localization of them in cellular media, by the use of fluorescent colorants.

68 T. Hirata, T. Terai, H. Yamamura, M. Shimonishi, T. Komatsu, K. Hanaoka, T. Ueno, Y. Imaizumi, T. Nagano and Y. Urano, Anal. Chem. 2016, 88, 2693-2700. 


\section{SYNTHESIS OF A MATERIAL FOR DETECTION OF LEAD (II)}

The initial purpose was modifying a polymer with a derivative from JG76 for the development of materials with the same sensitivity that the soluble probe.

\subsection{Synthesis of a PMI-crown ether derivative containing a free amino group}

So as to join the PMI+Receptor to a material (JG76+polymer), the most straightforward and simple procedure was the modification of the imide group to a substituent with enough reactivity to be anchored to a material.

In doing so, the procedure of re-imidization (Chapter 2) was adapted to JG76 (Figure 87).
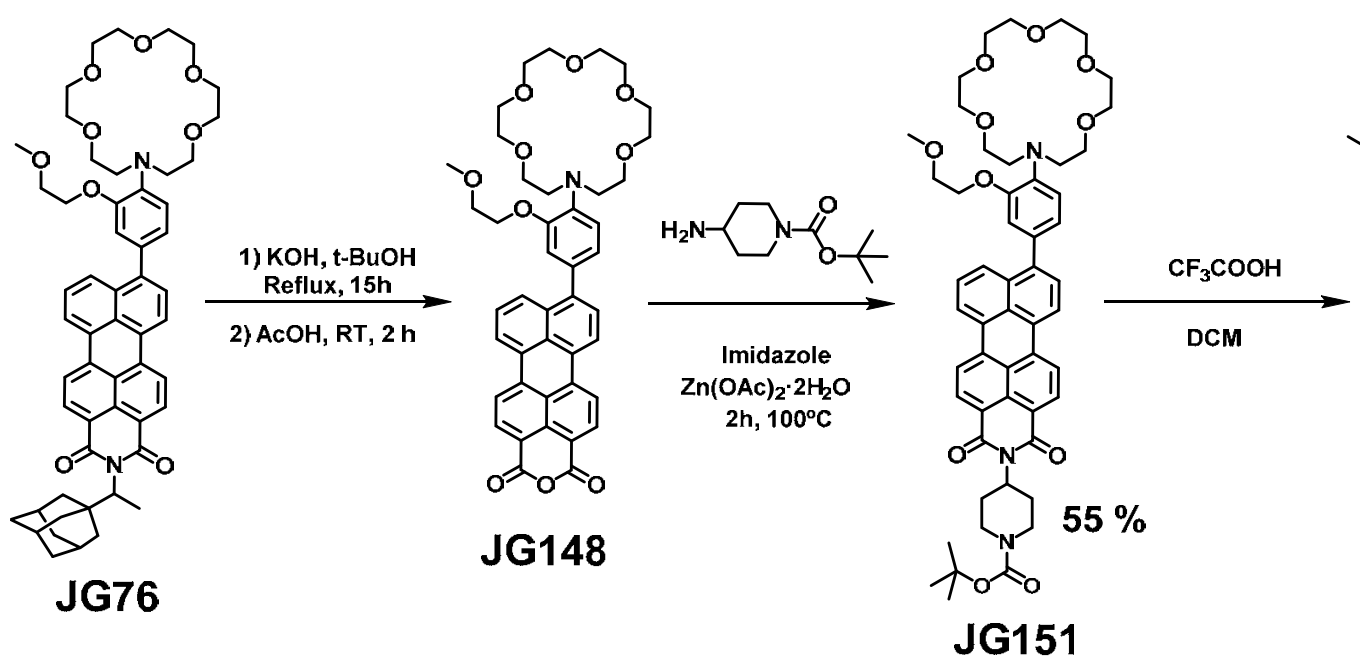

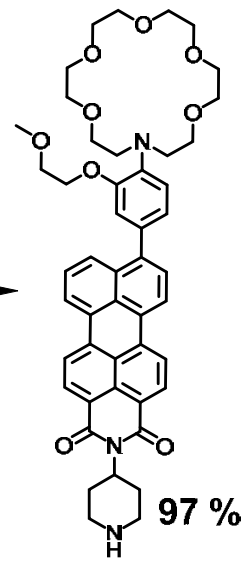

JG151d

Figure 77. Synthesis of the free amine soluble probe JG151d from JG76.

The procedure started by saponification of JG76 in concentrated $\mathrm{KOH}$ in $t$-BuOH under reflux for 2 hours. Once the reaction time had passed, the product was quenched with acetic acid at room temperature, filtered, dissolved in DCM and washed with water. The amine group of interest, in this case 4-amine-1-bocpiperidine was introduced by dissolving all the components in imidazole in the presence of zinc acetate, heating at $100^{\circ} \mathrm{C}$ for two hours. The product was purified by column chromatography and deprotected by using a strong acid in solution. 


\subsection{Composition and synthesis of the modified polymer}

The monomers that formed the polymer and the photoinitiator (DMPA), Figure 78, gave the polymer shown in Figure 79, that was modified with JG151d to obtain JG151dp (Figure 80):<smiles>COC(OC)(C(=O)c1ccccc1)c1ccccc1</smiles>

2,2-dimethoxy-1,2-diphenylethan-1-one<smiles>C=CN1CCCC1COC(=O)C1CCCCC1</smiles><smiles>C=C(C)C(=O)OC</smiles><smiles>C=CCCCBr</smiles><smiles>C=C(C)C(=O)OC(=O)C(=C)C</smiles>

1-vinylpyrrolidin-2-one methyl methacrylate 5-bromopent-1-ene methacrylic anhydride

Figure 78: Monomers and photoinitiator of the synthesised polymer.

49VP/49MM1/pVBr//MA1/1.56Photoi (DMPA)

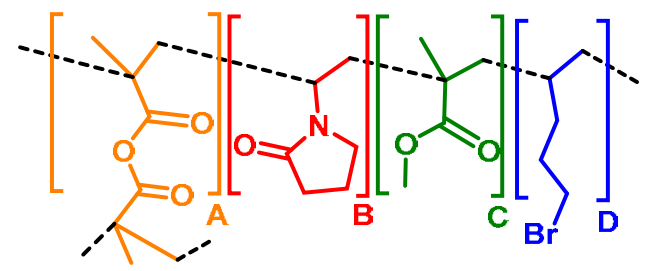

$(A / B / C / D=1 / / 49 / 49 / 2)$

Figure 79: Composition of the polymer without the probe (JGIF).

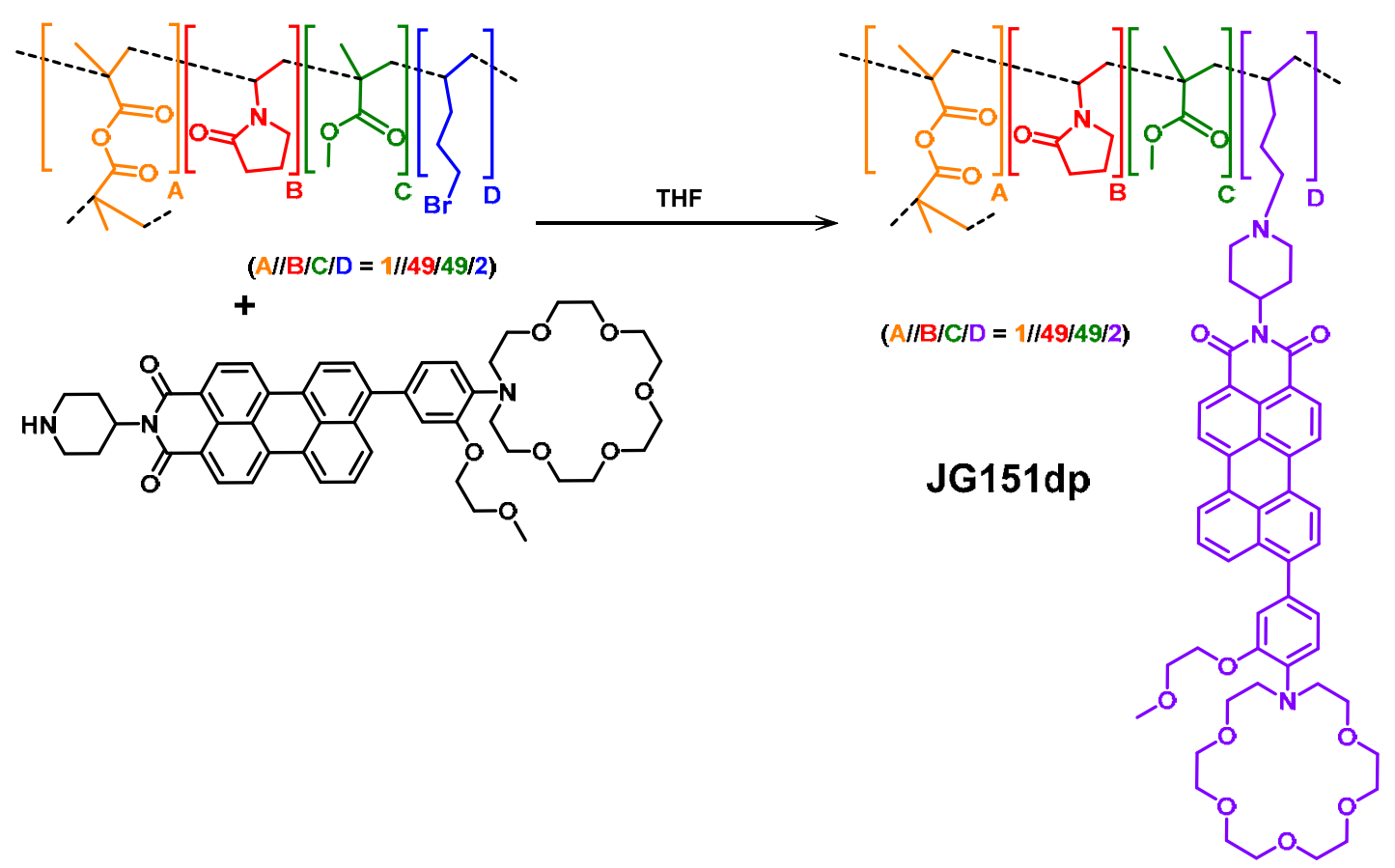

Figure 80: Reaction scheme for the modification of the film with JG151d to JG151dp. 
The synthesis of the polymer via photopolymerization with DMPA provided a colourless film (Figure 79) ${ }^{69}$ with high swelling in water $(50 \%)$ and even more in THF $(250 \%)$. The pristine film was introduced in a THF solution of JG151d $(10 \times 10 \mathrm{~cm}$ and $3 \mathrm{mg}$ of JG151d in $340 \mathrm{ml}$ of THF) and remained at $50^{\circ} \mathrm{C}$ for 24 hours. This process allowed to obtain a pink film (Figure 81) that was washed with $30 \mathrm{ml}$ of THF, $\mathrm{MeOH}$ and water, four times with each solvent, until the solution became colourless.

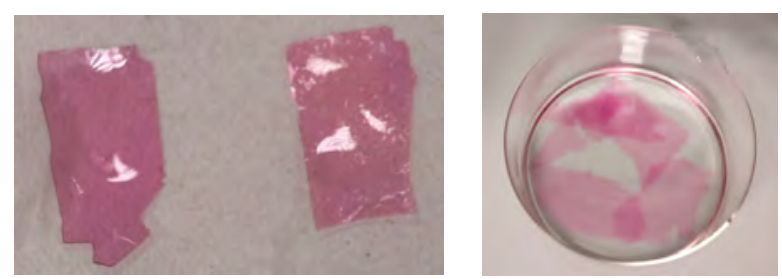

Figure 81. Pictures of the modified polymer JG151dp dried (left) and in water solution (right).

The reaction was checked for possible absorption processes by washing the polymer to check that there was not remaining colour in solution. Additionally, a blank experiment with JG76 instead of JG151d was performed and no change in colour was observed in the polymer.

\section{Characterization:}

The synthesized polymer was characterized by TGA, IR, its characteristic absorption and fluorescent spectra (Figure 82) were registered and the composition on the surface was determined by EDX. From the TGA and IR the characteristic decomposition band at $420^{\circ} \mathrm{C}$ and the IR bands were obtained; although not significative difference was observed in the spectra or in the temperature of decomposition after the modification of the polymer. (See Experimental Appendix 3)
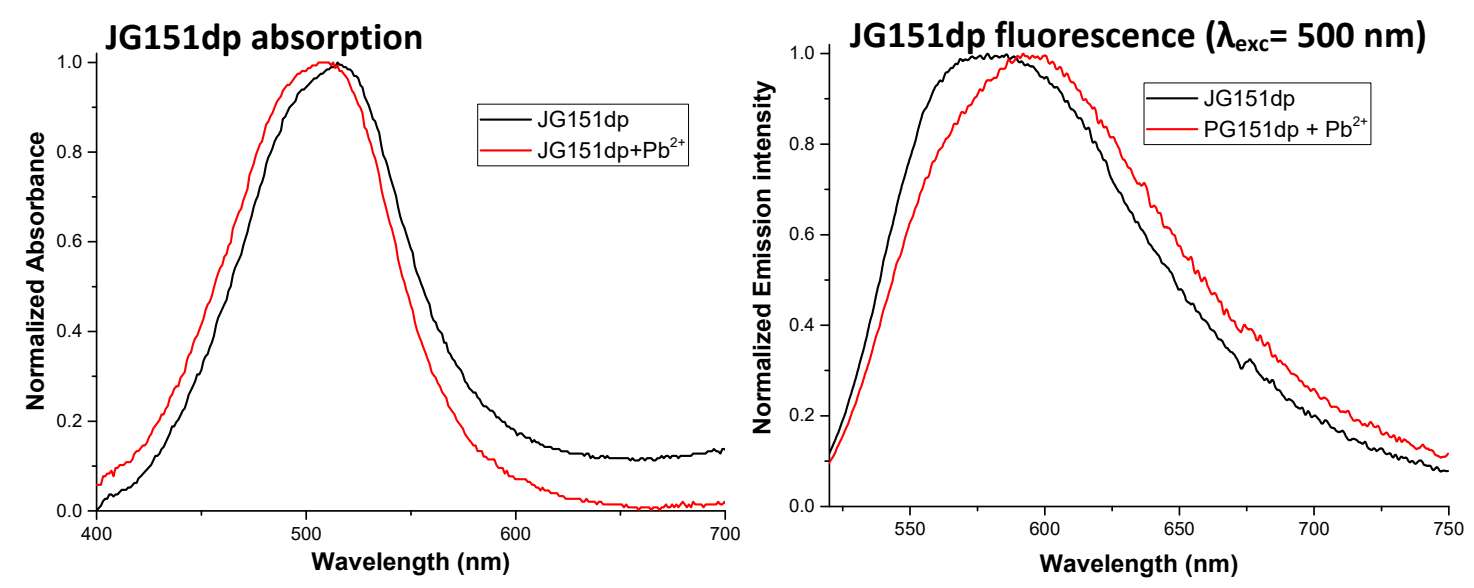

Figure 82. Absorbance of JG151dp (left) and fluorescence with $\lambda_{\mathrm{exc}}=500 \mathrm{~nm}$ (right).

To test the ability of the material to detect $\mathrm{Pb}(\mathrm{II})$, extensive SEM and EDX analyses were performed in pristine samples and samples submerged in $\mathrm{Pb}(\mathrm{II})$ solutions. From different sections observed by SEM (Figure 84), the EDX analysis (Figure 85) gave the elemental composition of the films on the surface. From that, the proportions were calculated for $\mathrm{C} / \mathrm{O} / \mathrm{Pb}$. The experiments were performed for 4 pieces of polymer (Figure 83), one of the non-functionalized starting material (named as JGIF), one after soaking the starting material in a $1 \mathrm{mM} \mathrm{Pb}\left(\mathrm{ClO}_{4}\right)_{2}$ solution in water (once dried) and the other two for pristine JG151dp and when placed in the presence of $\mathrm{Pb}(\mathrm{II})$, respectively.

\footnotetext{
${ }^{69}$ The synthesis of the pristine polymer was performed by Saúl Vallejos, from the Group of Polymers of Burgos University.
} 


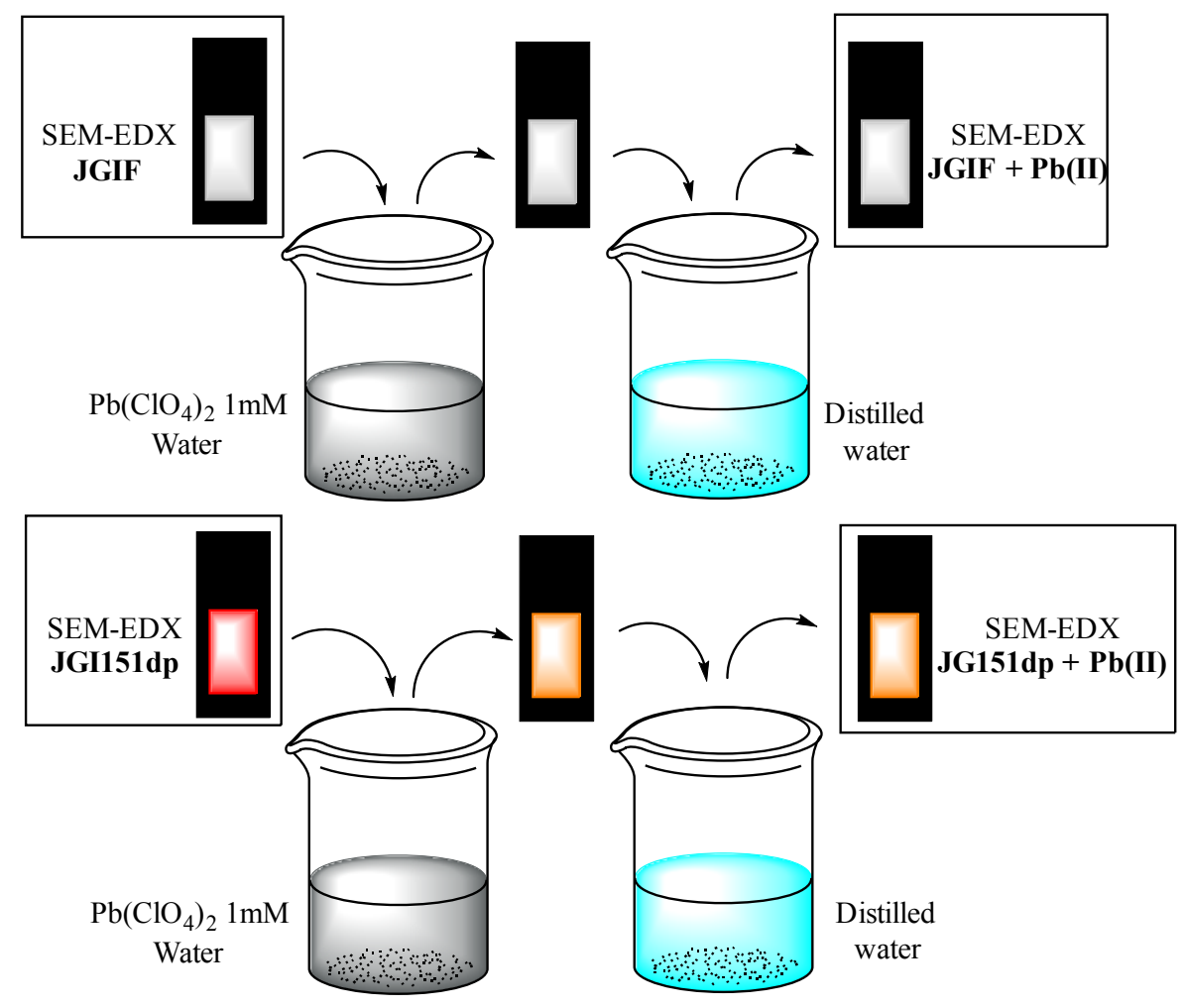

Figure 83. Samples analyzed by SEM-EDX.

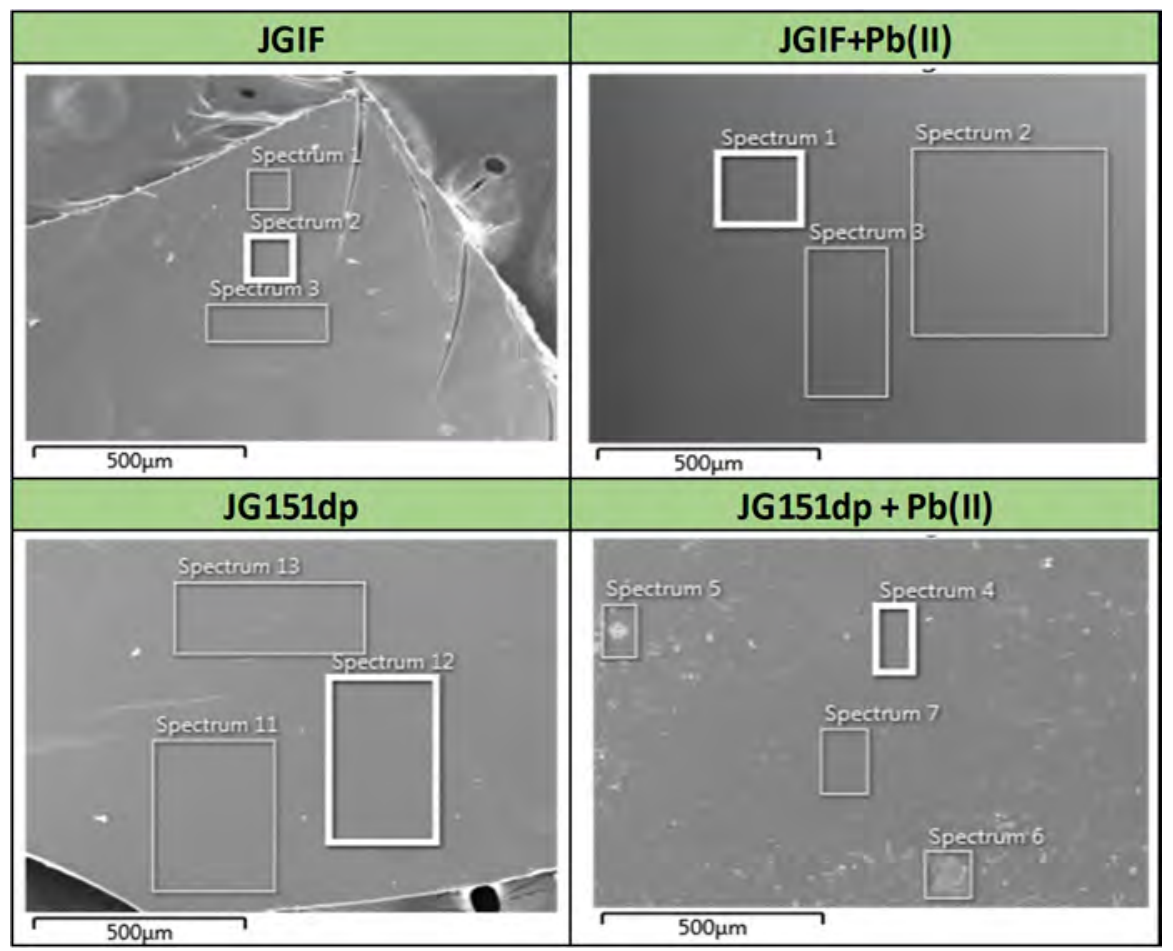

Figure 84. SEM images of the studied polymers, JGIF and JG151dp. 


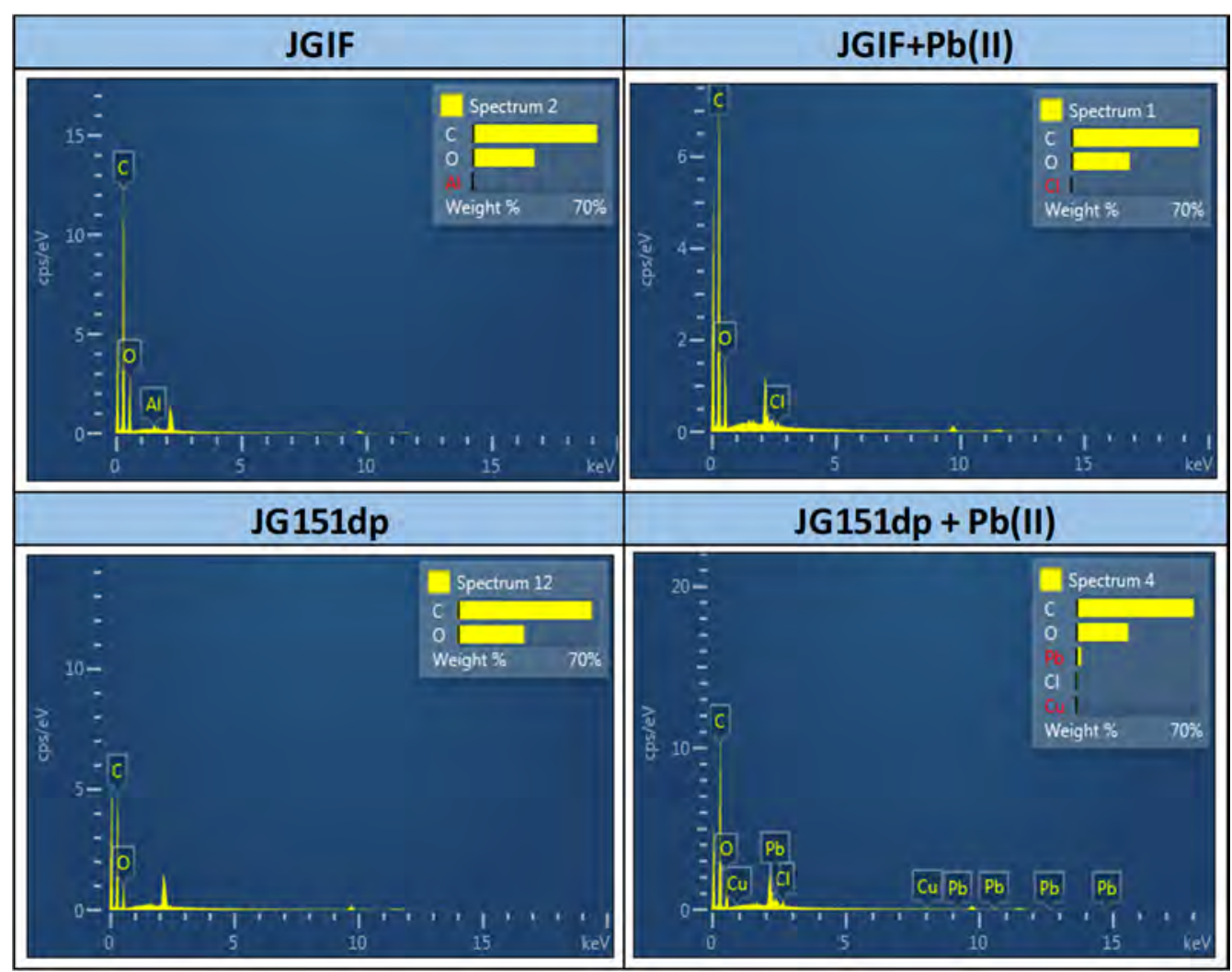

Figure 85. Composition obtained from the EDX representation of the SEM images of the studied polymers.

\begin{tabular}{|l|c|c|c|}
\hline \multicolumn{4}{|c|}{ Elemental proportions (weight) } \\
\hline & $\mathbf{O} / \mathbf{C}$ & $\mathbf{P b} / \mathbf{O}$ & $\mathbf{P b} / \mathbf{C}$ \\
\hline Theoretical JGIF & 0.359 & 0 & 0 \\
\hline Theoretical JG151 dp & 0.343 & 0.077 & 0.026 \\
\hline JGIF & 0.5 & 0 & 0 \\
\hline JGIF + Pb(II) & 0.463 & 0 & 0 \\
\hline JG151dp & 0.499 & 0 & 0 \\
\hline JG151dp + Pb(II) & 0.442 & 0.094 & 0.042 \\
\hline
\end{tabular}

Figure 86. Table of proportions $\mathrm{O} / \mathrm{C} / \mathrm{Pb}$.

$\mathrm{Pb}$ was only detected for $\mathbf{J G 1 5 1 d p}+\mathbf{P b}$ (II), and the proportions of $\mathrm{Pb} / \mathrm{O}$ were very close to the theoretical proportions, what is more, no $\mathrm{Pb}$ (II) was detected in any of the other samples (Figure 86). However, the method presented important limitations. The samples had to be covered with gold, and the EDX analysis gave relative composition within the penetration depth of the laser (usually around $2 \mu \mathrm{m})$.

\subsection{JG151dp ions test}

Pieces of polymer were introduced in different cations solutions in water or buffer solutions $(0.5$ $\mathrm{mL}, 50 \mu \mathrm{M}$ ) and pictures were taken (Figures 87, 88, 89 and 90). In the same way observed for molecular probes solutions (JG76), not using buffered solutions led to response to acidic $\mathrm{pH}$ and Lewis acids. 


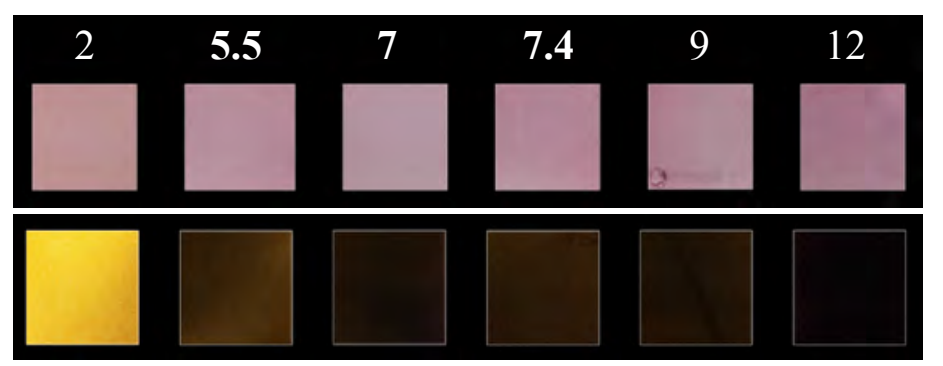

Figure 87. JG15dp in buffer water HEPES buffer solution $(10 \mathrm{mM})$ at different $\mathrm{pH}$ values, under visible (up) and $366 \mathrm{~nm}$ UV light (down).

In HEPES $10 \mathrm{mM}$ solutions, the response to different analytes was studied:

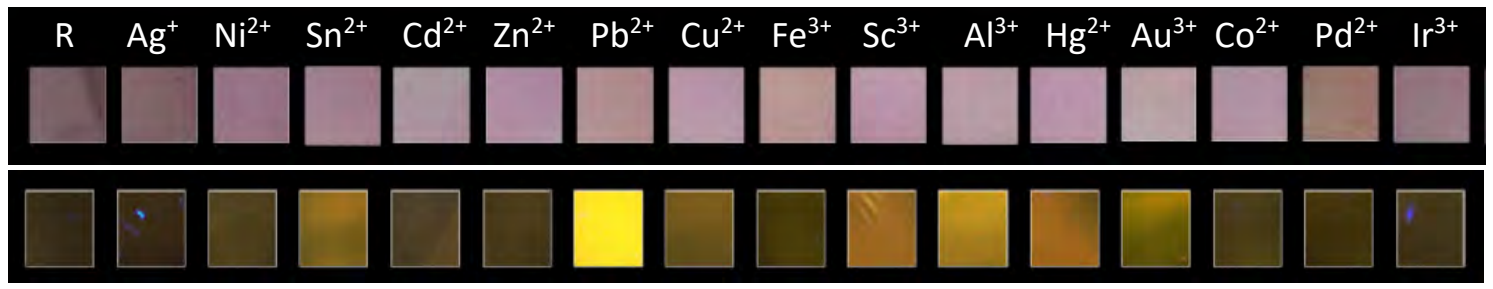

Figure 88. JG151dp in water, in presence of different cations (50 $\mu \mathrm{M})$ under visible light (up) and under UV light (down). $0.2 \mathrm{~mL}$ of $\mathrm{pH}$ buffer 7 HEPES $10 \mathrm{mM}$. The counterions were noncoordinative species like $\mathrm{CF}_{3} \mathrm{SO}_{3}{ }^{-}, \mathrm{ClO}_{4}{ }^{-}$and $\mathrm{Cl}^{-}$in case of $\mathrm{Pd}^{2+}$.

The response to anions was negative by testing $\mathrm{F}^{-}-\mathrm{Cl}^{-}-\mathrm{Br}^{-}-\mathrm{I}^{-}-\mathrm{BzO}^{-}-\mathrm{NO}_{3}^{-}-\mathrm{H}_{2} \mathrm{PO}_{4}^{-}-\mathrm{HSO}_{4}^{-}-\mathrm{AcO}^{-}$ $-\mathrm{CN}^{-}-\mathrm{SCN}^{-}$, being the cation $\mathrm{Bu}_{4} \mathrm{~N}^{+}$.

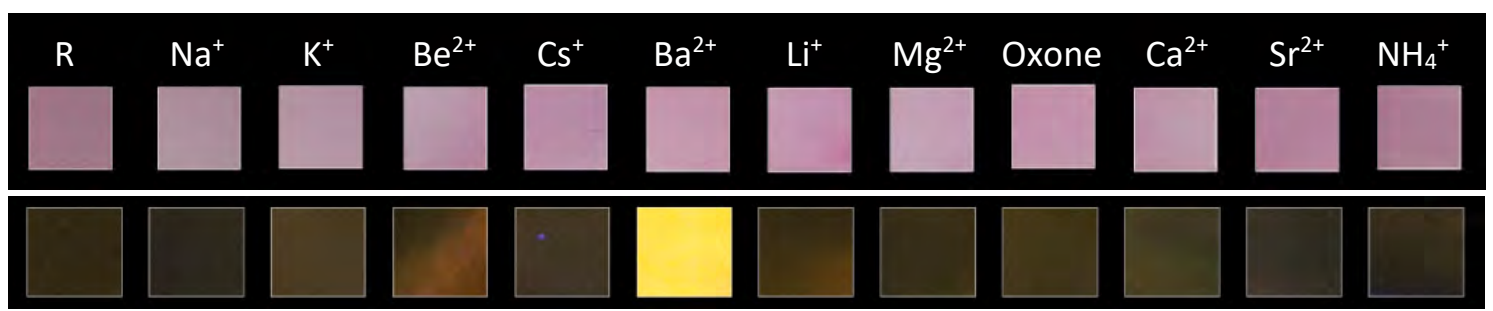

Figure 89. JG151dp in water, in the presence of different species $(50 \mu \mathrm{M})$ under visible light (up) and under UV light (down). Ions sequence: Reference - Water- $\mathrm{Li}^{+}-\mathrm{Na}^{+}-\mathrm{K}^{+}-\mathrm{Rb}^{+}-\mathrm{Cs}^{+}-\mathrm{Mg}^{2+}-\mathrm{Ca}^{2+}-$ $\mathrm{Sr}^{2+}-\mathrm{Ba}^{2+}-\mathrm{NH}_{4}^{+}$. Counterions: $\mathrm{ClO}_{4}^{-}, \mathrm{CO}_{3}^{2-}\left(\mathrm{Cs}^{+}\right), \mathrm{NO}_{3}^{-}\left(\mathrm{Rb}^{+}\right)$

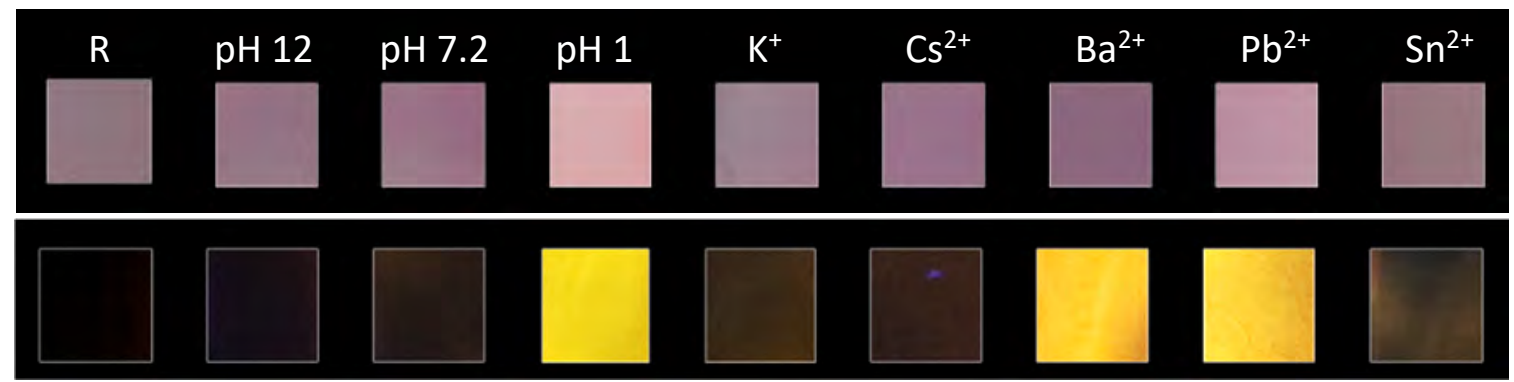

Figure 90. JG151dp in water at buffer $\mathrm{pH} 7$ HEPES solutions $(10 \mathrm{mM})$, changing the $\mathrm{pH}$ and in presence of cations at controlled $\mathrm{pH}$ 7. Under visible (up) and $366 \mathrm{~nm}$ UV light (down). Sequence: Reference - pH 12 - pH 7.2 - pH 1 - K( $\left(\mathrm{CF}_{3} \mathrm{SO}_{3}\right)-\mathrm{Cs}\left(\mathrm{CO}_{3}\right)_{2}-\mathrm{Ba}\left(\mathrm{NO}_{3}\right)_{2}-\mathrm{Pb}\left(\mathrm{ClO}_{4}\right)_{2}-\mathrm{Sn}\left(\mathrm{ClO}_{4}\right)_{2}$ 


\section{Characteristics of the polymer JG151dp:}

- JG151dp is very sensitive to extreme $\mathrm{pH}$.

- Cations with Lewis acid behavior result in increased fluorescence if the $\mathrm{pH}$ was not controlled.

- Potassium cations did not increase fluorescence of the polymer, in contrast with the soluble probe JG76.

- The fluorescence increased under extreme acidic $\mathrm{pH}$ or in presence of $\mathrm{Ba}^{2+}$ or $\mathrm{Pb}^{2+}$, therefore, the $\mathrm{pH}$ had to be controlled.

JG151dp emission was also registered quantitatively in the presence of the most representative species in solution and compared before and after adding lead cation, Figure 91.

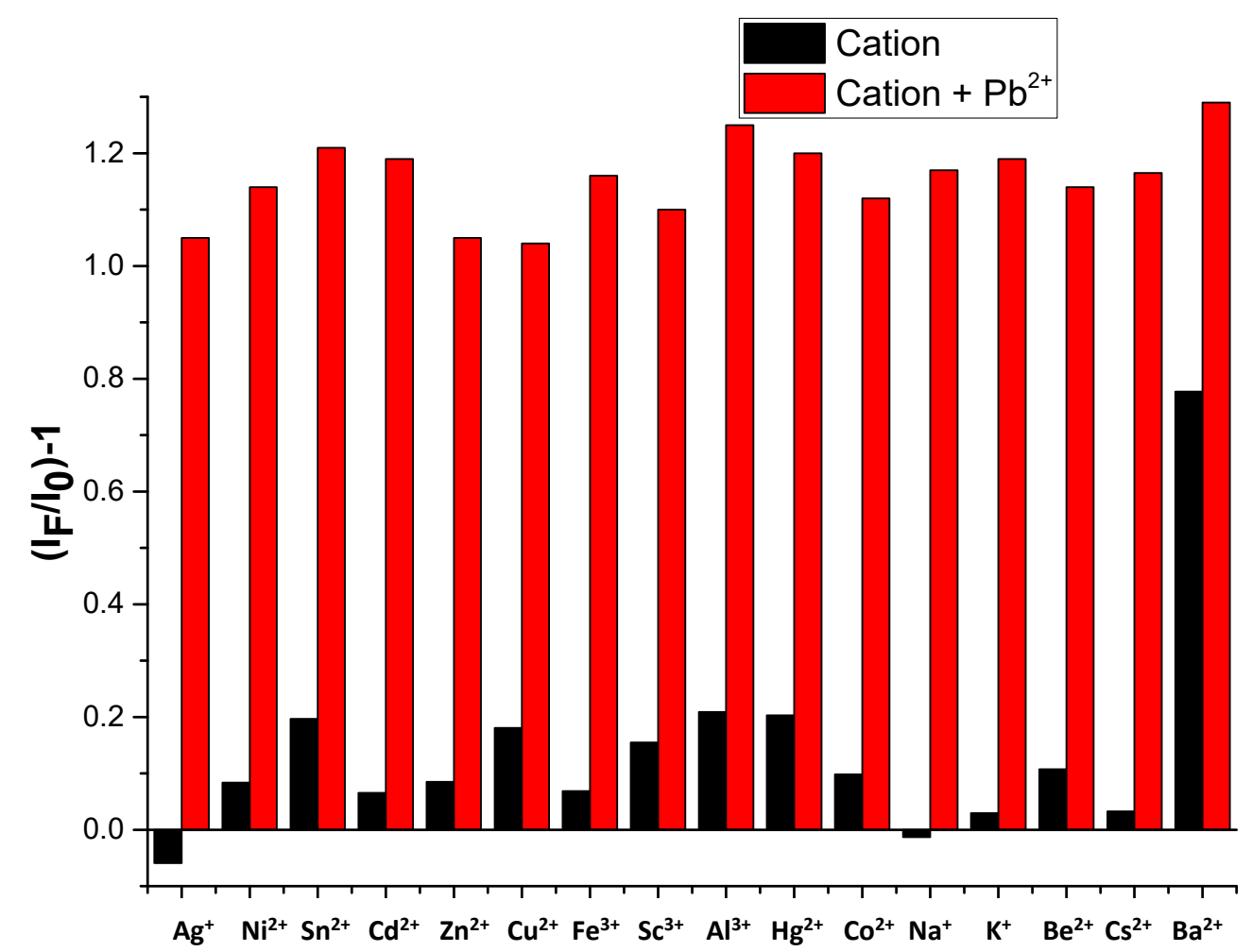

Figure 91. Fluorescent emission increase in the presence of different cations $(50 \mu \mathrm{M})$, dissolved in $\mathrm{pH}$ 7 HEPES water solutions $(10 \mathrm{mM}) .\left(\lambda_{\mathrm{exc}}=500 \mathrm{~nm}, \lambda_{\mathrm{em}}=593 \mathrm{~nm}\right)$.

\subsection{Fluorescence quantum yields $\left(\Phi_{\mathrm{F}}\right)$ and decay lifetimes $(\tau)$ of JG151dp}

The polymer fluorescence was measured dry, before and after being in a concentrated $\mathrm{Pb}\left(\mathrm{ClO}_{4}\right)_{2}$ solution in water, obtaining the next fluorescence quantum yields (calculated with an integration sphere):

$$
\begin{aligned}
& \Phi_{\text {F JG151dp }}=0.22 \pm 0.02 \\
& \Phi_{\text {F JG151dp }+\mathrm{Pb}^{2+}=0.39 \pm 0.02}
\end{aligned}
$$

*The fluorescent response is not the same dry than wet. LODs and the experiments for $\mathrm{Pb}(\mathrm{II})$ detection were measured with the sample in water solution. 
The fluorescence lifetime decay of JG151dp was calculated and compared with JG76 dissolved in EtOH (Figure 92):
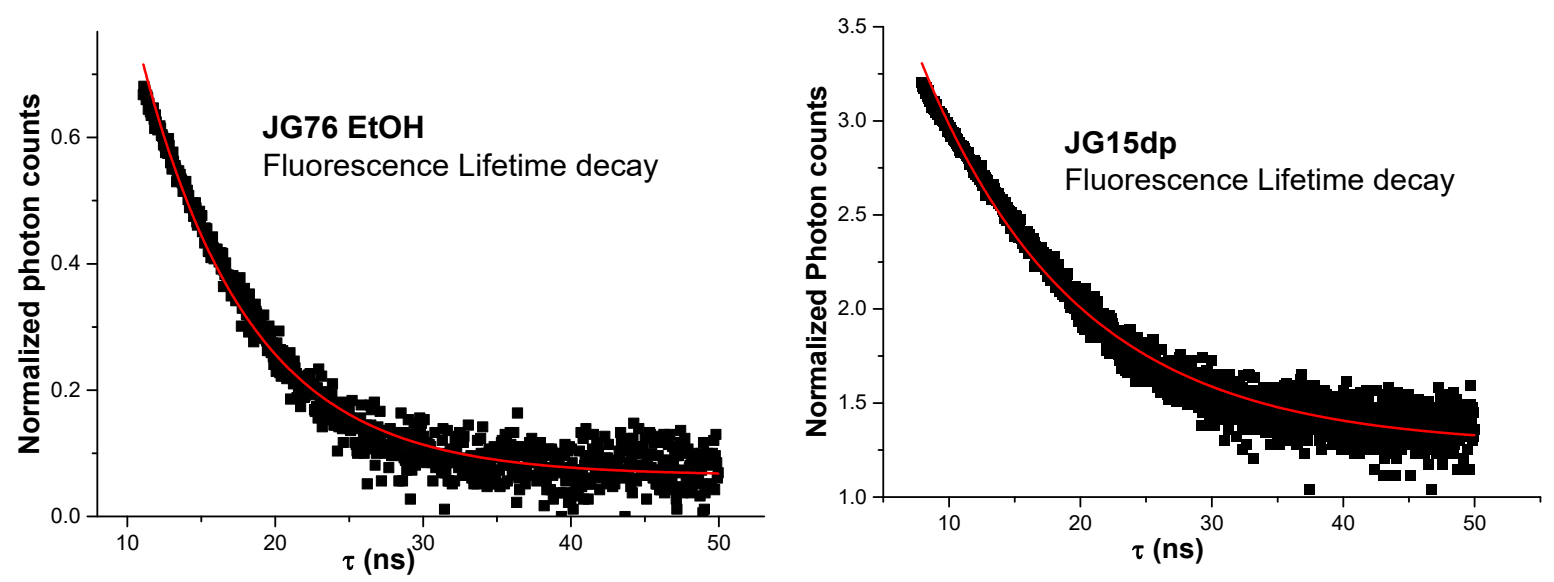

\begin{tabular}{|c|c|c|c|}
\hline Probe & Solvent & Lifetime (ns) & $\boldsymbol{\chi}^{\mathbf{2}}$ \\
\hline JG76 & EtOH & 3.58 & 1.138 \\
\hline JG151dp & - & 4.35 & 1.151 \\
\hline
\end{tabular}

Figure 92. Lifetime decay, JG76 dissolved in EtOH (left), JG151dp (right). Table of Lifetime decays, JG76 and JG151. (down)

The conclusions of the analysis were:

- Both JG76 and JG151dp had lifetime decays between 3-4.5 ns.

- It wasn't observed any change between measuring with and without lead cations.

\subsection{Limit of detection of $\mathrm{Pb}^{2+}$}

\subsubsection{With JG151dp in deionized water:}
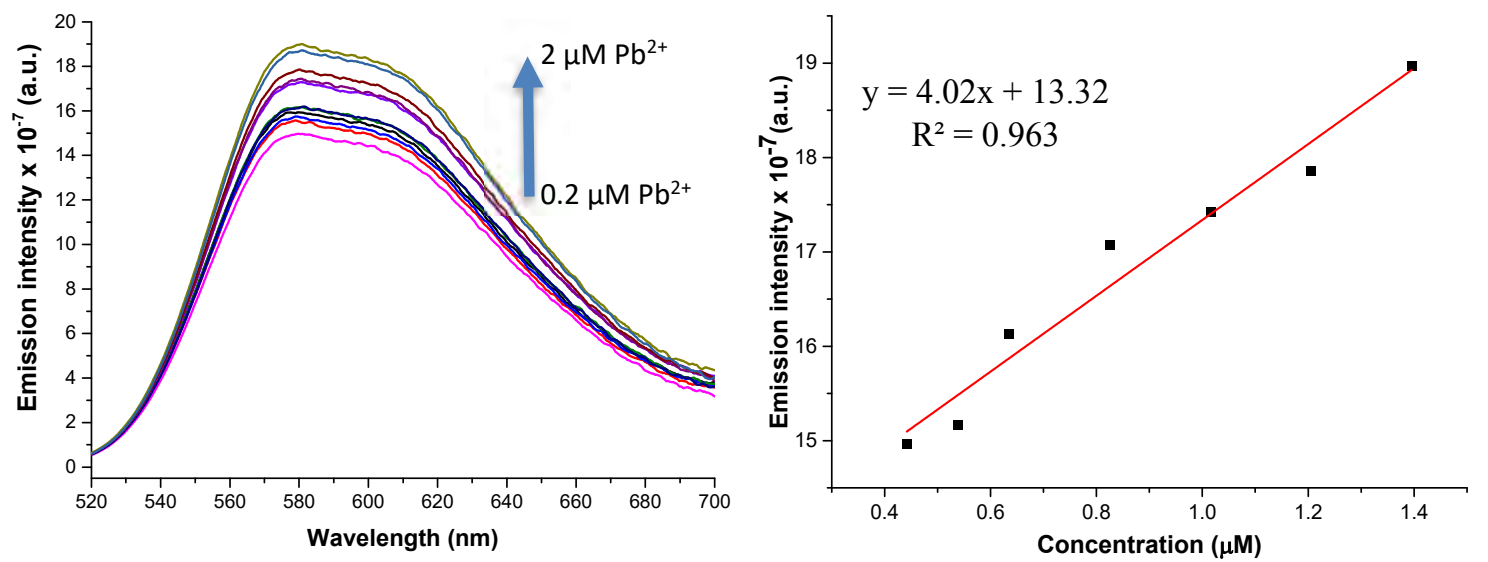

Figure 93. Fluorescence spectra of JG151dp in water solution while increasing $\mathrm{Pb}^{2+}$ concentration (left) and linear regression of a titration of JG151dp with $\mathrm{Pb}^{2+}$ in water, studying the increase in the fluorescent emission. $(\lambda \mathrm{exc}=500 \mathrm{~nm}, \lambda \mathrm{em}=580 \mathrm{~nm})$.

The limit of detection for $\mathbf{P b}^{2+}$ with JG151dp in deionized water was $610 \mathrm{nM}$ or 130 ppb. (From Linear Regression in Figure 93) 
There were two main issues to take into account from this titration:

- $\mathrm{Pb}(\mathrm{II})$ in water solution precipitated when $\mathrm{pH}$ was higher than 7.8.

- $\mathrm{Pb}(\mathrm{II})$ presence affected the media $(\mathrm{pH})$, decreasing the fluorescence when the concentrations of the metal were low. (Figure 94)

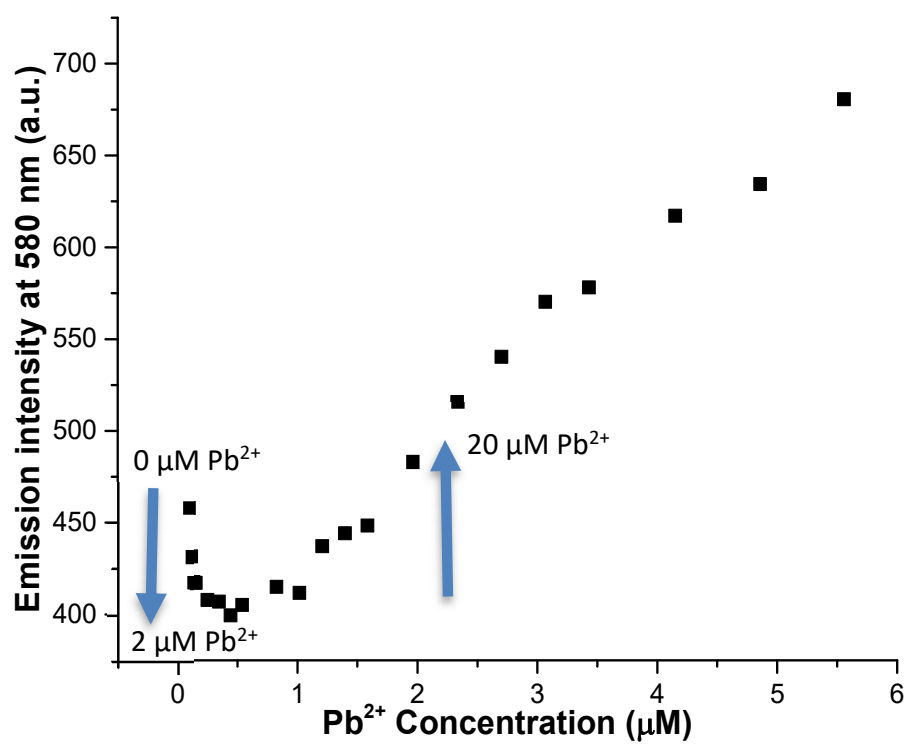

Figure 94. Fluorescence spectra of JG151dp in water solution while increasing $\mathrm{Pb}^{2+}$ concentration (left) and linear regression of a titration of JG151dp with $\mathrm{Pb}^{2+}$ in water, studying the increase in the fluorescent emission. $(\lambda \mathrm{exc}=500 \mathrm{~nm}, \lambda \mathrm{em}=580 \mathrm{~nm})$.

As a consequence, measurements of the presence of $\mathrm{Pb}^{2+}$ were repeated in buffered media.

\subsubsection{Limit of detection of $\mathrm{Pb}^{2+}$ with $\mathrm{JG151dp}$ in buffer $\mathrm{pH} 7$}

The LOD was calculated in HEPES buffered water solution $(0.5 \mathrm{mM})$.
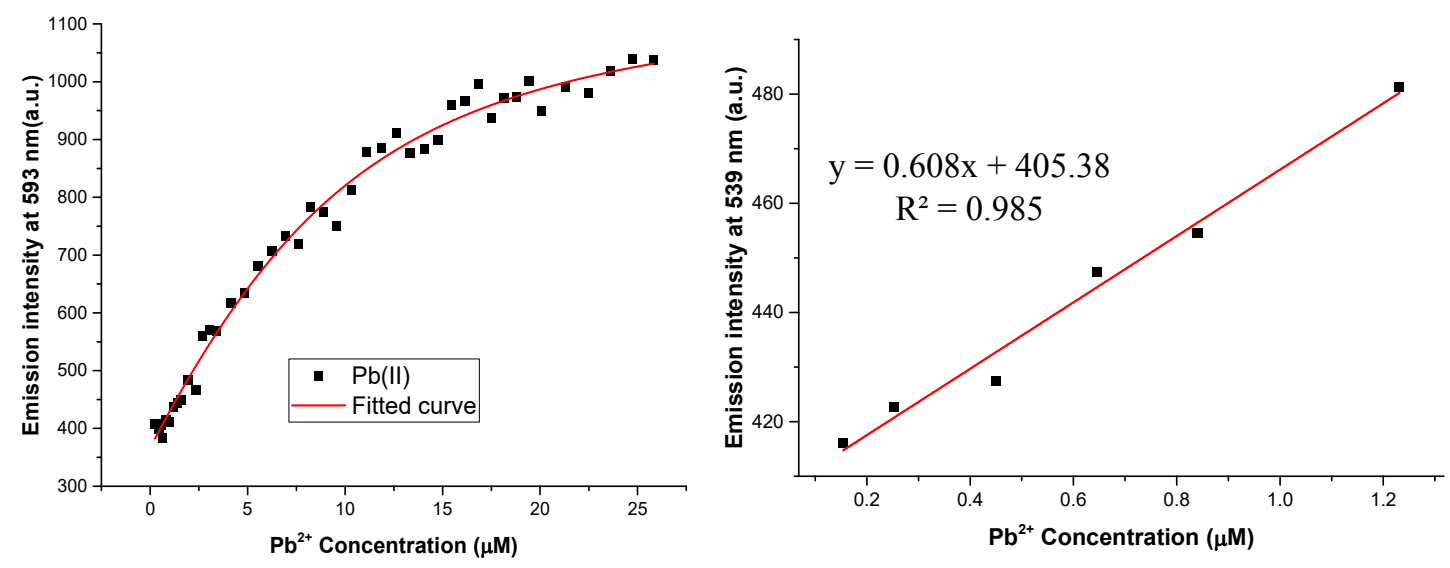

Figure 95. Linear regression of a titration of JG151dp with $\mathrm{Pb}^{2+}$ in HEPES $\mathrm{pH} 7$ solution, studying the increase in the fluorescent emission $(\lambda \mathrm{exc}=500 \mathrm{~nm}, \lambda \mathrm{em}=593 \mathrm{~nm})$.

The limit of detection for $\mathbf{P b}^{2+}$ with JG151dp in deionized HEPES buffer was $290 \mathbf{n M}$ or 66 ppb. (From Linear Regression in Figure 95) 


\subsection{Recyclability of the polymer JG151dp}

The recyclability of the polymer was checked by reusing different pieces of polymer. First, the polymer was placed in a $\mathrm{Pb}\left(\mathrm{ClO}_{4}\right)_{2}$ solution in water $0.1 \mathrm{mM}$. The fluorescence before and after being in solution was registered. After that, JG151dp was placed in an EDTA solution, $0.1 \mathrm{M}$. Then, it was washed up with distilled water (Figure 96).

This procedure allowed to use the polymer again having equivalent results. The recyclability was checked seven times by calculating the quantum yield before and after being in presence of $\mathrm{Pb}(\mathrm{II})$ cations (Figure 97). No significant change was observed.

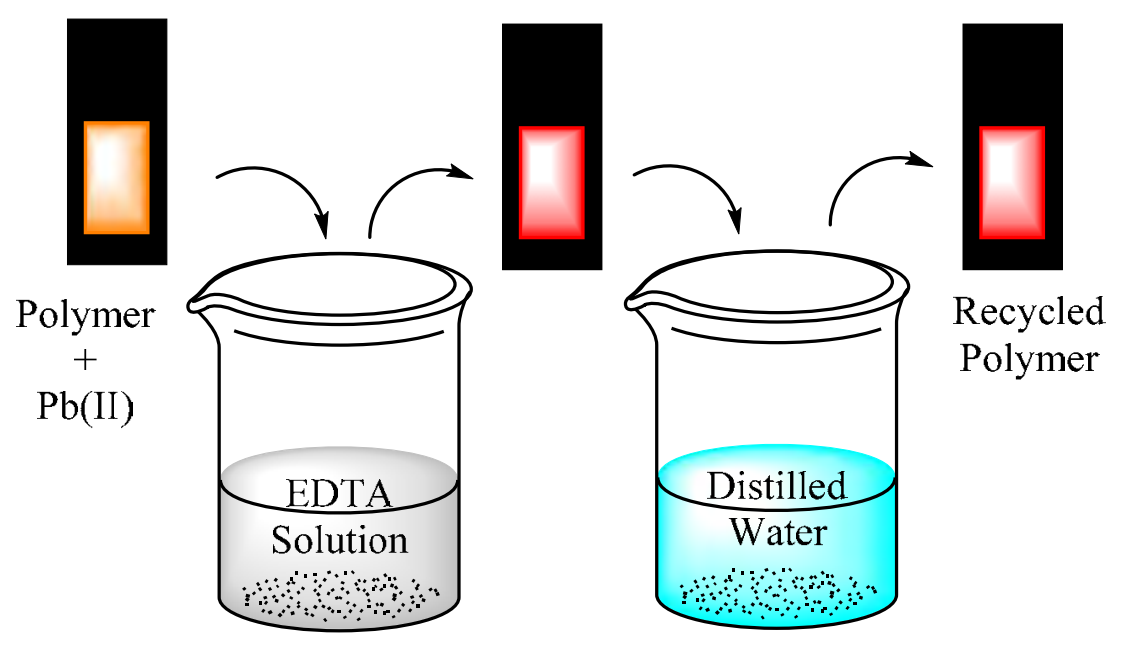

Figure 96. Scheme of the recycling process for the polymer JG151dp.

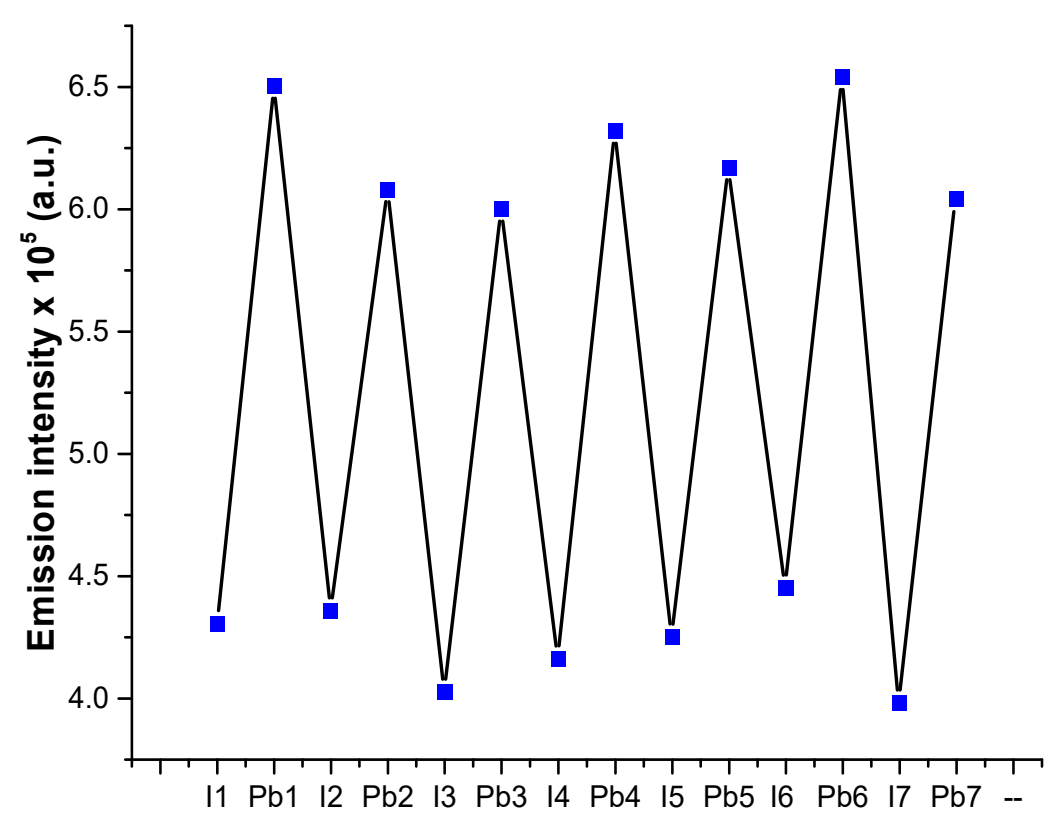

Figure 97. Fluorescence increase of a recycled piece of JG151 dp. $\left(\lambda_{\mathrm{exc}}=500 \mathrm{~nm}, \lambda_{\mathrm{em}}=593 \mathrm{~nm}\right)$ 


\section{DETECTION OF Pb(II) IN COLOURED SOLUTIONS}

The interests of detecting $\mathrm{Pb}^{2+}$ in solution were numerous: in tap water, in poisoned beverages, mud or balsamic vinegar. In case of colourless tap water, the probe JG76 would be a very sensitive option, because it was able to detect traces of less than $6 \mathrm{ppb}$, although the solvent should be changed to other but water. However, for measuring other solutions, such as coffee or muddy water, it is not possible to introduce a dissolved fluorescent probe to quantify the cation in solution, because of the intrinsic colour.

A solution to this issue was found by the use of polymer supported probes, following the procedure schematized in Figure 98.

- The polymer was hold between two magnetic surfaces.

- This polymer was introduced in a distilled water solution and the fluorescence was registered.

- The polymer was taken from this solution and introduced in the solution contaminated with $\mathrm{Pb}^{2+}$.

- After 5 minutes in this solution, the polymer was retired from the solution and washed with distilled water.

- The presence of $\mathrm{Pb}^{2+}$ was detected by measuring the fluorescence in the same conditions than initially.

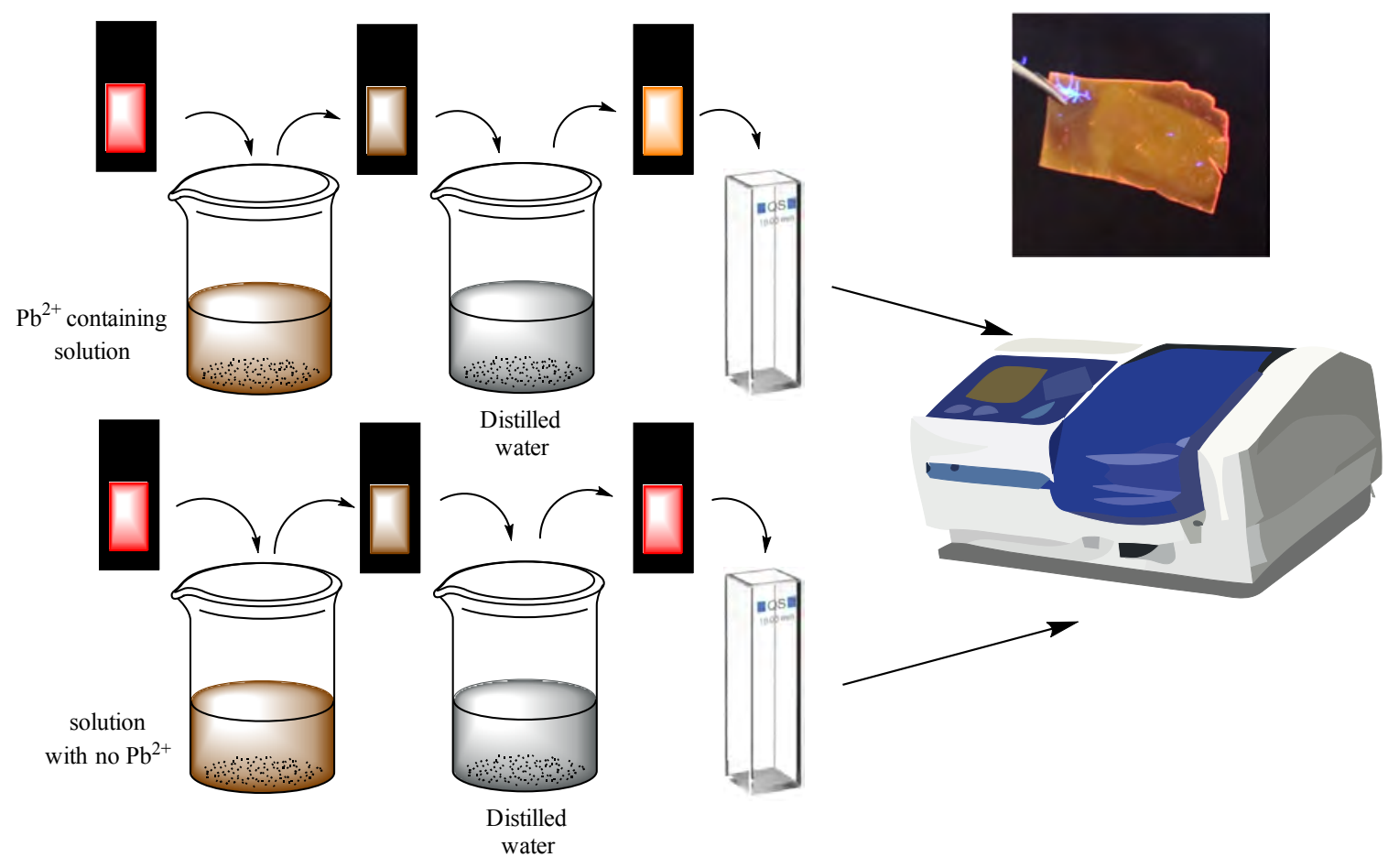

Figure 98. Reaction scheme of $\mathrm{Pb}(\mathrm{II})$ detection in colored solutions with JG151dp solutions. 


\section{Solutions tested}

The samples were tested in solutions of coffee and tea (Figure 99).
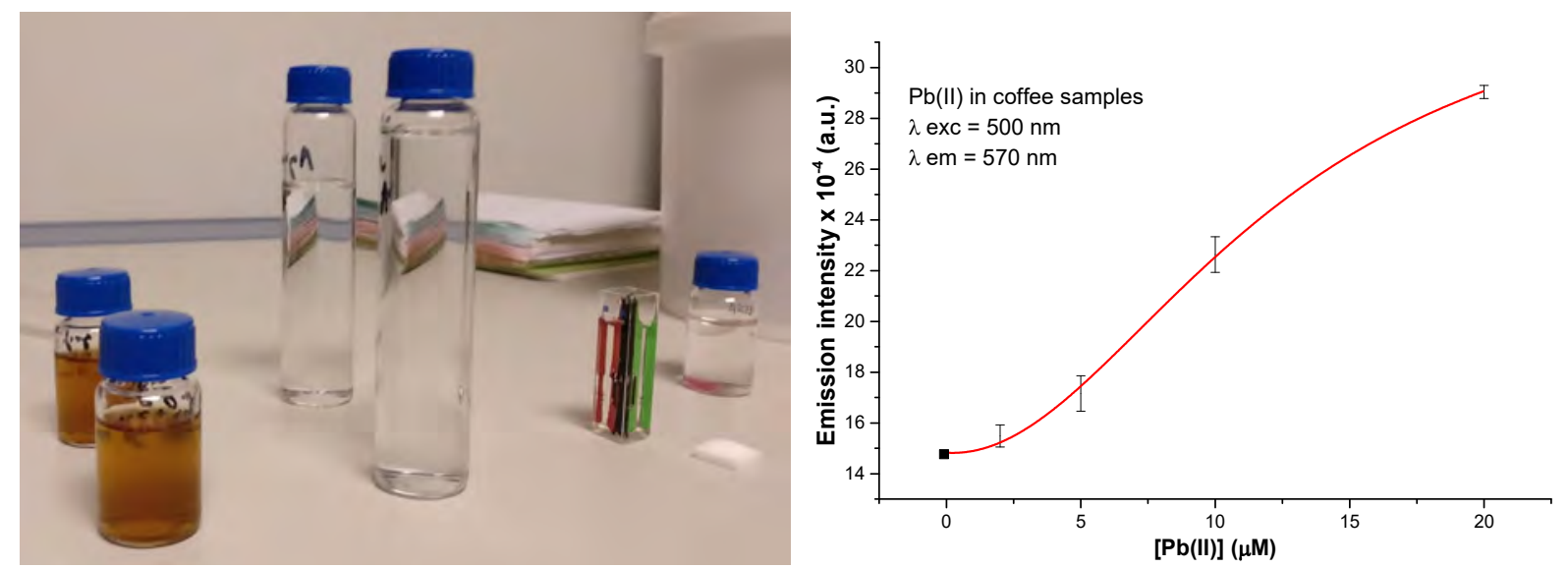

Figure 99. Coffee solutions containing one of them a concentration of $\mathrm{Pb}$ (II) $0.02 \mathrm{mM}$ and the other as a blank (left) and fluorescence titration results increasing $\mathrm{Pb}^{2+}$ concentration (right).

This procedure allowed us to measure the presence of $\mathrm{Pb}^{2+}$ when the concentration was higher than $1 \mu \mathrm{M}$. In any case, it was an example and it should be adapted to each different matrix, which could lead to different limits when working in low concentrations.

\section{Limits of the technique}

The possibility of measuring by this process is limited by the solution to measure. If the solution contains complexed $\mathrm{Pb}(\mathrm{II})$, such as high concentration of acetate, or $\mathrm{pH}$ too acid or basic, the measurements are not so reliable.

Additionally, to ensure the possibility of the quantification of the process, the concentration must be compared with a blank reference with no $\mathrm{Pb}$ (II) in solution and by a calibration with a linear regression. For example, it was checked to work by comparison between coffee and tea samples with and without lead (Figure 99). 


\section{SUMMARY OF THE CHAPTER}

In summary, the fluorescent probe JG76 may be considered as a very sensitive probe for detection of lead and potassium cations in alcohol-water mixtures. Moreover, it was a useful tool for the visualization of cereulide in live cells as well as the localization of potassium rich structures, from live cells.

Cereulide and the rest of cyclic depsipeptides with potassium affinity have also demonstrated to be a research field to be exploited. During this chapter it has been shown how little modifications may change their behaviour, opening the possibility to tune the potassium affinity and, therefore, the applications.

Finally, a new material, JG151dp was synthetized with the possibility to detect lead cations in water colourful samples. In addition, it was optimized to have an outstanding recyclability, going up to 7 times without showing any degradation. Between the many possible applications, it may be useful for monitoring industrial processes, the possible pollution of a river or the water from lead containing pipes.

\section{Data summary}

Soluble probe JG76.

\begin{tabular}{|c|c|c|c|c|c|c|}
\hline \multicolumn{2}{|c|}{ JG76 } & Solvent & Value & Units & \multicolumn{2}{|c|}{ Error } \\
\hline Fluor. Lifetime Decay & $\tau($ JG76) & $\mathrm{EtOH}$ & 3.58 & ns & 1.138 & $\left(\chi^{2}\right)$ \\
\hline \multirow{2}{*}{ Therm. Eq. constant } & K $\left(J G 76+K^{+}\right)$ & EtOH & 22 & $\mathrm{M}^{-1} \times 10^{-5}$ & 1 & $\mathrm{M}^{-1} \times 10^{5}$ \\
\hline & K $\left(\mathrm{JG76}+\mathrm{Pb}^{2+}\right)$ & $\mathrm{EtOH}$ & 15.5 & $\mathrm{M}^{-1} \times 10^{-5}$ & 1 & $\mathrm{M}^{-1} \times 10^{5}$ \\
\hline \multirow{3}{*}{ Fluor. Quantum Yield } & $\Phi($ (JG76) & $\mathrm{EtOH}$ & 0.12 & - & 0.02 & - \\
\hline & $\Phi_{\mathbf{F}}\left(\mathrm{JG} 76+\mathbf{K}^{+}\right)$ & EtOH & 0.48 & - & 0.02 & - \\
\hline & $\Phi_{\mathrm{F}}\left(\mathrm{JG76}+\mathrm{Pb}^{2+}\right)$ & EtOH & 0.84 & - & 0.02 & - \\
\hline \multirow{4}{*}{ Limits of detection } & $\operatorname{LOD}\left(\mathrm{K}^{+}\right)$ & EtOH & 60 & $\mathrm{nM}$ & - & - \\
\hline & LOD $\left(\mathrm{Pb}^{2+}\right)$ & $\mathrm{EtOH}$ & 30 & $\mathrm{nM}$ & - & - \\
\hline & LOD (Val) & EtOH & $540 * *$ & $\mathrm{nM}$ & - & - \\
\hline & LOD (Cer) & $\mathrm{EtOH}$ & $210 * *$ & $\mathrm{nM}$ & - & - \\
\hline
\end{tabular}

**JG76 concentration between $2-5 \mu \mathrm{M}$ and $[\mathrm{JG} 76] /\left[\mathrm{K}^{+}\right]=1.25$

Figure 100. Table that summarizes the calculated parameters for JG76, including fluorescent lifetime decays, thermodynamic equilibrium constants, fluorescence quantum yields and limits of detection. 
Cyclic depsipeptide potassium ionophores:

\begin{tabular}{|c|c|c|c|c|c|c|}
\hline \multicolumn{2}{|c|}{ Cyclic depsipeptide potassium ionophores } & \multirow{2}{*}{$\frac{\text { Solvent }}{\mathrm{EtOH}}$} & \multirow{2}{*}{$\begin{array}{c}\text { Value } \\
3.75\end{array}$} & \multirow{2}{*}{$\begin{array}{c}\text { Units } \\
\mathrm{ns}\end{array}$} & \multicolumn{2}{|c|}{ Error } \\
\hline Fluor. Lifetime Decay & $\tau($ JG121) & & & & 1.07 & $\left(\chi^{2}\right)$ \\
\hline \multirow{5}{*}{ Therm. Eq. constant } & $\mathbf{K}\left(\right.$ Val. $\left.+\mathbf{K}^{+}\right)$ & $\mathrm{EtOH}$ & 9.4 & $\mathrm{M}^{-1} \times 10^{-5}$ & 0.2 & $\mathrm{M}^{-1} \times 10^{5}$ \\
\hline & $\mathbf{K}\left(\right.$ Cer. $\left.+\mathbf{K}^{+}\right)$ & $\mathrm{EtOH}$ & 9.7 & $\mathrm{M}^{-1} \times 10^{-5}$ & 0.2 & $\mathrm{M}^{-1} \times 10^{5}$ \\
\hline & K $\left(\mathbf{J G 1 1 5}+\mathrm{K}^{+}\right)$ & $\mathrm{EtOH}$ & 9.3 & $\mathrm{M}^{-1} \times 10^{-5}$ & 0.2 & $\mathrm{M}^{-1} \times 10^{5}$ \\
\hline & K $\left(\mathrm{JG115B}+\mathrm{K}^{+}\right)$ & EtOH & 0.4 & $\mathrm{M}^{-1} \times 10^{-5}$ & $0.01 *$ & $\mathrm{M}^{-1} \times 10^{5}$ \\
\hline & K $\left(\mathrm{JG121}+\mathrm{K}^{+}\right)$ & $\mathrm{EtOH}$ & $<\mathrm{JG} 115 \mathrm{~B}$ & $\mathrm{M}^{-1} \times 10^{-5}$ & - & - \\
\hline Fluor. Quantum Yield & $\Phi_{\mathrm{F}}(\mathrm{JG} 121)$ & $\mathrm{EtOH}$ & 0.99 & - & 0.01 & - \\
\hline
\end{tabular}

Figure 101. Table that summarizes the calculated parameters for different cyclic depsipetides, including fluorescent lifetime decays, thermodynamic equilibrium constants, fluorescence quantum yields and limits of detection.

Fluorescent material JG151dp:

\begin{tabular}{|c|c|c|c|c|c|c|}
\hline \multicolumn{2}{|c|}{ JG151dp } & \multirow{2}{*}{$\begin{array}{l}\text { Solvent } \\
\text { Water* }\end{array}$} & \multirow{2}{*}{$\frac{\text { Value }}{3.75}$} & \multirow{2}{*}{$\frac{\text { Units }}{\mathrm{ns}}$} & \multicolumn{2}{|c|}{ Error } \\
\hline $\begin{array}{c}\text { Fluor. Lifetime } \\
\text { Decay }\end{array}$ & $\tau($ JG151dp) & & & & 1.15 & $\left(\chi^{2}\right)$ \\
\hline \multirow{2}{*}{$\begin{array}{c}\text { Fluor. Quantum } \\
\text { Yield }\end{array}$} & $\Phi_{\mathrm{F}}(\mathrm{JG151dp})$ & Air & 0.22 & - & 0.01 & - \\
\hline & $\Phi_{\mathrm{F}}\left(\mathrm{JG} 151 \mathrm{dp}+\mathrm{Pb}^{2+}\right)$ & Air & 0.39 & - & 0.01 & - \\
\hline \multirow{2}{*}{ Limits of detection } & $\operatorname{LOD}\left(\mathrm{Pb}^{2+}\right)$ & Water & 610 & $\mathrm{nM}$ & - & - \\
\hline & $\operatorname{LOD}\left(\mathrm{Pb}^{2+}\right)$ & Water* & 290 & $\mathrm{nM}$ & - & - \\
\hline
\end{tabular}

*HEPES buffer water solution $10 \mathrm{mM}$.

Figure 102. Table that summarizes the calculated parameters for the film JG151dp, including fluorescent lifetime decays, fluorescence quantum yields and limits of detection. 


\section{RESUMEN DEL CAPÍTULO}

El objetivo de este capítulo consistía en la modificación de perilenomonoimidas (PMIs), con receptores que actuaran de complejantes, para la detección de analitos de interés mediante un cambio en fluorescencia. Concretamente se buscaba un receptor sensible y selectivo a cationes potasio.

El interés hacia este ámbito surgió debido a que el grupo de investigación trabajaba en un proyecto sobre detección de toxinas alimentarias; más concretamente la bacteria conocida como Bacillus cereus y su toxina, la cereulida. El modo de actuación de esta toxina consiste en la modificación de los equilibrios de potasio intra-extracelulares de tal modo que las células mueren, causando problemas gastrointestinales cuando se consumen alimentos contaminados con ella. De este tema surgió el interés de la detección de potasio, que además juega un papel fundamental en gran cantidad de procesos biológicos. Este objetivo se llevó a cabo mediante la búsqueda y síntesis de complejantes moleculares selectivos de cationes potasio. Dos grupos fundamentales fueron encontrados, un derivado de un éter corona y otro de un triazacriptando, el cual se descartó debido a la mayor complejidad de síntesis y peores propiedades respecto al primero.

Gracias a estos derivados, que contenían una parte fluorescente (PMI) y un éter corona, se sintetizó un sensor altamente selectivo a cationes potasio, plomo y bario en disoluciones mezcla entre un alcohol y agua. Además, muestras que contenían cereulida fueron también medidas por competición en la complejación de potasio, siguiendo el proceso por métodos de fluorescencia, demostrando la validez de este tipo de pruebas.

Simultáneamente a la síntesis del éter corona fluorescente, la propia cereulida se sintetizó artificialmente en laboratorio, así como algunas variantes que permitieron determinar la importancia que tienen pequeñas modificaciones sobre su actividad complejante; un ámbito de investigación todavía bajo estudio y con resultados muy prometedores.

Como ya se ha explicado, el éter corona resultó ser sensible a otras especies, aparte de cationes potasio, como son los cationes de bario y plomo. Entre ellos, resultaba especialmente interesante el poder detectar este último. Hasta ahora, todos los sensores de plomo por colorimetría o fluorescencia presentaban problemas en su utilización, debido a que las disoluciones en las cuales es necesario utilizarlos suelen ser totalmente opacas o muy oscuras, haciendo imposible medir con un analito disuelto. De ahí surgió la idea de modificar un material con el colorante selectivo a plomo y medir el mismo en un soporte. De este modo, al modificar un material polimérico con el sensor resultó que el mayor interferente ya no actuaba como tal una vez soportado (catión potasio), con lo cual, la aplicación del mismo adquirió un mayor interés. Este material sensible a cationes plomo(II) se probó satisfactoriamente para su detección, con límites de detección cercanos a los $60 \mu \mathrm{g} / \mathrm{L}$, en distintas bebidas y disoluciones coloreadas, como té o café. 


\section{CHAPTER 4}

\section{SUPPORTED GOLD AND PALLADIUM NANOPARTICLES FOR CATALYSIS}
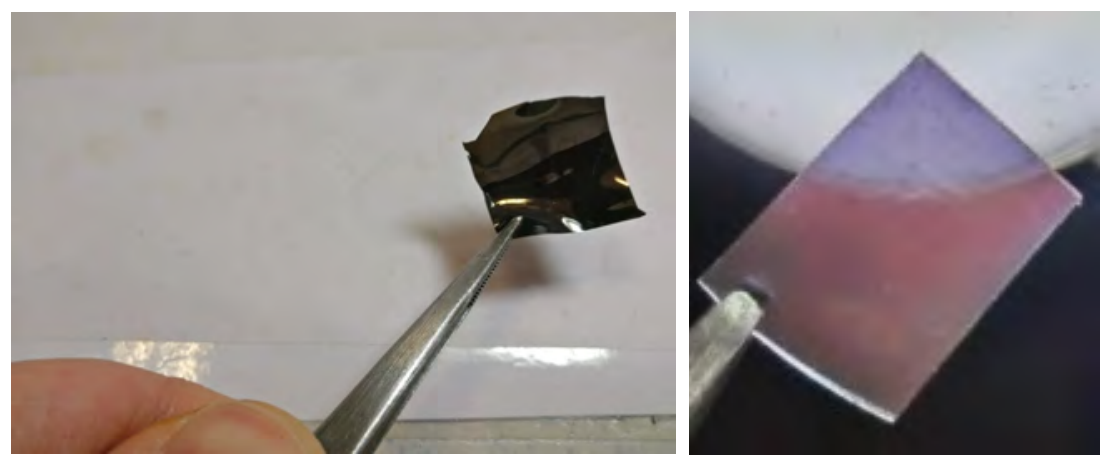

\section{ABSTRACT}

This chapter aims to introduce the synthesis, characteristics and applications of materials modified in one-step with gold or palladium metallic micro- and/or nanoparticles over its surface. The topic has plenty of applications going from the creation of electronic devices, to sensors or heterogeneous catalysts; in which this thesis was focused. ${ }^{1}$

\footnotetext{
${ }^{1}$ J. García-Calvo, V. García-Calvo, S. Vallejos, F. C. García, M. Avella, J.- M. García, T. Torroba, ACS Appl. Mater. Interfaces, 2016, 8, 24999-25004.

José García-Calvo, Patricia Calvo-Gredilla, Saúl Vallejos, José-Miguel García, José V. Cuevas, Gabriel GarcíaHerbosa, Manuel Avella, Tomás Torroba, Green Chem. 2018, 20, 3875-3883.
} 



\section{INTRODUCTION. SUPPORTED NANOPARTICLES}

In the last years, the use of nanomaterials has become an essential part in materials science. A nano-structured material is defined as the one formed or containing atomic substructures in a scale between 1-100 nm, in at least one of its dimensions. Then, there are many materials that are considered nano-structured, containing nanoparticles (0D), nanotubes (1D) or nanolayers (2D).

Micro or nanostructured materials get different properties from molecules or bulky solids, ${ }^{2}$ a characteristic that opens a new field of research and possible applications. The greatest advantage of nanostructures is the possibility to tune their electronical properties without changing the atomic composition, due to the variation of their structure in the quantum scale. This phenomenon comes from the coupling between different atoms/molecules in nanoscale, producing the so called plasmons. Plasmons electron density lead to final photoelectronical properties that differ from the isolated atoms/molecules and bulk materials. What is more, the properties may change depending on the characteristics of the nanostructure (size, shape, dimension...). ${ }^{3}$
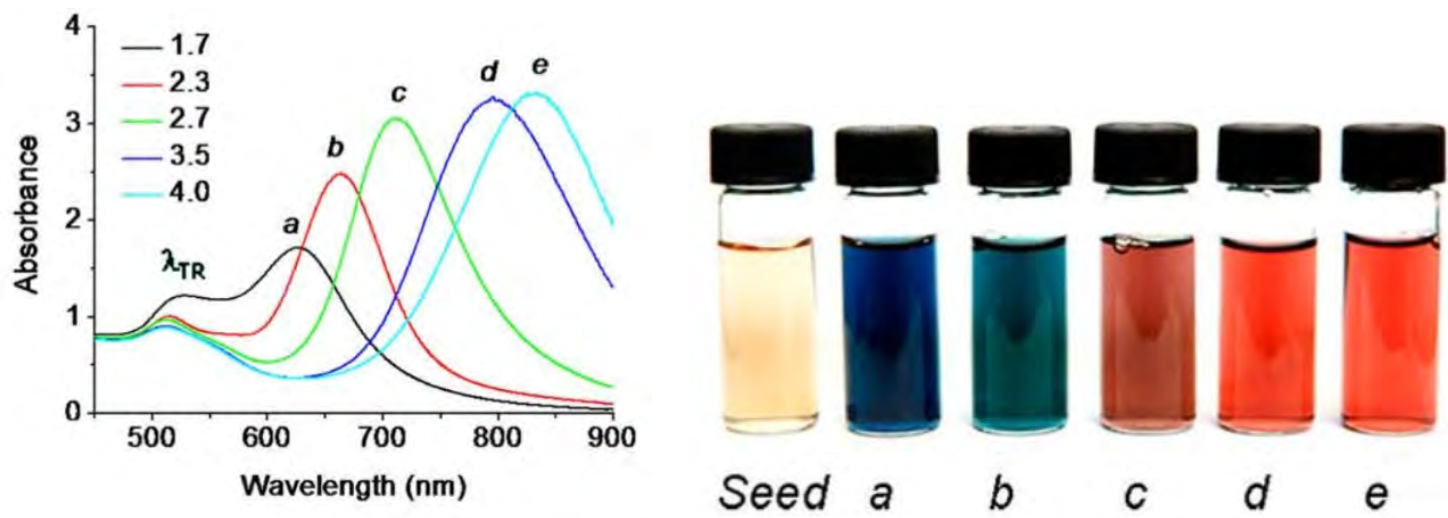

Figure 1. Gold nanorods (Gold-NRs) with different diameter to tune optical absorptions in visible and IR. Optical absorption spectra of gold NRs with different length/with ratio (a-e). ${ }^{4}$

The field of application for nanostructured materials covers a wide range of possible components, going from metallic nanoparticles to organic nanoparticles or bidimensional nanostructures, such as graphene. For each one of them, the properties are completely different to raw materials and they are studied specifically.

In consequence, to focus such broad scope, this work was centred in the properties of some specific metallic particles. These particles were formed by aggregation of $\operatorname{Au}(0)$ or $\operatorname{Pd}(0)$ in the nanomicroscale. Particularly, they possessed a combination of properties that gave them unusual applications in chemistry, physics, biomedical science and engineering (Figure 2).

\footnotetext{
${ }^{2}$ C. B. Murray, C. R. Kagan, M. G. Bawendi, An. Rev. Mat. Sci. 2000, 30, 545-610.

${ }^{3}$ V. V. Mody, R. Siwale, A. Singh, H. R. Mody. J. Pharm. Bioallied. Sci. 2010, 2, 282-289.

${ }^{4}$ L. Tong, Q. Wei, A. Wei, J. -X. Cheng. Photochem. Photobiol. 2009, 85, 21-32.
} 


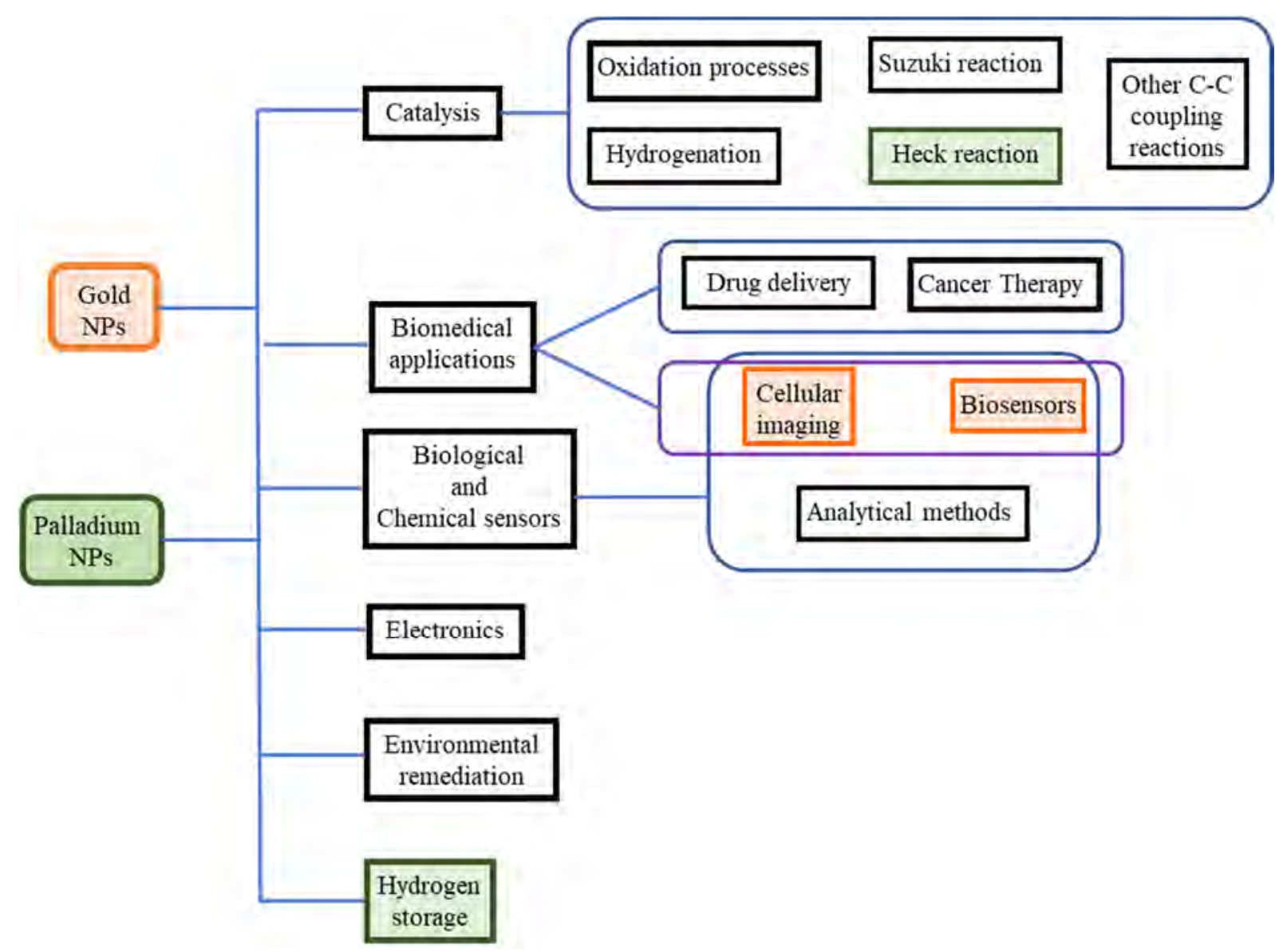

Figure 2. Scheme of the different applications for gold and palladium NPs.

As it is shown in Figure 2; most of the applications are shared, ${ }^{5}$ by changing conditions and presenting different advantages and disadvantages with respect to the other. Comparing what may be found in literature, palladium nanoparticles (Pd-NPs) have given better results in catalysis and, until now, they are the only ones in which hydrogen storage was performed. In contrast, gold nanoparticles (Au-NPs) have been more useful in the research of biomedical applications, fundamentally because of the toxicity of elemental Pd for living beings, which has limited the applications in this regard.

${ }^{5}$ G. Freemantle, M. Liu, W. Guo, S. O. Obare, Metallic Nanomaterials, Edited by C. S. S. R. Kumar, WILEYVCH Verlag GmbH \& Co. Weinheim, Volume 1, pp. 572, 2009. 


\section{GOLD NANOPARTICLES}

\subsection{Historical background}

The use of gold dispersions occurs, at least, since Ancient Egypt-China. In that time, they were used mostly unknowingly, for giving different colours to glass or associated to curative properties. What is more, tracks of gold nanoparticles and their special properties were also found throughout history in many other different cultures, such as the Roman or Islamic cultures. ${ }^{6}$

Michael Faraday, around 1850, was the first to take a scientific approach, associating the colour to the shape and size of the particles, performing several experiments with gold salts. ${ }^{7}$ Due to the multiple possibilities, gold NPs exhibited a range of physical and chemical properties that were promising for potential applications in a new generation of optical, electronic, and chemical devices. ${ }^{3}$

\subsection{Synthesis}

Starting from the previously mentioned studies of Faraday, since the beginning of $20^{\text {th }}$ century many researchers have worked in the controlled synthesis of gold nanoparticles. One of the first synthetic procedures to be standardized was developed by Turkevich in $1951 .^{8}$ A chemical synthesis that has been deeply studied, adapted and improved to work under different conditions. ${ }^{9}$

In general, gold-NPs synthesis techniques are divided into physical or chemical synthesis. Physical synthesis most common techniques are gold sputtering and condensation of metal vapours. They have been very useful to create nanostructures in a surface without chemical reagents. Nevertheless, they are very expensive, not manageable and require from specific equipment and facilities. In contrast, chemical synthesis processes are adapted to the controlled reduction of $\mathrm{Au}$ (III) salts in solution. Most methods are based on Turkevich synthesis, which consists of using citrate as reductive reagent in a water boiling solution of $\mathrm{AuCl}_{4}^{-}$. Afterwards, many variations have been developed by using other solvents, temperatures and/or reductive reagents. For instance, $\mathrm{NaBH}_{4}$ in toluene solution, by Brust and Schiffrin, ${ }^{10}$ or just HEPES buffer in water solutions. ${ }^{11}$ In fact, the procedure was demonstrated to be robust, obtaining gold nanoparticles even with complex matrixes. ${ }^{12}$

Besides, there are many ways to optimize not only the synthesis but the stability of the NPs. These so called stabilizers are species with known affinity to the composition of the nanoparticle (like goldsulfur), which allows to obtain nanoparticles with controlled shapes and sizes. Some examples of AuNPs stabilizers are bromides, thiol groups or surfactants as PVP. Thanks to them, it has been possible

\footnotetext{
${ }^{6}$ M.- C. Daniel, D. Astruc, Chem. Rev. 2004, 104, 293-346.

${ }^{7}$ M. Faraday, Phil. Trans. R. Soc. Lond. 1857, 147, 145-181.

${ }^{8}$ J. Turkevich, P. Stevenson, J. Hillier. Discuss. Faraday Soc. 1951, 11, 55-75.

${ }^{9}$ J. Kimling, M. Maier, B. Okenve, V. Kotaidis, H. Ballot, A. Plech, J. Phys. Chem. B, 2006, 110, 15700-15707.

${ }^{10}$ A. Uehara, S. G. Booth, S. Y. Chang, S. L. M. Schroeder, T. Imai, T. Hashimoto, J. F. W. Mosselmans, R. A. W. Dryfe, J. Am. Chem. Soc., 2015, 137, 15135-15144.

${ }^{11}$ R. Chen, J. Wu, H. Li, G. Cheng, Z. Lu, C.-Mi. Che, Rare Metals. 2010, 29, 180-186.

${ }^{12}$ Y. Gao, Y. Hu, C. Li, X. Zhao, X. Huang, R. Liu, Nanosci. Nanotech. Lett. 2014, 6, 118-123.
} 
to control polydispersity, shape and size (Figure 3); by smart control of several parameters simultaneously; such as proportions, concentration, solvent, reductive agent... ${ }^{13}$

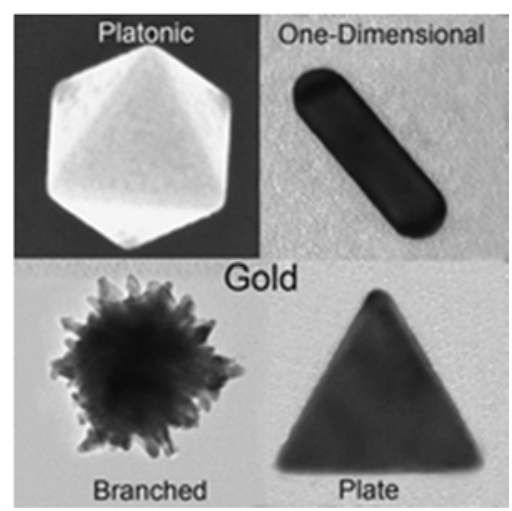

Figure 3. Different morphologies for gold nanoparticles changing reduction reagent, stabilizer and solvent. Picture by Liz-Marzán et al. ${ }^{13}$

\subsection{Physical and chemical properties-applications of gold nanoparticles}

Chemical properties: gold NPs might have different stability depending on their particular characteristics. Being usually synthetized by reduction of $\mathrm{Au}(\mathrm{III})$, gold NPs are not highly reactive to oxidative reagents. Nevertheless, they are particularly sensitive to the presence of some compounds; such as cyanide, thiol groups, highly acidic solution ("aqua regia") or to the presence of some other cations (for example, $\mathrm{Ag}^{+}$or $\mathrm{Hg}^{2+}$ ).

As it was previously mentioned the sensitivity-shape/size is directly related to the stabilizers of the nanoparticles. For example, when surrounded by a sulfur-PEG stabilizer (very common in medical research) the sensitivity is much lower against other gold-appealing groups than when, for example, having only citrate as stabilizer. Additionally, the "stabilizers" may have other functions due to the capacity to surround gold NPs. For instance, it is possible to put fluorescent molecules or drugs with pharmacological activity. This feature is useful for using the NPs as drug carriers or as markers / sensors. $^{14}$

Other related and interesting applications are associated to the electronical properties, their high surface (compared to bulk atoms ratio) and their overall chemical inertness. These characteristics confer to gold nanoparticles catalytical properties for redox processes. For instance, gold nanoparticles have been used successfully for oxidation of $\mathrm{CO}$ and $\mathrm{H}_{2}$, reduction of $\mathrm{NO}$ and other catalytic reactions, ${ }^{15}$ such as a variety of C-C coupling reactions.

Physical properties: they have significantly different properties compared to bulk gold; for instance, lower melting temperature (related with diameter of the particle) ${ }^{16}$ or higher thermal conductivity (high surface/volume). On top of them, the electronical properties are directly related with what is called surface plasmon absorptions (SPA), which is simultaneously related to their size.

\footnotetext{
${ }^{13}$ a) M. Grzelczak, J. Pérez-Juste, P. Mulvaney, L. M. Liz-Marzán, Chem. Soc. Rev. 2008, 37, 1783-1791. b) T.

H. Ha, H.-J. Koo, B. H. Chung, J. Phys. Chem. C, 2007, 111, 1123.

${ }^{14}$ L. M. Liz-Marzan. Materials Today, 2004, 2, 26-31.

${ }^{15}$ K, Kvitek, R. Prucek. J. Mat. Sci. 2005, 22, 2461-2473.

${ }^{16}$ C. Burda, X. Chen, R. Narayanan, M. A. El-Sayed. Chem. Rev. 2005, 105, 1025-1102.
} 
Moreover, the variation in the SPA has not only influence over the final absorption-colour, but other properties such as the conductivity are also affected. ${ }^{13}$ In consequence, when working with solutions of nanoparticles, studying their absorption might be directly related with the size and shape of the particle, which might be very useful for getting a raw estimation of their characteristics. As an example, for gold (Figure 4), the absorption between 20-100 nm particles produces an increase in its wavelength of emission, simultaneously to size.
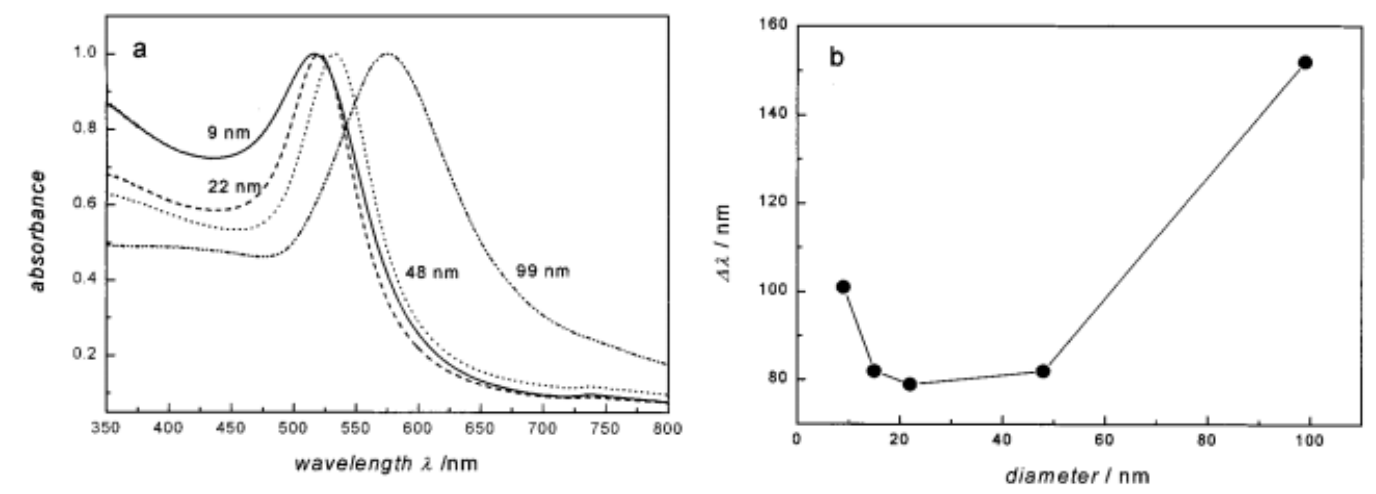

Figure 4. Normalized UV-vis absorption of nanoparticles of different size in water solution (left), and variation of the plasmon bandwith as a function of particle diameter (right). From Link et al. ${ }^{17}$

\subsection{Supported gold NPs}

Gold nanoparticles (Au-NPs) display a range of physical and chemical properties that are promising for the development of optical, electronic, and chemical devices. In doing so, many of these devices would require from immobilization of gold nanoparticles in a single layer, or in multilayers, over surfaces. ${ }^{18}$

Up to now, the process has been performed in two steps. First, the gold salt is reduced by adding a reductive reagent to the solution (for example, citrate). Secondly, the immobilization is performed by modification of the surface with functional groups that provide attractive interaction to gold nanoparticles. ${ }^{19}$ However, these procedures have no lead to optimal routes for the formation of monolayer ensembles on various substrates. In contrast, a homogeneous nanostructured system (Figure 5) may get unique optical and electronic properties, which make them a good prospect for future application in microelectronics, solid state chemical, biological sensors or catalysis. ${ }^{20}$

In spite of the potential applications, the formation of continuous films of metal nanoparticles on a solid substrate is not a simple task, because the size of metal clusters and their concentration on the surface are rather difficult to control.

\footnotetext{
${ }^{17}$ S. Link. M. A. El-Sayed, J. Phys. Chem. B 1999, 103, 4212-4217.

${ }^{18}$ A. X. Wang, X. Kong, Materials 2015, 8, 3024-3052.

19 a) M. S. Onses, C. J: Thode, C. -C. Liu, S. Ji, P. L. Cook, F. J. Himpsel, P. F. Nealey, Adv. Funct. Mater. 2011, 21, 3074-3082; b) F. L. Yap, P. Thoniyot, S. Krishnan, S. Krishnamoorthy, ACS Nano 2012, 6, 2056 2070 .

20 a) M. A. Mahmoud, D. O'Neil, M. A. El-Sayed, Chem. Mater. 2014, 26, 44-58. b) L. Prati, A. Villa, Acc. Chem. Res. 2014, 47, 855-863; c) E. C. Dreaden, A. M. Alkilany, X. Huang, C. J. Murphy, M. A. El-Sayed, Chem. Soc. Rev. 2012, 41, 2740-2779. d) F. Mitschang, H. Schmalz, S. Agarwal, A. Greiner, Angew. Chem. Int. Ed. 2014, 53, 4972-4975.
} 


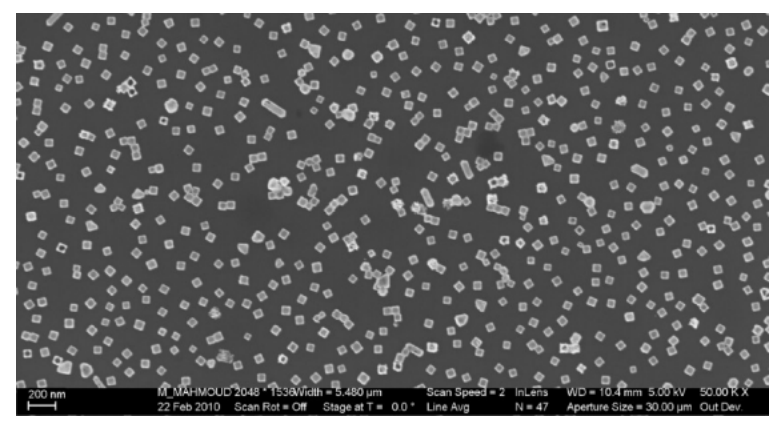

Figure 5. Au nanocubes layer over a silicon wafer, synthetized by Mahmoud and coworkers. ${ }^{20}$

Until now, the adsorption of the metallic nanoparticles from their colloidal solutions has been employed for modifying surfaces with nanoparticles, ${ }^{21}$ but this method presents major drawbacks. Highly dispersed gold nanoparticles are difficult to obtain in a solution phase without using organic stabilizers and reducing reagents such as citric acid, alkylamine, alkylthiol, and cationic surfactants, complicating the adsorption step. Therefore, the approaches with prebound reductants and stabilizers on the same material are quite desirable.

${ }^{21}$ Z. Zhang, C. Liu, J. Bai, C. Wu, Y. Xiao, Y. Li, J. Zheng, R. Yang, W. Tan, ACS Appl. Mater. Interf. 2015, 7, 6211-6219. 


\section{PALLADIUM NANOPARTICLES}

\subsection{Historical background}

$\operatorname{Pd}(0)$ derivatives have been mostly used in the fields of catalysis and hydrogen detection, purification and storage. ${ }^{22}$ As a consequence, Pd nanoparticles with a high relation surface/volume have the potential to provide a cost-effective solution to the requirements of evolving catalytical and electrochemical applications. In contrast with gold or silver, $\operatorname{Pd}(0)$ nanoparticles are not so well known by the different colour variations with size, usually black or brown (Figure 6), but they also have different morphologies that may tune the $\operatorname{Pd}(0)$ applications.

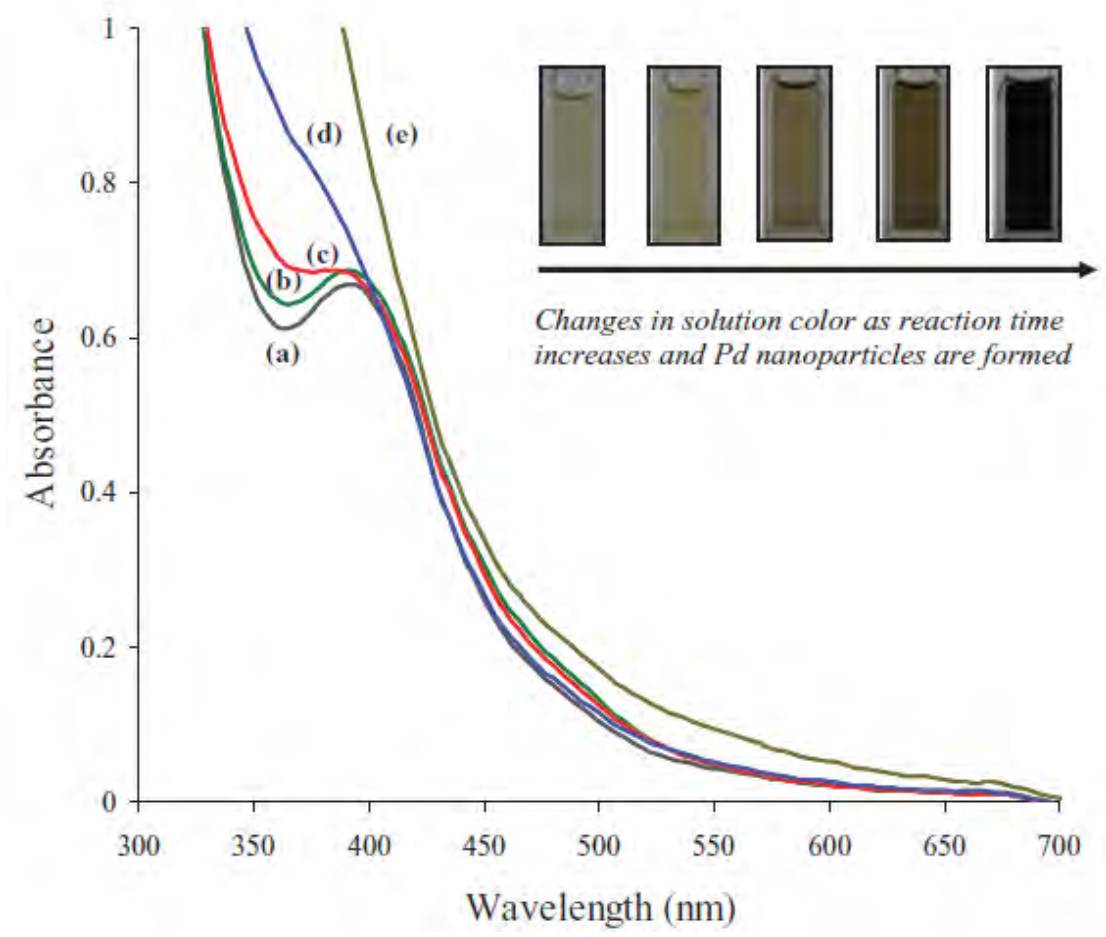

Figure 6. Absorbance of $\mathrm{Pd}$ nanoparticles formed from $\left[\mathrm{Pd}_{3}(\mathrm{OAc})_{6}\right]$ and $n$-dodecyl sulphide by heating from room temperature to $80^{\circ} \mathrm{C}$ and waiting from 0 (a) to 40 minutes (e), by Obare et al. ${ }^{23}$ The band at $400 \mathrm{~nm}$ indicates the presence of Pd-NPs.

Due to the difficulty of distinguishing the nano-structure from the palladium bulk or molecular derivatives it was not until the end of 90 s when the development of highly accurate characterization systems, such as transmission electron microscopy (TEM), allowed a deeper study of this kind of metallic particles. Therefore, in the last 30 years, the process has been perfectioned to relate internal structure - synthesis methods (Figure 7) and electronical properties, managing to achieve the optimization of its use for many applications.

\footnotetext{
22 A. Chen, C. Ostrom, Chem. Rev. 2015, 115, 11999-12044

${ }^{23}$ M. Ganesan, R.G. Freemantle, S. O. Obare, Chem. Mater. 2007, 19, 3464-3471.
} 


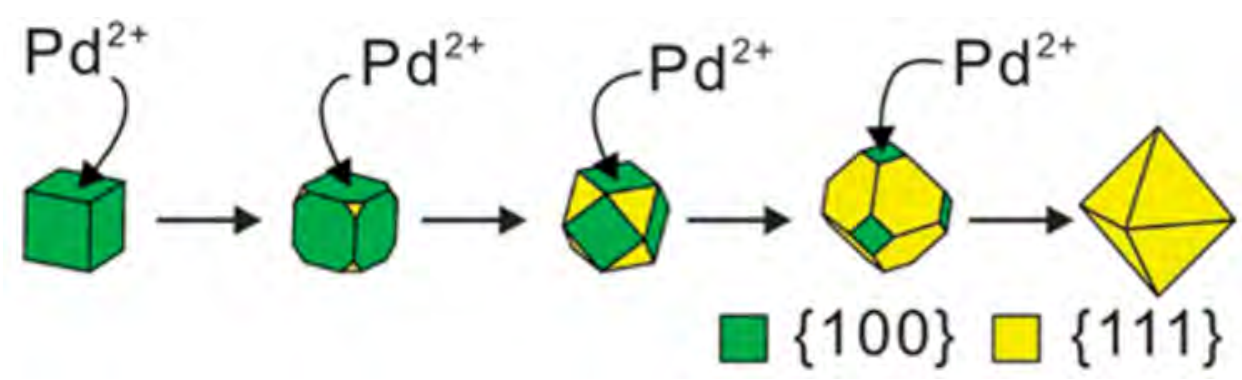

Figure 7. Scheme shape transformation of Pd nanoparticles when growing. From Chen and Ostrom review. $^{22}$

\subsection{Synthesis of Pd nanoparticles}

\subsubsection{Synthesis procedures:}

Pd-NPs synthesis, just as for other metals, are synthetized by physical or chemical techniques. Within the physical techniques there are sputtering methods, ion- and electron-beam-induced deposition or laser ablation. All of them, combined with stabilizing surfactants may achieve controlled compositions, morphologies and other attributes.

Within the chemical methods, the most common is the electrochemical deposition which, controlling the potential or current density, produces deposition of $\operatorname{Pd}(0)$ from the oxidized form in solution. Other method is what is called hydrothermal deposition, in which a chemical reaction occurs above the solvent boiling point and at pressures higher than 1 bar.

Finally, there are synthetic procedures involving photoreductants and/or chemical reducing agents. These electroless deposition methods are based on displacement deposition or autocatalytic deposition. Displacement deposition is reliant on the reduction potential of metallic precursors (such as $\left.\mathrm{Fe}^{2+}\right) .{ }^{24}$ While autocatalytic deposition proceeds by using chemical reducing agents, including ascorbic acid, ethylene glycol, citric acid or sodium borohydride, ${ }^{25}$ along with stabilizers, such as PVP. Chemically obtained Pd-NPs may have a wide variety of shapes, usually as octahedral or truncated octahedral nanocrystals (Figure 7); that are attached to one another depending on the media and stabilizers.

\subsubsection{Shape and size regulation:}

Shape controlled Pd-NPs need careful nucleation and growth conditions, ${ }^{26}$ which is achieved throughout the manipulation of kinetic parameters; ${ }^{27}$ especially in aqueous solutions. ${ }^{28}$ For controlling growth conditions, the presence of an organic stabilizer/additive is associated to the control on the reaction kinetics and hence, the shape/size of the nanostructures: ${ }^{29}$

\footnotetext{
${ }^{24}$ R. Ojani, Z. Abkar, E. Hasheminejad, J. -B. Raoof, Int. J. Hydrogen Energy 2014, 39, 7788-7797.

${ }^{25} \mathrm{~V}$. Raghuveer, P. Ferreira, A. Manthiram, Electrochem. Commun. 2006, 8, 807-814.

${ }^{26}$ Y. Xia, Y. Xiong, B. Lim, S. E. Skrabalak, Angew. Chem. Int. Ed. 2009, 48, 60-103.

${ }^{27}$ Y. Wang, H. -C. Peng, J. Liu, C. Z. Huang, Y. Xia, Nano Lett. 2015, 15, 1445-1450.

${ }^{28}$ B. Lim, M. Jiang, J. Tao, P. H. C. Camargo, Y. Zhu, Y. Xia, Adv. Funct. Mater. 2009, 19, 189-200.

${ }^{29}$ J. Watt, S. Cheong, M. F. Toney, B. Ingham, J. Cookson, P. T. Bishop, R. D. Tilley, ACS Nano, 2010, 4, 396402.
} 
- Using additives (such as polyol and sulfate): they guide the reaction kinetics and give control over shape.$^{30}$ Furthermore, they often serve as reducing and capping agents, for instance hexacarbonylmetals, ${ }^{31}$ acids, amines and $\mathrm{CO}^{32}$ or EDTA. ${ }^{33}$

- Stabilizing agents, like PVP, the function of which is directly related to the control of NPs size. $^{34}$

- Some agents act as reducing, stabilizing and additives simultaneously; for instance, some peptides. It usually helps for the generation of monodisperse, water-soluble palladium nanoparticles of controlled size. ${ }^{35}$

Controlling nucleation and using seed-mediated growth of palladium nanocrystals is a well stablished way to prepare size-controlled NPs. ${ }^{36}$ Nevertheless, significant achievements have been obtained by the seedless growth of palladium nanocrystals getting tuneable structures ${ }^{37}$ and ultrathin palladium nanosheets. ${ }^{38}$

\subsection{Supported palladium nanoparticles, synthesis and applications}

To take advantage of Pd-NPs the next step is the immobilization in solid supports, as it would permit recovery when involved in catalysis, and easier work-up and purification processes. ${ }^{39}$ Until now, most $\operatorname{Pd}(0)$ supported materials have been synthesized by electrochemical methods and, thanks to their characteristics, used with catalytical purposes. In contrast, when they are not directly supported by electrochemical methods, the usual way to do it is similar to other nanoparticles (as gold NPs). Preformed Pd-NPs are put in presence of a material that also acts as stabilizer.

Some of the most common applications of Pd-NPs, apart from hydrogen storage, has been as catalyst in a variety of chemical reactions, some examples are:

- Carbon-carbon coupling reaction ${ }^{40}$ (especially in water). ${ }^{41}$

- Hydrogenation of oxoderivatives, ${ }^{42}$ with recyclable materials. ${ }^{43}$

- Electrocatalysis, ${ }^{17}$ often as bimetallic nanocrystals. ${ }^{44}$ For ethanol oxidation, ${ }^{45}$ nitroaryl reduction ${ }^{46}$ or $\mathrm{CO}_{2}$ storage. $^{47}$

\footnotetext{
${ }^{30}$ H. Huang, Y. Wang, A. Ruditskiy, H.-C. Peng, X. Zhao, L. Zhang, J. Liu, Z. Ye, Y. Xia, ACS Nano 2014, 8, 7041-7050.

${ }^{31}$ Y. Li, Y. Yan, Y. Li, H. Zhang, D. Li, D. Yang, CrystEngComm, 2015, 17, 1833-1838.

${ }^{32}$ X. Yin, J. Wu, P. Li, M. Shi, H. Yang, ChemNanoMat 2016, 2, 37 - 41.

${ }^{33}$ C. Shang, W. Hong, Y. Guo, J. Wang, E. Wang, Chem. Eur. J. 2017, 23, 5799 - 5803.

${ }^{34}$ C. Evangelisti, N. Panziera, A. D’Alessio, L. Bertinetti, M. Botavina, G. Vitulli, J. Catal. 2010, $272,246-252$.

${ }^{35}$ S. Corra, U. Lewandowska, E. M. Benetti, H. Wennemers, Angew. Chem. Int. Ed. 2016, 55, 8542 -8545.

${ }^{36}$ Y. Xia, K. D. Gilroy, H.-C. Peng, X. Xia, Angew. Chem. Int. Ed. 2017, 56, 60-95.

${ }^{37}$ Y. Zhang, M. Wang, E. Zhu, Y. Zheng, Y. Huang, X. Huang, Nano Lett. 2015, 15, 7519-7525.

${ }^{38}$ X. Yin, X. Liu, Y.-T. Pan, K. A. Walsh, H. Yang, Nano Lett. 2014, 14, 7188-7194.

${ }^{39}$ D. Astruc, F. Lu, J. Ruiz Aranzaes, Angew. Chem. Int. Ed. 2005, 44, 7852-7872.

${ }^{40}$ A. Fihri, M. Bouhrara, B. Nekoueishahraki, J.-M. Basset, V. Polshettiwar, Chem. Soc. Rev. 2011, 40, 51815203.

${ }^{41}$ G. Yun, Z. Hassan, J. Lee, J. Kim, N.-S. Lee, N. H. Kim, K. Baek, I. Hwang, C. G. Park, K. Kim, Angew. Chem. Int. Ed. 2014, 53, 6414-6418.

42 A. Balouch, A. A. Umar, A. A. Shah, M. M. Salleh, M. Oyama, ACS Appl. Mater. Interfac. 2013, 5, 9843-9849.

${ }^{43}$ E. Hariprasad, T. P. Radhakrishnan, ACS Catal. 2012, 2, 1179-1186.

${ }^{44}$ K. D. Gilroy, A. Ruditskiy, H.-C. Peng, D. Qin, Y. Xia, Chem. Rev. 2016, 116, 10414-10472.
} 
When using supported Pd-NPs as catalysts, the molecular mechanisms are linked to the characteristics of the nanoparticles. ${ }^{40-47}$ Hence, the performance of palladium nanocatalysts is influenced by all the parameters that characterize the NPs; shape, size, stabilizers and support.

A particular case of catalytic procedure by supported palladium is the reduction by hydrogenation of alkenes ${ }^{48}$ and alkynes. ${ }^{49} \mathrm{~A}$ very efficient method but with low selectivity. ${ }^{50}$ In contrast, the importance of a selective, clean and cheap hydrogenation of internal alkynes to $(Z)$-alkenes is of great importance in pharmaceutical and industrial compounds. ${ }^{51}$ Regarding that selectivity, some methods with Pd nanoparticles have achieved high selectivity in DMF dispersion ${ }^{52}$ or supported with other nanomaterials. ${ }^{53}$ Existing extensive research in the mechanism of the (Z)-selectivity ${ }^{54}$ or the $\mathrm{Z} / \mathrm{E}$ interconversion of the initially obtained olefins. ${ }^{55}$

In summary, having well-defined structures, in shape and size, is the goal to achieve for new palladium nanomaterials, giving them clear-cut properties for innovative catalytic processes. ${ }^{22}$

${ }^{45}$ L. Ren, L. Yang, P. Yu, Y. Wang, L. Mao, ACS Appl. Mater. Interfac. 2013, 5, 11471-11478.

${ }^{46}$ E. D. Sultanova, V. V. Salnikov, R. K. Mukhitova, Y. F. Zuev, Y. N. Osin, L. Y. Zakharova, A. Y. Ziganshina, A. I. Konovalov, Chem. Commun. 2015, 51, 13317-13320.

47 A. Modak, M. Pramanik, S. Inagakib, A. Bhaumik, J. Mater. Chem. A, 2014, 2, 11642-11650

48 a) S. K. Mahato, R. U. Islam, C. Acharya, M. J. Witcomb, K. Mallick, ChemCatChem 2014, 6, 1419-1426. b) M. Iwanow, J. Finkelmeyer, A. Söldner, M. Kaiser, T. Gärtner, V. Sieber, B. König, Chem. Eur. J. 2017, 23, 12467-12470. c) S. K. Surmiak, C. Doerenkamp, P. Selter, M. Peterlechner, A. H. Schäfer, H. Eckert, A. Studer, Chem. Eur. J. 2017, 23, 6019-6028.

${ }^{49}$ Y. Gao, C.-A. Chen, H.-M. Gau, J. A. Bailey, E. Akhadov, D. Williams, H.-L. Wang, Chem. Mater. 2008, 20, $2839-2844$.

${ }^{50}$ A. S. Reddy, K. C. K. Swamy, Angew. Chem. Int. Ed. 2017, 56, 6984-6988.

${ }^{51}$ K. Chernichenko, A. Madarász, I. Pápai, M. Nieger, M. Leskelä, T. Repo, Nat. Chem. 2013, 5, 718-723.

52 J. Hori, K. Murata, T. Sugai, H. Shinohara, R. Noyori, N. Arai, N. Kurono, T. Ohkuma, Adv. Synth. Catal. 2009, 351, 3143-3149.

${ }_{53}$ T. Mitsudome, Y. Takahashi, S. Ichikawa, T. Mizugaki, K. Jitsukawa, K. Kaneda, Angew. Chem. Int. Ed. 2013, 52, 1481-1485.

${ }^{54}$ F. Zaera, ACS Catal. 2017, 7, 4947-4967.

${ }_{55}$ a) S. Furukawa, T. Komatsu, ACS Catal. 2016, 6, 2121-2125. b) K. Tokmic, A. R. Fout, J. Am. Chem. Soc. 2016, 138, 13700-13705. 


\section{CHARACTERIZATION OF SUPPORTED NPS}

The reference technique for studying NPs is transmission electron microscopy (TEM) and its high resolution version (HRTEM). This kind of analysis gives accurate data of shape, size and dispersity of the particles, or even the crystalline structure in high resolution. There are even more precise methods, such as atomic force microscopy (AFM), in which the structure of the nanomaterials could be observed with atomic resolution.

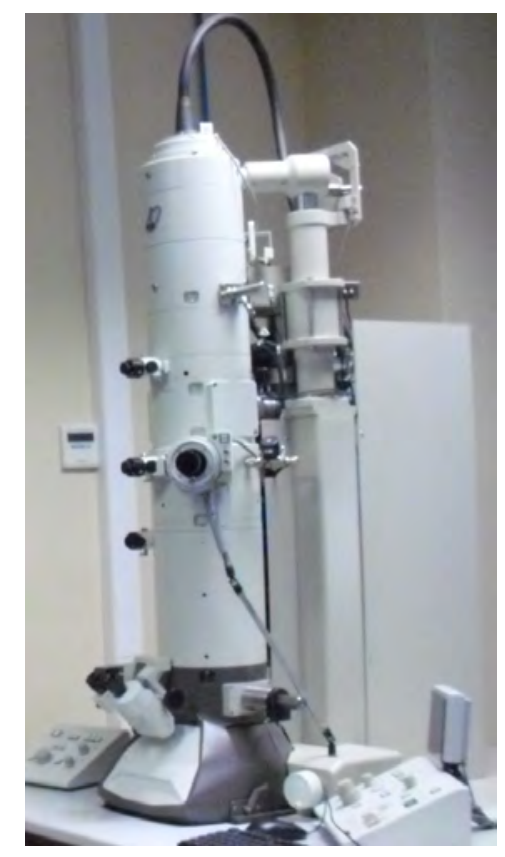

Figure 8. TEM apparatus from Valladolid University.

When the particles are deposited over a surface, the shape and size is observed and measured by scanning electron microscopy (SEM), usually combined with X-Ray photoelectron microscopy (XPS) for the composition of the external layer or energy-dispersive X-ray spectroscopy (EDX) for getting composition with more penetration into de material. SEM analysis has the disadvantage of lower resolution than TEM, but the sample preparation is simpler. Moreover, XPS and EDX analysis give the atomic composition, which is related to the amount and number of stabilizers presented in the sample (such as sulfur containing molecules).

Cheaper and more straightforward techniques are UV-Vis and NIR absorption. Metal nanoparticles have characteristic plasmons (SPA) that give different absorption depending on shape and size, giving raw information when measured.

Additionally, techniques such as Raman dispersion or fluorescence give complementary information to the absorption and dynamic light scattering may give information about aggregation. Besides, other techniques could give additional information related to specific properties of different nanoparticles, solubility, additives, stabilizers and stability; techniques such as NMR, mass spectrometry, melting point or calorimetric studies. 


\section{OBJECTIVES OF THE CHAPTER}

For some time, the group had been working in the detection of $\mathrm{Hg}(\mathrm{II})$, which is a known thiophilic cation. As it was explained in Chapter 1, detection of $\mathrm{Hg}(\mathrm{II})$ was performed by fluorogenic reagents having sulfur atoms. If the results of qualitative tests are carefully observed (Section 5.3.3 of Chapter 1) the characteristic colour of Au-NPs was obtained in solution. In addition, the change in colour also happened in solid materials, like silica NPs or polymeric films.

The colour and its intensity depended on many factors such as the composition of the material, Au(III) concentration, solvent, the relation between volume (solution)/ size (material) and the time of contact. Once checked in literature, the interest of getting Au-NPs supported in a material became clear. In consequence, the purpose was to reach different objectives:

- Optimization of materials to act as reductants, stabilizers and support for the nanoparticles.

- Evaluation and characterization of the synthetized gold nanoparticles.

- Optimization of the material-conditions to obtain gold nano-microparticles, controlling time, concentration and solvents while following the effect over size and shape of the particles; in solution and supported.

- Looking for a practical application of the supported gold nanoparticles. This experimental was aimed to get applications as a catalyst for organic synthesis; in particular, C-C coupling by substitution of the Pd catalyst used in Suzuki-Miyaura reactions.

After obtaining satisfactory results, the idea of getting similar synthetical procedures for a variety of supported metallic nanoparticles led us to try with other metals and copolymers. From all of them, there was a series of polymeric derivatives from what $\operatorname{Pd}(0)$ nanoparticles were easily obtained, giving to the material outstanding applications as it was subsequently noticed.

The objectives for supported Pd-NPs were:

- Simplify the preparation, by just adding the polymers to a $\operatorname{Pd}(\mathrm{II})$ solution in water. The aim was to improve pre-existent procedures in which the nanoparticles were preformed and stabilized in a surface.

- Adaptation and control of the synthesis to obtain monodisperse polycrystalline palladium nanoparticles uniformly distributed over the surface of polymers.

- Evaluation and characterization of the results.

- Search for a straightforward application of the supported NPs. In this case, it was potentially viable to use them as an efficient, portable and reusable catalyst for the stereoselective semihydrogenation reaction of internal alkynes to $(Z)$-alkenes. 


\section{EXPERIMENTAL SYNTHESIS OF GOLD NPS}

\subsection{Antecedents}

In Chapter 1, it was introduced how an excess of PEG containing probe in solution was capable to reduce $\mathrm{Au}(\mathrm{III})$ in solution to gold NPs. What is more, the silica modified solids with terminal triple bonds led to the formation of gold nanoparticles in solution (Figure 9), although they precipitated with time.

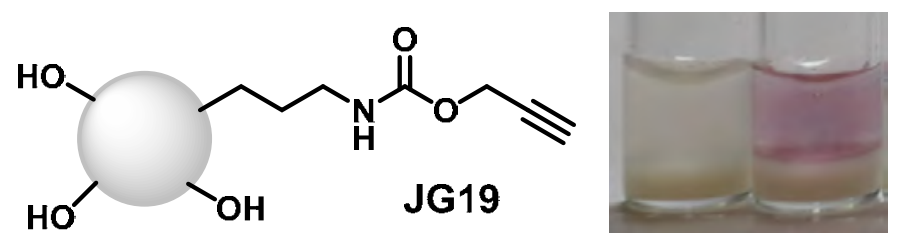

Figure 9. JG19 in presence of $\mathrm{HAuCl}_{4}(0.5 \mathrm{mM})$ after 5 hours.

Later, the reduction synthesis was optimized for solutions and polymeric films.

\subsection{Synthesis and characterization of gold NPs in solution}

In order to test the action of solutions containing triazole, PEGs and carbothioamide probe, several experiments were performed in solution. The water-soluble derivatives JG45 and JG47, $(\mathrm{Hg}(\mathrm{II})$ probes from Chapter 1) were tested in presence of $\mathrm{Au}(\mathrm{III})$ solutions.

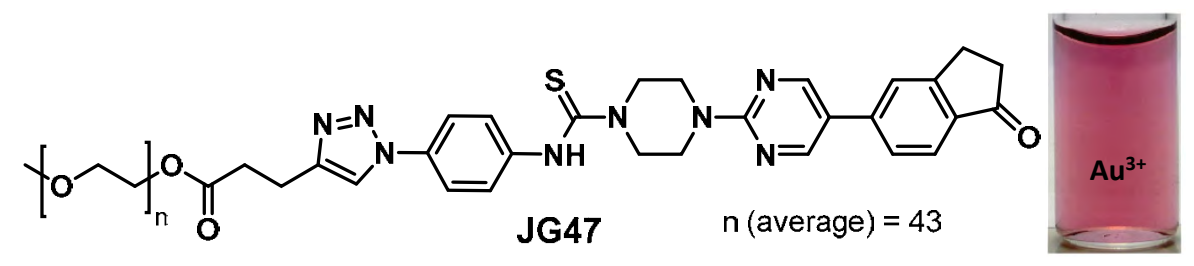

Figure 10. Au-NPs formed in presence of JG47 (0.5 mM JG47 and $0.2 \mathrm{mM} \mathrm{Au(III)).} \mathrm{After} 48$ hours.

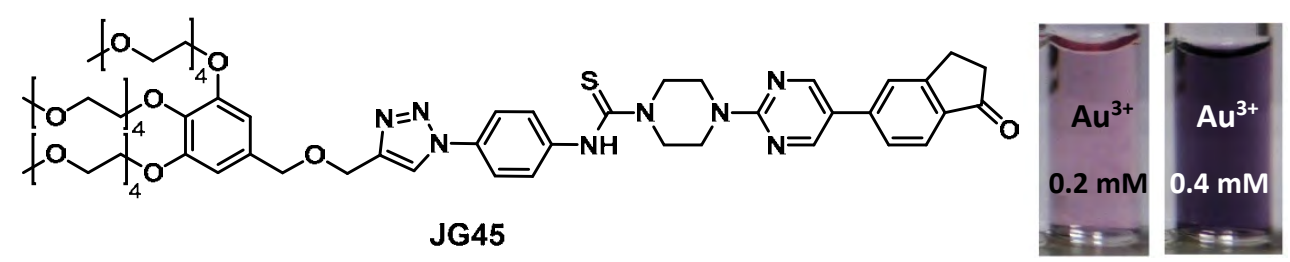

Figure 11. Au-NPs formed in presence of JG45 (0.1 mM JG45 and $0.2 \mathrm{mM}$ and $0.4 \mathrm{mM} \mathrm{Au}(\mathrm{III}))$.

After 24 hours.

As it may be observed in Figures 10 and 11, this method required high concentration of the analyte to stabilize the NPs and, if the proportion of $\mathrm{Au}(\mathrm{III})$ added was too high, it precipitated (dark purple). In consequence, to get a proof of the compound acting not only as reductant but as stabilizer, the solution was added to HEPES buffer - Au-NPs. HEPES gold nanoparticles are characterised as not very stable and with tendency to agglomeration and precipitation. ${ }^{56}$ However, in the presence of JG45 solution their colour changed from purple to pink, evidencing stabilization of the AuNP by JG45.

\footnotetext{
${ }^{56}$ R. Cheng, J. Wu, H. Li, G. Cheng, Z. Lu, C.-M. Che, Rare Metals, 2010, 29, 180-186.
} 
In a representative experiment (Figure 12), a solution of probe $(0.4 \mathrm{mM})$, in water, was added to an aqueous solution of HEPES $5 \mathrm{mM}(\mathrm{pH} 7.9)$ in a $0.5 \mathrm{~mL}$ tube, afterwards a solution of $\mathrm{HAuCl}_{4}$ in water was added to it $(0.4 \mathrm{mM})$. Furthermore, to get better demonstration of the tuneability of the particles (Figure 13), other thiophilic cation was added, $\mathrm{Hg}\left(\mathrm{ClO}_{4}\right)_{2}$, after 24 hours, in a concentration of 0.4 $\mathrm{mM}$.

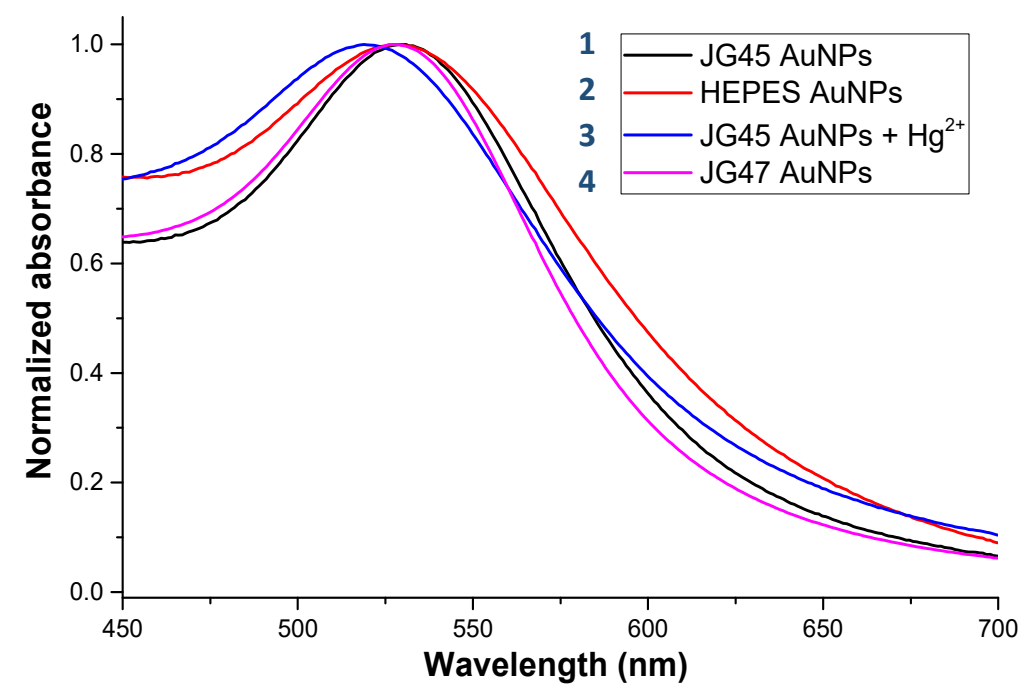

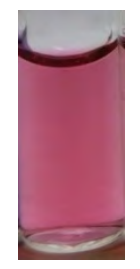

1

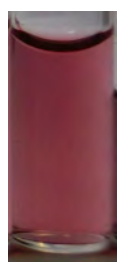

3

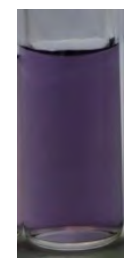

2

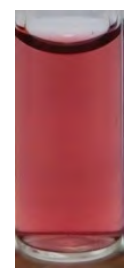

4

Figure 12. UV spectra of Au-NPs solutions obtained from JG45 (0.4 mM), JG47 (0.4 mM), HEPES $(5 \mathrm{mM})$ and $\mathrm{Au}^{3+}(0.4 \mathrm{mM})$ or $\mathrm{Au}^{3+}$ and $\mathrm{Hg}^{2+}$ solutions. Inset: solutions under white light.
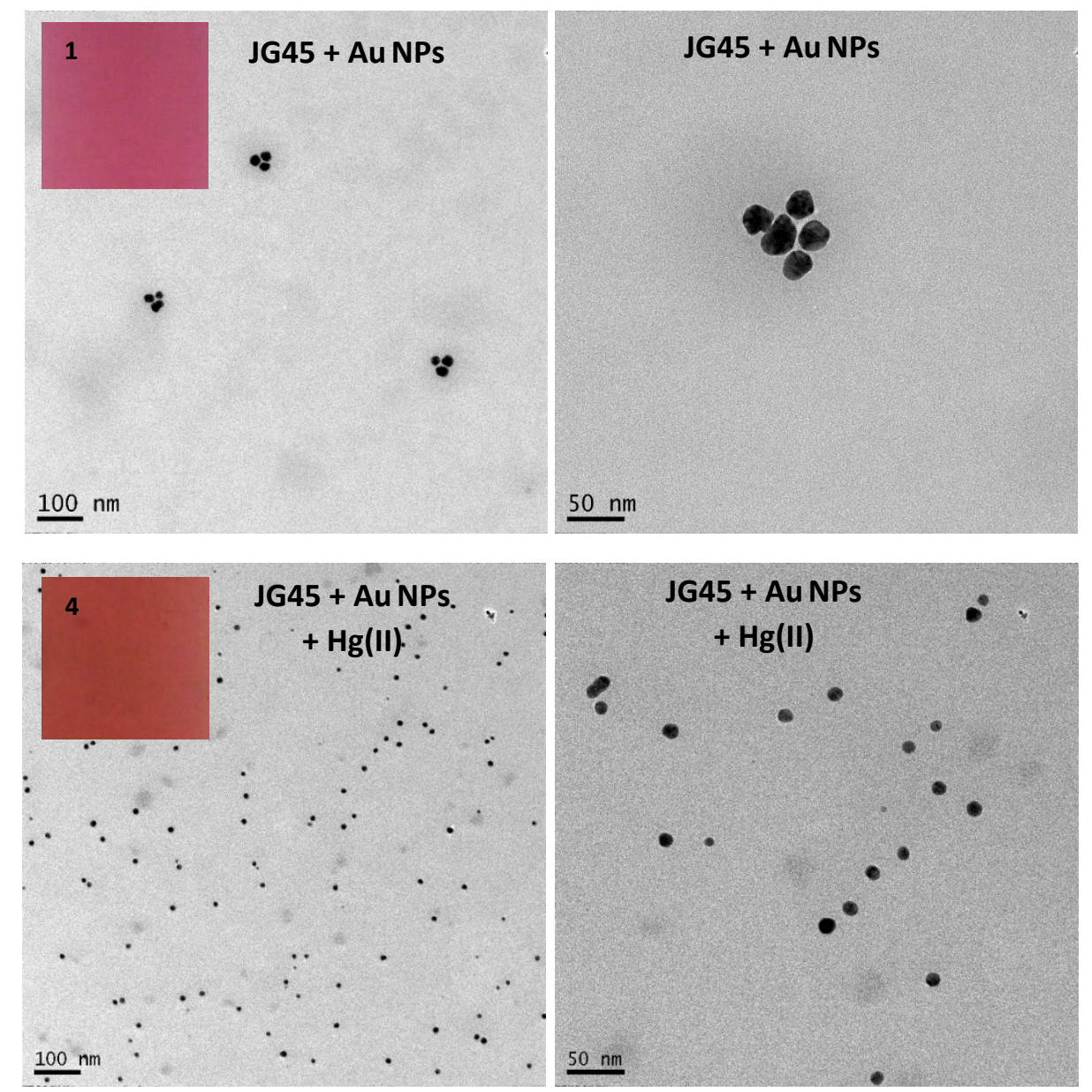

Figure 13. SEM images of Au-NPs from JG45 and pictures of the solutions. Solutions 1 and 4 from Figure 12. 
From this experiment several conclusions were obtained:

- The soluble probes containing PEGs, triazole and carbothioamide group led to Au-NPs in water solution.

- The molecules also acted as stabilizers for the NPs.

- The NPs formed were spherical-amorphous of around 20-25 nm in JG45 solution and spherical around 5-10 nm when $\mathrm{Hg}(\mathrm{II})$ was added.

- The colour given to the solution was red-pink when stable. It may be tuned by changing proportions $\mathrm{Au}(\mathrm{III}) /$ probe or adding some other species, such as $\mathrm{Hg}(\mathrm{II})$ (additive).

\subsection{Synthesis and characterization of supported gold particles}

First results for supported Au-NPs came from silica NPs. However, due to unsatisfactory results in their optimization and the preliminary search for applications, and the better performance of polymeric membranes, the work was focused in the latter, a film surface to be modified.

The film-shaped functional membrane (JG25_SA2) preparation was already explained in Chapter 1 and consisted of the photochemical initiated radical polymerization of the hydrophilic monomer 2-hydroxyethylacrylate (2HEA) plus propargyl methacrylate (PGM) and ethylene; using glycol dimethacrylate (EGDMA) as cross-linking agent (Figure 14).

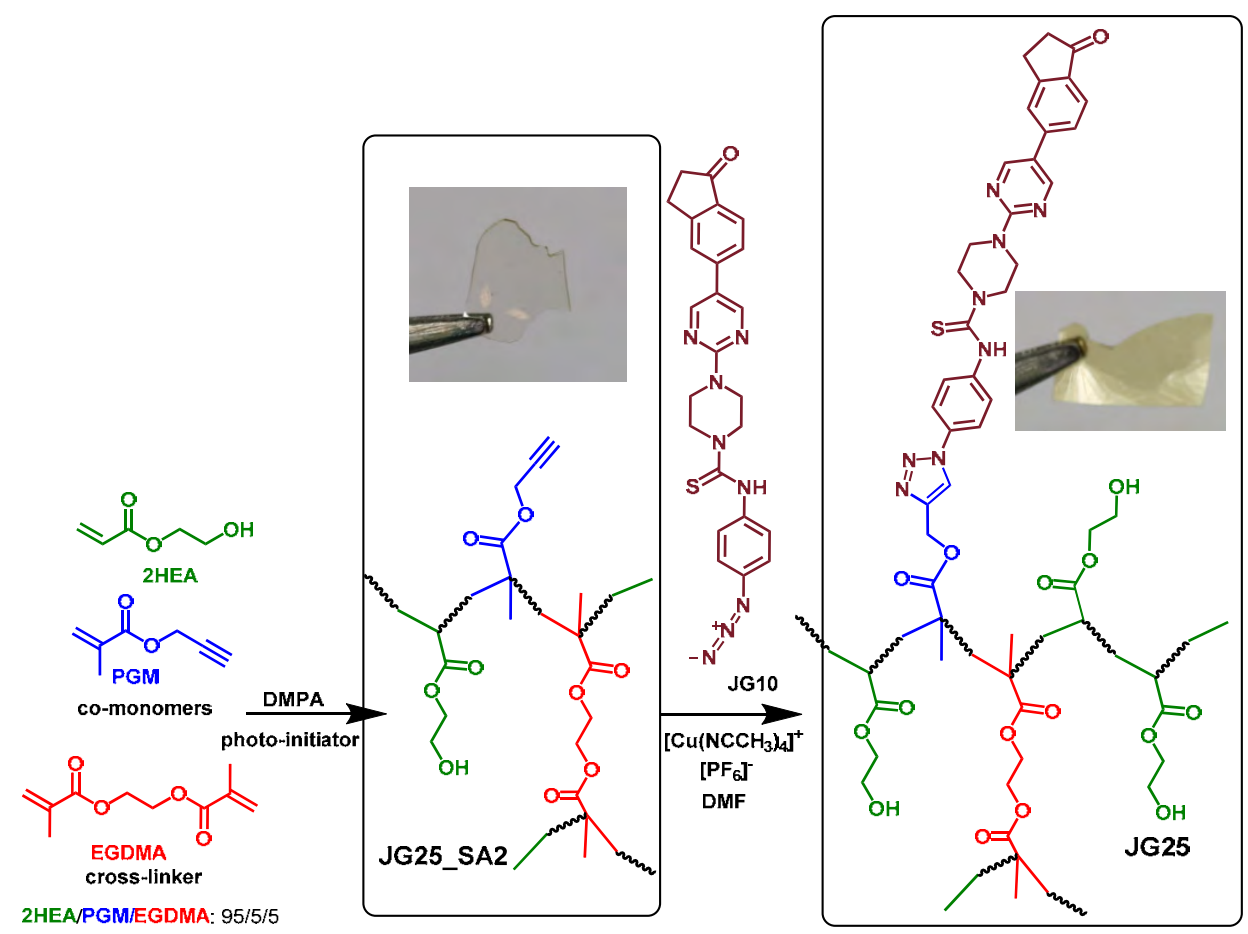

Figure 14. Synthesis of the polymeric film Mem and its fluorogenic derivative JG25. Pictures taken under white light.

The co-monomer molar ratio 2HEA/PGM/EGDMA was 95/5/5, respectively. 2,2-Dimethoxy-2phenylacetophenone (DMPA, 1.5\% wt) was employed as a photochemical initiator.

Next, fragments of JG25 and JG25_SA2 were submitted to $\mathrm{HAuCl}_{4}$ solutions in water until the polymer became purple. The optimization of the conditions was carried out systematically: 
- Using JG25_SA2 or JG25.

- Evaluating the effect of using different $A u(I I I)$ concentrations.

- Changing the relation volume/polymer surface.

- Regulating the time of contact of the polymer with the $A u(I I I)$ solution.

- Changing the solvent.

Different degrees of intensity of the purple colour were obtained and, therefore, different patterns of covering of the polymer with gold particles. The proportions of polymer surface/[ $\left.\mathrm{Au}^{3+}\right]$ and the time to form the nanoparticles were correlated. While the particles grew, the concentration or $\mathrm{Au}(\mathrm{III})$ in solution decreased, so the process could be stopped at any time by removing the polymer from the solution and washing it.

Something to take into account is that, when the polymer was introduced in water, it swelled. If this process was controlled, for example by adding very small amounts of water at specific concentrations, the shape of the particles could be regulated, even obtaining different colour on the surface of the polymer. Many examples were carried out in which all of these changes could be observed, as it is detailed in subsequent sections.

In a representative experiment, $100 \mu \mathrm{L}$ of $\mathrm{Au}^{3+}\left(5 \mathrm{mM} \mathrm{HAuCl}_{4}\right.$ in water $)$ were added to a $1 \times 1$ $\mathrm{cm}$ square piece of JG25 and it was left standing for $24 \mathrm{~h}$, becoming dark purple. This samples were analysed by SEM and absorption of the film (Figures 15 and 16).
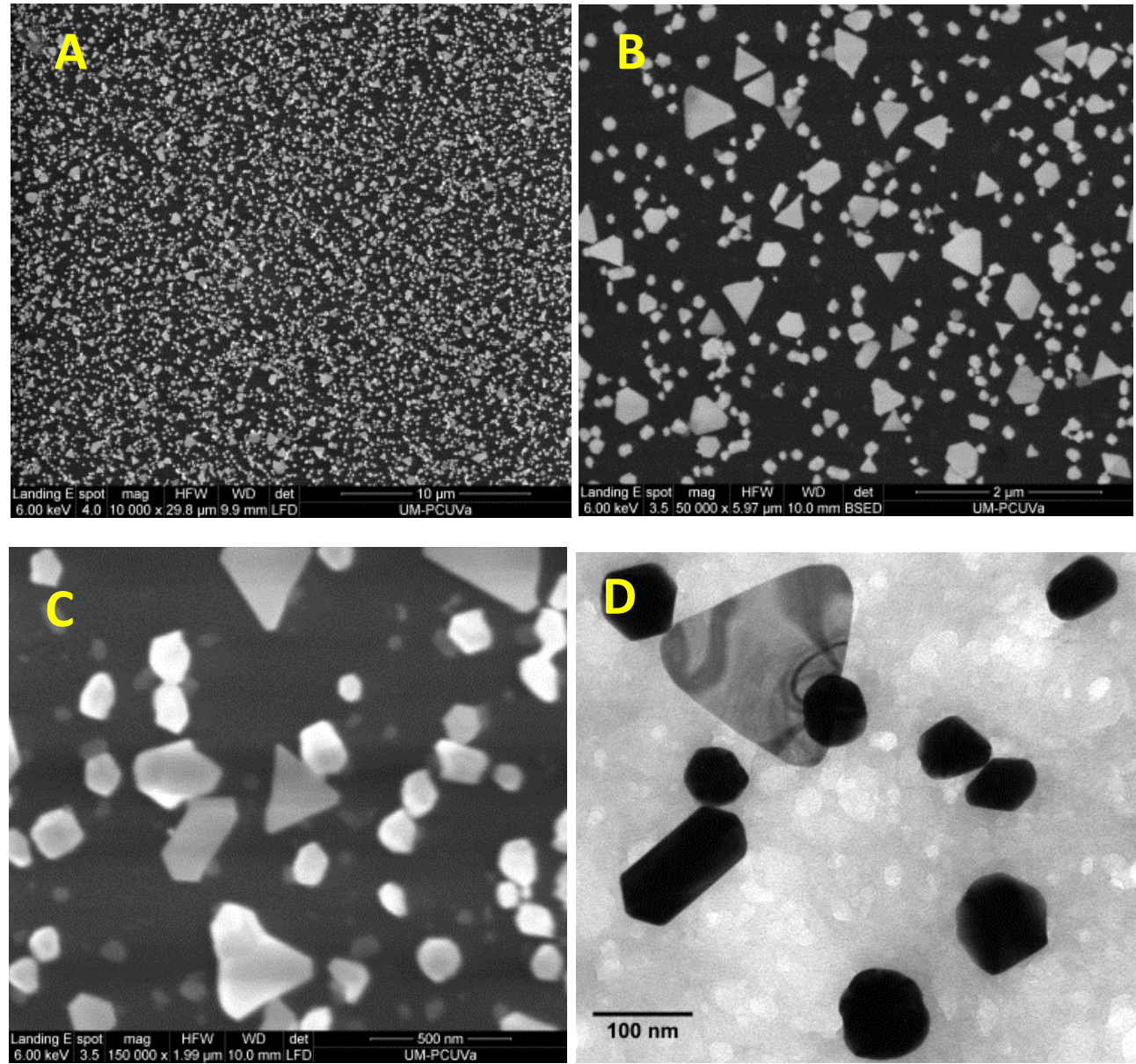

Figure 15. (A-C), SEM images of the homogeneous coating of the polymer JG25 by Au-Ps $(100 \mu \mathrm{L}$ $\mathrm{Au}^{3+}, 5 \mathrm{mM} \mathrm{HAuCl}_{4}$ in water). A) scale bar $\left.10 \mu \mathrm{m}, \mathrm{B}\right)$ scale bar $\left.2 \mu \mathrm{m}, \mathrm{C}\right)$ scale bar $500 \mu \mathrm{m}$. D) TEM image of related gold nanoparticles obtained in solution, scale bar $100 \mathrm{~nm}$. 

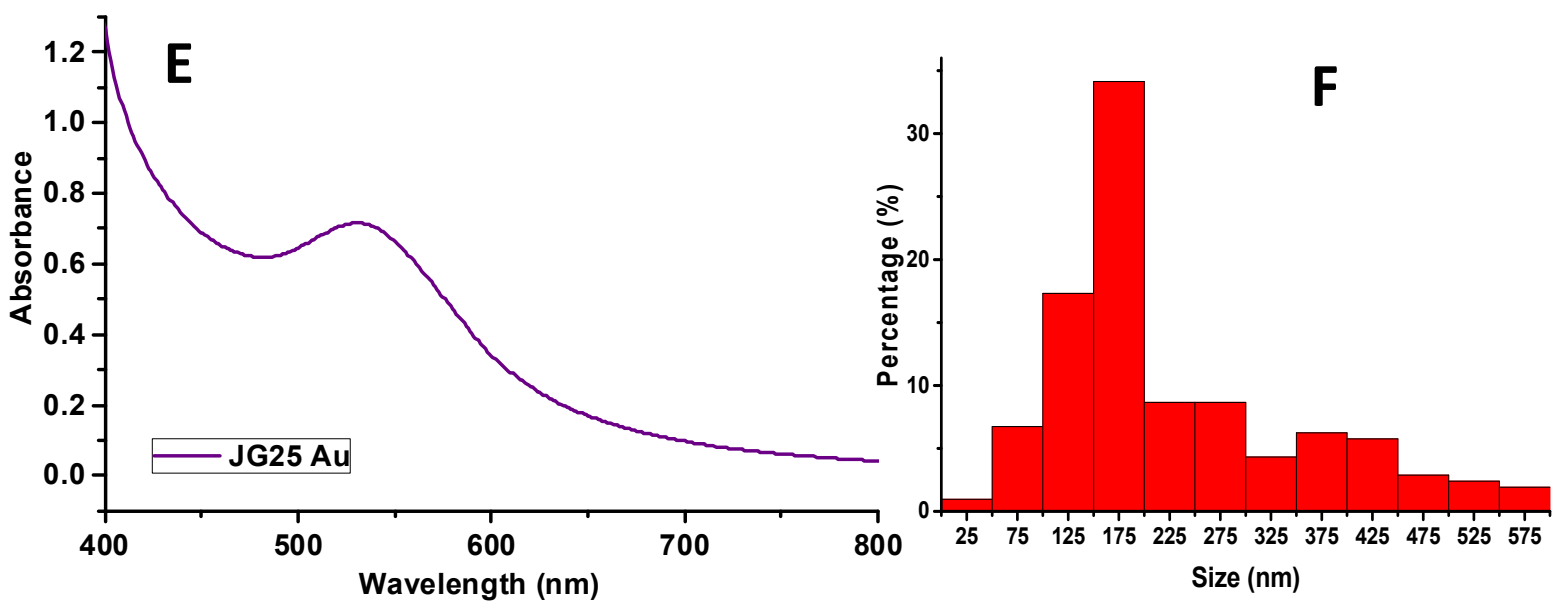

Figure 16. E) UV-vis spectrum of JG25 covered with Au-Ps (E). F) Distribution of the particle size.

By scanning electron microscopy (SEM) analysis, the surface of the polymer was observed as uniformly coated by gold nano and/or microparticles, as well as some flat gold nanoplates and short gold nanorods (Figure 15A-D). The covering area of the gold nanoparticles was around 27\%. A more detailed view of the gold nanoparticles was obtained by performing an analysis by transmission electron microscopy (TEM) of the related types of nanoparticles obtained in solution (Figure 15D). In this case, the UV-vis spectrum showed a maximum of absorbance at $540 \mathrm{~nm}$ (Figure 16F).

\subsubsection{Effect of increasing time:}

If the time in solution was increased and the rest of conditions were the same, the number and the size of the particles increased, becoming darker.

All the samples of Figure 17 were obtained in the presence of $100 \mu \mathrm{L}$ of $\mathrm{Au}^{3+}$ solution $\left(5 \mathrm{mM} \mathrm{HAuCl}_{4}\right.$ in water).

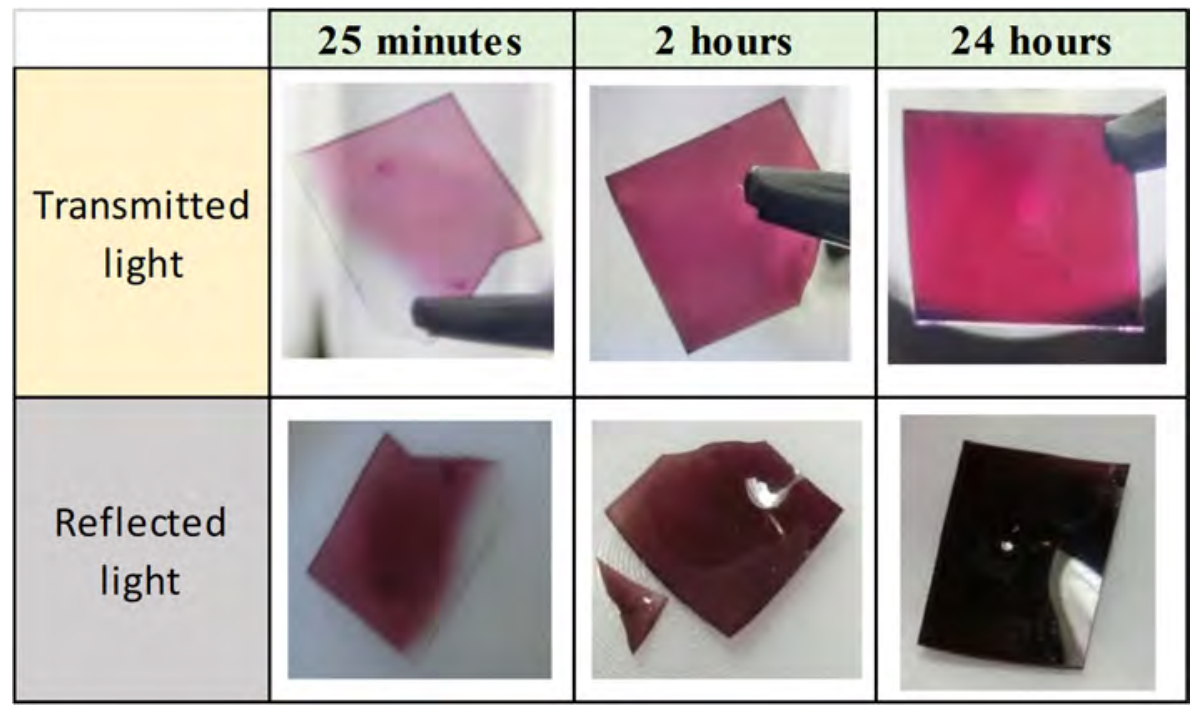

Figure 17. Colour changes by deposition of $\mathrm{Au}(0)$ at different reaction times, JG25 $+\mathrm{Au}(\mathrm{III})$ solutions $(5 \mathrm{mM})$. 


\subsubsection{Tests with JG25 SA2 (film without the carbothioamide probe):}

The film-shaped functional membrane (JG25_SA2) was tested in similar conditions to verify if the presence of the fluorogenic dye was required for the generation of the Au-Ps.

As a result, only smaller particles were obtained with very few nanoplates (Figure 18). Albeit, in contrast with JG25, the distribution of the size was more disperse under the same conditions. The gold nanoparticles were well distributed and covered a relative area of around $23 \%$.

Complementary information was given by cryofracture and SEM imaging of the transversal section of one of the samples of polymer coated by gold NPs. The NPs were located only on the surface of the polymer, with very few and very small nanoparticles inside the bulk (Figure 19G-H).
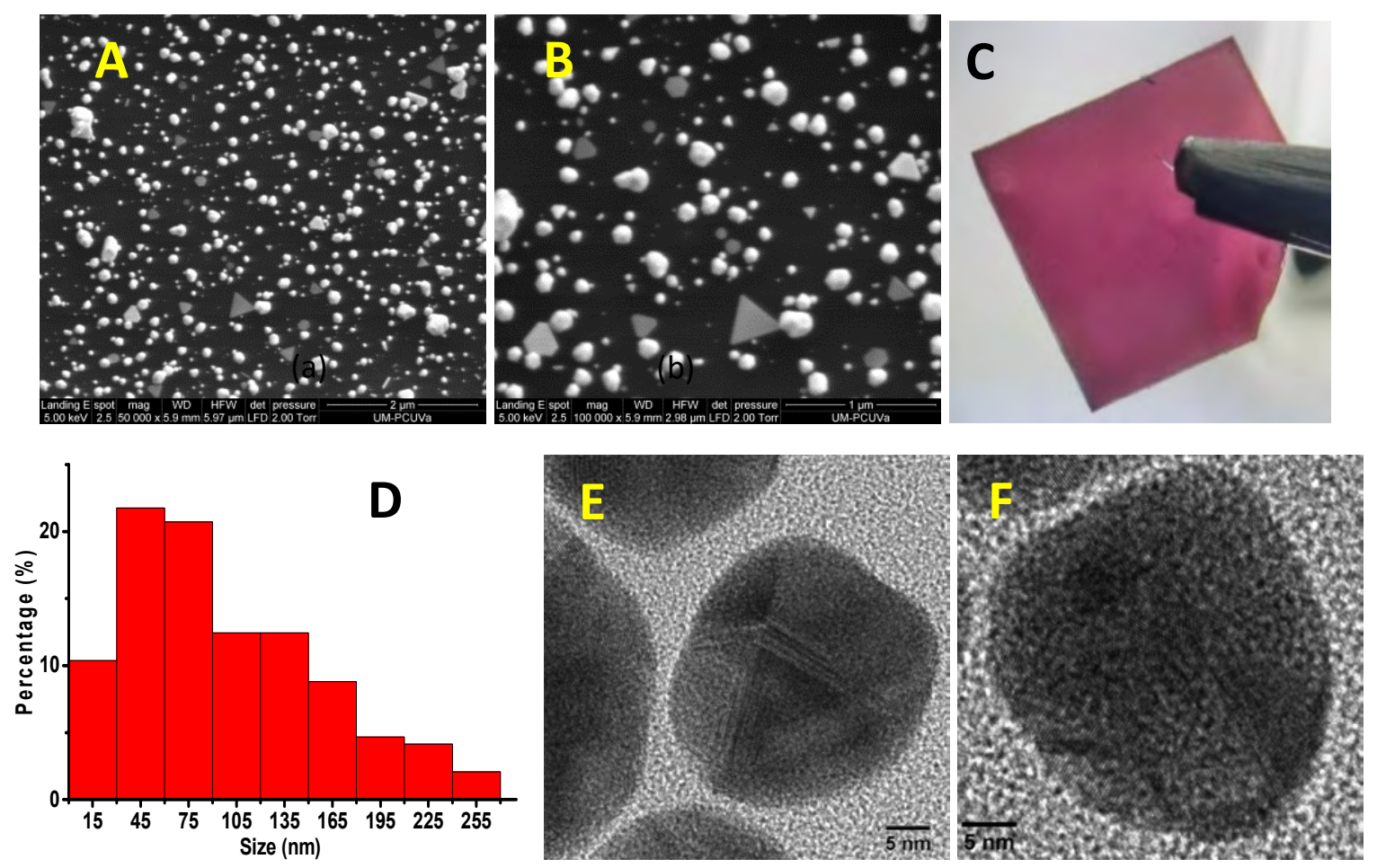

Figure 18. SEM images of the polymer membrane surface (JG25_SA2) homogeneously coated by gold NPs $\left(100 \mu \mathrm{L} \mathrm{Au}^{3+}, 5 \mathrm{mM} \mathrm{HAuCl}_{4}\right.$ in water). A) scale bar $\left.2 \mu \mathrm{m}, \mathrm{B}\right)$ scale bar $1 \mu \mathrm{m}$. C) Image showing the colour of the polymer by transmitted light, D) distribution of the particle size. E-F) TEM images of related gold nanoparticles obtained in solution, scale bar $5 \mathrm{~nm}$.
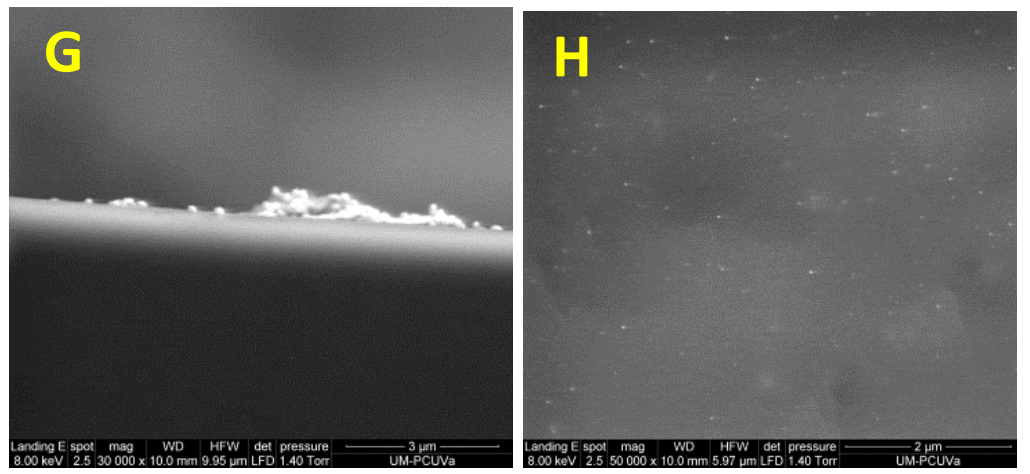

Figure 19. G) SEM images of the polymer membrane surface (JG25_SA2) and section on which gold nanoparticles were grown, scale bar $3 \mu \mathrm{m}$. H) SEM image of the cryofracture section of the polymer JG25_SA2 with very few small gold nanoparticles, scale bar $2 \mu \mathrm{m}$. 


\subsubsection{Changing the solvent:}

Au-NPs may be synthesised in different solvents, getting different size and shape. In addition, the swelling of the polymer was completely different so the properties were likely to change. For instance, the formation of NPs was measured in DMF, which produced a homogeneous coating of the polymer by gold nanoparticles.

A representative experiment consisted of the addition of $100 \mu \mathrm{L} \mathrm{Au}^{3+}\left(\mathrm{HAuCl}_{4} 5 \mathrm{mM}\right.$ in DMF) to a $1 \times 1 \mathrm{~cm}$ square piece of JG25_SA2 and then, left it standing for 24 hours. In this case, the polymer appeared blue translucent with purple reflection (Figure 21A-C). In comparison to water, the Au-NPs formed in DMF were very small, homogeneous and covered a very small relative area of $3 \%$ approximately (Figure 20A-B). Parameters such as time were equally important, having influence over size and giving larger nanoparticle sizes at longer times.
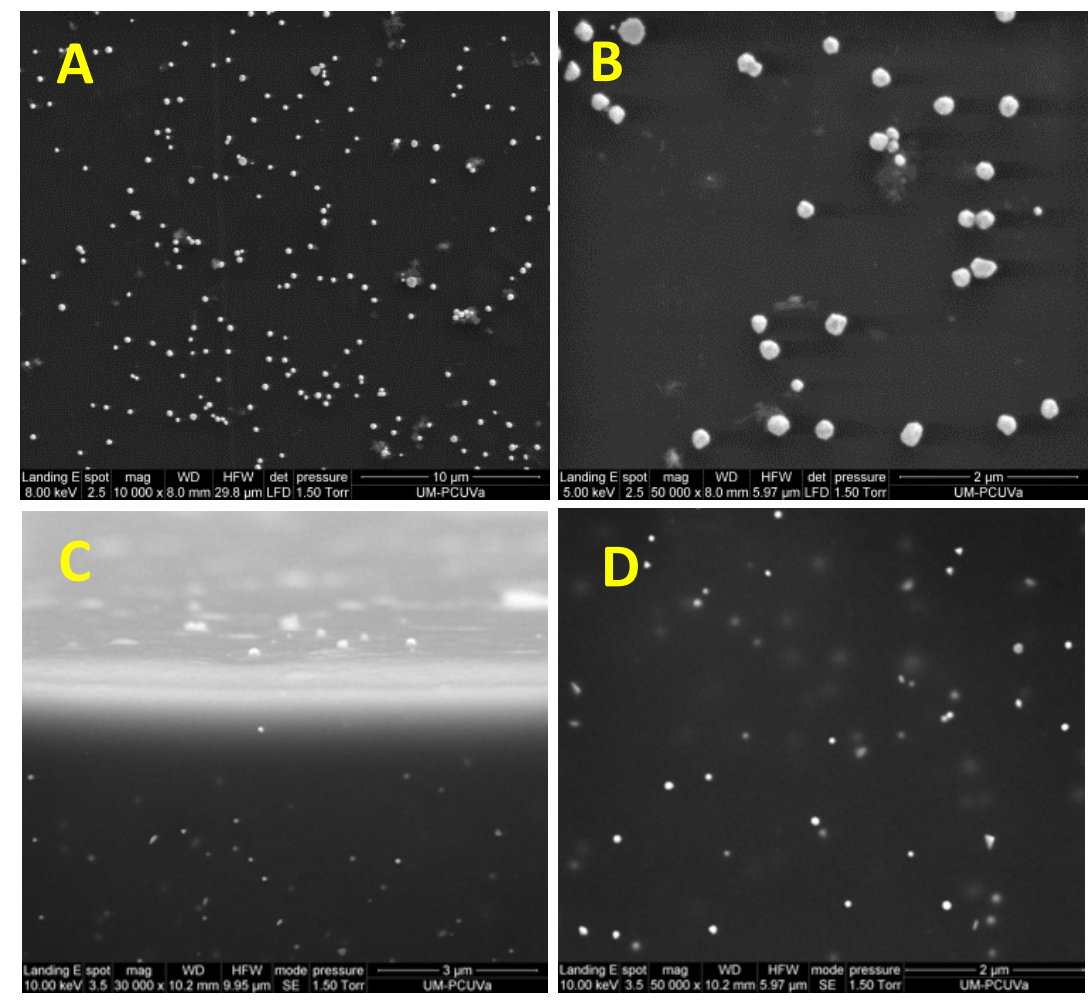

Figure 20. A-B) SEM images of the polymer membrane (JG25_SA2) surface in which Au-NPs were grown by using DMF (100 $\mu \mathrm{L} \mathrm{Au}^{3+}, \mathrm{HAuCl}_{4} 5 \mathrm{mM}$ in DMF). A) Scale bar $10 \mu \mathrm{m}$. B) Scale bar $2 \mu \mathrm{m}$.

C) SEM image of the surface and the cryofracture section of the polymer (JG25_SA2) by gold nanoparticles, scale bar $3 \mu \mathrm{m}$. D) SEM image of the cryofracture section of the polymer (JG25_SA2) with gold nanoparticles in the bulk of the polymer, scale bar $2 \mu \mathrm{m}$.
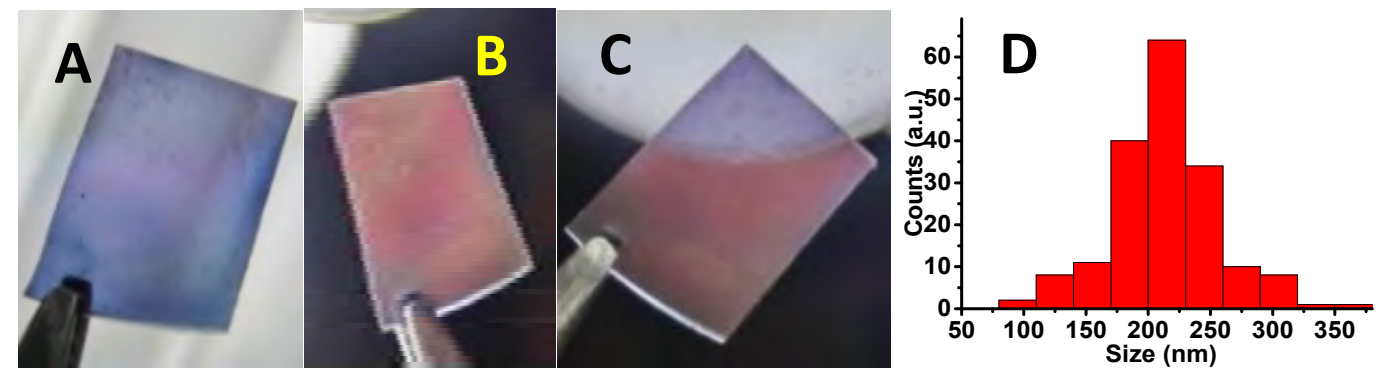

Figure 21. Images showing the colour of the polymer (JG25_SA2) by means of transmitted light (A), reflected light (B), transmitted and reflected light (E). Distribution of the particle size (F). 
Cryofracture and SEM imaging of the transversal section were also performed to one of the samples. It was verified that the Au-NPs were, in this case, evenly distributed also inside the bulk of the polymer (Figure 20D). Nevertheless, they were much smaller $(50-90 \mathrm{~nm}$ average) than the particles on the surface (200-250 nm). This fact explained the optical characteristics found for polymer membranes on which gold nanoparticles were grown by using DMF (Figure 21).

\subsubsection{Additional experiments:}

The importance of having "triple bonds" in the material or adding very low volumes of gold solutions were facts that could give additional information.

In a first experiment (Figure 22), it was checked whether a simple 2HEA (2hydroxyethylacrilate) polymer, a polymer with the same characteristics but without the $5 \%$ of propargyl methacrylate; could deposit Au-NPs. Negative results were obtained, even if the concentration and time were changed. Therefore, the presence of bonded JG10 or the triple bonds on the surface of the polymers were necessary for the formation of gold nanoparticles.
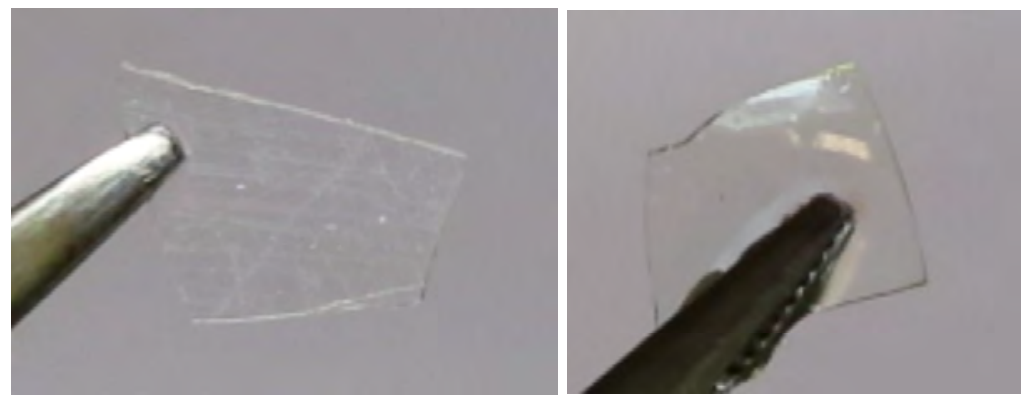

Figure 22. 2HEA polymer (left) and 2HEA polymer after 2 days in contact to a water solution $0.5 \mathrm{mM}$ $\mathrm{HAuCl}_{4}$.

Additionally, an experiment was performed in which a drop of $\mathrm{Au}(\mathrm{III})$ from a concentrated solution was added to the corner of a polymer (Figure 23). The aim was to show in one single piece of polymer the importance and relation between $[\mathrm{Au}(\mathrm{III})] /$ surface - swelling - time.

From the experiment, long nanorods up to $1.5 \mu \mathrm{m}$ were obtained (blue) with very few flat gold nanoplates, triangles and hexagons. ${ }^{57}$ However, the distribution was not homogeneous in this case; the gold nanorods, with lengths that ranged from $100 \mathrm{~nm}$ to 1.5 microns, where the major product for some areas of the polymer while Au-NPs similar to the other tests, were obtained within the place where the drop was added (pink-purple).

\footnotetext{
57 a) N. Li, P. Zhao, D. Astruc, Angew. Chem. Int. Ed. 2014, 53, 1756-1789. b) I. Pastoriza-Santos, R. A. Alvarez-Puebla, L. M. Liz-Marzán, Eur. J. Inorg. Chem. 2010, 4288-4297. c) Y. Wang, K. Sentosun, A. Li, M. Coronado-Puchau, A. Sánchez-Iglesias, S. Li, X. Su, S. Bals, L. M. Liz-Marzán, Chem. Mater. 2015, 27, 8032-8040.
} 

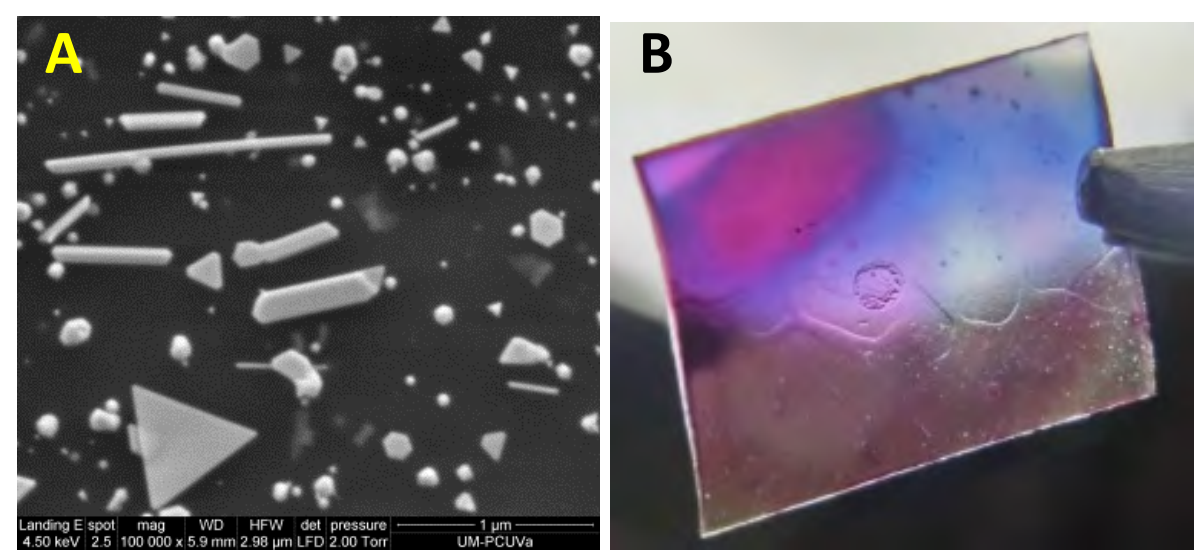

Figure 23. A) SEM image of the polymer membrane surface coated by gold nanorods, nanoplates and nanoparticles ( $40 \mu \mathrm{L} \mathrm{Au}^{3+}, \mathrm{HAuCl}_{4} 5 \mathrm{mM}$ in water), scale bar $1 \mu \mathrm{m}$. B) Image showing the colour of the polymer by transmitted and reflected light.

\subsubsection{Summary:}

The results of the different tests for the formation of film supported Au-NPs were:

$>$ In the presence of the fluorescent probe there was higher dispersion in particle size.

$>$ Using high concentrations of the salt increased faster the number of particles than the size.

$>$ Low relation volume/surface led to more variation in the shape of the nanoparticles.

$>$ The size of the particles was between $20 \mathrm{~nm}$ to $400 \mathrm{~nm}$, depending on the conditions.

$>$ The different shapes encountered were tridimensional, amorphous, spherical, tetrahedral or tetrahedral truncated; bidimensional, triangles; and monodimensional (or with a preferential grow direction), bars with different thickness.

$>$ In water, the particles were located over the polymer while using DMF led to NPs inside the film. 


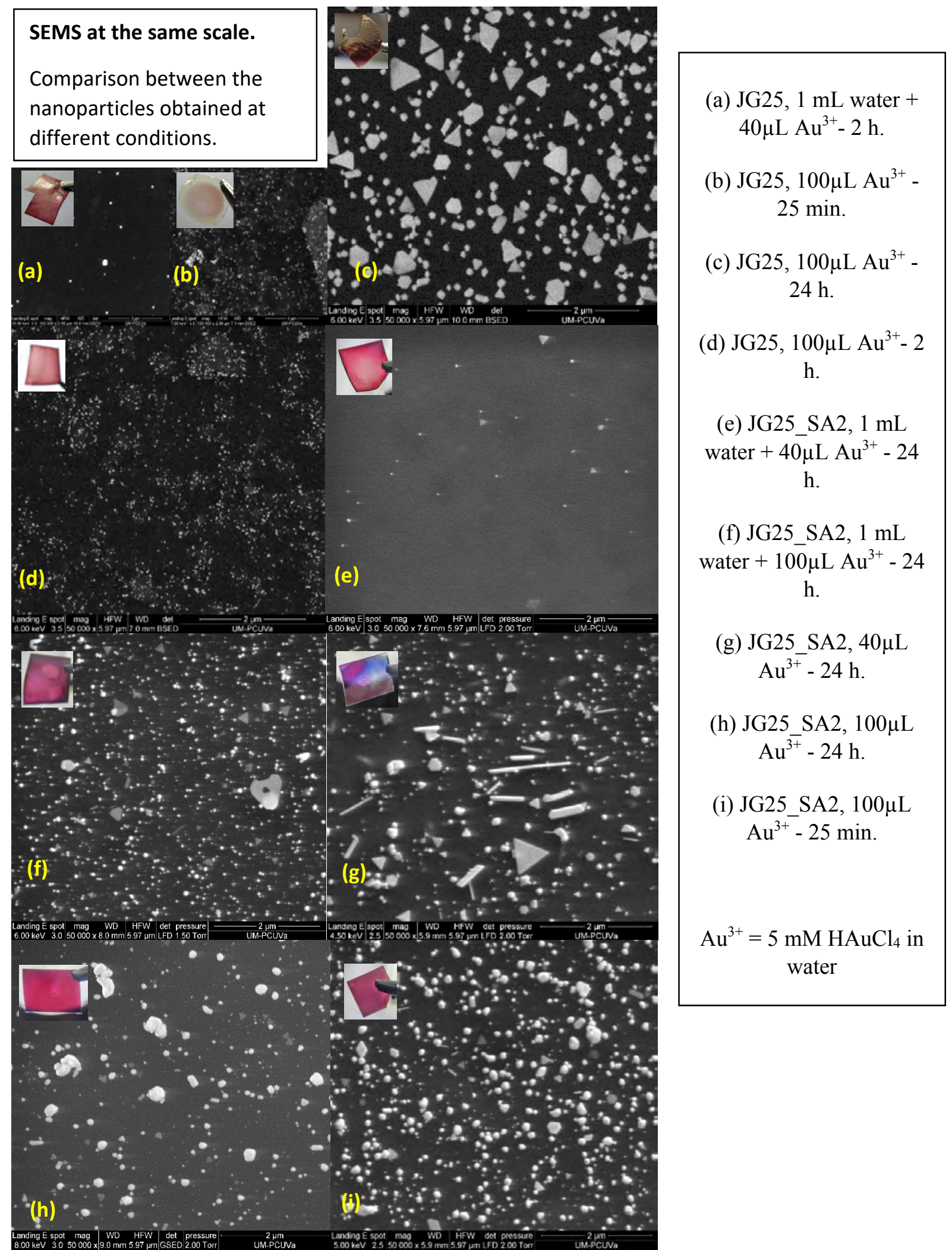

Figure 24. Gold nanoparticles formation at different time and reaction conditions. 


\section{APPLICATION IN CATALYSIS OF GOLD-NPs}

Once the coating of the surface of the polymer by gold nanoparticles was sufficiently studied, practical applications were the next step to be addressed. Between the many reactions catalysed by solid supported gold nanoparticles ${ }^{58}$ poly(2-aminothiophenol) supported gold nanoparticles had shown excellent catalytic activity for Suzuki-Miyaura cross-coupling reactions. ${ }^{59}$

The polymer supported Au-NPs material was found to be useful as a solid reusable catalyst. The conditions to perform these reactions were very similar to the classical reactions performed with $\mathrm{Pd}\left(\mathrm{PPh}_{3}\right)_{3}$ as the catalyst.

\subsection{Synthesis and yields}

As a representative experiment, a $25 \mathrm{~mL}$ round bottom flask was filled with $30 \mathrm{mg}(0.14 \mathrm{mmol})$ of 5-bromoindan-1-one, one equivalent of the boronic ester or acid, $75 \mathrm{mg}(0.7 \mathrm{mmol})$ of $\mathrm{Na}_{2} \mathrm{CO}_{3}$ and a $0.3 \times 0.3 \mathrm{~cm}$ polymer piece coated with gold nanoparticles obtained from previous synthesis. After that, a solvent mixture composed of $4 \mathrm{~mL}$ of THF and $0.5 \mathrm{~mL}$ of water was added. The mixture was refluxed for 20 hours, extracted by partition in DCM:Water and purified by column chromatography.

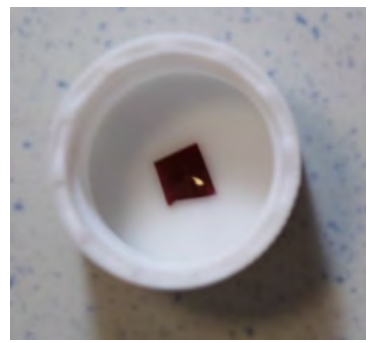

(a)

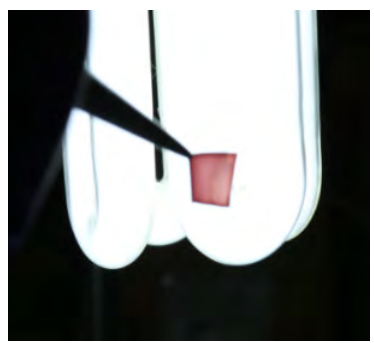

(b)

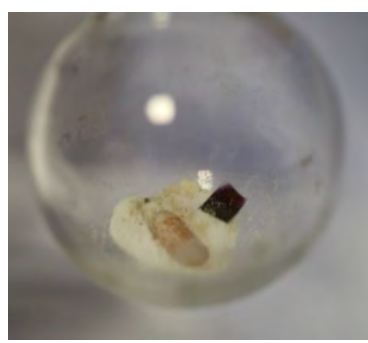

(c)

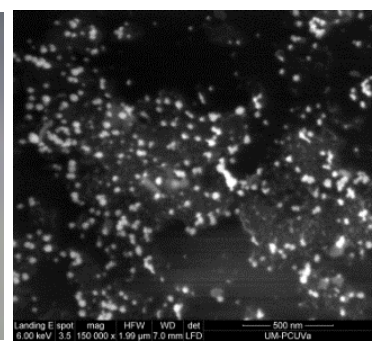

(d)

Figure 25. An example of polymer used in the catalytic synthesis. (a) Preparation. (b) Colour under transmitted white light. (c) Round bottom flask with the reagents before adding the solvent. (d) SEM image of the polymer before the reaction.

The corresponding spectral and physical characterization of every obtained product was checked in each case to be identical to previously reported compounds. Yields are compared in Figure 26 with the reported yields for every compound, $\mathbf{1 - 3},{ }^{60} 4,{ }^{61} \mathbf{5}^{62}$ and $\mathbf{6}^{63}$.

\footnotetext{
58 a) Y. Zhang, X. Cui, F. Shi, Y. Deng, Chem. Rev. 2012, 112, 2467-2505. b) M. Stratakis, H. Garcia, Chem. Rev. 2012, 112, 4469-4506.

59 J. Han, Y. Liu, R. Guo, J. Am. Chem. Soc. 2009, 131, 2060-2061.

${ }^{60}$ B. Díaz de Greñu, J. García-Calvo, J. V. Cuevas, G. García-Herbosa, B. García, N. Busto, N.; S. Ibeas, T. Torroba, B. Torroba, A. Herrera, S. Pons, Chem. Sci. 2015, 6, 3757-3764.

${ }^{61}$ B. Díaz de Greñu, D. Moreno, T. Torroba, A. Berg, J. Gunnars, T. Nilsson, R. Nyman, M. Persson, J. Pettersson, I. Eklind, P. Wästerby, J. Am. Chem. Soc. 2014, 136, 4125-4128.

${ }^{62}$ T. Gómez, D. Moreno, B. Díaz de Greñu, A. C. Fernández, T. Rodríguez, J. Rojo, J. V. Cuevas, T. Torroba, Chem. Asian J. 2013, 8, 1271-1278.

${ }^{63}$ M. D. Chordia, M. Zigler, L. J. Murphree, H. Figler, T. L. Macdonald, R. A. Olsson, J. Linden, J. Med. Chem. 2005, 48, 5131-5139.
} 


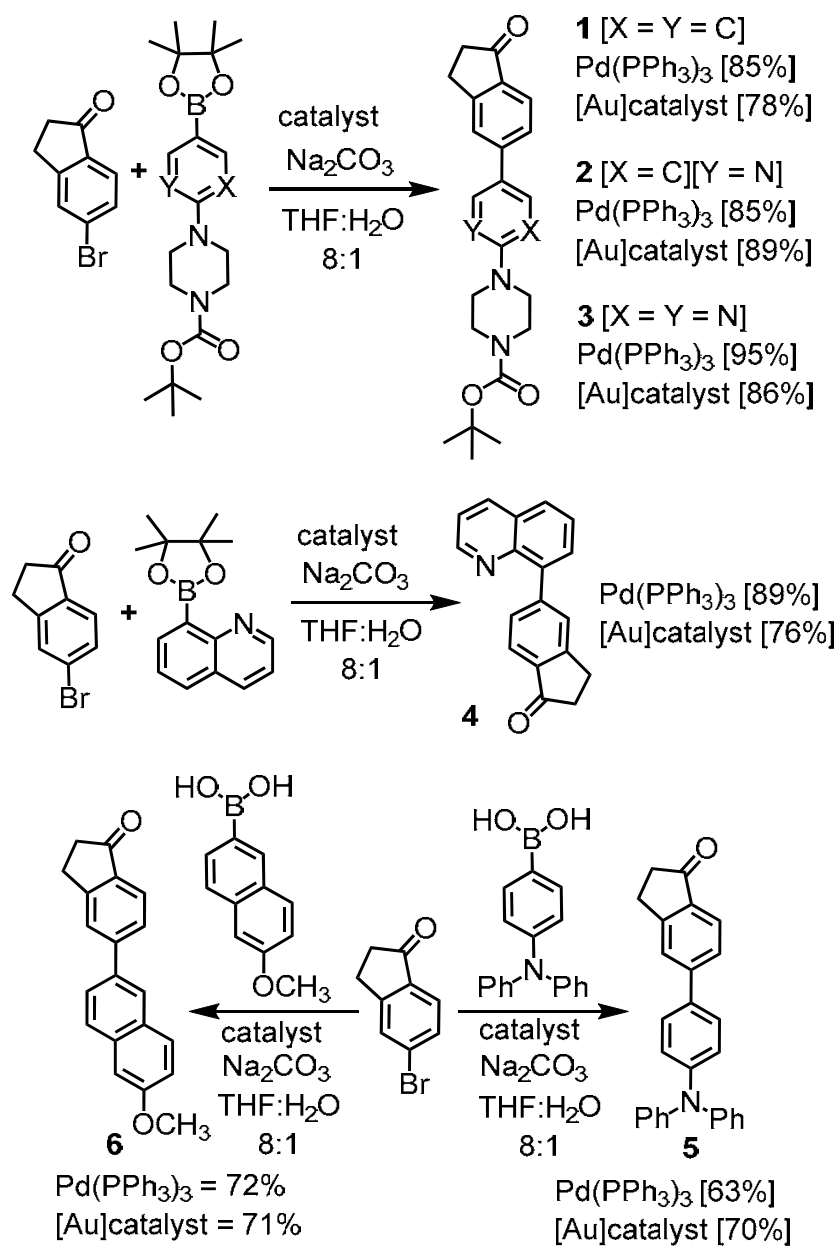

Figure 26. Representative experiments of Suzuki reactions by using the Au-NPs modified polymer as catalyst.

\subsection{Recyclability of the catalyst}

The modified polymers used as heterogeneous catalysts can be recovered from the solution and reused again in other synthetic processes. To know exactly how many times it could be used, and how it affected its catalytical properties, the polymer was recycled several times in different conditions. It was found that the polymeric catalyst worked properly after subsequent use of the polymer, by using $40 \%$ of water and $60 \%$ of THF as solvent. Albeit, having different water proportions of the solvent in the Suzuki reactions led different percentages of swelling:

$$
\text { 8:1 THF: } \mathrm{H}_{2} \mathrm{O}, 330 \% \quad 6: 4 \mathrm{THF}: \mathrm{H}_{2} \mathrm{O}, 310 \% \quad 4: 6 \text { THF: } \mathrm{H}_{2} \mathrm{O}, 280 \%
$$

The reaction was tested at different percentages of THF in order to get the best results. However, although higher percentage of THF slightly increased the yield of the reaction, it decreased the recyclability of the catalyst, because the stiffness of the polymer was lower. In the end, if the percentage of THF was too high, the polymer broke easily into pieces and was impossible to recover it.

No significant change in yield was found when using the same piece of polymer up to 4 times (4:6 THF: $\mathrm{H}_{2} \mathrm{O}$ ). In order to use the polymer for more cycles, it would have been necessary to optimize the mechanical properties by using thicker layers, and/or adding more crosslinker to the polymer. 
Additionally, it was noticed that polymers with high concentration of gold in the surface were more efficient in getting faster reactions with higher yields of the product than polymers with a lower surface concentration of gold nanoparticles.

Complementary to the catalytic behaviour it was also evaluated the possibility that the catalysis occurred by the Au-NPs, that may dissolve into the solution. In fact, a small fraction of Au-NPs dissolved in the solution (Figure 27) when it was heated to temperatures over $70{ }^{\circ} \mathrm{C}$, and/or the percentage of organic solvent was too high with respect to water (more than $70 \%$ THF). But, contrary to what was expected, for those tests, the yield of the reaction was lower than when it was performed under the standard conditions.

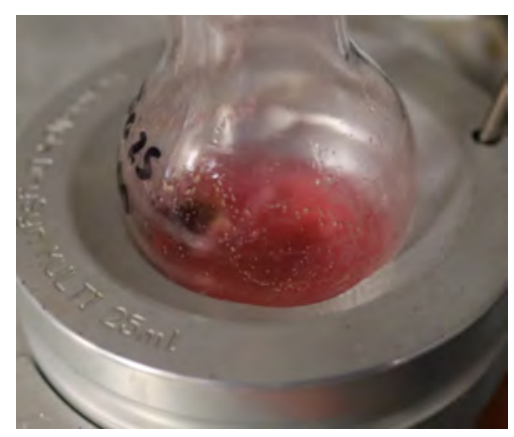

Figure 27. Catalytic solution, $80 \%$ THF after 3 days at $70{ }^{\circ} \mathrm{C}$.

In contrast, when conditions were optimal, the polymer was recovered almost unchanged (Figures 28 and 29) from the solution after the reaction had finished.

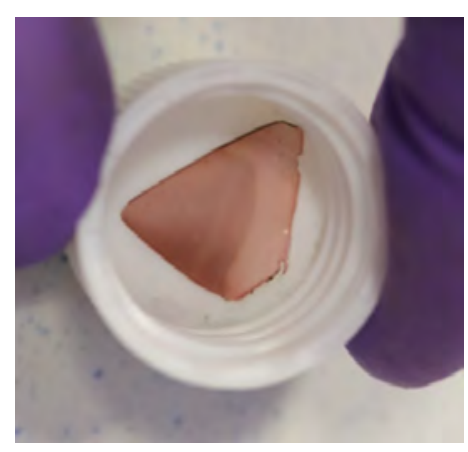

Figure 28. Piece of polymer recovered after the catalysis process ( 16 hours, $65{ }^{\circ} \mathrm{C}$, THF: $\mathrm{H}_{2} \mathrm{O} 60: 40$ )
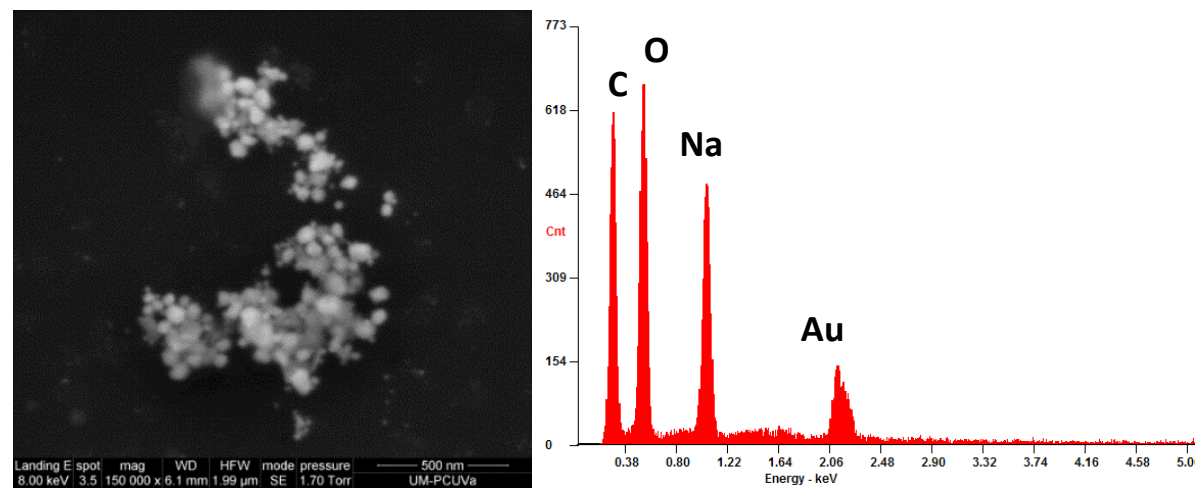

Figure 29. SEM image and EDS analysis of the piece of polymer recovered after the catalysis process (16 hours, $65^{\circ} \mathrm{C}, \mathrm{THF}: \mathrm{H}_{2} \mathrm{O} 60: 40$ ). Sodium was absorbed from the sodium carbonate used for the reaction. 


\subsection{Turn-over number (TON) and turn-over frequency (TOF)}

The turn over number (TON) of a catalyst is defined as:

$$
\text { TON }=\frac{\text { total product }(\mathrm{mol})}{\text { surface area }\left(\mathrm{cm}^{2}\right)} \quad \text { Equation [1] }
$$

The results with the area of the polymer. TON $=0.36$ mmoles $/ 0.18 \mathrm{~cm}^{2}=\mathbf{2} \mathbf{m m o l e s} / \mathrm{cm}^{2}$, probably superior because the main problem was the fragility of the polymer.

The turn over frequency (TOF) is defined as the turnover number per time unit. The calculation is performed through the equation:

$$
T O F=\frac{\text { product }(\mathrm{mol})}{\text { surface area }\left(\mathrm{cm}^{2}\right) \times \min } \quad \text { Equation [2] }
$$

In order to calculate this number, it would be necessary to perform the reaction several times with different periods of reflux and different pieces of polymer. In case of the polymer, it was not possible more than an estimation, due to the limited amount and the difficulties on performing it with exactly the same conditions. With the available data it was concluded that the time is lower than 16 hours. So the TOF would be superior to $0.0021 \mathrm{mmoles} /\left(\mathrm{cm}^{2} \times \mathrm{min}\right)$.

\subsection{Conclusions of the material as catalyst}

The polymer with supported Au-Ps was used as an efficient portable and reusable catalyst for Suzuki reactions in mixed organic-aqueous solvents.

- The yields obtained were similar to classic methods, that use $\mathrm{Pd}\left(\mathrm{PPh}_{3}\right)_{4}$ as catalyst.

- The working conditions were optimized to mixtures THF:Water (between 40 or $60 \%$ THF), a base and temperature around $65^{\circ} \mathrm{C}$ overnight.

- It was tested to work for at least 4 reactions with the same piece of polymer

In conclusion, the simplicity in the preparation of the catalyst, with no need of additional reagents for reduction stabilization, ${ }^{64}$ makes the system competitive for Suzuki reactions against gold NPs suspended in solution, ${ }^{65}$ supported on paper, ${ }^{66}$ or to other solid supported gold NPs catalysts. ${ }^{67}$ Therefore, these polymer-supported gold NPs may be considered as a useful material for green catalysis in the synthesis of fine chemicals by heterogeneous catalysis. ${ }^{68}$

\footnotetext{
${ }^{64}$ G. Li, R. Jin, Nanotechnol. Rev 2013, 2, 529-545.

${ }^{65}$ T. Chen, G. Li, H. Qian, R. Jin, Catalysis by Atomically Precise Gold Nanoclusters, in: Z. Wu, S. H. Overbury, Catalysis by Materials with Well-Defined Structures, Elsevier, New York, Chapter 8, pp. 239-262. 2015.

${ }^{66}$ a) G. Zheng, L. Polavarapu, L. M. Liz-Marzán, I. Pastoriza-Santos, J. Pérez-Juste, Chem. Commun. 2015, 51, 4572-4575. b) G. Zheng, K. Kaefer, S. Mourdikoudis, L. Polavarapu, B. Vaz, S. E. Cartmell, A. Bouleghlimat, N. J. Buurma, L. Yate, A. R. de Lera, L. M. Liz-Marzán, I. Pastoriza-Santos, J. Pérez-Juste, J. Phys. Chem. Lett. 2015, 6, 230-238.

${ }^{67}$ a) Y. Li, X. Fan, J. Qi, J. Ji, S. Wang, G. Zhang, F. Zhang, Mater. Res. Bull. 2010, 45, 1413-1418. b) M. G. Speziali, A. G. M. da Silva, D. M. V. de Miranda, A. L. Monteiro, P. A. Robles-Dutenhefner, Appl. Catal. AGen. 2013, 462- 463, 39- 45.

${ }^{68}$ X. Liu, L. He, Y.-M. Liu, Y. Cao, Acc. Chem. Res. 2014, 47, 793-804.
} 


\section{EXPERIMENTAL SYNTHESIS OF PALLADIUM-NPs}

After the satisfactory results with Au-NPs, the possibility of getting different supported metallic NPs was tested with other metals - crosslinked polymers. From these tests it is worth to remark the case of $\mathrm{Pd}(\mathrm{II})$. From the different tests, $\mathrm{PdCl}_{2} \cdot 2 \mathrm{NaCl}$ salt had good response when reacted in the presence of some crosslinked polymeric films.

The components of the polymers tested are shown in Figure 30.<smiles>COC(OC)(C(=O)c1ccccc1)c1ccccc1</smiles>

2,2-dimethoxy-1,2-diphenylethan-1one

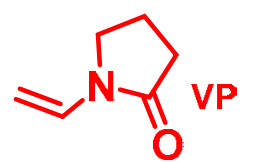

1-vinylpyrrolidin-2-one<smiles>C=CC(=O)OC</smiles>

methyl acrylate ethyl methacrylate<smiles>C=C(C)C(=O)OCCOC(=O)C(=C)C</smiles>

Ethylene glycol dimethacrylate

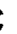<smiles>C=CC(=O)OCCCC</smiles>

butyl acrylate<smiles>C=C(C)C(=O)OCCOCCOCC</smiles><smiles>C=CC(=O)OCCCCCCCCCCCC</smiles><smiles>C=CC(=O)OCCO</smiles><smiles>C#CCOC(=O)C(=C)C</smiles>

2-hydroxyethyl acrylate

prop-2-yn-1-yl methacrylate

\section{XVP:yMM // zC \\ xHE:yMP // zC}

Figure 30. Components of the film monomers and photoinitiator (DMPA), " $x$ ", " $y$ " and " $z$ " represent the relations between components; $\mathrm{x}+\mathrm{y}=100 \%, \mathrm{z}$ is the percentage respect to $\mathrm{x}+\mathrm{y}$. 
From the monomeric structures, several mixtures were tested, as it is detailed in the table below (Figure 31).

\begin{tabular}{|c|c|c|c|c|c|c|c|c|c|}
\hline Films (\%) & VP & A12 & A4 & A1 & M2 & MEGMA & MP & A2HE & C \\
\hline PBM2 & 50 & $\mathrm{x}$ & $\mathrm{x}$ & $\mathrm{x}$ & 50 & $\mathrm{x}$ & $\mathrm{x}$ & $\mathrm{x}$ & 0 \\
\hline PBMEGMA & 50 & $\mathrm{x}$ & $\mathrm{x}$ & $\mathrm{x}$ & $\mathrm{x}$ & 50 & $\mathrm{x}$ & $\mathrm{x}$ & 0 \\
\hline PB0 & 60 & $\mathrm{x}$ & 40 & $\mathrm{x}$ & $\mathrm{x}$ & $\mathrm{x}$ & $\mathrm{x}$ & $\mathrm{x}$ & 0 \\
\hline PB20_80A12 & 20 & 80 & $\mathrm{x}$ & $\mathrm{x}$ & $\mathrm{x}$ & $\mathrm{x}$ & $\mathrm{x}$ & $\mathrm{x}$ & 10 \\
\hline PB20_80A4 & 20 & $\mathrm{x}$ & 80 & $\mathrm{x}$ & $\mathrm{x}$ & $\mathrm{x}$ & $\mathrm{x}$ & $\mathrm{x}$ & 10 \\
\hline PB20_80A1 & 20 & $\mathrm{x}$ & $\mathrm{x}$ & 80 & $\mathrm{x}$ & $\mathrm{x}$ & $\mathrm{x}$ & $\mathrm{x}$ & 10 \\
\hline PB0_100A4 & $\mathrm{x}$ & $\mathrm{x}$ & 100 & $\mathrm{x}$ & $\mathrm{x}$ & $\mathrm{x}$ & $\mathrm{x}$ & $\mathrm{x}$ & 10 \\
\hline PB0_100A1 & $\mathrm{x}$ & $\mathrm{x}$ & $\mathrm{x}$ & 100 & $\mathrm{x}$ & $\mathrm{x}$ & $\mathrm{x}$ & $\mathrm{x}$ & 10 \\
\hline PB80_20A1 & 80 & $\mathrm{x}$ & $\mathrm{x}$ & 20 & $\mathrm{x}$ & $\mathrm{x}$ & $\mathrm{x}$ & $\mathrm{x}$ & 10 \\
\hline PB80_20A4 & 80 & $\mathrm{x}$ & 20 & $\mathrm{x}$ & $\mathrm{x}$ & $\mathrm{x}$ & $\mathrm{x}$ & $\mathrm{x}$ & 10 \\
\hline A2HE5 & $\mathrm{x}$ & $\mathrm{x}$ & $\mathrm{x}$ & $\mathrm{x}$ & $\mathrm{x}$ & $\mathrm{x}$ & $\mathrm{x}$ & 100 & 10 \\
\hline JG25_SA2 & $\mathrm{x}$ & $\mathrm{x}$ & $\mathrm{x}$ & $\mathrm{x}$ & $\mathrm{x}$ & $\mathrm{x}$ & 5 & 95 & 10 \\
\hline
\end{tabular}

Figure 31. Name and composition of some of the tested polymeric structures.

These colourless films $(1 \times 1 \mathrm{~cm})$ were put in $\mathrm{PdCl}_{2} \cdot 2 \mathrm{NaCl}$ solution $(5 \mathrm{mM}, 3 \mathrm{~mL})$ for 20 hours, and washed with water. Afterwards, changes in the colour of the polymer were observed for some of them, Figure 32:

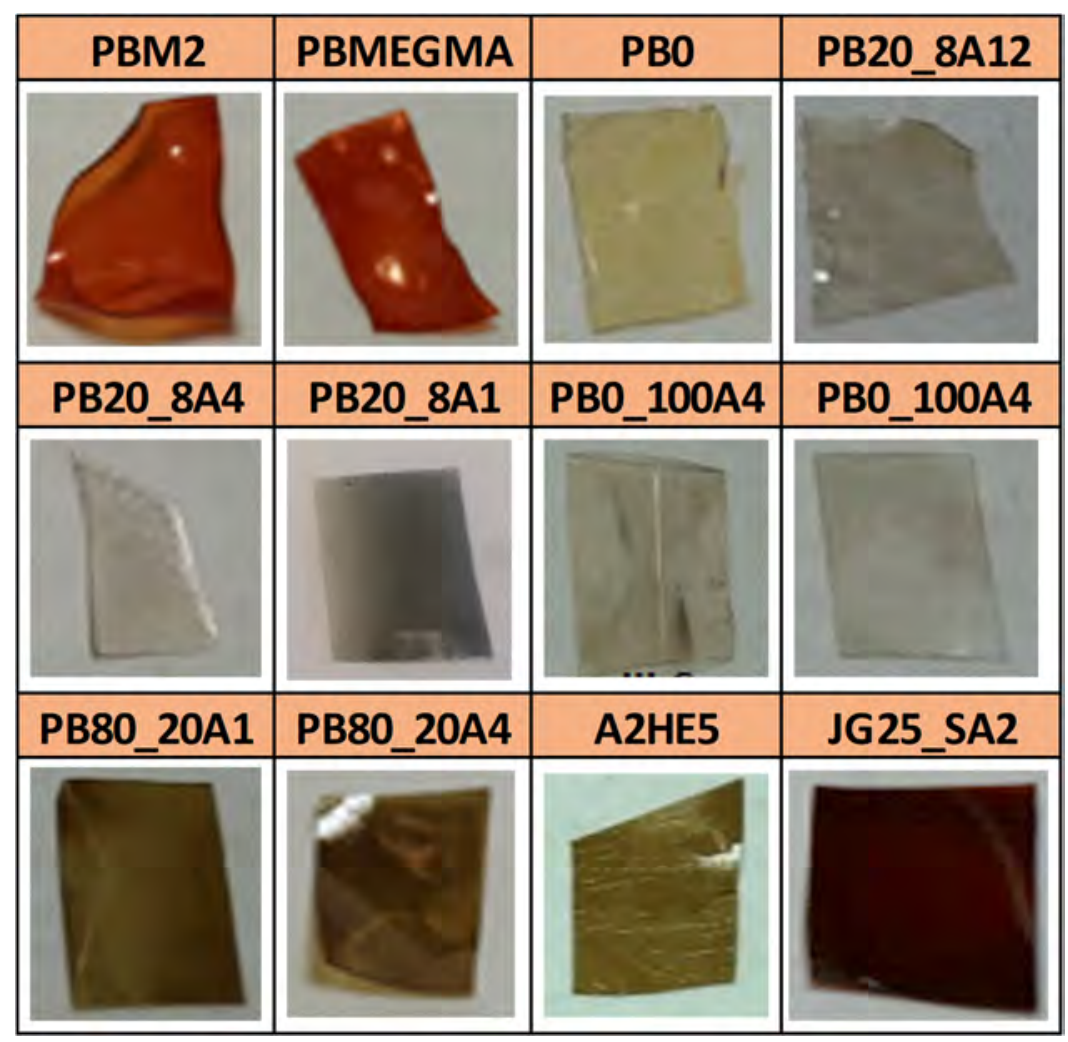

Figure 32. Pictures of the polymeric films after in presence of $\mathrm{PdCl}_{2} \cdot 2 \mathrm{NaCl}$ solution. 
Four different behaviours were observed. The polymer remained unaffected, it took $\operatorname{Pd}(0)$ on the surface or it absorbed $\mathrm{Pd}(\mathrm{II})$, or a mixture of $\mathrm{Pd}(0)+\mathrm{Pd}(\mathrm{II})$.

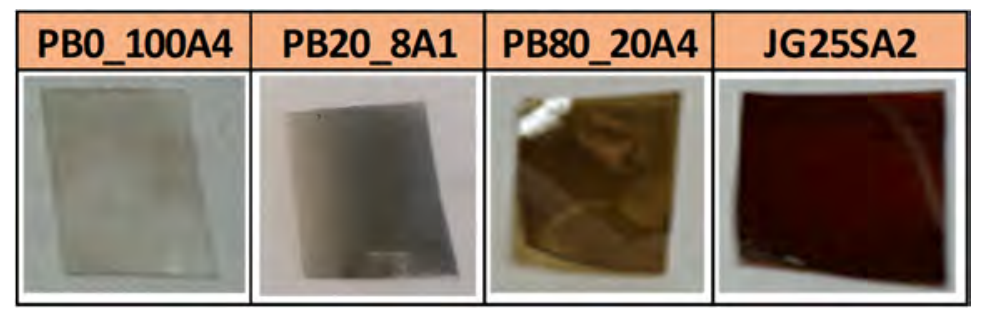

Figure 33. Visual appearance of the films after in presence of $\mathrm{PdCl}_{2} \cdot 2 \mathrm{NaCl} 5 \mathrm{mM}$ for 20 hours. From left to right; no effect, $\mathrm{Pd}(0)$ and $\mathrm{Pd}(\mathrm{II})$ or $\mathrm{Pd}(0)+\mathrm{Pd}(\mathrm{II})$.

- The polymers that contained only acrylate derivatives (PB0_100) or a high percentage of the acrylate with a long aliphatic chain (PB20_80A4 and PB20_80A12) showed no change after being in the presence of palladium solutions. The film remained colourless, first picture on Figure 33 .

- PB20_80A1 presented a black layer over its surface; once analysed, it was concluded that they were $\operatorname{Pd}(0)$ nanoparticles. Second picture in Figure 33.

- PB0, PB80_20A4, PB80_20A1 and A2HE5 acquired orange-brown colour. Being a mixture of $\operatorname{Pd}(\mathrm{II})$ and $\operatorname{Pd}(0)$. Third picture in Figure 33.

- PBM2, PBMEGMA and JGSA2 acquired a reddish colour. Afterwards, it was checked hat they also presented a mixture between $\mathrm{Pd}(\mathrm{II})$ and $\mathrm{Pd}(0)$. Fourth picture in Figure 33.

\subsection{Characterization of the films}

The polymeric films were characterized by infrared and TGA, presenting a degradation point at $430{ }^{\circ} \mathrm{C}$ in TGA, and not remarkable changes in the infrared although there was $\operatorname{Pd}(0)$ or $\operatorname{Pd}(\mathrm{II})$ absorbed. In contrast, UV-Vis absorption and SEM-TEM analysis gave information about the supported Pd:

UV-Vis absorption spectra:
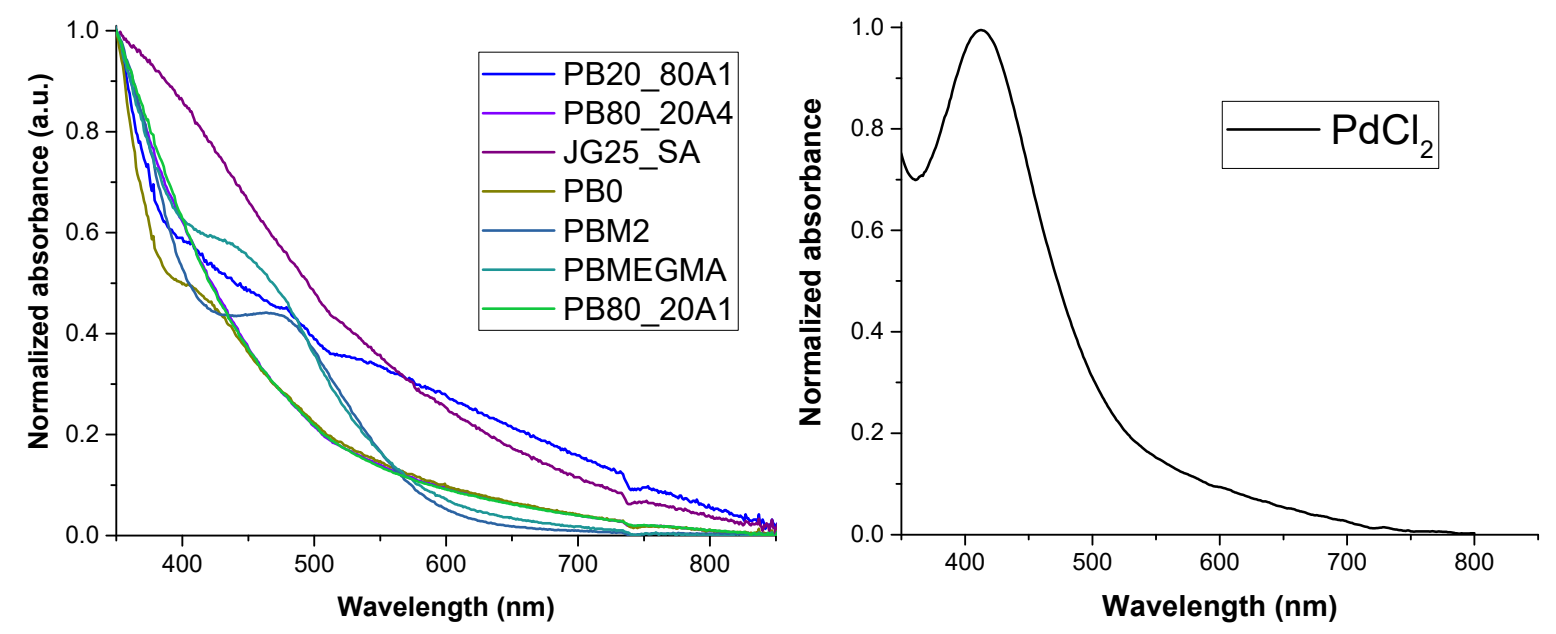

Figure 34. Normalized absorption spectra of different synthesized polymers (left) and $\mathrm{PdCl}_{2} \cdot 2 \mathrm{NaCl}$ $5 \mathrm{mM}$ solution in water (right). 
The colour - absorption is a way to characterize the formation of $\operatorname{Pd}(0)$, by comparing the results obtained and the differences between $\mathrm{PdCl}_{4}{ }^{2-}$ and $\mathrm{Pd}(0)$ solutions, which may be found in literature. ${ }^{69}$ $\mathrm{PdCl}_{4}{ }^{2-}$ in water solution provided a yellow-orange colour with a characteristic absorption at $420 \mathrm{~nm}$, as it can be seen in Figure 34. In contrast, $\operatorname{Pd}(0)$ particles led to the disappearance of this band, getting a wider absorption that decays continuously from $350 \mathrm{~nm}$ to $850 \mathrm{~nm}$.

The results of the tests led to different absorption depending on the polymer. In conclusion, high absorbances at 400-500 nm indicated the presence of starting $\mathrm{PdCl}_{4}{ }^{2-}$ which is clearly shown in $\mathrm{PB} 0$, PBMEGMA and PBM2. However, in other films is difficult to distinguish if the colour is due to the presence of $\mathrm{Pd}(0), \mathrm{PdCl}_{2}$ or a mixture of both, such as $\mathrm{PB} 80 \_20$ or JG25_SA2, needing verification by other techniques.

\section{SEM and EDX analysis:}

First, some representative samples were measured by SEM+EDX (Figures 35, 36, 37 and 38) to get information about the particle agglomeration and composition:

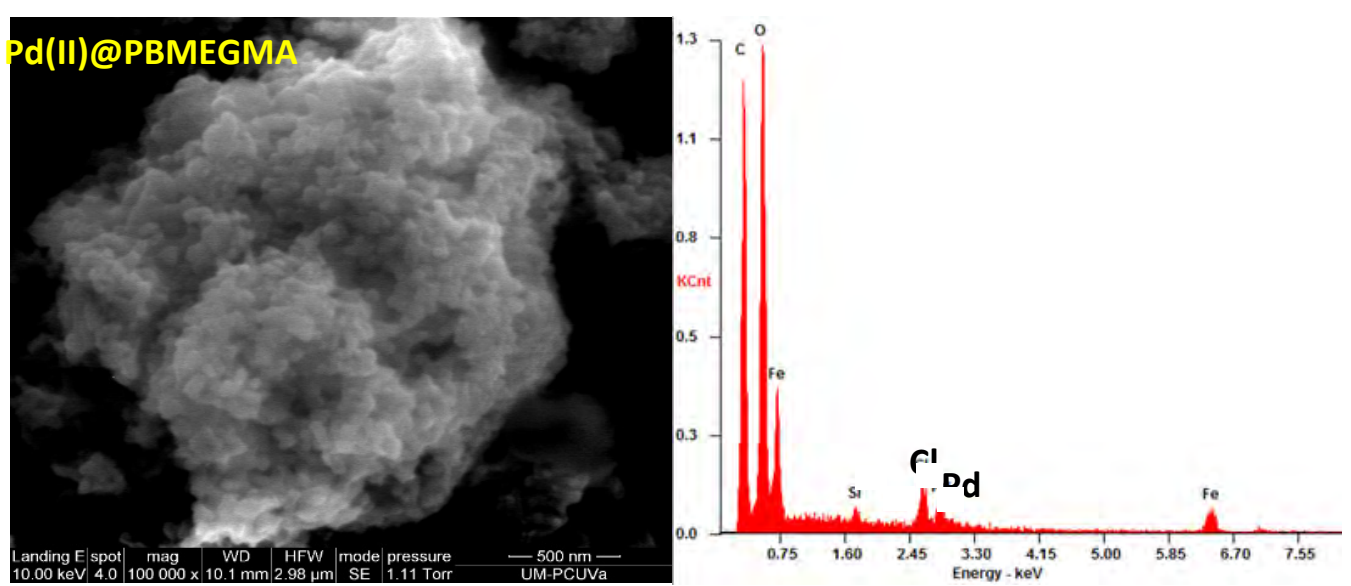

Figure 35. SEM image of Pd(II)@PBMEGMA (left). EDX analysis (right). $1 \mu \mathrm{m}$ aggregates of $\mathrm{PdCl}_{2}$.

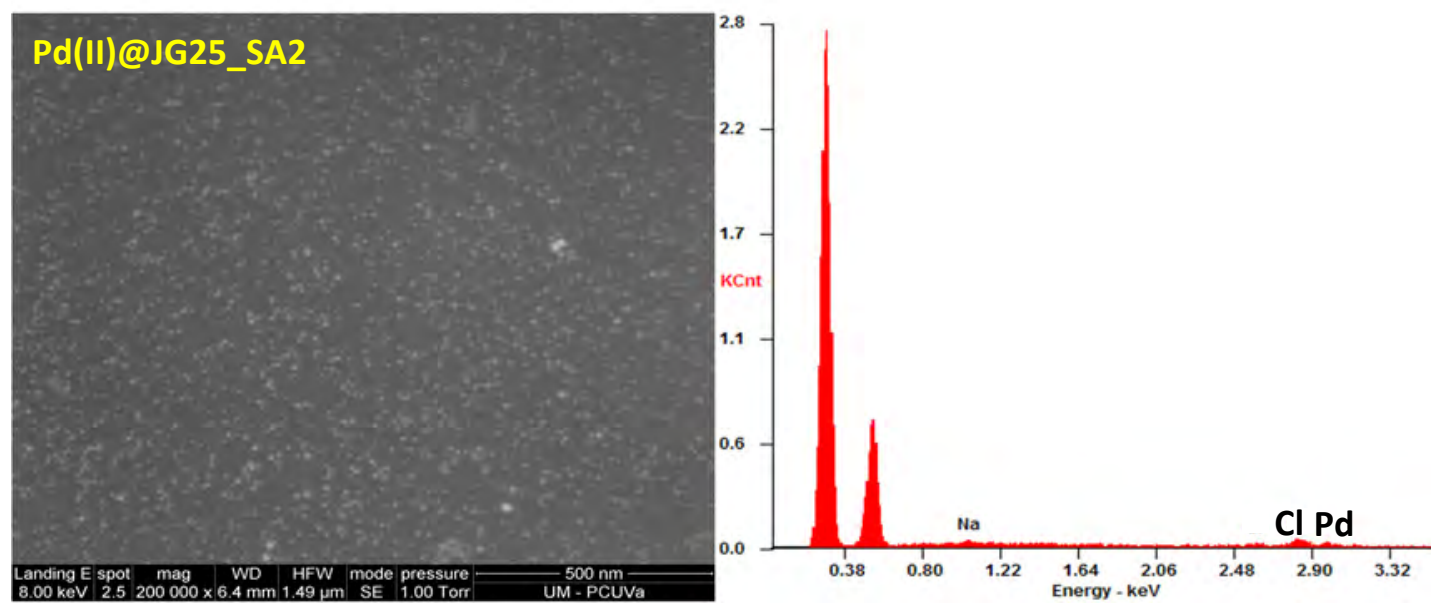

Figure 36. SEM image of Pd(II)@JG25_SA2 (left). EDX analysis (right). $20 \mathrm{~nm}$ aggregates of $\mathrm{PdCl}_{2}$.

\footnotetext{
${ }^{69}$ R. G. Freemantle, M. Liu, W. Guo, S. O. Obare. Metallic Nanomaterials, Edited by C. S. S. R. Kumar,
} WILEY-VCH Verlag GmbH \& Co. Weinheim, Volume 1, Chapter 9, pp. 572, 2009. 


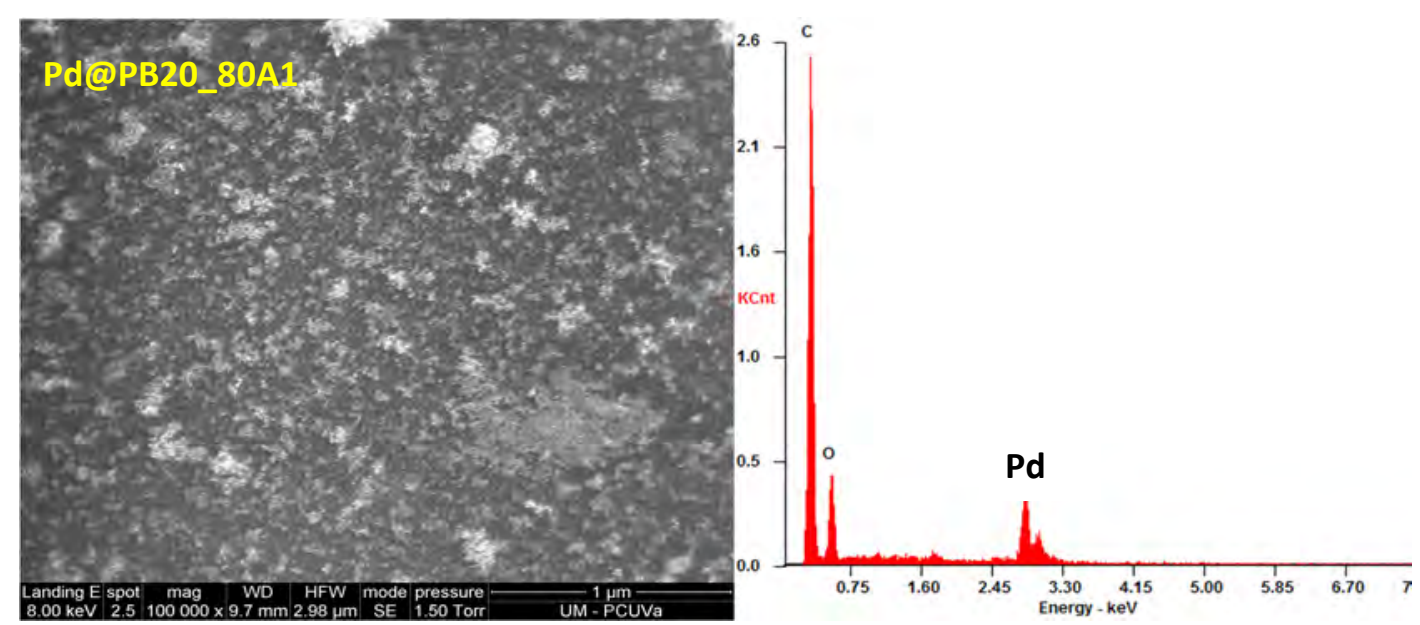

Figure 37. SEM image of Pd@PB20_80A1 (left). EDX analysis (right). NPs aggregated of Pd(0).

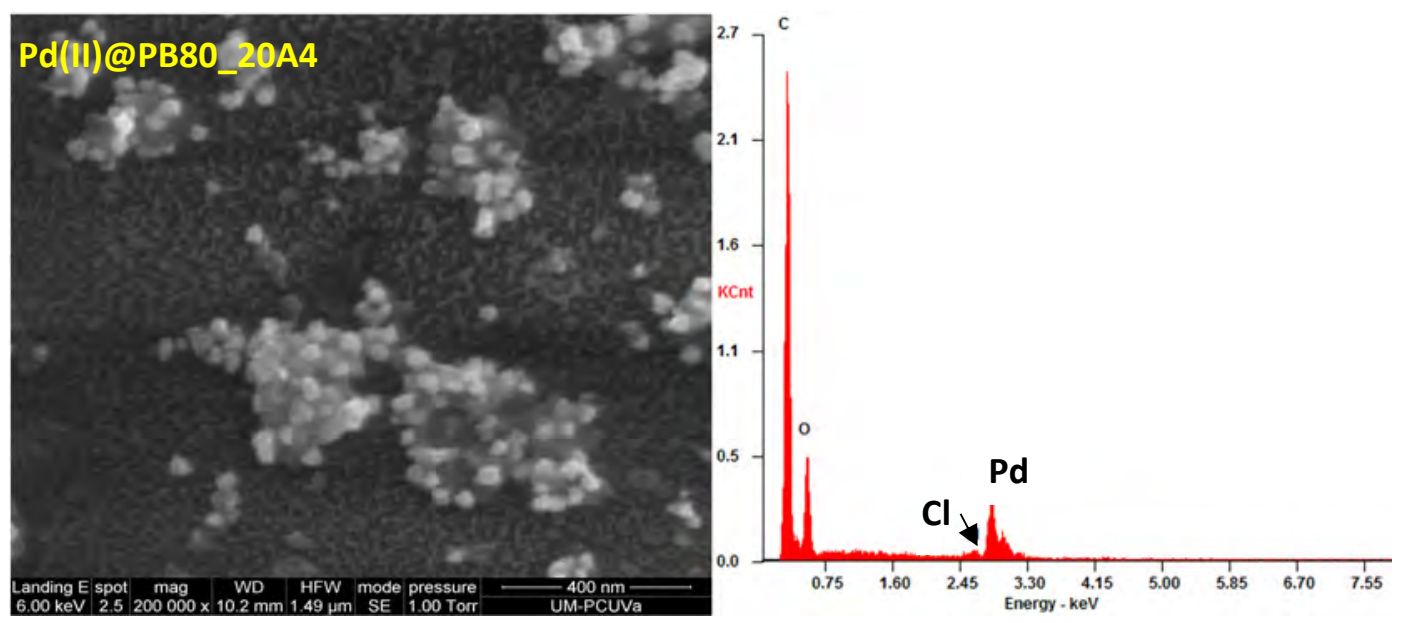

Figure 38. SEM image of Pd(II)@PB80_20A4 (left). EDX analysis (right). 20 nm aggregates of $\mathrm{PdCl}_{2}$ and $\mathrm{Pd}(0)$.

Different aggregation patterns were observed depending on the polymer. Moreover, from the EDX, it was obtained that some aggregates are $\mathrm{Pd}(\mathrm{II})$, or mixtures $\mathrm{Pd}(0)+\mathrm{Pd}(\mathrm{II})$, as salts containing chloride, in all cases except Pd@PB20_80A1 in which there was no trace of $\mathrm{Cl}^{-}$.

\subsection{Deep study of PB20_80A1 and PB80_20 modifications with palladium}

The polymers bearing acrylate esters (A) and vinylpyrrolidone (VP) as components gave rise to deposits of $\mathrm{Pd}$ nanoparticles on the surface. Apart from the results showed in previous characterization methods, there were two derivatives that gave the best performance:

- PB20_80A1 was capable to give supported $\operatorname{Pd}(0)$-NPs without any additional treatment. In addition, the polymer had good mechanical properties and could be used as heterogeneous catalyst in a wide variety of solvents, such as water or alcoholic solvents.

- PB80_20A1 and PB80_20A4 absorbed Pd(II) chloride salts. It had also good mechanical properties and could be used in water and alcoholic solutions. In addition, $\mathrm{Pd}(\mathrm{II})$ might be reduced to $\mathrm{Pd}(0)$ in presence of $\mathrm{H}_{2}$, giving NPs in the polymer; the process was explained in Section 8.2.2. Both PB80_20A1 and PB80_20A4 had similar results in the tests, being more deeply studied PB80_20A4. 


\subsubsection{PB20 80A1:}

PB20_80A1 gave the best performance under the conditions described for the direct deposit of Pd nanoparticles. They are presented as homogeneous covering in addition of some isolated spots of agglomeration on the surface (Figure 39).

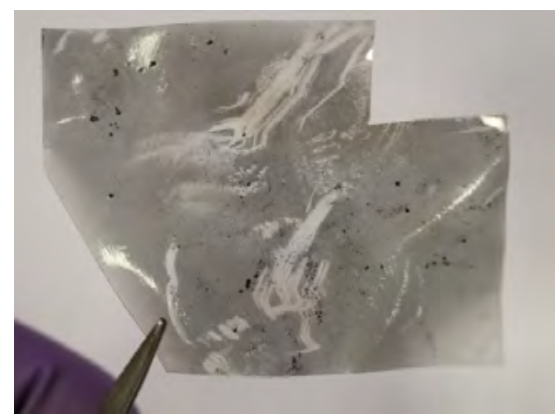

Figure 39. Picture of the polymer PB20_80A1 covered by Pd-NPs.

Scanning electron microscopy (SEM), showed the surface of the polymer uniformly coated by clusters of homogeneously sized Pd-NPs (Figure 40).

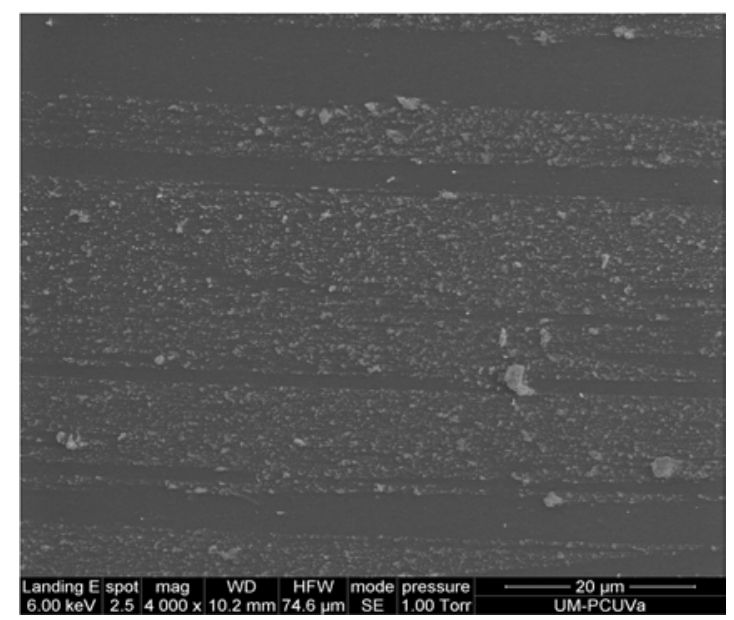

Figure 40. SEM image of Pd-NPs aggregates over the polymer PB20_80A1, SEM measurement. from 2.3 to $4.2 \mathrm{keV}$ scale.

In the figure above (Figure 40), it is showed that some areas had no deposition of NPs. What is more, the NPs might be easily transferred; by simply pressing the polymers to a carbon band for high vacuum TEM (Figure 41) or even by using some glass or sticky surface. The preparation of deposited Pd-NPs on film, Pd@PB20_80A1, and the transfer to carbon band, Pd@Cband, is shown in Figure 41. 


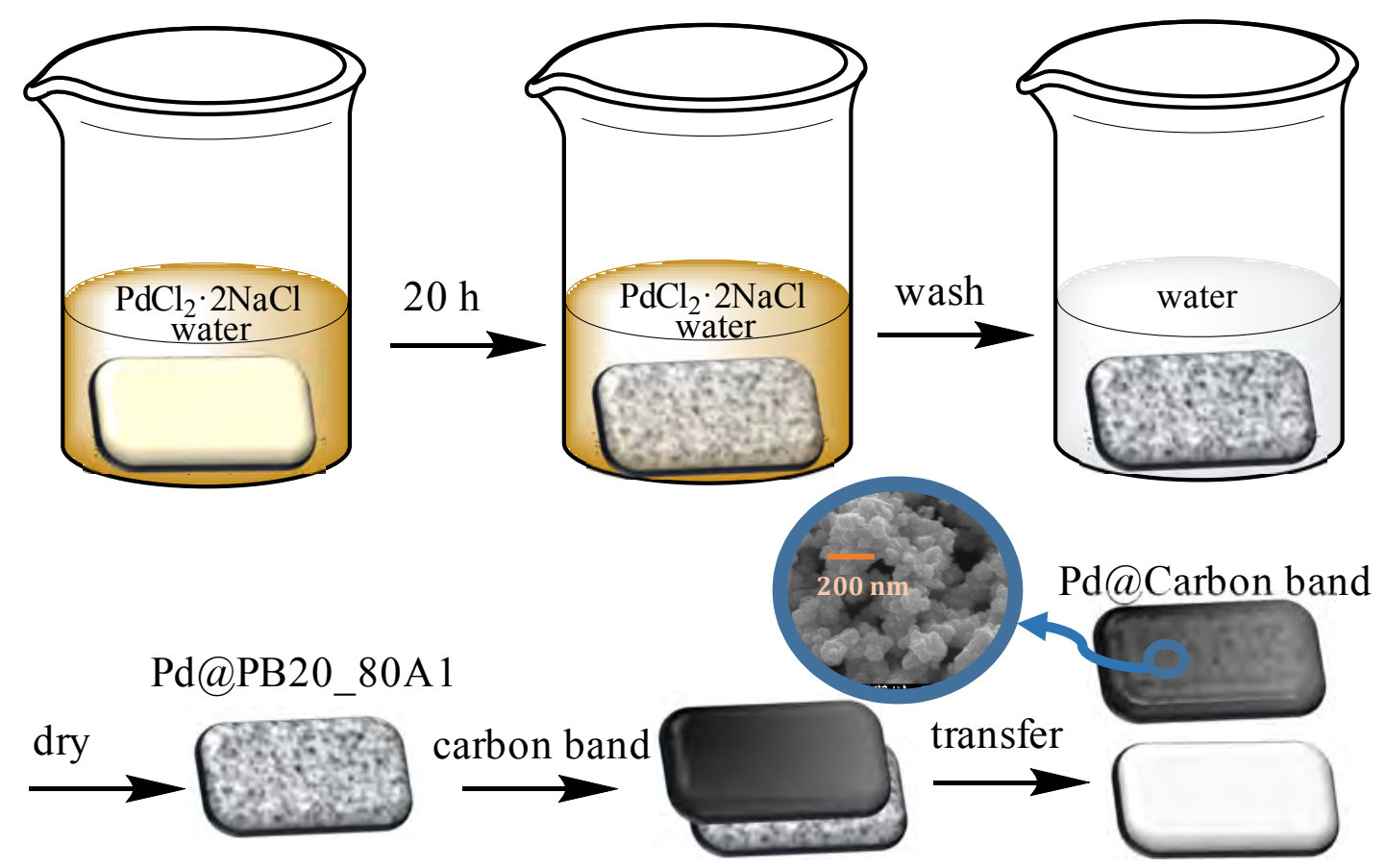

Figure 41. Scheme of a representative experiment for the preparation of polymer supported palladium nanoparticles Pd@PB20_80A1 and the dry transfer to carbon band Pd@Cband.

Due to the transfer process, the study of the Pd clusters was more easily and accurately performed, by analysing the particles on the surface of carbon band, Pd@Cband (Figure 42).
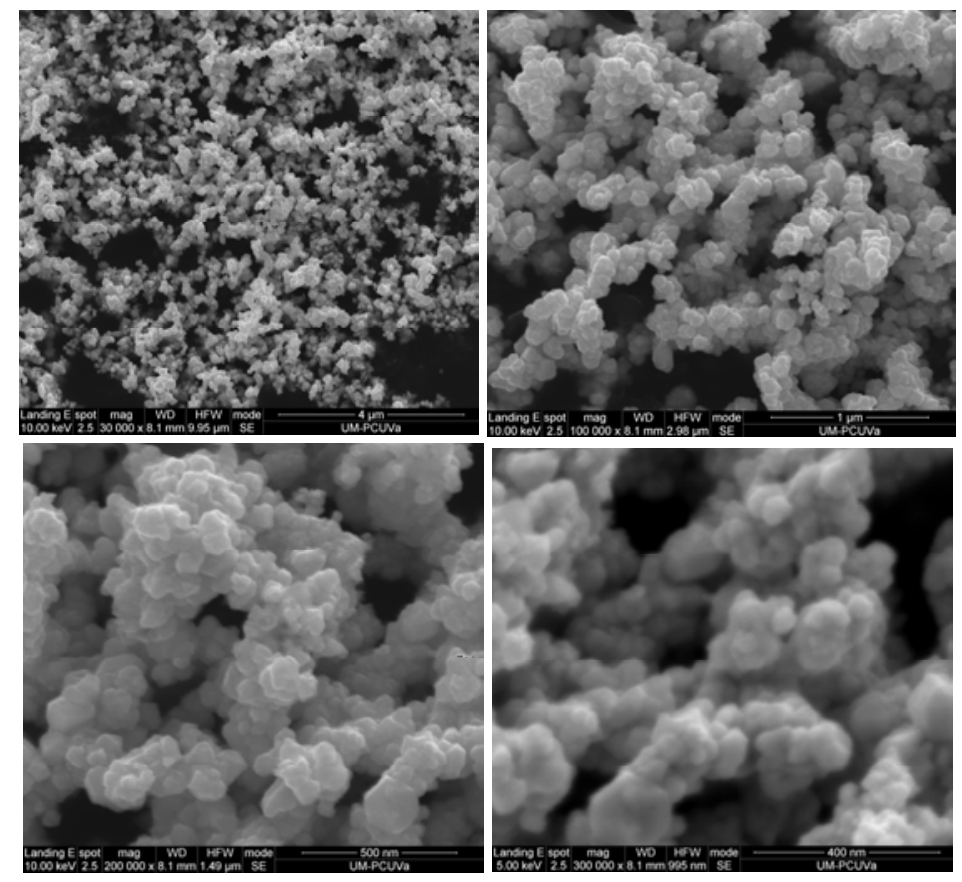

Figure 42. SEM images of palladium nanoclusters on carbon band, Pd@Cband, after homogeneous coating of polymer PB20_80A1 by palladium nanoclusters and carbon band transfer, (upper left) scale bar $4 \mu \mathrm{m}$, (upper right) scale bar $1 \mu \mathrm{m}$, (down left) scale bar $500 \mathrm{~nm}$, (down right) scale bar $400 \mathrm{~nm}$ (lower left).

The properties of the NPs were studied by TEM, further detailed in section 8.2.3. 


\subsubsection{PB80 20A4 (or PB80 20A1):}

Following a similar procedure than the used for Pd@PB20_80A1, the film PB80_20A4 $(1 \times 1$ $\mathrm{cm}$ ) was submerged in a $5 \mathrm{mM} \mathrm{PdCl}{ }_{2} \cdot 2 \mathrm{NaCl}$ solution in water $(3 \mathrm{~mL})$ for 20 hours. In this case, there was no initial deposition of Pd nanoparticles but, instead, palladium chloride was adsorbed uniformly on the surface of the material.

However, after just 5-10 minutes hydrogenation at $5 \mathrm{~atm}$, it gave rise to size-controlled deposition of uniformly distributed Pd-NPs on the surface of the polymer (Figure 43). Similar results were also obtained for PB80_20A1; therefore, the results in this case were not so tightly dependent on the acrylate ester as in the previous case.
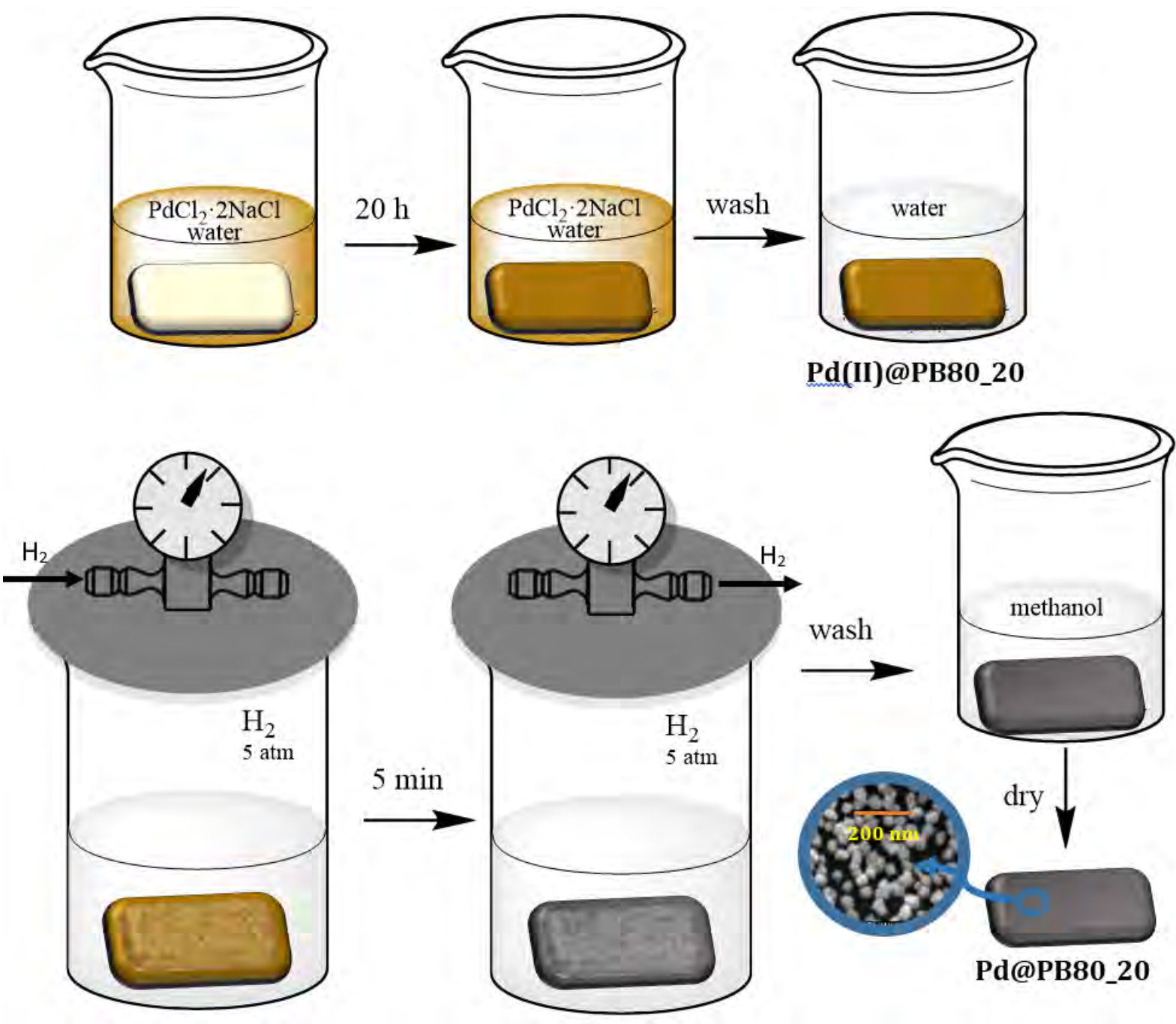

Figure 43. Schematic process of the Reduction of supported Pd(II) over PB80_20. Hydrogenation and cleaning process to obtain Pd@PB80_20. 


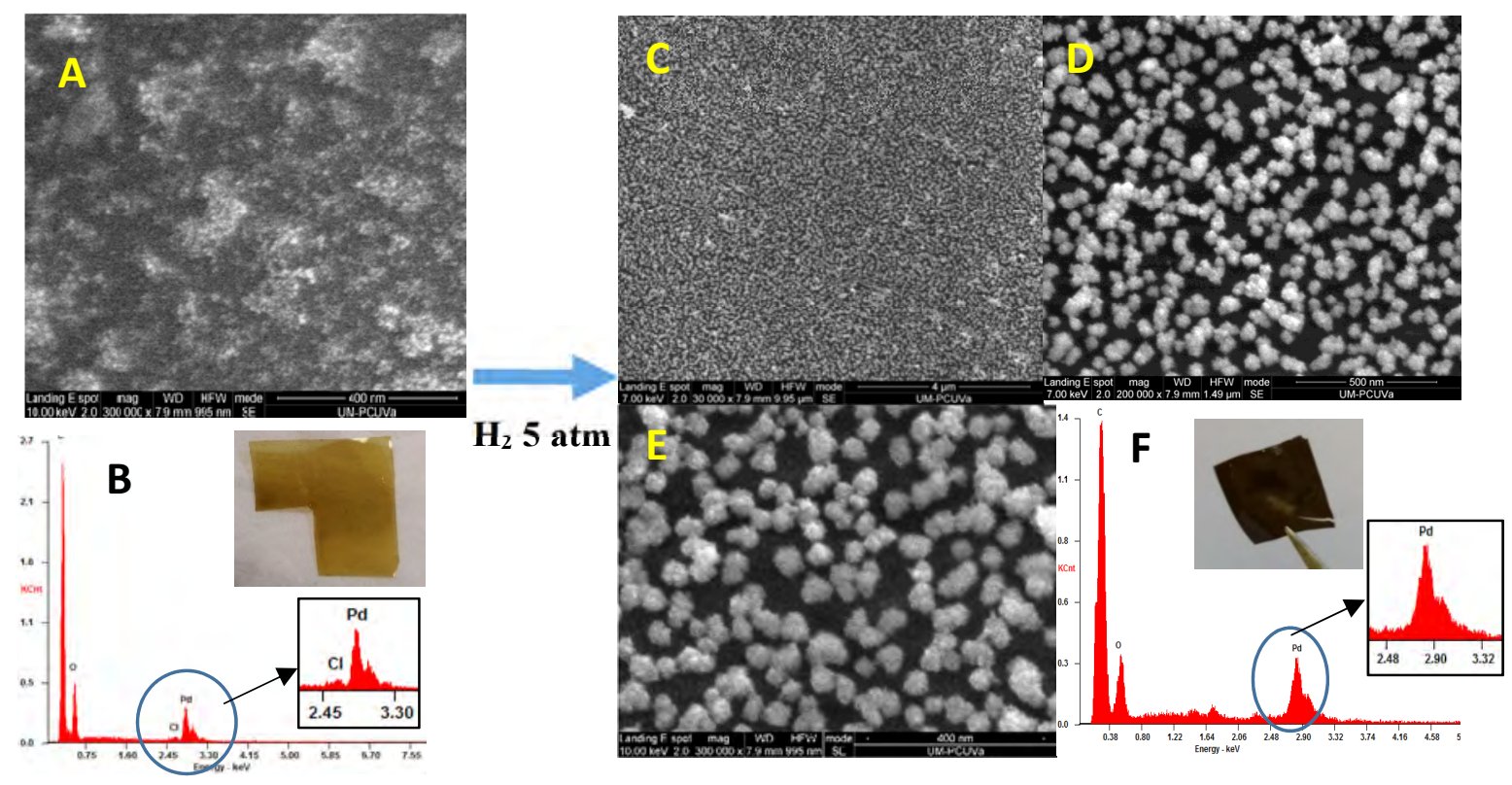

Figure 44. SEM images of A) Pd(II)@PB80_20A4 and C-E) Pd@PB80_20A4. EDX and photograph of B) Pd(II)@PB80_20A4 and, F) Pd@PB80_20A4.

Previously, it was explained that Pd(II)PB80_20A4 deposited Pd(II) or a mixture between Pd(0) and $\mathrm{Pd}(\mathrm{II})$. As it can be seen in the Figure 44, the reduction with $\mathrm{H}_{2}$ led to the formation of homogeneous Pd(0)-NPs (Figure 45) with the same characteristics obtained for PB20_80A1 on the surface of the polymer but without aggregation.

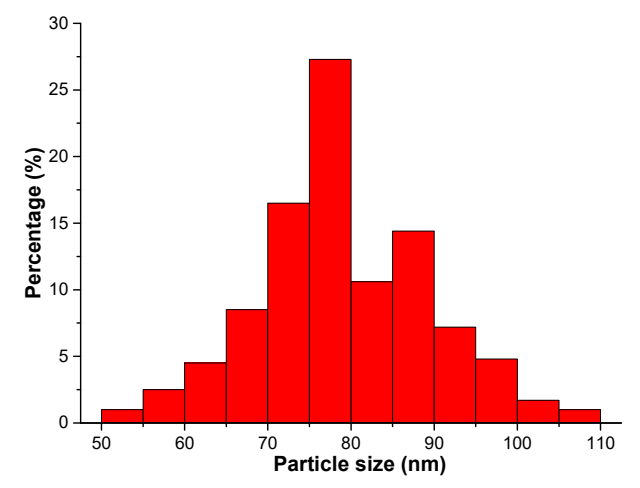

Figure 45. Distribution of the particle size. ${ }^{70}$ In the polymer Pd@PB80_20A4.

\subsubsection{TEM study of Pd-NPs}

Both Pd@PB80_20A4 and Pd@PB20_80A1 (more aggregated) had very similar Pd-NPs size distribution. The main difference was that, in the case of the polymer with high percentage of VP, the NPs were not transferable, being dispersed in the film and not only in the surface. However, a low percentage of both may be dispersed in solution (when washing the polymer after performing the modification) presenting characteristics that may be measured by TEM (Figures 46 and 47), being similar to the NPs on the surface. The resolution of TEM is much higher than when using SEM and the lower quantity of particles transferred allowed the study of them individually.

${ }^{70}$ C. A. Schneider, W. S. Rasband, K. W. Eliceiri, Nat. Meth. 2012, 9, 671-675. 

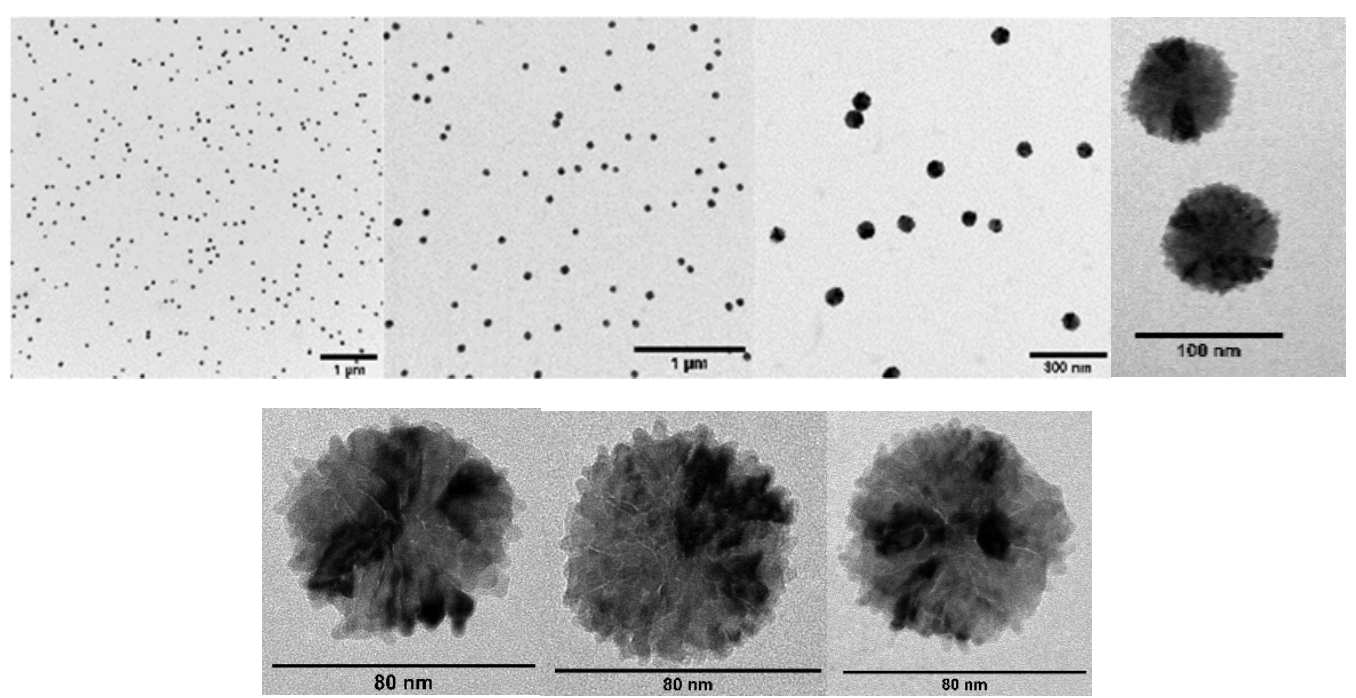

Figure 46. TEM images of the Pd-NPs. First row: medium resolution TEM, scale bar $1 \mu \mathrm{m}, 1 \mu \mathrm{m}, 300$ $\mathrm{nm}, 100 \mathrm{~nm}$ - Second row: high resolution TEM of individual nanoparticles, scale bar $80 \mathrm{~nm}$.
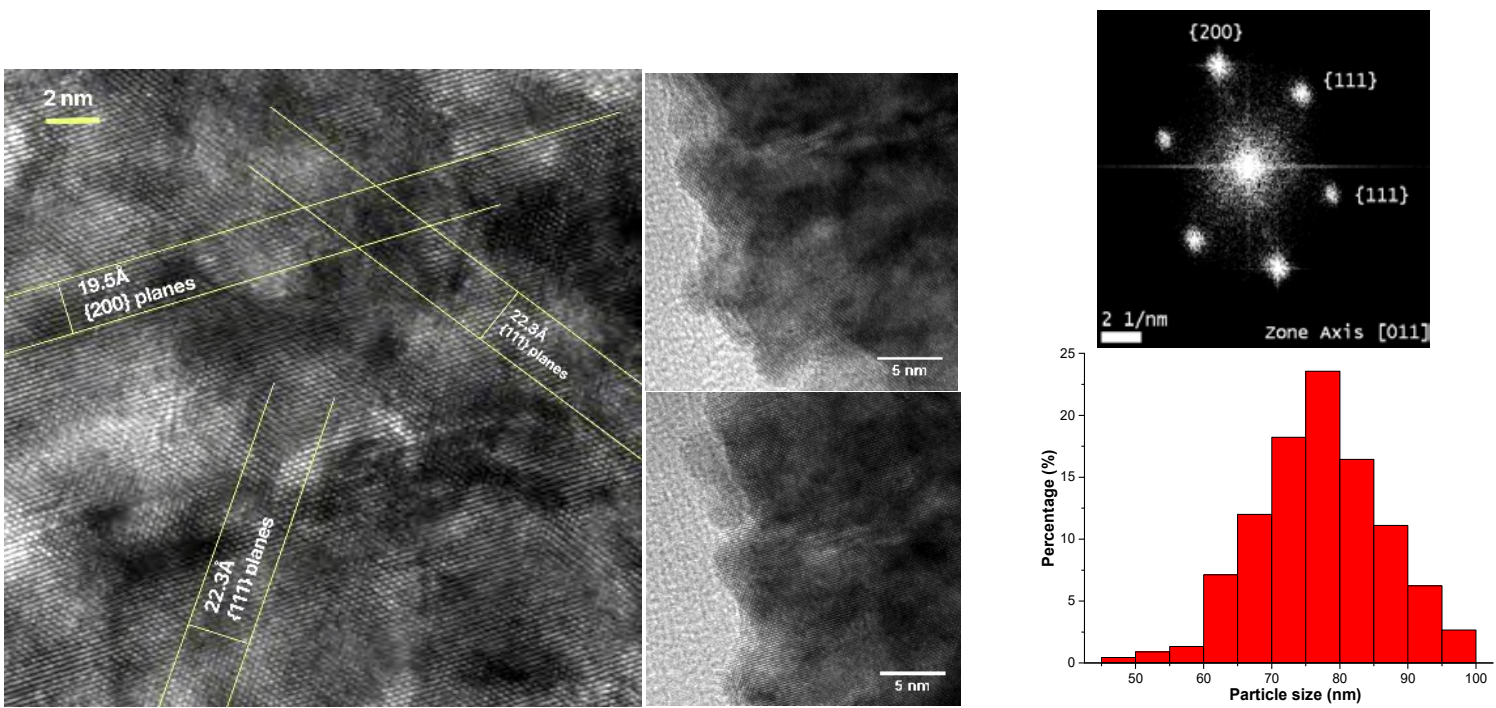

Figure 47. Left, TEM of an individual nanoparticle, the lattice spacing intervals of 19.5 and $22.3 \AA$ correspond to blocks of 10 planes each and can be indexed as $\{200\}$ and $\{111\}$ of fcc Pd, respectively. Middle, high resolution TEM of individual nanoparticles showing the boundaries of the nanoparticle, scale bar $5 \mathrm{~nm}$. Right up: FFT of the HRTEM image showing the interplanar distances corresponding to $\{111\}$ and $\{222\}$ family planes, a pattern corresponding to a f.c.c. crystal in the zone axis [011].

Right down: Distribution of the particle size. ${ }^{69}$

The NPs were deeply studied by HRTEM. These nanoparticles appeared as polycrystalline dendrites showing internal structure from radial growth and an average diameter of $75 \mathrm{~nm}$. The Figure 47, left figure, shows the image of a Pd particle obtained by means of high resolution transmission electron microscopy (HRTEM). The fringes with lattice of 1.95 and $2.23 \AA$ are calculated measuring the separation of 10 planes and dividing these values by 10 . These values can be indexed as $\{111\}$ and $\{200\}$ of fcc Pd, respectively. The size distribution was the same than the obtained by SEM for Pd@PB80_20A4 and Pd@PB20_80A1, the small difference were likely to be because of the aggregation and the three-dimensional position of the particles in SEM, complicating an accurate measurement of isolated particles. 


\subsubsection{Summary of Pd-NPs structures:}

The results for the optimized Pd modified films from Section 8 might be summarized as follows:

$>$ The films containing mixtures VP/A lead to Pd-NPs providing that the ester was a short aliphatic chain. It was only necessary to use a $\mathrm{PdCl}_{2} \cdot 2 \mathrm{NaCl}$ solution in water.

$>$ The oxidation state of Pd was detected by UV-Vis absorbance and EDX analysis.

$>$ For PB20_80A1 the Pd-NPs were formed spontaneously and deposited in the surface of the polymer.

$>$ It was possible to transfer to other surfaces the Pd-NPs from Pd@PB20_80A1.

$>$ PB80_20 polymers absorbed $\mathrm{Pd}(\mathrm{II})$. They needed for $\mathrm{H}_{2}$ atmosphere to reduce $\mathrm{Pd}(\mathrm{II})$ to $\mathrm{Pd}$ NPs.

>Pd@PB80_20 presented Pd-NPs distributed in the polymer with less aggregation than Pd@PB20_80A1, and not only in the surface.

$>$ The Pd-NPs for these two polymers were polycrystalline nanodendrites with an average diameter of $75 \mathrm{~nm}$. 


\section{PALLADIUM SUPPORTED POLYMERS AS CATALYSTS}

Dendritic-like palladium nanostructures have found use in many fields, such as the preparation of electrodes for electrocatalytic ethanol oxidation, ${ }^{71}$ formic acid oxidation, ${ }^{72}$ or lithium-oxygen batteries. ${ }^{73}$ Albeit, there are more possible applications; regarding that, this work is oriented to the use of supported Pd catalyst on the highly interesting ${ }^{74}$ semihydrogenation of disubstituted alkynes with molecular hydrogen. The importance of this new catalyst comes from the easiness to prepare these materials and their special characteristics. An ideal Pd heterogenous catalyst should possess the next characteristic:

- Using green solvents (avoiding DCM or DMSO).

- Having high recyclability of the catalyst.

- Getting no leaching of Pd to the solutions.

- The relation $[\mathrm{Pd}(\mathrm{mol})] /$ polymer surface $\left(\mathrm{cm}^{2}\right)$ has to be low.

- The turnover number (TON), (number of moles converted to product)/(surface of the heterogeneous catalyst), should be as high as possible.

In literature, there are several examples of supported catalysts for the reduction of triple bonds. The most common one, and the industrial standard, was $\mathrm{Pd} / \mathrm{C}$, which has been widely use, although it is very expensive. In addition, most of these catalysts have some issues such as requiring a filtration, big amounts of the catalyst and low (if some) recyclability. As a consequence, it was of upmost importance the development of a new catalyst that overcome those problems.

With this aim in mind, $\operatorname{Pd}(0)$ modified polymers were found as a solution for getting very efficient and recyclable heterogenic catalysts for selective reduction of triple bonds. After some preliminary testing, some variations of the different polymers were selected as representative examples. In particular, from all the polymers that contained palladium in its surface the ones with better properties were selected:

- Pd@PB20_80A1 which had Pd nanoparticles transferable to other surfaces.

- Pd@PB80_20A1 and A4, which had Pd nanoparticles anchored to the polymer.

- Pd@JG25SA, which was, apparently, very similar to PB80_20.

Furthermore, a reagent was selected as a model to compare the results in the reduction, optimizing the reaction conditions as follows:

- The solvent had to be a good solvent for most organic compounds. With that purpose, the reaction was tested in $\mathrm{MeOH}$ (a green and common solvent), DCM and THF.

- The yields were calculated by NMR and/or column purification, depending on the byproducts.

- The film was $0.5 \times 0.5 \mathrm{~cm} / 5 \mathrm{~mL}$ solution.

\footnotetext{
${ }^{71}$ A) K. Qi, Q. Wang, W. Zheng, W. Zhang, X. Cui, Nanoscale 2014, 6, 15090-15097. B) S. J. Ye, D. Y. Kim, S. W. Kang, K. W. Choi, S. W. Han, O. O. Park, Nanoscale 2014, 6, 4182-4187.

72 A) A. Klinkova, P. D. Luna, E. H. Sargent, E. Kumacheva, P. V. Cherepanov, J. Mater. Chem. A 2017, 5, 11582-11585. B) T. Huang, S. K. Moon, J.-M. Lee, Sustainable Energy Fuels 2017, 1, 450-457.

${ }^{73}$ S. J. Ye, D. Y. Kim, D. W. Kim, O. O. Park, Y. Kang, J. Mater. Chem. A 2016, 4, 578-586.

${ }^{74}$ D. B. Burueva, K. V. Kovtunov, A. V. Bukhtiyarov, D. A. Barskiy, I. P. Prosvirin, I. S. Mashkovsky, G. N. Baeva, V. I. Bukhtiyarov, A. Y. Stakheev, I. V. Koptyug, Chem. Eur. J. 2018, 24, 2547-2553.
} 
For the initial experiments, the selected sample for reduction was dimethyl acetylenedicarboxylate (DMAD), that was added as much as $500 \mathrm{mg}$ each $5 \mathrm{ml}$ of solvent. DMAD was interesting for several reasons so as to check the capabilities of the polymer as a catalyst:

o It was soluble in common solvents, such as methanol or dichloromethane.

o It was simple to analyse, it is liquid and there is only one signal in the ${ }^{1} \mathrm{HNMR}$ spectra.

o It was not an isolated internal triple bond, DMAD is a Michael acceptor with an electrondeficient triple bond, conjugated with the ester groups. However, it is not a large conjugation which means that the reaction would not be totally favoured nor disfavoured.

\section{Specific conditions for the tests:}

Thus, in $10 \mathrm{~mL}$ vials, $500 \mathrm{mg}$ of DMAD were dissolved in $5 \mathrm{~mL}$ of solvent, a piece of polymer, $0.5 \times 0.5 \mathrm{~cm}$, was added to the solution and the vial was placed in a reactor, then $\mathrm{H}_{2}$ was introduced to the chamber until reaching $5 \mathrm{~atm}$ and the mixture remained under $\mathrm{H}_{2}$ for 15 hours. After that, excess hydrogen was released, the solid catalyst was removed and the solvent evaporated (Figure 48).
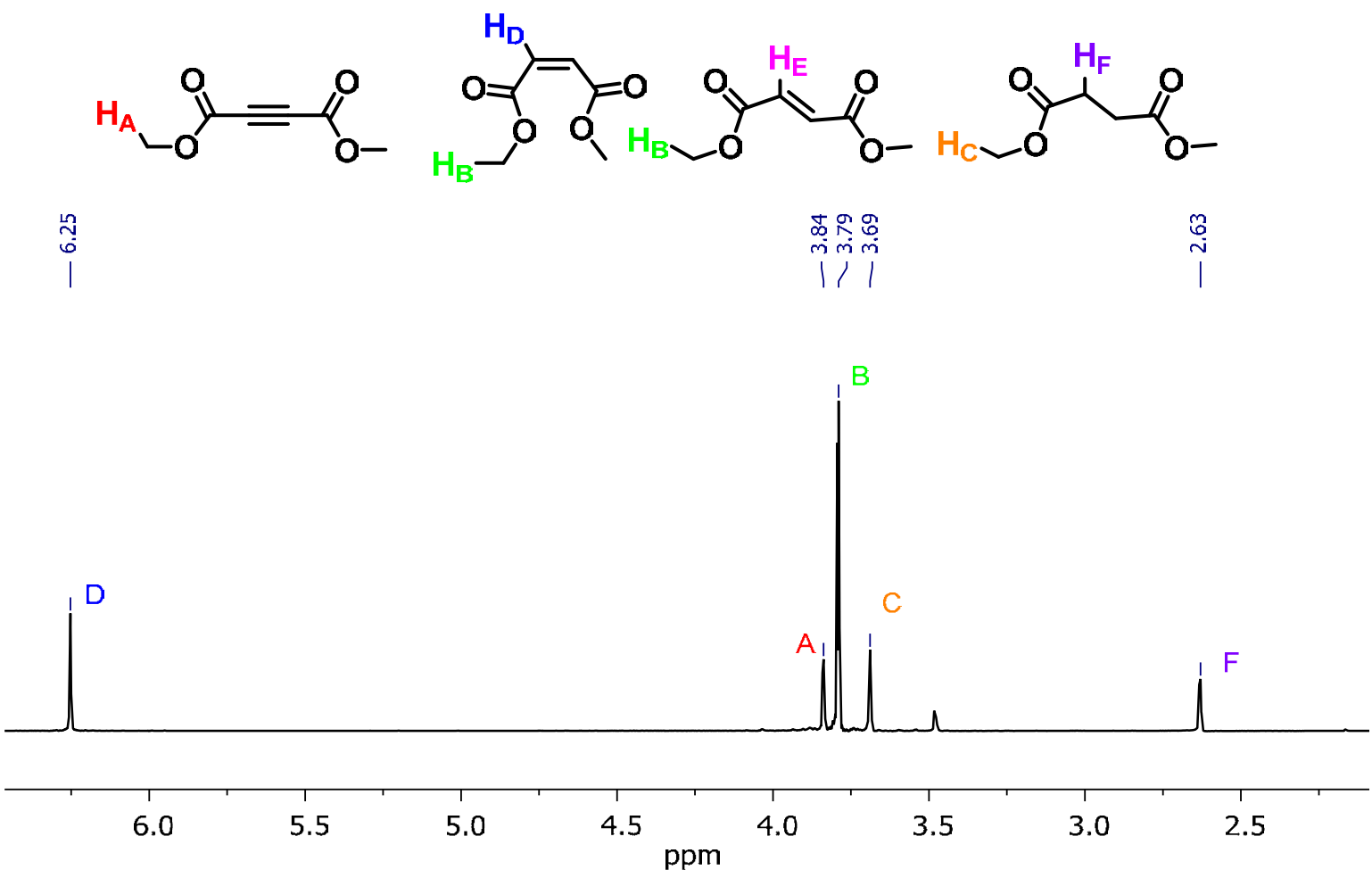

Figure 48. Structure of the possible products of the reduction of the triple bond from DMAD (up) and ${ }^{1}$ HNMR spectrum of the crude obtained from using the polymer as catalyst (down). The film used for the example was Pd(II)PB20_80A4, without previous cleaning nor reduction.

In ${ }^{1}$ HNMR from the Figure 48 it can be seen the presence of a mixture between the starting material and the products cis (maleate) and reduced to the simple bond (succinate). The trans product (fumarate) was rarely obtained (THF solutions) and in very low yields. As a result, all the expected products were easily detected by ${ }^{1} \mathrm{HNMR}$. 


\subsection{Reduction of DMAD with palladium modified films}

The following table (Figure 49) summarizes the results:

\begin{tabular}{|l|l|ccc|c|}
\hline Polymer & Solvent & Initial $(\%)$ & Cis (\%) & Trans (\%) & Simple (\%) \\
\hline Pd@PB80_20A4* & - & 93 & 7 & 0 & 0 \\
& $\mathrm{MeOH}$ & 10 & 90 & 0 & 0 \\
& $\mathrm{DCM}$ & 26 & 74 & 0 & 0 \\
& $\mathrm{THF}$ & 37 & 46 & 9 & 8 \\
\hline Pd@PB20_80A1 & - & 90 & 10 & 0 & 0 \\
& $\mathrm{MeOH}$ & 6 & 94 & 0 & 0 \\
& $\mathrm{DCM}$ & 71 & 29 & 0 & 0 \\
& $\mathrm{THF}$ & 83 & 8 & 9 & 0 \\
\hline Pd@JG25SA2 & - & 93 & 7 & 0 & 0 \\
& $\mathrm{MeOH}$ & 56 & 40 & 0 & 4 \\
& $\mathrm{DCM}$ & 97 & 3 & 0 & 0 \\
& $\mathrm{THF}$ & 9 & 8 & 6 & 0 \\
\hline
\end{tabular}

*Pd@PB80_20A4 palladium was previously reduced under $\mathrm{H}_{2}$ atmosphere $5 \mathrm{~atm}, 1$ hour and washed 5 times with $10 \mathrm{~mL}$ of $\mathrm{MeOH}$.

Figure 49. Reduction of the triple bond from DMAD in presence of 3 different heterogenous catalysts, 3 different solvents and with no solvent.

Previously to give an analysis of the results, there were several characteristics to take into account:

- Best yields (\% reacted) were reached for PB20_80A1 and PB80_20A4 and using MeOH as solvent. In addition, the yields of several repetitions are shown in the table in Figure 50.

\begin{tabular}{|l|l|ccc|}
\cline { 2 - 5 } \multicolumn{1}{l|}{} & Polymer & Initial (\%) & Cis(\%) & Simple(\%) \\
\hline 1st & Pd(II)@PB80_20A4* & 5 & 72 & 23 \\
& Pd@PB20_80A1 & 6 & 94 & 0 \\
\hline 2nd & Pd@PB80_20A4* & 9 & 85 & 6 \\
& Pd@PB20_80A1 & 21 & 79 & 0 \\
\hline \multirow{3}{*}{ rd } & Pd@PB80_20A4 & 5 & 95 & 0 \\
& Pd@PB20_80A1 & 32 & 68 & 0 \\
\hline 4th & Pd@PB80_20A4 & 8 & 92 & 0 \\
& Pd@PB20_80A1 & 65 & 35 & 0 \\
\hline
\end{tabular}

*The films were not previously reduced and washed before the reactions. If done so, the yields are similar to the reactions done afterwards.

Figure 50. Yields after repeating the reduction of the triple bond from DMAD, 4 times in $\mathrm{MeOH}$ as solvent.

- Pd@PB20_80A1 had the drawback (for catalytic purposes) of the transferable particles, if the polymer was scratched, or put in contact with some surfaces, the quantity of particles decreased. Also, there could be transference to the solutions (although they were not soluble in $\mathrm{MeOH})$ loosing effectiveness and reducing the recyclability. 
- Pd(II)@PB80_20A4 had the simple bond (succinate) as a by-product in the first two reactions. This is the consequence of using the not reduced-not washed polymer. After the second reaction, it is constant at least for 4 reactions more with yields of the cis product (maleate) superior $90 \%$.

- Pd@PB80_20A4 has the best yield/recyclability but, in order to not having by-products, such as the succinate, the polymer should be carefully washed with $\mathrm{MeOH}$, and previously subjected to hydrogen atmosphere. As a result, it can be used as a catalyst with high yields.

- Other molecules reached also quantitative yields in $\mathrm{MeOH}$ (see Section 10, "catalytic reduction of compounds with biological interest"). This yield changes depending on the solvents and the molecule to reduce (the surroundings of the triple bond), apart from being limited by its TON.

- Pd(II)JG25SA2 gave similar results to PB80_20A4, but worse yield. Additionally, the mechanical properties were worse; therefore, it was not further studied.

\subsection{Leaching of the polymers}

One of the biggest problems of using Pd supported catalysts is the quantity of the palladium that may leach to the solutions. Several situations were studied, to do so an aliquot of the solution from the reaction explained in section 9.1. (1 mL) was analysed by ICP mass (three times each), calibrating with palladium solutions. The results were:

- $\quad$ d @PB20_80A1: the palladium in solution was negligible, due to the insolubility and despite being transferable. The quantity detected was inferior to $2 \mu \mathrm{M}$.

- $\quad$ Pd(II)@PB80_20A4, not reduced/washed film: the quantity detected was between $0.4-0.1$ $\mathrm{mM}$.

- $\quad$ Pd@PB80_20A4 after reducing and washing the polymer: the quantity detected was inferior to $6 \mu \mathrm{M}$.

These data were also important in order to show the necessity of using washed polymers and the low concentration of palladium in solution when doing so.

\subsection{Other important characteristics studied}

Reaction time: the reaction time was tested in $\mathrm{MeOH}$ solution for DMAD reductions. The yield was constant when the reactions lasted 5 hours of more. However, less time led to lower yields, although this parameter should be adjusted depending on the specific molecule to reduce, and also depended on other parameters like the concentration or temperature.

Polymers without crosslinker: PBM2, PBMEGMA and PBMA did not contain crosslinker, that caused that they were soluble in most of the organic solvents. Despite this fact, they were tested in solution but the reaction did not work.

Turnover number (TON): the TON was defined as (product moles)/surface. It was calculated based on the studied reduction reaction. In this regard, the catalyst turnover number was calculated considering a yield of $90 \%$ and 6 reactions; obtaining a TON of $\mathbf{7 5} \mathbf{~ m m o l e s} / \mathbf{c m}^{2}$. 


\section{CATALYTIC REDUCTION OF COMPOUNDS WITH BIOLOGICAL INTEREST}

With the polymer with better results (Pd@PB80_20A4) and the optimized conditions, a series of compounds were chosen as potential substrates for semihydrogenation reactions because of their properties and applications in different fields (Figure 51). For showing the importance of this kind of reactions, it was necessary to check if the procedure permitted the quantitative and selective transformation of several important pharmacological drugs or intermediates into related compounds. In this regard, the catalyst had to have no by-products and restricted reactivity to triple bonds, and not halogens or double bonds.

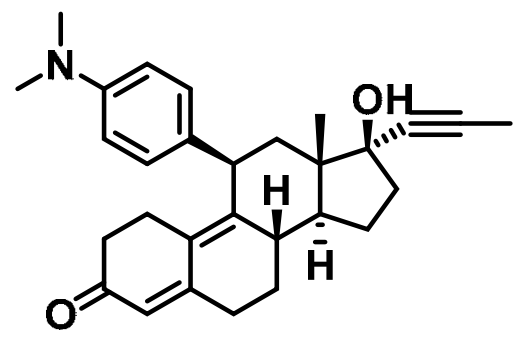

Mifepristone<smiles>CC#CCn1c(Br)nc2c1c(=O)[nH]c(=O)n2C</smiles>

(Br-R)<smiles>C[C@]1(C#CC2CC2)OC(=O)Nc2ccc(Cl)c(F)c21</smiles>

Efavirenz<smiles>CCOC(=O)c1ccc(C#Cc2ccc3c(c2)C(C)(C)CCS3)nc1</smiles>

Tazarotene

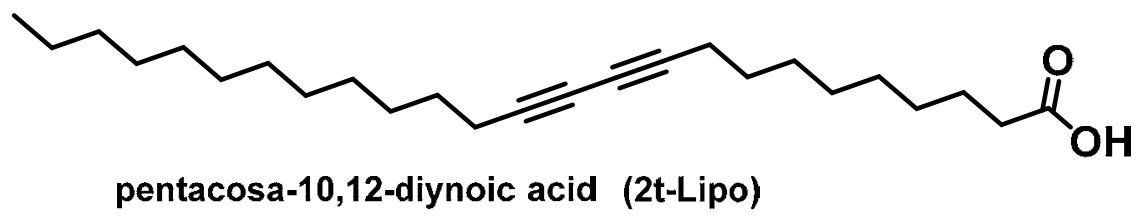

Figure 51. Molecules with triple bonds studied

- Mifepristone: a steroidal progesterone ${ }^{75}$ and glucocorticoid receptor antagonist, ${ }^{76}$ used as contraceptive agent and in the treatment of breast cancer. ${ }^{77}$ By cis-semihydrogenation reduction, was transformed into aglepristone,$^{78}$ a related progesterone antagonist used for the

\footnotetext{
${ }^{75}$ A. Yamada, Y. Kazui, H. Yoshioka, A. Tanatani, S. Mori, H. Kagechika, S. Fujii, ACS Med. Chem. Lett. 2016, 7, 1028-1033.

${ }^{76}$ C. A. Sanhueza, M. M. Baksh, B. Thuma, M. D. Roy, S. Dutta, C. Préville, B. A. Chrunyk, K. Beaumont, R. Dullea, M. Ammirati, S. Liu, D. Gebhard, J. E. Finley, C. T. Salatto, A. King-Ahmad, I. Stock, K. Atkinson, B. Reidich, W. Lin, R. Kumar, M. Tu, E. Menhaji-Klotz, D. A. Price, S. Liras, M. G. Finn, V. Mascitti, J. Am. Chem. Soc. 2017, 139, 3528-3536.

${ }^{77}$ Y. Lin, R. Liu, P. Zhao, J. Ye, Z. Zheng, J. Huang, Y. Zhang, Y. Gao, H. Chen, S. Liu, J. Zhou, C. Chen, H. Chen, Eur. J. Med. Chem. 2018, 146, 354-367.

${ }^{78}$ A. Gogny, F. Fiéni, Theriogenology 2016, 85, 555-566.
} 
treatment of various progesterone-dependent physiological or pathologic conditions in veterinary medicine.

- Efavirenz: ${ }^{79}$ a non-nucleoside reverse transcriptase inhibitor used as a first-line anti-HIV drug. ${ }^{80}$ It was transformed into (Z)-dihydroefavirenz, ${ }^{81}$ a predicted but yet unavailable efavirenz analogue. Thus, expanding the pharmaceutical possibilities of fluorine-containing pharmaceuticals. $^{82}$

- Tazarotene: ${ }^{83}$ a receptor selective retinoid that specifically binds to retinoid receptors in the skin after ester hydrolysis. ${ }^{84}$ It is currently used for topical treatment of psoriasis, it was then converted into (Z)-dihydrotazarotene [ethyl (Z)-6-(2-(4,4-dimethylthiochroman-6yl)vinyl)nicotinate] a new potential retinoid. ${ }^{85}$

- $\quad$-Bromo-7-(2-butyn-1-yl)-3-methylxanthine (Br-R): a key intermediate for the synthesis of linagliptin, ${ }^{86}$ a xanthine dipeptidyl peptidase-4 (DPP-4) inhibitor for the treatment of type 2 diabetes. ${ }^{87}$ It was transformed into (Z)-8-bromo-7-(2-buten-1-yl)-3-methylxanthine, a new intermediate on the way to new DPP-4 inhibitors. ${ }^{88}$ The presence of the sensitive bromo substituent in the reduced product constitutes a remarkable proof of the selectivity of the catalytic semihydrogenation selectivity of the described process, although in this case a purification step was required.

- Pentacosa-10,12-diynoic acid (2t-Lipo): a carboxylic acid with a lepidic chain with triple bonds, it serves as an example of the capabilities for reducing triple bonds in molecules with potential applicability in creating lipidic membranes for vesicles. In view of the importance of all-(Z) polyunsaturated lipids for biological purposes, the available pentacosa-10,12-diynoic acid was selected and subjected the compound to semihydrogenation conditions, from which a scarce yield was obtained for the corresponding $(Z),(Z)$-pentacosa-10,12-dienoic acid due to the natural tendency of the starting material to form Langmuir-Blodgett structures, that polymerize under the light giving blue and red solutions. ${ }^{89}$

To compare the selectivity of the reaction with reported conditions of a common palladium/carbon catalyst, the same reactions were performed in the presence of commercial palladium on carbon $(10 \%)$, obtaining in all cases mixtures of products coming from different hydrogenation patterns, usually triple bonds to saturated hydrocarbons as well as producing the reduction of internal double bonds, the dehalogenation products and mixtures of several of the possible products of reduction. In contrast, the selectivity of the heterogenic catalysis with modified films was verified.

\footnotetext{
${ }^{79}$ S. Li, J.-A. Ma, Chem. Soc. Rev. 2015, 44, 7439-7448.

${ }^{80}$ M. M. Bastos, C. C. P. Costa, T. C. Bezerra, F. de C. da Silva, N. Boechat, Eur. J. Med. Chem. 2016, 108, 455-465.

${ }^{81}$ D. D. Christ, A. J. Cocuzza, S. S. Ko, J. A. Markwalder, A. E. Mutlib, R. L. Jr. Parsons, M. Patel, S. P. Seitz, U.S. patent 1999, US 5874430 A 19990223.

${ }^{82}$ Y. Zhou, J. Wang, Z. Gu, S. Wang, W. Zhu, J. L. Aceña, V. A. Soloshonok, K. Izawa, H. Liu, Chem. Rev. 2016, 116, 422-518.

${ }^{83}$ I. S. Makarov, C. E. Brocklehurst, K. Karaghiosoff, G. Koch, P. Knochel, Angew. Chem. Int. Ed. 2017, 56, $12774-12777$.

${ }^{84}$ A. M. Mansour, Polyhedron 2016, 109, 99-106.

${ }^{85}$ R. Álvarez, B. Vaz, H. Gronemeyer, Á. R. de Lera, Chem. Rev. 2014, 114, 1-125.

${ }^{86}$ M. Eckhardt, T. Klein, H. Nar, S. Thiemann, Discovery of Linagliptin for the Treatment of Type 2 Diabetes Mellitus, in: J. Fischer, D. P. Rotella, Eds., Successful Drug Discovery, Wiley-VCH Verlag, 2015, Ch. 7.

${ }^{87}$ W.-L. Wu, J. Hao, M. Domalski, D. A. Burnett, D. Pissarnitski, Z. Zhao, A. Stamford, G. Scapin, Y.-D. Gao, A. Soriano, T. M. Kelly, Z. Yao, M. A. Powles, S. Chen, H. Mei, J. Hwa, ACS Med. Chem. Lett. 2016, 7, $498-501$.

${ }^{88}$ L. Juillerat-Jeanneret, J. Med. Chem. 2014, 57, 2197-2212.

${ }^{89}$ S. Balakrishnan, S. Lee. J.-M. Kim, J. Mater. Chem. 2010, 20, 2302-2304.
} 


\subsection{Conditions of the reaction and yields obtained}

The reaction conditions were similar to the DMAD reduction. 50-100 $\mathrm{mg}$ of the different analytes were dissolved in 2-5 $\mathrm{mL}$ of $\mathrm{MeOH}$ and put under $5 \mathrm{~atm}$ of $\mathrm{H}_{2}$ overnight.

\begin{tabular}{cc}
\hline Pd@PB80_20A4 & cis (\%) \\
\hline Efavirenz & $>99$ \\
Mifepristone & $>99$ \\
Br-R & 90 \\
Tazarotene & 85 \\
2t-Lipo* $^{*}$ & $\geq 50$ \\
\hline
\end{tabular}

Figure 52. Yields (\%) for the cis product for the selected molecules.

These yields were calculated after the purification by column chromatography of the product except for 2t-Lipo. Characteristics of each reaction:

- Efavirenz and Mifepristone had a favoured reduction reaction, with yields superior to $99 \%$ and no by-products.

- $\quad \underline{B r-R}$ had several minor by-products, needing column chromatography for the separation of the cis product.

- Tazarotene was less reactive under the same reaction conditions. Therefore, an easy separation from the remaining starting material, that was reused, was also needed.

- 2t-Lipo was only slightly soluble in $\mathrm{MeOH}$ and chloroform. Nevertheless, the reaction occurred with high yields as it can be deduced from ${ }^{1} \mathrm{H}$ NMR, which demonstrated its applicability for the purpose of reducing triple bonds in lipidic chains. In this case, the yield was estimated from the ${ }^{1} \mathrm{H}$ NMR because a column chromatography was not possible due to its low solubility.

\subsection{Results for $\mathrm{Pd}$ films reduction and comparison with $\mathrm{Pd} / \mathrm{C}$ catalyst}

In this section, the results of using the polymeric heterogenic catalyst and $\mathrm{Pd} / \mathrm{C}$ are compared. The main differences are showed by the purified NMR spectra and the results of high resolution mass spectra analysis. In addition, the compounds were fully characterized, although the specifics are more detailed in the Experimental Appendix 4.

In this way, the reaction was performed with the catalysts under similar conditions to previous synthesis; $100 \mathrm{mg}$ of analyte, $5 \mathrm{~mL}$ of $\mathrm{MeOH}$ and $100 \mathrm{mg}$ of $\mathrm{Pd} / \mathrm{C}$ or a $0.5 \times 0.5$ piece of Pd@PB80_20A4. All components were put at 5 atm pressure and stirred for 16 hours. 


\subsubsection{Mifepristone:}
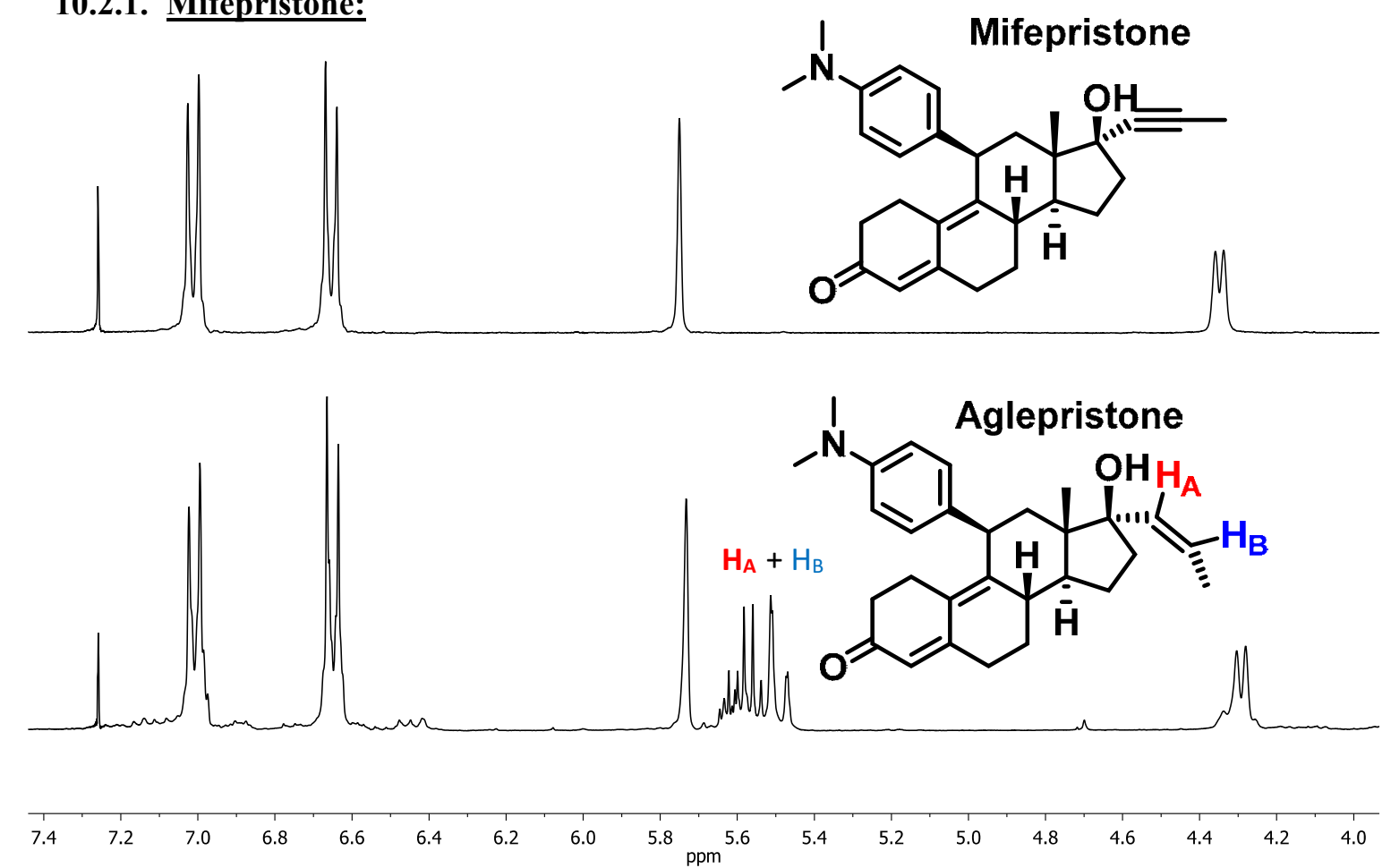

Figure 53. ${ }^{1} \mathrm{H}$ NMR spectra $\left(\mathrm{CDCl}_{3}\right)$, comparison between Mifepristone and Aglepristone, its reduced specie. Analysis performed for the product of Pd@PB80_20A4 reduction.

In this case, the product obtained under $\mathrm{Pd} / \mathrm{C}$, was a mixture of different reductions, as it was showed by the mass spectra. Mifepristone was likely to be reduced in different positions due to the presence of double bonds in its structure. The major product was the reduction with six more hydrogen atoms (probably the triple bond to simple and one of the double bonds) and in less quantity the reductions adding four and eight hydrogen atoms.

Pd@PB80_20A4: HRMS (ESI-TOF) m/z calcd for $\mathrm{C}_{29} \mathrm{H}_{38} \mathrm{NO}_{2}\left(\mathrm{M}^{+}\right)$: 432.2897; found: 432.2911 .

Pd/C: HRMS (ESI-TOF): (a) $\mathrm{m} / \mathrm{z}$ calcd for $\mathrm{C}_{29} \mathrm{H}_{40} \mathrm{NO}_{2}\left(\mathrm{M}^{+}\right)$: 434.3054; found: 434.3057. (b) $\mathrm{m} / \mathrm{z}$ calcd for $\mathrm{C}_{29} \mathrm{H}_{42} \mathrm{NO}_{2}\left(\mathrm{M}^{+}\right)$: 436.3210; found: 436.3217. (c) $\mathrm{m} / \mathrm{z}$ calcd for $\mathrm{C}_{29} \mathrm{H}_{44} \mathrm{NO}_{2}\left(\mathrm{M}^{+}\right): 438.3367$; found: 438.3367 . 


\subsubsection{Efavirenz:}

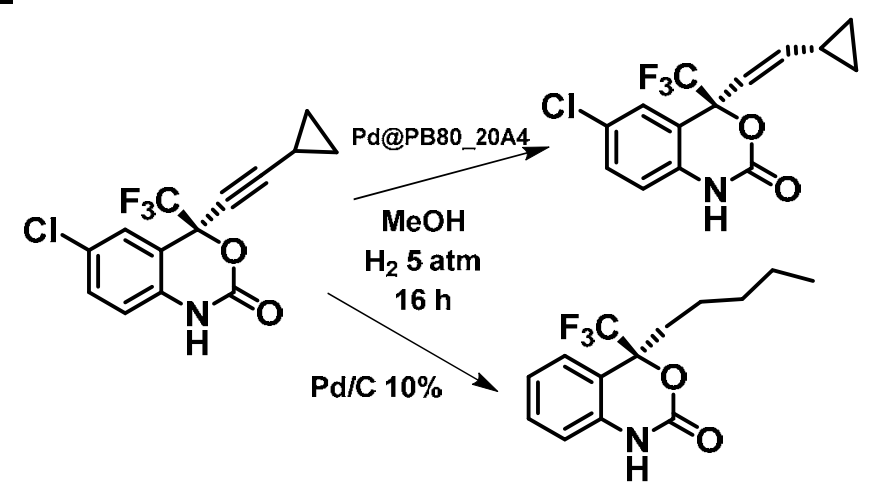

Figure 54. Efavirenz scheme reactions.

The reaction with $\mathrm{Pd} / \mathrm{C}$ led to the reduction of the triple bond to simple, the aperture of the cyclopropyl ring and the dehalogenation of the aryl-chloride (Figure 54), in quantitative yield.

a) Comparison starting material- polymer reduction:

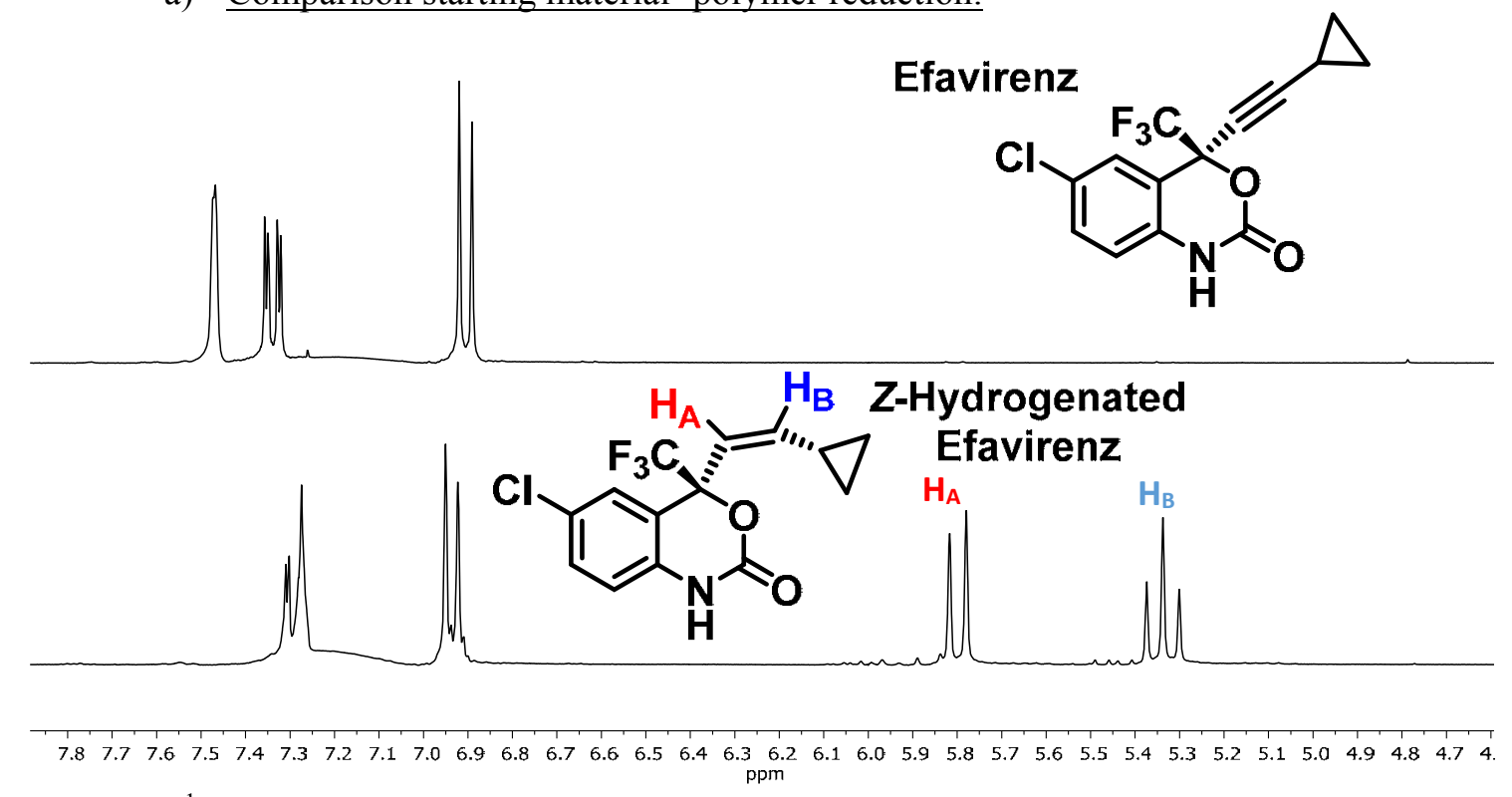

Figure 55. ${ }^{1} \mathrm{H}$ NMR spectra $\left(\mathrm{CDCl}_{3}\right)$, comparison between Efavirenz and dihydro efavirenz, its cis reduced specie. Analysis performed for the product of Pd@PB80_20A4 reduction.

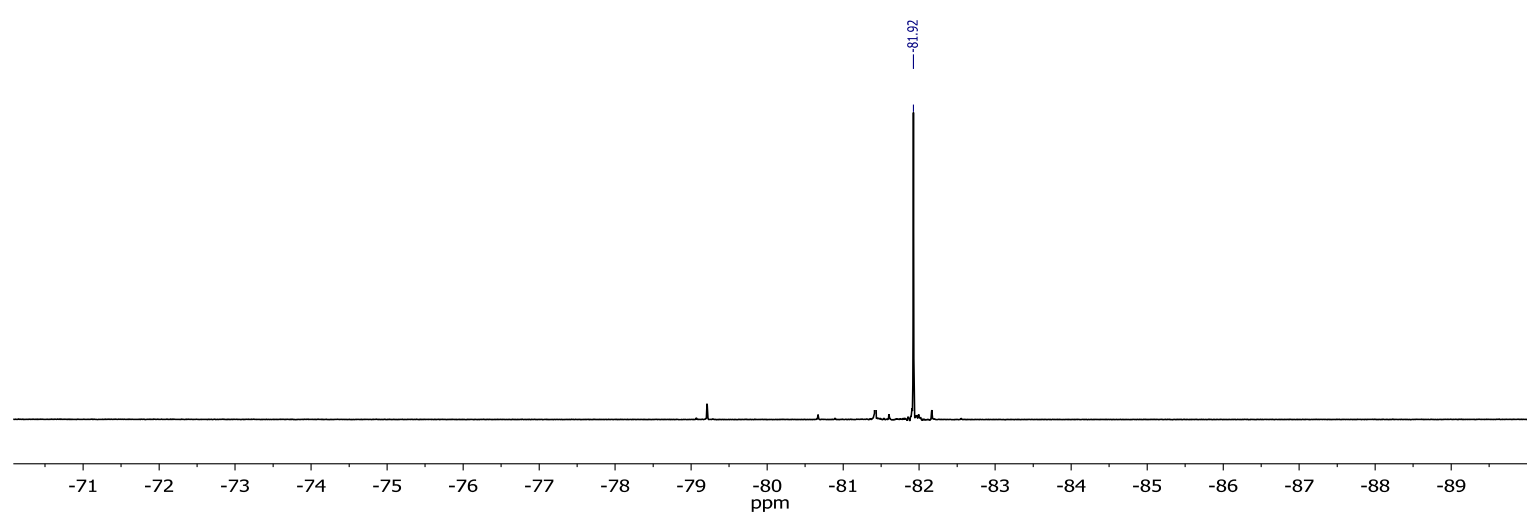

Figure 56. ${ }^{19} \mathrm{~F}$ NMR spectra $\left(\mathrm{CDCl}_{3}\right)$ of dihydro Efavirenz.

Pd@PB80_20A4: HRMS (ESI-TOF) m/z calcd for $\mathrm{C}_{34} \mathrm{H}_{29} \mathrm{NO}_{2}\left(\mathrm{M}^{+}\right)$: 483.2193; found: 483.2224 .

Pd/C: HRMS (ESI-TOF) m/z calcd for $\mathrm{C}_{14} \mathrm{H}_{13} \mathrm{~F}_{3} \mathrm{NO}_{2}\left(\mathrm{M}^{+}\right)$: 284.0904; found: 284.0896 . 


\subsubsection{8-Bromo-7-(2-butyn-1-yl)-3-methylxanthine (Br-R):}

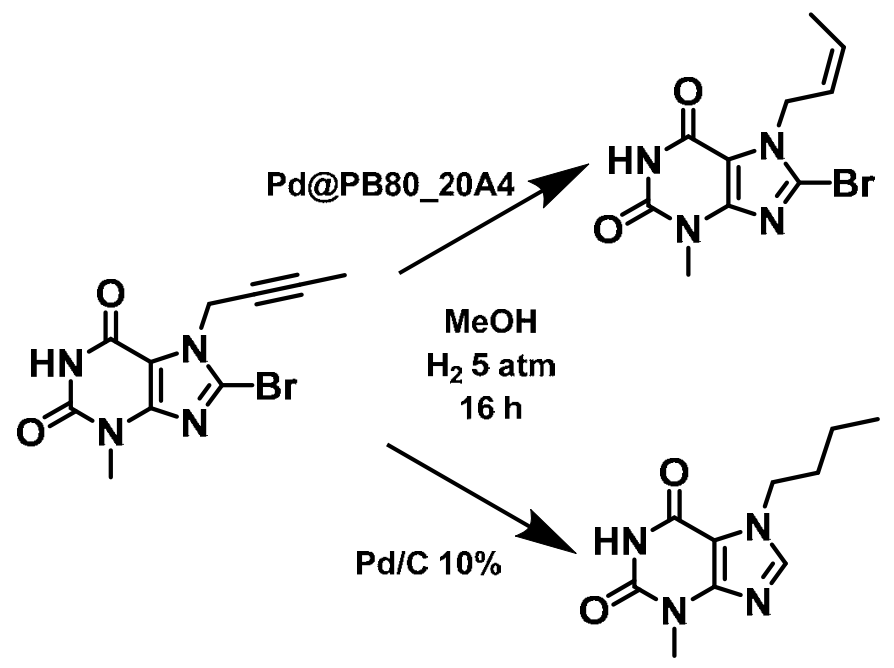

Figure 57. Br-R reduction, reactions scheme.

Just as Efavirenz, the reaction with $\mathrm{Pd} / \mathrm{C}$ led to the reduction of the triple bond to simple and the dehalogenation of the aryl-bromide (Figure 57). The $\mathrm{Pd} / \mathrm{C}$ product was obtained in quantitative yield but the cis reduction needed for a purification step for separation.<smiles>CC#CC(C)n1c(Br)nc2c1c(=O)[nH]c(=O)n2C</smiles><smiles>[R]CCC#CCC</smiles>

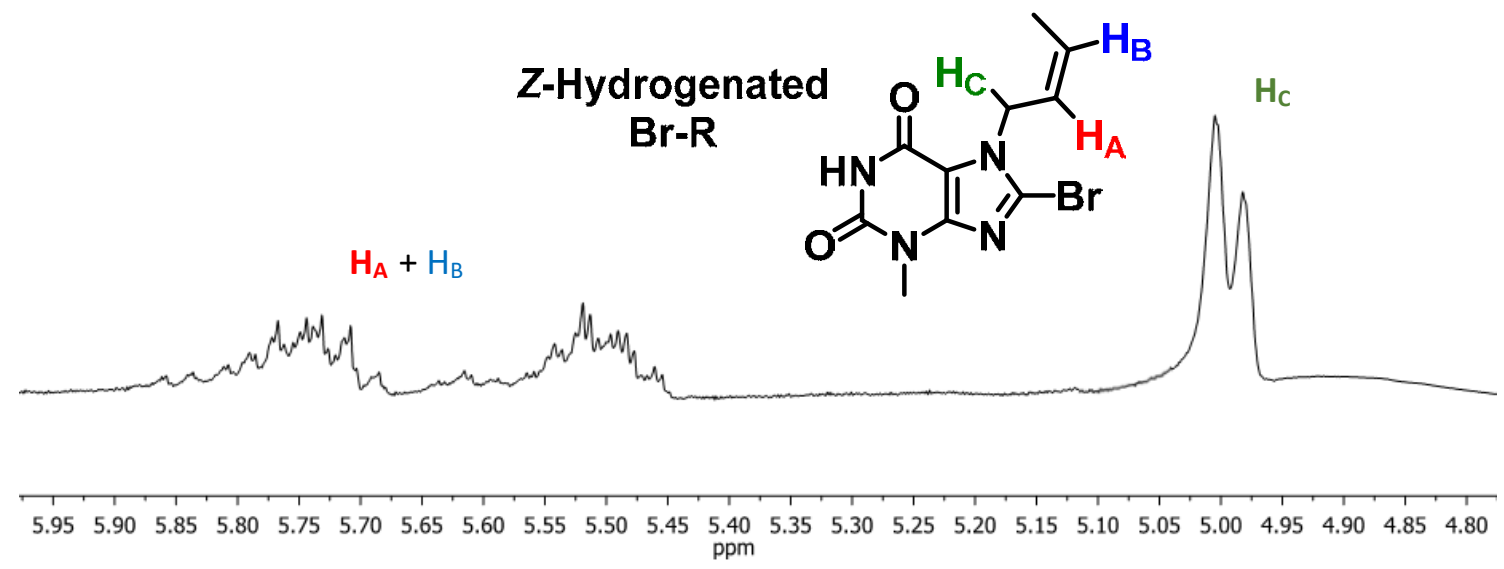

Figure 58. ${ }^{1} \mathrm{H}$ NMR spectra $\left(\mathrm{CDCl}_{3}\right)$, comparison between $\mathrm{Br}-\mathrm{R}$ and its cis reduced specie. Analysis performed for the product of Pd@PB80_20A4 reduction.

Pd@PB80_20A4: HRMS (ESI-TOF) $\mathrm{m} / \mathrm{z}$ calcd for $\mathrm{C}_{10} \mathrm{H}_{11} \mathrm{BrN}_{4} \mathrm{NaO}_{2}\left(\mathrm{M}^{+}\right)$: 320.9958; found: 320.9952 .

Pd/C: HRMS (ESI-TOF) m/z calcd for $\mathrm{C}_{10} \mathrm{H}_{15} \mathrm{~N}_{4} \mathrm{O}_{2}\left(\mathrm{M}^{+}\right)$: 223.1190 ; found: 223.1191 . 


\subsubsection{Tazarotene:}

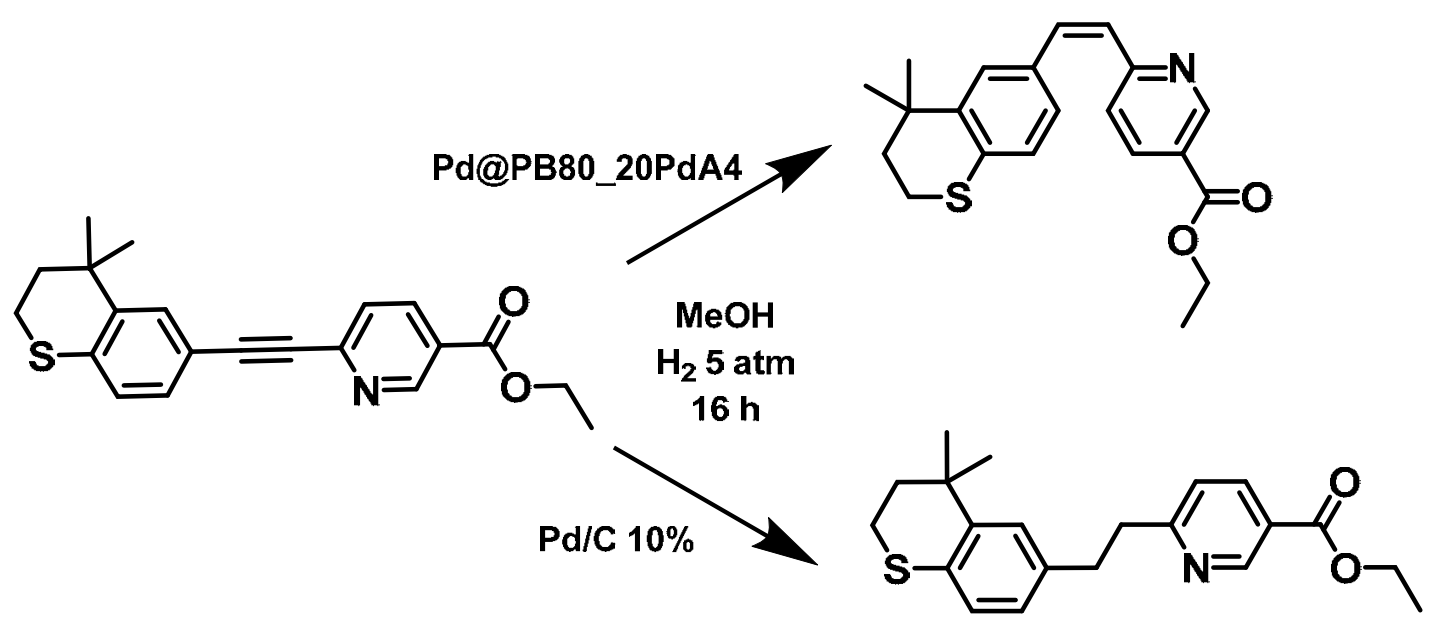

Figure 59. Tazarotene reduction, reactions scheme.

The reaction with $\mathrm{Pd} / \mathrm{C}$ led to the reduction of the triple bond to simple (Figure 59). The $\mathrm{Pd} / \mathrm{C}$ product was obtained in quantitative yield. The (Z)-dihydro tazarotene product required from column chromatography, although the product was easily separated.

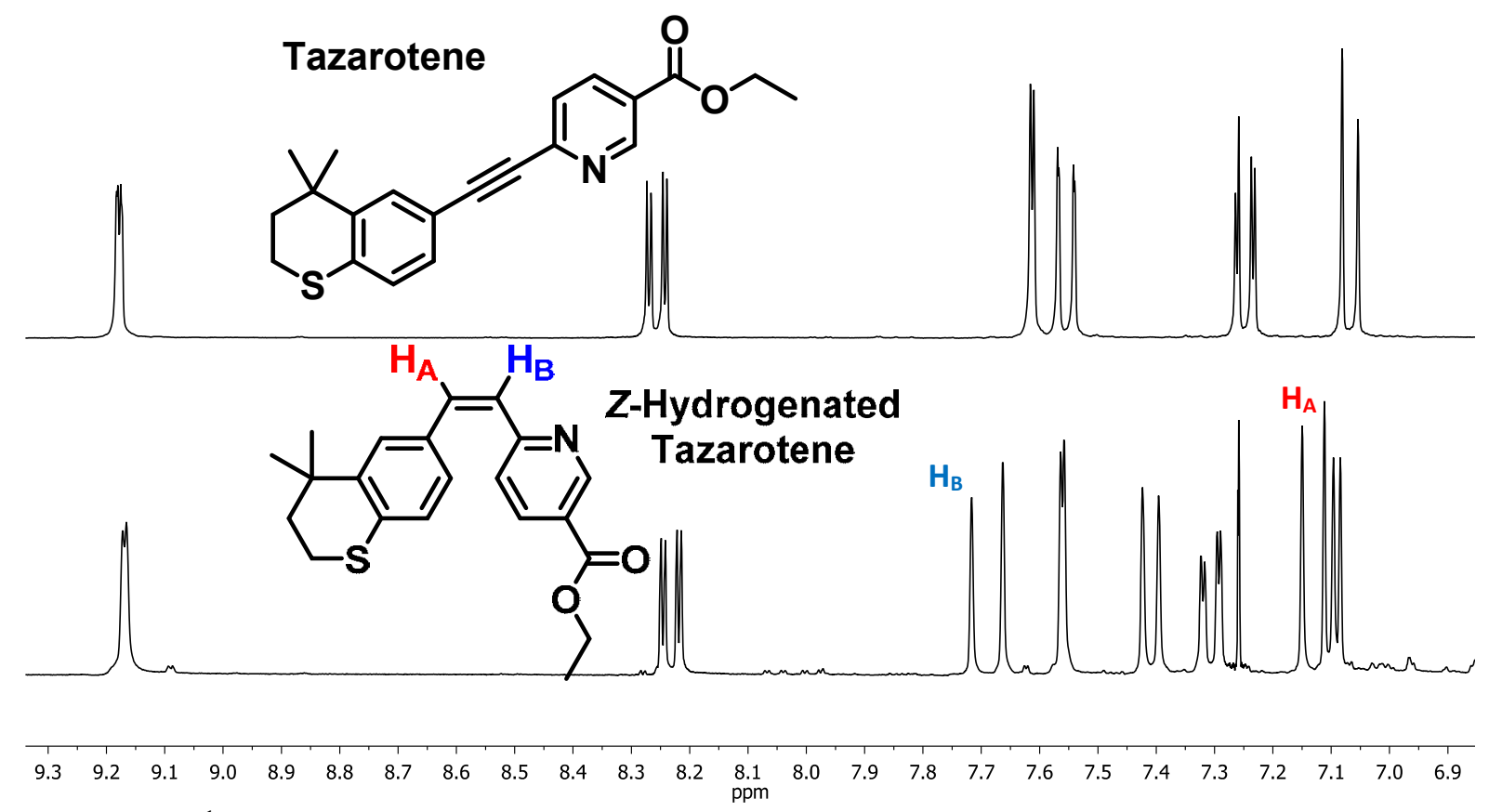

Figure 60. ${ }^{1} \mathrm{H}$ NMR spectra $\left(\mathrm{CDCl}_{3}\right)$, comparison between tazarotene and $\mathrm{Z}$ - dihydro tazarotene, its cis reduced specie. Analysis performed for the product of Pd@PB80_20A4 reduction.

Pd@PB80_20A4: HRMS (ESI-TOF) m/z calcd for $\mathrm{C}_{21} \mathrm{H}_{24} \mathrm{NO}_{2} \mathrm{~S}\left(\mathrm{M}^{+}\right)$: 354.1522; found: 354.1531.

Pd/C: HRMS (ESI-TOF) m/z calcd for $\mathrm{C}_{21} \mathrm{H}_{26} \mathrm{NO}_{2} \mathrm{~S}\left(\mathrm{M}^{+}\right)$: 356.1679; found: 356.1690 . 


\subsubsection{Pentacosa-10,12-diynoic acid (2t-Lipo):}

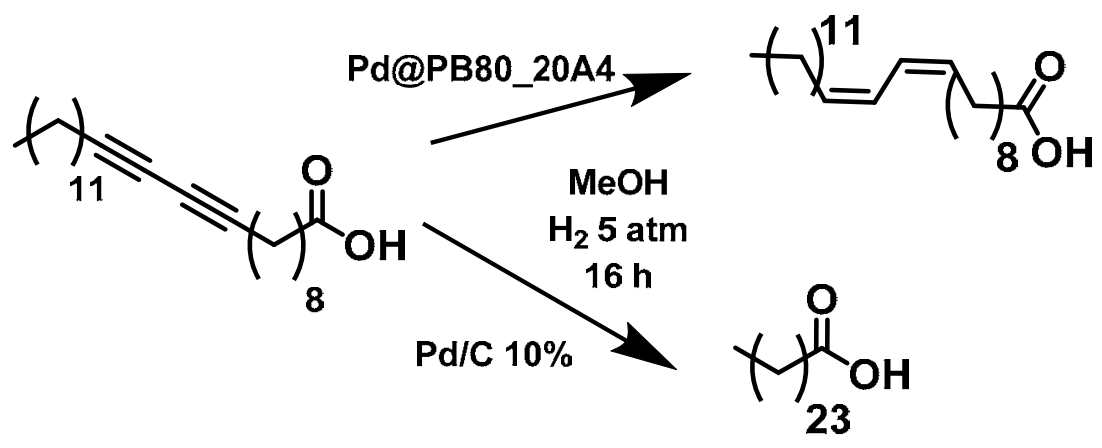

Figure 61. 2t-Lipo reduction, reactions scheme.

In this case, when using $\mathrm{Pd} / \mathrm{C}$ the product became a colourless grease, which was an evidence of the quantitative transformation into the aliphatic chain (Figure 61). When using the film, the reaction was not total, and it was not possible to perform purification by column chromatography, because of the low solubility; then, the yield was estimated from the ${ }^{1} \mathrm{H}$ NMR to be around $50 \%$.

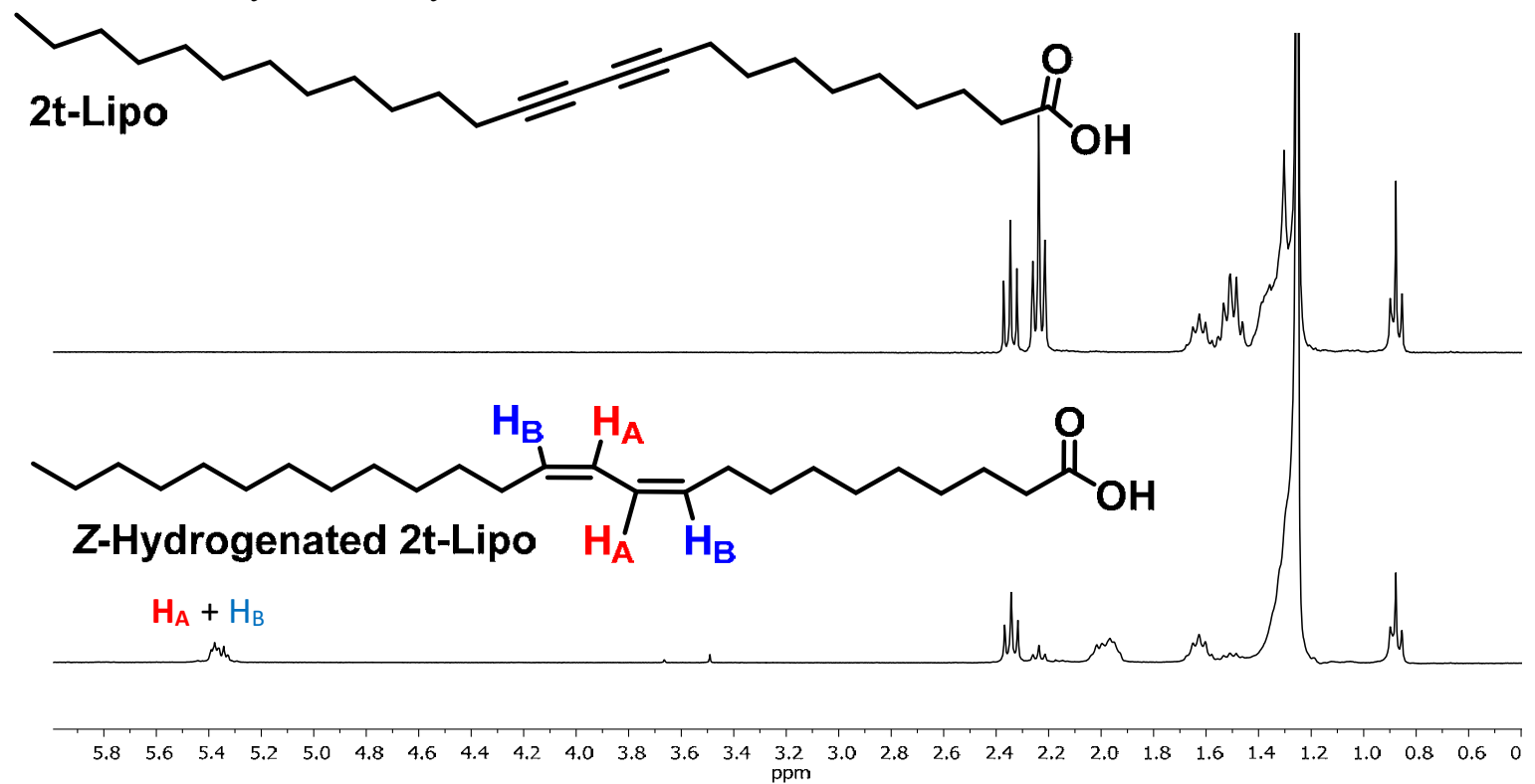

Figure 62. ${ }^{1} \mathrm{H}$ NMR spectra $\left(\mathrm{CDCl}_{3}\right)$, comparison between $2 \mathrm{t}$-Lipo and its cis reduced specie. Analysis performed for the product of Pd@PB80_20A4 reduction.

Pd@PB80_20A4: HRMS (ESI-TOF) m/z calcd for $\mathrm{C}_{25} \mathrm{H}_{47} \mathrm{O}_{2}\left(\mathrm{M}^{+}\right)$: 379.3582; found: 379.3584 .

Pd/C: HRMS (ESI-TOF) m/z calcd for $\mathrm{C}_{25} \mathrm{H}_{49} \mathrm{O}_{2}\left(\mathrm{M}^{+}\right)$: 381.3738; found: 381.3729 . 


\section{PALLADIUM-SUPPORTED REDUCTION. MECHANISM AND CALCULATIONS}

The semihydrogenation selectivity is not easily explained. Pd catalysed reductions lead to different products depending on the Pd oxidation state and the atomic distribution. For that reason, the interactions of the different substrates were simulated using DFT calculations.

- All calculations were carried out using the quantum chemical software ORCA 4.0.1.2. ${ }^{90}$

- Pd was described using the relativistic effective-core potentials (LANL2DZ). ${ }^{91}$

- The basis sets for $\mathrm{C}, \mathrm{O}$ and $\mathrm{H}$ were the Ahlrichs basis def2-SVP. ${ }^{92}$

- $\mathrm{B} 3 \mathrm{LYP}^{93}$ was selected as an exchange-correlation energy functional.

The combination B3LYP/LANL2DZ model chemistry was chosen because it has been found reliable for systems formed by organic molecules adsorbed on palladium. ${ }^{94}$ For the simulations, several parameters were set:

- The geometry of the single-layer Pd lattice was frozen during geometry optimization and frequency calculation.

- The interaction energies were computed as $\Delta \mathrm{E}=\mathrm{E}_{\mathrm{sub} / \mathrm{Pd} 10}-\left(\mathrm{E}_{\mathrm{Pd} 10}+\mathrm{E}_{\mathrm{sub}}\right)$, with "sub" being the adsorbed substrate. The Cartesian coordinates of the optimized geometries can be found in the DFT Calculations Appendix.

- For this simulation, a single layer of 10 atoms of Pd was used (Pd10).

- A simplified model of mifepristone molecule was used. It was singly adsorbed by the $C \equiv C$ bond on the plane sites of the $\{111\}$ faces, interacting by bridge coordination with three Pd atoms of the cluster. By means of this interaction the carbon-carbon bond distance was 138 pm.

To simulate the interaction of the product of hydrogenation, throughout the $\mathrm{C}=\mathrm{C}$ bond with the Pd layer, two possibilities were explored. The difference between these two possibilities was the relative orientation of the added hydrogen atoms with the $\mathrm{OH}$ group. In one of them, these two hydrogen atoms were oriented at the opposite side of the $\mathrm{OH}$ group (Figure 63B) and, in the other possibility these hydrogen atoms were oriented at the same side of the $\mathrm{OH}$ group (Figure 63C). Keeping in mind that the free rotation around the $\mathrm{C}-\mathrm{C}$ single bond $\mathrm{HOC}-\mathrm{C}(\mathrm{H}) \mathrm{C}$ was a way of conversion between both structures, once the interaction to the metal layer is broken and the steric hindrance reduced. It was observed that, in both cases, the interaction of the product of hydrogenation with the same plane was slightly different because there was not bridging interaction between the carbon atoms and the Pd atoms. All these facts were in good agreement with reported similar systems. ${ }^{92}$

\footnotetext{
${ }^{90}$ N. Frank, The ORCA program system, WIREs Comput. Mol. Sci. 2012, 2, 73-78.

${ }^{91}$ P.Hay, W. Wadt, J. Chem. Phys. 1985, 82, 299-310.

${ }^{92}$ F. Weigend, R. Ahlrichs, Phys. Chem. 2005, 7, 3297-3305.
}

${ }_{93}^{9}$ A) A. Becke, Density-functional thermochemistry. III. The role of exact exchange, J. Chem. Phys. 1993, 98, 5648-5652. B) C. Lee, W. Yang, R. Parr, Development of the Colle-Salvetti correlation-energy formula into a functional of the electron density, Phys. Rev. B 1988, 37, 785-789.

${ }^{94}$ A) S. Omar, J. Palomar, L. M. Gómez, M. A. Álvarez-Montero, J. J. Rodriguez, J. Phys. Chem. C, 2011, 115, 14180-14192. B) A. Prestianni, M. Crespo-Quesada, R. Cortese, F. Ferrante, L. Kiwi-Minsker, D. Duca, J. Phys. Chem. C 2014, 118, 3119-3128. 


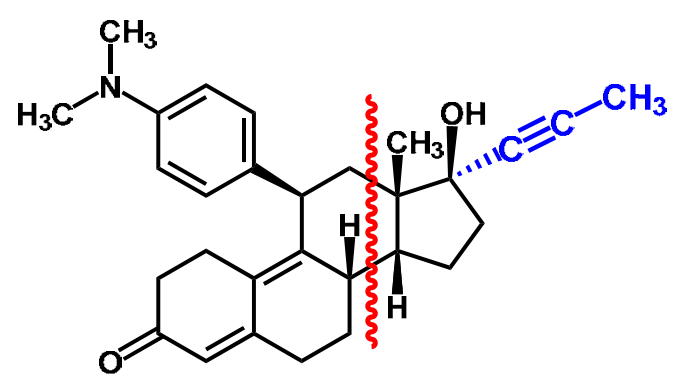

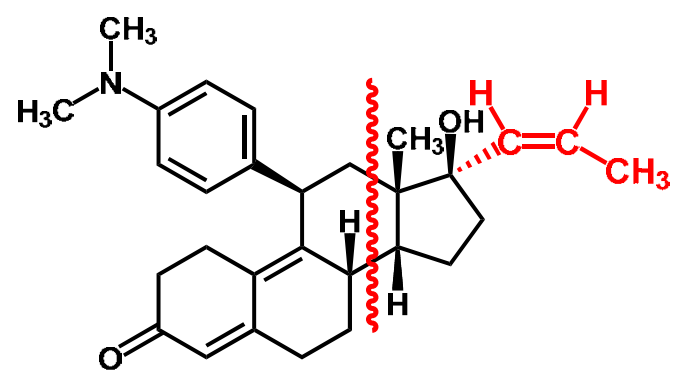

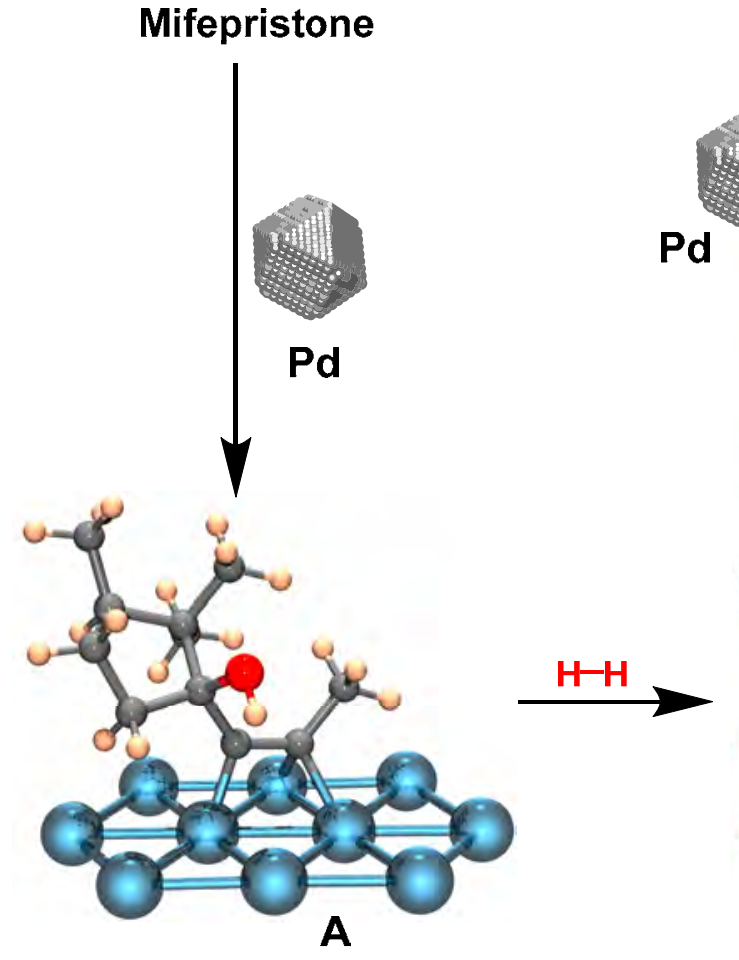

Aglepristone

d

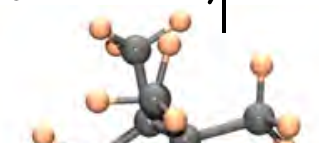

$-\mathrm{H}$ at opposite

face $-\mathrm{OH}$

group

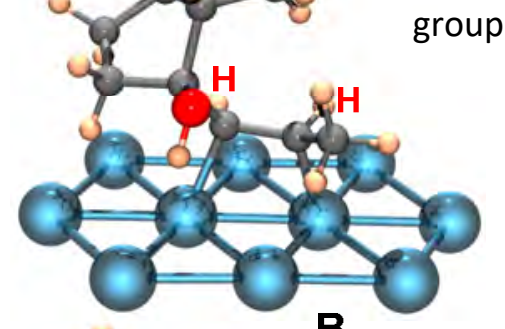

B

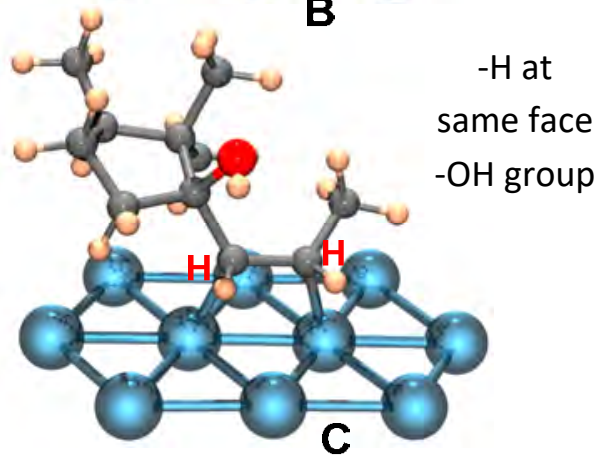

Figure 63. Interaction with a layer of ten atoms of palladium corresponding to a plane $\{111\}$ of the simplified model of mifepristone (A), the product of hydrogenation at the opposite side of the $\mathrm{OH}$ group (B) and the product of hydrogenation at the same side to the $\mathrm{OH}$ group (C).

The difference of the energy of interaction between the model of mifepristone with $\mathrm{Pd}_{10}$ and alkene substrates with $\mathrm{Pd}_{10}$ was estimated. In addition, the interaction energies resulted more marked for the mifepristone model than the observed for the product of its hydrogenation by $49.6 \mathrm{kcal} / \mathrm{mol}$ for the structure of Figure 63B and by $50.3 \mathrm{kcal} / \mathrm{mol}$ for the structure of Figure 63C. The higher stabilization found for the mifepristone model is in good agreement with a higher affinity of the $\mathrm{C} \equiv \mathrm{C}$ bond than the $\mathrm{C}=\mathrm{C}$ bond for the adsorption of the mifepristone. This higher affinity is related with the experimentally observed semihydrogenation of the mifepristone, when compared with the lack of reduction of the semihydrogenation product. 


\section{SUPPORTED GOLD AND PALLADIUM PARTICLES. SUMMARY}

\section{Supported Au(0):}

Properties of the Au substituted polymer:

- Composition:

0 JG25_SA2 is formed by hydroxyethylacrylate (2HEA, 95\%) and propargyl methacrylate (PGM, 5\%). ethylene glycol dimethacrylate (EGDMA, 5\%) was used as cross-linking agent.

o JG25 was as JG25_SA2, but JG10 substituted which formed a triazole.

- In the presence of the fluorescent probe, JG25, the shape, size and dispersion of $\mathrm{Au}(0)$ particles were higher.

- High concentrations of $\mathrm{Au}(\mathrm{III})$ solutions increased faster the number of particles than their size.

- Low relation volume/surface led to more variation in the shape of the nanoparticles, not so much in their number.

- The size of the nanoparticles was between $20 \mathrm{~nm}$ to $400 \mathrm{~nm}$ of diameter, depending on the conditions.

- The different shapes encountered were tridimensional, amorphous, spherical, tetrahedral, tetrahedral truncated...; bidimensional, triangles; and monodimensional (or with a preferential grow direction), bars with different thickness.

- In water Au-NPs were located over the polymer while, when using DMF, NPs inside the film were also obtained.

\section{Properties as catalyst of the supported $\mathrm{Au}(0)$ films:}

- They were checked to catalyse Suzuki reactions, with similar yields compared to general conditions used in published results (using $\mathrm{Pd}\left(\mathrm{PPh}_{3}\right)_{4}$ as catalyst).

- They should be used under mild working conditions, THF:Water between 40 or $60 \% \mathrm{THF}$ and temperature around $65^{\circ} \mathrm{C}$ overnight, to maximize the relation yield/recyclability.

- They were tested to work for at least 4 reactions with the same piece of polymer.

- The TON for the Suzuki coupling was $2 \mathrm{mmol} / \mathrm{cm}^{2}$.

\section{Properties of the Pd modified films: (best results)}

Composition of the films:

o PB20_80A1 contained vinylpyrrolidone (VP, 20\%) and methyl acrylate (A1, 80\%). The crosslinker was ethylene glycol dimethacrylate (EGDMA, 5\%).

o PB80_20A4, contained vinylpyrrolidone (VP, 80\%) and $n$-butyl acrylate (A4, 20\%). The crosslinker was ethylene glycol dimethacrylate (EGDMA, 5\%).

- $\mathrm{PB} 20 \_80 \mathrm{~A} 1$, when in presence of $\mathrm{PdCl}_{2} \cdot 2 \mathrm{NaCl}$ water solution formed a black $\mathrm{Pd}(0)$ layer over its surface; once analysed, it was concluded that they were $\operatorname{Pd}(0)$-NPs. 
- PB80_20A4, when in presence of $\mathrm{PdCl}_{2} \cdot 2 \mathrm{NaCl}$ solution was capable to absorb $\mathrm{Pd}(\mathrm{II})$. These $\mathrm{Pd}$ may be easily reduced to $\operatorname{Pd}(0)-\mathrm{NPs}$, by being in the presence of hydrogen gas for several minutes.

- The aggregation of the Pd-NPs is much higher for Pd@PB20_80A1, although they may be easily transferred to other surfaces.

- The nanoparticles formed were polycristalline nanodendrites with an average diameter of 75 nm.

\section{Properties as catalyst of supported Pd-NPs:}

- They were checked to be an efficient heterogenic catalyst for semihydrogenation reduction of alkynes in green solvents, ${ }^{95}$ such as methanol.

- Dimethylacetylenedicarboxylate (DMAD) was studied as standard for regio- and stereoselective reduction to its $(Z)$ hydrogenated product, dimethyl maleate. It occurred under hydrogen atmosphere $(5 \mathrm{~atm})$ in less than 15 hours, with yields around $95 \%$.

- The recyclability of the product was checked to be of at least 6 times when using Pd@PB80_20A4.

- The leach of palladium to the solution was detected to be inferior to $6 \mu \mathrm{M}$.

- The calculated TON was $75 \mathrm{mmoles} / \mathrm{cm}^{2}$.

- The cis selective reduction was performed successfully to products with pharmaceutical interest. As example 5 products were tested, mifepristone, efavirenz, tazarotene, 8-Bromo7-(2-butyn-1-yl)-3-methylxanthine and pentacosa-10,12-diynoic acid. Obtaining $100 \%$ selectivity in the transformation from alkynes to $(Z)$-alkenes, against alkanes.

- The drugs mifepristone and efavirenz were quantitively reduced. Tazarotene and the xanthine derivative needed for purification; despite this fact, they were obtained in high yields.

In conclusion, the remarkable selectivity and the simplicity in the preparation of the catalysts, with no need for additional ligands, makes the systems competitive to known solid supported metallic nanoparticles catalysts for Suzuki coupling and the semihydrogenation reaction. The polymer supported metallic nanoparticles material can be very useful for heterogeneous catalysis in the synthesis of important drugs, or intermediates for drugs, in a highly sustainable chemistry.

\footnotetext{
${ }^{95}$ C. Capello, U. Fischer, K. Hungerbühler, Green Chem. 2007, 9, 927-934.
} 


\section{RESUMEN DEL CAPÍTULO}

El objetivo de este capítulo consistía en mostrar un nuevo procedimiento para la modificación de superficies con partículas metálicas de oro y paladio; así como ilustrar alguna de las posibles aplicaciones de estos materiales modificados.

Durante el desarrollo de materiales poliméricos modificados para la detección de $\mathrm{Hg}(\mathrm{II})$, (Capítulo 1) se observó que estos polímeros, en contacto con disoluciones acuosas de Au(III), eran capaces de producir espontáneamente nano y micro-partículas de oro (0) unidas a su superficie. Posteriormente, un estudio en profundidad permitió determinar que tanto la forma como el tamaño de estas partículas eran regulables cambiando las condiciones de trabajo. Así, entre otros parámetros, se estudió la dependencia entre el disolvente, la concentración de $\mathrm{Au}(\mathrm{III})$ o la proporción volumen/superficie lo cual permitió la modificación controlada de estos polímeros; posteriormente estudiados por técnicas como absorción o microscopia electrónica.

El siguiente paso fue la búsqueda de una aplicación a estos materiales. De las múltiples posibilidades, la investigación se centró en la utilización de los mismos como catalizadores heterogéneos. Para ello, se eligió la reacción de Suzuki de acoplamiento C-C. Los resultados fueron satisfactorios, obteniendo rendimientos similares a los obtenidos por métodos clásicos, pero con claras ventajas como la posibilidad de reciclar el catalizador.

De igual modo que para la modificación de superficies con oro se planteó la posibilidad de usar otros metales. Entre ellos paladio. Este metal se caracteriza por poseer propiedades que lo hacen de gran utilidad en aplicaciones como catálisis o almacenamiento de hidrógeno. Tras el testeo y la optimización de condiciones con diferentes polímeros se escogieron dos variantes que contenían mezclas de polivinilpirrolidona y un alquil-acrilato. Por un lado, estos polímeros fueron capaces de ser modificados de manera sencilla con nanopartículas de $\operatorname{Pd}(0)$, e incluso con la posibilidad de transferir estas partículas una vez formadas.

En cuanto a la utilidad de los materiales modificados con nanopartículas de $\mathrm{Pd}(0)$, el estudio se centró en sus aplicaciones como catalizadores, en este caso para la semi-hidrogenación selectiva de triples enlaces internos a dobles (Z-alquenos). Esta propiedad, dio resultado para un conjunto de moléculas con distinto interés biológico (precursores de medicamentos y hormonas) presentando a su vez altos rendimientos y siendo un catalizador reutilizable y que funciona en disolventes verdes, principalmente alcoholes. 
FINAL CONCLUSIONS 

As it was explained in the thesis, the objectives and achievements were extensive. In the next part, the conclusions are summarized by chapters:

\section{Chapter 0: Introduction to fluorescent probes.}

- The properties and ideal characteristics of molecular sensors were described. It was highlighted the importance of the specificity, selectivity, reusability, low-cost, fast operation mechanism, cheap maintenance and portability.

- The importance of fluorescent sensors and how they work was explained. From their many characteristics it is worth to remark the importance of having OFF-ON sensors for higher selectivity, as well as how the different types of sensors work (complexation or reaction) and how they may be properly designed (system fluorophore-receptor).

- A protocol was elaborated so as to work with fluorescent probes, for getting repeatable and reliable results. In doing so, different studies give characteristics and parameters that are of great importance:

o Solvatochromism (response to different solvents).

o Tests with different species, cations, anions, oxidative, reductive species, amines...

0 Work concentration and molar extinction coefficients $(\varepsilon)$.

o Kinetic effects.

o Titration methods.

o Thermodynamic equilibrium constant calculation $(\mathrm{K})$.

o Limits of detection (LODs).

o Fluorescence quantum yields $(\Phi)$.

o Fluorescence decay lifetime $(\tau)$.

\section{Chapter 1: Fluorescent probes for detection of $\mathrm{Hg}(\mathrm{II})$ derivatives.}

- The importance of having probes for detection of $\mathrm{Hg}(\mathrm{II})$ derivatives was explained. It was remarked the importance of the organic species, such as $\mathrm{MeHg}(\mathrm{II})$, being a bioaccumulative cation presented in some fish samples and very toxic for living organisms.

- A series of water soluble fluorescent probes, highly selective to $\mathrm{Hg}(\mathrm{II})$ derivatives, were synthetized and characterized.

- Selective detection of $\mathrm{Hg}(\mathrm{II})$ and $\mathrm{MeHg}$ (II) in cellular environments was achieved.

- Polymeric modified materials were created, capable of detecting $\mathrm{Hg}(\mathrm{II})$ presence in water.

o The LOD of the polymer soluble in water, JG32, for $\mathrm{Hg}(\mathrm{II})$ was $1.5 \mathrm{ppm}$ and for $\mathrm{MeHg}(\mathrm{II})$ it was $6.5 \mathrm{ppm}$.

o The film with water affinity, JG25, had a LOD for $\mathrm{Hg}$ (II) of $1.3 \mathrm{ppm}$ and for $\mathrm{MeHg}(\mathrm{II})$ it was $0.3 \mathrm{ppm}$.

- The supported probe JG25 was adapted to work for detection and quantification of $\mathrm{Hg}$ (II) from fish. Concentrations around $1 \mathrm{ppm}$ of mercury cations were detected on dogfish, swordfish and tuna. 


\section{Chapter 2: Perylenemonoimides. Introduction and general properties.}

- The advantages and properties of perylene imide derivatives as fluorescent backbones were explained. It was of particular importance to explain their stacking properties, highly dependent on the different substituents and their position.

- The synthesis for PMI derivatives as starting materials was optimized.

- A comparison between PMIs substituted in different positions was performed in the same solvent (chloroform).

0 The wavelength with the maximum of absorption was around 500-520 nm. Except for the ruthenium complex JG10L (576 nm).

o The molar extinction coefficient was maximized by creating a combination PMIBodipy (JGBod). It makes them a potential candidate for applications in sensing and photoelectronics.

o The fluorescence lifetime decays were between 3.5-5.1 ns.

o The fluorescence stokes shift, when comparing between more and less polar solvents, was maximized in probes such as JG116 and JG125 that were the examples containing a pyrimidine or pyridine group.

- JG119c2 resulted to have applications as cellular biomarker with interaction with Gquadruplex DNA. It was selected for future studies, currently under development.

\section{Chapter 3A. PMI derivatives for detection of explosives.}

TATP is a white powder that could act as explosive in terrorist attacks; because of that and some particular properties that it possesses, such as the difficulty to give a controlled explosion, its detection is of high interest.

- A PMI derivative, JG125, was selected as the fluorescent probe with response to TATP oxidation, by increasing fluorescence.

o The characteristics for detection in solution were measured, and it was determined that it was possible to detect $0.27 \mathrm{mg}$ of TATP with JG125 $(2.5 \mu \mathrm{M})$ in $\mathrm{CHCl}_{3}: \mathrm{MeOH}$ 9:1, in $2.5 \mathrm{~mL}$ solution.

o The detection was selective to other oxidants such as water peroxide, and with the possibility to be distinguished from acid vapours.

- A series of materials, silica derivatives, were modified for the detection of TATP in the gas phase, $0.12 \mathrm{mg}$ was the limit of detection of TATP gas.

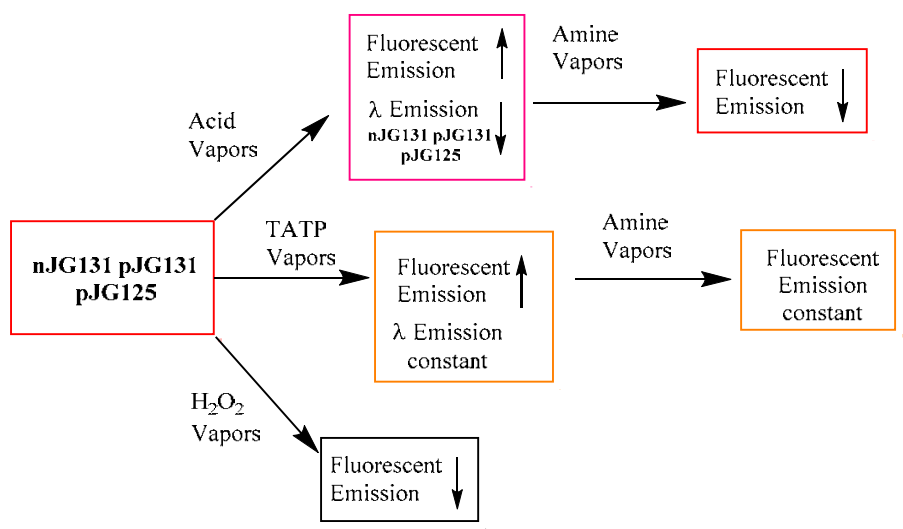

Scheme showing the interpretation of the results and the application of the different probes. 


\section{Chapter 3B. PMI-Ru(II) complexes for detection of CO.}

- The interest and antecedents for using $\mathrm{Ru}(\mathrm{II})$ complexes for $\mathrm{CO}$ sensing were: its toxicity as gas (being a semicombustion residue) and its crucial role in some biological processes.

- $\mathrm{Ru}(\mathrm{II})$ complexes for detection of $\mathrm{CO}$ have high selectivity in comparison to electrochemical methods, being more sensitive and cheaper. In addition, they are less toxic and with better results compared with other metallic complexes, such as Pd.

- It was performed the synthesis of three different complexes Ru(II)-PMI; JG10L, JG11L and JG12L; containing a PEG to increase the final solubility in water.

- $\mathrm{The} \mathrm{Ru}(\mathrm{II})$ complexes were useful for $\mathrm{CO}$ detection, but not soluble in $100 \%$ water media nor in cellular environments.

- It was explained the potential applicability of the complexes not only as $\mathrm{CO}$ detectors but as light sensitive derivatives. This characteristic may be useful for future research in photoelectronics and biological applications.

- JG10L presented selective response to other analytes of interest, such as isonitriles or cyanide.

- The next step would be the creation of derivatives changing the triphenylphosphine groups so as to improve the properties and applications.

\section{Chapter 3C. PMI derivatives for $\mathrm{K}^{+}$and $\mathrm{Pb}^{2+}$ sensing.}

- The importance of detecting K(I) was briefly summarized, being part of most biological processes; as a consequence, it is interesting not only in solution but in cellular environments too.

- Cereulide and similar derivatives from natural potassium ionophores are of great importance. They have direct influence in potassium transport between intra-extracellular media; acting by equilibrating concentrations or by disrupting the equilibria, which leads to the death of the cells.

- $\mathrm{Pb}(\mathrm{II})$ is a cation that is toxic for living organisms. Its presence may come from different sources, the most remarkable are the incorrectly treated tap water samples ("Flint water crisis"). Because of that, it was determined that measuring concentrations of $\mathrm{Pb}$ (II) in coloured water samples (containing solid from corrosion) would be of high interest.

- Two fluorescent PMI probes were synthetized, with selective sensitivity to $\mathrm{K}(\mathrm{I})$ and $\mathrm{Pb}(\mathrm{II})$; a triazacryptand (JG103) and a crown ether (JG76).

- Additionally, a natural ionophore of $\mathrm{K}^{+}$, cereulide, was synthetized in laboratory; along with some derivatives. They were employed to research about some of their possible biological applications and to determine how the properties of these ionophores could be modulated.

- The crown ether probe JG76 resulted to be an efficient probe for detection of $\mathrm{K}(\mathrm{I})$ and $\mathrm{Pb}(\mathrm{II})$ in ethanol solution ( $\mathrm{LOD}=2.3 \mathrm{ppb}$ of $\mathrm{K}(\mathrm{I})$ and $6 \mathrm{ppb}$ of $\mathrm{Pb}(\mathrm{II})$ ) and in cell cultures. Additionally, it was possible to detect the presence of cereulide from contaminated cultures $(\mathrm{LOD}=240 \mathrm{ppb})$.

- The studies of the effect of using several cereulide derivatives had promising results, being capable of tuning the potassium affinity and perform cellular location by fluorescent modification.

- The crown ether probe was modified to be anchored to a polymeric material (JG151dp).

- The modified polymer (JG151dp) was used successfully in detection of $\mathrm{Pb}$ (II) in coloured water samples with no interference from potassium cations. (LOD $=66 \mathrm{ppb})$ 


\section{Chapter 4. Supported gold and palladium nanoparticles for catalysis.}

- The different properties of gold and palladium nanostructured materials were summarized, being a state of the art topic with potential applications in catalysis, biomedicine (drug delivery of cancer therapy), biological and chemical sensors (cellular imaging and biosensors), electronics, environmental remediation and hydrogen storage (for Pd).

- The synthesis of supported gold and palladium particles was optimized to be a one-step synthesis in water over a modified surface.

- The polymer containing hydroxyethylacrylate (2HEA, 95\%) and propargyl methacrylate (PGM, 5\%), ethylene glycol dimethacrylate (EGDMA, 5\%), and its derivative with a triazole (+ JG10), was capable of the spontaneous formation of gold particles in their surface, in the presence of $\mathrm{Au}(\mathrm{III})$ in water. The conditions were optimized.

- For the synthesis of Pd modified polymers two derivatives were optimized and fully characterized; they contained vinylpyrrolidone (VP), an alkyl acrylate (A) and different Pd substitutions depending on their percentages. The most useful for catalysis was called Pd@PB80_20A4, which was covered of homogeneous 75-80 nm Pd-NPs.

- Gold covered polymers were used as an alternative catalyst for Suzuki reactions, getting the same yields in similar conditions (THF:water mixtures). The main advantage was the recyclability of the polymer; up to 4 times. The TON for the Suzuki coupling was 2 $\mathrm{mmol} / \mathrm{cm}^{2}$.

- Palladium covered polymers were used for selective semihydrogenation of triple bonds to obtain the $(Z)$-alkene derivative. The reduction process was tested to be selective against double bonds and halogenated molecules and it was successfully used for reduction of specific drugs with pharmacological interest. They presented high recyclability, up to 6 times, with no significant leaching of palladium to the reaction solvent and a TON of $75 \mathrm{mmol} / \mathrm{cm}^{2}$. 
ANNEX 



\section{REAGENTS AND SOLVENTS}

All materials and solvents were commercially available from different commercial brands; such as Sigma-Aldrich, TCI, Fisher, Merck or Fluorochem. Used as received, unless otherwise indicated. Column chromatography: $\mathrm{SiO}_{2}(40-63 \mu \mathrm{m})$ TLC plates coated with $\mathrm{SiO}_{2} 60 \mathrm{~F} 254$ were visualized by UV light. The solvents for spectroscopic studies were of spectroscopic grade and used as received. The silica nanoparticles used for modification were 10-20 nm particle size (Sigma Aldrich), the TLC plates for modification were acquired free of coating, with the same particle size $(40-63 \mu \mathrm{m})$.

\section{APPARATUS}

Circular dichroism (CD): the spectra were recorded on a MOS-450 Bio-Logic dichrograph (Claix, France). The measurements were performed in $1.0 \mathrm{~cm}$ path-length cells at $25^{\circ} \mathrm{C}$.

Confocal microscopy: The images were obtained using a NIKON A1R scanning confocal laser microscope equipped with TIRF module, laser lines $405 \mathrm{~nm}, 457 \mathrm{~nm}, 477 \mathrm{~nm}, 488 \mathrm{~nm}, 514 \mathrm{~nm}, 561$ $\mathrm{nm}, 638 \mathrm{~nm}$ microscope. Images were processed with the NIS-Elements Advanced Research software and J-image.

Elemental analysis of $\mathrm{C}, \mathrm{H}$ and $\mathrm{N}$ were taken in a Leco CHNS 932.

Fluorescence spectra were recorded with a F-7000 Hitachi Fluorescence fluorometer and/or FLS980 Series, Edinburgh instruments. The dissolved samples were measured with a in $1 \mathrm{~cm}$ path length quartz UV cells, at $25^{\circ} \mathrm{C}$.

Fluorescence decay lifetimes were measured using a time-correlated single photon counting instrument (FLS980 Series, Edinburgh instruments) with a pulsed LED (Edinburgh instruments, EPL) light source having between 100-200 ps. Fluorescence quantum yields were measured by coupling the system with an integration sphere from Edinburgh instruments.

ICP measurements were performed with a model ICP-MS Agilent Series 7500, after a calibration with the corresponding metal standard.

Infrared spectra (FT-IR) were recorded with a JASCO FT/IR-4200. Transmission spectra were collected from pressed $\mathrm{KBr}$ pellets. The spectrum has been yielded at a resolution of $4 \mathrm{~cm}^{-1}$.

Mass Spectra and High resolution Mass Spectra (MS and HRMS): were obtained from a Bruker Autoflex matrix-assisted laser desorption/ionization time of flight (MALDI-TOF) using dithranol (DIT) or trans-2-[3-(4-tert-butylphenyl)-2-methyl-2-propenylidene]malononitrile (DCTB) as matrix. Time of Flight Mass Spectrometry (MS-TOF) was performed on a Bruker Maxis Impact coupled to an ultraperformance liquid chromatography device Waters Acquity (UPLC-MS-TOF).

Thermogravimetric analysis (TGA) data were recorded using a 4-6 mg sample under a nitrogen atmosphere on a TA Instrument Q50 TGA analyser at a scan rate of $10^{\circ} \mathrm{C} \mathrm{min}^{-1}$.

Scanning electron microscopy (SEM) images were obtained from the gold-sputtered membranes using a JEOL JSM-6460LV instrument. In addition, the elemental composition of the surface was measured with an Oxford Instruments INCA EDS (Energy-dispersive X-ray spectroscopy). Images were processed with the NIS-Elements Advanced Research software and J-image. 
Transmission electron microscopy (TEM) were performed by dipping a holey carbon TEM grid into a colloidal suspension of the MWCNTs. Samples were imaged using two models: a) TEM JEOL JEM-FS2200 HRP) using Gatan Ultrascan camera of $2 \mathrm{k} \times 2$ with a range of accelerating voltage from 80 to $200 \mathrm{kV}$ (UVA University) and b) TEM JEOL- model JEM 1011, images were collected using a Gatan digital camera of high resolution (IDIVAL institute); by the other hand the optical microscopy is a Nikon A1R confocal microscope and an Nikon Ti epifluorescence microscope designed to make time lapse. Images were processed with J-image.

NMR spectra were recorded in a Varian Mercury-300 and/or Varian Unity Inova-400. Chemical shifts are reported in ppm with respect to residual solvent protons, coupling constants $(\mathrm{J})$ are reported in $\mathrm{Hz}$.

Confocal Raman Microscope-Atomic Force Microscope (AFM) model Alpha300R - Alpha300A AFM Witec system with a power of $0.5 \mathrm{~mW} 532 \mathrm{~nm}$ He-Ne laser and magnification 10X (NA 0.25) and $785 \mathrm{~nm}$ Ti:Sapphire laser.

Ultrasonic bath Elma D-78224, singen/htw.

Ultraviolet-visible (UV-Vis) absorption spectra were registered using a Hitachi U-3900. The samples were measured within a quartz cells of $1 \mathrm{~cm}$ path legth, at $25^{\circ} \mathrm{C}$ controlled temperature.

\section{MATERIAL AND SAMPLES FOR SENSORING STUDIES}

\section{a) Qualitative tests with anions and cations:}

The cations and anions used to characterise the behaviour of a novel probe as sensor are need for having a counterion that not affect the measurements directly. In doing so the specific cations, anions and their corresponding counterions were:

\begin{tabular}{|c|c|c|c|c|c|}
\hline \multicolumn{2}{|r|}{ Cations } & \multicolumn{2}{|r|}{ Cations } & \multicolumn{2}{|r|}{ Anions } \\
\hline $\mathrm{Ag}^{+}$ & $\mathrm{AgClO}_{4} \times \mathrm{xH}_{2} \mathrm{O}$ & $\mathrm{Co}^{2+}$ & $\mathrm{CoCl}_{2} \times 6 \mathrm{H}_{2} \mathrm{O}$ & $\mathrm{F}^{-}$ & {$[t-\mathrm{Bu}]_{4} \mathrm{NF}$} \\
\hline $\mathrm{Ni}^{2+}$ & $\mathrm{Ni}\left(\mathrm{ClO}_{4}\right)_{2} \times 6 \mathrm{H}_{2} \mathrm{O}$ & $\mathrm{Ir}^{3+}$ & $\mathrm{IrCl}_{3} \times \mathrm{xH}_{2} \mathrm{O}$ & $\mathrm{Cl}^{-}$ & {$[t-\mathrm{Bu}]_{4} \mathrm{NCl}$} \\
\hline $\mathrm{Sn}^{2+}$ & $\mathrm{Sn}\left(\mathrm{CF}_{3} \mathrm{SO}_{3}\right)_{2}$ & $\mathrm{Cu}^{+}$ & $\mathrm{Cu}\left(\mathrm{NCCH}_{3}\right)_{4} \times \mathrm{CF}_{3} \mathrm{SO}_{3}$ & $\mathrm{Br}^{-}$ & {$[t-\mathrm{Bu}]_{4} \mathrm{NBr}$} \\
\hline $\mathrm{Cd}^{2+}$ & $\mathrm{Cd}\left(\mathrm{ClO}_{4}\right)_{2}$ & $\mathrm{Li}^{+}$ & $\mathrm{LiClO}_{4}$ & $\mathrm{I}^{-}$ & {$[t-\mathrm{Bu}]_{4} \mathrm{NI}$} \\
\hline $\mathrm{Zn}^{2+}$ & $\mathrm{Zn}\left(\mathrm{CF}_{3} \mathrm{SO}_{3}\right)_{2}$ & $\mathrm{Na}^{+}$ & $\mathrm{NaClO}_{4}$ & $\mathrm{BzO}^{-}$ & {$[t-\mathrm{Bu}]_{4} \mathrm{~N}\left(\mathrm{OCOC}_{6} \mathrm{H}_{5}\right)$} \\
\hline $\mathrm{Pb}^{2+}$ & $\mathrm{Pb}\left(\mathrm{ClO}_{4}\right)_{2}$ & $\mathrm{~K}^{+}$ & $\mathrm{K}\left(\mathrm{CF}_{3} \mathrm{SO}_{3}\right)$ & $\mathrm{NO}_{3}^{-}$ & {$[t-\mathrm{Bu}]_{4} \mathrm{~N}\left(\mathrm{NO}_{3}\right)$} \\
\hline $\mathrm{Cu}^{2+}$ & $\mathrm{Cu}\left(\mathrm{ClO}_{4}\right)_{2} \times 6 \mathrm{H}_{2} \mathrm{O}$ & $\mathrm{Rb}^{+}$ & $\mathrm{RbCl}$ & $\mathrm{H}_{2} \mathrm{PO}_{4}^{-}$ & {$[t-\mathrm{Bu}]_{4} \mathrm{~N}\left(\mathrm{H}_{2} \mathrm{PO}_{4}\right)$} \\
\hline $\mathrm{Fe}^{3+}$ & $\mathrm{Fe}\left(\mathrm{ClO}_{4}\right)_{3} \times \mathrm{xH}_{2} \mathrm{O}$ & $\mathrm{Cs}^{+}$ & $\mathrm{CsClO}_{4}$ & $\mathrm{HSO}_{4}^{-}$ & {$[t-\mathrm{Bu}]_{4} \mathrm{~N}\left(\mathrm{HSO}_{4}\right)$} \\
\hline $\mathrm{Sc}^{3+}$ & $\mathrm{Sc}\left(\mathrm{CF}_{3} \mathrm{SO}_{3}\right)_{3}$ & $\mathrm{Be}^{2+}$ & $\mathrm{Be}\left(\mathrm{SO}_{4}\right)$ & $\mathrm{AcO}^{-}$ & {$[t-\mathrm{Bu}]_{4} \mathrm{~N}(\mathrm{OAc})$} \\
\hline $\mathrm{Al}^{3+}$ & $\mathrm{Al}\left(\mathrm{ClO}_{4}\right)_{3} \times 9 \mathrm{H}_{2} \mathrm{O}$ & $\mathrm{Mg}^{2+}$ & $\mathrm{Mg}\left(\mathrm{ClO}_{4}\right)_{2}$ & $\mathrm{CN}^{-}$ & {$[t-\mathrm{Bu}]_{4} \mathrm{~N}(\mathrm{CN})$} \\
\hline $\mathrm{Hg}^{2+}$ & $\mathrm{Hg}\left(\mathrm{ClO}_{4}\right)_{2} \times \mathrm{xH}_{2} \mathrm{O}$ & $\mathrm{Ca}^{2+}$ & $\mathrm{Ca}\left(\mathrm{ClO}_{4}\right)_{2}$ & $\mathrm{SCN}^{-}$ & {$[t-\mathrm{Bu}]_{4} \mathrm{~N}(\mathrm{SCN})$} \\
\hline $\mathrm{Au}^{3+}$ & $\mathrm{HAuCl}_{4} \times 3 \mathrm{H}_{2} \mathrm{O}$ & $\mathrm{Sr}^{2+}$ & $\mathrm{SrCl}_{2}$ & & \\
\hline $\mathrm{MeHg}^{+}$ & $\mathrm{MeHgCl}$ & $\mathrm{Ba}^{2+}$ & $\mathrm{Ba}\left(\mathrm{ClO}_{4}\right)_{2}$ & & \\
\hline $\mathrm{Pd}^{2+}$ & $\mathrm{PdCl}_{2} \times 2 \mathrm{NaCl}$ & $\mathrm{NH}_{4}^{+}$ & $\mathrm{NH}_{4} \mathrm{Cl}$ & & \\
\hline
\end{tabular}

Table 1. Salts employed during sensing measurements. 
Pictures were taken with a digital camera Canon EOS M3 with a macro of $0.15 \mathrm{~m}$; under white light $(230-240 \mathrm{~V} ; 50-60 \mathrm{~Hz})$ and UV light $(366 \mathrm{~nm})$. Then, the corresponding concentrations and solutions were prepared as it was detailed in Chapter 0, otherwise it was specified.

b) For general volumetric measurements,

Micropipettes Eppendorf Research Plus were used. The technical data are showed in the table below:

\begin{tabular}{|c|c|c|c|c|}
\hline Model & Ep T.I.P.S. & Volume & Systematic error & Random error \\
\hline $2-20 \mu \mathrm{L}$ & $2-200$ & $2 \mu \mathrm{L}$ & $\pm 5.0 \%$ & $\pm 1.5 \%$ \\
& & $10 \mu \mathrm{L}$ & $\pm 1.2 \%$ & $\pm 0.6 \%$ \\
& & $20 \mu \mathrm{L}$ & $\pm 1.0 \%$ & $\pm 0.3 \%$ \\
\hline $10-100 \mu \mathrm{L}$ & $2-200$ & $10 \mu \mathrm{L}$ & $\pm 3.0 \%$ & $\pm 1.0 \%$ \\
& & $50 \mu \mathrm{L}$ & $\pm 1.0 \%$ & $\pm 0.3 \%$ \\
& & $100 \mu \mathrm{L}$ & $\pm 0.8 \%$ & $\pm 0.2 \%$ \\
\hline $100-1000 \mu \mathrm{L}$ & $50-1000$ & $100 \mu \mathrm{L}$ & $\pm 3.0 \%$ & $\pm 0.6 \%$ \\
& & $500 \mu \mathrm{L}$ & $\pm 1.0 \%$ & $\pm 0.2 \%$ \\
& & $1000 \mu \mathrm{L}$ & $\pm 0.6 \%$ & $\pm 0.2 \%$ \\
\hline $500-5000 \mu \mathrm{L}$ & $100-5000$ & $500 \mu \mathrm{L}$ & $\pm 2.4 \%$ & $\pm 0.6 \%$ \\
& & $2500 \mu \mathrm{L}$ & $\pm 1.2 \%$ & $\pm 0.25 \%$ \\
& & $5000 \mu \mathrm{L}$ & $\pm 0.6 \%$ & $\pm 0.15 \%$ \\
\hline
\end{tabular}

Table 2. Technical data of Eppendorf micropipettes employed.

\section{c) Measurements in the absorbance-fluorescence cuvettes:}

The cuvettes, used for the measurements of absorbance - fluorescence, were always for volumes between 2.5-3 mL. When the sample was a film, in order to follow increments in the same sample, the film was put between two magnets and introduced into the solution within the cuvette. Next picture shows a sample prepared this way.
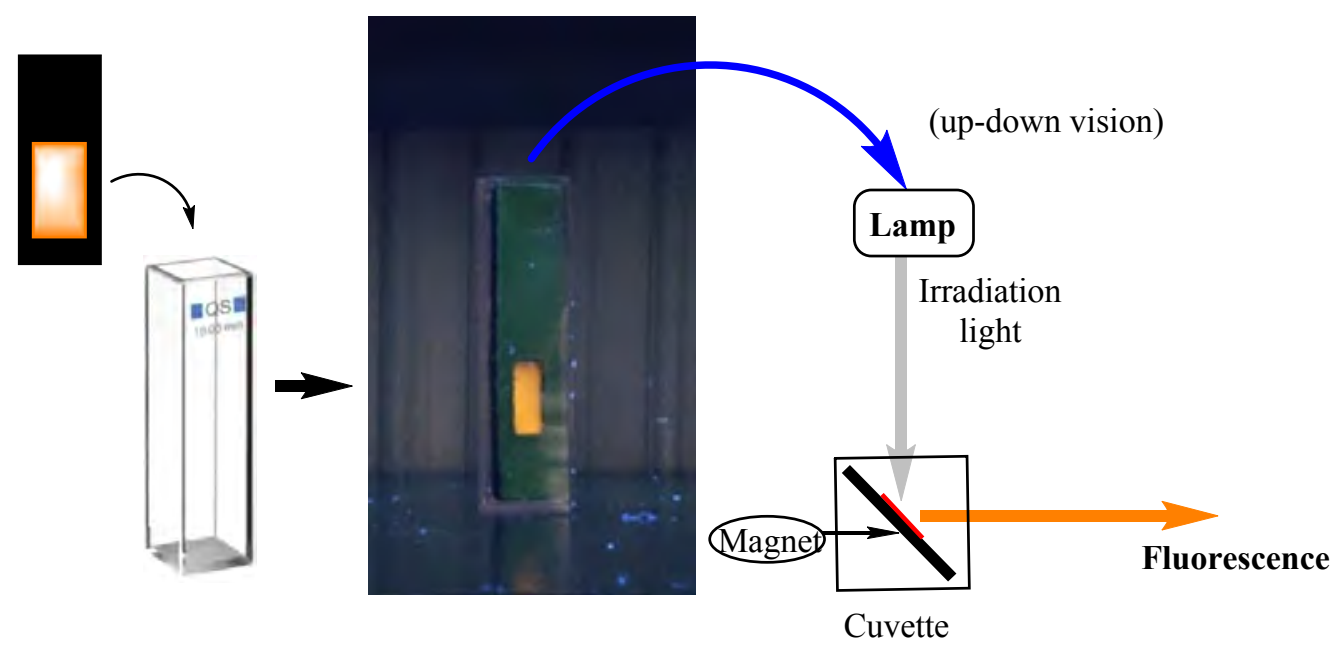

Figure 1. Representative figure for film sample measurements. 


\section{PUBLICATIONS}

1. Papers directly related to the thesis (7):

Palladium nanodendrites uniformly deposited on the surface of polymers as an efficient and recyclable catalyst for direct drug modification via $Z$-selective semihydrogenation of alkynes

J. García-Calvo, P. Calvo-Gredilla, S. Vallejos, J.-M. García, J. V. Cuevas, G. García-Herbosa, M. Avella, T. Torroba.

Green Chemistry, 2018, 20, 3875-3883.

Surface Functionalized Silica Nanoparticles for the Off-On Fluorogenic Detection of an Improvised Explosive, TATP, in a Vapour Flow

J. García-Calvo, P. Calvo-Gredilla, M. Ibáñez-Llorente, D. C. Romero, J. V. Cuevas, G. GarcíaHerbosa, M. Avella and T. Torroba.

Journal of Materials Chemistry A, 2018, 6, 4416-4423.

Solvent-Free Off-On Detection of the Improvised Explosive Triacetone Triperoxide TATP with Fluorogenic Materials

P. Calvo-Gredilla, J. García-Calvo, J. V. Cuevas, T. Torroba, J.-L. Pablos, F. C. García, J.-M. García, N. Zink-Lorre, E. Font-Sanchis, Á. Sastre-Santos, F. Fernández-Lázaro.

Chemistry, A European Journal, 2017, 23, 13973-13979.

Potassium-Ion-Selective Fluorescent Sensors To Detect Cereulide, the Emetic Toxin of B. cereus, in Food Samples and HeLa Cells

J. García-Calvo, S. Ibeas, E. C. Antón-García, T. Torroba, G. González-Aguilar, W. Antunes, E. González-Lavado and M. L. Fanarraga.

ChemistryOpen 2017, 12, 562-570.

A smart material for the in situ detection of mercury in fish

J. García-Calvo, S. Vallejos, F. C. García, J. Rojo, J. M. García and T. Torroba

Chemical Communications 2016, 52, 11915-11918.

Surface Coating by Gold Nanoparticles on Functional Polymers: On-Demand Portable Catalysts for Suzuki Reactions

J. García-Calvo, V. García-Calvo, S. Vallejos, F. C. García, M. Avella, J.-M. García and T. Torroba. ACS Applield Materials and Interfaces, 2016, 8, 24999-25004. 
Chemical Speciation of MeHg+ and $\mathrm{Hg} 2+$ in Water Solutions and HEK Cells Nuclei by means of DNA Interacting Fluorogenic Probes

B. Díaz de Greñu, J. García-Calvo, J. Cuevas, G. García-Herbosa, B. García, N. Busto, S. Ibeas, t. Torroba, B. Torroba, A. Herrera and S. Pons.

Chemical Science 2015, 6, 3757-3764.

\section{Other papers (2):}

Detection of Contaminants of High Environmental Impact by Means of Fluorogenic Probes

J. García-Calvo, P. Calvo-Gredilla, M. Ibáñez-Llorente, T. Rodríguez, T. Torroba.

The Chemical Record 2016, 16, 810-824.

Structural Conformers of (1,3-Dithiol-2-ylidene)ethanethioamides: The Balance Between Thioamide Rotation and Preservation of Classical Sulfur-Sulfur Hypervalent Bonds.

P. Fuertes, M. García-Valverde, R. Pascual, T. Rodríguez, J. Rojo, J. García-Calvo, P. Calvo, J. V. Cuevas, G. García-Herbosa and T. Torroba.

Journal of Organic Chemistry, 2015, 80, 30-39.

\section{Patents (3):}

Dispositivo para determinación fluorimétrica de aminas terciarias

José García Calvo; Víctor García Calvo; José Vicente Cuevas Vicario; Gabriel García Herbosa; Tomás Torroba Pérez

Universidad de Burgos; P201730530, España, Castilla y León

Register: 31/03/2017

Obtención de nano-partículas metálicas mediante uso de copolímeros en forma de membrana

José García Calvo; Patricia Calvo Gredilla; Saúl Vallejos Calzada; Felipe José Serna Arenas; Jose Miguel García Pérez; Manuel Pedro Avella Moreno; Tomás Torroba Pérez

Universidad de Burgos; P201730551, España, Castilla y León

Register: 31/03/2017

1-(Piperazin-N-Ilaril)-y

1,7-Di(Piperazin-N-Ilaril)Perileno-3,4:9,10-Tetracarboxidiimidas, procedimiento de preparación de las mismas y su uso como detectores de explosivos oxidantes. 
José García Calvo; Patricia Calvo Gredilla; Tomás Torroba Pérez; Nathalie Zink Lorre; Ángela Sastre Santos; Fernando Fernández Lázaro; Enrique Font Sanchís

PCT/ES2016/0709; España.

Register: 23/12/2015

\section{Oral communications (12; 6 different symposium):}

Versatility of polysubstituted PDI: Synthesis, optical properties and biological applications.

Alberto Diez-Varga, Tomás Torroba, Patricia Calvo-Gredilla, Daisy Romero-Velásquez, José GarcíaCalvo, Mónica L. Fanarraga, Eloísa G. Lavado.

XXVII Biennial Meeting in Organic Chemistry.

Santiago de Compostela, Galicia, España; 20/06/2018 - 22/06/2018.

A smart material for detection of mercury in fish.

José García Calvo; Patricia Calvo Gredilla; Víctor García Calvo; Jose Miguel García Pérez; Saúl Vallejos Calzada; Félix Clemente García; Maria José Rojo Cámara; Maria Teresa Rodríguez; Alberto Díez de la Varga; Daisy Carolina Romero Velásquez; Eva Clara Antón García; Marcos Ibáñez Llorente; Tomás Torroba Pérez.

XXXVI Reunión bienal de la sociedad española de química.

Sitges, Cataluña, España; 25/06/2017 - 29/06/2017.

Fluorescence Quenching of Perylenediimides by Absorption with Graphene Oxide.

Patricia Calvo Gredilla; José García Calvo; Daisy Carolina Romero Velásquez; Alberto Díez de la Varga; Eva Clara Antón García; Marcos Ibáñez Llorente; Víctor García Calvo; Tomás Torroba Pérez.

XXXVI Reunión bienal de la sociedad española de química.

Sitges, Cataluña, España; 25/06/2017 - 29/06/2017.

Surface coating by gold nanoparticles on functional polymers.

José García Calvo; Víctor García Calvo; Jose Miguel García Pérez; Saúl Vallejos Calzada; Félix Clemente García; Tomás Torroba Pérez.

XXXVI Reunión bienal de la sociedad española de química.

Sitges, Cataluña, España; 25/06/2017 - 29/06/2017.

Development of new sensors for process of fluorogenic recognition of cations and oxidizers. 
Eva Clara Antón García; Marcos Ibáñez Llorente; Daisy Carolina Romero Velásquez; José García Calvo; Patricia Calvo Gredilla; Tomás Torroba Pérez.

XIII Simposio de investigadores jóvenes de la RSEQ, ISBN 978-84-617-6244-6.

Logroño, La Rioja, España; 08/11/2016 - 11/11/2016.

Development of selective fluorogenic sensors based on perylenemonoimides.

Marcos Ibáñez Llorente; Eva Clara Antón García; Daisy Carolina Romero Velásquez; José García Calvo; Patricia Calvo Gredilla; Tomás Torroba Pérez.

XIII Simposio de investigadores jóvenes de la RSEQ, ISBN 978-84-617-6244-6.

Logroño, La Rioja, España; 08/11/2016 - 11/11/2016.

Fluorogenic polymers for mercury detection in fish samples.

José García Calvo; Patricia Calvo Gredilla; Víctor García Calvo; Jose Miguel García Pérez; Saúl Vallejos Calzada; Félix Clemente García; Maria José Rojo Cámara; Maria Teresa Rodríguez; Tomás Torroba Pérez.

XVI Reunión del grupo especializado de polímeros (GEP) de la RSEQ y RSEF.

Burgos, Castilla y León, España, 05/09/2016 - 08/09/2016.

Targeting G-quadruplex DNA with fluorescent perylene derivatives.

Natalia Busto; Patricia Calvo Gredilla; José García Calvo; Aurore Guédin; Jose María Leal Villalba; Tomás Torroba Pérez; Jean Louis Mergni; Begoña García.

International Symposia on Metal Complexes 2016, ISSN 2239-2459.

Barcelona, Cataluña, España; 07/06/2016 - 10/06/2016.

A new selective fluorogenic probe turn-on for cyanide.

Patricia Calvo Gredilla; Borja Díaz de Greñu Puertas; José García Calvo; Tomás Torroba Pérez.

XXV Reunión Bienal de Química Orgánica.

Alicante, Comunidad Valenciana, España; 04/06/2014 - 06/06/2014.

Fluorogenic materials for the environmental detection of neurotoxic compounds.

José García Calvo; Borja Díaz de Greñu Puertas; Patricia Calvo Gredilla; José Miguel García; Saúl Vallejos; Félix Clemente García; Miriam Trigo; Tomás Torroba Pérez.

XXV Reunión Bienal de Química Orgánica.

Alicante, Comunidad Valenciana, España; 04/06/2014 - 06/06/2014. 
New fluorogenic OFF-ON probes for the quantification and discrimination of $\mathrm{Hg}(\mathrm{II})$ and $\mathrm{MeHg}(\mathrm{II})$ in aqueous solution.

Tomás Torroba Pérez; Borja Díaz de Greñu Puertas; Jose Vicente Cuevas Vicario; Patricia Calvo Gredilla; José García Calvo; Sebastian Pons; Blanca Torroba.

International Symposium on Metal Complexes; ISSN 2239-2459.

Burgos, Castilla y León, España; 16/06/2013 - 20/06/2013.

Materiales fluorogénicos para la detección medioambiental de compuestos neurotóxicos.

Tomás Torroba Pérez; José García Calvo; José Miguel García; Saúl Vallejos Calzada.

XIV Escuela Nacional de Materiales Moleculares.

Ciudad Real, Castilla-La Mancha, España; 03/02/2013 - 07/02/2013.

\section{Poster presentations $(15,6$ different symposium):}

Perylenediimides: development of new fluorogenic sensors.

Daisy Carolina Romero Velásquez; Patricia Calvo Gredilla; José García Calvo; Víctor García Calvo; Alberto, Díez de la Varga; Eva Clara Antón García; Marcos Ibáñez Llorente; Tomás Torroba Pérez.

XXXVI Reunión bienal de la sociedad española de química.

Sitges, Cataluña, España; 25/06/2017 - 29/06/2017.

A new fluorogenic probe for the detection of biogenic amines in food.

María José Rojo Cámara; Franchesca Ovalle; Maria Teresa Rodríguez; Borja Díaz de Greñu Puertas; José García Calvo; Patricia Calvo Gredilla; Tomás Torroba Pérez.

XXXV Bienal RSEQ; ISBN 978-84-606-9786-2.

A Coruña, Galicia, España; 19/07/2015 - 23/07/2015.

Fluorogenic materials for the detection of mercury species.

Maria Teresa Rodríguez; María José Rojo Cámara; Borja Díaz de Greñu Puertas; José García Calvo; Patricia Calvo Gredilla; José Miguel García; Saúl Vallejos Calzada; Félix Clemente García; Miriam Trigo; Tomás Torroba Pérez.

XXXV Bienal RSEQ; ISBN 978-84-606-9786-2.

A Coruña, Galicia, España; 19/07/2015 - 23/07/2015. 
Modulating the Fluorescence of Bent Perylenediimides by Adsorption on Graphene Oxide.

Patricia Calvo Gredilla; José García Calvo; Tomás Torroba Pérez; Daisy Carolina Romero Velásquez. 16th International Symposium on Novel Aromatic Compounds; ISBN 978-84-606-9566-0.

Madrid, Comunidad de Madrid, España; 05/07/2015 - 10/07/2015.

Modulating the Fluorescent Solvatochromism of Perylenediimides by Suzuki Reactions.

José García Calvo; Patricia Calvo Gredilla; Tomás Torroba Pérez.

16th International Symposium on Novel Aromatic Compounds; ISBN 978-84-606-9566-0

Madrid, Comunidad de Madrid, España; 05/07/2015 - 10/07/2015.

Valinomycin-perylenediimide nanocomposites as potassium.

Tomás Torroba Pérez; José García Calvo; Patricia Calvo Gredilla; Gerardo González Aguilar.

16th International Symposium on Novel Aromatic Compounds; ISBN 978-84-606-9566-0

Madrid, Comunidad de Madrid, España; 05/07/2015 - 10/07/2015.

A New Selective Turn-on Fluorogenic Probe for Cyanide.

Patricia Calvo Gredilla; Borja Díaz de Greñu Puertas; José García Calvo; Tomás Torroba Pérez.

X Simposio de Investigadores Jóvenes.

Madrid, Comunidad de Madrid, España; 06/11/2013 - 09/11/2013.

Fluorogenic materials for the environmental detection of neurotoxic mercury derivatives.

José García Calvo; Borja Díaz de Greñu Puertas; Patricia Calvo Gredilla; José Miguel García; Saúl Vallejos; Félix Clemente García; Miriam Trigo; Tomás Torroba Pérez.

X Simposio de Investigadores Jóvenes.

Madrid, Comunidad de Madrid, España; 06/11/2013 - 09/11/2013

Estudio de una nueva sonda fluorogénica OFF-ON selectiva de $\mathrm{Hg}(\mathrm{II})$ en medio acuoso.

Patricia Calvo Gredilla; Jose Vicente Cuevas Vicario; Borja Díaz de Greñu Puertas; José García Calvo; Tomás Torroba Pérez.

VIII Reunión Científica de Bioinorgánica; BU-187-2013.

Burgos, Castilla y León, España; 07/07/2013 - 10/07/2013.

Materiales fluorogénicos para la detección medioambiental de compuestos neurotóxicos. 
José García Calvo; Borja Díaz de Greñu Puertas; Patricia Calvo Gredilla; José Miguel García; Saúl Vallejos Calzada; Félix Clemente García; Miriam Trigo; Tomás Torroba Pérez.

VIII Reunión Científica de Bioinorgánica; BU-187-2013.

Burgos, Castilla y León, España; 07/07/2013 - 10/07/2013.

Nuevas sondas fluorogénicas OFF-ON para la cuantificación y discriminación de $\mathrm{Hg}$ (II) y $\mathrm{MeHg}(\mathrm{II})$ en medio acuoso.

Borja Díaz de Greñu Puertas; Patricia Calvo Gredilla; José García Calvo; Sebastián Pons; Blanca Torroba; Tomás Torroba Pérez.

VIII Reunión Científica de Bioinorgánica; BU-187-2013.

Burgos, Castilla y León, España; 07/07/2013 - 10/07/2013.

Una nueva sonda fluorogénica OFF-ON para la detección selectiva de $\mathrm{Cu}(\mathrm{II})$.

Tomás Torroba Pérez; Borja Díaz de Greñu Puertas; Patricia Calvo Gredilla; José García Calvo; Maria José Rojo; Teresa Gómez; Teresa Rodríguez.

VIII Reunión Científica de Bioinorgánica; BU-187-2013.

Burgos, Castilla y León, España; 07/07/2013 - 10/07/2013.

A New Selective Chromogenic and Turn-on Fluorogenic Probe for Copper(II).

Patricia Calvo Gredilla; Borja Díaz de Greñu Puertas; José García Calvo; Tomás Torroba Pérez.

International Symposium on Metal Complexes; ISSN 2239-2459.

Burgos, Castilla y León, España; 16/06/2013 - 20/06/2013.

A novel OFF-ON selective fluorogenic probe for $\mathrm{Hg}(\mathrm{II})$ in aqueous media.

Borja Díaz de Greñu Puertas; Jose Vicente Cuevas Vicario; Patricia Calvo Gredilla; José García Calvo; Tomás Torroba Pérez.

International Symposium on Metal Complexes; ISSN 2239-2459.

Burgos, Castilla y León, España; 16/06/2013 - 20/06/2013.

Fluorogenic materials for the environmental detection of neurotoxic compounds.

José García Calvo; Borja Díaz de Greñu Puertas; Patricia Calvo Gredilla; José Miguel García; Saúl Vallejos; Félix Clemente García; Miriam Trigo; Tomás Torroba Pérez.

International Symposium on Metal Complexes; ISSN 2239-2459.

Burgos, Castilla y León, España; 16/06/2013 - 20/06/2013. 